\title{
Low energy effective field theory operator basis at
} $d \leq 9$

\author{
Hao-Lin Li, ${ }^{a}$ Zhe Ren, ${ }^{a, b}$ Ming-Lei Xiao, ${ }^{a}$ Jiang-Hao Yu ${ }^{a, b, c, d, e}$ and Yu-Hui Zheng ${ }^{a, b}$ \\ ${ }^{a}$ CAS Key Laboratory of Theoretical Physics, Institute of Theoretical Physics, \\ Chinese Academy of Sciences, \\ Beijing 100190, P.R. China \\ ${ }^{b}$ School of Physical Sciences, University of Chinese Academy of Sciences, \\ Beijing 100049, P.R. China \\ ${ }^{c}$ School of Fundamental Physics and Mathematical Sciences, \\ Hangzhou Institute for Advanced Study, University of Chinese Academy of Sciences, \\ Hangzhou 310024, P.R. China \\ ${ }^{d}$ International Centre for Theoretical Physics Asia-Pacific, \\ Beijing/Hangzhou, P.R. China \\ ${ }^{e}$ Center for High Energy Physics, Peking University, \\ Beijing 100871, P.R. China \\ E-mail: lihaolin@itp.ac.cn, renzhe@itp.ac.cn, mingleix@itp.ac.cn, \\ jhyu@itp.ac.cn, zhengyuhui@itp.ac.cn
}

ABstract: We obtain the complete operator bases at mass dimensions 5, 6, 7, 8, 9 for the low energy effective field theory (LEFT), which parametrize various physics effects between the QCD scale and the electroweak scale. The independence of the operator basis regarding the equation of motion, integration by parts and flavor relations, is guaranteed by our algorithm [1,2], whose validity for the LEFT with massive fermions involved is proved by a generalization of the amplitude-operator correspondence. At dimension 8 and 9 , we list the 35058 (756) and 704584 (3686) operators for three (one) generations of fermions categorized by their baryon and lepton number violations $(\Delta B, \Delta L)$, as these operators are of most phenomenological relevance.

Keywords: Beyond Standard Model, Effective Field Theories

ArXIV EPRINT: 2012.09188 


\section{Contents}

1 Introduction 1

2 General framework 3

2.1 Amplitude basis including massive fermions 3

2.2 Amplitude-operator correspondence including massive fermions 5

2.3 The operator basis 8

3 Lists of operators in LEFT $\quad 11$

$\begin{array}{ll}3.1 \text { Lists of the dim-5 operators } & 14\end{array}$

$\begin{array}{lll}3.2 & \text { Lists of the dim- } 6 \text { operators } & 14\end{array}$

3.3 Lists of the dim-7 operators 20

3.4 Lists of the dim-8 operators 24

3.4.1 Classes involving bosons only 24

3.4.2 Classes involving two-fermions 24

3.4.3 Classes involving four-fermions 26

3.5 Lists of the dim-9 operators 46

3.5.1 Classes involving two-fermions 46

3.5.2 Classes involving four-fermions $\quad 52$

3.5.3 Classes involving six-fermions: $\psi^{6} \quad 92$

$\begin{array}{lr}\text { 3.5.4 Classes involving six-fermions: } \psi^{4} \psi^{\dagger 2} & 109\end{array}$

$\begin{array}{llr}4 \text { Conclusion } & 157\end{array}$

\section{Introduction}

The standard model (SM) has been proved to be a success according to current experiment data at the LHC, yet several unsolved basic problems about our universe motivate both theorists and experimentalists to search for new physics beyond the SM, which have yielded a null result up to $\Lambda_{\mathrm{NP}} \sim \mathrm{TeV}$ scale. Under the circumstances, the effective field theory (EFT) provides a model-independent way to parameterize new physics above $\Lambda_{\mathrm{NP}}$ using interactions involving higher dimensional operators at energy scale $<\Lambda_{\mathrm{NP}}$.

While the physics above the electroweak scale $\Lambda_{\mathrm{EW}}$ is described by the standard model effective field theory (SMEFT) [1-7], we also need the low-energy effective field theory (LEFT) below $\Lambda_{\mathrm{EW}}$, which has gauge symmetry $\mathrm{SU}(3)_{C} \times \mathrm{U}(1)_{\mathrm{EM}}$ and all the SM fermions but the top quark. Part of the LEFT operators can be derived from the SM by integrating out the massive vectors $W^{ \pm}, Z$, the top quark $t$ and the Higgs boson $h$. For example, the historical Fermi interactions generated by integrating out the $W^{ \pm}$boson is essentially a part of the LEFT operators at the dimension- 6 level, and had been applied to various processes 
like the $\beta$ decay, the muon decay, and the lepton flavor violation [8-11]. Furthermore, flavor physics such as $B$-physics and $K$-physics utilizes these LEFT operators to parameterize new physics contributions in the $B$ and $K$ meson decay processes, e.g. [12, 13]. However, generic LEFT operators are only subject to Lorentz invariance and gauge symmetry, which is far more rich than those derived in the SM.

The low energy probes of new physics, complementary to collider searches for new physics, have been gradually gaining importance. Various low energy processes, such as neutrino-less double beta decay $[14,15]$, neutron-antineutron oscillation [16-19], nucleon exotic decays [20, 21], dinucleon decays [22], electric dipole moment [23, 24], have been investigated to examine fundamental global symmetries, such as charge conjugate and parity violation, baryon and lepton number violation [25]. Below the electroweak scale, various new physics effects in these low energy processes could be systematically parameterized in the LEFT. For the baryon and lepton number violation, ${ }^{1}$ the neutronanti-neutron oscillation and dinucleon decay process only start to appear at dimension 9 with $(\Delta B, \Delta L)=( \pm 2,0)$. It is also possible that the dimension 9 operators with $(\Delta B, \Delta L)=(0, \pm 2)$ dominate the neutrino-less double beta decay process [15]. There are also exotic nucleon decay processes dominated at dimension 9 with $(\Delta B, \Delta L)=( \pm 1, \mp 3)$. Therefore, it is necessary to write down the complete set of the higher dimensional LEFT operators. Recently [26-28], the LEFT operators have been written up to dimension 7.

The traditional way to obtain the operator basis is to consider all possible operators, and then eliminate the operator redundancies caused by equation of motion (EOM), integration by parts (IBP), repeated flavor structure and Fierz identities, e.g. [4], which is quite involved when the number of possible structures blows up at higher dimensions. So far the traditional way has been applied to dimension 5,6 in ref. [26] and dimension 7 in ref. [28], which present the result in the form of a set of operators with complicated flavor relations. On the other hand, the systematical procedure proposed in refs. [1,2] is capable of generating independent structures of operators automatically, and presents the flavor-specified operators without extra redundancies. However, the procedure makes use of the amplitude-operator correspondence [29], and was designed only for massless amplitudes, which seems to be not compatible with LEFT. In this work, we present the massive amplitudes in the form introduced in refs. [30,31], which have a one-to-one correspondence to a set of massless amplitudes when the spin $S \leq 1 / 2$, while the correspondence between massive amplitudes and operators remains valid. Hence we argue that the procedure should work as well for EFTs with massive fermions, such as LEFT.

We sketch the procedure as follows. We first use the amplitude operator correspondence to convert the problem of finding the operator basis into finding the amplitude basis, which is further divided into separate tasks as to find: 1. a basis of Lorentz factors expressed as functions of spinor-helicity variables; 2 . a basis of gauge factors expressed in terms of invariant group tensors. The basis of Lorentz factors is generated via the method

\footnotetext{
${ }^{1}$ At dimension 5 , we find the only violation pattern is $(\Delta B, \Delta L)=(0, \pm 2)$, while there are $(0, \pm 2)$, $(0, \pm 4),( \pm 1, \pm 1),( \pm 1, \mp 1)$ at dimension 6 and $(0, \pm 2),( \pm 1, \pm 1),( \pm 1, \mp 1)$ at dimension 7 . The violation patterns at dimension 8 are the same as that at dimension 6 , while there are new violation patterns $(0, \pm 6)$, $( \pm 1, \pm 3),( \pm 1, \mp 3),( \pm 2,0)$ at dimension 9 .
} 
in $[2,32]$, translated from semi-standard Young tableau (SSYT), which after converting to the operators are automatically free from the IBP and EOM redundancy. Meanwhile, using the Littlewood-Richardson rules, we construct a set of singlet Young tableau of the gauge group indices from the constituting particles, which induces the basis of gauge group factors. A complete and independent flavor-blind operator basis are obtained by direct product of these two factors. Afterwards, we symmetrize the flavor indices of the repeated fields to obtain the so-called p-basis operators, where the ones with permutation symmetries allowed by the flavor number make up our final results as an independent basis of flavorspecified operators. As a key feature, our form of operators include a flavor Young tableau, which indicates the independent components in the Wilson coefficient tensor, hence no more flavor relations are necessary.

In this work, we apply the procedure above to the LEFT. The dimension 5, 6, 7 operator bases are reproduced with new form of flavor structures as indicated above, compared to the previous literature $[26,28]$. More importantly, we also generate the dimension 8,9 operator bases for the first time, which constitutes the main part of this work. We adopt the chiral fermion notation in order to be consistent with other literature [26], as well as to keep the correspondence with the spinor helicity amplitudes explicit.

The paper is organized as follows. In section 2, We introduce the notation and discuss the validity of amplitude-operator correspondence in the massive spinor case. In particular, we describe the main ideas of how to deal with the amplitude bases, group structures and permutation symmetries in section 2.3. In the section 3 , We list the complete operator bases in order of dimension from 5 to 9 . Our conclusion is presented in section 4 .

\section{General framework}

In this section, we describe the framework of writing down the operator basis of the LEFT. We start by reviewing the massive spinor helicity formalism [30, 31], and then illustrate a one to one correspondence between the massless and massive amplitude basis for particles with spin $S \leq 1 / 2$. Together with the massive version of the amplitude operator correspondence that we formulate later, we extend the framework for massless EFTs introduced in $[1,2]$ to include massive fermions, and summarize the procedure of enumerating the complete and independent operator basis of LEFT. We strongly recommend readers to gain preliminary information in refs. $[1,2]$ where we elaborate the algorithm for enumerating and constructing of operator basis for massless fields, in this work, we only outline the important ingredients of the algorithm in section. 2.3.

\subsection{Amplitude basis including massive fermions}

Let us first review the massive spinor helicity formalism developed recently in [31], and build the connection between massive spinor and amplitude basis in this subsection. In spinor formalism, a momentum vector can always be decomposed into two spinors, i.e. the spinor helicity variables,

$$
p_{\alpha \dot{\alpha}}=p_{\mu} \sigma_{\alpha \dot{\alpha}}^{\mu}=\left(\begin{array}{cc}
E+p_{3} & p_{1}-i p_{2} \\
p_{1}+i p_{2} & E-p_{3}
\end{array}\right)=\lambda_{\alpha}^{I} \tilde{\lambda}_{\dot{\alpha} I}
$$


where $\alpha, \dot{\alpha}$ are the $\mathrm{SU}(2)_{L} \times \mathrm{SU}(2)_{R}$ Lorentz indices, $I$ is the little group indices, which is $\mathrm{SU}(2)$ for massive particle, and $\mathrm{U}(1)$ for massless particle ( $I$ can be omitted). Note that $\operatorname{det} p_{\alpha \dot{\alpha}}=p^{\mu} p_{\mu}=m^{2}$. Therefore $p_{\alpha \dot{\alpha}}$ has rank 1 in massless case and rank 2 for massive particles. These indices are raised and lowered by the 2-index Levi-Civita symbols defined as,

$$
\epsilon^{12}=-\epsilon^{21}=\epsilon_{21}=-\epsilon_{12}=1, \quad \lambda^{\alpha I}=\epsilon^{\alpha \beta} \lambda_{\beta}^{I}, \quad \lambda_{\alpha I}=\epsilon_{I J} \lambda_{\alpha}^{J}, \quad \tilde{\lambda}^{\dot{\alpha} I}=\epsilon^{\dot{\alpha}} \dot{\lambda^{\beta}} \tilde{\beta}_{\dot{\beta}}^{I}, \quad \tilde{\lambda}_{\dot{\alpha} I}=\epsilon_{I J} \tilde{\lambda}_{\dot{\alpha}}^{J} .
$$

We normalize the spinors as

$$
\lambda^{I \alpha} \lambda_{\alpha}^{J}=-m \epsilon^{I J}, \quad \tilde{\lambda}_{\dot{\alpha}}^{I} \tilde{\lambda}^{J \dot{\alpha}}=m \epsilon^{I J}, \quad \lambda_{\alpha}^{I} \lambda_{\beta I}=m \epsilon_{\alpha \beta}, \quad \tilde{\lambda}_{\dot{\alpha}}^{I} \tilde{\lambda}_{\dot{\beta} I}=m \epsilon_{\dot{\alpha} \dot{\beta}}
$$

In the standard polar coordinates where $p^{\mu}=(E, p \sin \theta \cos \phi, p \sin \theta \sin \phi, p \cos \theta)$, the general solutions of the spinors are the following,

$$
\begin{array}{lll}
\lambda_{\alpha}^{I}=\lambda_{\alpha} \zeta^{-I}+\eta_{\alpha} \zeta^{+I}, & \text { with } \lambda_{\alpha}=\sqrt{E+p}\left(\begin{array}{c}
-s_{\theta}^{*} \\
c_{\theta}
\end{array}\right), \quad \eta_{\alpha}=\sqrt{E-p}\left(\begin{array}{c}
c_{\theta} \\
s_{\theta}
\end{array}\right), \\
\tilde{\lambda}_{\dot{\alpha}}^{I}=\tilde{\lambda}_{\dot{\alpha}} \zeta^{+I}-\tilde{\eta}_{\dot{\alpha}} \zeta^{-I}, \quad \text { with } \tilde{\lambda}^{\dot{\alpha}}=\sqrt{E+p}\left(\begin{array}{c}
c_{\theta} \\
s_{\theta}
\end{array}\right), \quad \tilde{\eta}^{\dot{\alpha}}=\sqrt{E-p}\left(\begin{array}{c}
s_{\theta}^{*} \\
-c_{\theta}
\end{array}\right),
\end{array}
$$

where $\zeta^{ \pm}$satisfy $\epsilon_{I J} \zeta^{-I} \zeta^{+J}=1$ and abbreviation $c_{\theta} \equiv \cos \frac{\theta}{2}, s_{\theta} \equiv \sin \frac{\theta}{2} e^{i \phi} . \zeta^{ \pm}$are determined by the direction along which we take the spin components, for instance $\zeta^{+}=$ $(1,0), \zeta^{-}=(0,1)$ mean that we take the spin components along the momentum direction. Because we work in the in-coming convention in this paper, the out-going physical momenta are thus written as in-coming momentum with $E<0$. For such momentum, we define $\lambda_{\alpha}^{I}(-p)=-\lambda_{\alpha}^{I}(p)$ and $\tilde{\lambda}_{\dot{\alpha}}^{I}(-p)=\tilde{\lambda}_{\dot{\alpha}}^{I}(p)$. In the massless limit, $E-p, \eta, \tilde{\eta} \rightarrow 0$, the $\mathrm{SU}(2)$ little group reduces to the transverse $\mathrm{U}(1)$ rotation, while $I$ can be omitted. Only two of spinors in eq. (2.4), (2.5) are still valid,

$$
\lambda_{\alpha}=\sqrt{2 E}\left(\begin{array}{c}
-s_{\theta}^{*} \\
c_{\theta}
\end{array}\right), \quad \tilde{\lambda}^{\dot{\alpha}}=\sqrt{2 E}\left(\begin{array}{c}
c_{\theta} \\
s_{\theta}
\end{array}\right) .
$$

The resulting Lorentz scalar can be represented by angle spinor brackets and square spinor brackets

$$
\begin{aligned}
\left\langle 1^{I} 2^{J}\right\rangle & \equiv \lambda_{1}^{\alpha I} \lambda_{2 \alpha}^{J}=\lambda_{1 \alpha}^{I} \epsilon^{\beta \alpha} \lambda_{2 \beta}^{J}=-\lambda_{1 \alpha}^{I} \epsilon^{\alpha \beta} \lambda_{2 \beta}^{J}=-\left\langle 2^{J} 1^{I}\right\rangle, \\
{\left[1^{I} 2^{J}\right] } & \equiv \tilde{\lambda}_{1 \dot{\alpha}}^{I} \tilde{\lambda}_{2}^{\dot{\alpha} J}=\tilde{\lambda}_{1 \dot{\alpha}}^{I} \epsilon^{\dot{\alpha} \dot{\beta}} \tilde{\lambda}_{2 \dot{\beta}}^{J}=-\tilde{\lambda}_{1 \dot{\alpha}}^{I} \epsilon^{\dot{\beta} \dot{\alpha}} \tilde{\lambda}_{2 \dot{\beta}}^{J}=-\left[2^{J} 1^{I}\right] .
\end{aligned}
$$

The same definitions apply to the massless case with the $I, J$ indices removed. The Lorentz invariant amplitude can be constructed from the spinor brackets. The little group representations of each particle constrains the form of amplitude as follows

helicity $h$ massless particle with spinor variables $(\lambda, \tilde{\lambda}): \mathcal{A} \sim \begin{cases}\lambda_{\{\alpha\}}^{r-2 h} \tilde{\lambda}_{\{\dot{\alpha}\}}^{r}, & h \leq 0 \\ \lambda_{\{\alpha\}}^{r} \tilde{\lambda}_{\{\dot{\alpha}\}}^{r+2 h}, & h \geq 0\end{cases}$

spin $S$ massive particle with spinor variables $\left(\lambda^{I}, \tilde{\lambda}^{I}\right): \quad \mathcal{A} \sim\left(\lambda_{\{\alpha\}}^{r+2 S-n} \tilde{\lambda}_{\{\dot{\alpha}\}}^{r+n}\right)^{\left\{I_{1} \cdots I_{2 S}\right\}}$,

$$
0 \leq n \leq 2 S
$$


where $\{\cdot\}$ denotes totally symmetric indices. The total symmetries among the spinor indices are superfluous for the massless amplitudes, while those for massive amplitudes result from the removal of terms with factor of masses via eq. (2.3). For example

$$
\left\langle 1^{J} 2\right\rangle\left\langle 1^{I} 3\right\rangle\left[1_{J} 4\right]=[\left(\lambda_{1}^{\{\alpha} \lambda_{1}^{\beta\}} \tilde{\lambda}_{1 \dot{\alpha}}\right)^{I}+\underbrace{\left(\lambda_{1}^{[\alpha} \lambda_{1}^{\beta]} \tilde{\lambda}_{1 \dot{\alpha}}\right)^{I}}_{=m_{1} \epsilon^{\alpha \beta} \tilde{\lambda}_{1 \dot{\alpha}}^{I}}] \lambda_{2, \alpha} \lambda_{3, \beta} \tilde{\lambda}_{4}^{\dot{\alpha}} .
$$

By this rule, we have a well-defined dimensionality of the amplitudes as the highest power of spinor brackets in it. Under this definition, the complete amplitude basis at a certain dimension is defined modulo lower dimensional amplitudes. It will be clear shortly that the EOM redundancy of the corresponding operators is eliminated through this removal. Also due to the total symmetry of the spinor indices, the $r$ pairs of little group indices in eq. (2.10) must be contracted between $\lambda^{I}$ and $\tilde{\lambda}^{I}$, because otherwise the amplitude would vanish. Therefore the integer $r$ stands for the number of spinor pairs $\left(\lambda^{(I)}, \tilde{\lambda}^{(I)}\right)$ in both cases that constitute factors of momentum due to eq. (2.1). The $2 S+1$ choices of $n$ represent independent forms ${ }^{2}$ of contribution the spin- $S$ massive particle could have.

It is easy to observe the correspondence between the massless factor and the massive factor when $S=|h|$ and $n=0,2 S$, which maps momenta to momenta, and the rest of the massless spinors to massive spinors with totally symmetric little group indices. ${ }^{3}$ The mapping covers all the possible massive amplitudes only when $S \leq \frac{1}{2}$, in that $n=0,2 S$ are the only two choices. Therefore, we have the explicit form of the mapping as

$$
\begin{array}{lcrlrl}
h=0 & \lambda^{r} \tilde{\lambda}^{r} \longrightarrow\left(\lambda^{J} \tilde{\lambda}_{J}\right)^{r} & S=0, & n=0 \\
h=-\frac{1}{2} & \lambda^{1+r} \tilde{\lambda}^{r} \longrightarrow \lambda^{I}\left(\lambda^{J} \tilde{\lambda}_{J}\right)^{r} & S=\frac{1}{2}, & & n=0 \\
h=\frac{1}{2} & \lambda^{r} \tilde{\lambda}^{1+r} \longrightarrow \tilde{\lambda}^{I}\left(\lambda^{J} \tilde{\lambda}_{J}\right)^{r} & S=\frac{1}{2}, & & &
\end{array}
$$

For higher spin massive particles, the map could not be established, and our framework would fail. However, it is already enough for us to enumerate the amplitude basis involving massive scalars and fermions by enumerating the massless ones and then simply adding the little group indices according to eq. (2.12).

\subsection{Amplitude-operator correspondence including massive fermions}

With the massive local amplitude basis in hand, we still need the massive version of the amplitude operator correspondence to finally obtain the operator basis. First we convert the Lorentz indices $\mu$ in the operators into the spinor indices $\alpha$ and $\dot{\alpha}$ and we have the

\footnotetext{
${ }^{2}$ It is true that $\lambda^{I}$ and $\tilde{\lambda}^{I}$ are not independent due to the Dirac equations. However, given the standard form in eq. (2.10), by removing the terms with factor of masses, the amplitude with various $n$ are indeed independent.

${ }^{3}$ The mapping is referred to as the "first reduction" in [33], which is claimed to be valid only for the "maximal helicity categories", namely $n=0,2 S$. Our formulation is from a different perspective, which better fits in our framework.
} 
following definitions of various notations used in constructing operator basis:

$$
\begin{aligned}
& \gamma^{\mu}=\left(\begin{array}{cc}
0 & \sigma_{\alpha \dot{\alpha}}^{\mu} \\
\bar{\sigma}^{\mu \dot{\alpha} \alpha} & 0
\end{array}\right), \quad \Psi=\left(\begin{array}{c}
\xi_{\alpha} \\
\chi^{\dagger \dot{\alpha}}
\end{array}\right), \quad \Psi_{M}=\left(\begin{array}{c}
\zeta_{\alpha} \\
\zeta^{\dagger \dot{\alpha}}
\end{array}\right), \\
& \Psi_{\mathrm{L}}=\frac{1-\gamma_{5}}{2} \Psi=\left(\begin{array}{c}
\xi_{\alpha} \\
0
\end{array}\right), \quad \Psi_{\mathrm{R}}=\frac{1+\gamma_{5}}{2} \Psi=\left(\begin{array}{c}
0 \\
\chi^{\dagger \dot{\alpha}}
\end{array}\right) \text {, } \\
& D^{\mu}=\frac{1}{2} D_{\alpha \dot{\alpha}} \bar{\sigma}^{\mu \dot{\alpha} \alpha}, \quad F_{\mathrm{L} \mu \nu}=\frac{1}{4} F_{\mathrm{L} \alpha \beta} \epsilon_{\dot{\alpha} \dot{\beta}} \bar{\sigma}^{\mu \dot{\alpha} \alpha} \bar{\sigma}^{\nu \dot{\beta} \beta}, \quad F_{\mathrm{R} \mu \nu}=\frac{1}{4} F_{\mathrm{R} \dot{\alpha} \dot{\beta}} \epsilon_{\alpha \beta} \bar{\sigma}^{\mu \dot{\alpha} \alpha} \bar{\sigma}^{\nu \dot{\beta} \beta},
\end{aligned}
$$

where $\xi, \chi, \zeta$ are the Weyl fermions, $\Psi$ and $\Psi_{M}$ denote the Dirac and Majorana fermion respectively, $F_{\mathrm{L} / \mathrm{R}}=\frac{1}{2}(F \pm i \tilde{F})$ are the chiral basis of the gauge bosons. Note that in terms of Weyl components, we need a pair of them to represent the independent chiral components of the Dirac fermion, but only one for a Majorana fermion. Nevertheless, all the Weyl degrees of freedom share the same form of spinor wave functions

$$
u^{I}=\left(\begin{array}{c}
\lambda_{\alpha}^{I} \\
\tilde{\lambda}^{\dot{\alpha} I}
\end{array}\right), \bar{u}_{I}=\left(-\lambda_{I}^{\alpha}, \tilde{\lambda}_{\dot{\alpha} I}\right), \quad v_{I}=\left(\begin{array}{c}
-\lambda_{\alpha I} \\
\tilde{\lambda}_{I}^{\dot{\alpha}}
\end{array}\right), \quad \bar{v}^{I}=\left(\lambda^{\alpha I}, \tilde{\lambda}_{\dot{\alpha}}^{I}\right)
$$

which solve the Dirac equations

$$
\begin{array}{llll}
(\not p-m) u^{I}=0, & \bar{u}_{I}(\not p-m)=0, & \bar{u}_{I} u^{J}=2 m \delta_{I}^{J}, & u^{I} \bar{u}_{I}=\not p+m, \\
(\not p+m) v_{I}=0, & \bar{v}^{I}(\not p+m)=0, & \bar{v}^{I} v_{J}=2 m \delta_{J}^{I}, & v_{I} \bar{v}^{I}=\not p-m .
\end{array}
$$

We focus on $u^{I}$ and $\bar{v}^{I}$ associated with incoming fermions and anti-fermions, since all momenta are incoming in our convention. The amplitudes generated by fermion bilinears are given by the following Feynman rules.

$$
\begin{aligned}
\bar{\Psi}_{1} \Psi_{2} & =\bar{\Psi}_{\mathrm{L} 1} \Psi_{\mathrm{R} 2}+\bar{\Psi}_{\mathrm{R} 1} \Psi_{\mathrm{L} 2} & \rightarrow & \bar{v}_{1}^{I} u_{2}^{J}=\lambda_{1}^{\alpha I} \lambda_{2 \alpha}^{J}+\tilde{\lambda}_{1 \dot{\alpha}}^{I} \tilde{\lambda}_{2}^{\dot{\alpha} J}=\left\langle 1^{I} 2^{J}\right\rangle+\left[1^{I} 2^{J}\right], \\
\bar{\Psi}_{1} \gamma_{5} \Psi_{2} & =\bar{\Psi}_{\mathrm{L} 1} \Psi_{\mathrm{R} 2}-\bar{\Psi}_{\mathrm{R} 1} \Psi_{\mathrm{L} 2} & \rightarrow & \bar{v}_{1}^{I} \gamma_{5} u_{2}^{J}=\left[1^{I} 2^{J}\right]-\left\langle 1^{I} 2^{J}\right\rangle, \\
\bar{\Psi}_{1} \gamma^{\mu} \Psi_{2} & =\bar{\Psi}_{\mathrm{R} 1} \gamma^{\mu} \Psi_{\mathrm{R} 2}+\bar{\Psi}_{\mathrm{L} 1} \gamma^{\mu} \Psi_{\mathrm{L} 2} & \rightarrow & \bar{v}_{1}^{I} \gamma^{\mu} u_{2}^{J}=\left\langle 1^{I}\left|\sigma^{\mu}\right| 2^{J}\right]+\left[1^{I}\left|\bar{\sigma}^{\mu}\right| 2^{J}\right\rangle, \\
\bar{\Psi}_{1} \gamma^{\mu} \gamma_{5} \Psi_{2} & =\bar{\Psi}_{\mathrm{R} 1} \gamma^{\mu} \Psi_{\mathrm{R} 2}-\bar{\Psi}_{\mathrm{L} 1} \gamma^{\mu} \Psi_{\mathrm{L} 2} & \rightarrow & \bar{v}_{1}^{I} \gamma^{\mu} \gamma_{5} u_{2}^{J}=\left\langle 1^{I}\left|\sigma^{\mu}\right| 2^{J}\right]-\left[1^{I}\left|\bar{\sigma}^{\mu}\right| 2^{J}\right\rangle .
\end{aligned}
$$

Therefore the amplitude operator correspondence between spinor variables with free little group indices and four-component spinor fields can be read from the second and the last expressions in above equations:

$$
\lambda_{i}^{I} \rightarrow \psi_{i}=\Psi_{\mathrm{L} i}, \bar{\Psi}_{\mathrm{R} i} \text { or } \Psi_{M i}, \quad \tilde{\lambda}_{i}^{I} \rightarrow \psi_{i}^{\dagger}=\bar{\Psi}_{\mathrm{L} i}, \Psi_{\mathrm{R} i} \text { or } \bar{\Psi}_{M i} .
$$

The pair of spinor helicity variables $\lambda_{i}^{J} \tilde{\lambda}_{i J}$ with contracted little group indices can be translated into derivative acting on particle $i$ yielding following correspondences:

$$
\begin{aligned}
\lambda_{i}^{n+2} \tilde{\lambda}_{i}^{n} & \Leftrightarrow D^{n} F_{\mathrm{L} i}, & \lambda_{i}^{n} \tilde{\lambda}^{n+2} & \Leftrightarrow D^{n} F_{\mathrm{R} i}, \\
\lambda_{i}^{n+1} \tilde{\lambda}_{i}^{n}, \lambda_{i}^{I}\left(\lambda_{i}^{J} \tilde{\lambda}_{i J}\right)^{n} & \Leftrightarrow D^{n} \psi_{i}, & \lambda_{i}^{n} \tilde{\lambda}_{i}^{n+1}, \tilde{\lambda}_{i}^{I}\left(\lambda_{i}^{J} \tilde{\lambda}_{i J}\right)^{n} & \Leftrightarrow D^{n} \psi_{i}^{\dagger},
\end{aligned}
$$


where for completeness we also include the massless case denoted by spinor variables without $I$ or $J$ indices. One may question about do the presence of the covariant derivatives generate other local amplitudes with more gauge bosons, which exist in ordinary Feynman rules. However, these vertices are not gauge invariant, and the final gauge invariant amplitudes with contributions from these operators are non-local, and our amplitude operator correspondence applies to local amplitudes only.

Let us take a closer look at the derivatives in eq. (2.24) and (2.25). The total symmetries among the spinor indices in amplitudes are very clear. Otherwise, the resulting amplitude must vanish or reduce the dimension due to the on-shell condition $\lambda_{i[\alpha}^{I} \lambda_{i \beta]}^{J}=m_{i} \epsilon^{I J} \epsilon_{\alpha \beta}$. Therefore the derivatives acting on each fields in the operators also take the totally symmetric spinor indices. It is easy to see that any pair of anti-symmetric spinor indices if associated with derivatives can be always converted to other types or reduced dimesion with EOM and the relation $i\left[D_{\mu}, D_{\nu}\right]=\sum F_{\mu \nu}$, where we are supposed to have obtained a complete basis:

$$
\begin{aligned}
D_{[\alpha \dot{\alpha}} D_{\beta] \dot{\beta}} & =D_{\mu} D_{\nu} \sigma_{[\alpha \dot{\alpha}}^{\mu} \sigma_{\beta] \dot{\beta}}^{\nu}=-D^{2} \epsilon_{\alpha \beta} \epsilon_{\dot{\alpha} \dot{\beta}}+\frac{i}{2}\left[D_{\mu}, D_{\nu}\right] \epsilon_{\alpha \beta} \bar{\sigma}_{\dot{\alpha} \dot{\beta}}^{\mu \nu}, \\
D_{[\alpha \dot{\alpha}} \psi_{\beta]} & =D_{\mu} \sigma_{[\alpha \dot{\alpha}}^{\mu} \psi_{\beta]}=-\epsilon_{\alpha \beta}(\not D \psi)_{\dot{\alpha}} \\
D_{[\alpha \dot{\alpha}} F_{\mathrm{L} \beta] \gamma} & =D_{\mu} F_{\nu \rho} \sigma_{[\alpha \dot{\alpha}}^{\mu} \sigma_{\beta] \gamma}^{\nu \rho}=2 D^{\mu} F_{\mu \nu} \epsilon_{\alpha \beta} \sigma_{\gamma \dot{\alpha}}^{\nu} .
\end{aligned}
$$

Therefore, the building block in constructing the operator basis is in the following form:

$$
\left(D^{r_{i}-\left|h_{i}\right|} \Phi_{i}\right)_{\alpha_{i}^{r_{i}-h_{i}}}^{\dot{\alpha}_{r_{i}+h_{i}}} \in\left(j_{l}+r_{i}-\left|h_{i}\right|, j_{r}+r_{i}-\left|h_{i}\right|\right)
$$

where the powers of the indices $\alpha_{i}$ and $\dot{\alpha}_{i}$ indicate that they are already totally symmetrized, thus the whole object transforms as $\left(j_{l}+r_{i}-\left|h_{i}\right| 2, j_{r}+r_{i}-\left|h_{i}\right|\right)$ under $s l(2, \mathbb{C})=$ $s u(2)_{l} \times s u(2)_{r}$, given that $\Phi$ transforms as $\left(j_{l}, j_{r}\right)$.

The IBP redundancy of operators is solved by the manifest momentum conservation in the amplitude, which can be illustrated by the following example:

$$
\left\langle 1^{I} 2\right\rangle\left[23^{J}\right]=\left\langle 1^{I}\left|p_{2}\right| 3^{J}\right]=-\left\langle 1^{I}\left|\left(p_{1}+p_{3}+p_{4}\right)\right| 3^{J}\right] \sim-\left\langle 1^{I} 4\right\rangle\left[43^{J}\right]
$$

corresponds to the operator equivalence

$$
\left(\psi_{1} \sigma^{\mu} \psi_{3}^{\dagger}\right) D_{\mu} \phi_{2} \phi_{4} \sim-\left(\psi_{1} \sigma^{\mu} \psi_{3}^{\dagger}\right) \phi_{2} D_{\mu} \phi_{4}
$$

where terms that convert to other type steming from $\left\langle 1^{I} 1^{J}\right\rangle=m_{1} \epsilon^{I J}$ s by EOM are omitted. Hence, taking momentum conservation into account, the amplitude basis corresponds to an IBP non-redundant basis of operators.

Finally, taking into account the $\mathrm{SU}(3)$ group factors $T^{a_{1}, \ldots a_{N}}$, the amplitude-operator correspondence can be represented by:

$$
\begin{aligned}
T^{a_{1}, \ldots, a_{N}} \mathcal{B}\left(\lambda_{1}^{(I)}, \ldots, \lambda_{N}^{(I)}\right) & \sim T^{a_{1}, \ldots, a_{N}} \mathcal{B}\left(\Phi_{1}, \cdots, \Phi_{N}\right)_{a_{1}, \cdots, a_{N}} \\
\mathcal{B}\left(\Phi_{1}, \cdots, \Phi_{N}\right)_{a_{1}, \cdots, a_{N}} & =\left(\epsilon^{\alpha_{i} \alpha_{j}}\right)^{\otimes n}\left(\tilde{\epsilon}_{\dot{\alpha}_{i} \dot{\alpha}_{j}}\right)^{\otimes \tilde{n}} \prod_{i=1}^{N}\left(D^{r_{i}-\left|h_{i}\right|} \Phi_{i, a_{i}}\right)_{\alpha_{i}^{\dot{\alpha}_{i}-h_{i}}}^{\dot{\alpha}_{i}^{r_{i}+h_{i}}}
\end{aligned}
$$


where $\mathcal{B}$ and $T$ determine the Lorentz structure and gauge structure of the operators respectively, and our goal to find complete and independent operator basis is equivalent to find independent T's and B's given the type of the operators. Note that we have suppressed the flavor indices on the right hand side of eq. (2.28) for the moment, and thus call them as "flavor-blind" operators, where we effectively treat all the particles as distinguishable ones, the redundancy related to the repeated fields or equivalently constraints from spin-statistics among the identical particles are tackled in the next section.

\subsection{The operator basis}

Having established the correspondence, the next step is to construct concrete amplitude basis satisfying momentum conservation and spin-statistics constraint. Such amplitude basis could be translated into operator basis following eq. (2.28).

We start by reviewing the operator classification at different levels as introduced in refs. $[1,2]$ :

- Class: a (Lorentz) class is defined by the numbers of fields of each Lorentz irreducible representations and the definite number of covariant derivatives in a operator.

- Type: for each Lorentz Class, an assignment of each Lorentz irreducible representations with SM field contents that conserve the electric charge is called a type.

- Term (flavor-blind operator): for each type, we organize operators into different irreducible representations of the symmetric groups of permuting flavor indices of repeated fields. Each irreducible representation corresponds to a term of operators, and our result in the following sections are all presented at this level.

- Operator (flavor-specified operator): for each term, when the flavor indices are taken for a specific number in a SSYT of the corresponding Young diagram, it becomes what we called flavor-specified operator.

Since our results are presented at the term level, in the following we briefly summarize the method for finding terms for each type of operators, which has been elaborated in refs. [1, 2]. We encourage readers to refer refs. [1, 2] for details and the systematic explanation of the method. In the following, we first separate the task for finding complete and independent Lorentz and gauge factors, then illustrate how to obtain the term with definite flavor permutation symmetry.

In general, the Lorentz factor of the amplitude, determined only by the helicities of the constituting particles, can be expressed as

$$
\mathcal{B}=\langle\cdot\rangle^{n}[\cdot]^{\tilde{n}}
$$

where $n$ and $\tilde{n}$ denote the numbers of $\lambda$ and $\tilde{\lambda}$ pairs, as shown in eq. (2.29).

To find the complete and independent amplitude basis, we use the method introduced by ref. [32] and further developed by ref. [1]. It is proved in ref. [32] that the amplitudes 
modulo total momenta form irreducible representations of $\mathrm{SU}(N)$ group are denoted by the primary Young diagrams,

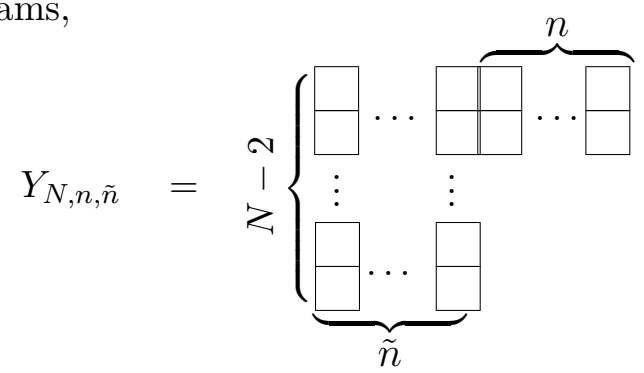

where $N$ is the number of particles in the amplitudes. The base vectors of the irreducible representations are given by $\mathrm{SU}(N)$ semi-standard Young tableaus (SSYTs). The number of indices $i$ to fill in the Young diagram, denoted by $\# i$, is determined by $\# i=\tilde{n}-2 h_{i}$, where $\left\{h_{i}\right\}$ denote the set of helicity of the $i$ th particle in the class and are sorted in the order $h_{i} \leq h_{i+1}, i=1, \cdots, N-1$. Going through all possible ways to fill in the numbers and obtain a SSYT will give us the complete and non-redundant amplitude basis expressed by SSYTs. In fact, the Fock's condition of Young tableaus corresponds to IBP and Fierz/Schouten identities of operators. Thus choosing SSYTs means picking out all independent Young tableaus, which corresponds to picking out all independent Lorentz structures of operators.

The SSYTs can be translated to amplitudes using following relations for each columns,

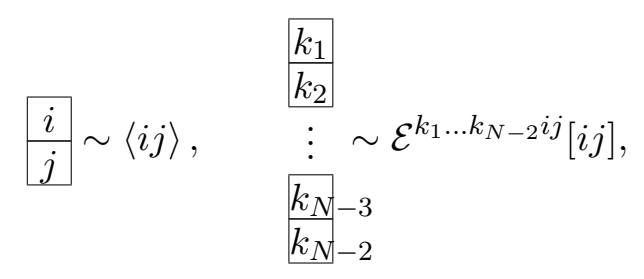

where $\mathcal{E}$ is the Levi-Civita tensor of the $\mathrm{SU}(N)$ group. These amplitudes can be further translated to Lorentz structures of operators using amplitude-operator correspondence.

The gauge group factors are expressed by Levi-Civita tensors that contract with the fundamental indices of the fields. Anti-fundamental representation and adjoint representation of fields can be converted to fundamental representation by

$$
\begin{aligned}
& \epsilon_{a c d}\left(\lambda^{A}\right){ }_{b}^{d} G^{A}=G_{a b c} \sim \begin{array}{|l|l|}
\hline a & b \\
\hline c
\end{array}, \\
& \epsilon_{a b c} u^{\dagger, c}=u_{a b}^{\dagger} \sim \frac{a}{b} .
\end{aligned}
$$

Following the procedures in ref. [1], the gauge group factors can be obtained by constructing the singlet Young tableau using the modified Littlewood-Richardson rule with the corresponding indices of each field filled in.

Finally, we need to find the flavor structure in the presence of repeated fields. To achieve that, we utilize the basis $b_{x=1, \ldots, d_{\lambda}}^{[\lambda]}$ in the irreducible left ideal of the group algebra $\tilde{S}_{m}$, which is a set of independent symmetrizers spanning an irreducible representation space of the permutation group $S_{m}$. By acting them on the flavor indices of an operator, 
we obtain either zero or an irreducible flavor tensor, whose independent components are given by the SU $\left(n_{f}\right)$ SSYT's. We thus express the result as such irreducible tensors in the form $\mathcal{Y}_{1}^{[\lambda]} \circ \mathcal{O}$, where $\mathcal{Y}_{1}^{[\lambda]} \equiv b_{1}^{[\lambda]}$ is the famous Young symmetrizer ${ }^{4}$ and $\mathcal{O}$ is some monomial operator. Take $S_{3}$ group for example, we show the action of Young symmetrizer as following

$$
\begin{aligned}
\mathcal{Y}_{1}^{[3]} O^{p r s} & =\mathcal{Y}\left[\frac{p|r| s]}{p r s}=O^{p r s}+O^{r p s}+O^{p s r}+O^{r s p}+O^{s r p}+O^{s p r},\right. \\
\mathcal{Y}_{1}^{[2,1]} O^{p r s} & =\mathcal{Y}\left[\left[\begin{array}{l}
p r \\
s
\end{array}\right] O^{p r s}=O^{p r s}+O^{r p s}-O^{s r p}-O^{s p r},\right. \\
\mathcal{Y}_{1}^{\left[1^{3}\right]} O^{p r s} & =\mathcal{Y}\left[\begin{array}{l}
p \\
\frac{p}{s}
\end{array}\right] O^{p r s}=O^{p r s}-O^{r p s}-O^{p s r}+O^{r s p}-O^{s r p}+O^{s p r} .
\end{aligned}
$$

To get all the independent irreducible representation spaces, one must find a collection of operators $\left\{\mathcal{O}_{\zeta}\right\}$ such that $\mathcal{Y}_{1}^{[\lambda]} \circ \mathcal{O}_{\zeta}$ are linearly independent and whose linear combinations exhaust all the possible $\mathcal{Y}_{1}^{[\lambda]} \circ \mathcal{O}$. Such projections form the so-called p-basis of the operators [2]. It is usually highly non-trivial considering the complexity of redundancy relations of the operators. But with the Young tableau basis obtained above, we could find the unique coordinates of any $\mathcal{Y}_{1}^{[\lambda]} \circ \mathcal{O}_{\zeta}$ which makes it easy to find the collection $\left\{\mathcal{O}_{\zeta}\right\}$.

In practice, the coordinates of $\mathcal{Y}_{1}^{[\lambda]} \circ \mathcal{O}_{\zeta}$ can be obtained by finding the matrix representation of the group elements $\mathcal{D}^{T}(\pi)$ and $\mathcal{D}^{\mathcal{B}}(\pi)$ on the complete and independent bases of $T_{i}$ and $\mathcal{B}_{j}$ obtained by the Young tableau approaches discussed above. As the permutation on the flavor indices is equivalent to permute the gauge and Lorentz structures as discussed in refs. $[1,2]$ :

$$
\underbrace{\pi \circ \mathcal{O}^{\left\{f_{k}, \ldots\right\}}}_{\text {permute flavor }}=\underbrace{\left(\pi \circ T_{\mathrm{SU} 3}^{\left\{g_{k}, \ldots\right\}}\right)}_{\text {permute gauge }} \underbrace{\left(\pi \circ \mathcal{B}_{\left\{g_{k}, \ldots\right\}}^{\left\{f_{k}, \ldots\right\}}\right)}_{\text {permute Lorentz }} .
$$

Thus for any operator $\mathcal{O}=C_{i j} T_{i} B_{j}$, the permutation yields a transformation of the coordinate $C_{i j}$ :

$$
\begin{aligned}
\pi \circ \mathcal{O} & =C_{i j}\left(\pi \circ T_{i}\right)\left(\pi \circ B_{j}\right) \\
& =C_{i j}\left(\mathcal{D}^{T}(\pi)_{i k} T_{k}\right)\left(\mathcal{D}^{\mathcal{B}}(\pi)_{i l} \mathcal{B}_{l}\right) \\
& =\left(C_{k l} \mathcal{D}^{T}(\pi)_{k i} \mathcal{D}^{\mathcal{B}}(\pi)_{l j}\right) T_{i} \mathcal{B}_{j} .
\end{aligned}
$$

Alternatively, one could obtain the p-basis by inner product decomposition as in refs. $[1,2]$, where one first finds the irreducible Lorentz factors $\mathcal{B}^{[\lambda]} x$ and gauge factors $T_{G}^{[\lambda]}$ and then combine them with the CG coefficients of the permutation group as

$$
\mathcal{O}_{(\lambda, x), \zeta}=\sum_{x_{1}, x_{2}} C_{(\lambda, x), \zeta}^{\left(\lambda_{1}, x_{1}\right),\left(\lambda_{2}, x_{2}\right)} \mathcal{B}_{x_{1}}^{\lambda_{1}} T_{\mathrm{SU} 3, x_{2}}^{\lambda_{2}}
$$

To get the final form as $\mathcal{O}_{(\lambda, 1), \zeta}=\mathcal{Y}_{1}^{[\lambda]} \circ \mathcal{O}_{\zeta}$, we could simply find the coordinate of $\mathcal{O}_{\zeta}$ in the p-basis which encode the combination of p-basis amplitudes with $[\lambda]$ symmetry one

\footnotetext{
${ }^{4}$ In our prescription, $\mathcal{Y}_{i \neq 1}^{[\lambda]} \circ \mathcal{O}$, if not vanishing, belongs to another irreducible tensor $\mathcal{Y}_{1}^{[\lambda]} \circ \mathcal{O}^{\prime}$, and should not be counted.
} 
should get from the projection of $\mathcal{Y}_{1}^{[\lambda]}$. It is then straightforward to select a set $\left\{\mathcal{O}_{\zeta}\right\}$ with independent projections, which we refer to as the "desymmetrization" process in [2].

When we contract the flavor tensor with the Wilson coefficient tensor $C_{p r s}$, the Young symmetrizer could be interpreted as acting on $C_{p r s}$ with inverse permutations

$$
\sum_{p r s} C_{p r s}\left(\mathcal{Y}^{[\lambda]} \circ \mathcal{O}^{\text {prs }}\right)=\sum_{\text {prs }}\left(\overline{\mathcal{Y}}[\lambda] \circ C_{\text {prs }}\right) \mathcal{O}^{\text {prs }}
$$

Thus the result can also be interpreted as the monomial operator $\mathcal{O}$ with symmetrized Wilson coefficient tensor. Note that instead of looking for flavor relations among the Wilson coefficients, we only define Wilson coefficients for the independent entries of the

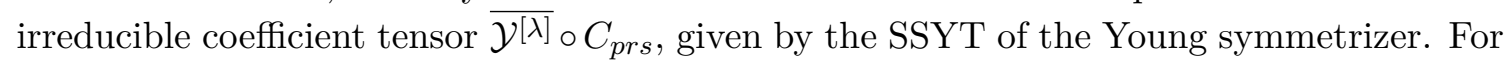
example, the independent Wilson coefficients of a $[\lambda]=[2,1]$ symmetrized p-basis operator $\mathcal{Y}\left[\frac{p r r}{s}\right] \circ \mathcal{O}^{p r s}$ are given by the 8 SSYT's:

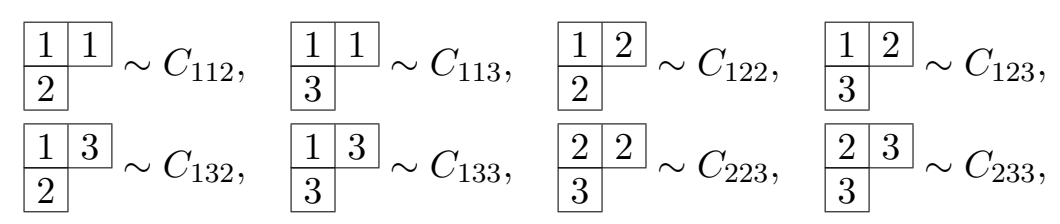

while all the other components in the tensor $C_{\text {prs }}$ are either 0 or related to the above ones by the tensor symmetry $[\lambda]$.

\section{$3 \quad$ Lists of operators in LEFT}

In this section, we first exhibit the field content in table 1 , and then list all the independent dimension 5, 6, 7, 8 and 9 operators in LEFT, and the number of operators with various lepton number violation $|\Delta L|$, are summarized for each dimension in table 2 and table 3 . Although the two-component Weyl spinors are used to construct the operator basis, we changed them into four-component Dirac spinors when presenting the result for user's convenience. The conversion relations between two-component Weyl spinors and fourcomponent Dirac spinors are as follows.

$$
\begin{array}{llll}
\nu_{\mathrm{L}}=\left(\begin{array}{c}
\nu \\
0
\end{array}\right), & e_{\mathrm{L}}=\left(\begin{array}{c}
e \\
0
\end{array}\right), & e_{\mathrm{R}}=\left(\begin{array}{c}
0 \\
e_{\mathbb{C}}^{\dagger}
\end{array}\right), & u_{\mathrm{L}}=\left(\begin{array}{l}
u \\
0
\end{array}\right), \\
u_{\mathrm{R}}=\left(\begin{array}{c}
0 \\
u_{\mathbb{C}}^{\dagger}
\end{array}\right), & d_{\mathrm{L}}=\left(\begin{array}{c}
d \\
0
\end{array}\right), & d_{\mathrm{R}}=\left(\begin{array}{c}
0 \\
d_{\mathbb{C}}^{\dagger}
\end{array}\right) . \\
\bar{\nu}_{\mathrm{L}}=\left(0, \nu^{\dagger}\right), & \bar{e}_{\mathrm{L}}=\left(0, e^{\dagger}\right), & \bar{e}_{\mathrm{R}}=\left(e_{\mathbb{C}}, 0\right), & \bar{u}_{\mathrm{L}}=\left(0, u^{\dagger}\right), \\
\bar{u}_{\mathrm{R}}=\left(u_{\mathbb{C}}, 0\right), & \bar{d}_{\mathrm{L}}=\left(0, d^{\dagger}\right), & \bar{d}_{\mathrm{R}}=\left(d_{\mathbb{C}}, 0\right) .
\end{array}
$$

In the following lists of operators, the $\psi\left(\psi^{\dagger}\right)$ in the name of each class indicates a twocomponent left-handed (right handed) spinor in this class, and the baryon-number and lepton-number violation pattern of each type is listed next to the type as $(\Delta B, \Delta L)$. We label the (anti)fundamental representation of group $\mathrm{SU}(3)_{C}$ and the adjoint representation 
of $\mathrm{SU}(3)_{C}$ by the indices $\{a, b, c, d, e, f\}$ and $\{A, B, C, D\}$, respectively, and the indices for flavor of the fermion fields are denoted by $\{p, r, s, t, u, v\}$.

After applying the method introduced in section 2 to LEFT, we are able to obtain the complete basis of independent operators in LEFT at various mass dimensions. In order to compare our result with others, we change the two-component spinors in our building blocks to four-component spinors using the correspondence in eq. (3.1) and (3.2). Furthermore, since the operator basis in LEFT can be different in the literature, the equivalence relations due to Schouten Identity, Fierz Identity and IBP need to be taken into account in the comparison.

To show the conversion between two-component spinors and four-component spinors and compare the result with others, let us take type $\bar{d}_{\mathrm{R}} d_{\mathrm{L}} \bar{u}_{\mathrm{R}} u_{\mathrm{L}}$ as an example. Operators of this type are written as two-component spinors at first,

$$
\begin{aligned}
& \left(d_{\mathbb{C} p}^{a} d_{r b}\right)\left(u_{\mathbb{C} s}^{b} u_{t a}\right), \\
& \left(d_{\mathbb{C} p}^{a} d_{r a}\right)\left(u_{\mathbb{C} s}^{c} u_{t c}\right), \\
& \left(d_{r b} u_{t a}\right)\left(d_{\mathbb{C} p}^{a} u_{\mathbb{C} s}^{b}\right), \\
& \left(d_{r a} u_{t c}\right)\left(d_{\mathbb{C} p}^{a} u_{\mathbb{C} s}^{c}\right) .
\end{aligned}
$$

These operators can be converted to the (chiral) four-component spinor notation eq. (3.1), (3.2) using

$$
\begin{aligned}
\bar{\Psi}_{1} \Psi_{2} & =\chi_{1}^{\alpha} \xi_{2 \alpha}+\xi_{1 \dot{\alpha}}^{\dagger} \chi_{2}^{\dagger \dot{\alpha}}, \\
\Psi_{1}^{\mathrm{T}} C \Psi_{2} & =\xi_{1}^{\alpha} \xi_{2 \alpha}+\chi_{1 \dot{\alpha}}^{\dagger} \chi_{2}^{\dagger \dot{\alpha}}, \\
\bar{\Psi}_{1} C \bar{\Psi}_{2}^{\mathrm{T}} & =\xi_{1 \dot{\alpha}}^{\dagger} \xi_{2}^{\dagger \dot{\alpha}}+\chi_{1}^{\alpha} \chi_{2 \alpha},
\end{aligned}
$$

where $\Psi$ s are four-component spinors and $\xi \mathrm{s}, \chi_{\mathrm{s}}$ are left-handed two-component spinors,

$$
\Psi=\left(\begin{array}{c}
\xi_{\alpha} \\
\chi^{\dagger \dot{\alpha}}
\end{array}\right), \quad \bar{\Psi}=\Psi^{\dagger} \gamma^{0}=\left(\chi^{\alpha}, \xi_{\dot{\alpha}}^{\dagger}\right)
$$

and $C=i \gamma^{0} \gamma^{2}=\left(\begin{array}{cc}\epsilon_{\alpha \beta} & 0 \\ 0 & \epsilon^{\dot{\alpha} \dot{\beta}}\end{array}\right)=\left(\begin{array}{cc}-\epsilon^{\alpha \beta} & 0 \\ 0 & -\epsilon_{\dot{\alpha} \dot{\beta}}\end{array}\right)$. Taking eq. (3.3) as an example, the pairs of fields in each parenthesis can be translated into four-components ones of the form $\bar{\Psi} \Psi$ using eq. (3.1) and (3.2), with the first line in eq. (3.7), the two-component notation is directly translated into the four component one: $\left(\bar{d}_{\mathrm{R} p}^{a} d_{\mathrm{L} r b}\right)\left(\bar{u}_{\mathrm{R}}^{b} u_{\mathrm{L} t a}\right)$. For the case in eq. (3.6) where the pairs of fields in each parenthesis have the forms of $\Psi \Psi$ or $\bar{\Psi} \bar{\Psi}$ after conversion to four-component ones, the charge conjugate operators $C$ and the proper transposition of spinors need to be inserted properly following the last two lines in eq. (3.7) yielding $\left(d_{\mathrm{L} r a}^{\mathrm{T}} C u_{\mathrm{L} t c}\right)\left(\bar{d}_{\mathrm{R} p}^{a} C \bar{u}_{\mathrm{R} s}^{\mathrm{T} c}\right)$. More examples of such conversions can be found in the appendix of ref. [1]. Thus the operators of type $\bar{d}_{\mathrm{R}} d_{\mathrm{L}} \bar{u}_{\mathrm{R}} u_{\mathrm{L}}$ can be presented in four- 
component spinors as

$$
\begin{aligned}
\left(\bar{d}_{\mathrm{R} p}^{a} d_{\mathrm{L} r b}\right)\left(\bar{u}_{\mathrm{R} s}^{b} u_{\mathrm{L} t a}\right) & =\left(d_{\mathbb{C} p}^{a} d_{r b}\right)\left(u_{\mathbb{C} s}^{b} u_{t a}\right), \\
\left(\bar{d}_{\mathrm{R} p}^{a} d_{\mathrm{L} r a}\right)\left(\bar{u}_{\mathrm{R} s}^{c} u_{\mathrm{L} t c}\right) & =\left(d_{\mathbb{C} p}^{a} d_{r a}\right)\left(u_{\mathbb{C} s}^{c} u_{t c}\right), \\
\left(d_{\mathrm{L} r b}^{\mathrm{T}} C u_{\mathrm{L} t a}\right)\left(\bar{d}_{\mathrm{R} p}^{a} C \bar{u}_{\mathrm{R} s}^{\mathrm{T} b}\right) & =\left(d_{r b} u_{t a}\right)\left(d_{\mathbb{C} p}^{a} u_{\mathbb{C} s}^{b}\right), \\
\left(d_{\mathrm{L} r a}^{\mathrm{T}} C u_{\mathrm{L} t c}\right)\left(\bar{d}_{\mathrm{R} p}^{a} C \bar{u}_{\mathrm{R} s}^{\mathrm{T} c}\right) & =\left(d_{r a} u_{t c}\right)\left(d_{\mathbb{C} p}^{a} u_{\mathbb{C} s}^{c}\right) .
\end{aligned}
$$

These operators on the left-hand side are the final form presented in our result in eq. (3.34), where the transpose symbol $\mathrm{T}$ is omitted for simplicity.

To compare our bases with their counterparts in ref. [26], Fierz identities in the following equations are needed:

$$
\begin{aligned}
\left(d_{\mathrm{L}}^{\mathrm{T}} C u_{\mathrm{L}}\right)\left(\bar{d}_{\mathrm{R}} C \bar{u}_{\mathrm{R}}^{\mathrm{T}}\right) & =-\left(\bar{d}_{\mathrm{R}} u_{\mathrm{L}}\right)\left(\bar{u}_{\mathrm{R}} d_{\mathrm{L}}\right)-\left(\bar{d}_{\mathrm{R}} d_{\mathrm{L}}\right)\left(\bar{u}_{\mathrm{R}} u_{\mathrm{L}}\right), \\
\sum_{A}\left(T^{A}\right)_{a b}\left(T^{A}\right)_{c d} & =\delta_{a d} \delta_{c b}-\frac{1}{N} \delta_{a b} \delta_{c d}
\end{aligned}
$$

and it can be shown that the following basis operators are equivalent to ours

$$
\begin{aligned}
& \left(\bar{d}_{\mathrm{R} p} d_{\mathrm{L} r}\right)\left(\bar{u}_{\mathrm{R} s} u_{\mathrm{L} t}\right), \\
& \left(\bar{d}_{\mathrm{R} p} T^{A} d_{\mathrm{L} r}\right)\left(\bar{u}_{\mathrm{R} s} T^{A} u_{\mathrm{L} t}\right), \\
& \left(\bar{d}_{\mathrm{R} p} u_{\mathrm{L} t}\right)\left(\bar{u}_{\mathrm{R} s} d_{\mathrm{L} r}\right), \\
& \left(\bar{d}_{\mathrm{R} p} T^{A} u_{\mathrm{L} t}\right)\left(\bar{u}_{\mathrm{R} s} T^{A} d_{\mathrm{L} r}\right),
\end{aligned}
$$

which are exactly the Hermitian conjugate of operators $\mathcal{O}_{u d}^{S 1, R R}, \mathcal{O}_{u d}^{S 8, R R}, \mathcal{O}_{u d d u}^{S 1, R R}$ and $\mathcal{O}_{u d d u}^{S 8, R R}$ in ref. [26].

In addition to the conversion between two-component and four-component spinors, we used some identities of $\sigma$ matrices to convert spinor indices to Lorentz indices, for example,

$$
F_{L}^{\alpha \beta} F_{L \alpha \beta}=-\frac{1}{4} F_{L \mu \nu} F_{L \rho \lambda}\left(\sigma^{\mu \nu}\right)^{\alpha \beta}\left(\sigma^{\rho \lambda}\right)_{\alpha \beta}=\frac{1}{4} F_{L \mu \nu} F_{L \rho \lambda} \operatorname{Tr}\left(\sigma^{\mu} \bar{\sigma}^{\nu} \sigma^{\rho} \bar{\sigma}^{\lambda}\right)
$$

where

$$
F_{\mathrm{L} \alpha \beta}=\frac{i}{2} F_{\mu \nu}\left(\sigma^{\mu \nu}\right)_{\alpha \beta}=\frac{i}{2} F_{L \mu \nu}\left(\sigma^{\mu \nu}\right)_{\alpha \beta},
$$

and the following identities are used,

$$
\begin{aligned}
& \left(\sigma^{\mu} \bar{\sigma}^{\nu}\right)_{\alpha}{ }^{\beta}=g^{\mu \nu} \delta_{\alpha}^{\beta}-i\left(\sigma^{\mu \nu}\right)_{\alpha}{ }^{\beta}, \\
& \left(\bar{\sigma}^{\mu} \sigma^{\nu}\right)^{\dot{\alpha}}{ }_{\dot{\beta}}=g^{\mu \nu} \delta_{\dot{\beta}}^{\dot{\alpha}}-i\left(\bar{\sigma}^{\mu \nu}\right)^{\dot{\alpha}}{ }_{\dot{\beta}} .
\end{aligned}
$$

The trace of $\sigma$ matrices in eq. (3.19) can be simplified in a systematic way using our code and yields

$$
\operatorname{Tr}\left(\sigma^{\mu} \bar{\sigma}^{\nu} \sigma^{\rho} \bar{\sigma}^{\lambda}\right)=2 g^{\mu \nu} g^{\rho \lambda}-2 g^{\mu \rho} g^{\nu \lambda}+2 g^{\nu \rho} g^{\mu \lambda}+2 i \epsilon^{\mu \nu \rho \lambda} .
$$

Finally we get

$$
F_{L}^{\alpha \beta} F_{L \alpha \beta}=\frac{1}{2} F_{L}^{\mu}{ }_{\mu} F_{L}{ }^{\rho}{ }_{\rho}-\frac{1}{2} F_{L}{ }^{\mu \nu} F_{L \mu \nu}+\frac{1}{2} F_{L}{ }^{\mu \nu} F_{L \nu \mu}-F_{L}{ }^{\mu \nu} F_{L \mu \nu}=-2 F_{L}{ }^{\mu \nu} F_{L \mu \nu} .
$$




\begin{tabular}{|c|cc|cc|ccc|}
\hline Fields & $\mathrm{SU}(2)_{l} \times \mathrm{SU}(2)_{r}$ & $h$ & $\mathrm{SU}(3)_{C}$ & $\mathrm{U}(1)_{\mathrm{EM}}$ & Flavor & $B$ & $L$ \\
\hline$G_{\mathrm{L} \alpha \beta}^{A}$ & $(1,0)$ & -1 & $\mathbf{8}$ & 0 & 1 & 0 & 0 \\
$F_{\mathrm{L} \alpha \beta}$ & $(1,0)$ & -1 & $\mathbf{1}$ & 0 & 1 & 0 & 0 \\
\hline$\nu_{\alpha}$ & $\left(\frac{1}{2}, 0\right)$ & $-\frac{1}{2}$ & $\mathbf{1}$ & 0 & $n_{e}$ & 0 & 1 \\
$e_{\alpha}$ & $\left(\frac{1}{2}, 0\right)$ & $-\frac{1}{2}$ & $\mathbf{1}$ & -1 & $n_{e}$ & 0 & 1 \\
$e_{\mathbb{C} \alpha}$ & $\left(\frac{1}{2}, 0\right)$ & $-\frac{1}{2}$ & $\mathbf{1}$ & 1 & $n_{e}$ & 0 & -1 \\
$u_{\alpha a}$ & $\left(\frac{1}{2}, 0\right)$ & $-\frac{1}{2}$ & $\mathbf{3}$ & $\frac{2}{3}$ & $n_{u}$ & $\frac{1}{3}$ & 0 \\
$u_{\mathbb{C} \alpha}^{a}$ & $\left(\frac{1}{2}, 0\right)$ & $-\frac{1}{2}$ & $\overline{3}$ & $-\frac{2}{3}$ & $n_{u}$ & $-\frac{1}{3}$ & 0 \\
$d_{\alpha a}$ & $\left(\frac{1}{2}, 0\right)$ & $-\frac{1}{2}$ & $\mathbf{3}$ & $-\frac{1}{3}$ & $n_{d}$ & $\frac{1}{3}$ & 0 \\
$d_{\mathbb{C} \alpha}^{a}$ & $\left(\frac{1}{2}, 0\right)$ & $-\frac{1}{2}$ & $\overline{\mathbf{3}}$ & $\frac{1}{3}$ & $n_{d}$ & $-\frac{1}{3}$ & 0 \\
\hline
\end{tabular}

Table 1. The field content of the LEFT, along with their representations under the Lorentz and gauge symmetries. The representation under Lorentz group is denoted by $\left(j_{l}, j_{r}\right)$, while the helicity of the field is given by $h=j_{r}-j_{l}$. The numbers of lepton flavors, u-type quark flavors and d-type quark flavors are denoted as $n_{e}, n_{u}$ and $n_{d}$ respectively with $n_{e}=3, n_{u}=2$ and $n_{d}=3$ in LEFT. Their global charges, baryon number $B$ and lepton number $L$ are also listed. All of the fields are accompanied with their Hermitian conjugates that are omitted, $\left(F_{\mathrm{L} \alpha \beta}\right)^{\dagger}=F_{\mathrm{R} \dot{\alpha} \dot{\beta}}$ for gauge bosons and $\left(\psi_{\alpha}\right)^{\dagger}=\left(\psi^{\dagger}\right)_{\dot{\alpha}}$ for fermions, which are under the conjugate representations of all the groups.

\subsection{Lists of the dim-5 operators}

\section{Class $F_{\mathrm{L}} \psi^{2}: 6$ types}

$$
\begin{aligned}
& \mathcal{O}_{G_{\mathrm{L}} \bar{u}_{\mathrm{R}} u_{\mathrm{L}}}(0,0) \mid i\left(\lambda^{A}\right)_{a}^{b} G_{\mathrm{L}}^{A \mu \nu}\left(\bar{u}_{\mathrm{R} p}^{a} \sigma_{\mu \nu} u_{\mathrm{L} r b}\right) \\
& \mathcal{O}_{G_{\mathrm{L}} \bar{d}_{\mathrm{R}} d_{\mathrm{L}}}(0,0) \mid i\left(\lambda^{A}\right)_{a}^{b} G_{\mathrm{L}}^{A \mu \nu}\left(\bar{d}_{\mathrm{R}}^{a} \sigma_{\mu \nu} d_{\mathrm{L} r b}\right) \\
& \mathcal{O}_{F_{\mathrm{L}} \bar{u}_{\mathrm{R}} u_{\mathrm{L}}}(0,0) \mid i F_{\mathrm{L}}^{\mu \nu}\left(\bar{u}_{\mathrm{R}}^{a} \sigma_{\mu \nu} u_{\mathrm{L} r a}\right) \\
& \mathcal{O}_{F_{\mathrm{L}} \bar{d}_{\mathrm{R}} d_{\mathrm{L}}}(0,0) \mid i F_{\mathrm{L}}^{\mu \nu}\left(\bar{d}_{\mathrm{R} p}^{a} \sigma_{\mu \nu} d_{\mathrm{L} r a}\right) \\
& \mathcal{O}_{F_{\mathrm{L}} \bar{e}_{\mathrm{R}} e_{\mathrm{L}}}(0,0) \mid i F_{\mathrm{L}}^{\mu \nu}\left(\bar{e}_{\mathrm{R} p} \sigma_{\mu \nu} e_{\mathrm{L} r}\right) \\
& \mathcal{O}_{F_{\mathrm{L}} \nu_{\mathrm{L}}^{2}}(0,2) \mid \mathcal{Y}\left[\left[\frac{p}{r}\right] i F_{\mathrm{L}}^{\mu \nu}\left(\nu_{\mathrm{L} p} C \sigma_{\mu \nu} \nu_{\mathrm{L} r}\right)\right.
\end{aligned}
$$

\subsection{Lists of the dim-6 operators}

Class $F_{\mathrm{L}}^{3}: 1$ types

$$
\mathcal{O}_{G_{\mathrm{L}}{ }^{3}}(0,0) \mid f^{A B C} G_{\mathrm{L} \mu \nu}^{A} G_{\mathrm{L}}^{B \mu}{ }_{\lambda} G_{\mathrm{L}}^{C \nu \lambda}
$$




\begin{tabular}{|c|c|c|c|c|c|c|}
\hline \multicolumn{7}{|c|}{ Dim-5 operators } \\
\hline & $(n, \tilde{n})$ & Classes & $\mathcal{N}_{\text {type }}$ & $\mathcal{N}_{\text {term }}$ & $\mathcal{N}_{\text {operator }}$ & Equations \\
\hline 3 & $(2,0)$ & $F_{\mathrm{L}} \psi^{2}+$ h.c. & $10+0+2+0$ & 12 & 76 & $(3.25)-(3.30)$ \\
\hline \multicolumn{7}{|c|}{ Dim-6 operators } \\
\hline & $(n, \tilde{n})$ & Classes & $\mathcal{N}_{\text {type }}$ & $\mathcal{N}_{\text {term }}$ & $\mathcal{N}_{\text {operator }}$ & Equations \\
\hline 3 & $(3,0)$ & $F_{\mathrm{L}}^{3}+$ h.c. & $2+0+0+0$ & 2 & 2 & $(3.31)$ \\
\hline \multirow[t]{2}{*}{4} & $(2,0)$ & $\psi^{4}+$ h.c. & $14+12+8+2$ & 78 & 2362 & $(3.32)-(3.49)$ \\
\hline & $(1,1)$ & $\psi^{2} \psi^{\dagger 2}$ & $40+20+12+0$ & 84 & 3511 & $(3.50)-(3.99)$ \\
\hline & Total & 5 & $56+32+20+2$ & 164 & 5875 & \\
\hline \multicolumn{7}{|c|}{ Dim-7 operators } \\
\hline & $(n, \tilde{n})$ & Classes & $\mathcal{N}_{\text {type }}$ & $\mathcal{N}_{\text {term }}$ & $\mathcal{N}_{\text {operator }}$ & Equations \\
\hline \multirow[t]{3}{*}{4} & $(3,0)$ & $F_{\mathrm{L}}^{2} \psi^{2}+$ h.c. & $16+0+4+0$ & 32 & 216 & $(3.100)-(3.109)$ \\
\hline & $(2,1)$ & $F_{\mathrm{L}}^{2} \psi^{\dagger 2}+h . c$. & $16+0+4+0$ & 24 & 164 & $(3.110)-(3.119)$ \\
\hline & & $\psi^{3} \psi^{\dagger} D+$ h.c. & $50+32+22+0$ & 120 & 4738 & $(3.120)-(3.171)$ \\
\hline \multicolumn{2}{|c|}{ Total } & 6 & $82+32+30+0$ & 176 & 5118 & \\
\hline \multicolumn{7}{|c|}{ Dim-8 operators } \\
\hline & $(n, \tilde{n})$ & Classes & $\mathcal{N}_{\text {type }}$ & $\mathcal{N}_{\text {term }}$ & $\mathcal{N}_{\text {operator }}$ & Equations \\
\hline \multirow[t]{6}{*}{4} & $(4,0)$ & $F_{\mathrm{L}}^{4}+$ h.c. & $8+0+0+0$ & 14 & 14 & $(3.172)-(3.175)$ \\
\hline & $(3,1)$ & $F_{\mathrm{L}}^{2} \psi \psi^{\dagger} D+$ h.c. & $16+0+0+0$ & 16 & 104 & $(3.181)-(3.188)$ \\
\hline & & $\psi^{4} D^{2}+$ h.c. & $14+12+8+2$ & 120 & 3552 & $(3.207)-(3.224)$ \\
\hline & $(2,2)$ & $F_{\mathrm{L}}^{2} F_{\mathrm{R}}^{2}$ & $7+0+0+0$ & 9 & 9 & $(3.176)-(3.180)$ \\
\hline & & $F_{\mathrm{L}} F_{\mathrm{R}} \psi \psi^{\dagger} D$ & $22+0+0+0$ & 30 & 210 & $(3.189)-(3.206)$ \\
\hline & & $\psi^{2} \psi^{\dagger 2} D^{2}$ & $40+20+12+0$ & 168 & 7061 & $(3.225)-(3.274)$ \\
\hline \multirow[t]{2}{*}{5} & $(3,0)$ & $F_{\mathrm{L}} \psi^{4}+$ h.c. & $26+24+14+2$ & 290 & 8024 & $(3.275)-(3.307)$ \\
\hline & $(2,1)$ & $F_{\mathrm{L}} \psi^{2} \psi^{\dagger 2}+$ h.c. & $148+80+44+0$ & 408 & 16084 & $(3.308)-(3.443)$ \\
\hline & Total & 13 & $281+136+78+4$ & 1055 & 35058 & \\
\hline
\end{tabular}

Table 2. The complete statistics of dimension 5, 6, 7, 8 LEFT operators. $N$ in the leftmost column shows the number of particles. $(n, \tilde{n})$ are the numbers of $\epsilon$ and $\tilde{\epsilon}$ in the Lorentz structure. $\mathcal{N}_{\text {type }}, \mathcal{N}_{\text {term }}$, and $\mathcal{N}_{\text {operator }}$ show the number of types, terms and Hermitian operators respectively (independent conjugates are counted), while the numbers under $\mathcal{N}_{\text {type }}$ describe the sum of each possible $|\Delta L|$ types/operators with $\mathcal{N}=\mathcal{N}(|\Delta L|=0)+\mathcal{N}(|\Delta L|=1)+\mathcal{N}(|\Delta L|=2)+\mathcal{N}(|\Delta L|=4)$. The links in the rightmost column refer to the list(s) of the terms in given classes. 


\begin{tabular}{|c|c|c|c|c|c|c|}
\hline \multicolumn{7}{|c|}{ Dim-9 operators } \\
\hline & $(n, \tilde{n})$ & Classes & $\mathcal{N}_{\text {type }}$ & $\mathcal{N}_{\text {term }}$ & $\mathcal{N}_{\text {operator }}$ & Equations \\
\hline \multirow{4}{*}{\multicolumn{2}{|c|}{$4 \quad(3,2)$}} & $F_{\mathrm{L}}^{2} \psi^{2} D^{2}+$ h.c. & $16+0+4+0+0+0$ & 56 & 380 & $(3.444)-(3.453)$ \\
\hline & & $F_{\mathrm{L}} F_{\mathrm{R}} \psi^{2} D^{2}+$ h.c. & $20+0+4+0+0+0$ & 32 & 216 & $(3.454)-(3.465)$ \\
\hline & & $F_{\mathrm{L}}^{2} \psi^{\dagger 2} D^{2}+h . c$. & $16+0+4+0+0+0$ & 32 & 216 & $(3.466)-(3.475)$ \\
\hline & & $\psi^{3} \psi^{\dagger} D^{3}+h . c$. & $50+32+22+0+0+0$ & 234 & 9344 & $(3.516)-(3.567)$ \\
\hline \multirow[t]{5}{*}{5} & $(4,0)$ & $F_{\mathrm{L}}^{3} \psi^{2}+$ h.c. & $22+0+6+0+0+0$ & 84 & 542 & $(3.476)-(3.489)$ \\
\hline & $(3,1)$ & $F_{\mathrm{L}}^{3} \psi^{\dagger 2}+h . c$. & $10+0+2+0+0+0$ & 16 & 108 & $(3.490)-(3.495)$ \\
\hline & & $F_{\mathrm{L}} \psi^{3} \psi^{\dagger} D+$ h.c. & $94+64+38+0+0+0$ & 1150 & 43002 & $(3.568)-(3.665)$ \\
\hline & $\overline{(2,2)}$ & $F_{\mathrm{L}} F_{\mathrm{R}}^{2} \psi^{2}+$ h.c. & $32+0+8+0+0+0$ & 68 & 434 & $(3.496)-(3.515)$ \\
\hline & & $F_{\mathrm{R}} \psi^{3} \psi^{\dagger} D+h . c$. & $94+64+38+0+0+0$ & 862 & 32208 & $(3.666)-(3.763)$ \\
\hline \multirow[t]{2}{*}{6} & $(3,0)$ & $\psi^{6}+$ h.c. & $30+42+24+10+8+2$ & 908 & 88136 & $(3.764)-(3.821)$ \\
\hline & $(2,1)$ & $\psi^{4} \psi^{\dagger 2}+h . c$. & $272+318+184+34+12+0$ & 3012 & 529998 & $(3.822)-(3.1231)$ \\
\hline & Total & 22 & $656+520+334+44+20+2$ & 6454 & 704584 & \\
\hline
\end{tabular}

Table 3. The complete statistics of dimension 9 LEFT operators. The numbers under $\mathcal{N}_{\text {type }}$ describe the sum of each possible $|\Delta L|$ types/operators with $\mathcal{N}=\mathcal{N}(|\Delta L|=0)+\mathcal{N}(|\Delta L|=$ $1)+\mathcal{N}(|\Delta L|=2)+\mathcal{N}(|\Delta L|=3)+\mathcal{N}(|\Delta L|=4)+\mathcal{N}(|\Delta L|=6)$.

\section{Class $\psi^{4}: 18$ types}

$$
\begin{aligned}
& \mathcal{Y}\left[\begin{array}{c}
\frac{p}{r}, \frac{s}{t} \\
t
\end{array}\right]\left(u_{\mathrm{L} s a} C u_{\mathrm{L} t b}\right)\left(\bar{u}_{\mathrm{R} p}^{a} C \bar{u}_{\mathrm{R} r}^{b}\right) \\
& \mathcal{O}_{\bar{u}_{\mathrm{R}}^{2} u_{\mathrm{L}}^{2}}^{(1 \sim 4)}(0,0) \mathcal{Y}\left[\begin{array}{l}
\left.\frac{p}{r}, s\right] \\
\left.\frac{s}{t}\right]
\end{array}\right]\left(\bar{u}_{\mathrm{R} p}^{a} u_{\mathrm{L} s a}\right)\left(\bar{u}_{\mathrm{R} r}^{b} u_{\mathrm{L} t b}\right) \\
& \mathcal{Y}[\overline{p \mid r}, s \mid t]]\left(u_{\mathrm{L} s a} C u_{\mathrm{L} t b}\right)\left(\bar{u}_{\mathrm{R}}^{a} C \bar{u}_{\mathrm{R}}{ }_{r}^{b}\right) \\
& \mathcal{Y}[\overline{p \mid r}, s \mid t]]\left(\bar{u}_{\mathrm{R}}^{a} u_{\mathrm{L} s a}\right)\left(\bar{u}_{\mathrm{R} r}^{b} u_{\mathrm{L} t b}\right)
\end{aligned}
$$

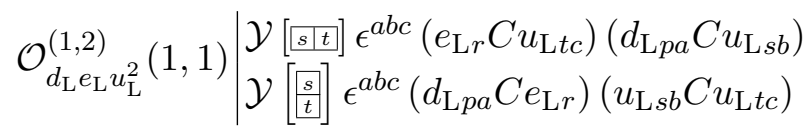

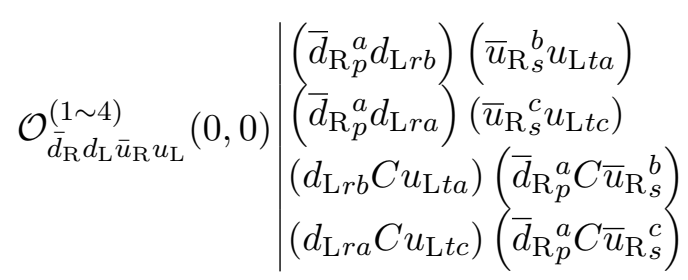

$$
\begin{aligned}
& \mathcal{O}_{\bar{e}_{\mathrm{R}} e_{\mathrm{L}} \bar{u}_{\mathrm{R}} u_{\mathrm{L}}}^{(1,2,0)} \mid \begin{array}{l}
\left(\bar{e}_{\mathrm{R} p} e_{\mathrm{L} r}\right)\left(\bar{u}_{\mathrm{R} s}^{a} u_{\mathrm{L} t a}\right) \\
\left(e_{\mathrm{L} r} C u_{\mathrm{L} t a}\right)\left(\bar{e}_{\mathrm{R} p} C \bar{u}_{\mathrm{R}}{ }_{s}^{a}\right)
\end{array}
\end{aligned}
$$

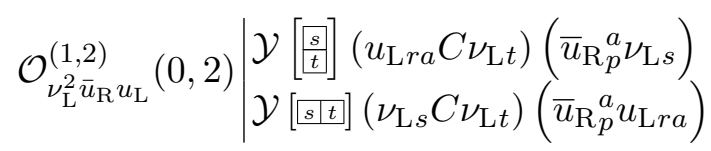




$$
\begin{aligned}
& \mathcal{O}_{d_{\mathrm{L}}^{2} \nu_{\mathrm{L}} u_{\mathrm{L}}}^{(1,2)}(1,1) \mid \begin{array}{l}
\mathcal{Y}[[p \mid r]] \\
\mathcal{Y}\left[\frac{p}{[r}\right] \epsilon^{a b c}\left(d_{\mathrm{L} r b} C \nu_{\mathrm{L} t}\right)\left(d_{\mathrm{L} p a} C u_{\mathrm{L} p s c} C d_{\mathrm{L} r b}\right)\left(u_{\mathrm{L} s c} C \nu_{\mathrm{L} t}\right)
\end{array} \\
& \left.\mathcal{O}_{\bar{d}_{\mathrm{R}} e_{\mathrm{L}} \nu_{\mathrm{L}} u_{\mathrm{L}}}^{(1,2)}(0,2) \mid \begin{array}{l}
\left(u_{\mathrm{L} s a} C \nu_{\mathrm{L} t}\right)\left(\bar{d}_{\mathrm{R}}^{a} e_{\mathrm{L} r}\right) \\
\left(e_{\mathrm{L} r} C \nu_{\mathrm{L} t}\right)\left(\bar{d}_{\mathrm{R} p}^{a} u_{\mathrm{L} s a}\right.
\end{array}\right) \\
& \left.\mathcal{O}_{\bar{d}_{\mathrm{R}} \bar{e}_{\mathrm{R}} \bar{u}_{\mathrm{R}}^{2}(-1,-1)} \mid \begin{array}{l}
\mathcal{Y}[s \mid t] \epsilon_{a b c}\left(\bar{e}_{\mathrm{R} r} C \bar{u}_{\mathrm{R}}^{c}\right)\left(\bar{d}_{\mathrm{R}}^{a} C \bar{u}_{\mathrm{R}}^{b}\right) \\
\mathcal{Y}\left[\begin{array} { l } 
{ s ] } \\
{ \underline { t } ] }
\end{array} \epsilon _ { a b c } ( \overline { d } _ { \mathrm { R } p } ^ { a } C \overline { e } _ { \mathrm { R } r } ) \left(\bar{u}_{\mathrm{R}}^{b} C \bar{u}_{\mathrm{R}}^{c}{ }_{t}^{c}\right.\right.
\end{array}\right) \\
& \mathcal{O}_{d_{\mathrm{L}} \bar{e}_{\mathrm{R}} \nu_{\mathrm{L}} \bar{u}_{\mathrm{R}}}^{(1,2,0)} \mid \begin{array}{l}
\left(\bar{e}_{\mathrm{R} r} d_{\mathrm{L} p a}\right)\left(\bar{u}_{\mathrm{R}}^{a} \nu_{\mathrm{L} t}\right) \\
\left(\bar{e}_{\mathrm{R} r} \nu_{\mathrm{L} t}\right)\left(\bar{u}_{\mathrm{R}}^{a}{ }^{a} d_{\mathrm{L} p a}\right)
\end{array} \\
& \mathcal{O}_{\bar{d}_{\mathrm{R}}^{2} \nu_{\mathrm{L}} \bar{u}_{\mathrm{R}}}^{(1,2)}(-1,1) \mid \begin{array}{l}
\mathcal{Y}\left[[p p] \epsilon_{a b c}\left(\bar{d}_{\mathrm{R}}^{b} \nu_{\mathrm{L} t}\right)\left(\bar{d}_{\mathrm{R}}^{a} C \bar{u}_{\mathrm{R}}^{c}\right)\right. \\
\mathcal{Y}\left[\begin{array}{l}
p \\
\left.\frac{p}{r}\right]
\end{array} \epsilon_{a b c}\left(\bar{u}_{\mathrm{R}}^{c} \nu_{\mathrm{L} t}\right)\left(\bar{d}_{\mathrm{R}}^{a} C \bar{d}_{\mathrm{R}}^{b}\right)\right.
\end{array} \\
& \mathcal{O}_{d_{\mathrm{L}}^{3} \bar{e}_{\mathrm{R}}}(1,-1) \mid \mathcal{Y}\left[\frac{p p r}{s \mid}\right] \epsilon^{a b c}\left(\bar{e}_{\mathrm{R} t} d_{\mathrm{L} s c}\right)\left(d_{\mathrm{L} p a} C d_{\mathrm{L} r b}\right) \\
& \mathcal{Y}\left[\frac{p}{r}, \frac{s}{t}\right]\left(d_{\mathrm{L} s a} C d_{\mathrm{L} t b}\right)\left(\bar{d}_{\mathrm{R} p}^{a} C \bar{d}_{\mathrm{R}}^{b}\right) \\
& \mathcal{O}_{\bar{d}_{\mathrm{R}}^{2} d_{\mathrm{L}}^{2}}^{(1 \sim 4)}(0,0) \mathcal{Y}\left[\frac{p}{r}, \frac{s}{t}\right]\left(\bar{d}_{\mathrm{R}}^{a} d_{\mathrm{L} s a}\right)\left(\bar{d}_{\mathrm{R}}{ }_{r}^{b} d_{\mathrm{L} t b}\right) \\
& \mathcal{Y}[\underline{p \mid r,}, s \mid t]]\left(d_{\mathrm{L} s a} C d_{\mathrm{L} t b}\right)\left(\bar{d}_{\mathrm{R} p}^{a} C \bar{d}_{\mathrm{R} r}^{b}\right) \\
& \mathcal{Y}[\underline{p \mid r,}, \underline{s \mid t]}]\left(\bar{d}_{\mathrm{R} p}^{a} d_{\mathrm{L} s a}\right)\left(\bar{d}_{\mathrm{R} r}^{b} d_{\mathrm{L} t b}\right) \\
& \mathcal{O}_{\bar{d}_{\mathrm{R}} d_{\mathrm{L}} \bar{e}_{\mathrm{R}} e_{\mathrm{L}}}^{(1,2)}(0,0) \mid \begin{array}{l}
\left(\bar{e}_{\mathrm{R} s} e_{\mathrm{L} t}\right)\left(\bar{d}_{\mathrm{R}}^{a} d_{\mathrm{L} r a}\right) \\
\left(d_{\mathrm{L} r a} C e_{\mathrm{L} t}\right)\left(\bar{d}_{\mathrm{R} p}^{a} C \bar{e}_{\mathrm{R} s}\right)
\end{array}
\end{aligned}
$$

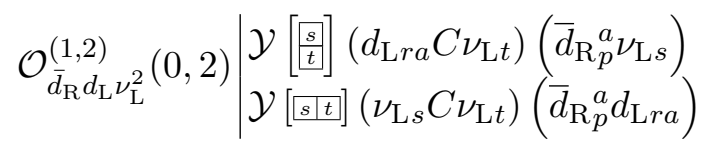

$$
\begin{aligned}
& \mathcal{O}_{\bar{d}_{\mathrm{R}}^{3} e_{\mathrm{L}}}(-1,1) \mid \mathcal{Y}\left[\frac{p r r}{s}\right] \epsilon_{a b c}\left(\bar{d}_{\mathrm{R}}^{c} e_{\mathrm{L} t}\right)\left(\bar{d}_{\mathrm{R}}^{a} C \bar{d}_{\mathrm{R}}^{b}\right) \\
& \mathcal{O}_{\bar{e}_{\mathrm{R}}^{2} e_{\mathrm{L}}^{2}}^{(1,2)}(0,0) \mid \begin{array}{l}
\mathcal{Y}\left[\begin{array}{l}
p, s] \\
{[r, s} \\
t
\end{array}\right]\left(\bar{e}_{\mathrm{R} p} e_{\mathrm{L} s}\right)\left(\bar{e}_{\mathrm{R} r} e_{\mathrm{L} t}\right) \\
\mathcal{Y}[\underline{[p \mid r}, \overline{s \mid t}]\left(e_{\mathrm{L} s} C e_{\mathrm{L} t}\right)\left(\bar{e}_{\mathrm{R} p} C \bar{e}_{\mathrm{R} r}\right)
\end{array}
\end{aligned}
$$

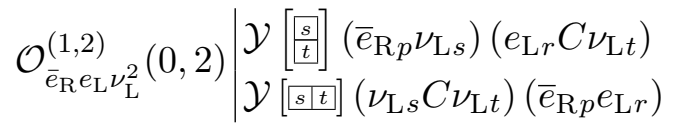

$$
\begin{aligned}
& \mathcal{O}_{\nu_{\mathrm{L}}^{4}}(0,4) \mid \mathcal{Y}\left[\begin{array}{l}
p r r \\
s, t
\end{array}\right]\left(\nu_{\mathrm{L} p} C \nu_{\mathrm{L} r}\right)\left(\nu_{\mathrm{L} s} C \nu_{\mathrm{L} t}\right)
\end{aligned}
$$

Class $\psi^{2} \psi^{\dagger 2}: 50$ types

$$
\mathcal{O}_{u_{\mathrm{L}}^{2} \bar{u}_{\mathrm{L}}^{2}(0,0)}^{(1,2)} \mid \begin{aligned}
& \mathcal{Y}\left[[p|r, s| t]\left(u_{\mathrm{L} p a} C u_{\mathrm{L} r b}\right)\left(\bar{u}_{\mathrm{L} s}^{a} C \bar{u}_{\mathrm{L} t}^{b}\right)\right. \\
& \mathcal{Y}\left[\begin{array}{l}
{\left[\frac{p}{r}, s\right]} \\
t
\end{array}\right]\left(u_{\mathrm{L} p a} C u_{\mathrm{L} r b}\right)\left(\bar{u}_{\mathrm{L} s}^{a} C \bar{u}_{\mathrm{L} t}^{b}\right)
\end{aligned}
$$




$$
\begin{aligned}
& \mathcal{O}_{d_{\mathrm{R}} e_{\mathrm{R}} u_{\mathrm{L}}^{2}}(1,1) \mid \mathcal{Y}\left[\frac{p}{r}\right] \epsilon^{a b c}\left(u_{\mathrm{L} p a} C u_{\mathrm{L} r b}\right)\left(d_{\mathrm{R} s c} C e_{\mathrm{R} t}\right)
\end{aligned}
$$

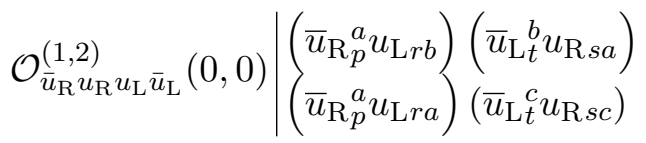

$$
\begin{aligned}
& \mathcal{O}_{d_{\mathrm{R}} \bar{d}_{\mathrm{L}} \bar{u}_{\mathrm{R}} u_{\mathrm{L}}}^{(1,2,0)} \mid\left(\begin{array}{ll}
\left(\bar{d}_{\mathrm{L} t}^{b} d_{\mathrm{R} s a}\right) & \left(\bar{u}_{\mathrm{R}}^{a} u_{\mathrm{L} r b}\right. \\
\left.\bar{u}_{\mathrm{R}}^{a} u_{\mathrm{L} r a}\right) & \left(\bar{d}_{\mathrm{L}}^{c} d_{\mathrm{R} s c}\right.
\end{array}\right) \\
& \mathcal{O}_{e_{\mathrm{R}} \bar{e}_{\mathrm{L}} \bar{u}_{\mathrm{R}} u_{\mathrm{L}}}(0,0) \mid\left(\bar{e}_{\mathrm{L} t} e_{\mathrm{R} s}\right)\left(\bar{u}_{\mathrm{R} p}^{a} u_{\mathrm{L} r a}\right) \\
& \mathcal{O}_{\bar{\nu}_{\mathrm{L}}^{2} \bar{u}_{\mathrm{R}} u_{\mathrm{L}}}(0,-2) \mid \mathcal{Y}\left[s^{s \mid t}\right]\left(\bar{\nu}_{\mathrm{L} s} C \bar{\nu}_{\mathrm{L} t}\right)\left(\bar{u}_{\mathrm{R} p}^{a} u_{\mathrm{L} r a}\right) \\
& \mathcal{O}_{d_{\mathrm{L}} \bar{d}_{\mathrm{L}} u_{\mathrm{L}} \bar{u}_{\mathrm{L}}}^{(1,2)}(0,0) \mid \begin{array}{l}
\left(d_{\mathrm{L} p a} C u_{\mathrm{L} r b}\right)\left(\bar{d}_{\mathrm{L} s}^{a} C \bar{u}_{\mathrm{L} t}^{b}\right) \\
\left(d_{\mathrm{L} p a} C u_{\mathrm{L} r b}\right)\left(\bar{d}_{\mathrm{L} s}^{b} C \bar{u}_{\mathrm{L} t}^{a}\right)
\end{array} \\
& \mathcal{O}_{d_{\mathrm{L}} e_{\mathrm{R}} u_{\mathrm{R}} u_{\mathrm{L}}}(1,1) \mid \epsilon^{a b c}\left(e_{\mathrm{R} s} C u_{\mathrm{R} t c}\right)\left(d_{\mathrm{L} p a} C u_{\mathrm{L} r b}\right) \\
& \mathcal{O}_{d_{\mathrm{R}} d_{\mathrm{L}} \bar{\nu}_{\mathrm{L}} u_{\mathrm{L}}}(1,-1) \mid \epsilon^{a b c}\left(\bar{\nu}_{\mathrm{L} t} d_{\mathrm{R} s c}\right)\left(d_{\mathrm{L} p a} C u_{\mathrm{L} r b}\right) \\
& \mathcal{O}_{\bar{d}_{\mathrm{R}} d_{\mathrm{R}} u_{\mathrm{L}} \bar{u}_{\mathrm{L}}}^{(1,2,0) \mid} \mid \begin{array}{l}
\left(\bar{d}_{\mathrm{R}}^{a} u_{\mathrm{L} r b}\right) \\
\left(\bar{u}_{\mathrm{L}}^{b}{ }_{\mathrm{L}}^{b} u_{\mathrm{L} r a}\right) \\
\left(\bar{u}_{\mathrm{L} t}^{c} d_{\mathrm{R} s c}\right)
\end{array} \\
& \mathcal{O}_{\bar{d}_{\mathrm{R}} e_{\mathrm{R}} \bar{\nu}_{\mathrm{L}} u_{\mathrm{L}}}(0,0) \mid\left(\bar{\nu}_{\mathrm{L} t} e_{\mathrm{R} s}\right)\left(\bar{d}_{\mathrm{R}}^{a} u_{\mathrm{L} r a}\right)
\end{aligned}
$$

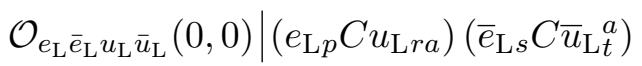

$$
\begin{aligned}
& \mathcal{O}_{d_{\mathrm{R}} e_{\mathrm{L}} u_{\mathrm{R}} u_{\mathrm{L}}}(1,1) \mid \epsilon^{a b c}\left(e_{\mathrm{L} p} C u_{\mathrm{L} r a}\right)\left(d_{\mathrm{R} s b} C u_{\mathrm{R} t c}\right) \\
& \mathcal{O}_{\bar{d}_{\mathrm{L}} e_{\mathrm{L}} \bar{\nu}_{\mathrm{L}} u_{\mathrm{L}}}(0,0) \mid\left(e_{\mathrm{L} p} C u_{\mathrm{L} r a}\right)\left(\bar{d}_{\mathrm{L} s}^{a} C \bar{\nu}_{\mathrm{L} t}\right)
\end{aligned}
$$

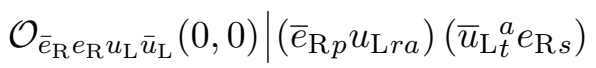

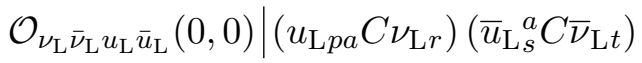

$$
\begin{aligned}
& \mathcal{O}_{\bar{d}_{\mathrm{L}} e_{\mathrm{R}} \nu_{\mathrm{L}} u_{\mathrm{L}}}(0,2) \mid\left(u_{\mathrm{L} p a} C \nu_{\mathrm{L} r}\right)\left(\bar{d}_{\mathrm{L} s}^{a} e_{\mathrm{R} t}\right) \\
& \mathcal{O}_{d_{\mathrm{R}}^{2} \nu_{\mathrm{L}} u_{\mathrm{L}}}(1,1) \mid \mathcal{Y}\left[\begin{array}{l}
s \\
t
\end{array}\right] \epsilon^{a b c}\left(u_{\mathrm{L} p a} C \nu_{\mathrm{L} r}\right)\left(d_{\mathrm{R} s b} C d_{\mathrm{R} t c}\right)
\end{aligned}
$$

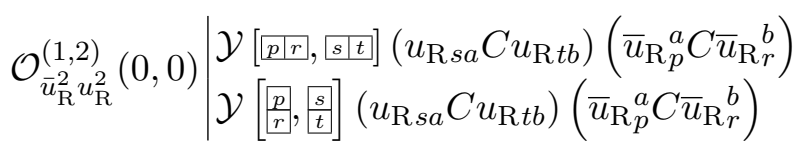

$$
\begin{aligned}
& \mathcal{O}_{\bar{d}_{\mathrm{L}} \bar{e}_{\mathrm{L}} \bar{u}_{\mathrm{R}}^{2}}(-1,-1) \mid \mathcal{Y}\left[\frac{p}{r}\right] \epsilon_{a b c}\left(\bar{u}_{\mathrm{R}}^{a} C \bar{u}_{\mathrm{R} r}^{b}\right)\left(\bar{d}_{\mathrm{L} s}^{c} C \bar{e}_{\mathrm{L} t}\right)
\end{aligned}
$$




$$
\begin{aligned}
& \mathcal{O}_{d_{\mathrm{L}} \bar{d}_{\mathrm{L}} \bar{u}_{\mathrm{R}} u_{\mathrm{R}}}^{(1,2,0)}\left|\begin{array}{ll}
\left(\bar{u}_{\mathrm{R}}{ }_{r}^{b} d_{\mathrm{L} p a}\right) & \left(\bar{d}_{\mathrm{L}}{ }^{a} u_{\mathrm{R} t b}\right) \\
\left(\bar{u}_{\mathrm{R}}{ }_{r}^{a} d_{\mathrm{L} p a}\right) & \left(\bar{d}_{\mathrm{L}_{s}}^{c} u_{\mathrm{R} t c}\right)
\end{array}\right|
\end{aligned}
$$

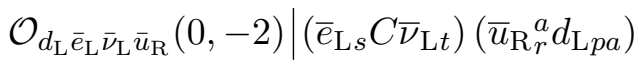

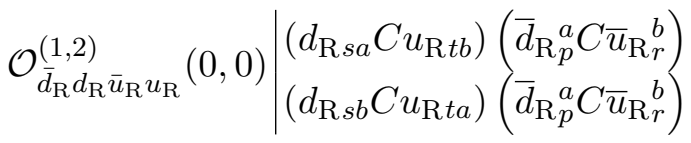

$$
\begin{aligned}
& \mathcal{O}_{\bar{d}_{\mathrm{R}} \bar{d}_{\mathrm{L}} \bar{\nu}_{\mathrm{L}} \bar{u}_{\mathrm{R}}}(-1,-1) \mid \epsilon_{a b c}\left(\bar{d}_{\mathrm{L}}^{c} C \bar{\nu}_{\mathrm{L} t}\right)\left(\bar{d}_{\mathrm{R}}^{a} C \bar{u}_{\mathrm{R}}^{b}\right)
\end{aligned}
$$

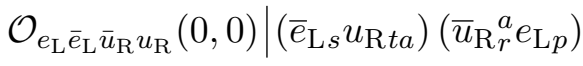

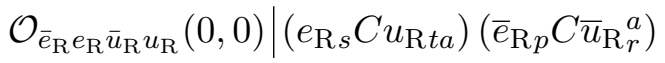

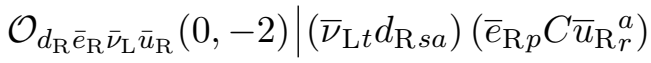

$$
\begin{aligned}
& \mathcal{O}_{\nu_{\mathrm{L}} \bar{\nu}_{\mathrm{L}} \bar{u}_{\mathrm{R}} u_{\mathrm{R}}}(0,0) \mid\left(\bar{\nu}_{\mathrm{L} t} u_{\mathrm{R} s a}\right)\left(\bar{u}_{\mathrm{R} p}^{a} \nu_{\mathrm{L} r}\right) \\
& \mathcal{O}_{\bar{d}_{\mathrm{L}}^{2} \nu_{\mathrm{L}} \bar{u}_{\mathrm{R}}}(-1,1) \mid \mathcal{Y}\left[\begin{array}{l}
s \\
\underline{t}
\end{array}\right] \epsilon_{a b c}\left(\bar{u}_{\mathrm{R} p}^{a} \nu_{\mathrm{L} r}\right)\left(\bar{d}_{\mathrm{L} s}^{b} C \bar{d}_{\mathrm{L} t}^{c}\right) \\
& \mathcal{O}_{d_{\mathrm{R}} \bar{e}_{\mathrm{L}} \nu_{\mathrm{L}} \bar{u}_{\mathrm{R}}}(0,0) \mid\left(\bar{e}_{\mathrm{L} t} d_{\mathrm{R} s a}\right)\left(\bar{u}_{\mathrm{R} p}^{a} \nu_{\mathrm{L} r}\right)
\end{aligned}
$$

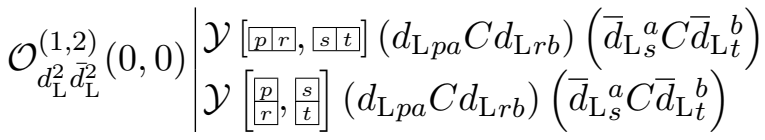

$$
\begin{aligned}
& \mathcal{O}_{d_{\mathrm{R}} d_{\mathrm{L}}^{2} \bar{e}_{\mathrm{L}}}(1,-1) \mid \mathcal{Y}\left[\frac{p}{r}\right] \epsilon^{a b c}\left(\bar{e}_{\mathrm{L} t} d_{\mathrm{R} s c}\right)\left(d_{\mathrm{L} p a} C d_{\mathrm{L} r b}\right)
\end{aligned}
$$

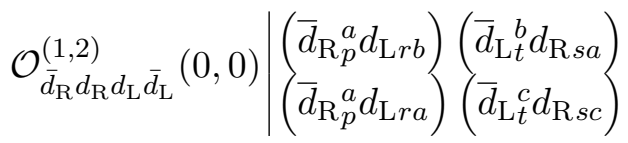

$$
\begin{aligned}
& \mathcal{O}_{\bar{d}_{\mathrm{R}} d_{\mathrm{L}} e_{\mathrm{R}} \bar{e}_{\mathrm{L}}}(0,0) \mid\left(\bar{e}_{\mathrm{L} t} e_{\mathrm{R} s}\right)\left(\bar{d}_{\mathrm{R} p}^{a} d_{\mathrm{L} r a}\right) \\
& \left.\mathcal{O}_{\bar{d}_{\mathrm{R}} d_{\mathrm{L}} \bar{\nu}_{\mathrm{L}}^{2}}(0,-2) \mid \mathcal{Y}[s \mid t]\right]\left(\bar{\nu}_{\mathrm{L} s} C \bar{\nu}_{\mathrm{L} t}\right)\left(\bar{d}_{\mathrm{R}}{ }_{p}^{a} d_{\mathrm{L} r a}\right) \\
& \mathcal{O}_{d_{\mathrm{L}} \bar{d}_{\mathrm{L}} e_{\mathrm{L}} \bar{e}_{\mathrm{L}}}(0,0) \mid\left(d_{\mathrm{L} p a} C e_{\mathrm{L} r}\right)\left(\bar{d}_{\mathrm{L}_{s}^{a}}^{a} C \bar{e}_{\mathrm{L} t}\right) \\
& \mathcal{O}_{d_{\mathrm{L}} \bar{d}_{\mathrm{L}} \bar{e}_{\mathrm{R}} e_{\mathrm{R}}}(0,0) \mid\left(\bar{e}_{\mathrm{R} r} d_{\mathrm{L} p a}\right)\left(\bar{d}_{\mathrm{L}}^{a} e_{\mathrm{R} t}\right) \\
& \mathcal{O}_{d_{\mathrm{R}}^{2} d_{\mathrm{L}} \bar{e}_{\mathrm{R}}}(1,-1) \mid \mathcal{Y}\left[\begin{array}{c}
s \\
\underline{t}
\end{array}\right] \epsilon^{a b c}\left(\bar{e}_{\mathrm{R} r} d_{\mathrm{L} p a}\right)\left(d_{\mathrm{R} s b} C d_{\mathrm{R} t c}\right) \\
& \mathcal{O}_{d_{\mathrm{L}} \bar{d}_{\mathrm{L}} \nu_{\mathrm{L}} \bar{\nu}_{\mathrm{L}}}(0,0) \mid\left(d_{\mathrm{L} p a} C \nu_{\mathrm{L} r}\right)\left(\bar{d}_{\mathrm{L}}^{a} C \bar{\nu}_{\mathrm{L} t}\right)
\end{aligned}
$$




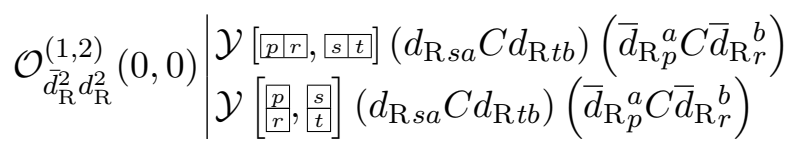

$$
\begin{aligned}
& \mathcal{O}_{\bar{d}_{\mathrm{R}} d_{\mathrm{R}} e_{\mathrm{L}} \bar{e}_{\mathrm{L}}}(0,0) \mid\left(\bar{e}_{\mathrm{L} t} d_{\mathrm{R} s a}\right)\left(\bar{d}_{\mathrm{R} p}^{a} e_{\mathrm{L} r}\right) \\
& \mathcal{O}_{\bar{d}_{\mathrm{R}} d_{\mathrm{R}} \bar{e}_{\mathrm{R}} e_{\mathrm{R}}}(0,0) \mid\left(d_{\mathrm{R} s a} C e_{\mathrm{R} t}\right)\left(\bar{d}_{\mathrm{R} p}^{a} C \bar{e}_{\mathrm{R} r}\right) \\
& \mathcal{O}_{\bar{d}_{\mathrm{R}} d_{\mathrm{R}} \nu_{\mathrm{L}} \bar{\nu}_{\mathrm{L}}}(0,0) \mid\left(\bar{\nu}_{\mathrm{L} t} d_{\mathrm{R} s a}\right)\left(\bar{d}_{\mathrm{R}}^{a} \nu_{\mathrm{L} r}\right) \\
& \mathcal{O}_{e_{\mathrm{L}}^{2} \bar{e}_{\mathrm{L}}^{2}}(0,0) \mid \mathcal{Y}[\underline{p \mid r}, \underline{s \mid t}]\left(e_{\mathrm{L} p} C e_{\mathrm{L} r}\right)\left(\bar{e}_{\mathrm{L} s} C \bar{e}_{\mathrm{L} t}\right) \\
& \mathcal{O}_{\bar{e}_{\mathrm{R}} e_{\mathrm{R}} e_{\mathrm{L}} \bar{e}_{\mathrm{L}}}(0,0) \mid\left(\bar{e}_{\mathrm{R} p} e_{\mathrm{L} r}\right)\left(\bar{e}_{\mathrm{L} t} e_{\mathrm{R} s}\right) \\
& \mathcal{O}_{\bar{e}_{\mathrm{R}} e_{\mathrm{L}} \bar{\nu}_{\mathrm{L}}^{2}}(0,-2) \mid \mathcal{Y}[\underline{s \mid t}]\left(\bar{\nu}_{\mathrm{L} s} C \bar{\nu}_{\mathrm{L} t}\right)\left(\bar{e}_{\mathrm{R} p} e_{\mathrm{L} r}\right) \\
& \mathcal{O}_{e_{\mathrm{L}} \bar{e}_{\mathrm{L}} \nu_{\mathrm{L}} \bar{\nu}_{\mathrm{L}}}(0,0) \mid\left(e_{\mathrm{L} p} C \nu_{\mathrm{L} r}\right)\left(\bar{e}_{\mathrm{L} s} C \bar{\nu}_{\mathrm{L} t}\right) \\
& \mathcal{O}_{\bar{e}_{\mathrm{R}}^{2} e_{\mathrm{R}}^{2}}(0,0) \mid \mathcal{Y}\left[[p \mid r, \overline{s \mid t}]\left(e_{\mathrm{R} s} C e_{\mathrm{R} t}\right)\left(\bar{e}_{\mathrm{R} p} C \bar{e}_{\mathrm{R} r}\right)\right. \\
& \mathcal{O}_{\bar{e}_{\mathrm{R}} e_{\mathrm{R}} \nu_{\mathrm{L}} \bar{\nu}_{\mathrm{L}}}(0,0) \mid\left(\bar{e}_{\mathrm{R} p} \nu_{\mathrm{L} r}\right)\left(\bar{\nu}_{\mathrm{L} t} e_{\mathrm{R} s}\right) \\
& \left.\mathcal{O}_{\nu_{\mathrm{L}}^{2} \bar{\nu}_{\mathrm{L}}^{2}}(0,0) \mid \mathcal{Y}[\overline{p r r},, s \mid t]\right]\left(\nu_{\mathrm{L} p} C \nu_{\mathrm{L} r}\right)\left(\bar{\nu}_{\mathrm{L} s} C \bar{\nu}_{\mathrm{L} t}\right)
\end{aligned}
$$

\subsection{Lists of the dim-7 operators}

\section{Class $F_{\mathrm{L}}{ }^{2} \psi^{2}: 10$ types}

$$
\begin{aligned}
& \mathcal{O}_{G_{\mathrm{L}}{ }^{2} \bar{u}_{\mathrm{R}} u_{\mathrm{L}}}^{(1 \sim 3)}(0,0) \mid \begin{array}{l}
G_{\mathrm{L} \mu \nu}^{A} G_{\mathrm{L}}^{A \mu \nu}\left(\bar{u}_{\mathrm{R} p}{ }^{a} u_{\mathrm{L} r a}\right) \\
d^{A B C}\left(\lambda^{C}\right)_{a}^{b} G_{\mathrm{L} \mu \nu}^{A} G_{\mathrm{L}}^{B \mu \nu}\left(\bar{u}_{\mathrm{R} p}{ }^{a} u_{\mathrm{L} r b}\right)
\end{array} \\
& i f^{A B C}\left(\lambda^{C}\right)_{a}^{b} G_{\mathrm{L} \mu \nu}^{A} G_{\mathrm{L}}^{B \mu}{ }_{\lambda}\left(\bar{u}_{\mathrm{R}}{ }_{p}^{a} \sigma^{\nu \lambda} u_{\mathrm{L} r b}\right) \\
& \mathcal{O}_{G_{\mathrm{L}}{ }^{2} \bar{d}_{\mathrm{R}} d_{\mathrm{L}}}^{(1 \sim 3,0)}(0,0) \mid \begin{array}{l}
G_{\mathrm{L} \mu \nu}^{A} G_{\mathrm{L}}^{A \mu \nu}\left(\bar{d}_{\mathrm{R} p}^{a} d_{\mathrm{L} r a}\right) \\
d^{A B C}\left(\lambda^{C}\right)_{a}^{b} G_{\mathrm{L} \mu \nu}^{A} G_{\mathrm{L}}^{B \mu \nu}\left(\bar{d}_{\mathrm{R} p}^{a} d_{\mathrm{L} r b}\right) \\
i f^{A B C}\left(\lambda^{C}\right)_{a}^{b} G_{\mathrm{L} \mu \nu}^{A} G_{\mathrm{L}}^{B \mu}{ }_{\lambda}\left(\bar{d}_{\mathrm{R} p}^{a} \sigma^{\nu \lambda} d_{\mathrm{L} r b}\right)
\end{array} \\
& \mathcal{O}_{G_{\mathrm{L}}{ }^{2} \bar{e}_{\mathrm{R}} e_{\mathrm{L}}}(0,0) \mid\left(\bar{e}_{\mathrm{R} p} e_{\mathrm{L} r}\right) G_{\mathrm{L} \mu \nu}^{A} G_{\mathrm{L}}^{A \mu \nu} \\
& \mathcal{O}_{G_{\mathrm{L}}{ }^{2} \nu_{\mathrm{L}}^{2}}(0,2) \mid \mathcal{Y}[p p r]\left(\nu_{\mathrm{L} p} C \nu_{\mathrm{L} r}\right) G_{\mathrm{L} \mu \nu}^{A} G_{\mathrm{L}}^{A \mu \nu} \\
& \mathcal{O}_{F_{\mathrm{L}} G_{\mathrm{L}} \bar{u}_{\mathrm{R}} u_{\mathrm{L}}}^{(1,2,0)} \mid \begin{array}{l}
\left(\lambda^{A}\right)_{a}^{b} F_{\mathrm{L} \mu \nu} G_{\mathrm{L}}^{A \mu \nu}\left(\bar{u}_{\mathrm{R}}^{a} u_{\mathrm{L} r b}\right) \\
i\left(\lambda^{A}\right)_{a}^{b} F_{\mathrm{L} \mu \nu} G_{\mathrm{L}}^{A \mu}{ }_{\lambda}\left(\bar{u}_{\mathrm{R}}^{a}{ }_{p} \sigma^{\nu \lambda} u_{\mathrm{L} r b}\right)
\end{array} \\
& \mathcal{O}_{F_{\mathrm{L}} G_{\mathrm{L}} \bar{d}_{\mathrm{R}} d_{\mathrm{L}}}^{(1,2)}(0,0) \mid \begin{array}{l}
\left(\lambda^{A}\right)_{a}^{b} F_{\mathrm{L} \mu \nu} G_{\mathrm{L}}^{A \mu \nu}\left(\bar{d}_{\mathrm{R} p}^{a} d_{\mathrm{L} r b}\right) \\
i\left(\lambda^{A}\right)_{a}^{b} F_{\mathrm{L} \mu \nu} G_{\mathrm{L}}^{A \mu}{ }_{\lambda}\left(\bar{d}_{\mathrm{R} p}^{a} \sigma^{\nu \lambda} d_{\mathrm{L} r b}\right)
\end{array}
\end{aligned}
$$




$$
\begin{aligned}
& \mathcal{O}_{F_{\mathrm{L}}{ }^{2} \bar{u}_{\mathrm{R}} u_{\mathrm{L}}}(0,0) \mid F_{\mathrm{L} \mu \nu} F_{\mathrm{L}}^{\mu \nu}\left(\bar{u}_{\mathrm{R} p}^{a} u_{\mathrm{L} r a}\right) \\
& \mathcal{O}_{F_{\mathrm{L}}{ }^{2} \bar{d}_{\mathrm{R}} d_{\mathrm{L}}}(0,0) \mid F_{\mathrm{L} \mu \nu} F_{\mathrm{L}}{ }^{\mu \nu}\left(\bar{d}_{\mathrm{R} p}^{a} d_{\mathrm{L} r a}\right) \\
& \mathcal{O}_{F_{\mathrm{L}}{ }^{2} \bar{e}_{\mathrm{R}} e_{\mathrm{L}}}(0,0) \mid F_{\mathrm{L} \mu \nu} F_{\mathrm{L}}{ }^{\mu \nu}\left(\bar{e}_{\mathrm{R} p} e_{\mathrm{L} r}\right) \\
& \mathcal{O}_{F_{\mathrm{L}}{ }^{2} \nu_{\mathrm{L}}^{2}}(0,2) \mid \mathcal{Y}[\overline{p r r}] F_{\mathrm{L} \mu \nu}\left(\nu_{\mathrm{L} p} C \nu_{\mathrm{L} r}\right) F_{\mathrm{L}}^{\mu \nu}
\end{aligned}
$$

\section{Class $F_{\mathrm{L}}^{2} \psi^{\dagger 2}: 10$ types}

$$
\begin{aligned}
& \mathcal{O}_{G_{\mathrm{L}}{ }^{2} u_{\mathrm{R}} \bar{u}_{\mathrm{L}}}^{(1,2)}(0,0) \mid \begin{array}{l}
G_{\mathrm{L} \mu \nu}^{A} G_{\mathrm{L}}^{A \mu \nu}\left(\bar{u}_{\mathrm{L} r}^{a} u_{\mathrm{R} p a}\right) \\
d^{A B C}\left(\lambda^{C}\right)_{b}^{a} G_{\mathrm{L} \mu \nu}^{A} G_{\mathrm{L}}^{B \mu \nu}\left(\bar{u}_{\mathrm{L} r}^{b} u_{\mathrm{R} p a}\right)
\end{array} \\
& \mathcal{O}_{G_{\mathrm{L}}{ }^{2} d_{\mathrm{R}} \bar{d}_{\mathrm{L}}}^{(1,2)}(0,0) \mid \begin{array}{l}
G_{\mathrm{L} \mu \nu}^{A} G_{\mathrm{L}}^{A \mu \nu}\left(\bar{d}_{\mathrm{L} r}^{a} d_{\mathrm{R} p a}\right) \\
d^{A B C}\left(\lambda^{C}\right)_{b}^{a} G_{\mathrm{L} \mu \nu}^{A} G_{\mathrm{L}}^{B \mu \nu}\left(\bar{d}_{\mathrm{L} r}^{b} d_{\mathrm{R} p a}\right)
\end{array} \\
& \mathcal{O}_{G_{\mathrm{L}}{ }^{2} e_{\mathrm{R}} \bar{e}_{\mathrm{L}}}(0,0) \mid\left(\bar{e}_{\mathrm{L} r} e_{\mathrm{R} p}\right) G_{\mathrm{L} \mu \nu}^{A} G_{\mathrm{L}}^{A \mu \nu} \\
& \mathcal{O}_{G_{\mathrm{L}}{ }^{2} \bar{\nu}_{\mathrm{L}}^{2}}(0,-2) \mid \mathcal{Y}[\overline{p r r}]\left(\bar{\nu}_{\mathrm{L} p} C \bar{\nu}_{\mathrm{L} r}\right) G_{\mathrm{L} \mu \nu}^{A} G_{\mathrm{L}}^{A \mu \nu} \\
& \mathcal{O}_{F_{\mathrm{L}} G_{\mathrm{L}} u_{\mathrm{R}} \bar{u}_{\mathrm{L}}}(0,0) \mid\left(\lambda^{A}\right)_{b}^{a} F_{\mathrm{L} \mu \nu} G_{\mathrm{L}}^{A \mu \nu}\left(\bar{u}_{\mathrm{L}}^{b} u_{\mathrm{R} p a}\right) \\
& \mathcal{O}_{F_{\mathrm{L}} G_{\mathrm{L}} d_{\mathrm{R}} \bar{d}_{\mathrm{L}}}(0,0) \mid\left(\lambda^{A}\right)_{b}^{a} F_{\mathrm{L} \mu \nu} G_{\mathrm{L}}^{A \mu \nu}\left(\bar{d}_{\mathrm{L} r}^{b} d_{\mathrm{R} p a}\right) \\
& \mathcal{O}_{F_{\mathrm{L}}{ }^{2} u_{\mathrm{R}} \bar{u}_{\mathrm{L}}}(0,0) \mid F_{\mathrm{L} \mu \nu} F_{\mathrm{L}}{ }^{\mu \nu}\left(\bar{u}_{\mathrm{L} r}{ }^{a} u_{\mathrm{R} p a}\right) \\
& \mathcal{O}_{F_{\mathrm{L}}^{2} d_{\mathrm{R}} \bar{d}_{\mathrm{L}}}(0,0) \mid F_{\mathrm{L} \mu \nu} F_{\mathrm{L}}^{\mu \nu}\left(\bar{d}_{\mathrm{L} r}^{a} d_{\mathrm{R} p a}\right) \\
& \mathcal{O}_{F_{\mathrm{L}}{ }^{2} e_{\mathrm{R}} \bar{e}_{\mathrm{L}}}(0,0) \mid F_{\mathrm{L} \mu \nu} F_{\mathrm{L}}^{\mu \nu}\left(\bar{e}_{\mathrm{L} r} e_{\mathrm{R} p}\right) \\
& \mathcal{O}_{F_{\mathrm{L}}{ }^{2} \bar{\nu}_{\mathrm{L}}^{2}}(0,-2) \mid \mathcal{Y}[\underline{p p r}] F_{\mathrm{L} \mu \nu} F_{\mathrm{L}}{ }^{\mu \nu}\left(\bar{\nu}_{\mathrm{L} p} C \bar{\nu}_{\mathrm{L} r}\right)
\end{aligned}
$$

\section{Class $\psi^{3} \psi^{\dagger} D: 52$ types}

$$
\begin{aligned}
& \mathcal{O}_{\bar{u}_{\mathrm{R}} u_{\mathrm{L}}^{2} \bar{u}_{\mathrm{L}} D}^{(1,2,0)} \mid \begin{array}{l}
\mathcal{Y}\left[\frac{r}{s]}\right] i\left(u_{\mathrm{L} r b} C D^{\mu} u_{\mathrm{L} s a}\right)\left(\bar{u}_{\mathrm{R}}^{a} \gamma_{\mu} C \bar{u}_{\mathrm{L} t}^{b}\right) \\
\mathcal{Y}[\overline{r s s}] i\left(u_{\mathrm{L} r b} C D^{\mu} u_{\mathrm{L} s a}\right)\left(\bar{u}_{\mathrm{R}}^{a} \gamma_{\mu} C \bar{u}_{\mathrm{L} t}^{b}\right)
\end{array} \\
& \mathcal{O}_{d_{\mathrm{L}} e_{\mathrm{R}} u_{\mathrm{L}}^{2} D}(1,1) \mid \mathcal{Y}[\overline{r r s}] i \epsilon^{a b c}\left(d_{\mathrm{L} p a} C \gamma_{\mu} e_{\mathrm{R} t}\right)\left(u_{\mathrm{L} r b} C D^{\mu} u_{\mathrm{L} s c}\right) \\
& \mathcal{O}_{d_{\mathrm{R}} e_{\mathrm{L}} u_{\mathrm{L}}^{2} D}(1,1) \mid \mathcal{Y}[\overline{r r s}] i \epsilon^{a b c}\left(e_{\mathrm{L} p} C \gamma_{\mu} d_{\mathrm{R} t c}\right)\left(u_{\mathrm{L} r a} C D^{\mu} u_{\mathrm{L} s b}\right)
\end{aligned}
$$

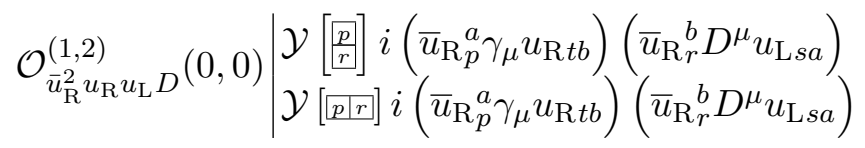

$$
\begin{aligned}
& \mathcal{O}_{d_{\mathrm{L}} \overline{\mathrm{L}}_{\mathrm{L}} \bar{u}_{\mathrm{R}} u_{\mathrm{L}} D}^{(0,0)} \mid \begin{array}{ll}
i\left(\bar{d}_{\mathrm{L} t}^{a} \gamma_{\mu} d_{\mathrm{L} p a}\right) & \left(\bar{u}_{\mathrm{R} r}^{b} D^{\mu} u_{\mathrm{L} s b}\right) \\
i\left(\bar{d}_{\mathrm{L} t}^{c} \gamma_{\mu} d_{\mathrm{L} p a}\right) & \left(\bar{u}_{\mathrm{R} r}^{a} D^{\mu} u_{\mathrm{L} s c}\right)
\end{array}
\end{aligned}
$$




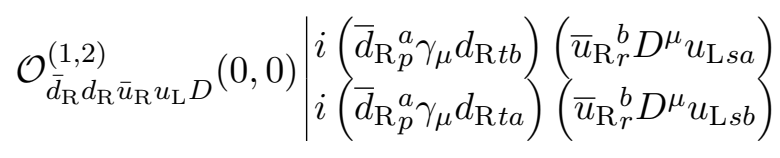

$$
\begin{aligned}
& \mathcal{O}_{e_{\mathrm{L}} \bar{e}_{\mathrm{L}} \bar{u}_{\mathrm{R}} u_{\mathrm{L}} D}(0,0) \mid i\left(\bar{e}_{\mathrm{L} t} \gamma_{\mu} e_{\mathrm{L} p}\right)\left(\bar{u}_{\mathrm{R} r}^{a} D^{\mu} u_{\mathrm{L} s a}\right) \\
& \mathcal{O}_{\bar{e}_{\mathrm{R}} e_{\mathrm{R}} \bar{u}_{\mathrm{R}} u_{\mathrm{L}} D}(0,0) \mid i\left(\bar{e}_{\mathrm{R} p} \gamma_{\mu} e_{\mathrm{R} t}\right)\left(\bar{u}_{\mathrm{R} r}^{a} D^{\mu} u_{\mathrm{L} s a}\right) \\
& \mathcal{O}_{\nu_{\mathrm{L}} \bar{\nu}_{\mathrm{L}} \bar{u}_{\mathrm{R}} u_{\mathrm{L}} D}(0,0) \mid i\left(u_{\mathrm{L} r a} C D^{\mu} \nu_{\mathrm{L} s}\right)\left(\bar{u}_{\mathrm{R}}^{a} \gamma_{\mu} C \bar{\nu}_{\mathrm{L} t}\right) \\
& \mathcal{O}_{d_{\mathrm{L}}^{2} \bar{\nu}_{\mathrm{L}} u_{\mathrm{L}} D}(1,-1) \mid \mathcal{Y}[\overline{p p r}] i \epsilon^{a b c}\left(\bar{\nu}_{\mathrm{L} t} \gamma_{\mu} d_{\mathrm{L} p a}\right)\left(d_{\mathrm{L} r b} C D^{\mu} u_{\mathrm{L} s c}\right) \\
& \mathcal{O}_{\bar{d}_{\mathrm{R}} d_{\mathrm{L}} u_{\mathrm{L}} \bar{u}_{\mathrm{L}} D}^{(0,0)} \mid \begin{array}{l}
i\left(d_{\mathrm{L} r b} C D^{\mu} u_{\mathrm{L} s a}\right)\left(\bar{d}_{\mathrm{R}}^{a} \gamma_{\mu} C \bar{u}_{\mathrm{L} t}^{b}\right) \\
i\left(d_{\mathrm{L} r a} C D^{\mu} u_{\mathrm{L} s c}\right)\left(\bar{d}_{\mathrm{R}}^{a} \gamma_{\mu} C \bar{u}_{\mathrm{L} t}^{c}\right)
\end{array} \\
& \mathcal{O}_{d_{\mathrm{L}} e_{\mathrm{L}} u_{\mathrm{R}} u_{\mathrm{L}} D}(1,1) \mid i \epsilon^{a b c}\left(d_{\mathrm{L} p a} C \gamma_{\mu} u_{\mathrm{R} t c}\right)\left(e_{\mathrm{L} r} C D^{\mu} u_{\mathrm{L} s b}\right) \\
& \mathcal{O}_{d_{\mathrm{R}} d_{\mathrm{L}} \nu_{\mathrm{L}} u_{\mathrm{L}} D}(1,1) \mid i \epsilon^{a b c}\left(d_{\mathrm{L} p a} C \gamma_{\mu} d_{\mathrm{R} t c}\right)\left(u_{\mathrm{L} r b} C D^{\mu} \nu_{\mathrm{L} s}\right) \\
& \mathcal{O}_{\bar{d}_{\mathrm{R}} e_{\mathrm{L}} \bar{\nu}_{\mathrm{L}} u_{\mathrm{L}} D}(0,0) \mid i\left(e_{\mathrm{L} r} C D^{\mu} u_{\mathrm{L} s a}\right)\left(\bar{d}_{\mathrm{R}}^{a} \gamma_{\mu} C \bar{\nu}_{\mathrm{L} t}\right) \\
& \mathcal{O}_{\bar{d}_{\mathrm{R}} e_{\mathrm{R}} \nu_{\mathrm{L}} u_{\mathrm{L}} D}(0,2) \mid i\left(\bar{d}_{\mathrm{R} p}^{a} \gamma_{\mu} e_{\mathrm{R} t}\right)\left(u_{\mathrm{L} r a} C D^{\mu} \nu_{\mathrm{L} s}\right) \\
& \mathcal{O}_{\bar{e}_{\mathrm{R}} e_{\mathrm{L}} u_{\mathrm{L}} \bar{u}_{\mathrm{L}} D}(0,0) \mid i\left(e_{\mathrm{L} r} C D^{\mu} u_{\mathrm{L} s a}\right)\left(\bar{e}_{\mathrm{R} p} \gamma_{\mu} C \bar{u}_{\mathrm{L} t}^{a}\right) \\
& \mathcal{O}_{\bar{d}_{\mathrm{L}} e_{\mathrm{L}} \nu_{\mathrm{L}} u_{\mathrm{L}} D}(0,2) \mid i\left(\bar{d}_{\mathrm{L} t}^{a} \gamma_{\mu} e_{\mathrm{L} p}\right)\left(u_{\mathrm{L} r a} C D^{\mu} \nu_{\mathrm{L} s}\right) \\
& \mathcal{O}_{\nu_{\mathrm{L}}^{2} u_{\mathrm{L}} \bar{u}_{\mathrm{L}} D}(0,2) \mid \mathcal{Y}\left[\frac{r}{s}\right] i\left(\nu_{\mathrm{L} r} C D^{\mu} \nu_{\mathrm{L} s}\right)\left(\bar{u}_{\mathrm{L} t}^{a} \gamma_{\mu} u_{\mathrm{L} p a}\right) \\
& \mathcal{O}_{\bar{d}_{\mathrm{R}} \bar{e}_{\mathrm{L}} \bar{u}_{\mathrm{R}}^{2} D}(-1,-1) \mid \mathcal{Y}[[r s]] i \epsilon_{a b c}\left(\bar{d}_{\mathrm{R} p}^{a} \gamma_{\mu} C \bar{e}_{\mathrm{L} t}\right)\left(\bar{u}_{\mathrm{R} r}^{b} C D^{\mu} \bar{u}_{\mathrm{R}}^{c}\right) \\
& \mathcal{O}_{\bar{d}_{\mathrm{L}} \bar{e}_{\mathrm{R}} \bar{u}_{\mathrm{R}}^{2} D}(-1,-1) \mid \mathcal{Y}[[r] s] i \epsilon_{a b c}\left(\bar{u}_{\mathrm{R} r}^{a} C D^{\mu} \bar{u}_{\mathrm{R} s}^{b}\right)\left(\bar{e}_{\mathrm{R} p} \gamma_{\mu} C \bar{d}_{\mathrm{L} t}^{c}\right) \\
& \mathcal{O}_{\bar{d}_{\mathrm{R}} d_{\mathrm{L}} \bar{u}_{\mathrm{R}} u_{\mathrm{R}} D}^{(0,0)} \mid \begin{array}{l}
i\left(\bar{d}_{\mathrm{R}}^{a} \gamma_{\mu}{u_{\mathrm{R} t a}}\right)\left(D^{\mu} \bar{u}_{\mathrm{R}}^{b} d_{\mathrm{L} r b}\right) \\
i\left(D^{\mu} \bar{u}_{\mathrm{R} s}^{c} d_{\mathrm{L} r a}\right)\left(\bar{d}_{\mathrm{R} p}^{a} \gamma_{\mu} u_{\mathrm{R} t c}\right)
\end{array} \\
& \mathcal{O}_{d_{\mathrm{L}} \bar{e}_{\mathrm{R}} \bar{\nu}_{\mathrm{L}} \bar{u}_{\mathrm{R}} D}(0,-2) \mid i\left(\bar{\nu}_{\mathrm{L} t} \gamma_{\mu} d_{\mathrm{L} p a}\right)\left(\bar{e}_{\mathrm{R} r} C D^{\mu} \bar{u}_{\mathrm{R}}^{a}\right) \\
& \mathcal{O}_{d_{\mathrm{L}} \bar{e}_{\mathrm{L}} \nu_{\mathrm{L}} \bar{u}_{\mathrm{R}} D}(0,0) \mid i\left(\bar{e}_{\mathrm{L} t} \gamma_{\mu} d_{\mathrm{L} p a}\right)\left(\bar{u}_{\mathrm{R}}{ }_{r}^{a} D^{\mu} \nu_{\mathrm{L} s}\right) \\
& \mathcal{O}_{\bar{d}_{\mathrm{R}}^{2} \bar{\nu}_{\mathrm{L}} \bar{u}_{\mathrm{R}} D}(-1,-1) \mid \mathcal{Y}\left[[\overline{p r r}] i \epsilon_{a b c}\left(\bar{d}_{\mathrm{R}}^{a} \gamma_{\mu} C \bar{\nu}_{\mathrm{L} t}\right)\left(\bar{d}_{\mathrm{R}}^{b} C D^{\mu} \bar{u}_{\mathrm{R}}^{c}\right)\right. \\
& \mathcal{O}_{\bar{d}_{\mathrm{R}} \bar{e}_{\mathrm{R}} \bar{u}_{\mathrm{R}} \bar{u}_{\mathrm{L}} D}(-1,-1) \mid i \epsilon_{a b c}\left(\bar{e}_{\mathrm{R} r} C D^{\mu} \bar{u}_{\mathrm{R}}^{b}\right)\left(\bar{d}_{\left.\mathrm{R}_{p}^{a} \gamma_{\mu} C \bar{u}_{\mathrm{L}}^{c}\right)}\right) \\
& \mathcal{O}_{\bar{d}_{\mathrm{R}} \bar{d}_{\mathrm{L}} \nu_{\mathrm{L}} \bar{u}_{\mathrm{R}} D}(-1,1) \mid i \epsilon_{a b c}\left(\bar{u}_{\mathrm{R}}{ }_{r}^{b} D^{\mu} \nu_{\mathrm{L} s}\right)\left(\bar{d}_{\mathrm{R}}^{a} \gamma_{\mu} C \bar{d}_{\mathrm{L} t}^{c}\right)
\end{aligned}
$$




$$
\begin{aligned}
& \mathcal{O}_{\bar{e}_{\mathrm{R}} e_{\mathrm{L}} \bar{u}_{\mathrm{R}} u_{\mathrm{R}} D}(0,0) \mid i\left(\bar{e}_{\mathrm{R} p} \gamma_{\mu} u_{\mathrm{R} t a}\right)\left(D^{\mu} \bar{u}_{\mathrm{R} s}^{a} e_{\mathrm{L} r}\right) \\
& \mathcal{O}_{d_{\mathrm{R}} \bar{e}_{\mathrm{R}} \nu_{\mathrm{L}} \bar{u}_{\mathrm{R}} D}(0,0) \mid i\left(\bar{e}_{\mathrm{R} p} \gamma_{\mu} d_{\mathrm{R} t a}\right)\left(\bar{u}_{\mathrm{R} r}^{a} D^{\mu} \nu_{\mathrm{L} s}\right) \\
& \mathcal{O}_{\nu_{\mathrm{L}}^{2} \bar{u}_{\mathrm{R}} u_{\mathrm{R}} D}(0,2) \mid \mathcal{Y}\left[\frac{r}{s}\right] i\left(\nu_{\mathrm{L} r} C D^{\mu} \nu_{\mathrm{L} s}\right)\left(\bar{u}_{\mathrm{R}}^{a} \gamma_{\mu} u_{\mathrm{R} t a}\right) \\
& \mathcal{O}_{d_{\mathrm{L}}^{3} \bar{e}_{\mathrm{L}} D}(1,-1) \mid \mathcal{Y}\left[\overline{p p r \mid s]} i \epsilon^{a b c}\left(\bar{e}_{\mathrm{L} t} \gamma_{\mu} d_{\mathrm{L} p a}\right)\left(d_{\mathrm{L} r b} C D^{\mu} d_{\mathrm{L} s c}\right)\right.
\end{aligned}
$$

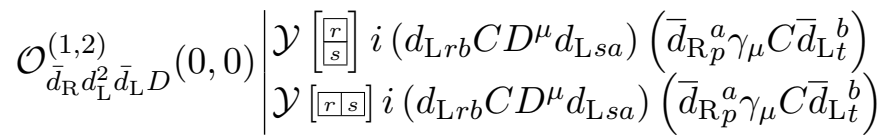

$$
\begin{aligned}
& \mathcal{O}_{d_{\mathrm{R}} d_{\mathrm{L}}^{2} \bar{e}_{\mathrm{R}} D}(1,-1) \mid \mathcal{Y}\left[[p \mid r] i \epsilon^{a b c}\left(D^{\mu} \bar{e}_{\mathrm{R} s} d_{\mathrm{L} r b}\right)\left(d_{\mathrm{L} p a} C \gamma_{\mu} d_{\mathrm{R} t c}\right)\right. \\
& \mathcal{O}_{d_{\mathrm{L}}^{2} \nu_{\mathrm{L}} u_{\mathrm{R}} D}(1,1) \mid \mathcal{Y}\left[[p r r] i \epsilon^{a b c}\left(d_{\mathrm{L} p a} C \gamma_{\mu} u_{\mathrm{R} t c}\right)\left(d_{\mathrm{L} r b} C D^{\mu} \nu_{\mathrm{L} s}\right)\right.
\end{aligned}
$$

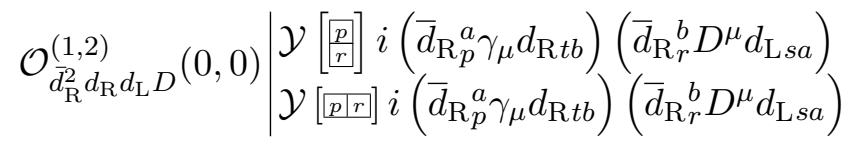

$$
\begin{aligned}
& \mathcal{O}_{\bar{d}_{\mathrm{R}} d_{\mathrm{L}} e_{\mathrm{L}} \bar{e}_{\mathrm{L}} D}(0,0) \mid i\left(d_{\mathrm{L} r a} C D^{\mu} e_{\mathrm{L} s}\right)\left(\bar{d}_{\mathrm{R} p}^{a} \gamma_{\mu} C \bar{e}_{\mathrm{L} t}\right) \\
& \mathcal{O}_{\bar{d}_{\mathrm{R}} d_{\mathrm{L}} \bar{e}_{\mathrm{R}} e_{\mathrm{R}} D}(0,0) \mid i\left(D^{\mu} \bar{e}_{\mathrm{R} s} d_{\mathrm{L} r a}\right)\left(\bar{d}_{\mathrm{R}}^{a} \gamma_{\mu} e_{\mathrm{R} t}\right) \\
& \mathcal{O}_{\bar{d}_{\mathrm{R}} d_{\mathrm{L}} \nu_{\mathrm{L}} \bar{\nu}_{\mathrm{L}} D}(0,0) \mid i\left(d_{\mathrm{L} r a} C D^{\mu} \nu_{\mathrm{L} s}\right)\left(\bar{d}_{\mathrm{R} p}^{a} \gamma_{\mu} C \bar{\nu}_{\mathrm{L} t}\right) \\
& \mathcal{O}_{d_{\mathrm{L}} \bar{d}_{\mathrm{L}} \bar{e}_{\mathrm{R}} e_{\mathrm{L}} D}(0,0) \mid i\left(\bar{d}_{\mathrm{L} t}^{a} \gamma_{\mu} d_{\mathrm{L} p a}\right)\left(\bar{e}_{\mathrm{R} r} D^{\mu} e_{\mathrm{L} s}\right) \\
& \mathcal{O}_{d_{\mathrm{L}} \bar{e}_{\mathrm{R}} \nu_{\mathrm{L}} \bar{u}_{\mathrm{L}} D}(0,0) \mid i\left(\bar{e}_{\mathrm{R} r} D^{\mu} \nu_{\mathrm{L} s}\right)\left(\bar{u}_{\mathrm{L} t}^{a} \gamma_{\mu} d_{\mathrm{L} p a}\right) \\
& \mathcal{O}_{d_{\mathrm{L}} \bar{d}_{\mathrm{L}} \nu_{\mathrm{L}}^{2} D}(0,2) \mid \mathcal{Y}\left[\frac{r}{s}\right] i\left(\nu_{\mathrm{L} r} C D^{\mu} \nu_{\mathrm{L} s}\right)\left(\bar{d}_{\mathrm{L}}{ }_{t}^{a} \gamma_{\mu} d_{\mathrm{L} p a}\right)
\end{aligned}
$$

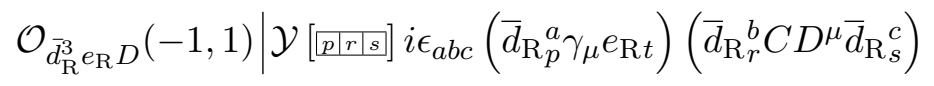

$$
\begin{aligned}
& \mathcal{O}_{\bar{d}_{\mathrm{R}}^{2} \bar{d}_{\mathrm{L}} e_{\mathrm{L}} D}(-1,1) \mid \mathcal{Y}\left[[\overline{p \mid r}] i \epsilon_{a b c}\left(\bar{d}_{\mathrm{R} r}^{b} D^{\mu} e_{\mathrm{L} s}\right)\left(\bar{d}_{\mathrm{R}}^{a} \gamma_{\mu} C \bar{d}_{\mathrm{L} t}^{c}\right)\right. \\
& \mathcal{O}_{\bar{d}_{\mathrm{R}}^{2} \nu_{\mathrm{L}} \bar{u}_{\mathrm{L}} D}(-1,1) \mid \mathcal{Y}[\overline{p p r}] i \epsilon_{a b c}\left(\bar{d}_{\mathrm{R} r}^{b} D^{\mu} \nu_{\mathrm{L} s}\right)\left(\bar{d}_{\mathrm{R} p}^{a} \gamma_{\mu} C \bar{u}_{\mathrm{L} t}^{c}\right) \\
& \mathcal{O}_{\bar{d}_{\mathrm{R}} d_{\mathrm{R}} \bar{e}_{\mathrm{R}} e_{\mathrm{L}} D}(0,0) \mid i\left(\bar{d}_{\mathrm{R} p}^{a} \gamma_{\mu} d_{\mathrm{R} t a}\right)\left(\bar{e}_{\mathrm{R} r} D^{\mu} e_{\mathrm{L} s}\right) \\
& \mathcal{O}_{\bar{d}_{\mathrm{R}} e_{\mathrm{L}} \nu_{\mathrm{L}} u_{\mathrm{R}} D}(0,2) \mid i\left(e_{\mathrm{L} r} C D^{\mu} \nu_{\mathrm{L} s}\right)\left(\bar{d}_{\mathrm{R} p}^{a} \gamma_{\mu} u_{\mathrm{R} t a}\right) \\
& \mathcal{O}_{\bar{d}_{\mathrm{R}} d_{\mathrm{R}} \nu_{\mathrm{L}}^{2} D}(0,2) \mid \mathcal{Y}\left[\frac{r}{s}\right] i\left(\nu_{\mathrm{L} r} C D^{\mu} \nu_{\mathrm{L} s}\right)\left(\bar{d}_{\mathrm{R} p}^{a} \gamma_{\mu} d_{\mathrm{R} t a}\right) \\
& \mathcal{O}_{\bar{e}_{\mathrm{R}} e_{\mathrm{L}}^{2} \bar{e}_{\mathrm{L}} D}(0,0) \mid \mathcal{Y}\left[\frac{r}{s}\right] i\left(e_{\mathrm{L} r} C D^{\mu} e_{\mathrm{L} s}\right)\left(\bar{e}_{\mathrm{R} p} \gamma_{\mu} C \bar{e}_{\mathrm{L} t}\right)
\end{aligned}
$$




$$
\begin{aligned}
& \mathcal{O}_{\bar{e}_{\mathrm{R}}^{2} e_{\mathrm{R}} e_{\mathrm{L}} D}(0,0) \mid \mathcal{Y}\left[\frac{p}{r}\right] i\left(\bar{e}_{\mathrm{R} p} \gamma_{\mu} e_{\mathrm{R} t}\right)\left(\bar{e}_{\mathrm{R} r} D^{\mu} e_{\mathrm{L} s}\right) \\
& \mathcal{O}_{\bar{e}_{\mathrm{R}} e_{\mathrm{L}} \nu_{\mathrm{L}} \bar{\nu}_{\mathrm{L}} D}(0,0) \mid i\left(\bar{e}_{\mathrm{R} p} \gamma_{\mu} C \bar{\nu}_{\mathrm{L} t}\right)\left(e_{\mathrm{L} r} C D^{\mu} \nu_{\mathrm{L} s}\right) \\
& \mathcal{O}_{e_{\mathrm{L}} \bar{e}_{\mathrm{L}} \nu_{\mathrm{L}}^{2} D}(0,2) \mid \mathcal{Y}\left[\begin{array}{l}
\left.\frac{r}{s}\right] \\
s
\end{array}\right]\left(\bar{e}_{\mathrm{L} t} \gamma_{\mu} e_{\mathrm{L} p}\right)\left(\nu_{\mathrm{L} r} C D^{\mu} \nu_{\mathrm{L} s}\right) \\
& \mathcal{O}_{\bar{e}_{\mathrm{R}} e_{\mathrm{R}} \nu_{\mathrm{L}}^{2} D}(0,2) \mid \mathcal{Y}\left[\begin{array}{l}
\frac{r}{s} \\
]
\end{array}\right] i\left(\bar{e}_{\mathrm{R} p} \gamma_{\mu} e_{\mathrm{R} t}\right)\left(\nu_{\mathrm{L} r} C D^{\mu} \nu_{\mathrm{L} s}\right) \\
& \mathcal{O}_{\nu_{\mathrm{L}}^{3} \bar{\nu}_{\mathrm{L}} D}(0,2) \mid \mathcal{Y}\left[\begin{array}{l}
\frac{p}{r} \\
\frac{r}{s}
\end{array}\right] i\left(\bar{\nu}_{\mathrm{L} t} \gamma_{\mu} \nu_{\mathrm{L} p}\right)\left(\nu_{\mathrm{L} r} C D^{\mu} \nu_{\mathrm{L} s}\right)
\end{aligned}
$$

\subsection{Lists of the dim-8 operators}

\subsubsection{Classes involving bosons only}

Class $F_{\mathrm{L}}{ }^{4}: 4$ types

$$
\begin{aligned}
& \mathcal{O}_{G_{\mathrm{L}}{ }^{4}}^{(1 \sim 3)}(0,0) \mid \begin{array}{l}
d^{A C E} d^{B D E} G_{\mathrm{L} \mu \nu}^{A} G_{\mathrm{L}}^{B \mu \nu} G_{\mathrm{L} \lambda \rho}^{C} G_{\mathrm{L}}^{D \lambda \rho} \\
G_{\mathrm{L} \lambda \rho}^{A} G_{\mathrm{L} \mu \nu}^{A} G_{\mathrm{L}}^{B \lambda \rho} G_{\mathrm{L}}^{B \mu \nu} \\
f^{A C E} f^{B D E} G_{\mathrm{L} \mu \nu}^{A} G_{\mathrm{L}}^{B \mu \nu} G_{\mathrm{L} \lambda \rho}^{C} G_{\mathrm{L}}^{D \lambda \rho}
\end{array} \\
& \mathcal{O}_{F_{\mathrm{L}} G_{\mathrm{L}}{ }^{3}}(0,0) \mid d^{A B C} F_{\mathrm{L} \mu \nu} G_{\mathrm{L}}^{A \mu \nu} G_{\mathrm{L} \lambda \rho}^{B} G_{\mathrm{L}}^{C \lambda \rho} \\
& \mathcal{O}_{F_{\mathrm{L}}{ }^{2} G_{\mathrm{L}}{ }^{2}}^{(1,2)}(0,0) \mid \begin{array}{l}
F_{\mathrm{L} \mu \nu} F_{\mathrm{L}}{ }^{\mu \nu} G_{\mathrm{L} \lambda \rho}^{A} G_{\mathrm{L}}^{A \lambda \rho} \\
F_{\mathrm{L} \lambda \rho} F_{\mathrm{L} \mu \nu} G_{\mathrm{L}}^{A \lambda \rho} G_{\mathrm{L}}^{A \mu \nu}
\end{array} \\
& \mathcal{O}_{F_{\mathrm{L}}{ }^{4}(0,0) \mid}\left(F_{\mathrm{L} \lambda \rho} F_{\mathrm{L} \mu \nu} F_{\mathrm{L}}{ }^{\lambda \rho} F_{\mathrm{L}}{ }^{\mu \nu}\right.
\end{aligned}
$$

Class ${F_{\mathrm{L}}}^{2} \boldsymbol{F}_{\mathrm{R}}^{2}: 5$ types

$$
\begin{aligned}
& \mathcal{O}_{G_{\mathrm{L}}{ }^{2} G_{\mathrm{R}}{ }^{2}}^{(1 \sim 3)}(0) \mid \begin{array}{l}
d^{A C E} d^{B D E} G_{\mathrm{L} \mu \nu}^{A} G_{\mathrm{L}}^{B \mu \nu} G_{\mathrm{R} \lambda \rho}^{C} G_{\mathrm{R}}^{D \lambda \rho} \\
G_{\mathrm{L} \mu \nu}^{A} G_{\mathrm{R} \lambda \rho}^{A} G_{\mathrm{L}}^{B \mu \nu} G_{\mathrm{R}}^{B \lambda \rho} \\
f^{A C E} f^{B D E} G_{\mathrm{L} \mu \nu}^{A} G_{\mathrm{L}}^{B \mu \nu} G_{\mathrm{R} \lambda \rho}^{C} G_{\mathrm{R}}^{D \lambda \rho}
\end{array} \\
& \mathcal{O}_{F_{\mathrm{R}} G_{\mathrm{L}}{ }^{2} G_{\mathrm{R}}}(0,0) \mid d^{A B C} F_{\mathrm{R} \lambda \rho} G_{\mathrm{L} \mu \nu}^{A} G_{\mathrm{L}}^{B \mu \nu} G_{\mathrm{R}}^{C \lambda \rho} \\
& \mathcal{O}_{F_{\mathrm{R}}{ }^{2} G_{\mathrm{L}}{ }^{2}}(0,0) \mid F_{\mathrm{R} \lambda \rho} F_{\mathrm{R}}{ }^{\lambda \rho} G_{\mathrm{L} \mu \nu}^{A} G_{\mathrm{L}}^{A \mu \nu} \\
& \mathcal{O}_{F_{\mathrm{L}} F_{\mathrm{R}} G_{\mathrm{L}} G_{\mathrm{R}}}(0,0) \mid F_{\mathrm{L} \mu \nu} F_{\mathrm{R} \lambda \rho} G_{\mathrm{L}}^{A \mu \nu} G_{\mathrm{R}}^{A \lambda \rho} \\
& \mathcal{O}_{F_{\mathrm{L}}{ }^{2} F_{\mathrm{R}}{ }^{2}}(0,0) \mid F_{\mathrm{L} \mu \nu} F_{\mathrm{R} \lambda \rho} F_{\mathrm{L}}{ }^{\mu \nu} F_{\mathrm{R}}{ }^{\lambda \rho}
\end{aligned}
$$

\subsubsection{Classes involving two-fermions}

\section{Class $F_{\mathrm{L}}^{2} \psi \psi^{\dagger} D: 8$ types}

$$
\begin{aligned}
& \mathcal{O}_{G_{\mathrm{L}}{ }^{2} u_{\mathrm{L}} \bar{u}_{\mathrm{L}} D}(0,0) \mid i f^{A B C}\left(\lambda^{C}\right)_{b}^{a} G_{\mathrm{L} \nu \lambda}^{A} G_{\mathrm{L}}^{B \lambda \mu}\left(\bar{u}_{\mathrm{L} r}^{b} \gamma^{\nu} D_{\mu} u_{\mathrm{L} p a}\right) \\
& \mathcal{O}_{G_{\mathrm{L}}{ }^{2} \bar{u}_{\mathrm{R}} u_{\mathrm{R}} D}(0,0) \mid i f^{A B C}\left(\lambda^{C}\right)_{a}^{b} G_{\mathrm{L} \nu \lambda}^{A} G_{\mathrm{L}}^{B \lambda \mu}\left(D_{\mu} \bar{u}_{\mathrm{R}}^{a} \gamma^{\nu} u_{\mathrm{R} r b}\right)
\end{aligned}
$$




$$
\begin{aligned}
& \mathcal{O}_{G_{\mathrm{L}}^{2} d_{\mathrm{L}} \bar{d}_{\mathrm{L}} D}(0,0) \mid i f^{A B C}\left(\lambda^{C}\right)_{b}^{a} G_{\mathrm{L} \nu \lambda}^{A} G_{\mathrm{L}}^{B \lambda \mu}\left(\bar{d}_{\mathrm{L}}^{b} \gamma^{\nu} D_{\mu} d_{\mathrm{L} p a}\right) \\
& \mathcal{O}_{G_{\mathrm{L}}{ }^{2} \bar{d}_{\mathrm{R}} d_{\mathrm{R}} D}(0,0) \mid i f^{A B C}\left(\lambda^{C}\right)_{a}^{b} G_{\mathrm{L} \nu \lambda}^{A} G_{\mathrm{L}}^{B \lambda \mu}\left(D_{\mu} \bar{d}_{\mathrm{R} p}^{a} \gamma^{\nu} d_{\mathrm{R} r b}\right) \\
& \mathcal{O}_{F_{\mathrm{L}} G_{\mathrm{L}} u_{\mathrm{L}} \bar{u}_{\mathrm{L}} D}(0,0) \mid i\left(\lambda^{A}\right)_{b}^{a} F_{\mathrm{L} \nu \lambda} G_{\mathrm{L}}^{A \lambda \mu}\left(\bar{u}_{\mathrm{L} r}^{b} \gamma^{\nu} D_{\mu} u_{\mathrm{L} p a}\right) \\
& \mathcal{O}_{F_{\mathrm{L}} G_{\mathrm{L}} \bar{u}_{\mathrm{R}} u_{\mathrm{R}} D}(0,0) \mid i\left(\lambda^{A}\right)_{a}^{b} F_{\mathrm{L} \nu \lambda} G_{\mathrm{L}}^{A \lambda \mu}\left(D_{\mu} \bar{u}_{\mathrm{R}}^{a} \gamma^{\nu} u_{\mathrm{R} r b}\right) \\
& \mathcal{O}_{F_{\mathrm{L}} G_{\mathrm{L}} d_{\mathrm{L}} \bar{d}_{\mathrm{L}} D}(0,0) \mid i\left(\lambda^{A}\right)_{b}^{a} F_{\mathrm{L} \nu \lambda} G_{\mathrm{L}}^{A \lambda \mu}\left(\bar{d}_{\mathrm{L} r}^{b} \gamma^{\nu} D_{\mu} d_{\mathrm{L} p a}\right) \\
& \mathcal{O}_{F_{\mathrm{L}} G_{\mathrm{L}} \bar{d}_{\mathrm{R}} d_{\mathrm{R}} D}(0,0) \mid i\left(\lambda^{A}\right)_{a}^{b} F_{\mathrm{L} \nu \lambda} G_{\mathrm{L}}^{A \lambda \mu}\left(D_{\mu} \bar{d}_{\mathrm{R}}^{a} \gamma^{\nu} d_{\mathrm{R} r b}\right)
\end{aligned}
$$

\section{Class $F_{\mathrm{L}} \boldsymbol{F}_{\mathrm{R}} \psi \psi^{\dagger} D: 18$ types}

$$
\begin{aligned}
& \mathcal{O}_{G_{\mathrm{L}} G_{\mathrm{R}} u_{\mathrm{L}} \bar{u}_{\mathrm{L} D}(0,0)}^{(1 \sim 3)} \mid \begin{array}{l}
i d^{A B C}\left(\lambda^{C}\right)_{b}^{a} G_{\mathrm{L}}^{A \mu}{ }_{\nu} G_{\mathrm{R}}^{B \nu}{ }_{\lambda}\left(D_{\mu} \bar{u}_{\mathrm{L} r}^{b} \gamma^{\lambda} u_{\mathrm{L} p a}\right) \\
i f^{A B C}\left(\lambda^{C}\right)_{b}^{a} G_{\mathrm{L}}^{A \mu}{ }_{\nu} G_{\mathrm{R}}^{B \nu}{ }_{\lambda}\left(D_{\mu} \bar{u}_{\mathrm{L}}{ }^{b} \gamma^{\lambda} u_{\mathrm{L} p a}\right) \\
i G_{\mathrm{L}}^{A \mu}{ }_{\nu} G_{\mathrm{R}}^{A \nu}{ }_{\lambda}\left(D_{\mu} \bar{u}_{\mathrm{L}}{ }_{r} \gamma^{\lambda} u_{\mathrm{L} p a}\right)
\end{array} \\
& \mathcal{O}_{F_{\mathrm{R}} G_{\mathrm{L}} u_{\mathrm{L}} \bar{u}_{\mathrm{L}} D}(0,0) \mid i\left(\lambda^{A}\right)_{b}^{a} F_{\mathrm{R}}{ }_{\lambda}{ }_{\lambda} G_{\mathrm{L}}^{A \mu}{ }_{\nu}\left(D_{\mu} \bar{u}_{\mathrm{L}}{ }_{r}^{b} \gamma^{\lambda} u_{\mathrm{L} p a}\right) \\
& \mathcal{O}_{G_{\mathrm{L}} G_{\mathrm{R}} \bar{u}_{\mathrm{R}} u_{\mathrm{R}} D}^{(1 \sim 3,0)} \mid \begin{array}{l}
i d^{A B C}\left(\lambda^{C}\right)_{q}^{b} G_{\mathrm{L}}^{A \mu}{ }_{\nu} G_{\mathrm{R}}^{B \nu}{ }_{\lambda}\left(\bar{u}_{\mathrm{R} p}^{a} \gamma^{\lambda} D_{\mu} u_{\mathrm{R} r b}\right) \\
i f^{A B C}\left(\lambda^{C}\right)_{a}^{b} G_{\mathrm{L}}^{A \mu}{ }_{\nu} G_{\mathrm{R}}^{B \nu}{ }_{\lambda}\left(\bar{u}_{\mathrm{R} p}^{a} \gamma^{\lambda} D_{\mu} u_{\mathrm{R} r b}\right) \\
i G_{\mathrm{L}}^{A \mu}{ }_{\nu} G_{\mathrm{R}}^{A \nu}{ }_{\lambda}\left(\bar{u}_{\mathrm{R}}{ }_{p}^{a} \gamma^{\lambda} D_{\mu} u_{\mathrm{R} r a}\right)
\end{array} \\
& \mathcal{O}_{F_{\mathrm{R}} G_{\mathrm{L}} \bar{u}_{\mathrm{R}} u_{\mathrm{R}} D}(0,0) \mid i\left(\lambda^{A}\right)_{a}^{b} F_{\mathrm{R}}{ }^{\nu}{ }_{\lambda} G_{\mathrm{L}}^{A \mu}{ }_{\nu}\left(\bar{u}_{\mathrm{R}}{ }_{p}^{a} \gamma^{\lambda} D_{\mu} u_{\mathrm{R} r b}\right) \\
& \mathcal{O}_{G_{\mathrm{L}} G_{\mathrm{R}} d_{\mathrm{L}} \bar{d}_{\mathrm{L}} D}^{(1 \sim, 0)} \mid \begin{array}{l}
i d^{A B C}\left(\lambda^{C}\right)_{b}^{a} G_{\mathrm{L}}^{A \mu}{ }_{\nu} G_{\mathrm{R}}^{B \nu}{ }_{\lambda}\left(D_{\mu} \bar{d}_{\mathrm{L} r}^{b} \gamma^{\lambda} d_{\mathrm{L} p a}\right) \\
i f^{A B C}\left(\lambda^{C}\right)_{b}^{a} G_{\mathrm{L}}^{A \mu}{ }_{\nu} G_{\mathrm{R}}^{B{ }{ }_{\lambda} \lambda}\left(D_{\mu} \bar{d}_{\mathrm{L}}^{b} \gamma^{\lambda} d_{\mathrm{L} p a}\right) \\
i G_{\mathrm{L}}^{A \mu}{ }_{\nu} G_{\mathrm{R}}^{A \nu}{ }_{\lambda}\left(D_{\mu} \bar{d}_{\mathrm{L} r}{ }^{a} \gamma^{\lambda} d_{\mathrm{L} p a}\right)
\end{array} \\
& \mathcal{O}_{F_{\mathrm{R}} G_{\mathrm{L}} d_{\mathrm{L}} \bar{d}_{\mathrm{L}} D}(0,0) \mid i\left(\lambda^{A}\right)_{b}^{a} F_{\mathrm{R}}{ }_{\lambda}{ }_{\lambda} G_{\mathrm{L}}^{A \mu}{ }_{\nu}\left(D_{\mu} \bar{d}_{\mathrm{L} r}^{b} \gamma^{\lambda} d_{\mathrm{L} p a}\right) \\
& \mathcal{O}_{G_{\mathrm{L}} G_{\mathrm{R}} \bar{d}_{\mathrm{R}} d_{\mathrm{R} D}}^{(1 \sim 33)}(0,0) \mid \begin{array}{l}
i d^{A B C}\left(\lambda^{C}\right)_{a}^{b} G_{\mathrm{L}}^{A \mu}{ }_{\nu} G_{\mathrm{R}}^{B \nu}{ }_{\lambda}\left(\bar{d}_{\mathrm{R}}{ }^{a} \gamma^{\lambda} D_{\mu} d_{\mathrm{R} r b}\right) \\
i f^{A B C}\left(\lambda^{C}\right)_{a}^{b} G_{\mathrm{L}}^{A \mu}{ }_{\nu} G_{\mathrm{R}}^{B \nu}{ }_{\lambda}\left(\bar{d}_{\mathrm{R} p}^{a} \gamma^{\lambda} D_{\mu} d_{\mathrm{R} r b}\right) \\
i G_{\mathrm{L}}^{A \mu}{ }_{\nu} G_{\mathrm{R}}^{A \nu}{ }_{\lambda}\left(\bar{d}_{\mathrm{R}}{ }_{p}^{a} \gamma^{\lambda} D_{\mu} d_{\mathrm{R} r a}\right)
\end{array} \\
& \mathcal{O}_{F_{\mathrm{R}} G_{\mathrm{L}} \bar{d}_{\mathrm{R}} d_{\mathrm{R}} D}(0,0) \mid i\left(\lambda^{A}\right)_{a}^{b} F_{\mathrm{R}}{ }_{\lambda}{ }_{\lambda} G_{\mathrm{L}}^{A \mu}{ }_{\nu}\left(\bar{d}_{\mathrm{R}}{ }_{p}^{a} \gamma^{\lambda} D_{\mu} d_{\mathrm{R} r b}\right) \\
& \mathcal{O}_{G_{\mathrm{L}} G_{\mathrm{R}} e_{\mathrm{L}} \bar{e}_{\mathrm{L}} D}(0,0) \mid i G_{\mathrm{L}}^{A \mu}{ }_{\nu} G_{\mathrm{R}}^{A \nu}{ }_{\lambda}\left(D_{\mu} \bar{e}_{\mathrm{L} r} \gamma^{\lambda} e_{\mathrm{L} p}\right) \\
& \mathcal{O}_{G_{\mathrm{L}} G_{\mathrm{R}} \bar{e}_{\mathrm{R}} e_{\mathrm{R}} D}(0,0) \mid i G_{\mathrm{L}}^{A \mu}{ }_{\nu} G_{\mathrm{R}}^{A \nu}{ }_{\lambda}\left(\bar{e}_{\mathrm{R} p} \gamma^{\lambda} D_{\mu} e_{\mathrm{R} r}\right)
\end{aligned}
$$




$$
\begin{aligned}
& \mathcal{O}_{G_{\mathrm{L}} G_{\mathrm{R}} \nu_{\mathrm{L}} \bar{\nu}_{\mathrm{L}} D}(0,0) \mid i G_{\mathrm{L}}^{A \mu}{ }_{\nu} G_{\mathrm{R}}^{A \nu}{ }_{\lambda}\left(D_{\mu} \bar{\nu}_{\mathrm{L} r} \gamma^{\lambda} \nu_{\mathrm{L} p}\right) \\
& \mathcal{O}_{F_{\mathrm{L}} F_{\mathrm{R}} u_{\mathrm{L}} \bar{u}_{\mathrm{L}} D}(0,0) \mid i F_{\mathrm{L}}{ }_{\nu}{ }_{\nu} F_{\mathrm{R}}{ }^{\nu}{ }_{\lambda}\left(D_{\mu} \bar{u}_{\mathrm{L}}{ }^{a} \gamma^{\lambda} u_{\mathrm{L} p a}\right) \\
& \mathcal{O}_{F_{\mathrm{L}} F_{\mathrm{R}} \bar{u}_{\mathrm{R}} u_{\mathrm{R}} D}(0,0) \mid i F_{\mathrm{L}}{ }^{\mu}{ }_{\nu} F_{\mathrm{R}}{ }^{\nu}{ }_{\lambda}\left(\bar{u}_{\mathrm{R}}{ }^{a} \gamma^{\lambda} D_{\mu} u_{\mathrm{R} r a}\right) \\
& \mathcal{O}_{F_{\mathrm{L}} F_{\mathrm{R}} d_{\mathrm{L}} \bar{d}_{\mathrm{L}} D}(0,0) \mid i F_{\mathrm{L}}{ }_{\nu}{ }_{\nu} F_{\mathrm{R}}{ }^{\nu}{ }_{\lambda}\left(D_{\mu} \bar{d}_{\mathrm{L}}{ }_{r}^{a} \gamma^{\lambda} d_{\mathrm{L} p a}\right) \\
& \mathcal{O}_{F_{\mathrm{L}} F_{\mathrm{R}} \bar{d}_{\mathrm{R}} d_{\mathrm{R}} D}(0,0) \mid i F_{\mathrm{L}}{ }_{\nu}{ }_{\nu} F_{\mathrm{R}}{ }^{\nu}{ }_{\lambda}\left(\bar{d}_{\mathrm{R} p}{ }^{a} \gamma^{\lambda} D_{\mu} d_{\mathrm{R} r a}\right) \\
& \mathcal{O}_{F_{\mathrm{L}} F_{\mathrm{R}} e_{\mathrm{L}} \bar{e}_{\mathrm{L}} D}(0,0) \mid i F_{\mathrm{L}}{ }^{\mu}{ }_{\nu} F_{\mathrm{R}}{ }^{\nu}{ }_{\lambda}\left(D_{\mu} \bar{e}_{\mathrm{L} r} \gamma^{\lambda} e_{\mathrm{L} p}\right) \\
& \mathcal{O}_{F_{\mathrm{L}} F_{\mathrm{R}} \bar{e}_{\mathrm{R}} e_{\mathrm{R}} D}(0,0) \mid i F_{\mathrm{L}}{ }_{\nu}{ }_{\nu} F_{\mathrm{R}}{ }^{\nu}{ }_{\lambda}\left(\bar{e}_{\mathrm{R} p} \gamma^{\lambda} D_{\mu} e_{\mathrm{R} r}\right) \\
& \mathcal{O}_{F_{\mathrm{L}} F_{\mathrm{R}} \nu_{\mathrm{L}} \bar{\nu}_{\mathrm{L}} D}(0,0) \mid i F_{\mathrm{L}}{ }_{\nu}{ }_{\nu} F_{\mathrm{R}}{ }^{\nu}{ }_{\lambda}\left(D_{\mu} \bar{\nu}_{\mathrm{L} r} \gamma^{\lambda} \nu_{\mathrm{L} p}\right)
\end{aligned}
$$

\subsubsection{Classes involving four-fermions}

\section{Class $\psi^{4} D^{2}: 18$ types}

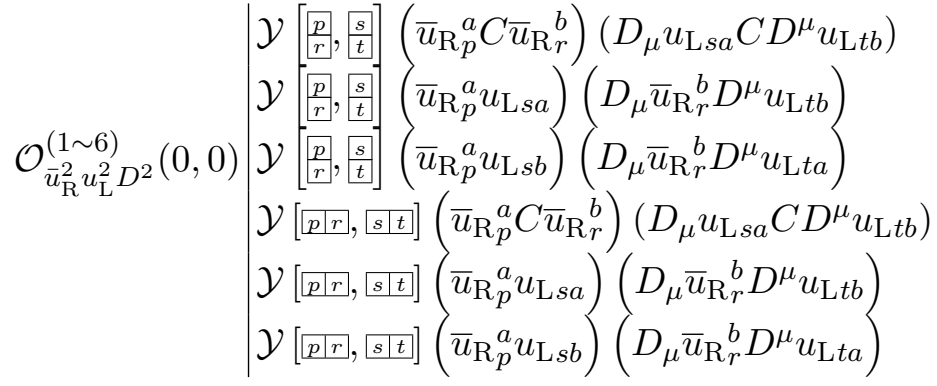

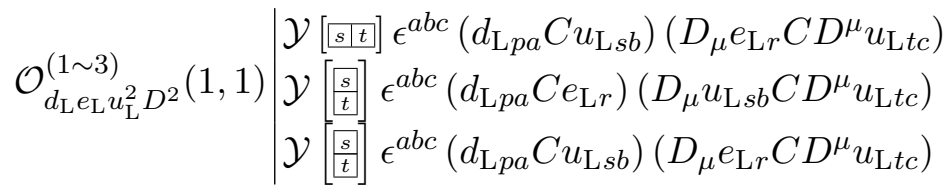

$$
\begin{aligned}
& \mathcal{O}_{\bar{d}_{\mathrm{R}} d_{\mathrm{L}} \bar{u}_{\mathrm{R}} u_{\mathrm{L}} D^{2}}^{(1 \sim 6,0)} \mid \begin{array}{ll}
\left(\bar{d}_{\mathrm{R}}^{a} d_{\mathrm{L} r b}\right) & \left(D_{\mu} \bar{u}_{\mathrm{R}}^{b} D^{\mu} u_{\mathrm{L} t a}\right) \\
\left.\bar{d}_{\mathrm{R}}^{a} d_{\mathrm{L} r a}\right)\left(D_{\mu} \bar{u}_{\mathrm{R}}^{c} D^{\mu} D^{\mu} u_{\mathrm{L} t c}\right) \\
\left.\bar{d}_{\mathrm{R}}^{a} C \bar{u}_{\mathrm{R}}^{b}\right)\left(D_{\mu} d_{\mathrm{L} r b} C D^{\mu} u_{\mathrm{L} t a}\right) \\
\left(\bar{d}_{\mathrm{R} p}^{a} C \bar{u}_{\mathrm{R} s}^{c}\right)\left(D_{\mu} d_{\mathrm{L} r a} C D^{\mu} u_{\mathrm{Ltc}}\right) \\
i\left(D_{\mu} \bar{u}_{\mathrm{R}}^{b} D_{\nu} u_{\mathrm{L} t a}\right)\left(\bar{d}_{\mathrm{R}}^{a} \sigma^{\mu \nu} d_{\mathrm{L} r b}\right) \\
i\left(\bar{d}_{\mathrm{R} p}^{a} \sigma^{\mu \nu} d_{\mathrm{L} r a}\right)\left(D_{\mu} \bar{u}_{\mathrm{R}}^{c} D_{\nu} u_{\mathrm{L} t c}\right)
\end{array} \\
& \mathcal{O}_{\bar{e}_{\mathrm{R}} e_{\mathrm{L}} \bar{u}_{\mathrm{R}} u_{\mathrm{L}} D^{2}}^{(1 \sim 3)}(0,0) \mid \begin{array}{l}
\left(\bar{e}_{\mathrm{R} p} e_{\mathrm{L} r}\right)\left(D_{\mu} \bar{u}_{\mathrm{R}}^{a} D^{\mu} D^{\mu} u_{\mathrm{L} t a}\right) \\
\left(\bar{e}_{\mathrm{R} p} C \bar{u}_{\mathrm{R} s}^{a}\right)\left(D_{\mu} e_{\mathrm{L} r} C D^{\mu} u_{\mathrm{L} t a}\right) \\
i\left(\bar{e}_{\mathrm{R} p} \sigma^{\mu \nu} e_{\mathrm{L} r}\right)\left(D_{\mu} \bar{u}_{\mathrm{R}}^{a} D_{\nu} D_{\mathrm{L} t a}\right)
\end{array}
\end{aligned}
$$




$$
\begin{aligned}
& \mathcal{O}_{\nu_{\mathrm{L}}^{2} \bar{u}_{\mathrm{R}} u_{\mathrm{L}} D^{2}}^{(1 \sim 2)} \mid \begin{array}{l}
\mathcal{Y}\left[\begin{array}{l}
{[s]} \\
t]
\end{array}\right]\left(\bar{u}_{\mathrm{R}}^{a} \nu_{\mathrm{L} s}\right)\left(D_{\mu} u_{\mathrm{L} r a} C D^{\mu} \nu_{\mathrm{L} t}\right) \\
\mathcal{Y}[s] t]\left(\bar{u}_{\mathrm{R}}^{a} u_{\mathrm{L} r a}\right)\left(D_{\mu} \nu_{\mathrm{L} s} C D^{\mu} \nu_{\mathrm{L} t}\right) \\
\mathcal{Y}[\overline{s \mid t]}]\left(\bar{u}_{\mathrm{R}}^{a}{ }_{p} \nu_{\mathrm{L} s}\right)\left(D_{\mu} u_{\mathrm{L} r a} C D^{\mu} \nu_{\mathrm{L} t}\right)
\end{array}
\end{aligned}
$$

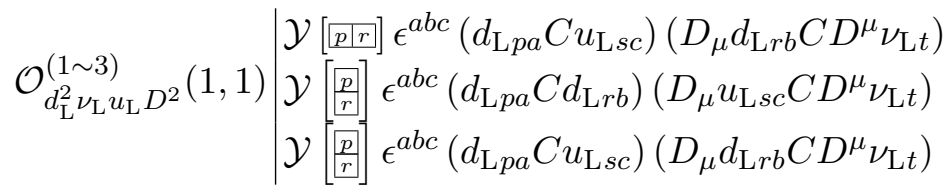

$$
\begin{aligned}
& \mathcal{O}_{\bar{d}_{\mathrm{R}} e_{\mathrm{L}} \nu_{\mathrm{L}} u_{\mathrm{L}} D^{2}}^{(1 \sim 3)}(0,2) \mid \begin{array}{l}
\left(\bar{d}_{\mathrm{R}}^{a} e_{\mathrm{L} r}\right)\left(D_{\mu} u_{\mathrm{L} s a} C D^{\mu} \nu_{\mathrm{L} t}\right) \\
\left(\bar{d}_{\mathrm{R}}^{a} u_{\mathrm{L} s a}\right)\left(D_{\mu} e_{\mathrm{L} r} C D^{\mu} \nu_{\mathrm{L} t}\right) \\
i\left(D_{\mu} u_{\mathrm{L} s a} C D_{\nu} \nu_{\mathrm{L} t}\right)\left(\bar{d}_{\mathrm{R}}^{a} \sigma^{\mu \nu} e_{\mathrm{L} r}\right)
\end{array}
\end{aligned}
$$

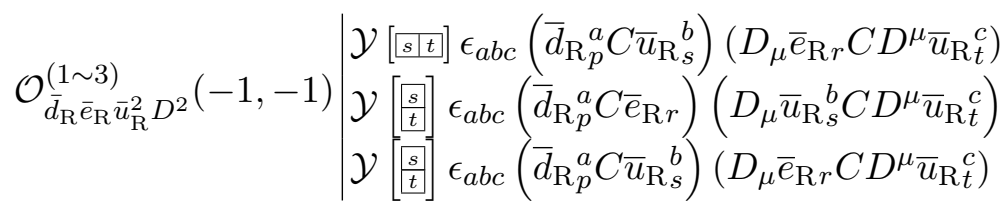

$$
\begin{aligned}
& \mathcal{O}_{d_{\mathrm{L}} \bar{e}_{\mathrm{R}} \nu_{\mathrm{L}} \bar{u}_{\mathrm{R}} D^{2}}(0,0) \mid \begin{array}{l}
\left(\bar{e}_{\mathrm{R} r} d_{\mathrm{L} p a}\right)\left(D_{\mu} \bar{u}_{\mathrm{R} s}{ }^{a} D^{\mu} \nu_{\mathrm{L} t}\right) \\
\left(\bar{u}_{\mathrm{R}}{ }_{s}^{a} d_{\mathrm{L} p a}\right)\left(D_{\mu} \bar{e}_{\mathrm{R} r} D^{\mu} \nu_{\mathrm{L} t}\right) \\
i\left(D_{\mu} \bar{u}_{\mathrm{R}}{ }_{s}^{a} D_{\nu} \nu_{\mathrm{L} t}\right)\left(\bar{e}_{\mathrm{R} r} \sigma^{\mu \nu} d_{\mathrm{L} p a}\right)
\end{array}
\end{aligned}
$$

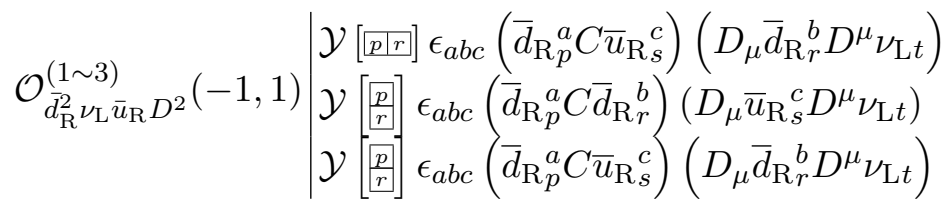

$$
\begin{aligned}
& \mathcal{O}_{d_{\mathrm{L}}^{(3)} \overline{\mathrm{e}}_{\mathrm{R}} D^{2}}^{(1,-1)} \mid \begin{array}{l}
\mathcal{Y}\left[\begin{array}{l}
\left.\frac{p r}{p r}\right] \\
\frac{s}{a}
\end{array}\right] \epsilon^{a b c}\left(d_{\mathrm{L} p a} C d_{\mathrm{L} r b}\right)\left(D^{\mu} \bar{e}_{\mathrm{R} t} D_{\mu} d_{\mathrm{L} s c}\right) \\
\mathcal{Y}\left[\begin{array}{l}
\frac{p}{r} \\
\frac{r}{s}
\end{array}\right] \epsilon^{a b c}\left(d_{\mathrm{L} p a} C d_{\mathrm{L} r b}\right)\left(D^{\mu} \bar{e}_{\mathrm{R} t} D_{\mu} d_{\mathrm{L} s c}\right)
\end{array}
\end{aligned}
$$

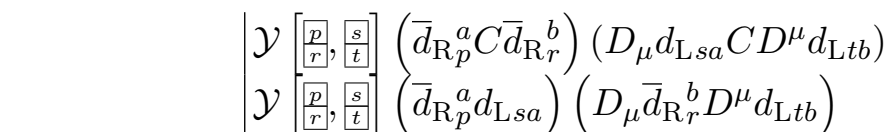

$$
\begin{aligned}
& \mathcal{O}_{\bar{d}_{\mathrm{R}}^{2} d_{\mathrm{L}}^{2} D^{2}}^{(1 \sim 6)}(0,0) \mathcal{Y}\left[\frac{p}{r}, \frac{s}{t}\right] .\left(\bar{d}_{\mathrm{R}}^{a}{ }_{p}^{a} d_{\mathrm{L} s b}\right)\left(D_{\mu} \bar{d}_{\mathrm{R} r}^{b} D^{\mu} d_{\mathrm{L} t a}\right) \\
& \mathcal{Y}[\underline{p|r, s| t]}]\left(\bar{d}_{\mathrm{R} p}^{a} C \bar{d}_{\mathrm{R}}{ }_{r}^{b}\right)\left(D_{\mu} d_{\mathrm{L} s a} C D^{\mu} d_{\mathrm{L} t b}\right) \\
& \mathcal{Y}[\underline{p \mid r,}, s \mid t]]\left(\bar{d}_{\mathrm{R} p}^{a} d_{\mathrm{L} s a}\right)\left(D_{\mu} \bar{d}_{\mathrm{R} r}^{b} D^{\mu} d_{\mathrm{L} t b}\right) \\
& \mathcal{Y}[\underline{p p r, s, t]}]\left(\bar{d}_{\mathrm{R} p}^{a} d_{\mathrm{L} s b}\right)\left(D_{\mu} \bar{d}_{\mathrm{R} r}^{b} D^{\mu} d_{\mathrm{L} t a}\right) \\
& \mathcal{O}_{\bar{d}_{\mathrm{R}} d_{\mathrm{L}} \bar{e}_{\mathrm{R}} e_{\mathrm{L}} D^{2}}^{(1 \sim 3)}(0,0) \mid \begin{array}{l}
\left(\bar{d}_{\mathrm{R} p}^{a} d_{\mathrm{L} r a}\right)\left(D_{\mu} \bar{e}_{\mathrm{R} s} D^{\mu} e_{\mathrm{L} t}\right) \\
\left(\bar{d}_{\mathrm{R} p}^{a} C \bar{e}_{\mathrm{R} s}\right)\left(D_{\mu} d_{\mathrm{L} r a} C D^{\mu} e_{\mathrm{L} t}\right) \\
i\left(D_{\mu} \bar{e}_{\mathrm{R} s} D_{\nu} e_{\mathrm{L} t}\right)\left(\bar{d}_{\mathrm{R} p}^{a} \sigma^{\mu \nu} d_{\mathrm{L} r a}\right)
\end{array}
\end{aligned}
$$




$$
\begin{aligned}
& \mathcal{O}_{\bar{d}_{\mathrm{R}} d_{\mathrm{L}} \nu_{\mathrm{L}}^{2} D^{2}}^{(1 \sim 3)}(0,2) \mid \begin{array}{l}
\mathcal{Y}\left[\begin{array}{l}
s] \\
\mathcal{t}
\end{array}\right]\left(\bar{d}_{\mathrm{R} p}^{a} \nu_{\mathrm{L} s}\right)\left(D_{\mu} d_{\mathrm{L} r a} C D^{\mu} \nu_{\mathrm{L} t}\right) \\
\mathcal{Y}[s \mid t]\left(\bar{d}_{\mathrm{R} p}^{a} d_{\mathrm{L} r a}\right)\left(D_{\mu} \nu_{\mathrm{L} s} C D^{\mu} \nu_{\mathrm{L} t}\right) \\
\mathcal{Y}[s \mid s]\left(\bar{d}_{\mathrm{R} p}^{a} \nu_{\mathrm{L} s}\right)\left(D_{\mu} d_{\mathrm{L} r a} C D^{\mu} \nu_{\mathrm{L} t}\right)
\end{array}
\end{aligned}
$$

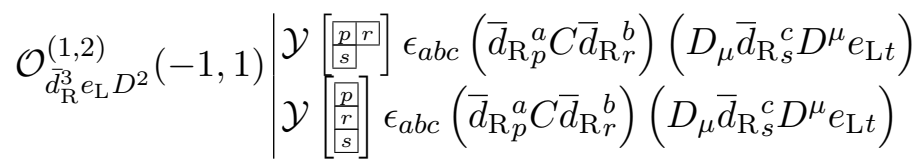

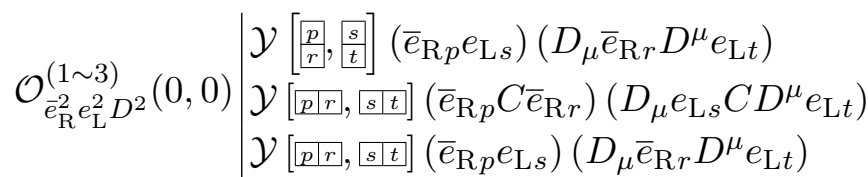

$$
\begin{aligned}
& \mathcal{O}_{\bar{e}_{\mathrm{R}} e_{\mathrm{L}} \nu_{\mathrm{L}}^{2} D^{2}}^{(1 \sim 3)}(0,2) \mid \begin{array}{l}
\mathcal{Y}\left[\begin{array}{l}
s \\
{\left[\begin{array}{l}
s \\
t
\end{array}\right]}
\end{array}\left(\bar{e}_{\mathrm{R} p} \nu_{\mathrm{L} s}\right)\left(D_{\mu} e_{\mathrm{L} r} C D^{\mu} \nu_{\mathrm{L} t}\right)\right. \\
\mathcal{Y}[s \mid t]\left(\bar{e}_{\mathrm{R} p} e_{\mathrm{L} r}\right)\left(D_{\mu} \nu_{\mathrm{L} s} C D^{\mu} \nu_{\mathrm{L} t}\right) \\
\mathcal{Y}[s \mid t]\left(\bar{e}_{\mathrm{R} p} \nu_{\mathrm{L} s}\right)\left(D_{\mu} e_{\mathrm{L} r} C D^{\mu} \nu_{\mathrm{L} t}\right)
\end{array} \\
& \mathcal{O}_{\nu_{\mathrm{L}}^{4} D^{2}}^{(1,2)}(0,4) \mid \begin{array}{l}
\mathcal{Y}\left[\begin{array}{l}
p r r \\
s, t
\end{array}\right]\left(\nu_{\mathrm{L} p} C \nu_{\mathrm{L} r}\right)\left(D_{\mu} \nu_{\mathrm{L} s} C D^{\mu} \nu_{\mathrm{L} t}\right) \\
\mathcal{Y}[\underline{p|r| s \mid t]}]\left(\nu_{\mathrm{L} p} C \nu_{\mathrm{L} r}\right)\left(D_{\mu} \nu_{\mathrm{L} s} C D^{\mu} \nu_{\mathrm{L} t}\right)
\end{array}
\end{aligned}
$$

\section{Class $\psi^{2} \psi^{\dagger 2} D^{2}: 50$ types}

$$
\begin{aligned}
& \mathcal{Y}\left[\begin{array}{l}
\frac{p}{r}, \frac{s}{t} \\
\frac{1}{t}
\end{array}\right]\left(u_{\mathrm{L} p a} C u_{\mathrm{L} r b}\right)\left(D_{\mu} \bar{u}_{\mathrm{L}}^{a} C D^{\mu} \bar{u}_{\mathrm{L} t}^{b}\right) \\
& \mathcal{O}_{u_{\mathrm{L}}^{2} \bar{u}_{\mathrm{L}}^{2} D^{2}}^{(1 \sim 4)}(0,0) \mathcal{Y}\left[\frac{p}{r}, \frac{s}{t}\right] i\left(u_{\mathrm{L} p a} C \sigma^{\mu \nu} u_{\mathrm{L} r b}\right)\left(D_{\mu} \bar{u}_{\mathrm{L}}^{a} C D_{\nu} \bar{u}_{\mathrm{L} t}^{b}\right) \\
& \mathcal{Y}[\underline{[p \mid r}, s|t|]]\left(u_{\mathrm{L} p a} C u_{\mathrm{L} r b}\right)\left(D_{\mu} \bar{u}_{\mathrm{L} s}^{a} C D^{\mu} \bar{u}_{\mathrm{L} t}^{b}\right) \\
& \mathcal{Y}[\underline{p \mid r}, \underline{s \mid t]}] i\left(u_{\mathrm{L} p a} C \sigma^{\mu \nu} u_{\mathrm{L} r b}\right)\left(D_{\mu} \bar{u}_{\mathrm{L} s}^{a} C D_{\nu} \bar{u}_{\mathrm{L} t}^{b}\right) \\
& \mathcal{O}_{d_{\mathrm{R}} e_{\mathrm{R}} u_{\mathrm{L}}^{2} D^{2}}^{(1,2)}(1,1) \mid \begin{array}{l}
\mathcal{Y}\left[\frac{p p r}{[p} i \epsilon^{a b c}\left(D_{\mu} d_{\mathrm{R} s c} C D_{\nu} e_{\mathrm{R} t}\right)\left(u_{\mathrm{L} p a} C \sigma^{\mu \nu} u_{\mathrm{L} r b}\right)\right. \\
\mathcal{Y}\left[\frac{p}{r}\right] \epsilon^{a b c}\left(u_{\mathrm{L} p a} C u_{\mathrm{L} r b}\right)\left(D_{\mu} d_{\mathrm{R} s c} C D^{\mu} e_{\mathrm{R} t}\right)
\end{array} \\
& \mathcal{O}_{\bar{u}_{\mathrm{R}} u_{\mathrm{R}} u_{\mathrm{L}} \bar{u}_{\mathrm{L}} D^{2}}^{(1 \sim 9,0)} \mid \begin{array}{ll}
\left(\bar{u}_{\mathrm{R}}^{a} u_{\mathrm{L} r b}\right) & \left(D^{\mu} \bar{u}_{\mathrm{L} t}^{b} D_{\mu} u_{\mathrm{R} s a}\right) \\
\left.\bar{u}_{\mathrm{R} p}^{a} u_{\mathrm{L} r a}\right)\left(D^{\mu} \bar{u}_{\mathrm{L} t}^{c} D_{\mu} u_{\mathrm{R} s c}\right) \\
i\left(D_{\nu} \bar{u}_{\mathrm{L} t}^{b} D_{\mu} u_{\mathrm{R} s a}\right)\left(\bar{u}_{\mathrm{R} p}^{a} \sigma^{\mu \nu} u_{\mathrm{L} r b}\right) \\
i\left(\bar{u}_{\mathrm{R} p}^{a} \sigma^{\mu \nu} u_{\mathrm{L} r a}\right)\left(D_{\nu} \bar{u}_{\mathrm{L} t}^{c} D_{\mu} u_{\mathrm{R} s c}\right)
\end{array} \\
& \left.\mathcal{O}_{d_{\mathrm{R}} \bar{d}_{\mathrm{L}} \bar{u}_{\mathrm{R}} u_{\mathrm{L}} D^{2}}^{(\sim, 0)} \mid \begin{array}{l}
\left(\bar{u}_{\mathrm{R}}^{a} u_{\mathrm{L} r b}\right)\left(D^{\mu} \bar{d}_{\mathrm{L} t}^{b} D_{\mu} d_{\mathrm{R} s a}\right) \\
\left(\bar{u}_{\mathrm{R}}^{a} u_{\mathrm{L} r a}\right)\left(D^{\mu} \bar{d}_{\mathrm{L} t}^{c} D_{\mu} d_{\mathrm{R} s c}\right) \\
i\left(D_{\nu} \bar{d}_{\mathrm{L} t}^{b} D_{\mu} d_{\mathrm{R} s a}\right)\left(\bar{u}_{\mathrm{R} p}^{a} \sigma^{\mu \nu} u_{\mathrm{L} r b}\right. \\
i\left(\bar{u}_{\mathrm{R}}^{a} \sigma^{\mu \nu} u_{\mathrm{L} r a}\right)\left(D_{\nu} \bar{d}_{\mathrm{L} t}^{c} D_{\mu} d_{\mathrm{R} s c}\right.
\end{array}\right) \\
& \mathcal{O}_{e_{\mathrm{R}} \bar{e}_{\mathrm{L}} \bar{u}_{\mathrm{R}} u_{\mathrm{L}} D^{2}}^{(1,0,0)} \mid \begin{array}{l}
\left(\bar{u}_{\mathrm{R}}^{a} u_{\mathrm{L} r a}\right)\left(D^{\mu} \bar{e}_{\mathrm{L} t} D_{\mu} e_{\mathrm{R} s}\right) \\
i\left(D_{\nu} \bar{e}_{\mathrm{L} t} D_{\mu} e_{\mathrm{R} s}\right)\left(\bar{u}_{\mathrm{R} p}^{a} \sigma^{\mu \nu} u_{\mathrm{L} r a}\right)
\end{array}
\end{aligned}
$$




$$
\begin{aligned}
& \mathcal{O}_{\bar{\nu}_{\mathrm{L}}^{2} \bar{u}_{\mathrm{R}} u_{\mathrm{L}} D^{2}}^{(1,2,-2)} \mid \begin{array}{l}
\mathcal{Y}\left[\begin{array}{l}
{[s]} \\
t
\end{array}\right] i\left(D_{\mu} \bar{\nu}_{\mathrm{L} s} C D_{\nu} \bar{\nu}_{\mathrm{L} t}\right)\left(\bar{u}_{\mathrm{R}}^{a} \sigma^{\mu \nu} u_{\mathrm{L} r a}\right) \\
\mathcal{Y}[\underline{s \mid t]}]\left(\bar{u}_{\mathrm{R}}{ }^{a} u_{\mathrm{L} r a}\right)\left(D_{\mu} \bar{\nu}_{\mathrm{L} s} C D^{\mu} \bar{\nu}_{\mathrm{L} t}\right)
\end{array} \\
& \mathcal{O}_{d_{\mathrm{L}} \bar{d}_{\mathrm{L}} u_{\mathrm{L}} \bar{u}_{\mathrm{L}} D^{2}}(0,0) \mid \begin{array}{l}
\left(d_{\mathrm{L} p a} C u_{\mathrm{L} r b}\right)\left(D_{\mu} \bar{d}_{\mathrm{L} s}^{a} C D^{\mu} \bar{u}_{\mathrm{L} t}^{b}\right) \\
\left(d_{\mathrm{L} p a} C u_{\mathrm{L} r b}\right)\left(D_{\mu} \bar{d}_{\mathrm{L}}^{b} C D^{\mu} \bar{u}_{\mathrm{L} t}^{a}\right) \\
i\left(d_{\mathrm{L} p a} C \sigma^{\mu \nu} u_{\mathrm{L} r b}\right)\left(D_{\mu} \bar{d}_{\mathrm{L} s}^{a} C D_{\nu} \bar{u}_{\mathrm{L} t}^{b}\right) \\
i\left(d_{\mathrm{L} p a} C \sigma^{\mu \nu} u_{\mathrm{L} r b}\right)\left(D_{\mu} \bar{d}_{\mathrm{L} s}^{b} C D_{\nu} \bar{u}_{\mathrm{L} t}^{a}\right)
\end{array} \\
& \mathcal{O}_{d_{\mathrm{L}} e_{\mathrm{R}} u_{\mathrm{R}} u_{\mathrm{L}} D^{2}}^{(1,2)}(1) \mid \begin{array}{l}
\epsilon^{a b c}\left(d_{\mathrm{L} p a} C u_{\mathrm{L} r b}\right)\left(D_{\mu} e_{\mathrm{R} s} C D^{\mu} u_{\mathrm{R} t c}\right) \\
i \epsilon^{a b c}\left(D_{\mu} e_{\mathrm{R} s} C D_{\nu} u_{\mathrm{R} t c}\right)\left(d_{\mathrm{L} p a} C \sigma^{\mu \nu} u_{\mathrm{L} r b}\right)
\end{array} \\
& \mathcal{O}_{d_{\mathrm{R}} d_{\mathrm{L}} \bar{\nu}_{\mathrm{L}} u_{\mathrm{L}} D^{2}}^{(1,2,-1)} \mid \begin{array}{l}
\epsilon^{a b c}\left(d_{\mathrm{L} p a} C u_{\mathrm{L} r b}\right)\left(D^{\mu} \bar{\nu}_{\mathrm{L} t} D_{\mu} d_{\mathrm{R} s c}\right) \\
i \epsilon^{a b c}\left(D_{\nu} \bar{\nu}_{\mathrm{L} t} D_{\mu} d_{\mathrm{R} s c}\right)\left(d_{\mathrm{L} p a} C \sigma^{\mu \nu} u_{\mathrm{L} r b}\right)
\end{array} \\
& \mathcal{O}_{\bar{d}_{\mathrm{R}} d_{\mathrm{R}} u_{\mathrm{L}} \bar{u}_{\mathrm{L}} D^{2}}^{(10,0)} \mid \begin{array}{ll}
\left(\bar{d}_{\mathrm{R}}^{a} u_{\mathrm{L} r b}\right) & \left(D^{\mu} \bar{u}_{\mathrm{L}}^{b} D_{\mu} d_{\mathrm{R} s a}\right) \\
\left(\bar{d}_{\mathrm{R}}^{a} u_{\mathrm{L} r a}\right)\left(D^{\mu} \bar{u}_{\mathrm{L} t}^{c} D_{\mu} d_{\mathrm{R} s c}\right) \\
i\left(D_{\nu} \bar{u}_{\mathrm{L} t}^{b} D_{\mu} d_{\mathrm{R} s a}\right)\left(\bar{d}_{\mathrm{R} p}^{a} \sigma^{\mu \nu} u_{\mathrm{L} r b}\right) \\
i\left(\bar{d}_{\mathrm{R} p}^{a} \sigma^{\mu \nu} u_{\mathrm{L} r a}\right)\left(D_{\nu} \bar{u}_{\mathrm{L} t}^{c} D_{\mu} d_{\mathrm{R} s c}\right)
\end{array} \\
& \mathcal{O}_{\bar{d}_{\mathrm{R}} e_{\mathrm{R}} \bar{\nu}_{\mathrm{L}} u_{\mathrm{L}} D^{2}}^{(1,2,0)} \mid \begin{array}{l}
\left(\bar{d}_{\mathrm{R}}^{a} u_{\mathrm{L} r a}\right)\left(D^{\mu} \bar{\nu}_{\mathrm{L} t} D_{\mu} e_{\mathrm{R} s}\right) \\
i\left(D_{\nu} \bar{\nu}_{\mathrm{L} t} D_{\mu} e_{\mathrm{R} s}\right)\left(\bar{d}_{\mathrm{R} p}^{a} \sigma^{\mu \nu} u_{\mathrm{L} r a}\right)
\end{array} \\
& \left.\mathcal{O}_{e_{\mathrm{L}} \bar{e}_{\mathrm{L}} u_{\mathrm{L}} \bar{u}_{\mathrm{L}} D^{2}}^{(0,0)} \mid \begin{array}{l}
\left(e_{\mathrm{L} p} C u_{\mathrm{L} r a}\right)\left(D_{\mu} \bar{e}_{\mathrm{L} s} C D^{\mu} \bar{u}_{\mathrm{L} t}^{a}\right) \\
i\left(e_{\mathrm{L} p} C \sigma^{\mu \nu} u_{\mathrm{L} r a}\right)\left(D_{\mu} \bar{e}_{\mathrm{L} s} C D_{\nu} \bar{u}_{\mathrm{L}}^{a}\right)
\end{array}\right) \\
& \mathcal{O}_{d_{\mathrm{R}} e_{\mathrm{L}} u_{\mathrm{R}} u_{\mathrm{L}} D^{2}}^{(1,2)}(1) \mid \begin{array}{l}
\epsilon^{a b c}\left(e_{\mathrm{L} p} C u_{\mathrm{L} r a}\right)\left(D_{\mu} d_{\mathrm{R} s b} C D^{\mu} u_{\mathrm{R} t c}\right) \\
i \epsilon^{a b c}\left(e_{\mathrm{L} p} C \sigma^{\mu \nu} u_{\mathrm{L} r a}\right)\left(D_{\mu} d_{\mathrm{R} s b} C D_{\nu} u_{\mathrm{R} t c}\right)
\end{array} \\
& \mathcal{O}_{\bar{d}_{\mathrm{L}} e_{\mathrm{L}} \bar{\nu}_{\mathrm{L}} u_{\mathrm{L}} D^{2}}^{(1,2)}(0,0) \mid \begin{array}{l}
\left(e_{\mathrm{L} p} C u_{\mathrm{L} r a}\right)\left(D_{\mu} \bar{d}_{\mathrm{L}}^{a} C D^{\mu} \bar{\nu}_{\mathrm{L} t}\right) \\
i\left(e_{\mathrm{L} p} C \sigma^{\mu \nu} u_{\mathrm{L} r a}\right)\left(D_{\mu} \bar{d}_{\mathrm{L} s}^{a} C D_{\nu} \bar{\nu}_{\mathrm{L} t}\right)
\end{array} \\
& \mathcal{O}_{\bar{e}_{\mathrm{R}} e_{\mathrm{R}} u_{\mathrm{L}} \bar{u}_{\mathrm{L}} D^{2}}^{(1,2,0)} \mid \begin{array}{l}
\left(\bar{e}_{\mathrm{R} p} u_{\mathrm{L} r a}\right)\left(D^{\mu} \bar{u}_{\mathrm{L}}^{a} D_{\mu} e_{\mathrm{R} s}\right) \\
i\left(D_{\nu} \bar{u}_{\mathrm{L} t}^{a} D_{\mu} e_{\mathrm{R} s}\right)\left(\bar{e}_{\mathrm{R} p} \sigma^{\mu \nu} u_{\mathrm{L} r a}\right)
\end{array} \\
& \mathcal{O}_{\nu_{\mathrm{L}} \bar{\nu}_{\mathrm{L}} u_{\mathrm{L}} \bar{u}_{\mathrm{L}} D^{2}}^{(1,2,0)} \mid \begin{array}{l}
\left(u_{\mathrm{L} p a} C \nu_{\mathrm{L} r}\right)\left(D_{\mu} \bar{u}_{\mathrm{L}}^{a} C D^{\mu} \bar{\nu}_{\mathrm{L} t}\right) \\
i\left(u_{\mathrm{L} p a} C \sigma^{\mu \nu} \nu_{\mathrm{L} r}\right)\left(D_{\mu} \bar{u}_{\mathrm{L} s}^{a} C D_{\nu} \bar{\nu}_{\mathrm{L} t}\right)
\end{array} \\
& \mathcal{O}_{\bar{d}_{\mathrm{L}} e_{\mathrm{R}} \nu_{\mathrm{L}} u_{\mathrm{L}} D^{2}}^{(1,2)}(0,2) \mid \begin{array}{l}
\left(u_{\mathrm{L} p a} C \nu_{\mathrm{L} r}\right)\left(D_{\mu} \bar{d}_{\mathrm{L}}^{a} D^{\mu} e_{\mathrm{R} t}\right) \\
i\left(D_{\mu} \bar{d}_{\mathrm{L} s}^{a} D_{\nu} e_{\mathrm{R} t}\right)\left(u_{\mathrm{L} p a} C \sigma^{\mu \nu} \nu_{\mathrm{L} r}\right)
\end{array}
\end{aligned}
$$

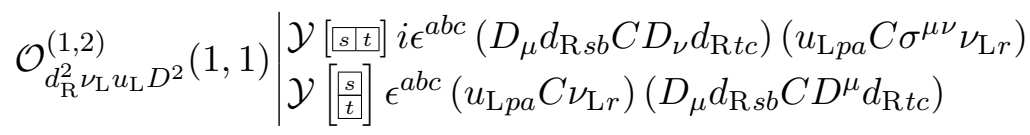




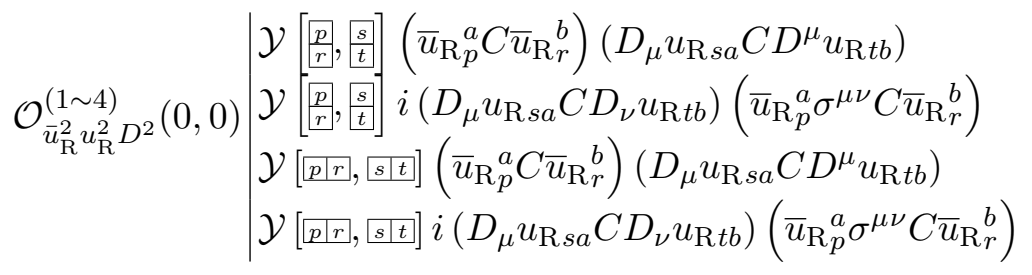

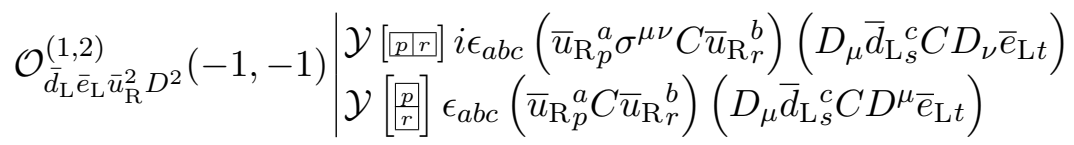

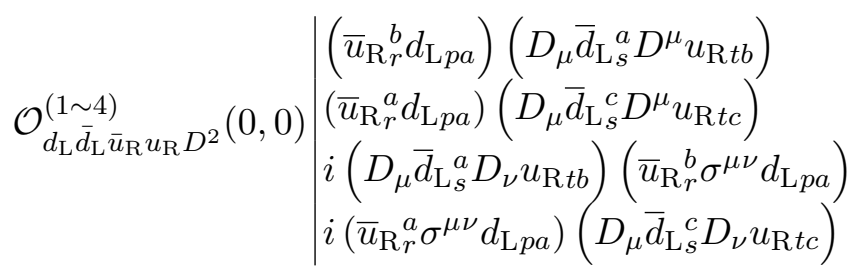

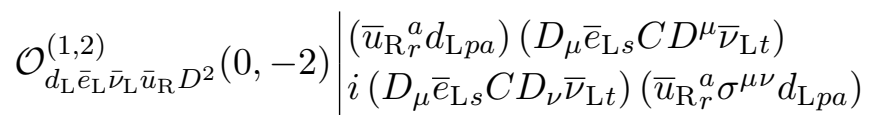

$$
\begin{aligned}
& \mathcal{O}_{\bar{d}_{\mathrm{R}} d_{\mathrm{R}} \bar{u}_{\mathrm{R}} u_{\mathrm{R}} D^{2}}^{(1 \sim 4)}(0) \mid \begin{array}{l}
\left(\bar{d}_{\mathrm{R} p}^{a} C \bar{u}_{\mathrm{R} r}^{b}\right)\left(D_{\mu} d_{\mathrm{R} s a} C D^{\mu} u_{\mathrm{R} t b}\right) \\
\left(\bar{d}_{\mathrm{R} p}^{a} C \bar{u}_{\mathrm{R}}^{b}\right)\left(D_{\mu} d_{\mathrm{R} s b} C D^{\mu} u_{\mathrm{R} t a}\right) \\
i\left(D_{\mu} d_{\mathrm{R} s a} C D_{\nu} u_{\mathrm{R} t b}\right)\left(\bar{d}_{\mathrm{R}_{p}^{a}}^{a} \sigma^{\mu \nu} C \bar{u}_{\mathrm{R}}{ }_{r}^{b}\right) \\
i\left(D_{\mu} d_{\mathrm{R} s b} C D_{\nu} u_{\mathrm{R} t a}\right)\left(\bar{d}_{\mathrm{R}_{p}^{a} \sigma^{\mu \nu} C \bar{u}_{\mathrm{R} r}^{b}}\right)
\end{array} \\
& \mathcal{O}_{\bar{d}_{\mathrm{R}} \bar{d}_{\mathrm{L}} \bar{\nu}_{\mathrm{L}} \bar{u}_{\mathrm{R}} D^{2}}^{(1,-1,-1)} \mid \begin{array}{l}
\epsilon_{a b c}\left(\bar{d}_{\mathrm{R}}^{a} C \bar{u}_{\mathrm{R}}^{b}\right)\left(D_{\mu} \bar{d}_{\mathrm{L}}^{c} C D^{\mu} \bar{\nu}_{\mathrm{L} t}\right) \\
i \epsilon_{a b c}\left(D_{\mu} \bar{d}_{\mathrm{L}_{s}^{c}}^{c} C D_{\nu} \bar{\nu}_{\mathrm{L} t}\right)\left(\bar{d}_{\mathrm{R}}^{a} \sigma^{\mu \nu} C \bar{u}_{\mathrm{R}}^{b}\right)
\end{array} \\
& \mathcal{O}_{e_{\mathrm{L}} \bar{e}_{\mathrm{L}} \bar{u}_{\mathrm{R}} u_{\mathrm{R}} D^{2}}^{(0,0)} \mid \begin{array}{l}
\left(\bar{u}_{\mathrm{R} r}^{a} e_{\mathrm{L} p}\right)\left(D_{\mu} \bar{e}_{\mathrm{L} s} D^{\mu} u_{\mathrm{R} t a}\right) \\
i\left(D_{\mu} \bar{e}_{\mathrm{L} s} D_{\nu} u_{\mathrm{R} t a}\right)\left(\bar{u}_{\mathrm{R}}^{a}{ }_{r}^{\mu \nu} \sigma_{\mathrm{L} p}\right)
\end{array} \\
& \mathcal{O}_{\bar{e}_{\mathrm{R}} e_{\mathrm{R}} \bar{u}_{\mathrm{R}} u_{\mathrm{R}} D^{2}}^{(0,0)} \mid \begin{array}{l}
\left(\bar{e}_{\mathrm{R} p} C \bar{u}_{\mathrm{R}}{ }_{r}^{a}\right)\left(D_{\mu} e_{\mathrm{R} s} C D^{\mu} u_{\mathrm{R} t a}\right) \\
i\left(D_{\mu} e_{\mathrm{R} s} C D_{\nu} u_{\mathrm{R} t a}\right)\left(\bar{e}_{\mathrm{R} p} \sigma^{\mu \nu} C \bar{u}_{\mathrm{R}}^{a}\right)
\end{array} \\
& \mathcal{O}_{d_{\mathrm{R}} \bar{e}_{\mathrm{R}} \bar{\nu}_{\mathrm{L}} \bar{u}_{\mathrm{R}} D^{2}}^{(1,2,-2)} \mid \begin{array}{l}
\left(\bar{e}_{\mathrm{R} p} C \bar{u}_{\mathrm{R}}^{a}\right)\left(D^{\mu} \bar{\nu}_{\mathrm{L} t} D_{\mu} d_{\mathrm{R} s a}\right) \\
i\left(D_{\nu} \bar{\nu}_{\mathrm{L} t} D_{\mu} d_{\mathrm{R} s a}\right)\left(\bar{e}_{\mathrm{R} p} \sigma^{\mu \nu} C \bar{u}_{\mathrm{R}}^{a}\right)
\end{array} \\
& \mathcal{O}_{\nu_{\mathrm{L}} \bar{\nu}_{\mathrm{L}} \bar{u}_{\mathrm{R}} u_{\mathrm{R}} D^{2}}^{(1,0,0)} \mid \begin{array}{l}
\left(\bar{u}_{\mathrm{R}}^{a} \nu_{\mathrm{L} r}\right)\left(D^{\mu} \bar{\nu}_{\mathrm{L} t} D_{\mu} u_{\mathrm{R} s a}\right) \\
i\left(D_{\nu} \bar{\nu}_{\mathrm{L} t} D_{\mu} u_{\mathrm{R} s a}\right)\left(\bar{u}_{\mathrm{R}}^{a} \sigma^{\mu \nu} \nu_{\mathrm{L} r}\right)
\end{array}
\end{aligned}
$$

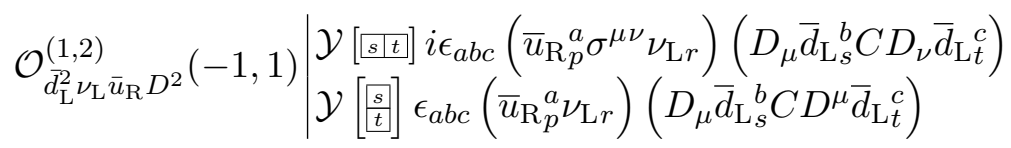

$$
\begin{aligned}
& \mathcal{O}_{d_{\mathrm{R}} \bar{e}_{\mathrm{L}} \nu_{\mathrm{L}} \bar{u}_{\mathrm{R}} D^{2}}^{(1,2,0)} \mid \begin{array}{l}
\left(\bar{u}_{\mathrm{R} p}^{a} \nu_{\mathrm{L} r}\right)\left(D^{\mu} \bar{e}_{\mathrm{L} t} D_{\mu} d_{\mathrm{R} s a}\right) \\
i\left(D_{\nu} \bar{e}_{\mathrm{L} t} D_{\mu} d_{\mathrm{R} s a}\right)\left(\bar{u}_{\mathrm{R} p}^{a} \sigma^{\mu \nu} \nu_{\mathrm{L} r}\right)
\end{array}
\end{aligned}
$$




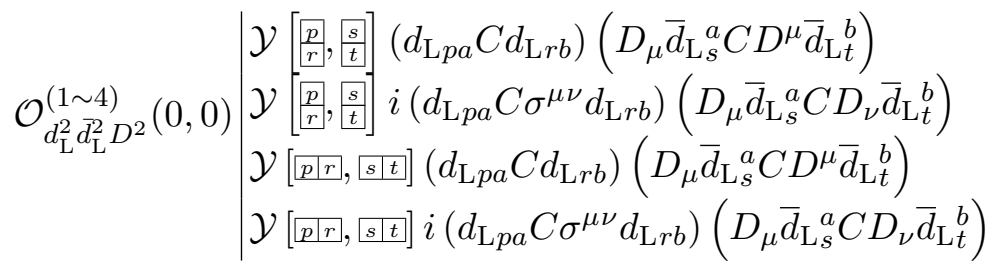

$$
\begin{aligned}
& \mathcal{O}_{d_{\mathrm{R}} d_{\mathrm{L}}^{2} \bar{e}_{\mathrm{L}} D^{2}}^{(1,2)-1)} \mid \begin{array}{l}
\mathcal{Y}\left[[p r r] i \epsilon^{a b c}\left(D_{\nu} \bar{e}_{\mathrm{L} t} D_{\mu} d_{\mathrm{R} s c}\right)\left(d_{\mathrm{L} p a} C \sigma^{\mu \nu} d_{\mathrm{L} r b}\right)\right. \\
\mathcal{Y}\left[\begin{array}{l}
p \\
{[r]}
\end{array} \epsilon^{a b c}\left(d_{\mathrm{L} p a} C d_{\mathrm{L} r b}\right)\left(D^{\mu} \bar{e}_{\mathrm{L} t} D_{\mu} d_{\mathrm{R} s c}\right)\right.
\end{array}
\end{aligned}
$$

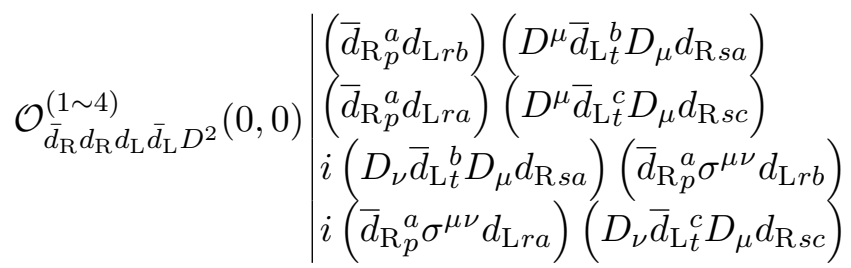

$$
\begin{aligned}
& \mathcal{O}_{\bar{d}_{\mathrm{R}} d_{\mathrm{L}} e_{\mathrm{R}} \bar{e}_{\mathrm{L}} D^{2}}^{(1,2)}(0,0) \mid \begin{array}{l}
\left(\bar{d}_{\mathrm{R}}^{a} d_{\mathrm{L} r a}\right)\left(D^{\mu} \bar{e}_{\mathrm{L} t} D_{\mu} e_{\mathrm{R} s}\right) \\
i\left(D_{\nu} \bar{e}_{\mathrm{L} t} D_{\mu} e_{\mathrm{R} s}\right)\left(\bar{d}_{\mathrm{R}}^{a} \sigma^{\mu \nu} d_{\mathrm{L} r a}\right)
\end{array} \\
& \mathcal{O}_{\bar{d}_{\mathrm{R}} d_{\mathrm{L}} \bar{\nu}_{\mathrm{L}}^{2} D^{2}}^{(1,2)}(0,-2) \mid \begin{array}{l}
\mathcal{Y}\left[\begin{array}{l}
s \\
{\left[\begin{array}{l}
s \\
t
\end{array}\right]}
\end{array}\right]\left(D_{\mu} \bar{\nu}_{\mathrm{L} s} C D_{\nu} \bar{\nu}_{\mathrm{L} t}\right)\left(\bar{d}_{\mathrm{R}}^{a} \sigma^{\mu \nu} d_{\mathrm{L} r a}\right) \\
\mathcal{Y}[s s t]]\left(\bar{d}_{\mathrm{R} p}^{a} d_{\mathrm{L} r a}\right)\left(D_{\mu} \bar{\nu}_{\mathrm{L} s} C D^{\mu} \bar{\nu}_{\mathrm{L} t}\right)
\end{array} \\
& \mathcal{O}_{d_{\mathrm{L}} \bar{d}_{\mathrm{L}} e_{\mathrm{L}} \bar{e}_{\mathrm{L}} D^{2}}^{(1,2,0)} \mid \begin{array}{l}
\left(d_{\mathrm{L} p a} C e_{\mathrm{L} r}\right)\left(D_{\mu} \bar{d}_{\mathrm{L}}^{a} C D^{\mu} \bar{e}_{\mathrm{L} t}\right) \\
i\left(d_{\mathrm{L} p a} C \sigma^{\mu \nu} e_{\mathrm{L} r}\right)\left(D_{\mu} \bar{d}_{\mathrm{L} s}^{a} C D_{\nu} \bar{e}_{\mathrm{L} t}\right)
\end{array} \\
& \mathcal{O}_{d_{\mathrm{L}} \overline{\mathrm{d}}_{\mathrm{L}} \bar{e}_{\mathrm{R}} e_{\mathrm{R}} D^{2}}^{(1,0,0)} \mid \begin{array}{l}
\left(\bar{e}_{\mathrm{R} r} d_{\mathrm{L} p a}\right)\left(D_{\mu} \bar{d}_{\mathrm{L} s}^{a} D^{\mu} e_{\mathrm{R} t}\right) \\
i\left(D_{\mu} \bar{d}_{\mathrm{L} s}^{a} D_{\nu} e_{\mathrm{R} t}\right)\left(\bar{e}_{\mathrm{R} r} \sigma^{\mu \nu} d_{\mathrm{L} p a}\right)
\end{array}
\end{aligned}
$$

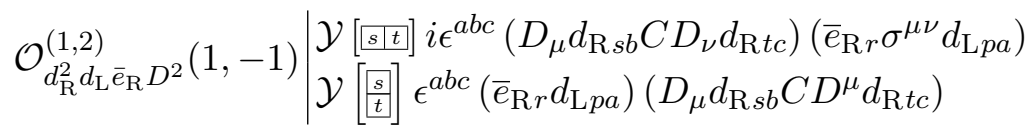

$$
\begin{aligned}
& \mathcal{O}_{d_{\mathrm{L}} \bar{d}_{\mathrm{L}} \nu_{\mathrm{L}} \bar{\nu}_{\mathrm{L}} D^{2}}^{(1,0,0)} \mid \begin{array}{l}
\left(d_{\mathrm{L} p a} C \nu_{\mathrm{L} r}\right)\left(D_{\mu} \bar{d}_{\mathrm{L}}^{a} C D^{\mu} \bar{\nu}_{\mathrm{L} t}\right) \\
i\left(d_{\mathrm{L} p a} C \sigma^{\mu \nu} \nu_{\mathrm{L} r}\right)\left(D_{\mu} \bar{d}_{\mathrm{L} s}^{a} C D_{\nu} \bar{\nu}_{\mathrm{L} t}\right)
\end{array}
\end{aligned}
$$

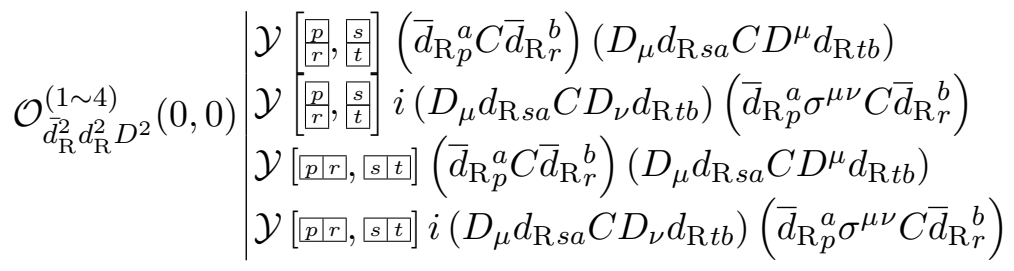

$$
\begin{aligned}
& \mathcal{O}_{\bar{d}_{\mathrm{R}} d_{\mathrm{R}} e_{\mathrm{L}} \bar{e}_{\mathrm{L}} D^{2}}^{(1,2)}(0,0) \mid \begin{array}{l}
\left(\bar{d}_{\mathrm{R}}^{a} e_{\mathrm{L} r}\right)\left(D^{\mu} \bar{e}_{\mathrm{L} t} D_{\mu} d_{\mathrm{R} s a}\right) \\
i\left(D_{\nu} \bar{e}_{\mathrm{L} t} D_{\mu} d_{\mathrm{R} s a}\right)\left(\bar{d}_{\mathrm{R} p}^{a} \sigma^{\mu \nu} e_{\mathrm{L} r}\right)
\end{array}
\end{aligned}
$$




$$
\begin{aligned}
& \mathcal{O}_{\bar{d}_{\mathrm{R}} d_{\mathrm{R}} \bar{e}_{\mathrm{R}} e_{\mathrm{R}} D^{2}}^{(1,2,0)} \mid \begin{array}{l}
\left(\bar{d}_{\mathrm{R}}^{a} C \bar{e}_{\mathrm{R} r}\right)\left(D_{\mu} d_{\mathrm{R} s a} C D^{\mu} e_{\mathrm{R} t}\right) \\
i\left(D_{\mu} d_{\mathrm{R} s a} C D_{\nu} e_{\mathrm{R} t}\right)\left(\bar{d}_{\mathrm{R} p}^{a} \sigma^{\mu \nu} C \bar{e}_{\mathrm{R} r}\right)
\end{array} \\
& \mathcal{O}_{\bar{d}_{\mathrm{R}} d_{\mathrm{R}} \nu_{\mathrm{L}} \bar{\nu}_{\mathrm{L}} D^{2}}^{(1,2)}(0,0) \mid \begin{array}{l}
\left(\bar{d}_{\mathrm{R}}^{a} \nu_{\mathrm{L} r}\right)\left(D^{\mu} \bar{\nu}_{\mathrm{L} t} D_{\mu} d_{\mathrm{R} s a}\right) \\
i\left(D_{\nu} \bar{\nu}_{\mathrm{L} t} D_{\mu} d_{\mathrm{R} s a}\right)\left(\bar{d}_{\mathrm{R}}^{a} \sigma^{\mu \nu} \nu_{\mathrm{L} r}\right)
\end{array}
\end{aligned}
$$

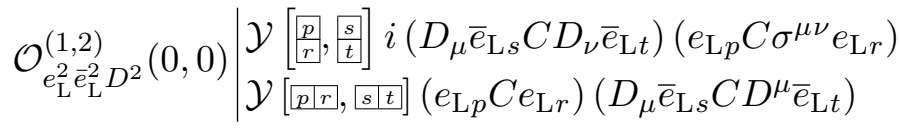

$$
\begin{aligned}
& \mathcal{O}_{\bar{e}_{\mathrm{R}} e_{\mathrm{R}} e_{\mathrm{L}} \bar{e}_{\mathrm{L}} D^{2}}^{(1,2)} \mid \begin{array}{l}
\left(\bar{e}_{\mathrm{R} p} e_{\mathrm{L} r}\right)\left(D^{\mu} \bar{e}_{\mathrm{L} t} D_{\mu} e_{\mathrm{R} s}\right) \\
i\left(D_{\nu} \bar{e}_{\mathrm{L} t} D_{\mu} e_{\mathrm{R} s}\right)\left(\bar{e}_{\mathrm{R} p} \sigma^{\mu \nu} e_{\mathrm{L} r}\right)
\end{array} \\
& \mathcal{O}_{\bar{e}_{\mathrm{R}} e_{\mathrm{L}} \bar{\nu}_{\mathrm{L}}^{2} D^{2}}^{(1,2),-2)} \mid \begin{array}{l}
\mathcal{Y}\left[\begin{array}{l}
{[s]} \\
{[t]}
\end{array}\right] i\left(D_{\mu} \bar{\nu}_{\mathrm{L} s} C D_{\nu} \bar{\nu}_{\mathrm{L} t}\right)\left(\bar{e}_{\mathrm{R} p} \sigma^{\mu \nu} e_{\mathrm{L} r}\right) \\
\mathcal{Y}\left[\begin{array}{l}
s] t] \\
{[s}
\end{array} \bar{e}_{\mathrm{R} p} e_{\mathrm{L} r}\right)\left(D_{\mu} \bar{\nu}_{\mathrm{L} s} C D^{\mu} \bar{\nu}_{\mathrm{L} t}\right)
\end{array} \\
& \mathcal{O}_{e_{\mathrm{L}} \bar{e}_{\mathrm{L}} \nu_{\mathrm{L}} \bar{\nu}_{\mathrm{L}} D^{2}}^{(1,2,0)} \mid \begin{array}{l}
\left(e_{\mathrm{L} p} C \nu_{\mathrm{L} r}\right)\left(D_{\mu} \bar{e}_{\mathrm{L} s} C D^{\mu} \bar{\nu}_{\mathrm{L} t}\right) \\
i\left(D_{\mu} \bar{e}_{\mathrm{L} s} C D_{\nu} \bar{\nu}_{\mathrm{L} t}\right)\left(e_{\mathrm{L} p} C \sigma^{\mu \nu} \nu_{\mathrm{L} r}\right)
\end{array}
\end{aligned}
$$

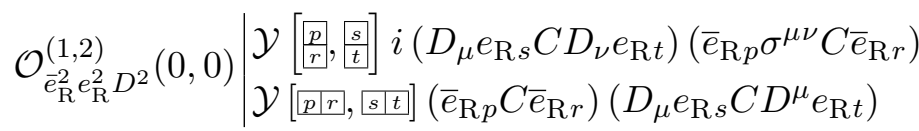

$$
\begin{aligned}
& \mathcal{O}_{\bar{e}_{\mathrm{R}} e_{\mathrm{R}} \nu_{\mathrm{L}} \bar{\nu}_{\mathrm{L}} D^{2}}^{(10,0)} \mid \begin{array}{l}
\left(\bar{e}_{\mathrm{R} p} \nu_{\mathrm{L} r}\right)\left(D^{\mu} \bar{\nu}_{\mathrm{L} t} D_{\mu} e_{\mathrm{R} s}\right) \\
i\left(D_{\nu} \bar{\nu}_{\mathrm{L} t} D_{\mu} e_{\mathrm{R} s}\right)\left(\bar{e}_{\mathrm{R} p} \sigma^{\mu \nu} \nu_{\mathrm{L} r}\right)
\end{array}
\end{aligned}
$$

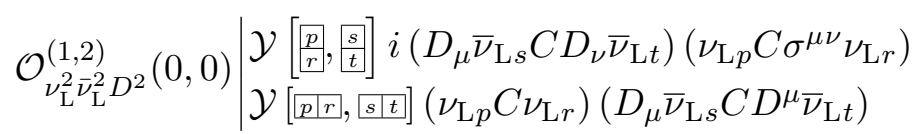

\section{Class $F_{\mathrm{L}} \psi^{4}: 33$ types}

$$
\begin{aligned}
& \mathcal{Y}\left[\frac{p}{r}, \frac{s}{t}\right] i\left(\lambda^{A}\right)_{b}^{d} G_{\mathrm{L}}^{A \mu \nu}\left(u_{\mathrm{L} s a} C u_{\mathrm{L} t d}\right)\left(\bar{u}_{\mathrm{R} p}^{a} \sigma_{\mu \nu} C \bar{u}_{\mathrm{R} r}^{b}\right) \\
& \mathcal{Y}\left[\frac{p}{r}, \frac{s}{t}\right] i\left(\lambda^{A}\right)_{b}^{d} G_{\mathrm{L}}^{A \mu \nu}\left(\bar{u}_{\mathrm{R} r}^{b} u_{\mathrm{L} t d}\right)\left(\bar{u}_{\mathrm{R} p}^{a} \sigma_{\mu \nu} u_{\mathrm{L} s a}\right) \\
& \mathcal{Y}\left[\begin{array}{l}
\left.\frac{p}{r}, \frac{s}{t}\right] \\
{[}
\end{array}\right] i\left(\lambda^{A}\right)_{a}^{d} G_{\mathrm{L}}^{A \mu \nu}\left(\bar{u}_{\mathrm{R}}{ }_{r}^{b} u_{\mathrm{L} t d}\right)\left(\bar{u}_{\mathrm{R}}^{a} \sigma_{\mu \nu} u_{\mathrm{L} s b}\right) \\
& \left.\mathcal{Y}\left[\frac{p}{r}, s, s\right]\right] i\left(\lambda^{A}\right)_{b}^{d} G_{\mathrm{L}}^{A \mu \nu}\left(u_{\mathrm{L} s a} C u_{\mathrm{L} t d}\right)\left(\bar{u}_{\mathrm{R}}^{a} \sigma_{\mu \nu} C \bar{u}_{\mathrm{R}}^{b}\right) \\
& \mathcal{O}_{G_{\mathrm{L}} \overline{\mathrm{R}}_{\mathrm{R}}^{2} u_{\mathrm{L}}^{2}}^{(1 \sim 12)}(0,0) \mathcal{Y}\left[\frac{p}{r}, s \mid t\right] i\left(\lambda^{A}\right)_{b}^{d} G_{\mathrm{L}}^{A \mu \nu}\left(\bar{u}_{\mathrm{R}}{ }_{r}^{b} u_{\mathrm{L} t d}\right)\left(\bar{u}_{\mathrm{R}}^{a} \sigma_{\mu \nu} u_{\mathrm{L} s a}\right) \\
& \mathcal{Y}\left[\frac{p}{r}, s \mid t\right] i\left(\lambda^{A}\right)_{a}^{d} G_{\mathrm{L}}^{A \mu \nu}\left(\bar{u}_{\mathrm{R} r}^{b} u_{\mathrm{L} t d}\right)\left(\bar{u}_{\mathrm{R} p}^{a} \sigma_{\mu \nu} u_{\mathrm{L} s b}\right) \\
& \mathcal{Y}\left[\underline{p p r}, \frac{s}{t}\right] i\left(\lambda^{A}\right)_{b}^{d} G_{\mathrm{L}}^{A \mu \nu}\left(u_{\mathrm{L} s a} C u_{\mathrm{L} t d}\right)\left(\bar{u}_{\mathrm{R} p}^{a} \sigma_{\mu \nu} C \bar{u}_{\mathrm{R} r}^{b}\right)
\end{aligned}
$$

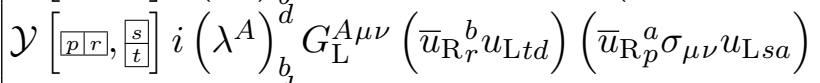

$$
\begin{aligned}
& \mathcal{Y}\left[\underline{p \mid r,}, \frac{s}{t}\right] i\left(\lambda^{A}\right)_{a}^{d} G_{\mathrm{L}}^{A \mu \nu}\left(\bar{u}_{\mathrm{R} r}^{b} u_{\mathrm{L} t d}\right)\left(\bar{u}_{\mathrm{R}}^{a} \sigma_{\mu \nu} u_{\mathrm{L} s b}\right) \\
& \mathcal{Y}\left[[p r,, s \mid t] i\left(\lambda^{A}\right)_{b}^{d} G_{\mathrm{L}}^{A \mu \nu}\left(u_{\mathrm{L} s a} C u_{\mathrm{L} t d}\right)\left(\bar{u}_{\mathrm{R}}^{a} \sigma_{\mu \nu} C \bar{u}_{\mathrm{R}}^{b}\right)\right. \\
& \mathcal{Y}\left[[p|r, s| t] i\left(\lambda^{A}\right)_{b}^{d} G_{\mathrm{L}}^{A \mu \nu}\left(\bar{u}_{\mathrm{R}}^{b} u_{\mathrm{L} t d}\right)\left(\bar{u}_{\mathrm{R} p}^{a} \sigma_{\mu \nu} u_{\mathrm{L} s a}\right)\right. \\
& \mathcal{Y}[\underline{p \mid r}, \underline{s \mid t]}] i\left(\lambda^{A}\right)_{a}^{d} G_{\mathrm{L}}^{A \mu \nu}\left(\bar{u}_{\mathrm{R}}^{b} u_{\mathrm{L} t d}\right)\left(\bar{u}_{\mathrm{R}}^{a} \sigma_{\mu \nu} u_{\mathrm{L} s b}\right)
\end{aligned}
$$




$$
\begin{aligned}
& \mathcal{Y}\left[\begin{array}{l}
s \\
\underline{t}
\end{array}\right] i\left(\lambda^{A}\right)_{d}^{b} \epsilon^{a c d} G_{\mathrm{L}}^{A \mu \nu}\left(u_{\mathrm{L} s b} C u_{\mathrm{L} t c}\right)\left(d_{\mathrm{L} p a} C \sigma_{\mu \nu} e_{\mathrm{L} r}\right) \\
& \mathcal{Y}\left[\begin{array}{l}
s \\
t
\end{array}\right] i\left(\lambda^{A}\right)_{d}^{b} \epsilon^{a c d} G_{\mathrm{L}}^{A \mu \nu}\left(e_{\mathrm{L} r} C u_{\mathrm{L} t c}\right)\left(d_{\mathrm{L} p a} C \sigma_{\mu \nu} u_{\mathrm{L} s b}\right) \\
& \mathcal{O}_{G_{\mathrm{L}} d_{\mathrm{L}} e_{\mathrm{L}} u_{\mathrm{L}}^{2}}^{(1 \sim 6)}(1,1) \mid \mathcal{Y}\left[\begin{array}{l}
s \\
\left.\frac{s}{t}\right]
\end{array}\right] i\left(\lambda^{A}\right)_{d}^{b} \epsilon^{a c d} G_{\mathrm{L} \mu \nu}^{A}\left(d_{\mathrm{L} p a} C u_{\mathrm{L} t c}\right)\left(e_{\mathrm{L} r} C \sigma^{\mu \nu} u_{\mathrm{L} s b}\right) \\
& \mathcal{Y}[s \mid t] i\left(\lambda^{A}\right)_{d}^{b} \epsilon^{a c d} G_{\mathrm{L}}^{A \mu \nu}\left(u_{\mathrm{L} s b} C u_{\mathrm{L} t c}\right)\left(d_{\mathrm{L} p a} C \sigma_{\mu \nu} e_{\mathrm{L} r}\right) \\
& \mathcal{Y}\left[s[t] i\left(\lambda^{A}\right)_{d}^{b} \epsilon^{a c d} G_{\mathrm{L}}^{A \mu \nu}\left(e_{\mathrm{L} r} C u_{\mathrm{L} t c}\right)\left(d_{\mathrm{L} p a} C \sigma_{\mu \nu} u_{\mathrm{L} s b}\right)\right. \\
& \mathcal{Y}\left[s[t] i\left(\lambda^{A}\right)_{d}^{b} \epsilon^{a c d} G_{\mathrm{L} \mu \nu}^{A}\left(d_{\mathrm{L} p a} C u_{\mathrm{L} t c}\right)\left(e_{\mathrm{L} r} C \sigma^{\mu \nu} u_{\mathrm{L} s b}\right)\right. \\
& \mathcal{O}_{G_{\mathrm{L}} \bar{d}_{\mathrm{R}} d_{\mathrm{L}} \bar{u}_{\mathrm{R}} u_{\mathrm{L}}}^{(1 \sim 12)}(0,0) i\left(\lambda^{A}\right)_{c}^{b} G_{\mathrm{L}}^{A \mu \nu}\left(d_{\mathrm{L} r b} C u_{\mathrm{L} t a}\right)\left(\bar{d}_{\mathrm{R}}^{a} \sigma_{\mu \nu} C \bar{u}_{\mathrm{R}}^{c}\right) \\
& i\left(\lambda^{A}\right)_{a}^{b} G_{\mathrm{L}}^{A \mu \nu}\left(d_{\mathrm{L} r b} C u_{\mathrm{L} t c}\right)\left(\bar{d}_{\mathrm{R} p}^{a} \sigma_{\mu \nu} C \bar{u}_{\mathrm{R}}^{c}\right) \\
& i\left(\lambda^{A}\right)_{c}^{d} G_{\mathrm{L}}^{A \mu \nu}\left(d_{\mathrm{L} r a} C u_{\mathrm{L} t d}\right)\left(\bar{d}_{\left.\mathrm{R}_{p}^{a} \sigma_{\mu \nu} C \bar{u}_{\mathrm{R}}^{c}\right)}\right) \\
& i\left(\lambda^{A}\right)_{a}^{d} G_{\mathrm{L}}^{A \mu \nu}\left(d_{\mathrm{L} r b} C u_{\mathrm{L} t d}\right)\left(\bar{d}_{\left.\mathrm{R}_{p}^{a} \sigma_{\mu \nu} C \bar{u}_{\mathrm{R}}^{b}\right)}^{b}\right. \\
& i\left(\lambda^{A}\right)_{c}^{b} G_{\mathrm{L} \mu \nu}^{A}\left(\bar{d}_{\mathrm{R} p}^{a} u_{\mathrm{L} t a}\right)\left(\bar{u}_{\mathrm{R}}^{c} \sigma^{\mu \nu} d_{\mathrm{L} r b}\right) \\
& i\left(\lambda^{A}\right)_{a}^{b} G_{\mathrm{L} \mu \nu}^{A}\left(\bar{d}_{\mathrm{R}}^{a} u_{\mathrm{L} t c}\right)\left(\bar{u}_{\mathrm{R}}^{c} \sigma^{\mu \nu} d_{\mathrm{L} r b}\right) \\
& i\left(\lambda^{A}\right)_{c}^{d} G_{\mathrm{L} \mu \nu}^{A}\left(\bar{d}_{\mathrm{R} p}^{a} u_{\mathrm{L} t d}\right)\left(\bar{u}_{\mathrm{R}}^{c} \sigma^{\mu \nu} d_{\mathrm{L} r a}\right) \\
& i\left(\lambda^{A}\right)_{a}^{d} G_{\mathrm{L} \mu \nu}^{A}\left(\bar{d}_{\mathrm{R} p}^{a} u_{\mathrm{L} t d}\right)\left(\bar{u}_{\mathrm{R}}^{b} \sigma^{\mu \nu} d_{\mathrm{L} r b}\right) \\
& \mathcal{O}_{G_{\mathrm{L}} \bar{e}_{\mathrm{R}} e_{\mathrm{L}} \bar{u}_{\mathrm{R}} u_{\mathrm{L}}}^{(1 \sim 2,0)} \mid \begin{array}{l}
i\left(\lambda^{A}\right)_{a}^{b} G_{\mathrm{L}}^{A \mu \nu}\left(\bar{u}_{\mathrm{R}}^{a} u_{\mathrm{L} t b}\right)\left(\bar{e}_{\mathrm{R} p} \sigma_{\mu \nu} e_{\mathrm{L} r}\right) \\
i\left(\lambda^{A}\right)_{a}^{b} G_{\mathrm{L}}^{A \mu \nu}\left(e_{\mathrm{L} r} C u_{\mathrm{L} t b}\right)\left(\bar{e}_{\mathrm{R} p} \sigma_{\mu \nu} C \bar{u}_{\mathrm{R}}^{a}\right) \\
i\left(\lambda^{A}\right)_{a}^{b} G_{\mathrm{L} \mu \nu}^{A}\left(\bar{e}_{\mathrm{R} p} u_{\mathrm{L} t b}\right)\left(\bar{u}_{\mathrm{R}}^{a} \sigma^{\mu \nu} e_{\mathrm{L} r}\right)
\end{array} \\
& \mathcal{O}_{G_{\mathrm{L}} \nu_{\mathrm{L}}^{2} \bar{u}_{\mathrm{R}} u_{\mathrm{L}}}^{(1 \sim 3)}(0,2) \mid \begin{array}{l}
\mathcal{Y}\left[\begin{array}{l}
s \\
\underline{t}
\end{array}\right] i\left(\lambda^{A}\right)_{a}^{b} G_{\mathrm{L}}^{A \mu \nu}\left(u_{\mathrm{L} r b} C \nu_{\mathrm{L} t}\right)\left(\bar{u}_{\mathrm{R}}^{a} \sigma_{\mu \nu} \nu_{\mathrm{L} s}\right) \\
\mathcal{Y}\left[\begin{array}{l}
s \\
t
\end{array}\right] i\left(\lambda^{A}\right)_{a}^{b} G_{\mathrm{L} \mu \nu}^{A}\left(\bar{u}_{\mathrm{R}}{ }_{p}^{a} \nu_{\mathrm{L} t}\right)\left(u_{\mathrm{L} r b} C \sigma^{\mu \nu} \nu_{\mathrm{L} s}\right) \\
\mathcal{Y}[\underline{s \mid t]}] i\left(\lambda^{A}\right)_{a}^{b}\left(\nu_{\mathrm{L} s} C \nu_{\mathrm{L} t}\right) G_{\mathrm{L}}^{A \mu \nu}\left(\bar{u}_{\mathrm{R} p}^{a} \sigma_{\mu \nu} u_{\mathrm{L} r b}\right)
\end{array}
\end{aligned}
$$




$$
\begin{aligned}
& \mathcal{Y}\left[\frac{p}{r}\right] i\left(\lambda^{A}\right)_{d}^{b} \epsilon^{a c d} G_{\mathrm{L}}^{A \mu \nu}\left(u_{\mathrm{L} s c} C \nu_{\mathrm{L} t}\right)\left(d_{\mathrm{L} p a} C \sigma_{\mu \nu} d_{\mathrm{L} r b}\right) \\
& \mathcal{Y}\left[\frac{p}{r}\right] i\left(\lambda^{A}\right)_{d}^{b} \epsilon^{a c d} G_{\mathrm{L}}^{A \mu \nu}\left(d_{\mathrm{L} r b} C \nu_{\mathrm{L} t}\right)\left(d_{\mathrm{L} p a} C \sigma_{\mu \nu} u_{\mathrm{L} s c}\right)
\end{aligned}
$$

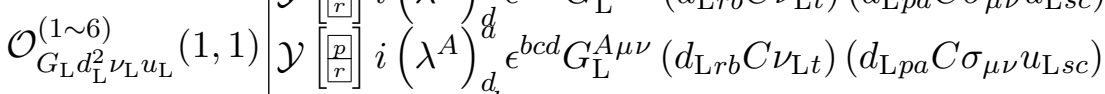

$$
\begin{aligned}
& \mathcal{Y}[\overline{p p r}] i\left(\lambda^{A}\right)_{d}^{b} \epsilon^{a c d} G_{\mathrm{L}}^{A \mu \nu}\left(u_{\mathrm{L} s c} C \nu_{\mathrm{L} t}\right)\left(d_{\mathrm{L} p a} C \sigma_{\mu \nu} d_{\mathrm{L} r b}\right) \\
& \mathcal{Y}[\overline{p p r}] i\left(\lambda^{A}\right)_{d}^{b} \epsilon^{a c d} G_{\mathrm{L}}^{A \mu \nu}\left(d_{\mathrm{L} r b} C \nu_{\mathrm{L} t}\right)\left(d_{\mathrm{L} p a} C \sigma_{\mu \nu} u_{\mathrm{L} s c}\right) \\
& \mathcal{Y}[\overline{p r r}] i\left(\lambda^{A}\right)_{d}^{d} \epsilon^{b c d} G_{\mathrm{L}}^{A \mu \nu}\left(d_{\mathrm{L} r b} C \nu_{\mathrm{L} t}\right)\left(d_{\mathrm{L} p a} C \sigma_{\mu \nu} u_{\mathrm{L} s c}\right) \\
& \mathcal{O}_{G_{\mathrm{L}} \bar{d}_{\mathrm{R}} e_{\mathrm{L}} \nu_{\mathrm{L}} u_{\mathrm{L}}}^{(1 \sim, 2)} \mid \begin{array}{l}
i\left(\lambda^{A}\right)_{a}^{b} G_{\mathrm{L}}^{A \mu \nu}\left(u_{\mathrm{L} s b} C \nu_{\mathrm{L} t}\right)\left(\bar{d}_{\mathrm{R} p}^{a} \sigma_{\mu \nu} e_{\mathrm{L} r}\right) \\
i\left(\lambda^{A}\right)_{a}^{b} G_{\mathrm{L}}^{A \mu \nu}\left(e_{\mathrm{L} r} C \nu_{\mathrm{L} t}\right)\left(\bar{d}_{\mathrm{R} p}^{a} \sigma_{\mu \nu} u_{\mathrm{L} s b}\right) \\
i\left(\lambda^{A}\right)_{a}^{b} G_{\mathrm{L} \mu \nu}^{A}\left(\bar{d}_{\mathrm{R}}{ }_{p}^{a} \nu_{\mathrm{L} t}\right)\left(e_{\mathrm{L} r} C \sigma^{\mu \nu} u_{\mathrm{L} s b}\right)
\end{array} \\
& \mathcal{Y}\left[\begin{array}{l}
s \\
t
\end{array}\right] i \epsilon_{a c d}\left(\lambda^{A}\right)_{b}^{d} G_{\mathrm{L}}^{A \mu \nu}\left(\bar{u}_{\mathrm{R}}^{b} C \bar{u}_{\mathrm{R}}^{c}\right)\left(\bar{d}_{\mathrm{R} p}^{a} \sigma_{\mu \nu} C \bar{e}_{\mathrm{R} r}\right) \\
& \mathcal{Y}\left[\begin{array}{l}
s] \\
t
\end{array}\right] i \epsilon_{a c d}\left(\lambda^{A}\right)_{b}^{d} G_{\mathrm{L}}^{A \mu \nu}\left(\bar{e}_{\mathrm{R} r} C \bar{u}_{\mathrm{R}}{ }_{t}^{c}\right)\left(\bar{d}_{\mathrm{R} p}^{a} \sigma_{\mu \nu} C \bar{u}_{\mathrm{R}}^{b}\right)
\end{aligned}
$$

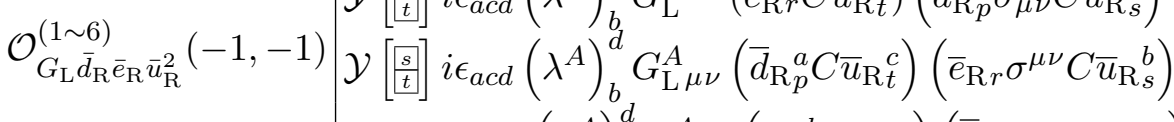

$$
\begin{aligned}
& \mathcal{Y}[s \mid t] i \epsilon_{a c d}\left(\lambda^{A}\right)_{b}^{d} G_{\mathrm{L}}^{A \mu \nu}\left(\bar{u}_{\mathrm{R}}^{b} C \bar{u}_{\mathrm{R}}^{c}\right)\left(\bar{d}_{\mathrm{R}}^{a} \sigma_{\mu \nu} C \bar{e}_{\mathrm{R} r}\right) \\
& \mathcal{Y}\left[\overline{s \mid t]} i \epsilon_{a c d}\left(\lambda^{A}\right)_{b}^{d} G_{\mathrm{L}}^{A \mu \nu}\left(\bar{e}_{\mathrm{R} r} C \bar{u}_{\mathrm{R} t}^{c}\right)\left(\bar{d}_{\mathrm{R} p}^{a} \sigma_{\mu \nu} C \bar{u}_{\mathrm{R} s}^{b}\right)\right. \\
& \mathcal{Y}[\underline{s \mid t}] i \epsilon_{a c d}\left(\lambda^{A}\right)_{b}^{d} G_{\mathrm{L} \mu \nu}^{A}\left(\bar{d}_{\mathrm{R}}^{a} C \bar{u}_{\mathrm{R} t}^{c}\right)\left(\bar{e}_{\mathrm{R} r} \sigma^{\mu \nu} C \bar{u}_{\mathrm{R}}^{b}\right) \\
& \mathcal{O}_{G_{\mathrm{L}} d_{\mathrm{L}} \bar{e}_{\mathrm{R}} \nu_{\mathrm{L}} \bar{u}_{\mathrm{R}}}^{(1 \sim 0)} \mid \begin{array}{l}
i\left(\lambda^{A}\right)_{b}^{a} G_{\mathrm{L}}^{A \mu \nu}\left(\bar{u}_{\mathrm{R}}^{b} \nu_{\mathrm{L} t}\right)\left(\bar{e}_{\mathrm{R} r} \sigma_{\mu \nu} d_{\mathrm{L} p a}\right) \\
i\left(\lambda^{A}\right)_{b}^{a}\left(\bar{e}_{\mathrm{R} r} \nu_{\mathrm{L} t}\right) G_{\mathrm{L}}^{A \mu \nu}\left(\bar{u}_{\mathrm{R} s}^{b} \sigma_{\mu \nu} d_{\mathrm{L} p a}\right) \\
i\left(\lambda^{A}\right)_{b}^{a} G_{\mathrm{L} \mu \nu}^{A}\left(d_{\mathrm{L} p a} C \nu_{\mathrm{L} t}\right)\left(\bar{e}_{\mathrm{R} r} \sigma^{\mu \nu} C \bar{u}_{\mathrm{R} s}^{b}\right)
\end{array} \\
& \mathcal{Y}\left[\frac{p}{r}\right] i \epsilon_{a c d}\left(\lambda^{A}\right)_{b}^{d} G_{\mathrm{L}}^{A \mu \nu}\left(\bar{u}_{\mathrm{R}}^{c} \nu_{\mathrm{L} t}\right)\left(\bar{d}_{\mathrm{R}}^{a} \sigma_{\mu \nu} C \bar{d}_{\mathrm{R}}^{b}\right) \\
& \mathcal{Y}\left[\frac{p}{r}\right] i \epsilon_{a c d}\left(\lambda^{A}\right)_{b}^{d} G_{\mathrm{L}}^{A \mu \nu}\left(\bar{d}_{\mathrm{R}}^{b} \nu_{\mathrm{L} t}\right)\left(\bar{d}_{\mathrm{R}}^{a} \sigma_{\mu \nu} C \bar{u}_{\mathrm{R}}^{c}{ }_{s}\right) \\
& \mathcal{O}_{G_{\mathrm{L}} \bar{d}_{\mathrm{R}}^{2} \nu_{\mathrm{L}} \bar{u}_{\mathrm{R}}}^{(1 \sim 6)}(-1,1) \mathcal{Y}\left[\begin{array}{l}
{\left[\frac{p}{r}\right]} \\
r
\end{array}\right] \epsilon_{a b d}\left(\lambda^{A}\right)_{c}^{d} G_{\mathrm{L}}^{A \mu \nu}\left(\bar{d}_{\mathrm{R}}{ }_{r}^{b} \nu_{\mathrm{L} t}\right)\left(\bar{d}_{\mathrm{R} p}^{a} \sigma_{\mu \nu} C \bar{u}_{\mathrm{R}}^{c}\right) \\
& \mathcal{Y}[\overline{p r r}] i \epsilon_{a c d}\left(\lambda^{A}\right)_{b}^{d} G_{\mathrm{L}}^{A \mu \nu}\left(\bar{u}_{\mathrm{R}}{ }_{s}^{c} \nu_{\mathrm{L} t}\right)\left(\bar{d}_{\mathrm{R} p}^{a} \sigma_{\mu \nu} C \bar{d}_{\mathrm{R} r}^{b}\right) \\
& \mathcal{Y}[\overline{p r r}] i \epsilon_{a c d}\left(\lambda^{A}\right)_{b}^{d} G_{\mathrm{L}}^{A \mu \nu}\left(\bar{d}_{\mathrm{R} r}^{b} \nu_{\mathrm{L} t}\right)\left(\bar{d}_{\mathrm{R}}^{a} \sigma_{\mu \nu} C \bar{u}_{\mathrm{R}}^{c}\right) \\
& \mathcal{Y}[\overline{p p r}] i \epsilon_{a b d}\left(\lambda^{A}\right)_{c}^{d} G_{\mathrm{L}}^{A \mu \nu}\left(\bar{d}_{\mathrm{R}}^{b} \nu_{\mathrm{L} t}\right)\left(\bar{d}_{\mathrm{R}}^{a} \sigma_{\mu \nu} C \bar{u}_{\mathrm{R}}^{c}\right)
\end{aligned}
$$

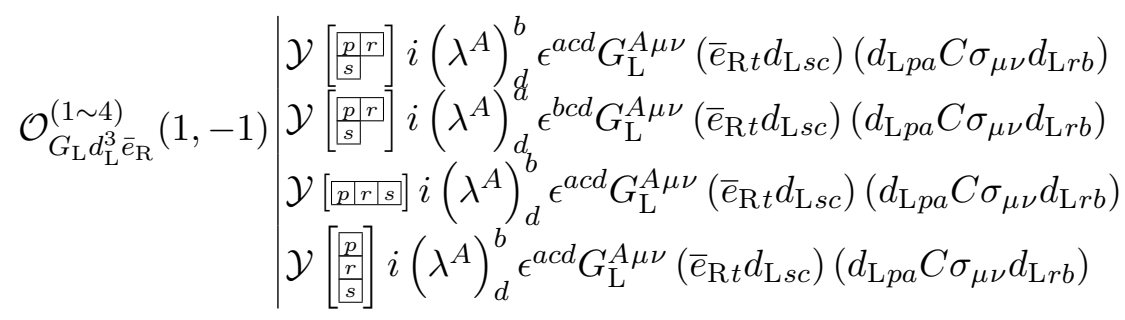




$$
\begin{aligned}
& \mathcal{Y}\left[\frac{p}{r}, \frac{s}{t}\right] i\left(\lambda^{A}\right)_{b}^{d} G_{\mathrm{L}}^{A \mu \nu}\left(d_{\mathrm{L} s a} C d_{\mathrm{L} t d}\right)\left(\bar{d}_{\mathrm{R}}^{a} \sigma_{\mu \nu} C \bar{d}_{\mathrm{R}}^{b}\right)
\end{aligned}
$$

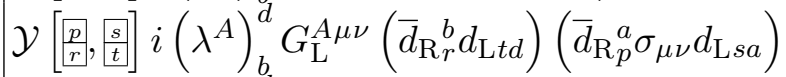

$$
\begin{aligned}
& \mathcal{Y}\left[\frac{p}{r}, \frac{s}{t}\right] i\left(\lambda^{A}\right)_{a}^{d} G_{\mathrm{L}}^{A \mu \nu}\left(\bar{d}_{\mathrm{R}}{ }_{r}^{b} d_{\mathrm{L} t d}\right)\left(\bar{d}_{\mathrm{R}_{p}^{a} \sigma_{\mu \nu}} d_{\mathrm{L} s b}\right) \\
& \mathcal{Y}\left[\frac{p}{r}, s \mid t\right] i\left(\lambda^{A}\right)_{b}^{d} G_{\mathrm{L}}^{A \mu \nu}\left(d_{\mathrm{L} s a} C d_{\mathrm{L} t d}\right)\left(\bar{d}_{\mathrm{R} p}^{a} \sigma_{\mu \nu} C \bar{d}_{\mathrm{R} r}^{b}\right) \\
& \mathcal{O}_{G_{\mathrm{L}} \bar{d}_{\mathrm{R}}^{2} d_{\mathrm{L}}^{2}}^{(1 \sim 12)}(0,0) \mathcal{Y}\left[\frac{p}{r}, s \mid t\right] i\left(\lambda^{A}\right)_{b}^{d} G_{\mathrm{L}}^{A \mu \nu}\left(\bar{d}_{\mathrm{R} r}^{b} d_{\mathrm{L} t d}\right)\left(\bar{d}_{\mathrm{R} p}^{a} \sigma_{\mu \nu} d_{\mathrm{L} s a}\right) \\
& \mathcal{Y}\left[\frac{p}{r}, s \mid t\right] i\left(\lambda^{A}\right)_{a}^{d} G_{\mathrm{L}}^{A \mu \nu}\left(\bar{d}_{\mathrm{R}}{ }_{r}^{b} d_{\mathrm{L} t d}\right)\left(\bar{d}_{\mathrm{R} p}^{a} \sigma_{\mu \nu} d_{\mathrm{L} s b}\right)
\end{aligned}
$$

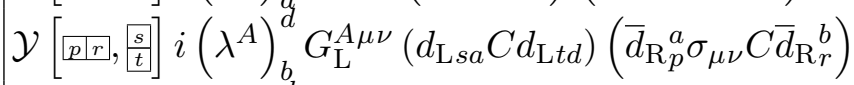

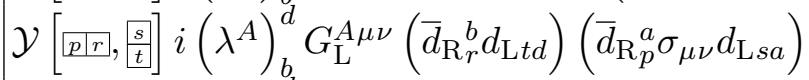

$$
\begin{aligned}
& \mathcal{Y}\left[\begin{array}{l}
p \mid r, \\
\left.\frac{s}{t}\right]
\end{array}\right] i\left(\lambda^{A}\right)_{a}^{d} G_{\mathrm{L}}^{A \mu \nu}\left(\bar{d}_{\mathrm{R}}{ }_{r}^{b} d_{\mathrm{L} t d}\right)\left(\bar{d}_{\mathrm{R}}^{a} \sigma_{\mu \nu} d_{\mathrm{L} s b}\right) \\
& \mathcal{Y}[\overline{p \mid r}, s \mid t]] i\left(\lambda^{A}\right)_{b}^{d} G_{\mathrm{L}}^{A \mu \nu}\left(d_{\mathrm{L} s a} C d_{\mathrm{L} t d}\right)\left(\bar{d}_{\mathrm{R} p}^{a} \sigma_{\mu \nu} C \bar{d}_{\mathrm{R}}{ }_{r}^{b}\right) \\
& \mathcal{Y}\left[[p|r, s| t] i\left(\lambda^{A}\right)_{b}^{d} G_{\mathrm{L}}^{A \mu \nu}\left(\bar{d}_{\mathrm{R}}^{b} d_{\mathrm{L} t d}\right)\left(\bar{d}_{\mathrm{R}_{p}^{a} \sigma_{\mu \nu}} d_{\mathrm{L} s a}\right)\right. \\
& \mathcal{Y}\left[[p r r, s s t] i\left(\lambda^{A}\right)_{a}^{d} G_{\mathrm{L}}^{A \mu \nu}\left(\bar{d}_{\mathrm{R}}^{b} d_{\mathrm{L} t d}\right)\left(\bar{d}_{\mathrm{R}}^{a} \sigma_{\mu \nu} d_{\mathrm{L} s b}\right)\right. \\
& \mathcal{O}_{G_{\mathrm{L}} \bar{d}_{\mathrm{R}} d_{\mathrm{L}} \bar{e}_{\mathrm{R}} e_{\mathrm{L}}}^{(1 \sim 3)}(0,0) \mid \begin{array}{l}
i\left(\lambda^{A}\right)_{a}^{b}\left(\bar{e}_{\mathrm{R} s} e_{\mathrm{L} t}\right) G_{\mathrm{L}}^{A \mu \nu}\left(\bar{d}_{\mathrm{R}}^{a} \sigma_{\mu \nu} d_{\mathrm{L} r b}\right) \\
i\left(\lambda^{A}\right)_{a}^{b} G_{\mathrm{L}}^{A \mu \nu}\left(d_{\mathrm{L} r b} C e_{\mathrm{L} t}\right)\left(\bar{d}_{\mathrm{R}}^{a} \sigma_{\mu \nu} C \bar{e}_{\mathrm{R} s}\right) \\
i\left(\lambda^{A}\right)_{a}^{b} G_{\mathrm{L} \mu \nu}^{A}\left(\bar{d}_{\mathrm{R}}^{a} e_{\mathrm{L} t}\right)\left(\bar{e}_{\mathrm{R} s} \sigma^{\mu \nu} d_{\mathrm{L} r b}\right)
\end{array}
\end{aligned}
$$

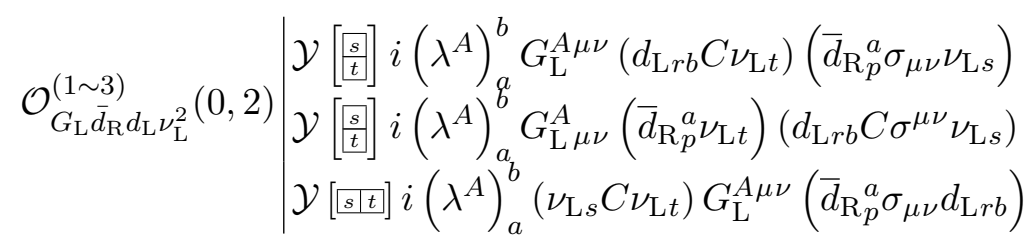

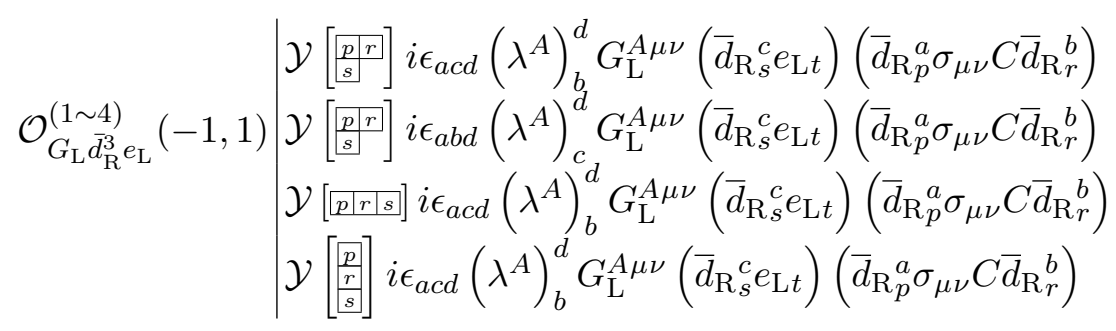

$$
\begin{aligned}
& \mathcal{Y}\left[\frac{p}{r}, \frac{s}{t}\right] i F_{\mathrm{L}}^{\mu \nu}\left(\bar{u}_{\mathrm{R}}^{b} u_{\mathrm{L} t b}\right)\left(\bar{u}_{\mathrm{R}}^{a} \sigma_{\mu \nu} u_{\mathrm{L} s a}\right) \\
& \mathcal{Y}[[p|r, s| t]] i F_{\mathrm{L}}^{\mu \nu}\left(\bar{u}_{\mathrm{R} r}^{b} u_{\mathrm{L} t b}\right)\left(\bar{u}_{\mathrm{R}}^{a} \sigma_{\mu \nu} u_{\mathrm{L} s a}\right) \\
& \left.\mathcal{O}_{F_{\mathrm{L}} \bar{u}_{\mathrm{R}}^{2} u_{\mathrm{L}}^{2}}^{(1 \sim 6)}(0,0) \mathcal{Y}\left[\frac{p}{r}, s\right] t\right] i F_{\mathrm{L}}{ }^{\mu \nu}\left(u_{\mathrm{L} s a} C u_{\mathrm{L} t b}\right)\left(\bar{u}_{\mathrm{R} p}^{a} \sigma_{\mu \nu} C \bar{u}_{\mathrm{R} r}^{b}\right) \\
& \left.\mathcal{Y}\left[\frac{p}{r}, s\right] t\right] i F_{\mathrm{L}}{ }^{\mu \nu}\left(\bar{u}_{\mathrm{R}}{ }_{r}^{b} u_{\mathrm{L} t b}\right)\left(\bar{u}_{\mathrm{R}}{ }_{p}^{a} \sigma_{\mu \nu} u_{\mathrm{L} s a}\right) \\
& \mathcal{Y}\left[\frac{p \mid r,}{s}, i F_{\mathrm{L}}^{\mu \nu}\left(u_{\mathrm{L} s a} C u_{\mathrm{L} t b}\right)\left(\bar{u}_{\mathrm{R} p}^{a} \sigma_{\mu \nu} C \bar{u}_{\mathrm{R}_{r}^{b}}^{b}\right)\right. \\
& \mathcal{Y}\left[\underline{\underline{p \mid r},}, \frac{s}{t}\right] i F_{\mathrm{L}}^{\mu \nu}\left(\bar{u}_{\mathrm{R}}^{b} u_{\mathrm{L} t b}\right)\left(\bar{u}_{\mathrm{R}}^{a} \sigma_{\mu \nu} u_{\mathrm{L} s a}\right)
\end{aligned}
$$




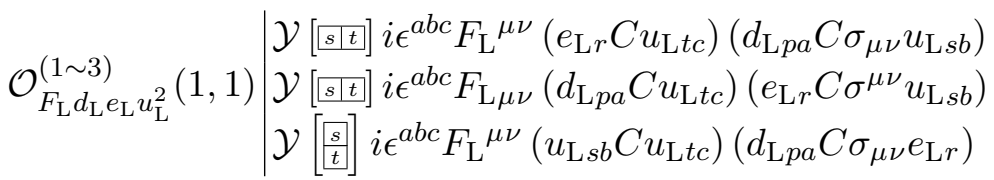

$$
\begin{aligned}
& i F_{\mathrm{L}}^{\mu \nu}\left(\bar{u}_{\mathrm{R}}^{b} u_{\mathrm{L} t a}\right)\left(\bar{d}_{\mathrm{R} p}^{a} \sigma_{\mu \nu} d_{\mathrm{L} r b}\right) \\
& i F_{\mathrm{L}}^{\mu \nu}\left(\bar{u}_{\mathrm{R}}^{c} u_{\mathrm{L} t c}\right)\left(\bar{d}_{\mathrm{R} p}^{a} \sigma_{\mu \nu} d_{\mathrm{L} r a}\right) \\
& \mathcal{O}_{F_{\mathrm{L}} \bar{d}_{\mathrm{R}} d_{\mathrm{L}} \bar{u}_{\mathrm{R}} u_{\mathrm{L}}}^{(\sim, \bar{s}}(0,0) i F_{\mathrm{L}}^{\mu \nu}\left(d_{\mathrm{L} r b} C u_{\mathrm{L} t a}\right)\left(\bar{d}_{\mathrm{R}}^{a} \sigma_{\mu \nu} C \bar{u}_{\mathrm{R}}{ }^{b}\right) \\
& i F_{\mathrm{L}}{ }^{\mu \nu}\left(d_{\mathrm{L} r a} C u_{\mathrm{L} t c}\right)\left(\bar{d}_{\mathrm{R} p}^{a} \sigma_{\mu \nu} C \bar{u}_{\mathrm{R} s}^{c}\right) \\
& i F_{\mathrm{L} \mu \nu}\left(\bar{d}_{\mathrm{R} p}^{a} u_{\mathrm{L} t a}\right)\left(\bar{u}_{\mathrm{R} s}^{b} \sigma^{\mu \nu} d_{\mathrm{L} r b}\right) \\
& i F_{\mathrm{L} \mu \nu}\left(\bar{d}_{\mathrm{R} p}^{a} u_{\mathrm{L} t c}\right)\left(\bar{u}_{\mathrm{R} s}^{c} \sigma^{\mu \nu} d_{\mathrm{L} r a}\right) \\
& \mathcal{O}_{F_{\mathrm{L}} \bar{e}_{\mathrm{R}} e_{\mathrm{L}} \bar{u}_{\mathrm{R}} u_{\mathrm{L}}}^{(\sim, 0)} \mid \begin{array}{l}
i F_{\mathrm{L}}{ }^{\mu \nu}\left(\bar{u}_{\mathrm{R}}{ }_{s}^{a} u_{\mathrm{L} t a}\right)\left(\bar{e}_{\mathrm{R} p} \sigma_{\mu \nu} e_{\mathrm{L} r}\right) \\
i F_{\mathrm{L}}{ }^{\mu \nu}\left(e_{\mathrm{L} r} C u_{\mathrm{L} t a}\right)\left(\bar{e}_{\mathrm{R} p} \sigma_{\mu \nu} C \bar{u}_{\mathrm{R}}{ }_{s}^{a}\right) \\
i F_{\mathrm{L} \mu \nu}\left(\bar{e}_{\mathrm{R} p} u_{\mathrm{L} t a}\right)\left(\bar{u}_{\mathrm{R}}{ }^{a} \sigma^{\mu \nu} e_{\mathrm{L} r}\right)
\end{array}
\end{aligned}
$$

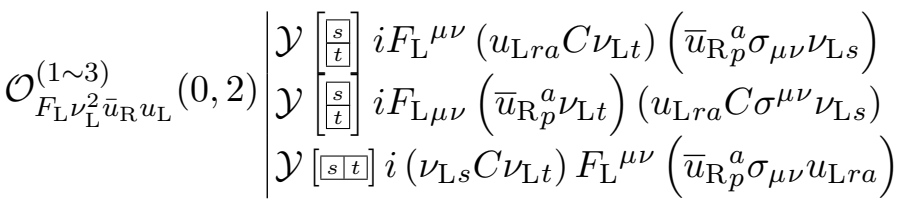

$$
\begin{aligned}
& \mathcal{O}_{F_{\mathrm{L}} d_{\mathrm{L}}^{2} \nu_{\mathrm{L}} u_{\mathrm{L}}}^{(1 \sim 3)} \mid \begin{array}{l}
\mathcal{Y}[1,1) i \epsilon^{a b c} F_{\mathrm{L}}{ }^{\mu \nu}\left(u_{\mathrm{L} s c} C \nu_{\mathrm{L} t}\right)\left(d_{\mathrm{L} p a} C \sigma_{\mu \nu} d_{\mathrm{L} r b}\right) \\
\mathcal{Y}\left[\underline{p \mid r]} i \epsilon^{a b c} F_{\mathrm{L}}{ }^{\mu \nu}\left(d_{\mathrm{L} r b} C \nu_{\mathrm{L} t}\right)\left(d_{\mathrm{L} p a} C \sigma_{\mu \nu} u_{\mathrm{L} s c}\right)\right. \\
\mathcal{Y}\left[\frac{p}{r}\right] i \epsilon^{a b c} F_{\mathrm{L}}{ }^{\mu \nu}\left(d_{\mathrm{L} r b} C \nu_{\mathrm{L} t}\right)\left(d_{\mathrm{L} p a} C \sigma_{\mu \nu} u_{\mathrm{L} s c}\right)
\end{array} \\
& \mathcal{O}_{F_{\mathrm{L}} \bar{d}_{\mathrm{R}} e_{\mathrm{L}} \nu_{\mathrm{L}} u_{\mathrm{L}}}^{(1 \sim 2)}(0,2) \mid \begin{array}{l}
i F_{\mathrm{L}}{ }^{\mu \nu}\left(u_{\mathrm{L} s a} C \nu_{\mathrm{L} t}\right)\left(\bar{d}_{\mathrm{R} p}^{a} \sigma_{\mu \nu} e_{\mathrm{L} r}\right) \\
i F_{\mathrm{L}}^{\mu \nu}\left(e_{\mathrm{L} r} C \nu_{\mathrm{L} t}\right)\left(\bar{d}_{\mathrm{R}}{ }^{a} \sigma_{\mu \nu} u_{\mathrm{L} s a}\right) \\
i F_{\mathrm{L} \mu \nu}\left(\bar{d}_{\mathrm{R} p}^{a} \nu_{\mathrm{L} t}\right)\left(e_{\mathrm{L} r} C \sigma^{\mu \nu} u_{\mathrm{L} s a}\right)
\end{array} \\
& \mathcal{O}_{F_{\mathrm{L}}(1 \sim 3)}^{\left(\bar{d}_{\mathrm{R}} \bar{e}_{\mathrm{R}} \bar{u}_{\mathrm{R}}^{2}(-1,-1)\right.} \mid \begin{array}{l}
\mathcal{Y}[s \mid t] i \epsilon_{a b c} F_{\mathrm{L}}^{\mu \nu}\left(\bar{e}_{\mathrm{R} r} C \bar{u}_{\mathrm{R} t}^{c}\right)\left(\bar{d}_{\mathrm{R} p}^{a} \sigma_{\mu \nu} C \bar{u}_{\mathrm{R} s}^{b}\right) \\
\mathcal{Y}\left[\begin{array}{l}
s \mid t] \\
\mathcal{Y}
\end{array} \epsilon_{a b c} F_{\mathrm{L} \mu \nu}\left(\bar{d}_{\mathrm{R} p}^{a} C \bar{u}_{\mathrm{R} t}^{c}\right)\left(\bar{e}_{\mathrm{R} r} \sigma^{\mu \nu} C \bar{u}_{\mathrm{R} s}^{b}\right)\right. \\
\mathcal{Y}\left[\begin{array}{l}
s \\
\underline{t}
\end{array}\right] i \epsilon_{a b c} F_{\mathrm{L}}{ }^{\mu \nu}\left(\bar{u}_{\mathrm{R}}^{b} C \bar{u}_{\mathrm{R}}^{c}\right)\left(\bar{d}_{\mathrm{R} p}^{a} \sigma_{\mu \nu} C \bar{e}_{\mathrm{R} r}\right)
\end{array}
\end{aligned}
$$

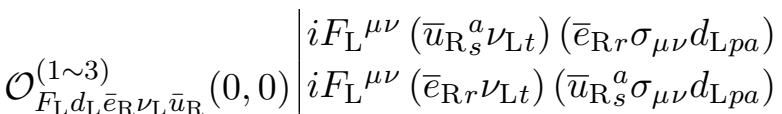

$$
\begin{aligned}
& i F_{\mathrm{L} \mu \nu}\left(d_{\mathrm{L} p a} C \nu_{\mathrm{L} t}\right)\left(\bar{e}_{\mathrm{R} r} \sigma^{\mu \nu} C \bar{u}_{\mathrm{R} s}^{a}\right)
\end{aligned}
$$

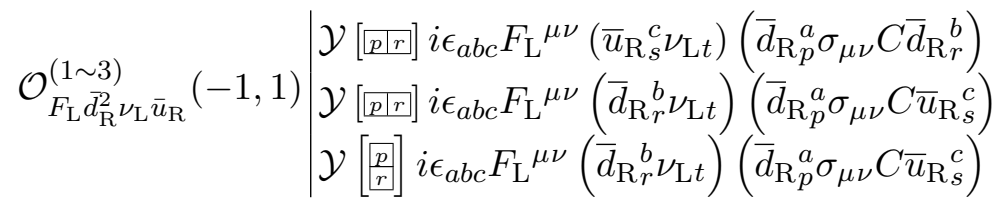

$$
\begin{aligned}
& \mathcal{O}_{F_{\mathrm{L}} d_{\mathrm{L}}^{3} \bar{e}_{\mathrm{R}}}^{(1,2,-1)} \mid \begin{array}{l}
\mathcal{Y}\left[\overline{[p|r| s]} i \epsilon^{a b c} F_{\mathrm{L}}^{\mu \nu}\left(\bar{e}_{\mathrm{R} t} d_{\mathrm{L} s c}\right)\left(d_{\mathrm{L} p a} C \sigma_{\mu \nu} d_{\mathrm{L} r b}\right)\right. \\
\mathcal{Y}\left[\frac{p r r}{[s]} i \epsilon^{a b c} F_{\mathrm{L}}{ }^{\mu \nu}\left(\bar{e}_{\mathrm{R} t} d_{\mathrm{L} s c}\right)\left(d_{\mathrm{L} p a} C \sigma_{\mu \nu} d_{\mathrm{L} r b}\right)\right.
\end{array}
\end{aligned}
$$




$$
\begin{aligned}
& \mathcal{Y}\left[\frac{p}{r}, \frac{s}{t}\right] i F_{\mathrm{L}}^{\mu \nu}\left(\bar{d}_{\mathrm{R} r}^{b} d_{\mathrm{L} t b}\right)\left(\bar{d}_{\mathrm{R}_{p}^{a} \sigma_{\mu \nu}} d_{\mathrm{L} s a}\right) \\
& \mathcal{Y}\left[[p|r, s| t] i F_{\mathrm{L}}^{\mu \nu}\left(\bar{d}_{\mathrm{R} r}^{b} d_{\mathrm{L} t b}\right)\left(\bar{d}_{\mathrm{R} p}^{a} \sigma_{\mu \nu} d_{\mathrm{L} s a}\right)\right. \\
& \mathcal{O}_{F_{\mathrm{L}} \bar{d}_{\mathrm{R}}^{2} d_{\mathrm{L}}^{2}}^{(1 \sim 6)}(0,0) \mathcal{Y}\left[\frac{p}{r}, s \mid t\right] i F_{\mathrm{L}}^{\mu \nu}\left(d_{\mathrm{L} s a} C d_{\mathrm{L} t b}\right)\left(\bar{d}_{\mathrm{R} p}^{a} \sigma_{\mu \nu} C \bar{d}_{\mathrm{R} r}^{b}\right) \\
& \left.\mathcal{Y}, \frac{p}{r}, s \mid t\right] i F_{\mathrm{L}}^{\mu \nu}\left(\bar{d}_{\mathrm{R}}^{b} d_{\mathrm{L} t b}\right)\left(\bar{d}_{\mathrm{R}}^{a} \sigma_{\mu \nu} d_{\mathrm{L} s a}\right) \\
& \mathcal{Y}\left[p p r, \frac{s}{t}\right] i F_{\mathrm{L}}^{\mu \nu}\left(d_{\mathrm{L} s a} C d_{\mathrm{L} t b}\right)\left(\bar{d}_{\mathrm{R} p}^{a} \sigma_{\mu \nu} C \bar{d}_{\mathrm{R}}^{b}\right) \\
& \mathcal{Y}\left[\overline{p \mid r}, \frac{s}{t}\right] i F_{\mathrm{L}}^{\mu \nu}\left(\bar{d}_{\mathrm{R} r}^{b} d_{\mathrm{L} t b}\right)\left(\bar{d}_{\mathrm{R} p}^{a} \sigma_{\mu \nu} d_{\mathrm{L} s a}\right) \\
& \mathcal{O}_{F_{\mathrm{L}}(1 \sim 3)}^{\left(\bar{d}_{\mathrm{R}} d_{\mathrm{L}} \bar{e}_{\mathrm{R}} e_{\mathrm{L}}\right.}(0,0) \mid \begin{array}{l}
i F_{\mathrm{L}}^{\mu \nu}\left(\bar{e}_{\mathrm{R} s} e_{\mathrm{L} t}\right)\left(\bar{d}_{\mathrm{R} p}^{a} \sigma_{\mu \nu} d_{\mathrm{L} r a}\right) \\
i F_{\mathrm{L}}^{\mu \nu}\left(d_{\mathrm{L} r a} C e_{\mathrm{L} t}\right)\left(\bar{d}_{\mathrm{R} p}^{a} \sigma_{\mu \nu} C \bar{e}_{\mathrm{R} s}\right) \\
i F_{\mathrm{L} \mu \nu}\left(\bar{d}_{\mathrm{R}}{ }^{a} e_{\mathrm{L}}\right)\left(\bar{e}_{\mathrm{R}} \sigma^{\mu \nu} d_{\mathrm{L}}\right)
\end{array} \\
& i F_{\mathrm{L} \mu \nu}\left(\bar{d}_{\mathrm{R} p}^{a} e_{\mathrm{L} t}\right)\left(\bar{e}_{\mathrm{R} s} \sigma^{\mu \nu} d_{\mathrm{L} r a}\right)
\end{aligned}
$$

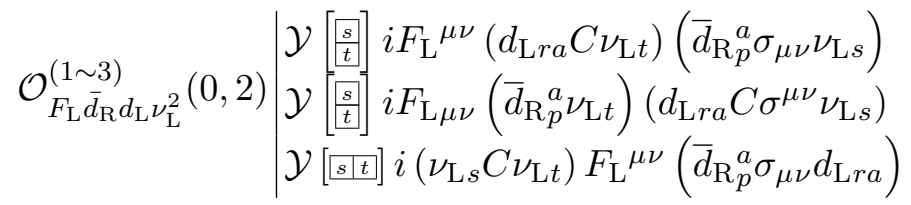

$$
\begin{aligned}
& \mathcal{O}_{F_{\mathrm{L}} \bar{d}_{\mathrm{R}}^{3} e_{\mathrm{L}}}^{(1,2)}(-1,1) \mid \begin{array}{l}
\mathcal{Y}\left[\overline{p|r| s]} i \epsilon_{a b c} F_{\mathrm{L}}^{\mu \nu}\left(\bar{d}_{\mathrm{R}}^{c} e_{s} e_{\mathrm{L} t}\right)\left(\bar{d}_{\mathrm{R} p}^{a} \sigma_{\mu \nu} C \bar{d}_{\mathrm{R} r}^{b}\right)\right. \\
\mathcal{Y}[\overline{p r r}] i \epsilon_{a b c} F_{\mathrm{L}}{ }^{\mu \nu}\left(\bar{d}_{\mathrm{R}_{s}^{c}}^{c} e_{\mathrm{L} t}\right)\left(\bar{d}_{\left.\mathrm{R}_{p}^{a} \sigma_{\mu \nu} C \bar{d}_{\mathrm{R} r}^{b}\right)}\right)
\end{array}
\end{aligned}
$$

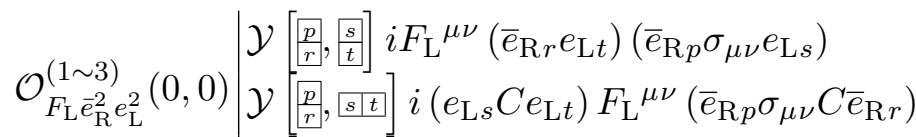

$$
\begin{aligned}
& \mathcal{Y}\left[\underline{p p r}, \frac{s}{t}\right] i F_{\mathrm{L}}^{\mu \nu}\left(\bar{e}_{\mathrm{R} r} e_{\mathrm{L} t}\right)\left(\bar{e}_{\mathrm{R} p} \sigma_{\mu \nu} e_{\mathrm{L} s}\right) \\
& \mathcal{O}_{F_{\mathrm{L}} \bar{e}_{\mathrm{R}} e_{\mathrm{L}} \nu_{\mathrm{L}}^{2}}^{(1 \sim 3)}(0,2) \mid \begin{array}{l}
\mathcal{Y}\left[\begin{array}{l}
{[s} \\
t
\end{array}\right] i F_{\mathrm{L}}^{\mu \nu}\left(e_{\mathrm{L} r} C \nu_{\mathrm{L} t}\right)\left(\bar{e}_{\mathrm{R} p} \sigma_{\mu \nu} \nu_{\mathrm{L} s}\right) \\
\mathcal{Y}\left[\begin{array}{l}
s \\
\bar{t}
\end{array}\right] i F_{\mathrm{L} \mu \nu}\left(\bar{e}_{\mathrm{R} p} \nu_{\mathrm{L} t}\right)\left(e_{\mathrm{L} r} C \sigma^{\mu \nu} \nu_{\mathrm{L} s}\right) \\
\mathcal{Y}\left[\begin{array}{l}
{[s \mid t]} \\
{[s \mid}
\end{array}\right] i\left(\nu_{\mathrm{L} s} C \nu_{\mathrm{L} t}\right) F_{\mathrm{L}}^{\mu \nu}\left(\bar{e}_{\mathrm{R} p} \sigma_{\mu \nu} e_{\mathrm{L} r}\right)
\end{array} \\
& \mathcal{O}_{F_{\mathrm{L}} \nu_{\mathrm{L}}^{4}}(0,4) \mid \mathcal{Y}\left[\begin{array}{c}
\frac{p r}{s} \\
\underline{s}
\end{array}\right] i\left(\nu_{\mathrm{L} r} C \nu_{\mathrm{L} t}\right) F_{\mathrm{L}}^{\mu \nu}\left(\nu_{\mathrm{L} p} C \sigma_{\mu \nu} \nu_{\mathrm{L} s}\right)
\end{aligned}
$$

\section{Class $F_{\mathrm{L}} \psi^{2} \psi^{\dagger 2}: 136$ types}

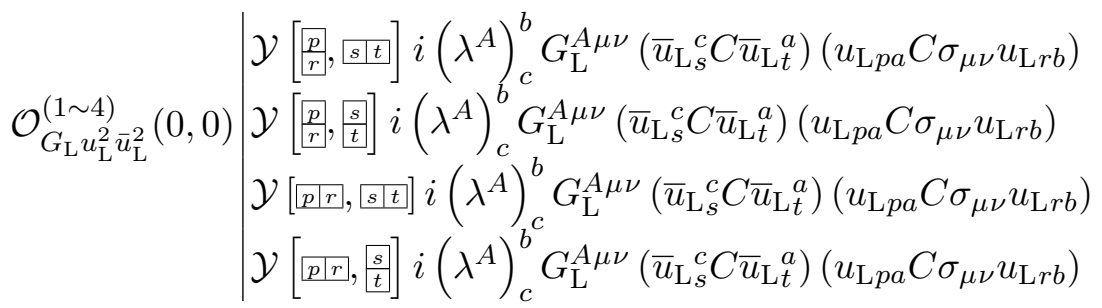

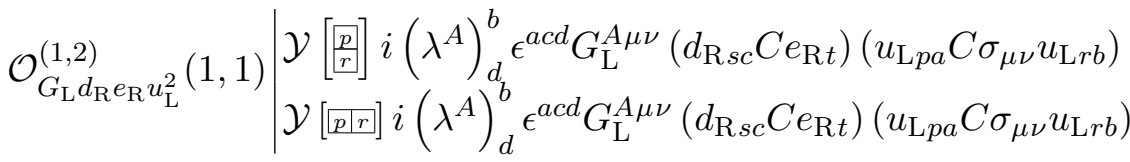




$$
\begin{aligned}
& \mid i\left(\lambda^{A}\right)_{d}^{b} G_{\mathrm{L}}^{A \mu \nu}\left(\bar{u}_{\mathrm{L} t}^{d} u_{\mathrm{R} s a}\right)\left(\bar{u}_{\mathrm{R} p}^{a} \sigma_{\mu \nu} u_{\mathrm{L} r b}\right) \\
& \mathcal{O}_{G_{\mathrm{L}} \bar{u}_{\mathrm{R}} u_{\mathrm{R}} u_{\mathrm{L}} \bar{u}_{\mathrm{L}}}^{(1 \sim 4)}(0,0) i\left(\lambda^{A}\right)_{q}^{b} G_{\mathrm{L}}^{A \mu \nu}\left(\bar{u}_{\mathrm{L} t}^{c} u_{\mathrm{R} s c}\right)\left(\bar{u}_{\mathrm{R}}^{a} \sigma_{\mu \nu} u_{\mathrm{L} r b}\right) \\
& i\left(\lambda^{A}\right)_{d}^{q} G_{\mathrm{L}}^{A \mu \nu}\left(\bar{u}_{\mathrm{L} t}{ }^{d} u_{\mathrm{R} s c}\right)\left(\bar{u}_{\mathrm{R} p}^{a} \sigma_{\mu \nu} u_{\mathrm{L} r a}\right) \\
& i\left(\lambda^{A}\right)_{a}^{c} G_{\mathrm{L}}^{A \mu \nu}\left(\bar{u}_{\mathrm{L} t}^{b} u_{\mathrm{R} s c}\right)\left(\bar{u}_{\mathrm{R} p}^{a} \sigma_{\mu \nu} u_{\mathrm{L} r b}\right) \\
& \mathcal{O}_{G_{\mathrm{L}} d_{\mathrm{R}} \overline{\mathrm{L}}_{\mathrm{L}} \bar{u}_{\mathrm{R}} u_{\mathrm{L}}}^{(1 \sim 4)}(0,0) \mid \begin{array}{ll}
i\left(\lambda^{A}\right)_{d}^{b} G_{\mathrm{L}}^{A \mu \nu}\left(\bar{d}_{\mathrm{L} t}^{d} d_{\mathrm{R} s a}\right) & \left(\bar{u}_{\mathrm{R}}^{a} \sigma_{\mu \nu} u_{\mathrm{L} r b}\right) \\
i\left(\lambda^{A}\right)_{a}^{b} G_{\mathrm{L}}^{A \mu \nu}\left(\bar{d}_{\mathrm{L}}^{c} d_{\mathrm{R} s c}\right) & \left(\bar{u}_{\mathrm{R}}^{a} \sigma_{\mu \nu} u_{\mathrm{L} r b}\right) \\
i\left(\lambda^{A}\right)_{d}^{q} G_{\mathrm{L}}^{A \mu \nu}\left(\bar{d}_{\mathrm{L} t}^{d} d_{\mathrm{R} s c}\right) & \left(\bar{u}_{\mathrm{R}}^{a} \sigma_{\mu \nu} u_{\mathrm{L} r a}\right) \\
i\left(\lambda^{A}\right)_{a}^{c} G_{\mathrm{L}}^{A \mu \nu}\left(\bar{d}_{\mathrm{L} t}^{b} d_{\mathrm{R} s c}\right)\left(\bar{u}_{\mathrm{R}}^{a} \sigma_{\mu \nu} u_{\mathrm{L} r b}\right)
\end{array} \\
& \mathcal{O}_{G_{\mathrm{L}} e_{\mathrm{R}} \bar{e}_{\mathrm{L}} \bar{u}_{\mathrm{R}} u_{\mathrm{L}}}(0,0) \mid i\left(\lambda^{A}\right)_{a}^{b}\left(\bar{e}_{\mathrm{L} t} e_{\mathrm{R} s}\right) G_{\mathrm{L}}^{A \mu \nu}\left(\bar{u}_{\mathrm{R} p}^{a} \sigma_{\mu \nu} u_{\mathrm{L} r b}\right) \\
& \mathcal{O}_{G_{\mathrm{L}} \bar{\nu}_{\mathrm{L}}^{2} \bar{u}_{\mathrm{R}} u_{\mathrm{L}}}(0,-2) \mid \mathcal{Y}[\underline{s \mid t]}] i\left(\lambda^{A}\right)_{a}^{b}\left(\bar{\nu}_{\mathrm{L} s} C \bar{\nu}_{\mathrm{L} t}\right) G_{\mathrm{L}}^{A \mu \nu}\left(\bar{u}_{\mathrm{R} p}^{a} \sigma_{\mu \nu} u_{\mathrm{L} r b}\right)
\end{aligned}
$$

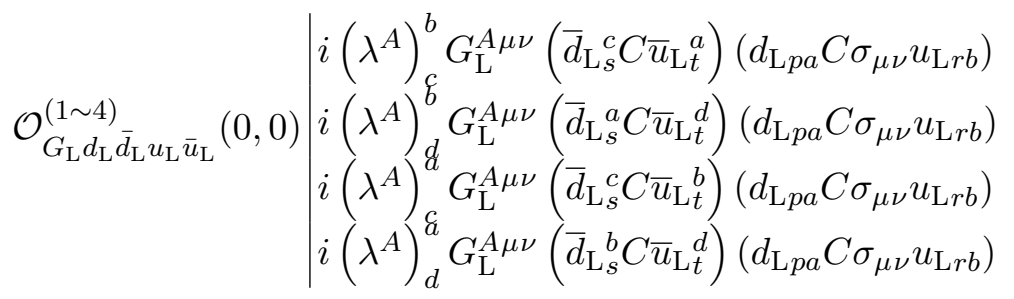

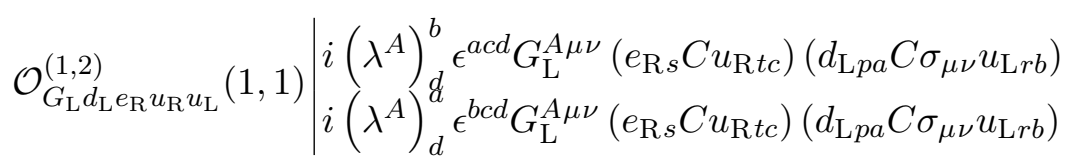

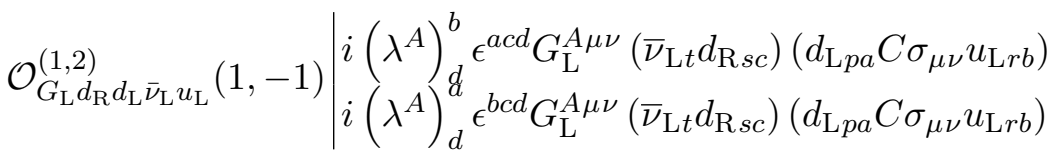

$$
\begin{aligned}
& \mathcal{O}_{G_{\mathrm{L}} \bar{d}_{\mathrm{R}} d_{\mathrm{R}} u_{\mathrm{L}} \bar{u}_{\mathrm{L}}}^{(1 \sim, 0)} \mid \begin{array}{ll}
i\left(\lambda^{A}\right)_{d}^{b} G_{\mathrm{L}}^{A \mu \nu}\left(\bar{u}_{\mathrm{L} t}^{d} d_{\mathrm{R} s a}\right)\left(\bar{d}_{\mathrm{R} p}^{a} \sigma_{\mu \nu} u_{\mathrm{L} r b}\right) \\
i\left(\lambda^{A}\right)_{a}^{b} G_{\mathrm{L}}^{A \mu \nu}\left(\bar{u}_{\mathrm{L} t}^{c} d_{\mathrm{R} s c}\right)\left(\bar{d}_{\mathrm{R} p}^{a} \sigma_{\mu \nu} u_{\mathrm{L} r b}\right) \\
i\left(\lambda^{A}\right)_{d}^{c} G_{\mathrm{L}}^{A \mu \nu}\left(\bar{u}_{\mathrm{L} t}^{d} d_{\mathrm{R} s c}\right)\left(\bar{d}_{\mathrm{R} p}^{a} \sigma_{\mu \nu} u_{\mathrm{L} r a}\right) \\
i\left(\lambda^{A}\right)_{a}^{d} G_{\mathrm{L}}^{A \mu \nu}\left(\bar{u}_{\mathrm{L} t}^{b} d_{\mathrm{R} s c}\right)\left(\bar{d}_{\mathrm{R} p}^{a} \sigma_{\mu \nu} u_{\mathrm{L} r b}\right)
\end{array} \\
& \mathcal{O}_{G_{\mathrm{L}} \bar{d}_{\mathrm{R}} e_{\mathrm{R}} \bar{\nu}_{\mathrm{L}} u_{\mathrm{L}}}(0,0) \mid i\left(\lambda^{A}\right)_{a}^{b}\left(\bar{\nu}_{\mathrm{L} t} e_{\mathrm{R} s}\right) G_{\mathrm{L}}^{A \mu \nu}\left(\bar{d}_{\mathrm{R}}^{a} \sigma_{\mu \nu} u_{\mathrm{L} r b}\right)
\end{aligned}
$$

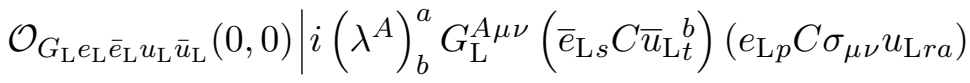

$$
\begin{aligned}
& \mathcal{O}_{G_{\mathrm{L}} d_{\mathrm{R}} e_{\mathrm{L}} u_{\mathrm{R}} u_{\mathrm{L}}}^{(1,2)}(1) \mid \begin{array}{ll}
i\left(\lambda^{A}\right)_{d}^{b} \epsilon^{a c d} G_{\mathrm{L}}^{A \mu \nu}\left(d_{\mathrm{R} s b} C u_{\mathrm{R} t c}\right) & \left(e_{\mathrm{L} p} C \sigma_{\mu \nu} u_{\mathrm{L} r a}\right) \\
i\left(\lambda^{A}\right)_{d}^{d} \epsilon^{b c d} G_{\mathrm{L}}^{A \mu \nu} & \left(d_{\mathrm{R} s b} C u_{\mathrm{R} t c}\right)\left(e_{\mathrm{L} p} C \sigma_{\mu \nu} u_{\mathrm{L} r a}\right)
\end{array}
\end{aligned}
$$




$$
\begin{aligned}
& \mathcal{O}_{G_{\mathrm{L}} \bar{d}_{\mathrm{L}} e_{\mathrm{L}} \bar{\nu}_{\mathrm{L}} u_{\mathrm{L}}}(0,0) \mid i\left(\lambda^{A}\right)_{b}^{a} G_{\mathrm{L}}^{A \mu \nu}\left(\bar{d}_{\mathrm{L} s}^{b} C \bar{\nu}_{\mathrm{L} t}\right)\left(e_{\mathrm{L} p} C \sigma_{\mu \nu} u_{\mathrm{L} r a}\right) \\
& \mathcal{O}_{G_{\mathrm{L}} \bar{e}_{\mathrm{R}} e_{\mathrm{R}} u_{\mathrm{L}} \bar{u}_{\mathrm{L}}}(0,0) \mid i\left(\lambda^{A}\right)_{b}^{a} G_{\mathrm{L}}^{A \mu \nu}\left(\bar{u}_{\mathrm{L}}^{b} e_{\mathrm{R} s}\right)\left(\bar{e}_{\mathrm{R} p} \sigma_{\mu \nu} u_{\mathrm{L} r a}\right) \\
& \mathcal{O}_{G_{\mathrm{L}} \nu_{\mathrm{L}} \bar{\nu}_{\mathrm{L}} u_{\mathrm{L}} \bar{u}_{\mathrm{L}}}(0,0) \mid i\left(\lambda^{A}\right)_{b}^{a} G_{\mathrm{L}}^{A \mu \nu}\left(\bar{u}_{\mathrm{L}}^{b} C \bar{\nu}_{\mathrm{L} t}\right)\left(u_{\mathrm{L} p a} C \sigma_{\mu \nu} \nu_{\mathrm{L} r}\right) \\
& \mathcal{O}_{G_{\mathrm{L}} \bar{d}_{\mathrm{L}} e_{\mathrm{R}} \nu_{\mathrm{L}} u_{\mathrm{L}}}(0,2) \mid i\left(\lambda^{A}\right)_{b}^{a} G_{\mathrm{L}}^{A \mu \nu}\left(\bar{d}_{\mathrm{L} s}^{b} e_{\mathrm{R} t}\right)\left(u_{\mathrm{L} p a} C \sigma_{\mu \nu} \nu_{\mathrm{L} r}\right) \\
& \mathcal{O}_{G_{\mathrm{L}} d_{\mathrm{R}}^{2} \nu_{\mathrm{L}} u_{\mathrm{L}}}^{(1,2)}(1,1) \mid \begin{array}{l}
\mathcal{Y}\left[\underline{[s \mid t]} i\left(\lambda^{A}\right)_{d}^{b} \epsilon^{a c d} G_{\mathrm{L}}^{A \mu \nu}\left(d_{\mathrm{R} s b} C d_{\mathrm{R} t c}\right)\left(u_{\mathrm{L} p a} C \sigma_{\mu \nu} \nu_{\mathrm{L} r}\right)\right. \\
\mathcal{Y}\left[\begin{array}{l}
s \\
\left.\frac{s}{t}\right]
\end{array}\right]\left(\lambda^{A}\right)_{d}^{b} \epsilon^{a c d} G_{\mathrm{L}}^{A \mu \nu}\left(d_{\mathrm{R} s b} C d_{\mathrm{R} t c}\right)\left(u_{\mathrm{L} p a} C \sigma_{\mu \nu} \nu_{\mathrm{L} r}\right)
\end{array} \\
& \mathcal{Y}\left[\left[\frac{p}{r}, s \mid t\right]\right] i\left(\lambda^{A}\right)_{b}^{d} G_{\mathrm{L}}^{A \mu \nu}\left(u_{\mathrm{R} s a} C u_{\mathrm{R} t d}\right)\left(\bar{u}_{\mathrm{R}}^{a} \sigma_{\mu \nu} C \bar{u}_{\mathrm{R}}^{b}\right) \\
& \mathcal{O}_{G_{\mathrm{L}} \bar{u}_{\mathrm{R}}^{2} u_{\mathrm{R}}^{2}}^{(1 \sim 4)}(0,0) \mathcal{Y}\left[\frac{p}{r}, \frac{s}{t}\right] i\left(\lambda^{A}\right)_{b}^{d} G_{\mathrm{L}}^{A \mu \nu}\left(u_{\mathrm{R} s a} C u_{\mathrm{R} t d}\right)\left(\bar{u}_{\mathrm{R} p}^{a} \sigma_{\mu \nu} C \bar{u}_{\mathrm{R} r}^{b}\right) \\
& \mathcal{Y}\left[\left[p \mid r,[s \mid t] i\left(\lambda^{A}\right)_{b}^{d} G_{\mathrm{L}}^{A \mu \nu}\left(u_{\mathrm{R} s a} C u_{\mathrm{R} t d}\right)\left(\bar{u}_{\mathrm{R} p}^{a} \sigma_{\mu \nu} C \bar{u}_{\mathrm{R} r}^{b}\right)\right.\right. \\
& \mathcal{Y}\left[\underline{p p r}, \frac{s}{t}\right] i\left(\lambda^{A}\right)_{b}^{d} G_{\mathrm{L}}^{A \mu \nu}\left(u_{\mathrm{R} s a} C u_{\mathrm{R} t d}\right)\left(\bar{u}_{\mathrm{R} p}^{a} \sigma_{\mu \nu} C \bar{u}_{\mathrm{R} r}^{b}\right) \\
& \mathcal{O}_{G_{\mathrm{L}} \bar{d}_{\mathrm{L}} \bar{e}_{\mathrm{L}} \bar{u}_{\mathrm{R}}^{2}(-1,-1)} \mid \begin{array}{l}
\mathcal{Y}\left[\left[\begin{array}{l}
p] \\
r
\end{array}\right] i \epsilon_{a c d}\left(\lambda^{A}\right)_{b}^{d} G_{\mathrm{L}}^{A \mu \nu}\left(\bar{d}_{\mathrm{L}}^{c} C \bar{e}_{\mathrm{L} t}\right)\left(\bar{u}_{\mathrm{R}}^{a} \sigma_{\mu \nu} C \bar{u}_{\mathrm{R}}^{b}\right)\right. \\
\mathcal{Y}[\underline{p r r}] i \epsilon_{a c d}\left(\lambda^{A}\right)_{b}^{d} G_{\mathrm{L}}^{A \mu \nu}\left(\bar{d}_{\mathrm{L}_{s}^{c}} C \bar{e}_{\mathrm{L} t}\right)\left(\bar{u}_{\mathrm{R}}^{a}{ }_{p}^{a} \sigma_{\mu \nu} C \bar{u}_{\mathrm{R}}^{b}\right)
\end{array}
\end{aligned}
$$

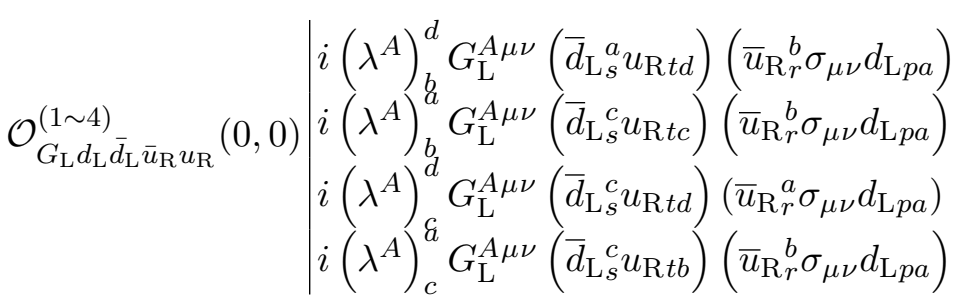

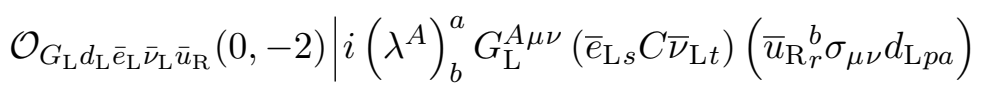

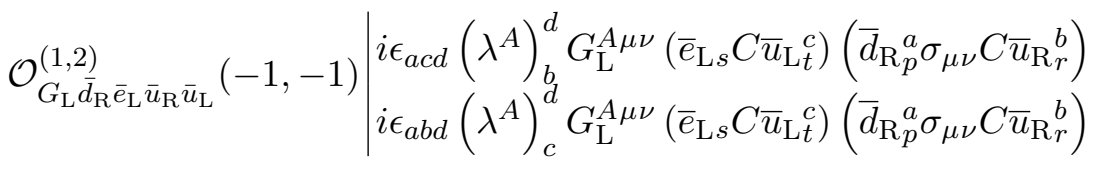

$$
\begin{aligned}
& \mid i\left(\lambda^{A}\right)_{b}^{d} G_{\mathrm{L}}^{A \mu \nu}\left(d_{\mathrm{R} s a} C u_{\mathrm{R} t d}\right)\left(\bar{d}_{\mathrm{R} p}^{a} \sigma_{\mu \nu} C \bar{u}_{\mathrm{R} r}^{b}\right) \\
& \mathcal{O}_{G_{\mathrm{L}} \bar{d}_{\mathrm{R}} d_{\mathrm{R}} \bar{u}_{\mathrm{R}} u_{\mathrm{R}}}^{(\sim 4)^{2}}(0,0) i\left(\lambda^{A}\right)_{q}^{d} G_{\mathrm{L}}^{A \mu \nu}\left(d_{\mathrm{R} s b} C u_{\mathrm{R} t d}\right)\left(\bar{d}_{\left.\mathrm{R}_{p}^{a} \sigma_{\mu \nu} C \bar{u}_{\mathrm{R}}^{b}\right)}^{b}\right)
\end{aligned}
$$

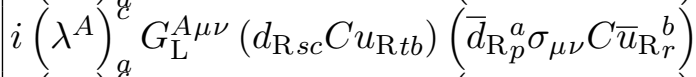

$$
\begin{aligned}
& i\left(\lambda^{A}\right)_{b}^{a} G_{\mathrm{L}}^{A \mu \nu}\left(d_{\mathrm{R} s c} C u_{\mathrm{R} t a}\right)\left(\bar{d}_{\mathrm{R} p}^{a} \sigma_{\mu \nu} C \bar{u}_{\mathrm{R}}^{b}\right)
\end{aligned}
$$

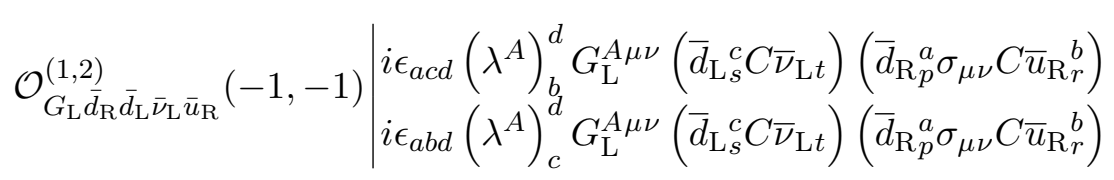




$$
\begin{aligned}
& \mathcal{O}_{G_{\mathrm{L}} e_{\mathrm{L}} \bar{e}_{\mathrm{L}} \bar{u}_{\mathrm{R}} u_{\mathrm{R}}}(0,0) \mid i\left(\lambda^{A}\right)_{a}^{b} G_{\mathrm{L}}^{A \mu \nu}\left(\bar{e}_{\mathrm{L} s} u_{\mathrm{R} t b}\right)\left(\bar{u}_{\mathrm{R} r}^{a} \sigma_{\mu \nu} e_{\mathrm{L} p}\right)
\end{aligned}
$$

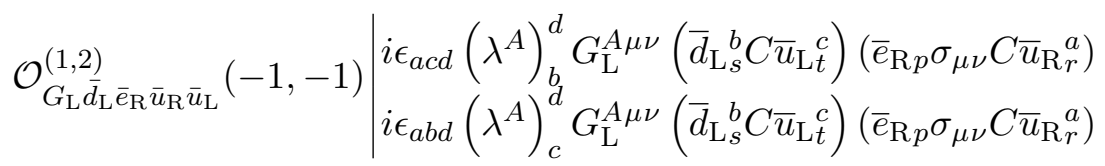

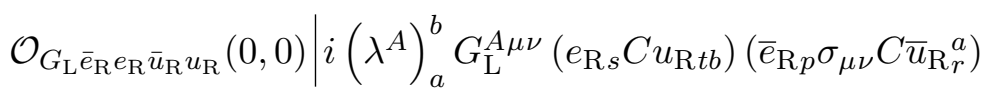

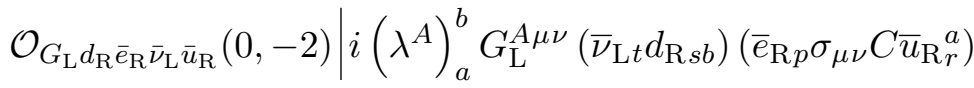

$$
\begin{aligned}
& \mathcal{O}_{G_{\mathrm{L}} \nu_{\mathrm{L}} \bar{\nu}_{\mathrm{L}} \bar{u}_{\mathrm{R}} u_{\mathrm{R}}}(0,0) \mid i\left(\lambda^{A}\right)_{a}^{b} G_{\mathrm{L}}^{A \mu \nu}\left(\bar{\nu}_{\mathrm{L} t} u_{\mathrm{R} s b}\right)\left(\bar{u}_{\mathrm{R}}^{a} \sigma_{\mu \nu} \nu_{\mathrm{L} r}\right)
\end{aligned}
$$

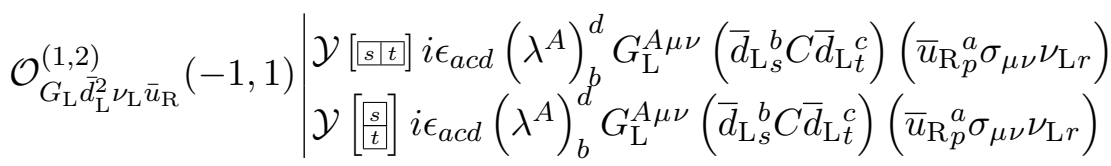

$$
\begin{aligned}
& \mathcal{O}_{G_{\mathrm{L}} d_{\mathrm{R}} \bar{e}_{\mathrm{L}} \nu_{\mathrm{L}} \bar{u}_{\mathrm{R}}}(0,0) \mid i\left(\lambda^{A}\right)_{a}^{b} G_{\mathrm{L}}^{A \mu \nu}\left(\bar{e}_{\mathrm{L} t} d_{\mathrm{R} s b}\right)\left(\bar{u}_{\mathrm{R} p}^{a} \sigma_{\mu \nu} \nu_{\mathrm{L} r}\right) \\
& \mathcal{O}_{G_{\mathrm{L}} d_{\mathrm{L}}^{2} \bar{\nu}_{\mathrm{L}} u_{\mathrm{R}}}^{(1,2,-1)} \mid \begin{array}{l}
\mathcal{Y}\left[\begin{array}{l}
p \\
\underline{r}]
\end{array}\right] i\left(\lambda^{A}\right)_{d}^{b} \epsilon^{a c d} G_{\mathrm{L}}^{A \mu \nu}\left(\bar{\nu}_{\mathrm{L} t} u_{\mathrm{R} s c}\right)\left(d_{\mathrm{L} p a} C \sigma_{\mu \nu} d_{\mathrm{L} r b}\right) \\
\mathcal{Y}\left[[p r r] i\left(\lambda^{A}\right)_{d}^{b} \epsilon^{a c d} G_{\mathrm{L}}^{A \mu \nu}\left(\bar{\nu}_{\mathrm{L} t} u_{\mathrm{R} s c}\right)\left(d_{\mathrm{L} p a} C \sigma_{\mu \nu} d_{\mathrm{L} r b}\right)\right.
\end{array}
\end{aligned}
$$

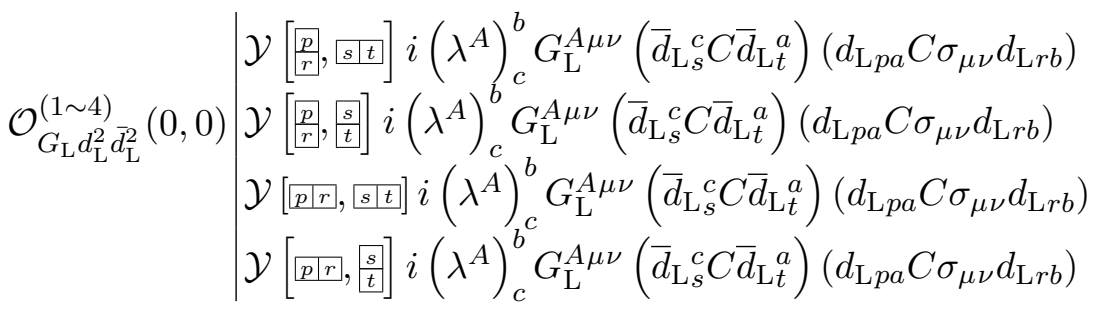

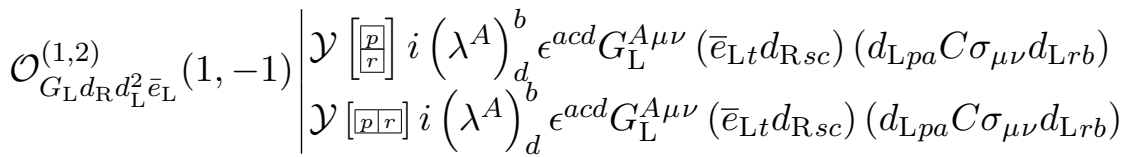

$$
\begin{aligned}
& \mathcal{O}_{G_{\mathrm{L}} \bar{d}_{\mathrm{R}} d_{\mathrm{L}} u_{\mathrm{R}} \bar{u}_{\mathrm{L}}}^{(1 \sim, 0)} \mid \begin{array}{l}
i\left(\lambda^{A}\right)_{d}^{b} G_{\mathrm{L}}^{A \mu \nu}\left(\bar{u}_{\mathrm{L} t}^{d} u_{\mathrm{R} s a}\right)\left(\bar{d}_{\mathrm{R} p}^{a} \sigma_{\mu \nu} d_{\mathrm{L} r b}\right) \\
i\left(\lambda^{A}\right)_{a}^{b} G_{\mathrm{L}}^{A \mu \nu}\left(\bar{u}_{\mathrm{L}}^{c}{ }_{t} u_{\mathrm{R} s c}\right)\left(\bar{d}_{\mathrm{R} p}^{a} \sigma_{\mu \nu} d_{\mathrm{L} r b}\right) \\
i\left(\lambda^{A}\right)_{d}^{c} G_{\mathrm{L}}^{A \mu \nu}\left(\bar{u}_{\mathrm{L} t}^{d} u_{\mathrm{R} s c}\right)\left(\bar{d}_{\mathrm{R} p}^{a} \sigma_{\mu \nu} d_{\mathrm{L} r a}\right) \\
i\left(\lambda^{A}\right)_{a}^{c} G_{\mathrm{L}}^{A \mu \nu}\left(\bar{u}_{\mathrm{L} t}^{b} u_{\mathrm{R} s c}\right)\left(\bar{d}_{\mathrm{R} p}^{a} \sigma_{\mu \nu} d_{\mathrm{L} r b}\right)
\end{array}
\end{aligned}
$$

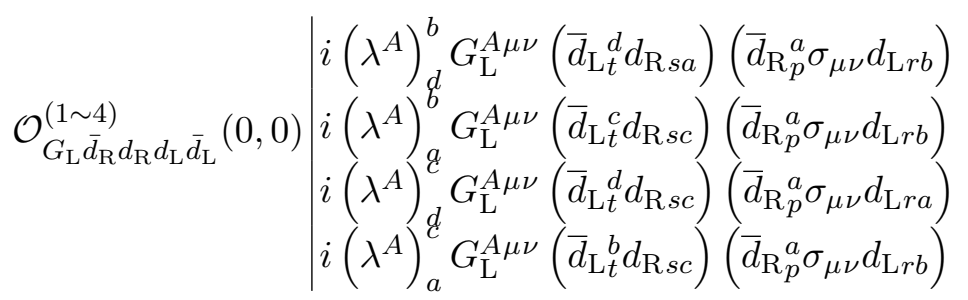




$$
\begin{aligned}
& \mathcal{O}_{G_{\mathrm{L}} \bar{d}_{\mathrm{R}} d_{\mathrm{L}} e_{\mathrm{R}} \bar{e}_{\mathrm{L}}}(0,0) \mid i\left(\lambda^{A}\right)_{a}^{b}\left(\bar{e}_{\mathrm{L} t} e_{\mathrm{R} s}\right) G_{\mathrm{L}}^{A \mu \nu}\left(\bar{d}_{\mathrm{R}}^{a} \sigma_{\mu \nu} d_{\mathrm{L} r b}\right) \\
& \left.\mathcal{O}_{G_{\mathrm{L}} \bar{d}_{\mathrm{R}} d_{\mathrm{L}} \bar{\nu}_{\mathrm{L}}^{2}}(0,-2) \mid \mathcal{Y}[s] t\right] i\left(\lambda^{A}\right)_{a}^{b}\left(\bar{\nu}_{\mathrm{L} s} C \bar{\nu}_{\mathrm{L} t}\right) G_{\mathrm{L}}^{A \mu \nu}\left(\bar{d}_{\mathrm{R}}^{a} \sigma_{\mu \nu} d_{\mathrm{L} r b}\right) \\
& \mathcal{O}_{G_{\mathrm{L}} d_{\mathrm{L}} e_{\mathrm{L}} u_{\mathrm{R}}^{2}}^{(1,2)}(1,1) \mid \begin{array}{l}
\mathcal{Y}[s \mid t] i\left(\lambda^{A}\right)_{d}^{b} \epsilon^{a c d} G_{\mathrm{L}}^{A \mu \nu}\left(u_{\mathrm{R} s b} C u_{\mathrm{R} t c}\right)\left(d_{\mathrm{L} p a} C \sigma_{\mu \nu} e_{\mathrm{L} r}\right) \\
\mathcal{Y}\left[\begin{array}{l}
s] \\
\underline{t}]
\end{array}\right]\left(\lambda^{A}\right)_{d}^{b} \epsilon^{a c d} G_{\mathrm{L}}^{A \mu \nu}\left(u_{\mathrm{R} s b} C u_{\mathrm{R} t c}\right)\left(d_{\mathrm{L} p a} C \sigma_{\mu \nu} e_{\mathrm{L} r}\right)
\end{array} \\
& \mathcal{O}_{G_{\mathrm{L}} d_{\mathrm{L}} \bar{d}_{\mathrm{L}} e_{\mathrm{L}} \bar{e}_{\mathrm{L}}}(0,0) \mid i\left(\lambda^{A}\right)_{b}^{a} G_{\mathrm{L}}^{A \mu \nu}\left(\bar{d}_{\mathrm{L} s}^{b} C \bar{e}_{\mathrm{L} t}\right)\left(d_{\mathrm{L} p a} C \sigma_{\mu \nu} e_{\mathrm{L} r}\right) \\
& \mathcal{O}_{G_{\mathrm{L}} d_{\mathrm{L}} \bar{e}_{\mathrm{R}} \bar{\nu}_{\mathrm{L}} \bar{u}_{\mathrm{L}}}(0,-2) \mid i\left(\lambda^{A}\right)_{b}^{a} G_{\mathrm{L}}^{A \mu \nu}\left(\bar{u}_{\mathrm{L}}^{b} C \bar{\nu}_{\mathrm{L} t}\right)\left(\bar{e}_{\mathrm{R} r} \sigma_{\mu \nu} d_{\mathrm{L} p a}\right) \\
& \mathcal{O}_{G_{\mathrm{L}} d_{\mathrm{L}} \bar{d}_{\mathrm{L}} \bar{e}_{\mathrm{R}} e_{\mathrm{R}}}(0,0) \mid i\left(\lambda^{A}\right)_{b}^{a} G_{\mathrm{L}}^{A \mu \nu}\left(\bar{d}_{\mathrm{L} s}^{b} e_{\mathrm{R} t}\right)\left(\bar{e}_{\mathrm{R} r} \sigma_{\mu \nu} d_{\mathrm{L} p a}\right)
\end{aligned}
$$

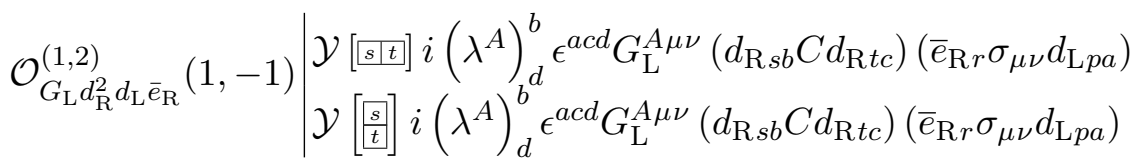

$$
\begin{aligned}
& \mathcal{O}_{G_{\mathrm{L}} d_{\mathrm{L}} \bar{e}_{\mathrm{L}} \nu_{\mathrm{L}} \bar{u}_{\mathrm{L}}}(0,0) \mid i\left(\lambda^{A}\right)_{b}^{a} G_{\mathrm{L}}^{A \mu \nu}\left(\bar{e}_{\mathrm{L} s} C \bar{u}_{\mathrm{L} t}^{b}\right)\left(d_{\mathrm{L} p a} C \sigma_{\mu \nu} \nu_{\mathrm{L} r}\right)
\end{aligned}
$$

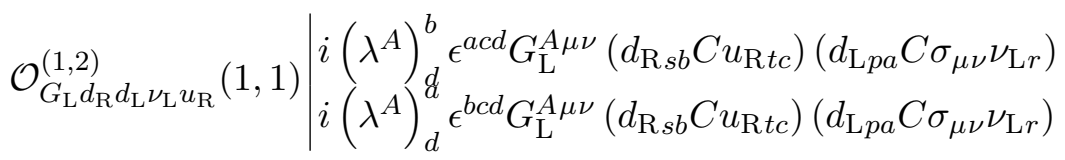

$$
\begin{aligned}
& \mathcal{O}_{G_{\mathrm{L}} d_{\mathrm{L}} \bar{d}_{\mathrm{L}} \nu_{\mathrm{L}} \bar{\nu}_{\mathrm{L}}}(0,0) \mid i\left(\lambda^{A}\right)_{b}^{a} G_{\mathrm{L}}^{A \mu \nu}\left(\bar{d}_{\mathrm{L}}^{b} C \bar{\nu}_{\mathrm{L} t}\right)\left(d_{\mathrm{L} p a} C \sigma_{\mu \nu} \nu_{\mathrm{L} r}\right) \\
& \mathcal{O}_{G_{\mathrm{L}} \bar{d}_{\mathrm{R}}^{2} \bar{\nu}_{\mathrm{L}} \bar{u}_{\mathrm{L}}(-1,-1)} \mid \begin{array}{l}
\mathcal{Y}\left[\left[\frac{p}{r}\right] i \epsilon_{a c d}\left(\lambda^{A}\right)_{b}^{d} G_{\mathrm{L}}^{A \mu \nu}\left(\bar{u}_{\mathrm{L}}^{c} C \bar{\nu}_{\mathrm{L} t}\right)\left(\bar{d}_{\mathrm{R}}^{a} \sigma_{\mu \nu} C \bar{d}_{\mathrm{R}}^{b}\right)\right. \\
\mathcal{Y}[\overline{p p r}] i \epsilon_{a c d}\left(\lambda^{A}\right)_{b}^{d} G_{\mathrm{L}}^{A \mu \nu}\left(\bar{u}_{\mathrm{L}}^{c} C \bar{\nu}_{\mathrm{L} t}\right)\left(\bar{d}_{\mathrm{R}}^{a} \sigma_{\mu \nu} C \bar{d}_{\mathrm{R} r}^{b}\right)
\end{array} \\
& \mathcal{O}_{G_{\mathrm{L}} \bar{d}_{\mathrm{R}}^{2} \bar{d}_{\mathrm{L}} e_{\mathrm{R}}(-1,1)} \mid \begin{array}{l}
\mathcal{Y}\left[\frac{p}{r}\right] i \epsilon_{a c d}\left(\lambda^{A}\right)_{b}^{d} G_{\mathrm{L}}^{A \mu \nu}\left(\bar{d}_{\mathrm{L}_{s}^{c}}^{c} e_{\mathrm{R} t}\right)\left(\bar{d}_{\mathrm{R}}^{a} \sigma_{\mu \nu} C \bar{d}_{\mathrm{R}}^{b}\right) \\
\mathcal{Y}[\underline{p p r}] i \epsilon_{a c d}\left(\lambda^{A}\right)_{b}^{d} G_{\mathrm{L}}^{A \mu \nu}\left(\bar{d}_{\mathrm{L}}^{c} e_{\mathrm{R} t}\right)\left(\bar{d}_{\mathrm{R}}^{a} \sigma_{\mu \nu} C \bar{d}_{\mathrm{R} r}^{b}\right)
\end{array}
\end{aligned}
$$

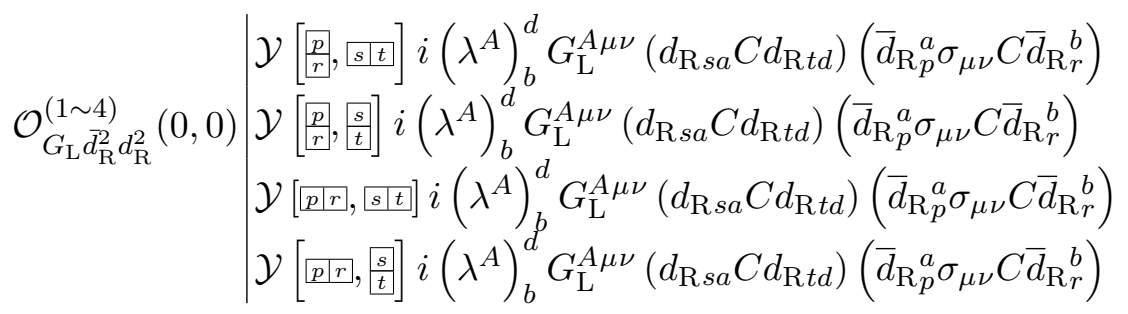

$$
\begin{aligned}
& \mathcal{O}_{G_{\mathrm{L}} \bar{d}_{\mathrm{R}} e_{\mathrm{L}} \bar{\nu}_{\mathrm{L}} u_{\mathrm{R}}}(0,0) \mid i\left(\lambda^{A}\right)_{a}^{b} G_{\mathrm{L}}^{A \mu \nu}\left(\bar{\nu}_{\mathrm{L} t} u_{\mathrm{R} s b}\right)\left(\bar{d}_{\mathrm{R} p}^{a} \sigma_{\mu \nu} e_{\mathrm{L} r}\right)
\end{aligned}
$$

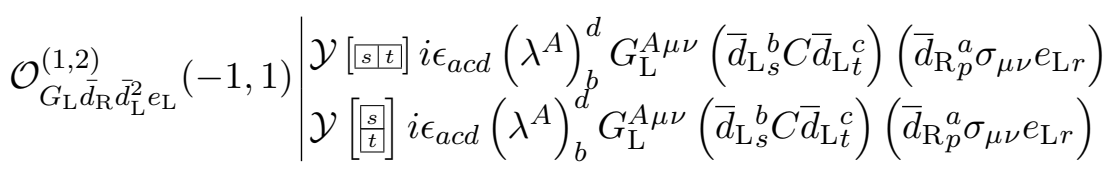




$$
\begin{aligned}
& \mathcal{O}_{G_{\mathrm{L}} \bar{d}_{\mathrm{R}} d_{\mathrm{R}} e_{\mathrm{L}} \bar{e}_{\mathrm{L}}}(0,0) \mid i\left(\lambda^{A}\right)_{a}^{b} G_{\mathrm{L}}^{A \mu \nu}\left(\bar{e}_{\mathrm{L} t} d_{\mathrm{R} s b}\right)\left(\bar{d}_{\mathrm{R}}^{a} \sigma_{\mu \nu} e_{\mathrm{L} r}\right)
\end{aligned}
$$

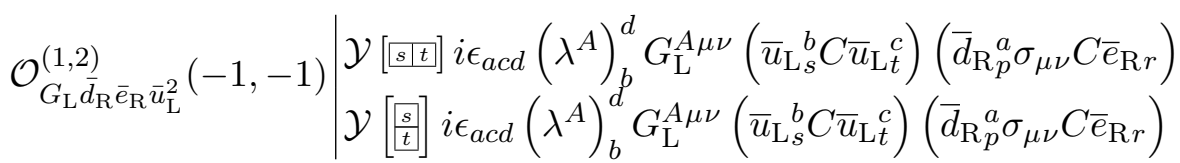

$$
\begin{aligned}
& \mathcal{O}_{G_{\mathrm{L}} \bar{d}_{\mathrm{R}} d_{\mathrm{R}} \bar{e}_{\mathrm{R}} e_{\mathrm{R}}}(0,0) \mid i\left(\lambda^{A}\right)_{a}^{b} G_{\mathrm{L}}^{A \mu \nu}\left(d_{\mathrm{R} s b} C e_{\mathrm{R} t}\right)\left(\bar{d}_{\mathrm{R}}^{a} \sigma_{\mu \nu} C \bar{e}_{\mathrm{R} r}\right)
\end{aligned}
$$

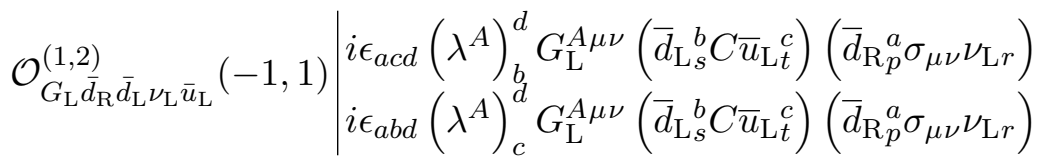

$$
\begin{aligned}
& \mathcal{O}_{G_{\mathrm{L}} \bar{d}_{\mathrm{R}} e_{\mathrm{R}} \nu_{\mathrm{L}} u_{\mathrm{R}}}(0,2) \mid i\left(\lambda^{A}\right)_{a}^{b} G_{\mathrm{L}}^{A \mu \nu}\left(e_{\mathrm{R} s} C u_{\mathrm{R} t b}\right)\left(\bar{d}_{\mathrm{R}}^{a} \sigma_{\mu \nu} \nu_{\mathrm{L} r}\right) \\
& \mathcal{O}_{G_{\mathrm{L}} \bar{d}_{\mathrm{R}} d_{\mathrm{R}} \nu_{\mathrm{L}} \bar{\nu}_{\mathrm{L}}}(0,0) \mid i\left(\lambda^{A}\right)_{a}^{b} G_{\mathrm{L}}^{A \mu \nu}\left(\bar{\nu}_{\mathrm{L} t} d_{\mathrm{R} s b}\right)\left(\bar{d}_{\mathrm{R}}^{a} \sigma_{\mu \nu} \nu_{\mathrm{L} r}\right) \\
& \mathcal{O}_{G_{\mathrm{L}} \bar{e}_{\mathrm{R}} e_{\mathrm{L}} u_{\mathrm{R}} \bar{u}_{\mathrm{L}}}(0,0) \mid i\left(\lambda^{A}\right)_{b}^{a} G_{\mathrm{L}}^{A \mu \nu}\left(\bar{u}_{\mathrm{L} t}^{b} u_{\mathrm{R} s a}\right)\left(\bar{e}_{\mathrm{R} p} \sigma_{\mu \nu} e_{\mathrm{L} r}\right) \\
& \mathcal{O}_{G_{\mathrm{L}} d_{\mathrm{R}} \bar{d}_{\mathrm{L}} \bar{e}_{\mathrm{R}} e_{\mathrm{L}}}(0,0) \mid i\left(\lambda^{A}\right)_{b}^{a} G_{\mathrm{L}}^{A \mu \nu}\left(\bar{d}_{\mathrm{L} t}^{b} d_{\mathrm{R} s a}\right)\left(\bar{e}_{\mathrm{R} p} \sigma_{\mu \nu} e_{\mathrm{L} r}\right) \\
& \mathcal{O}_{G_{\mathrm{L}} \bar{d}_{\mathrm{L}} e_{\mathrm{L}} \nu_{\mathrm{L}} u_{\mathrm{R}}}(0,2) \mid i\left(\lambda^{A}\right)_{a}^{b} G_{\mathrm{L}}^{A \mu \nu}\left(\bar{d}_{\mathrm{L} s}^{a} u_{\mathrm{R} t b}\right)\left(e_{\mathrm{L} p} C \sigma_{\mu \nu} \nu_{\mathrm{L} r}\right)
\end{aligned}
$$

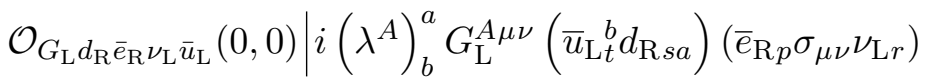

$$
\begin{aligned}
& \left.\mathcal{O}_{G_{\mathrm{L}} \nu_{\mathrm{L}}^{2} u_{\mathrm{R}} \bar{u}_{\mathrm{L}}}(0,2) \mid \mathcal{Y}\left[\frac{p}{r}\right]\right] i\left(\lambda^{A}\right)_{b}^{a} G_{\mathrm{L}}^{A \mu \nu}\left(\nu_{\mathrm{L} p} C \sigma_{\mu \nu} \nu_{\mathrm{L} r}\right)\left(\bar{u}_{\mathrm{L} t}^{b} u_{\mathrm{R} s a}\right) \\
& \mathcal{O}_{G_{\mathrm{L}} d_{\mathrm{R}} \bar{d}_{\mathrm{L}} \nu_{\mathrm{L}}^{2}}(0,2) \mid \mathcal{Y}\left[\frac{p}{r}\right] i\left(\lambda^{A}\right)_{b}^{a} G_{\mathrm{L}}^{A \mu \nu}\left(\nu_{\mathrm{L} p} C \sigma_{\mu \nu} \nu_{\mathrm{L} r}\right)\left(\bar{d}_{\mathrm{L} t}^{b} d_{\mathrm{R} s a}\right)
\end{aligned}
$$

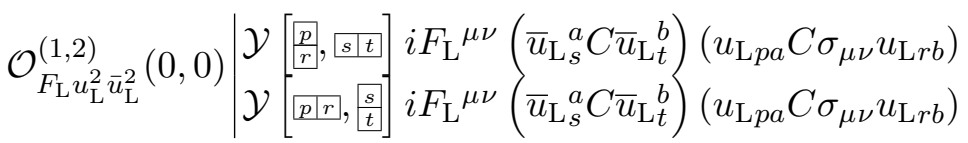

$$
\begin{aligned}
& \mathcal{O}_{F_{\mathrm{L}} d_{\mathrm{R}} e_{\mathrm{R}} u_{\mathrm{L}}^{2}}(1,1) \mid \mathcal{Y}\left[{ }_{[p r r}\right] i \epsilon^{a b c} F_{\mathrm{L}}^{\mu \nu}\left(d_{\mathrm{R} s c} C e_{\mathrm{R} t}\right)\left(u_{\mathrm{L} p a} C \sigma_{\mu \nu} u_{\mathrm{L} r b}\right)
\end{aligned}
$$

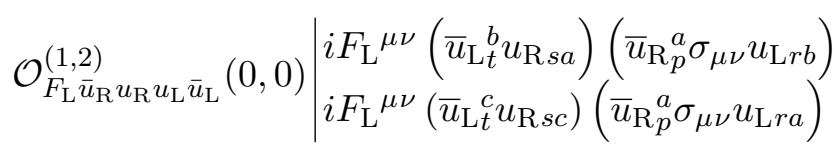

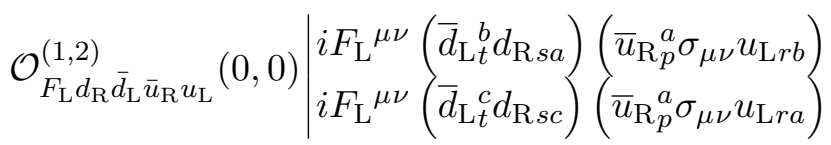

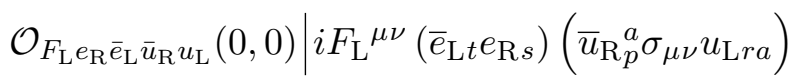

$$
\begin{aligned}
& \left.\mathcal{O}_{F_{\mathrm{L}} \bar{\nu}_{\mathrm{L}}^{2} \bar{u}_{\mathrm{R}} u_{\mathrm{L}}}(0,-2) \mid \mathcal{Y}[s \mid t]\right] i F_{\mathrm{L}}^{\mu \nu}\left(\bar{\nu}_{\mathrm{L} s} C \bar{\nu}_{\mathrm{L} t}\right)\left(\bar{u}_{\mathrm{R} p}^{a} \sigma_{\mu \nu} u_{\mathrm{L} r a}\right)
\end{aligned}
$$




$$
\begin{aligned}
& \mathcal{O}_{F_{\mathrm{L}} d_{\mathrm{L}} \bar{d}_{\mathrm{L}} u_{\mathrm{L}} \bar{u}_{\mathrm{L}}}^{(1,2,0)} \mid \begin{array}{ll}
i F_{\mathrm{L}}{ }^{\mu \nu}\left(\bar{d}_{\mathrm{L}}^{a} C \bar{u}_{\mathrm{L} t}^{b}\right) & \left(d_{\mathrm{L} p a} C \sigma_{\mu \nu} u_{\mathrm{L} r b}\right) \\
i F_{\mathrm{L}}{ }^{\mu \nu}\left(\bar{d}_{\mathrm{L}}^{b} C \bar{u}_{\mathrm{L} t}^{a}\right. & \left(d_{\mathrm{L} p a} C \sigma_{\mu \nu} u_{\mathrm{L} r b}\right)
\end{array} \\
& \mathcal{O}_{F_{\mathrm{L}} d_{\mathrm{L}} e_{\mathrm{R}} u_{\mathrm{R}} u_{\mathrm{L}}}(1,1) \mid i \epsilon^{a b c} F_{\mathrm{L}}{ }^{\mu \nu}\left(e_{\mathrm{R} s} C u_{\mathrm{R} t c}\right)\left(d_{\mathrm{L} p a} C \sigma_{\mu \nu} u_{\mathrm{L} r b}\right)
\end{aligned}
$$

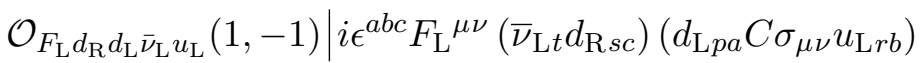

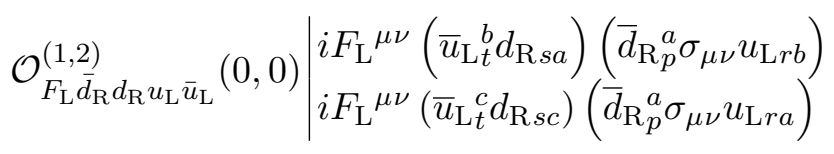

$$
\begin{aligned}
& \mathcal{O}_{F_{\mathrm{L}} \bar{d}_{\mathrm{R}} e_{\mathrm{R}} \bar{\nu}_{\mathrm{L}} u_{\mathrm{L}}}(0,0) \mid i F_{\mathrm{L}}^{\mu \nu}\left(\bar{\nu}_{\mathrm{L} t} e_{\mathrm{R} s}\right)\left(\bar{d}_{\mathrm{R} p}^{a} \sigma_{\mu \nu} u_{\mathrm{L} r a}\right)
\end{aligned}
$$

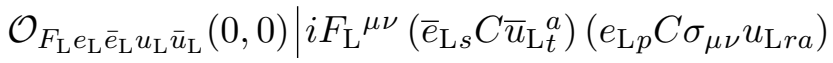

$$
\begin{aligned}
& \mathcal{O}_{F_{\mathrm{L}} d_{\mathrm{R}} e_{\mathrm{L}} u_{\mathrm{R}} u_{\mathrm{L}}}(1,1) \mid i \epsilon^{a b c} F_{\mathrm{L}}^{\mu \nu}\left(d_{\mathrm{R} s b} C u_{\mathrm{R} t c}\right)\left(e_{\mathrm{L} p} C \sigma_{\mu \nu} u_{\mathrm{L} r a}\right) \\
& \mathcal{O}_{F_{\mathrm{L}} \bar{d}_{\mathrm{L}} e_{\mathrm{L}} \bar{\nu}_{\mathrm{L}} u_{\mathrm{L}}}(0,0) \mid i F_{\mathrm{L}}^{\mu \nu}\left(\bar{d}_{\mathrm{L} s}^{a} C \bar{\nu}_{\mathrm{L} t}\right)\left(e_{\mathrm{L} p} C \sigma_{\mu \nu} u_{\mathrm{L} r a}\right)
\end{aligned}
$$

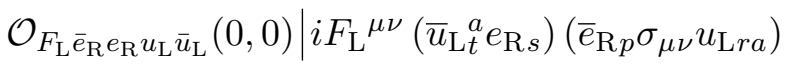

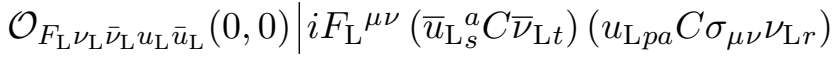

$$
\begin{aligned}
& \mathcal{O}_{F_{\mathrm{L}} \bar{d}_{\mathrm{L}} e_{\mathrm{R}} \nu_{\mathrm{L}} u_{\mathrm{L}}}(0,2) \mid i F_{\mathrm{L}}^{\mu \nu}\left(\bar{d}_{\mathrm{L} s}^{a} e_{\mathrm{R} t}\right)\left(u_{\mathrm{L} p a} C \sigma_{\mu \nu} \nu_{\mathrm{L} r}\right) \\
& \mathcal{O}_{F_{\mathrm{L}} d_{\mathrm{R}}^{2} \nu_{\mathrm{L}} u_{\mathrm{L}}}(1,1) \mid \mathcal{Y}\left[\begin{array}{l}
s \\
t
\end{array}\right] i \epsilon^{a b c} F_{\mathrm{L}}^{\mu \nu}\left(d_{\mathrm{R} s b} C d_{\mathrm{R} t c}\right)\left(u_{\mathrm{L} p a} C \sigma_{\mu \nu} \nu_{\mathrm{L} r}\right)
\end{aligned}
$$

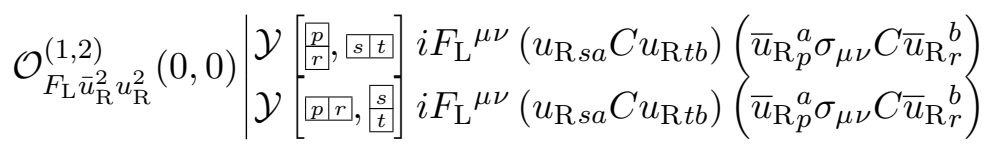

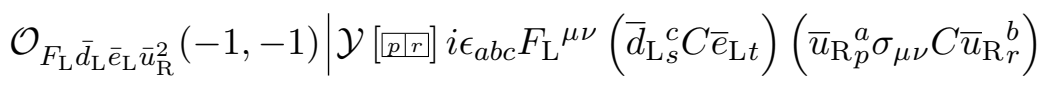

$$
\begin{aligned}
& \mathcal{O}_{F_{\mathrm{L}} d_{\mathrm{L}} \bar{d}_{\mathrm{L}} \bar{u}_{\mathrm{R}} u_{\mathrm{R}}}^{(1,2,0)}\left(\begin{array}{l}
i F_{\mathrm{L}}{ }^{\mu \nu}\left(\bar{d}_{\mathrm{L}}^{a} u_{\mathrm{R} t b}\right) \\
i F_{\mathrm{L}}{ }^{\mu \nu}\left(\bar{u}_{\mathrm{R} r}^{b} \sigma_{\mu \nu} d_{\mathrm{L} p a}\right) \\
\left.\bar{d}_{s}^{c} u_{\mathrm{R} t c}\right) \\
\left(\bar{u}_{\mathrm{R} r}^{a} \sigma_{\mu \nu} d_{\mathrm{L} p a}\right)
\end{array}\right.
\end{aligned}
$$

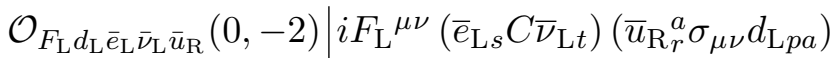

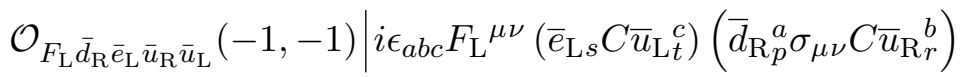

$$
\begin{aligned}
& \mathcal{O}_{F_{\mathrm{L}} \bar{d}_{\mathrm{R}} d_{\mathrm{R}} \bar{u}_{\mathrm{R}} u_{\mathrm{R}}}^{(1,0,0)} \mid \begin{array}{l}
i F_{\mathrm{L}}^{\mu \nu}\left(d_{\mathrm{R} s a} C u_{\mathrm{R} t b}\right) \\
i F_{\mathrm{L}}^{\mu \nu}\left(d_{\mathrm{R} s b} C u_{\mathrm{R} t a}\right)
\end{array}\left(\begin{array}{l}
\bar{d}_{\mathrm{R}}^{a} \sigma_{\mu \nu} C \bar{u}_{\mathrm{R}}^{b}{ }_{r}^{b} \\
\bar{d}_{\mathrm{R}}^{a} \sigma_{\mu \nu} C \bar{u}_{\mathrm{R}}^{b}
\end{array}\right)
\end{aligned}
$$

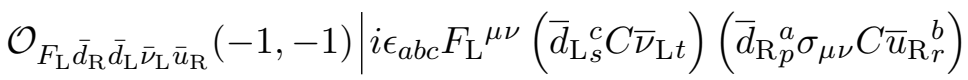

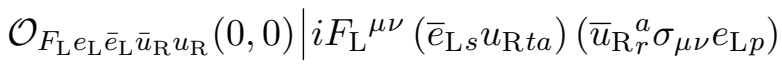




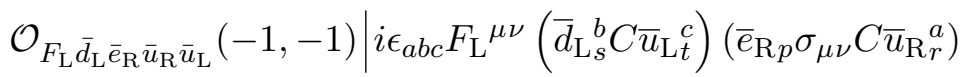

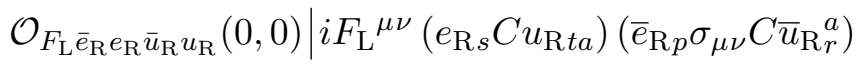

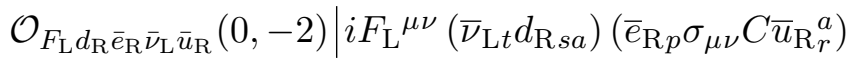

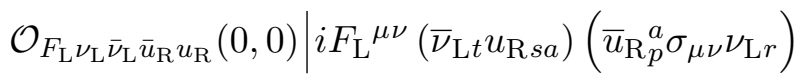

$$
\begin{aligned}
& \mathcal{O}_{F_{\mathrm{L}} \bar{d}_{\mathrm{L}}^{2} \nu_{\mathrm{L}} \bar{u}_{\mathrm{R}}}(-1,1) \mid \mathcal{Y}\left[\begin{array}{l}
s \\
\underline{t}
\end{array}\right] i \epsilon_{a b c} F_{\mathrm{L}}^{\mu \nu}\left(\bar{d}_{\mathrm{L}}^{b} C \bar{d}_{\mathrm{L} t}^{c}\right)\left(\bar{u}_{\mathrm{R}}^{a} \sigma_{\mu \nu} \nu_{\mathrm{L} r}\right)
\end{aligned}
$$

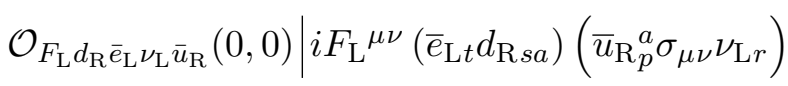

$$
\begin{aligned}
& \mathcal{O}_{F_{\mathrm{L}} d_{\mathrm{L}}^{2} \bar{\nu}_{\mathrm{L}} u_{\mathrm{R}}}(1,-1) \mid \mathcal{Y}[\overline{p p r}] i \epsilon^{a b c} F_{\mathrm{L}}^{\mu \nu}\left(\bar{\nu}_{\mathrm{L} t} u_{\mathrm{R} s c}\right)\left(d_{\mathrm{L} p a} C \sigma_{\mu \nu} d_{\mathrm{L} r b}\right)
\end{aligned}
$$

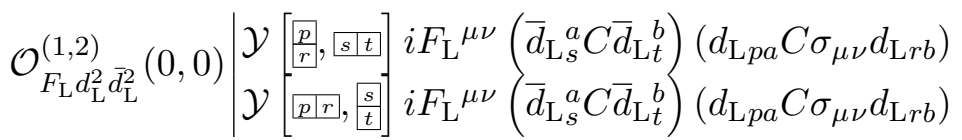

$$
\begin{aligned}
& \mathcal{O}_{F_{\mathrm{L}} d_{\mathrm{R}} d_{\mathrm{L}}^{2} \bar{e}_{\mathrm{L}}}(1,-1) \mid \mathcal{Y}[\overline{p p r}] i \epsilon^{a b c} F_{\mathrm{L}}^{\mu \nu}\left(\bar{e}_{\mathrm{L} t} d_{\mathrm{R} s c}\right)\left(d_{\mathrm{L} p a} C \sigma_{\mu \nu} d_{\mathrm{L} r b}\right) \\
& \mathcal{O}_{F_{\mathrm{L}} \bar{d}_{\mathrm{R}} d_{\mathrm{L}} u_{\mathrm{R}} \bar{u}_{\mathrm{L}}}^{(1,0,0)} \mid \begin{array}{l}
i F_{\mathrm{L}}{ }^{\mu \nu}\left(\bar{u}_{\mathrm{L} t}^{b} u_{\mathrm{R} s a}\right)\left(\bar{d}_{\mathrm{R}}^{a} \sigma_{\mu \nu} d_{\mathrm{L} r b}\right) \\
i F_{\mathrm{L}}{ }^{\mu \nu}\left(\bar{u}_{\mathrm{L} t}^{c} u_{\mathrm{R} s c}\right)\left(\bar{d}_{\mathrm{R} p}^{a} \sigma_{\mu \nu} d_{\mathrm{L} r a}\right)
\end{array}
\end{aligned}
$$

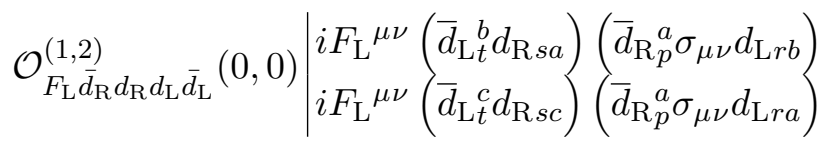

$$
\begin{aligned}
& \mathcal{O}_{F_{\mathrm{L}} \bar{d}_{\mathrm{R}} d_{\mathrm{L}} e_{\mathrm{R}} \bar{e}_{\mathrm{L}}}(0,0) \mid i F_{\mathrm{L}}^{\mu \nu}\left(\bar{e}_{\mathrm{L} t} e_{\mathrm{R} s}\right)\left(\bar{d}_{\mathrm{R} p}^{a} \sigma_{\mu \nu} d_{\mathrm{L} r a}\right) \\
& \left.\mathcal{O}_{F_{\mathrm{L}} \bar{d}_{\mathrm{R}} d_{\mathrm{L}} \bar{\nu}_{\mathrm{L}}^{2}}(0,-2) \mid \mathcal{Y}[s \mid t]\right] i F_{\mathrm{L}}^{\mu \nu}\left(\bar{\nu}_{\mathrm{L} s} C \bar{\nu}_{\mathrm{L} t}\right)\left(\bar{d}_{\mathrm{R} p}^{a} \sigma_{\mu \nu} d_{\mathrm{L} r a}\right) \\
& \mathcal{O}_{F_{\mathrm{L}} d_{\mathrm{L}} e_{\mathrm{L}} u_{\mathrm{R}}^{2}}(1,1) \mid \mathcal{Y}\left[\begin{array}{l}
s \\
t
\end{array}\right] i \epsilon^{a b c} F_{\mathrm{L}}^{\mu \nu}\left(u_{\mathrm{R} s b} C u_{\mathrm{R} t c}\right)\left(d_{\mathrm{L} p a} C \sigma_{\mu \nu} e_{\mathrm{L} r}\right) \\
& \mathcal{O}_{F_{\mathrm{L}} d_{\mathrm{L}} \bar{d}_{\mathrm{L}} e_{\mathrm{L}} \bar{e}_{\mathrm{L}}}(0,0) \mid i F_{\mathrm{L}}^{\mu \nu}\left(\bar{d}_{\mathrm{L} s}^{a} C \bar{e}_{\mathrm{L} t}\right)\left(d_{\mathrm{L} p a} C \sigma_{\mu \nu} e_{\mathrm{L} r}\right)
\end{aligned}
$$

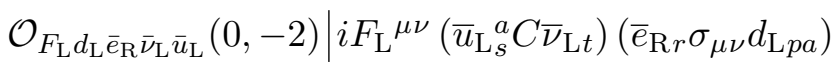

$$
\begin{aligned}
& \mathcal{O}_{F_{\mathrm{L}} d_{\mathrm{L}} \bar{d}_{\mathrm{L}} \bar{e}_{\mathrm{R}} e_{\mathrm{R}}}(0,0) \mid i F_{\mathrm{L}}^{\mu \nu}\left(\bar{d}_{\mathrm{L} s}^{a} e_{\mathrm{R} t}\right)\left(\bar{e}_{\mathrm{R} r} \sigma_{\mu \nu} d_{\mathrm{L} p a}\right) \\
& \mathcal{O}_{F_{\mathrm{L}} d_{\mathrm{R}}^{2} d_{\mathrm{L}} \bar{e}_{\mathrm{R}}}(1,-1) \mid \mathcal{Y}\left[\begin{array}{l}
s \\
\underline{t}
\end{array}\right] i \epsilon^{a b c} F_{\mathrm{L}}^{\mu \nu}\left(d_{\mathrm{R} s b} C d_{\mathrm{R} t c}\right)\left(\bar{e}_{\mathrm{R} r} \sigma_{\mu \nu} d_{\mathrm{L} p a}\right)
\end{aligned}
$$

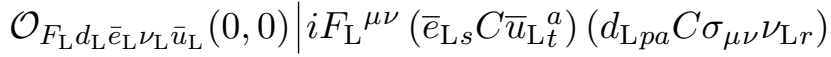

$$
\begin{aligned}
& \mathcal{O}_{F_{\mathrm{L}} d_{\mathrm{R}} d_{\mathrm{L}} \nu_{\mathrm{L}} u_{\mathrm{R}}}(1,1) \mid i \epsilon^{a b c} F_{\mathrm{L}}{ }^{\mu \nu}\left(d_{\mathrm{R} s b} C u_{\mathrm{R} t c}\right)\left(d_{\mathrm{L} p a} C \sigma_{\mu \nu} \nu_{\mathrm{L} r}\right) \\
& \mathcal{O}_{F_{\mathrm{L}} d_{\mathrm{L}} \bar{d}_{\mathrm{L}} \nu_{\mathrm{L}} \bar{\nu}_{\mathrm{L}}}(0,0) \mid i F_{\mathrm{L}}^{\mu \nu}\left(\bar{d}_{\mathrm{L}}^{a} C \bar{\nu}_{\mathrm{L} t}\right)\left(d_{\mathrm{L} p a} C \sigma_{\mu \nu} \nu_{\mathrm{L} r}\right)
\end{aligned}
$$




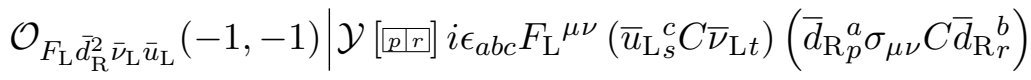

$$
\begin{aligned}
& \mathcal{O}_{F_{\mathrm{L}} \bar{d}_{\mathrm{R}}^{2} \bar{d}_{\mathrm{L}} e_{\mathrm{R}}}(-1,1) \mid \mathcal{Y}[[p r r]] i \epsilon_{a b c} F_{\mathrm{L}}^{\mu \nu}\left(\bar{d}_{\mathrm{L} s}^{c} e_{\mathrm{R} t}\right)\left(\bar{d}_{\mathrm{R} p}^{a} \sigma_{\mu \nu} C \bar{d}_{\mathrm{R}}^{b}\right)
\end{aligned}
$$

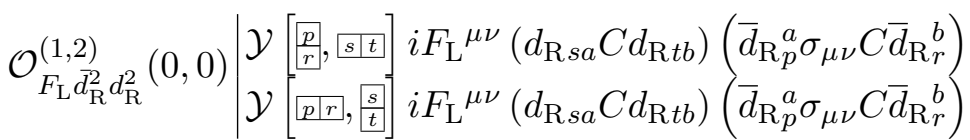

$$
\begin{aligned}
& \mathcal{O}_{F_{\mathrm{L}} \bar{d}_{\mathrm{R}} e_{\mathrm{L}} \bar{\nu}_{\mathrm{L}} u_{\mathrm{R}}}(0,0) \mid i F_{\mathrm{L}}^{\mu \nu}\left(\bar{\nu}_{\mathrm{L} t} u_{\mathrm{R} s a}\right)\left(\bar{d}_{\mathrm{R}}^{a} \sigma_{\mu \nu} e_{\mathrm{L} r}\right)
\end{aligned}
$$

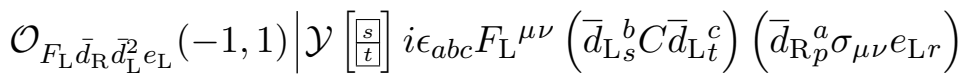

$$
\begin{aligned}
& \mathcal{O}_{F_{\mathrm{L}} \bar{d}_{\mathrm{R}} d_{\mathrm{R}} e_{\mathrm{L}} \bar{e}_{\mathrm{L}}}(0,0) \mid i F_{\mathrm{L}}^{\mu \nu}\left(\bar{e}_{\mathrm{L} t} d_{\mathrm{R} s a}\right)\left(\bar{d}_{\mathrm{R}}^{a} \sigma_{\mu \nu} e_{\mathrm{L} r}\right)
\end{aligned}
$$

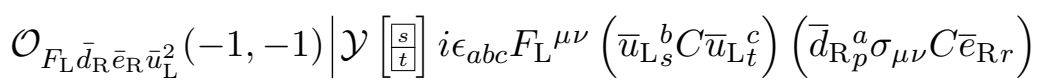

$$
\begin{aligned}
& \mathcal{O}_{F_{\mathrm{L}} \bar{d}_{\mathrm{R}} d_{\mathrm{R}} \bar{e}_{\mathrm{R}} e_{\mathrm{R}}}(0,0) \mid i F_{\mathrm{L}}^{\mu \nu}\left(d_{\mathrm{R} s a} C e_{\mathrm{R} t}\right)\left(\bar{d}_{\mathrm{R} p}^{a} \sigma_{\mu \nu} C \bar{e}_{\mathrm{R} r}\right)
\end{aligned}
$$

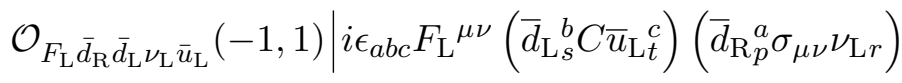

$$
\begin{aligned}
& \mathcal{O}_{F_{\mathrm{L}} \bar{d}_{\mathrm{R}} e_{\mathrm{R}} \nu_{\mathrm{L}} u_{\mathrm{R}}}(0,2) \mid i F_{\mathrm{L}}^{\mu \nu}\left(e_{\mathrm{R} s} C u_{\mathrm{R} t a}\right)\left(\bar{d}_{\mathrm{R} p}^{a} \sigma_{\mu \nu} \nu_{\mathrm{L} r}\right) \\
& \mathcal{O}_{F_{\mathrm{L}} \bar{d}_{\mathrm{R}} d_{\mathrm{R}} \nu_{\mathrm{L}} \bar{\nu}_{\mathrm{L}}}(0,0) \mid i F_{\mathrm{L}}^{\mu \nu}\left(\bar{\nu}_{\mathrm{L} t} d_{\mathrm{R} s a}\right)\left(\bar{d}_{\mathrm{R} p}^{a} \sigma_{\mu \nu} \nu_{\mathrm{L} r}\right) \\
& \mathcal{O}_{F_{\mathrm{L}} e_{\mathrm{L}}^{2} \bar{e}_{\mathrm{L}}^{2}}(0,0) \mid \mathcal{Y}\left[\frac{p}{r}, s \mid t\right] i F_{\mathrm{L}}{ }^{\mu \nu}\left(\bar{e}_{\mathrm{L} s} C \bar{e}_{\mathrm{L} t}\right)\left(e_{\mathrm{L} p} C \sigma_{\mu \nu} e_{\mathrm{L} r}\right)
\end{aligned}
$$

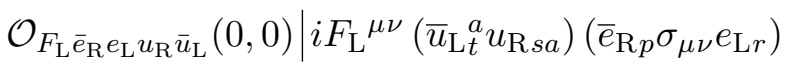

$$
\begin{aligned}
& \mathcal{O}_{F_{\mathrm{L}} d_{\mathrm{R}} \bar{d}_{\mathrm{L}} \bar{e}_{\mathrm{R}} e_{\mathrm{L}}}(0,0) \mid i F_{\mathrm{L}}^{\mu \nu}\left(\bar{d}_{\mathrm{L} t}^{a} d_{\mathrm{R} s a}\right)\left(\bar{e}_{\mathrm{R} p} \sigma_{\mu \nu} e_{\mathrm{L} r}\right) \\
& \mathcal{O}_{F_{\mathrm{L}} \bar{e}_{\mathrm{R}} e_{\mathrm{R}} e_{\mathrm{L}} \bar{e}_{\mathrm{L}}}(0,0) \mid i F_{\mathrm{L}}^{\mu \nu}\left(\bar{e}_{\mathrm{L} t} e_{\mathrm{R} s}\right)\left(\bar{e}_{\mathrm{R} p} \sigma_{\mu \nu} e_{\mathrm{L} r}\right) \\
& \mathcal{O}_{F_{\mathrm{L}} \bar{e}_{\mathrm{R}} e_{\mathrm{L}} \bar{\nu}_{\mathrm{L}}^{2}}(0,-2) \mid \mathcal{Y}[\overline{s \mid t}] i F_{\mathrm{L}}^{\mu \nu}\left(\bar{\nu}_{\mathrm{L} s} C \bar{\nu}_{\mathrm{L} t}\right)\left(\bar{e}_{\mathrm{R} p} \sigma_{\mu \nu} e_{\mathrm{L} r}\right) \\
& \mathcal{O}_{F_{\mathrm{L}} \bar{d}_{\mathrm{L}} e_{\mathrm{L}} \nu_{\mathrm{L}} u_{\mathrm{R}}}(0,2) \mid i F_{\mathrm{L}}^{\mu \nu}\left(\bar{d}_{\mathrm{L} s}^{a} u_{\mathrm{R} t a}\right)\left(e_{\mathrm{L} p} C \sigma_{\mu \nu} \nu_{\mathrm{L} r}\right)
\end{aligned}
$$

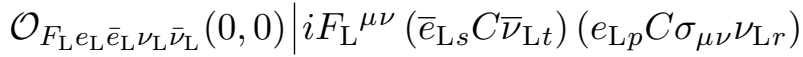

$$
\begin{aligned}
& \left.\mathcal{O}_{F_{\mathrm{L}} \bar{e}_{\mathrm{R}}^{2} e_{\mathrm{R}}^{2}}(0,0) \mid \mathcal{Y}\left[\frac{p}{r}, s\right] t\right] i\left(e_{\mathrm{R} s} C e_{\mathrm{R} t}\right) F_{\mathrm{L}}^{\mu \nu}\left(\bar{e}_{\mathrm{R} p} \sigma_{\mu \nu} C \bar{e}_{\mathrm{R} r}\right)
\end{aligned}
$$

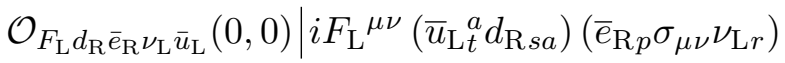

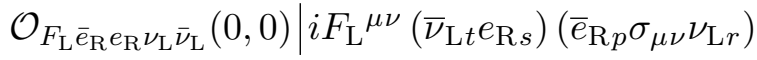

$$
\begin{aligned}
& \mathcal{O}_{F_{\mathrm{L}} \nu_{\mathrm{L}}^{2} u_{\mathrm{R}} \bar{u}_{\mathrm{L}}}(0,2) \mid \mathcal{Y}\left[\frac{p}{r}\right] i F_{\mathrm{L}}^{\mu \nu}\left(\bar{u}_{\mathrm{L} t}^{a} u_{\mathrm{R} s a}\right)\left(\nu_{\mathrm{L} p} C \sigma_{\mu \nu} \nu_{\mathrm{L} r}\right)
\end{aligned}
$$




$$
\begin{aligned}
& \mathcal{O}_{F_{\mathrm{L}} d_{\mathrm{R}} \bar{d}_{\mathrm{L}} \nu_{\mathrm{L}}^{2}}(0,2) \mid \mathcal{Y}\left[\frac{p}{r}\right] i F_{\mathrm{L}}{ }^{\mu \nu}\left(\bar{d}_{\mathrm{L} t}^{a} d_{\mathrm{R} s a}\right)\left(\nu_{\mathrm{L} p} C \sigma_{\mu \nu} \nu_{\mathrm{L} r}\right) \\
& \mathcal{O}_{F_{\mathrm{L}} e_{\mathrm{R}} \bar{e}_{\mathrm{L}} \nu_{\mathrm{L}}^{2}}(0,2) \mid \mathcal{Y}\left[\frac{p}{r}\right] i F_{\mathrm{L}}{ }^{\mu \nu}\left(\bar{e}_{\mathrm{L} t} e_{\mathrm{R} s}\right)\left(\nu_{\mathrm{L} p} C \sigma_{\mu \nu} \nu_{\mathrm{L} r}\right) \\
& \mathcal{O}_{F_{\mathrm{L}} \nu_{\mathrm{L}}^{2} \bar{\nu}_{\mathrm{L}}^{2}}(0,0) \mid \mathcal{Y}\left[\left[\frac{p}{r}, s\right] t\right] i F_{\mathrm{L}}{ }^{\mu \nu}\left(\bar{\nu}_{\mathrm{L} s} C \bar{\nu}_{\mathrm{L} t}\right)\left(\nu_{\mathrm{L} p} C \sigma_{\mu \nu} \nu_{\mathrm{L} r}\right)
\end{aligned}
$$

\subsection{Lists of the dim-9 operators}

\subsubsection{Classes involving two-fermions}

\section{Class $F_{\mathrm{L}}^{2} \psi^{2} D^{2}: 10$ types}

$$
\begin{aligned}
& \mathcal{O}_{G_{\mathrm{L}}{ }^{2} \bar{u}_{\mathrm{R}} u_{\mathrm{L}} D^{2}}^{(1 \sim 5)}(0,0) \mid \begin{array}{l}
G_{\mathrm{L} \nu \lambda}^{A} G_{\mathrm{L}}^{A \nu \lambda}\left(D_{\mu} \bar{u}_{\mathrm{R}}^{a} D^{\mu} u_{\mathrm{L} r a}\right) \\
d^{A B C}\left(\lambda^{C}\right)_{a}^{b} G_{\mathrm{L} \nu \lambda}^{A} G_{\mathrm{L}}^{B \nu \lambda}\left(D_{\mu} \bar{u}_{\mathrm{R} p}^{a} D^{\mu} u_{\mathrm{L} r b}\right) \\
f^{A B C}\left(\lambda^{C}\right)_{a}^{b} G_{\mathrm{L} \lambda}^{A}{ }^{\mu} G_{\mathrm{L}}^{B \lambda \nu}\left(D_{\mu} \bar{u}_{\mathrm{R} p}^{a} D_{\nu} u_{\mathrm{L} r b}\right) \\
i G_{\mathrm{L}}^{A \lambda \rho} G_{\mathrm{L} \mu \nu}^{A}\left(D_{\lambda} \bar{u}_{\mathrm{R} p}^{a} \sigma^{\mu \nu} D_{\rho} u_{\mathrm{L} r a}\right) \\
i d^{A B C}\left(\lambda^{C}\right)_{a}^{b} G_{\mathrm{L}}^{A \lambda \rho} G_{\mathrm{L} \mu \nu}^{B}\left(D_{\lambda} \bar{u}_{\mathrm{R}}^{a} \sigma^{\mu \nu} D_{\rho} u_{\mathrm{L} r b}\right)
\end{array} \\
& \mid G_{\mathrm{L} \nu \lambda}^{A} G_{\mathrm{L}}^{A \nu \lambda}\left(D_{\mu} \bar{d}_{\mathrm{R} p}^{a} D^{\mu} d_{\mathrm{L} r a}\right) \\
& \mathcal{O}_{G_{\mathrm{L}}{ }^{2} \bar{d}_{\mathrm{R}} d_{\mathrm{L}} D^{2}}^{(1 \sim 5)}(0,0) \mid \begin{array}{l}
d^{A B C}\left(\lambda^{C}\right)_{a}^{b} G_{\mathrm{L} \nu \lambda}^{A} G_{\mathrm{L}}^{B \nu \lambda}\left(D_{\mu} \bar{d}_{\mathrm{R} p}^{a} D^{\mu} d_{\mathrm{L} r b}\right) \\
f^{A B C}\left(\lambda^{C}\right)_{a}^{b} G_{\mathrm{L} \lambda^{\mu}}^{A} G_{\mathrm{L}}^{B \lambda \nu}\left(D_{\mu} \bar{d}_{\mathrm{R} p}^{a} D_{\nu} d_{\mathrm{L} r b}\right)
\end{array} \\
& i G_{\mathrm{L}}^{A \lambda \rho} G_{\mathrm{L} \mu \nu}^{A}\left(D_{\lambda} \bar{d}_{\mathrm{R} p}^{a} \sigma^{\mu \nu} D_{\rho} d_{\mathrm{L} r a}\right) \\
& i d^{A B C}\left(\lambda^{C}\right)_{a}^{b} G_{\mathrm{L}}^{A \lambda \rho} G_{\mathrm{L} \mu \nu}^{B}\left(D_{\lambda} \bar{d}_{\mathrm{R}}^{a} \sigma^{\mu \nu} D_{\rho} d_{\mathrm{L} r b}\right) \\
& \mathcal{O}_{G_{\mathrm{L}}^{2} \bar{e}_{\mathrm{R}} e_{\mathrm{L}} D^{2}}^{(1,2,0)} \mid \begin{array}{l}
G_{\mathrm{L} \nu \lambda}^{A} G_{\mathrm{L}}^{A \nu \lambda}\left(D_{\mu} \bar{e}_{\mathrm{R} p} D^{\mu} e_{\mathrm{L} r}\right) \\
i G_{\mathrm{L}}^{A \lambda \rho} G_{\mathrm{L} \mu \nu}^{A}\left(D_{\lambda} \bar{e}_{\mathrm{R} p} \sigma^{\mu \nu} D_{\rho} e_{\mathrm{L} r}\right)
\end{array} \\
& \mathcal{O}_{G_{\mathrm{L}}{ }^{2} \nu_{\mathrm{L}}^{2} D^{2}}^{(1,2)}(0,2) \mid \begin{array}{l}
\mathcal{Y}\left[[p \mid r] G_{\mathrm{L} \nu \lambda}^{A} G_{\mathrm{L}}^{A \nu \lambda}\left(D_{\mu} \nu_{\mathrm{L} p} C D^{\mu} \nu_{\mathrm{L} r}\right)\right. \\
\mathcal{Y}\left[[p r r] i G_{\mathrm{L}}^{A \lambda \rho} G_{\mathrm{L} \mu \nu}^{A}\left(D_{\lambda} \nu_{\mathrm{L} p} C \sigma^{\mu \nu} D_{\rho} \nu_{\mathrm{L} r}\right)\right.
\end{array} \\
& \mathcal{O}_{F_{\mathrm{L}} G_{\mathrm{L}} \bar{u}_{\mathrm{R}} u_{\mathrm{L}} D^{2}}^{(1 \sim 30)} \mid \begin{array}{l}
\left(\lambda^{A}\right)_{a}^{b} F_{\mathrm{L} \nu \lambda} G_{\mathrm{L}}^{A \nu \lambda}\left(D_{\mu} \bar{u}_{\mathrm{R}}^{a} D^{\mu} u_{\mathrm{L} r b}\right) \\
\left(\lambda^{A}\right)_{a}^{b} F_{\mathrm{L} \lambda}{ }^{\mu} G_{\mathrm{L}}^{A \lambda \nu}\left(D_{\mu} \bar{u}_{\mathrm{R}}^{a} D_{\nu} u_{\mathrm{L} r b}\right) \\
i\left(\lambda^{A}\right)_{a}^{b} F_{\mathrm{L}}^{\lambda \rho} G_{\mathrm{L} \mu \nu}^{A}\left(D_{\lambda} \bar{u}_{\mathrm{R}}^{a}{ }^{a} \sigma^{\mu \nu} D_{\rho} u_{\mathrm{L} r b}\right)
\end{array} \\
& \mathcal{O}_{F_{\mathrm{L}} G_{\mathrm{L}} \bar{d}_{\mathrm{R}} d_{\mathrm{L}} D^{2}}^{(1 \sim 3)}(0,0) \mid \begin{array}{l}
\left(\lambda^{A}\right)_{a}^{b} F_{\mathrm{L} \nu \lambda} G_{\mathrm{L}}^{A \nu \lambda}\left(D_{\mu} \bar{d}_{\mathrm{R} p}^{a} D^{\mu} d_{\mathrm{L} r b}\right) \\
\left(\lambda^{A}\right)_{a}^{b} F_{\mathrm{L} \lambda}{ }^{\mu} G_{\mathrm{L}}^{A \lambda \nu}\left(D_{\mu} \bar{d}_{\mathrm{R} p}^{a} D_{\nu} d_{\mathrm{L} r b}\right) \\
i\left(\lambda^{A}\right)_{a}^{b} F_{\mathrm{L}}^{\lambda \rho} G_{\mathrm{L} \mu \nu}^{A}\left(D_{\lambda} \bar{d}_{\mathrm{R} p}^{a} \sigma^{\mu \nu} D_{\rho} d_{\mathrm{L} r b}\right)
\end{array} \\
& \mathcal{O}_{F_{\mathrm{L}}{ }^{2} \bar{u}_{\mathrm{R}} u_{\mathrm{L}} D^{2}}^{(1,2,0)} \mid \begin{array}{l}
F_{\mathrm{L} \nu \lambda} F_{\mathrm{L}}{ }^{\nu \lambda}\left(D_{\mu} \bar{u}_{\mathrm{R}}^{a}{ }^{a} D^{\mu} u_{\mathrm{L} r a}\right) \\
i F_{\mathrm{L} \mu \nu} F_{\mathrm{L}}{ }^{\lambda \rho}\left(D_{\lambda} \bar{u}_{\mathrm{R}}{ }^{a} \sigma^{\mu \nu} D_{\rho} u_{\mathrm{L} r a}\right)
\end{array}
\end{aligned}
$$




$$
\begin{aligned}
& \mathcal{O}_{F_{\mathrm{L}}{ }^{2} \bar{d}_{\mathrm{R}} d_{\mathrm{L}} D^{2}}^{(1,0,0)} \mid \begin{array}{l}
F_{\mathrm{L} \nu \lambda} F_{\mathrm{L}}^{\nu \lambda}\left(D_{\mu} \bar{d}_{\mathrm{R}}^{a} D^{\mu} d_{\mathrm{L} r a}\right) \\
i F_{\mathrm{L} \mu \nu} F_{\mathrm{L}}^{\lambda \rho}\left(D_{\lambda} \bar{d}_{\mathrm{R} p}^{a} \sigma^{\mu \nu} D_{\rho} d_{\mathrm{L} r a}\right)
\end{array} \\
& \mathcal{O}_{F_{\mathrm{L}}{ }^{2} \bar{e}_{\mathrm{R}} e_{\mathrm{L}} D^{2}}^{(1,2)}(0) \mid \begin{array}{l}
F_{\mathrm{L} \nu \lambda} F_{\mathrm{L}}{ }^{\nu \lambda}\left(D_{\mu} \bar{e}_{\mathrm{R} p} D^{\mu} e_{\mathrm{L} r}\right) \\
i F_{\mathrm{L} \mu \nu} F_{\mathrm{L}}^{\lambda \rho}\left(D_{\lambda} \bar{e}_{\mathrm{R} p} \sigma^{\mu \nu} D_{\rho} e_{\mathrm{L} r}\right)
\end{array} \\
& \mathcal{O}_{F_{\mathrm{L}}{ }^{2} \nu_{\mathrm{L}}^{2} D^{2}}^{(1,2)}(0,2) \mid \begin{array}{l}
\mathcal{Y}[\overline{p p r}] F_{\mathrm{L} \nu \lambda} F_{\mathrm{L}}{ }^{\nu \lambda}\left(D_{\mu} \nu_{\mathrm{L} p} C D^{\mu} \nu_{\mathrm{L} r}\right) \\
\mathcal{Y}[p p r]] i F_{\mathrm{L} \mu \nu} F_{\mathrm{L}}^{\lambda \rho}\left(D_{\lambda} \nu_{\mathrm{L} p} C \sigma^{\mu \nu} D_{\rho} \nu_{\mathrm{L} r}\right)
\end{array}
\end{aligned}
$$

Class $F_{\mathrm{L}} F_{\mathrm{R}} \psi^{2} D^{2}: 12$ types

$$
\begin{aligned}
& \mathcal{O}_{G_{\mathrm{L}} G_{\mathrm{R}} \bar{u}_{\mathrm{R}} u_{\mathrm{L}} D^{2}}^{(1 \sim 3)}(0,0) \mid \begin{array}{l}
d^{A B C}\left(\lambda^{C}\right)_{a}^{b} G_{\mathrm{L}}^{A \mu}{ }_{\lambda} G_{\mathrm{R}}^{B \nu \lambda}\left(\bar{u}_{\mathrm{R} p}^{a} D_{\mu} D_{\nu} u_{\mathrm{L} r b}\right) \\
f^{A B C}\left(\lambda^{C}\right)_{a}^{b} G_{\mathrm{L}}^{A \mu}{ }_{\lambda} G_{\mathrm{R}}^{B \nu \lambda}\left(\bar{u}_{\mathrm{R}}^{a} D_{\mu} D_{\nu} u_{\mathrm{L} r b}\right) \\
G_{\mathrm{L}}^{A \mu}{ }_{\lambda} G_{\mathrm{R}}^{A \nu \lambda}\left(\bar{u}_{\mathrm{R} p}^{a} D_{\mu} D_{\nu} u_{\mathrm{L} r a}\right)
\end{array}
\end{aligned}
$$

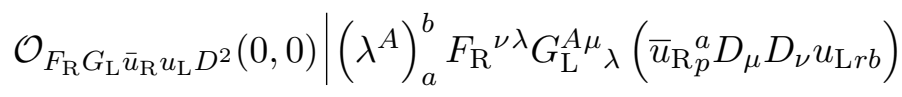

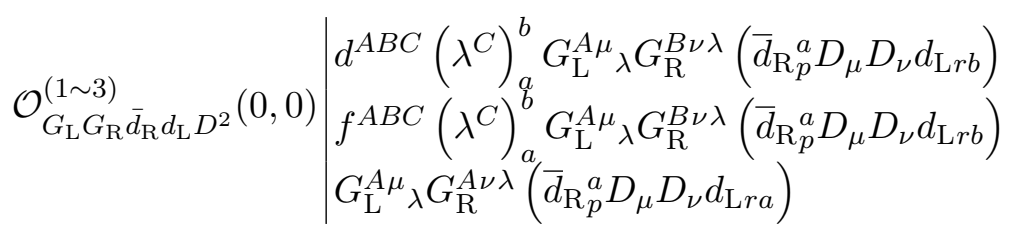

$$
\begin{aligned}
& \mathcal{O}_{F_{\mathrm{R}} G_{\mathrm{L}} \bar{d}_{\mathrm{R}} d_{\mathrm{L}} D^{2}}(0,0) \mid\left(\lambda^{A}\right)_{a}^{b} F_{\mathrm{R}}^{\nu \lambda} G_{\mathrm{L}}^{A \mu} \lambda\left(\bar{d}_{\mathrm{R} p}^{a} D_{\mu} D_{\nu} d_{\mathrm{L} r b}\right)
\end{aligned}
$$

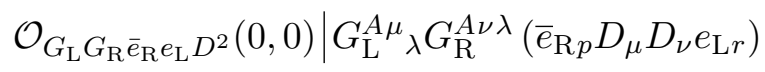

$$
\begin{aligned}
& \mathcal{O}_{G_{\mathrm{L}} G_{\mathrm{R}} \nu_{\mathrm{L}}^{2} D^{2}}(0,2) \mid \mathcal{Y}[\underline{p p r}] G_{\mathrm{L}}^{A \mu}{ }_{\lambda} G_{\mathrm{R}}^{A \nu \lambda}\left(\nu_{\mathrm{L} p} C D_{\mu} D_{\nu} \nu_{\mathrm{L} r}\right)
\end{aligned}
$$

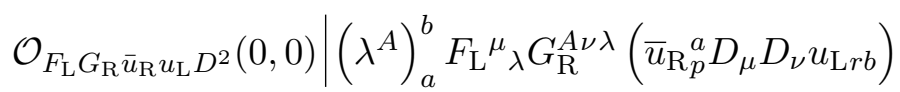

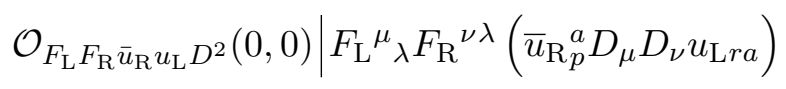

$$
\begin{aligned}
& \mathcal{O}_{F_{\mathrm{L}} G_{\mathrm{R}} \bar{d}_{\mathrm{R}} d_{\mathrm{L}} D^{2}}(0,0) \mid\left(\lambda^{A}\right)_{a}^{b} F_{\mathrm{L}}{ }_{\lambda}{ }_{\lambda} G_{\mathrm{R}}^{A \nu \lambda}\left(\bar{d}_{\mathrm{R}}^{a} D_{\mu} D_{\nu} d_{\mathrm{L} r b}\right) \\
& \mathcal{O}_{F_{\mathrm{L}} F_{\mathrm{R}} \bar{d}_{\mathrm{R}} d_{\mathrm{L}} D^{2}}(0,0) \mid F_{\mathrm{L}}{ }_{\lambda}{ }_{\lambda} F_{\mathrm{R}}{ }^{\nu \lambda}\left(\bar{d}_{\mathrm{R}}{ }_{p}^{a} D_{\mu} D_{\nu} d_{\mathrm{L} r a}\right)
\end{aligned}
$$

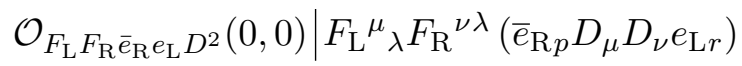

$$
\begin{aligned}
& \mathcal{O}_{F_{\mathrm{L}} F_{\mathrm{R}} \nu_{\mathrm{L}}^{2} D^{2}}(0,2) \mid \mathcal{Y}[\overline{p n r}] F_{\mathrm{L}}{ }_{\lambda}{ }_{\lambda} F_{\mathrm{R}}{ }^{\nu \lambda}\left(\nu_{\mathrm{L} p} C D_{\mu} D_{\nu} \nu_{\mathrm{L} r}\right)
\end{aligned}
$$

Class $F_{\mathrm{L}}^{2} \psi^{\dagger 2} D^{2}: 10$ types

$$
\mathcal{O}_{G_{\mathrm{L}}^{2} u_{\mathrm{R}} \bar{u}_{\mathrm{L}} D^{2}}^{(1 \sim 3)}(0,0) \mid \begin{aligned}
& G_{\mathrm{L} \nu \lambda}^{A} G_{\mathrm{L}}^{A \nu \lambda}\left(D^{\mu} \bar{u}_{\mathrm{L} r}^{a} D_{\mu} u_{\mathrm{R} p a}\right) \\
& d^{A B C}\left(\lambda^{C}\right)_{b}^{a} G_{\mathrm{L} \nu \lambda}^{A} G_{\mathrm{L}}^{B \nu \lambda}\left(D^{\mu} \bar{u}_{\mathrm{L} r}^{b} D_{\mu} u_{\mathrm{R} p a}\right) \\
& f^{A B C}\left(\lambda^{C}\right)_{b}^{a} G_{\mathrm{L} \lambda}^{A}{ }^{\mu} G_{\mathrm{L}}^{B \lambda \nu}\left(D_{\nu} \bar{u}_{\mathrm{L} r}^{b} D_{\mu} u_{\mathrm{R} p a}\right)
\end{aligned}
$$




$$
\begin{aligned}
& G_{\mathrm{L} \nu \lambda}^{A} G_{\mathrm{L}}^{A \nu \lambda}\left(D^{\mu} \bar{d}_{\mathrm{L} r}^{a} D_{\mu} d_{\mathrm{R} p a}\right) \\
& \mathcal{O}_{G_{\mathrm{L}}{ }^{2} d_{\mathrm{R}} \bar{d}_{\mathrm{L}} D^{2}}^{(1 \sim 3)}(0,0) \mid \begin{array}{l}
d^{A B C}\left(\lambda^{C}\right)_{b}^{a} G_{\mathrm{L} \nu \lambda}^{A} G_{\mathrm{L}}^{B \nu \lambda}\left(D^{\mu} \bar{d}_{\mathrm{L}}^{b} D_{\mu} d_{\mathrm{R} p a}\right) \\
f^{A B C}\left(\lambda^{C}\right)_{b}^{a} G_{\mathrm{L} \lambda}^{A}{ }^{\mu} G_{\mathrm{L}}^{B \lambda \nu}\left(D_{\nu} \bar{d}_{\mathrm{L} r}^{b} D_{\mu} d_{\mathrm{R} p a}\right)
\end{array} \\
& \mathcal{O}_{G_{\mathrm{L}}{ }^{2} e_{\mathrm{R}} \bar{e}_{\mathrm{L}} D^{2}}(0,0) \mid G_{\mathrm{L} \nu \lambda}^{A} G_{\mathrm{L}}^{A \nu \lambda}\left(D^{\mu} \bar{e}_{\mathrm{L} r} D_{\mu} e_{\mathrm{R} p}\right) \\
& \mathcal{O}_{G_{\mathrm{L}}{ }^{2} \bar{\nu}_{\mathrm{L}}^{2} D^{2}}(0,-2) \mid \mathcal{Y}\left[[p \mid r] G_{\mathrm{L} \nu \lambda}^{A} G_{\mathrm{L}}^{A \nu \lambda}\left(D_{\mu} \bar{\nu}_{\mathrm{L} p} C D^{\mu} \bar{\nu}_{\mathrm{L} r}\right)\right. \\
& \mathcal{O}_{F_{\mathrm{L}} G_{\mathrm{L}} u_{\mathrm{R}} \bar{u}_{\mathrm{L}} D^{2}}^{(1,2)}(0,0) \mid \begin{array}{l}
\left(\lambda^{A}\right)_{b}^{a} F_{\mathrm{L} \nu \lambda} G_{\mathrm{L}}^{A \nu \lambda}\left(D^{\mu} \bar{u}_{\mathrm{L} r}^{b} D_{\mu} u_{\mathrm{R} p a}\right) \\
\left(\lambda^{A}\right)_{b}^{a} F_{\mathrm{L} \lambda}^{\mu} G_{\mathrm{L}}^{A \lambda \nu}\left(D_{\nu} \bar{u}_{\mathrm{L} r}^{b} D_{\mu} u_{\mathrm{R} p a}\right)
\end{array} \\
& \mathcal{O}_{F_{\mathrm{L}} G_{\mathrm{L}} d_{\mathrm{R}} \bar{d}_{\mathrm{L}} D^{2}}^{(1,2)}(0,0) \mid \begin{array}{l}
\left(\lambda^{A}\right)_{b}^{a} F_{\mathrm{L} \nu \lambda} G_{\mathrm{L}}^{A \nu \lambda}\left(D^{\mu} \bar{d}_{\mathrm{L} r}^{b} D_{\mu} d_{\mathrm{R} p a}\right) \\
\left(\lambda^{A}\right)_{b}^{a} F_{\mathrm{L} \lambda}{ }^{\mu} G_{\mathrm{L}}^{A \lambda \nu}\left(D_{\nu} \bar{d}_{\mathrm{L} r}^{b} D_{\mu} d_{\mathrm{R} p a}\right)
\end{array} \\
& \mathcal{O}_{F_{\mathrm{L}}{ }^{2} u_{\mathrm{R}} \bar{u}_{\mathrm{L}} D^{2}}(0,0) \mid F_{\mathrm{L} \nu \lambda} F_{\mathrm{L}}^{\nu \lambda}\left(D^{\mu} \bar{u}_{\mathrm{L}}^{a} D_{\mu} u_{\mathrm{R} p a}\right) \\
& \mathcal{O}_{F_{\mathrm{L}}{ }^{2} d_{\mathrm{R}} \bar{d}_{\mathrm{L}} D^{2}}(0,0) \mid F_{\mathrm{L} \nu \lambda} F_{\mathrm{L}}^{\nu \lambda}\left(D^{\mu} \bar{d}_{\mathrm{L} r}^{a} D_{\mu} d_{\mathrm{R} p a}\right) \\
& \mathcal{O}_{F_{\mathrm{L}}{ }^{2} e_{\mathrm{R}} \bar{e}_{\mathrm{L}} D^{2}}(0,0) \mid F_{\mathrm{L} \nu \lambda} F_{\mathrm{L}}{ }^{\nu \lambda}\left(D^{\mu} \bar{e}_{\mathrm{L} r} D_{\mu} e_{\mathrm{R} p}\right) \\
& \mathcal{O}_{F_{\mathrm{L}}{ }^{2} \bar{\nu}_{\mathrm{L}}^{2} D^{2}}(0,-2) \mid \mathcal{Y}\left[\overline{p r r}^{\prime}\right] F_{\mathrm{L} \nu \lambda} F_{\mathrm{L}}{ }^{\nu \lambda}\left(D_{\mu} \bar{\nu}_{\mathrm{L} p} C D^{\mu} \bar{\nu}_{\mathrm{L} r}\right)
\end{aligned}
$$

\section{Class $F_{\mathrm{L}}{ }^{3} \psi^{2}: 14$ types}

$$
\begin{aligned}
& \mid \begin{array}{l}
i\left(\lambda^{A}\right)_{a}^{b} G_{\mathrm{L} \mu \nu}^{A} G_{\mathrm{L} \lambda \rho}^{B} G_{\mathrm{L}}^{B \mu \nu}\left(\bar{u}_{\mathrm{R} p}^{a} \sigma^{\lambda \rho} u_{\mathrm{L} r b}\right) \\
i\left(\lambda^{C}\right)_{a}^{b} G_{\mathrm{L} \mu \nu}^{A} G_{\mathrm{L}}^{A \mu \nu} G_{\mathrm{L} \lambda \rho}^{C}\left(\bar{u}_{\mathrm{R}}^{a} \sigma^{\lambda \rho} u_{\mathrm{L} r b}\right)
\end{array} \\
& \mathcal{O}_{G_{\mathrm{L}}{ }^{3} \bar{u}_{\mathrm{R}} u_{\mathrm{L}}}^{(1 \sim 7)}(0,0) i d^{A D E} d^{B C D}\left(\lambda^{E}\right)_{a}^{b} G_{\mathrm{L} \mu \nu}^{A} G_{\mathrm{L}}^{B \mu \nu} G_{\mathrm{L} \lambda \rho}^{C}\left(\bar{u}_{\mathrm{R}}{ }_{p}^{a} \sigma^{\lambda \rho} u_{\mathrm{L} r b}\right) \\
& i d^{B D E} f^{A C D}\left(\lambda^{E}\right)_{a}^{b} G_{\mathrm{L} \mu \nu}^{A} G_{\mathrm{L}}^{B \mu \nu} G_{\mathrm{L} \lambda \rho}^{C}\left(\bar{u}_{\mathrm{R}}^{a} \sigma^{\lambda \rho} u_{\mathrm{L} r b}\right) \\
& i d^{A B C} G_{\mathrm{L} \mu \nu}^{A} G_{\mathrm{L}}^{B \mu \nu} G_{\mathrm{L} \lambda \rho}^{C}\left(\bar{u}_{\mathrm{R}}{ }_{p}^{a} \sigma^{\lambda \rho} u_{\mathrm{L} r a}\right) \\
& d^{C D E} f^{A B D}\left(\lambda^{E}\right)_{a}^{b} G_{\mathrm{L} \mu \nu}^{A} G_{\mathrm{L}}^{B \mu}{ }_{\lambda} G_{\mathrm{L}}^{C \nu \lambda}\left(\bar{u}_{\mathrm{R} p}^{a} u_{\mathrm{L} r b}\right) \\
& f^{A B C} G_{\mathrm{L} \mu \nu}^{A} G_{\mathrm{L}}^{B \mu}{ }_{\lambda} G_{\mathrm{L}}^{C \nu \lambda}\left(\bar{u}_{\mathrm{R}}{ }_{p}^{a} u_{\mathrm{L} r a}\right) \\
& \mathcal{O}_{G_{\mathrm{L}}{ }^{3} \bar{d}_{\mathrm{R}} d_{\mathrm{L}}}^{(1 \sim 7,0)} \mid \begin{array}{l}
i\left(\lambda^{A}\right)_{a}^{b} G_{\mathrm{L} \mu \nu}^{A} G_{\mathrm{L} \lambda \rho}^{B} G_{\mathrm{L}}^{B \mu \nu}\left(\bar{d}_{\mathrm{R} p}^{a} \sigma^{\lambda \rho} d_{\mathrm{L} r b}\right) \\
i\left(\lambda^{C}\right)_{a}^{b} G_{\mathrm{L} \mu \nu}^{A} G_{\mathrm{L}}^{A \mu \nu} G_{\mathrm{L} \lambda \rho}^{C}\left(\bar{d}_{\mathrm{R} p}^{a} \sigma^{\lambda \rho} d_{\mathrm{L} r b}\right) \\
i d^{A D E} d^{B C D}\left(\lambda^{E}\right)_{a}^{b} G_{\mathrm{L} \mu \nu}^{A} G_{\mathrm{L}}^{B \mu \nu} G_{\mathrm{L} \lambda \rho}^{C}\left(\bar{d}_{\mathrm{R} p}^{a} \sigma^{\lambda \rho} d_{\mathrm{L} r b}\right) \\
i d^{B D E} f^{A C D}\left(\lambda^{E}\right)_{a}^{b} G_{\mathrm{L} \mu \nu}^{A} G_{\mathrm{L}}^{B \mu \nu} G_{\mathrm{L} \lambda \rho}^{C}\left(\bar{d}_{\mathrm{R} p}^{a} \sigma^{\lambda \rho} d_{\mathrm{L} r b}\right) \\
i d^{A B C} G_{\mathrm{L} \mu \nu}^{A} G_{\mathrm{L}}^{B \mu \nu} G_{\mathrm{L} \lambda \rho}^{C}\left(\bar{d}_{\mathrm{R} p}^{a} \sigma^{\lambda \rho} d_{\mathrm{L} r a}\right) \\
d^{C D E} f^{A B D}\left(\lambda^{E}\right)_{a}^{b} G_{\mathrm{L} \mu \nu}^{A} G_{\mathrm{L}}^{B \mu}{ }_{\lambda} G_{\mathrm{L}}^{C \nu \lambda}\left(\bar{d}_{\mathrm{R} p}^{a} d_{\mathrm{L} r b}\right) \\
f^{A B C} G_{\mathrm{L} \mu \nu}^{A} G_{\mathrm{L}}^{B \mu}{ }_{\lambda} G_{\mathrm{L}}^{C \nu \lambda}\left(\bar{d}_{\mathrm{R} p}^{a} d_{\mathrm{L} r a}\right)
\end{array}
\end{aligned}
$$




$$
\begin{aligned}
& \mathcal{O}_{G_{\mathrm{L}}{ }^{3} \bar{e}_{\mathrm{R}} e_{\mathrm{L}}}^{(1,2)}(0,0) \mid \begin{array}{l}
i d^{A B C} G_{\mathrm{L} \mu \nu}^{A} G_{\mathrm{L}}^{B \mu \nu} G_{\mathrm{L} \lambda \rho}^{C}\left(\bar{e}_{\mathrm{R} p} \sigma^{\lambda \rho} e_{\mathrm{L} r}\right) \\
f^{A B C}\left(\bar{e}_{\mathrm{R} p} e_{\mathrm{L} r}\right) G_{\mathrm{L} \mu \nu}^{A} G_{\mathrm{L}}^{B \mu}{ }_{\lambda} G_{\mathrm{L}}^{C \nu \lambda}
\end{array}
\end{aligned}
$$

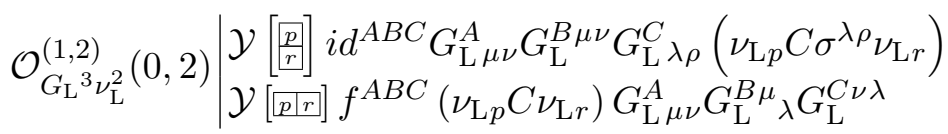

$$
\begin{aligned}
& \mathcal{O}_{F_{\mathrm{L}} G_{\mathrm{L}}{ }^{2} \bar{u}_{\mathrm{R}} u_{\mathrm{L}}}^{(1 \sim 6)}(0,0) \mid \begin{array}{l}
i F_{\mathrm{L} \mu \nu} G_{\mathrm{L} \lambda \rho}^{A} G_{\mathrm{L}}^{A \mu \nu}\left(\bar{u}_{\mathrm{R}}^{a} \sigma^{\lambda \rho} u_{\mathrm{L} r a}\right) \\
i d^{A B C}\left(\lambda^{C}\right)_{a}^{b} F_{\mathrm{L} \mu \nu} G_{\mathrm{L}}^{A \mu \nu} G_{\mathrm{L} \lambda \rho}^{B}\left(\bar{u}_{\mathrm{R} p}^{a} \sigma^{\lambda \rho} u_{\mathrm{L} r b}\right) \\
i f^{A B C}\left(\lambda^{C}\right)_{a}^{b} F_{\mathrm{L} \mu \nu} G_{\mathrm{L}}^{A \mu \nu} G_{\mathrm{L} \lambda \rho}^{B}\left(\bar{u}_{\mathrm{R}}{ }_{p}^{a} \sigma^{\lambda \rho} u_{\mathrm{L} r b}\right) \\
f^{A B C}\left(\lambda^{C}\right)_{a}^{b} F_{\mathrm{L} \mu \nu} G_{\mathrm{L}}^{A \mu}{ }_{\lambda} G_{\mathrm{L}}^{B \nu \lambda}\left(\bar{u}_{\mathrm{R} p}^{a} u_{\mathrm{L} r b}\right) \\
i F_{\mathrm{L} \mu \nu} G_{\mathrm{L}}^{A \mu}{ }_{\lambda} G_{\mathrm{L}}^{A \lambda}{ }_{\rho}\left(\bar{u}_{\mathrm{R} p}^{a} \sigma^{\nu \rho} u_{\mathrm{L} r a}\right) \\
i d^{A B C}\left(\lambda^{C}\right)_{a}^{b} F_{\mathrm{L} \mu \nu} G_{\mathrm{L}}^{A \mu}{ }_{\lambda} G_{\mathrm{L}}^{B \lambda}{ }_{\rho}\left(\bar{u}_{\mathrm{R} p}^{a} \sigma^{\nu \rho} u_{\mathrm{L} r b}\right)
\end{array} \\
& \mathcal{O}_{F_{\mathrm{L}} G_{\mathrm{L}}^{2} \bar{d}_{\mathrm{R}} d_{\mathrm{L}}}^{(1 \sim 6)}(0,0) \mid \begin{array}{l}
i F_{\mathrm{L} \mu \nu} G_{\mathrm{L} \lambda \rho}^{A} G_{\mathrm{L}}^{A \mu \nu}\left(\bar{d}_{\mathrm{R} p}^{a} \sigma^{\lambda \rho} d_{\mathrm{L} r a}\right) \\
i d^{A B C}\left(\lambda^{C}\right)_{a}^{b} F_{\mathrm{L} \mu \nu} G_{\mathrm{L}}^{A \mu \nu} G_{\mathrm{L} \lambda \rho}^{B}\left(\bar{d}_{\mathrm{R} p}^{a} \sigma^{\lambda \rho} d_{\mathrm{L} r b}\right) \\
i f^{A B C}\left(\lambda^{C}\right)_{a}^{b} F_{\mathrm{L} \mu \nu} G_{\mathrm{L}}^{A \mu \nu} G_{\mathrm{L} \lambda \rho}^{B}\left(\bar{d}_{\mathrm{R} p}^{a} \sigma^{\lambda \rho} d_{\mathrm{L} r b}\right) \\
f^{A B C}\left(\lambda^{C}\right)_{a}^{b} F_{\mathrm{L} \mu \nu} G_{\mathrm{L}}^{A \mu}{ }_{\lambda} G_{\mathrm{L}}^{B \nu \lambda}\left(\bar{d}_{\mathrm{R} p}{ }_{a}^{a} d_{\mathrm{L} r b}\right) \\
i F_{\mathrm{L} \mu \nu} G_{\mathrm{L}}^{A \mu}{ }_{\lambda} G_{\mathrm{L}}^{A \lambda}{ }_{\rho}\left(\bar{d}_{\mathrm{R} p}^{a} \sigma^{\nu \rho} d_{\mathrm{L} r a}\right) \\
i d^{A B C}\left(\lambda^{C}\right)_{a}^{b} F_{\mathrm{L} \mu \nu} G_{\mathrm{L}}^{A \mu}{ }_{\lambda} G_{\mathrm{L}}^{B \lambda}{ }_{\rho}\left(\bar{d}_{\mathrm{R} p}^{a} \sigma^{\nu \rho} d_{\mathrm{L} r b}\right)
\end{array} \\
& \mathcal{O}_{F_{\mathrm{L}} G_{\mathrm{L}}{ }^{2} \bar{e}_{\mathrm{R}} e_{\mathrm{L}}}^{(1,2,0)} \mid \begin{array}{l}
i F_{\mathrm{L} \mu \nu} G_{\mathrm{L} \lambda \rho}^{A} G_{\mathrm{L}}^{A \mu \nu}\left(\bar{e}_{\mathrm{R} p} \sigma^{\lambda \rho} e_{\mathrm{L} r}\right) \\
i F_{\mathrm{L} \mu \nu} G_{\mathrm{L}}^{A \mu}{ }_{\lambda} G_{\mathrm{L}}^{A \lambda} \rho\left(\bar{e}_{\mathrm{R} p} \sigma^{\nu \rho} e_{\mathrm{L} r}\right)
\end{array}
\end{aligned}
$$

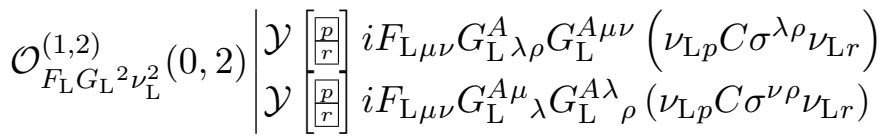

$$
\begin{aligned}
& \mathcal{O}_{F_{\mathrm{L}}{ }^{2} G_{\mathrm{L}} \bar{u}_{\mathrm{R}} u_{\mathrm{L}}}^{(1,2,0)} \mid \begin{array}{l}
i\left(\lambda^{A}\right)_{a}^{b} F_{\mathrm{L} \mu \nu} F_{\mathrm{L}}^{\mu \nu} G_{\mathrm{L} \lambda \rho}^{A}\left(\bar{u}_{\mathrm{R}}^{a} \sigma^{\lambda \rho} u_{\mathrm{L} r b}\right) \\
i\left(\lambda^{A}\right)_{a}^{b} F_{\mathrm{L} \lambda \rho} F_{\mathrm{L} \mu \nu} G_{\mathrm{L}}^{A \mu \nu}\left(\bar{u}_{\mathrm{R}}^{a} \sigma^{\lambda \rho} u_{\mathrm{L} r b}\right)
\end{array} \\
& \mathcal{O}_{F_{\mathrm{L}}{ }^{2} G_{\mathrm{L}} \bar{d}_{\mathrm{R}} d_{\mathrm{L}}}^{(1,2)}(0,0) \mid \begin{array}{l}
i\left(\lambda^{A}\right)_{a}^{b} F_{\mathrm{L} \mu \nu} F_{\mathrm{L}}^{\mu \nu} G_{\mathrm{L} \lambda \rho}^{A}\left(\bar{d}_{\mathrm{R} p}^{a} \sigma^{\lambda \rho} d_{\mathrm{L} r b}\right) \\
i\left(\lambda^{A}\right)_{a}^{b} F_{\mathrm{L} \lambda \rho} F_{\mathrm{L} \mu \nu} G_{\mathrm{L}}^{A \mu \nu}\left(\bar{d}_{\mathrm{R} p}^{a} \sigma^{\lambda \rho} d_{\mathrm{L} r b}\right)
\end{array} \\
& \mathcal{O}_{F_{\mathrm{L}}{ }^{3} \bar{u}_{\mathrm{R}} u_{\mathrm{L}}}(0,0) \mid i F_{\mathrm{L} \lambda \rho} F_{\mathrm{L} \mu \nu} F_{\mathrm{L}}^{\mu \nu}\left(\bar{u}_{\mathrm{R}}^{a} \sigma^{\lambda \rho} u_{\mathrm{L} r a}\right) \\
& \mathcal{O}_{F_{\mathrm{L}}{ }^{3} \bar{d}_{\mathrm{R}} d_{\mathrm{L}}}(0,0) \mid i F_{\mathrm{L} \lambda \rho} F_{\mathrm{L} \mu \nu} F_{\mathrm{L}}{ }^{\mu \nu}\left(\bar{d}_{\mathrm{R} p}^{a} \sigma^{\lambda \rho} d_{\mathrm{L} r a}\right) \\
& \mathcal{O}_{F_{\mathrm{L}}{ }^{3} \bar{e}_{\mathrm{R}} e_{\mathrm{L}}}(0,0) \mid i F_{\mathrm{L} \lambda \rho} F_{\mathrm{L} \mu \nu} F_{\mathrm{L}}{ }^{\mu \nu}\left(\bar{e}_{\mathrm{R} p} \sigma^{\lambda \rho} e_{\mathrm{L} r}\right)
\end{aligned}
$$




$$
\mathcal{O}_{F_{\mathrm{L}}{ }^{3} \nu_{\mathrm{L}}^{2}}(0,2) \mid \mathcal{Y}\left[\frac{p}{r}\right] i F_{\mathrm{L} \lambda \rho} F_{\mathrm{L} \mu \nu} F_{\mathrm{L}}{ }^{\mu \nu}\left(\nu_{\mathrm{L} p} C \sigma^{\lambda} \rho \nu_{\mathrm{L} r}\right)
$$

\section{Class $F_{\mathrm{L}}{ }^{3} \psi^{\dagger 2}: 6$ types}

$$
\begin{aligned}
& \mathcal{O}_{G_{\mathrm{L}}{ }^{3} u_{\mathrm{R}} \bar{u}_{\mathrm{L}}}^{(1,2)}(0,0) \mid \begin{array}{l}
d^{C D E} f^{A B D}\left(\lambda^{E}\right)_{b}^{a} G_{\mathrm{L} \mu \nu}^{A} G_{\mathrm{L}}^{B \mu}{ }_{\lambda} G_{\mathrm{L}}^{C \nu \lambda}\left(\bar{u}_{\mathrm{L} r}^{b} u_{\mathrm{R} p a}\right) \\
f^{A B C} G_{\mathrm{L} \mu \nu}^{A} G_{\mathrm{L}}^{B \mu}{ }_{\lambda} G_{\mathrm{L}}^{C \nu \lambda}\left(\bar{u}_{\mathrm{L} r}^{a} u_{\mathrm{R} p a}\right)
\end{array} \\
& \left.\mathcal{O}_{G_{\mathrm{L}}{ }^{3} d_{\mathrm{R}} \bar{d}_{\mathrm{L}}}^{(1,2)}(0,0) \mid \begin{array}{l}
d^{C D E} f^{A B D}\left(\lambda^{E}\right)_{b}^{a} G_{\mathrm{L} \mu \nu}^{A} G_{\mathrm{L}}^{B \mu}{ }_{\lambda} G_{\mathrm{L}}^{C \nu \lambda} \\
f^{A B C} G_{\mathrm{L} \mu \nu}^{A} G_{\mathrm{L}}^{B \mu}{ }_{\lambda} G_{\mathrm{L}}^{C \nu \lambda}\left(\bar{d}_{\mathrm{L} r}^{b}{ }_{\mathrm{L}}{ }^{a} d_{\mathrm{R} p a} d_{\mathrm{R} p a}\right)
\end{array}\right) \\
& \mathcal{O}_{G_{\mathrm{L}}{ }^{3} e_{\mathrm{R}} \bar{e}_{\mathrm{L}}}(0,0) \mid f^{A B C}\left(\bar{e}_{\mathrm{L} r} e_{\mathrm{R} p}\right) G_{\mathrm{L} \mu \nu}^{A} G_{\mathrm{L}}^{B \mu}{ }_{\lambda} G_{\mathrm{L}}^{C \nu \lambda} \\
& \mathcal{O}_{G_{\mathrm{L}}{ }^{3} \bar{\nu}_{\mathrm{L}}^{2}}(0,-2) \mid \mathcal{Y}\left[\underline{p p r]} f^{A B C}\left(\bar{\nu}_{\mathrm{L} p} C \bar{\nu}_{\mathrm{L} r}\right) G_{\mathrm{L} \mu \nu}^{A} G_{\mathrm{L}}^{B \mu}{ }_{\lambda} G_{\mathrm{L}}^{C \nu \lambda}\right. \\
& \mathcal{O}_{F_{\mathrm{L}} G_{\mathrm{L}}{ }^{2} u_{\mathrm{R}} \bar{u}_{\mathrm{L}}}(0,0) \mid f^{A B C}\left(\lambda^{C}\right)_{b}^{a} F_{\mathrm{L} \mu \nu} G_{\mathrm{L}}^{A \mu_{\lambda} G_{\mathrm{L}}^{B \nu \lambda}}\left(\bar{u}_{\mathrm{L}}^{b} u_{\mathrm{R} p a}\right) \\
& \mathcal{O}_{F_{\mathrm{L}} G_{\mathrm{L}}{ }^{2} d_{\mathrm{R}} \bar{d}_{\mathrm{L}}}(0,0) \mid f^{A B C}\left(\lambda^{C}\right)_{b}^{a} F_{\mathrm{L} \mu \nu} G_{\mathrm{L}}^{A \mu}{ }_{\lambda} G_{\mathrm{L}}^{B \nu \lambda}\left(\bar{d}_{\mathrm{L}}{ }_{r}^{b} d_{\mathrm{R} p a}\right)
\end{aligned}
$$

\section{Class $F_{\mathrm{L}} \boldsymbol{F}_{\mathrm{R}}^{2} \psi^{2}: 20$ types}

$$
\begin{aligned}
& \mid i\left(\lambda^{A}\right)_{a}^{b} G_{\mathrm{L} \mu \nu}^{A} G_{\mathrm{R} \lambda \rho}^{B} G_{\mathrm{R}}^{B \lambda \rho}\left(\bar{u}_{\mathrm{R} p}^{a} \sigma^{\mu \nu} u_{\mathrm{L} r b}\right) \\
& \mathcal{O}_{G_{\mathrm{L}} G_{\mathrm{R}}{ }^{2} \bar{u}_{\mathrm{R}} u_{\mathrm{L}}}^{(1 \sim 5,0)}\left(0, i\left(\lambda^{C}\right)_{a}^{b} G_{\mathrm{L} \mu \nu}^{A} G_{\mathrm{R} \lambda \rho}^{A} G_{\mathrm{R}}^{C \lambda \rho}\left(\bar{u}_{\mathrm{R} p}^{a} \sigma^{\mu \nu} u_{\mathrm{L} r b}\right)\right. \\
& i d^{A D E} d^{B C D}\left(\lambda^{E}\right)_{a}^{b} G_{\mathrm{L} \mu \nu}^{A} G_{\mathrm{R} \lambda \rho}^{B} G_{\mathrm{R}}^{C \lambda \rho}\left(\bar{u}_{\mathrm{R}}^{a} \sigma^{\mu \nu} u_{\mathrm{L} r b}\right) \\
& i d^{C D E} f^{A B D}\left(\lambda^{E}\right)_{a}^{b} G_{\mathrm{L} \mu \nu}^{A} G_{\mathrm{R} \lambda \rho}^{B} G_{\mathrm{R}}^{C \lambda \rho}\left(\bar{u}_{\mathrm{R}}^{a} \sigma^{\mu \nu} u_{\mathrm{L} r b}\right) \\
& i d^{A B C} G_{\mathrm{L} \mu \nu}^{A} G_{\mathrm{R} \lambda \rho}^{B} G_{\mathrm{R}}^{C \lambda \rho}\left(\bar{u}_{\mathrm{R} p}^{a} \sigma^{\mu \nu} u_{\mathrm{L} r a}\right) \\
& \mathcal{O}_{F_{\mathrm{R}} G_{\mathrm{L}} G_{\mathrm{R}} \bar{u}_{\mathrm{R}} u_{\mathrm{L}}}^{(1 \sim 0,0)}\left|\begin{array}{l}
i d^{A B C}\left(\lambda^{C}\right)_{a}^{b} F_{\mathrm{R} \lambda \rho} G_{\mathrm{L} \mu \nu}^{A} G_{\mathrm{R}}^{B \lambda \rho}\left(\bar{u}_{\mathrm{R} p}^{a} \sigma^{\mu \nu} u_{\mathrm{L} r b}\right) \\
i f^{A B C}\left(\lambda^{C}\right)_{a}^{b} F_{\mathrm{R} \lambda \rho} G_{\mathrm{L} \mu \nu}^{A} G_{\mathrm{R}}^{B \lambda \rho}\left(\bar{u}_{\mathrm{R}}^{a} \sigma^{\mu \nu} u_{\mathrm{L} r b}\right) \\
i F_{\mathrm{R} \lambda \rho} G_{\mathrm{L} \mu \nu}^{A} G_{\mathrm{R}}^{A \lambda \rho}\left(\bar{u}_{\mathrm{R}}^{a} \sigma^{\mu \nu} u_{\mathrm{L} r a}\right)
\end{array}\right| \\
& \mathcal{O}_{F_{\mathrm{R}}{ }^{2} G_{\mathrm{L}} \bar{u}_{\mathrm{R}} u_{\mathrm{L}}}(0,0) \mid i\left(\lambda^{A}\right)_{a}^{b} F_{\mathrm{R} \lambda \rho} F_{\mathrm{R}}^{\lambda \rho} G_{\mathrm{L} \mu \nu}^{A}\left(\bar{u}_{\mathrm{R}}^{a} \sigma^{\mu \nu} u_{\mathrm{L} r b}\right) \\
& \mathcal{O}_{G_{\mathrm{L}} G_{\mathrm{R}}{ }^{2} \bar{d}_{\mathrm{R}} d_{\mathrm{L}}}^{(1 \sim 5)}(0,0) \mid \begin{array}{l}
i\left(\lambda^{C}\right)_{a}^{b} G_{\mathrm{L} \mu \nu}^{A} G_{\mathrm{R} \lambda \rho}^{A} G_{\mathrm{R}}^{C \lambda \rho}\left(\bar{d}_{\mathrm{R} p}^{a} \sigma^{\mu \nu} d_{\mathrm{L} r b}\right) \\
i d^{A B D} d^{C D E}\left(\lambda^{E}\right)_{a}^{b} G_{\mathrm{L} \mu \nu}^{A} G_{\mathrm{R} \lambda \rho}^{B} G_{\mathrm{R}}^{C \lambda \rho}\left(\bar{d}_{\mathrm{R}}^{a} \sigma^{\mu \nu} d_{\mathrm{L} r b}\right) \\
i d^{C D E} f^{A B D}\left(\lambda^{E}\right)_{a}^{b} G_{\mathrm{L} \mu \nu}^{A} G_{\mathrm{R} \lambda \rho}^{B} G_{\mathrm{R}}^{C \lambda \rho}\left(\bar{d}_{\mathrm{R} p}^{a} \sigma^{\mu \nu} d_{\mathrm{L} r b}\right) \\
i f^{A B D} f^{C D E}\left(\lambda^{E}\right)_{a}^{b} G_{\mathrm{L} \mu \nu}^{A} G_{\mathrm{R} \lambda \rho}^{B} G_{\mathrm{R}}^{C \lambda \rho}\left(\bar{d}_{\mathrm{R} p}^{a} \sigma^{\mu \nu} d_{\mathrm{L} r b}\right) \\
i d^{A B C} G_{\mathrm{L} \mu \nu}^{A} G_{\mathrm{R} \lambda \rho}^{B} G_{\mathrm{R}}^{C \lambda \rho}\left(\bar{d}_{\mathrm{R} p}^{a} \sigma^{\mu \nu} d_{\mathrm{L} r a}\right)
\end{array}
\end{aligned}
$$




$$
\begin{aligned}
& \mathcal{O}_{F_{\mathrm{R}} G_{\mathrm{L}} G_{\mathrm{R}} \bar{d}_{\mathrm{R}} d_{\mathrm{L}}}^{(1 \sim 3)}(0,0) \mid \begin{array}{l}
i d^{A B C}\left(\lambda^{C}\right)_{a}^{b} F_{\mathrm{R} \lambda \rho} G_{\mathrm{L} \mu \nu}^{A} G_{\mathrm{R}}^{B \lambda \rho}\left(\bar{d}_{\mathrm{R} p}^{a} \sigma^{\mu \nu} d_{\mathrm{L} r b}\right) \\
i f^{A B C}\left(\lambda^{C}\right)_{a}^{b} F_{\mathrm{R} \lambda \rho} G_{\mathrm{L} \mu \nu}^{A} G_{\mathrm{R}}^{B \lambda \rho}\left(\bar{d}_{\mathrm{R}}^{a}{ }_{p}^{\mu \nu} d_{\mathrm{L} r b}\right) \\
i F_{\mathrm{R} \lambda \rho} G_{\mathrm{L} \mu \nu}^{A} G_{\mathrm{R}}^{A \lambda \rho}\left(\bar{d}_{\mathrm{R} p}^{a} \sigma^{\mu \nu} d_{\mathrm{L} r a}\right)
\end{array} \\
& \mathcal{O}_{F_{\mathrm{R}}{ }^{2} G_{\mathrm{L}} \bar{d}_{\mathrm{R}} d_{\mathrm{L}}}(0,0) \mid i\left(\lambda^{A}\right)_{a}^{b} F_{\mathrm{R} \lambda \rho} F_{\mathrm{R}}^{\lambda \rho} G_{\mathrm{L} \mu \nu}^{A}\left(\bar{d}_{\mathrm{R}}^{a} \sigma^{\mu \nu} d_{\mathrm{L} r b}\right) \\
& \mathcal{O}_{G_{\mathrm{L}} G_{\mathrm{R}}{ }^{2} \bar{e}_{\mathrm{R}} e_{\mathrm{L}}}(0,0) \mid i d^{A B C} G_{\mathrm{L} \mu \nu}^{A} G_{\mathrm{R} \lambda \rho}^{B} G_{\mathrm{R}}^{C \lambda \rho}\left(\bar{e}_{\mathrm{R} p} \sigma^{\mu \nu} e_{\mathrm{L} r}\right) \\
& \mathcal{O}_{F_{\mathrm{R}} G_{\mathrm{L}} G_{\mathrm{R}} \bar{e}_{\mathrm{R}} e_{\mathrm{L}}}(0,0) \mid i F_{\mathrm{R} \lambda \rho} G_{\mathrm{L} \mu \nu}^{A} G_{\mathrm{R}}^{A \lambda \rho}\left(\bar{e}_{\mathrm{R} p} \sigma^{\mu \nu} e_{\mathrm{L} r}\right) \\
& \mathcal{O}_{G_{\mathrm{L}} G_{\mathrm{R}}{ }^{2} \nu_{\mathrm{L}}^{2}}(0,2) \mid \mathcal{Y}\left[\frac{p}{r}\right] i d^{A B C} G_{\mathrm{L} \mu \nu}^{A} G_{\mathrm{R} \lambda \rho}^{B} G_{\mathrm{R}}^{C \lambda \rho}\left(\nu_{\mathrm{L} p} C \sigma^{\mu \nu} \nu_{\mathrm{L} r}\right) \\
& \mathcal{O}_{F_{\mathrm{R}} G_{\mathrm{L}} G_{\mathrm{R}} \nu_{\mathrm{L}}^{2}}(0,2) \mid \mathcal{Y}\left[\frac{p}{r}\right] i F_{\mathrm{R} \lambda \rho} G_{\mathrm{L} \mu \nu}^{A} G_{\mathrm{R}}^{A \lambda \rho}\left(\nu_{\mathrm{L} p} C \sigma^{\mu \nu} \nu_{\mathrm{L} r}\right) \\
& \mathcal{O}_{F_{\mathrm{L}} G_{\mathrm{R}}{ }^{2} \bar{u}_{\mathrm{R}} u_{\mathrm{L}}}^{(1,2)}(0,0) \mid \begin{array}{l}
i d^{A B C}\left(\lambda^{C}\right)_{a}^{b} F_{\mathrm{L} \mu \nu} G_{\mathrm{R} \lambda \rho}^{A} G_{\mathrm{R}}^{B \lambda \rho}\left(\bar{u}_{\mathrm{R} p}^{a} \sigma^{\mu \nu} u_{\mathrm{L} r b}\right) \\
i F_{\mathrm{L} \mu \nu} G_{\mathrm{R} \lambda \rho}^{A} G_{\mathrm{R}}^{A \lambda \rho}\left(\bar{u}_{\mathrm{R}}^{a} \sigma^{\mu \nu} u_{\mathrm{L} r a}\right)
\end{array}
\end{aligned}
$$

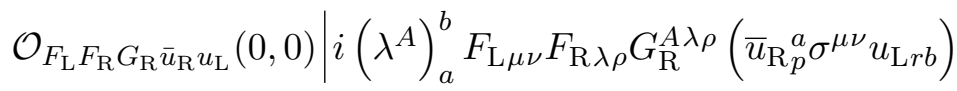

$$
\begin{aligned}
& \mathcal{O}_{F_{\mathrm{L}} F_{\mathrm{R}}{ }^{2} \bar{u}_{\mathrm{R}} u_{\mathrm{L}}}(0,0) \mid i F_{\mathrm{L} \mu \nu} F_{\mathrm{R} \lambda \rho} F_{\mathrm{R}}^{\lambda \rho}\left(\bar{u}_{\mathrm{R}}^{a} \sigma^{\mu \nu} u_{\mathrm{L} r a}\right) \\
& \mathcal{O}_{F_{\mathrm{L}} G_{\mathrm{R}}{ }^{2} \bar{d}_{\mathrm{R}} d_{\mathrm{L}}}^{(1,2,0)}\left(0 \begin{array}{l}
i d^{A B C}\left(\lambda^{C}\right)_{a}^{b} F_{\mathrm{L} \mu \nu} G_{\mathrm{R} \lambda \rho}^{A} G_{\mathrm{R}}^{B \lambda \rho}\left(\bar{d}_{\mathrm{R} p}^{a} \sigma^{\mu \nu} d_{\mathrm{L} r b}\right) \\
i F_{\mathrm{L} \mu \nu} G_{\mathrm{R} \lambda \rho}^{A} G_{\mathrm{R}}^{A \lambda \rho}\left(\bar{d}_{\mathrm{R} p}^{a} \sigma^{\mu \nu} d_{\mathrm{L} r a}\right)
\end{array}\right. \\
& \mathcal{O}_{F_{\mathrm{L}} F_{\mathrm{R}} G_{\mathrm{R}} \bar{d}_{\mathrm{R}} d_{\mathrm{L}}}(0,0) \mid i\left(\lambda^{A}\right)_{a}^{b} F_{\mathrm{L} \mu \nu} F_{\mathrm{R} \lambda \rho} G_{\mathrm{R}}^{A \lambda \rho}\left(\bar{d}_{\mathrm{R}}^{a} \sigma^{\mu \nu} d_{\mathrm{L} r b}\right) \\
& \mathcal{O}_{F_{\mathrm{L}} F_{\mathrm{R}}^{2} \bar{d}_{\mathrm{R}} d_{\mathrm{L}}}(0,0) \mid i F_{\mathrm{L} \mu \nu} F_{\mathrm{R} \lambda \rho} F_{\mathrm{R}}^{\lambda \rho}\left(\bar{d}_{\mathrm{R}}^{a} \sigma^{\mu \nu} d_{\mathrm{L} r a}\right) \\
& \mathcal{O}_{F_{\mathrm{L}} G_{\mathrm{R}}{ }^{2} \bar{e}_{\mathrm{R}} e_{\mathrm{L}}}(0,0) \mid i F_{\mathrm{L} \mu \nu} G_{\mathrm{R} \lambda \rho}^{A} G_{\mathrm{R}}^{A \lambda \rho}\left(\bar{e}_{\mathrm{R} p} \sigma^{\mu \nu} e_{\mathrm{L} r}\right) \\
& \mathcal{O}_{F_{\mathrm{L}} F_{\mathrm{R}}{ }^{2} \bar{e}_{\mathrm{R}} e_{\mathrm{L}}}(0,0) \mid i F_{\mathrm{L} \mu \nu} F_{\mathrm{R} \lambda \rho} F_{\mathrm{R}}^{\lambda \rho}\left(\bar{e}_{\mathrm{R} p} \sigma^{\mu \nu} e_{\mathrm{L} r}\right) \\
& \mathcal{O}_{F_{\mathrm{L}} G_{\mathrm{R}}{ }^{2} \nu_{\mathrm{L}}^{2}}(0,2) \mid \mathcal{Y}\left[\frac{p}{r}\right] i F_{\mathrm{L} \mu \nu} G_{\mathrm{R} \lambda \rho}^{A} G_{\mathrm{R}}^{A \lambda \rho}\left(\nu_{\mathrm{L} p} C \sigma^{\mu \nu} \nu_{\mathrm{L} r}\right) \\
& \mathcal{O}_{F_{\mathrm{L}} F_{\mathrm{R}}{ }^{2} \nu_{\mathrm{L}}^{2}}(0,2) \mid \mathcal{Y}\left[\frac{p}{r}\right] i F_{\mathrm{L} \mu \nu} F_{\mathrm{R} \lambda \rho} F_{\mathrm{R}}^{\lambda \rho}\left(\nu_{\mathrm{L} p} C \sigma^{\mu \nu} \nu_{\mathrm{L} r}\right)
\end{aligned}
$$




\subsubsection{Classes involving four-fermions}

\section{Class $\psi^{3} \psi^{\dagger} D^{3}: 52$ types}

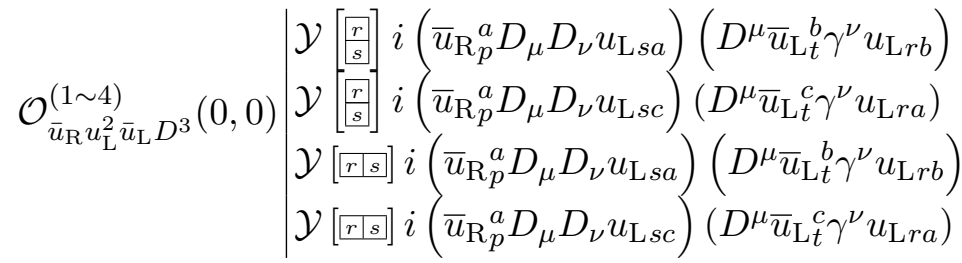

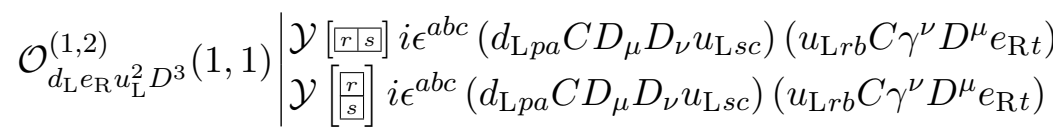

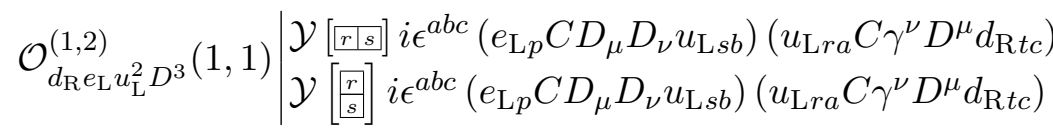

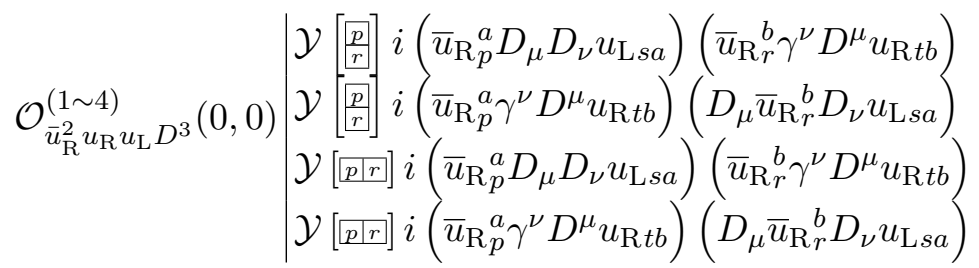

$$
\begin{aligned}
& \mathcal{O}_{d_{\mathrm{L}} \bar{d}_{\mathrm{L}} \bar{u}_{\mathrm{R}} u_{\mathrm{L}} D^{3}(0,0)} \mid \begin{array}{l}
i\left(d_{\mathrm{L} p a} C D_{\mu} D_{\nu} u_{\mathrm{L} s b}\right)\left(\bar{u}_{\mathrm{R} r}^{b} \gamma^{\nu} C D^{\mu} \bar{d}_{\mathrm{L} t}^{a}\right) \\
i\left(d_{\mathrm{L} p a} C D_{\mu} D_{\nu} u_{\mathrm{L} s c}\right)\left(\bar{u}_{\mathrm{R} r}^{a} \gamma^{\nu} C D^{\mu} \bar{d}_{\mathrm{L} t}^{c}\right) \\
i\left(D^{\mu} \bar{d}_{\mathrm{L} t}^{a} \gamma^{\nu} d_{\mathrm{L} p a}\right)\left(D_{\mu} \bar{u}_{\mathrm{R} r}^{b} D_{\nu} u_{\mathrm{L} s b}\right) \\
i\left(D^{\mu} \bar{d}_{\mathrm{L} t}^{c} \gamma^{\nu} d_{\mathrm{L} p a}\right)\left(D_{\mu} \bar{u}_{\mathrm{R} r}^{a} D_{\nu} u_{\mathrm{L} s c}\right)
\end{array} \\
& \left.\mathcal{O}_{\bar{d}_{\mathrm{R}} d_{\mathrm{R}} \bar{u}_{\mathrm{R}} u_{\mathrm{L}} D^{3}(0,0)}^{(1 \sim 4)} \begin{array}{l}
i\left(\bar{d}_{\mathrm{R}}^{a} D_{\mu} D_{\nu} u_{\mathrm{L} s a}\right)\left(\bar{u}_{\mathrm{R} r}^{b} \gamma^{\nu} D^{\mu} d_{\mathrm{R} t b}\right) \\
i\left(\bar{d}_{\mathrm{R} p}^{a} D_{\mu} D_{\nu} u_{\mathrm{L} s b}\right)\left(\bar{u}_{\mathrm{R}}^{b} \gamma^{\nu} D^{\mu} d_{\mathrm{R} t a}\right. \\
i\left(\bar{d}_{\mathrm{R} p}^{a} \gamma^{\nu} D^{\mu} d_{\mathrm{R} t b}\right)\left(D_{\mu} \bar{u}_{\mathrm{R} r}^{b} D_{\nu} u_{\mathrm{L} s a}\right. \\
i\left(\bar{d}_{\mathrm{R} p}^{a} \gamma^{\nu} D^{\mu} d_{\mathrm{R} t a}\right)\left(D_{\mu} \bar{u}_{\mathrm{R} r}^{b} D_{\nu} u_{\mathrm{L} s b}\right.
\end{array}\right) \\
& \mathcal{O}_{e_{\mathrm{L}} \bar{e}_{\mathrm{L}} \bar{u}_{\mathrm{R}} u_{\mathrm{L}} D^{3}(0,0)}^{(1,2)} \begin{array}{l}
i\left(e_{\mathrm{L} p} C D_{\mu} D_{\nu} u_{\mathrm{L} s a}\right)\left(\bar{u}_{\mathrm{R}}^{a} \gamma^{\nu} C D^{\mu} \bar{e}_{\mathrm{L} t}\right) \\
i\left(D^{\mu} \bar{e}_{\mathrm{L} t} \gamma^{\nu} e_{\mathrm{L} p}\right)\left(D_{\mu} \bar{u}_{\mathrm{R}}^{a} D_{\nu} u_{\mathrm{L} s a}\right)
\end{array} \\
& \mathcal{O}_{\bar{e}_{\mathrm{R}} e_{\mathrm{R}} \bar{u}_{\mathrm{R}} u_{\mathrm{L}} D^{3}}^{(0,0)} \mid \begin{array}{l}
i\left(\bar{e}_{\mathrm{R} p} D_{\mu} D_{\nu} u_{\mathrm{L} s a}\right)\left(\bar{u}_{\mathrm{R}}^{a} \gamma^{\nu} D^{\mu} e_{\mathrm{R} t}\right) \\
i\left(\bar{e}_{\mathrm{R} p} \gamma^{\nu} D^{\mu} e_{\mathrm{R} t}\right)\left(D_{\mu} \bar{u}_{\mathrm{R}}^{a} D_{\nu} u_{\mathrm{L} s a}\right)
\end{array} \\
& \mathcal{O}_{\nu_{\mathrm{L}} \bar{\nu}_{\mathrm{L}} \bar{u}_{\mathrm{R}} u_{\mathrm{L}} D^{3}}^{(1,2,0)} \mid \begin{array}{l}
i\left(\bar{u}_{\mathrm{R} p}^{a} D_{\mu} D_{\nu} \nu_{\mathrm{L} s}\right)\left(D^{\mu} \bar{\nu}_{\mathrm{L} t} \gamma^{\nu} u_{\mathrm{L} r a}\right) \\
i\left(D_{\mu} u_{\mathrm{L} r a} C D_{\nu} \nu_{\mathrm{L} s}\right)\left(\bar{u}_{\mathrm{R} p}^{a} \gamma^{\nu} C D^{\mu} \bar{\nu}_{\mathrm{L} t}\right)
\end{array}
\end{aligned}
$$




$$
\begin{aligned}
& \mathcal{O}_{d_{\mathrm{L}}^{2} \bar{\nu}_{\mathrm{L}} u_{\mathrm{L}} D^{3}}^{(1,-1)} \mid \begin{array}{l}
\mathcal{Y}[\overline{[p r}] i \epsilon^{a b c}\left(d_{\mathrm{L} p a} C D_{\mu} D_{\nu} u_{\mathrm{L} s c}\right)\left(D^{\mu} \bar{\nu}_{\mathrm{L} t} \gamma^{\nu} d_{\mathrm{L} r b}\right) \\
\mathcal{Y}\left[\begin{array}{l}
p \\
r
\end{array}\right] i \epsilon^{a b c}\left(D_{\mu} d_{\mathrm{L} r b} C D_{\nu} u_{\mathrm{L} s c}\right)\left(D^{\mu} \bar{\nu}_{\mathrm{L} t} \gamma^{\nu} d_{\mathrm{L} p a}\right)
\end{array} \\
& \left.\mathcal{O}_{\bar{d}_{\mathrm{R}} d_{\mathrm{L}} u_{\mathrm{L}} \bar{u}_{\mathrm{L}} D^{3}}^{(1 \sim 4)}(0) \mid \begin{array}{l}
i\left(\bar{d}_{\mathrm{R} p}^{a} D_{\mu} D_{\nu} u_{\mathrm{L} s a}\right)\left(D^{\mu} \bar{u}_{\mathrm{L} t}^{b} \gamma^{\nu} d_{\mathrm{L} r b}\right) \\
i\left(\bar{d}_{\mathrm{R}}^{a} D_{\mu} D_{\nu} u_{\mathrm{L} s c}\right)\left(D^{\mu} \bar{u}_{\mathrm{L} t}^{c} \gamma^{\nu} d_{\mathrm{L} r a}\right) \\
i\left(D_{\mu} d_{\mathrm{L} r b} C D_{\nu} u_{\mathrm{L} s a}\right)\left(\bar{d}_{\left.\mathrm{R}_{p}^{a} \gamma^{\nu} C D^{\mu} \bar{u}_{\mathrm{L} t}^{b}\right)}\right. \\
i\left(D_{\mu} d_{\mathrm{L} r a} C D_{\nu} u_{\mathrm{L} s c}\right)\left(\bar{d}_{\mathrm{R}_{p}^{a}}^{a} \gamma^{\nu} C D^{\mu} \bar{u}_{\mathrm{L} t}^{c}\right.
\end{array}\right) \\
& \mathcal{O}_{d_{\mathrm{L}} e_{\mathrm{L}} u_{\mathrm{R}} u_{\mathrm{L}} D^{3}}^{(1,1)} \mid \begin{array}{l}
i \epsilon^{a b c}\left(d_{\mathrm{L} p a} C D_{\mu} D_{\nu} u_{\mathrm{L} s b}\right)\left(e_{\mathrm{L} r} C \gamma^{\nu} D^{\mu} u_{\mathrm{R} t c}\right) \\
i \epsilon^{a b c}\left(D_{\mu} e_{\mathrm{L} r} C D_{\nu} u_{\mathrm{L} s b}\right)\left(d_{\mathrm{L} p a} C \gamma^{\nu} D^{\mu} u_{\mathrm{R} t c}\right)
\end{array} \\
& \mathcal{O}_{d_{\mathrm{R}} d_{\mathrm{L}} \nu_{\mathrm{L}} u_{\mathrm{L}} D^{3}}^{(1,1)} \mid \begin{array}{l}
i \epsilon^{a b c}\left(d_{\mathrm{L} p a} C D_{\mu} D_{\nu} \nu_{\mathrm{L} s}\right)\left(u_{\mathrm{L} r b} C \gamma^{\nu} D^{\mu} d_{\mathrm{R} t c}\right) \\
i \epsilon^{a b c}\left(D_{\mu} u_{\mathrm{L} r b} C D_{\nu} \nu_{\mathrm{L} s}\right)\left(d_{\mathrm{L} p a} C \gamma^{\nu} D^{\mu} d_{\mathrm{R} t c}\right)
\end{array} \\
& \mathcal{O}_{\bar{d}_{\mathrm{R}} e_{\mathrm{L}} \bar{\nu}_{\mathrm{L}} u_{\mathrm{L}} D^{3}}^{(1,2)}(0,0) \mid \begin{array}{l}
i\left(\bar{d}_{\mathrm{R} p}^{a} D_{\mu} D_{\nu} u_{\mathrm{L} s a}\right)\left(D^{\mu} \bar{\nu}_{\mathrm{L} t} \gamma^{\nu} e_{\mathrm{L} r}\right) \\
i\left(D_{\mu} e_{\mathrm{L} r} C D_{\nu} u_{\mathrm{L} s a}\right)\left(\bar{d}_{\mathrm{R} p}^{a} \gamma^{\nu} C D^{\mu} \bar{\nu}_{\mathrm{L} t}\right)
\end{array} \\
& \mathcal{O}_{\bar{d}_{\mathrm{R}} e_{\mathrm{R}} \nu_{\mathrm{L}} u_{\mathrm{L}} D^{3}}^{(1,2,2)} \mid \begin{array}{l}
i\left(\bar{d}_{\mathrm{R}}^{a} D_{\mu} D_{\nu} \nu_{\mathrm{L} s}\right)\left(u_{\mathrm{L} r a} C \gamma^{\nu} D^{\mu} e_{\mathrm{R} t}\right) \\
i\left(D_{\mu} u_{\mathrm{L} r a} C D_{\nu} \nu_{\mathrm{L} s}\right)\left(\bar{d}_{\mathrm{R} p}^{a} \gamma^{\nu} D^{\mu} e_{\mathrm{R} t}\right)
\end{array}
\end{aligned}
$$

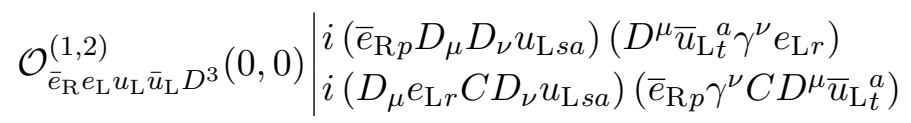

$$
\begin{aligned}
& \left.\mathcal{O}_{\bar{d}_{\mathrm{L}} e_{\mathrm{L}} \nu_{\mathrm{L}} u_{\mathrm{L}} D^{3}}^{(1,2)}(0,2) \mid \begin{array}{l}
i\left(e_{\mathrm{L} p} C D_{\mu} D_{\nu} \nu_{\mathrm{L} s}\right)\left(D^{\mu} \bar{d}_{\mathrm{L} t}^{a} \gamma^{\nu} u_{\mathrm{L} r a}\right) \\
i\left(D_{\mu} u_{\mathrm{L} r a} C D_{\nu} \nu_{\mathrm{L} s}\right)\left(D^{\mu} \bar{d}_{\mathrm{L}}^{a} \gamma^{\nu} e_{\mathrm{L} p}\right.
\end{array}\right)
\end{aligned}
$$

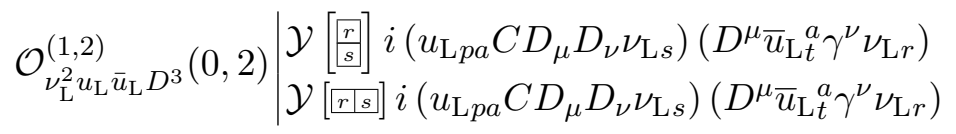

$$
\begin{aligned}
& \mathcal{O}_{\bar{d}_{\mathrm{R}} \bar{e}_{\mathrm{e}} \bar{u}_{\mathrm{R}}^{2} D^{3}(-1,-1)} \mid \begin{array}{l}
\mathcal{Y}\left[\underline{[r s]} i \epsilon_{a b c}\left(\bar{d}_{\mathrm{R}}^{a} C D_{\mu} D_{\nu} \bar{u}_{\mathrm{R}}^{c}\right)\left(\bar{u}_{\mathrm{R}}^{b} \gamma^{\nu} C D^{\mu} \bar{e}_{\mathrm{L} t}\right)\right. \\
\mathcal{Y}\left[\begin{array}{r}
{[s]} \\
s
\end{array}\right] \epsilon_{a b c}\left(\bar{d}_{\mathrm{R} p}^{a} C D_{\mu} D_{\nu} \bar{u}_{\mathrm{R} s}^{c}\right)\left(\bar{u}_{\mathrm{R}}^{b} \gamma^{\nu} C D^{\mu} \bar{e}_{\mathrm{L} t}\right)
\end{array} \\
& \mathcal{O}_{\bar{d}_{\mathrm{L}} \bar{e}_{\mathrm{R}} \bar{u}_{\mathrm{R}}^{2} D^{3}}^{(-1,-1)} \mid \begin{array}{l}
\mathcal{Y}[\overline{r s s}] i \epsilon_{a b c}\left(\bar{e}_{\mathrm{R} p} C D_{\mu} D_{\nu} \bar{u}_{\mathrm{R}}^{b}\right)\left(\bar{u}_{\mathrm{R}}^{a} \gamma^{\nu} C D^{\mu} \bar{d}_{\mathrm{L} t}^{c}\right) \\
\mathcal{Y}\left[\begin{array}{l}
\left.\frac{r}{s}\right] i \epsilon_{a b c}\left(\bar{e}_{\mathrm{R} p} C D_{\mu} D_{\nu} \bar{u}_{\mathrm{R}}^{b}\right)\left(\bar{u}_{\mathrm{R} r}^{a} \gamma^{\nu} C D^{\mu} \bar{d}_{\mathrm{L}}^{c}\right)
\end{array}\right.
\end{array} \\
& \mathcal{O}_{\bar{d}_{\mathrm{R}} d_{\mathrm{L}} \bar{u}_{\mathrm{R}} u_{\mathrm{R}} D^{3}}(0,0) \mid \begin{array}{l}
i\left(\bar{d}_{\mathrm{R} p}^{a} C D_{\mu} D_{\nu} \bar{u}_{\mathrm{R}}^{b}\right)\left(d_{\mathrm{L} r b} C \gamma^{\nu} D^{\mu} u_{\mathrm{R} t a}\right) \\
i\left(\bar{d}_{\mathrm{R} p}^{a} C D_{\mu} D_{\nu} \bar{u}_{\mathrm{R}}^{c}\right)\left(d_{\mathrm{L} r a} C \gamma^{\nu} D^{\mu} u_{\mathrm{R} t c}\right) \\
i\left(D_{\nu} \bar{u}_{\mathrm{R}}^{b} D_{\mu} d_{\mathrm{L} r b}\right)\left(\bar{d}_{\mathrm{R} p}^{a} \gamma^{\nu} D^{\mu} u_{\mathrm{R} t a}\right) \\
i\left(D_{\nu} \bar{u}_{\mathrm{R}}^{c} D_{\mu} D_{\mathrm{L} r a}\right)\left(\bar{d}_{\mathrm{R}}^{a} \gamma^{\nu} D^{\mu} u_{\mathrm{R} t c}\right)
\end{array}
\end{aligned}
$$




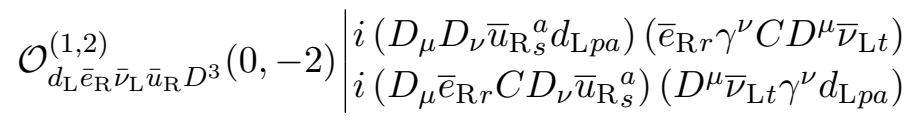

$$
\begin{aligned}
& \mathcal{O}_{d_{\mathrm{L}} \bar{e}_{\mathrm{L}} \nu_{\mathrm{L}} \bar{u}_{\mathrm{R}} D^{3}}^{(1,0,0)} \mid \begin{array}{l}
i\left(d_{\mathrm{L} p a} C D_{\mu} D_{\nu} \nu_{\mathrm{L} s}\right)\left(\bar{u}_{\mathrm{R}}{ }_{r}^{a} \gamma^{\nu} C D^{\mu} \bar{e}_{\mathrm{L} t}\right) \\
i\left(D_{\mu} \bar{u}_{\mathrm{R}}{ }_{r}^{a} D_{\nu} \nu_{\mathrm{L} s}\right)\left(D^{\mu} \bar{e}_{\mathrm{L} t} \gamma^{\nu} d_{\mathrm{L} p a}\right)
\end{array} \\
& \mathcal{O}_{\bar{d}_{\mathrm{R}}^{2} \bar{\nu}_{\mathrm{L}} \bar{u}_{\mathrm{R}} D^{3}}^{(1,-1,-1)} \mid \begin{array}{l}
\mathcal{Y}[\overline{p r r}] i \epsilon_{a b c}\left(\bar{d}_{\mathrm{R}}^{a} C D_{\mu} D_{\nu} \bar{u}_{\mathrm{R}}^{c}\right)\left(\bar{d}_{\mathrm{R}}^{b} \gamma^{\nu} C D^{\mu} \bar{\nu}_{\mathrm{L} t}\right) \\
\mathcal{Y}\left[\begin{array}{l}
{[p]} \\
r
\end{array}\right] i \epsilon_{a b c}\left(\bar{d}_{\mathrm{R}}^{a} \gamma^{\nu} C D^{\mu} \bar{\nu}_{\mathrm{L} t}\right)\left(D_{\mu} \bar{d}_{\mathrm{R} r}^{b} C D_{\nu} \bar{u}_{\mathrm{R}}^{c}\right)
\end{array}
\end{aligned}
$$

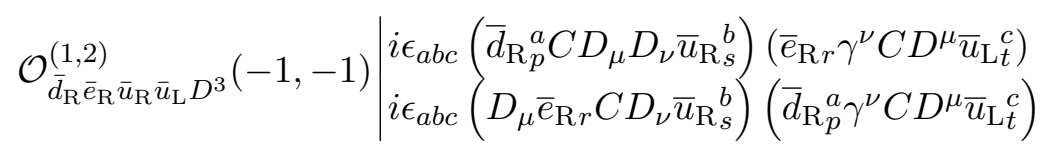

$$
\begin{aligned}
& \left.\mathcal{O}_{\bar{d}_{\mathrm{R}} \bar{d}_{\mathrm{L}} \nu_{\mathrm{L}} \bar{u}_{\mathrm{R}} D^{3}}^{(1,-1,1)} \mid \begin{array}{l}
i \epsilon_{a b c}\left(\bar{d}_{\mathrm{R}}^{a} D_{\mu} D_{\nu} \nu_{\mathrm{L} s}\right) \\
i \epsilon_{a b c}\left(\bar{D}_{\mu} \bar{u}_{\mathrm{R} r}^{b} D_{\nu} \nu_{\mathrm{L} s}\right.
\end{array}\right)\left(\begin{array}{l}
\bar{u}_{\mathrm{R}}^{b} \gamma^{\nu} C D^{\mu} \bar{d}_{\mathrm{L}}^{c} \\
\bar{d}_{\mathrm{R}}^{a} \gamma^{\nu} C D^{\mu} \bar{d}_{\mathrm{L} t}^{c}
\end{array}\right) \\
& \mathcal{O}_{\bar{e}_{\mathrm{R}} e_{\mathrm{L}} \bar{u}_{\mathrm{R}} u_{\mathrm{R}} D^{3}}^{(1,2,0)} \mid \begin{array}{l}
i\left(\bar{e}_{\mathrm{R} p} C D_{\mu} D_{\nu} \bar{u}_{\mathrm{R} s}^{a}\right)\left(e_{\mathrm{L} r} C \gamma^{\nu} D^{\mu} u_{\mathrm{R} t a}\right) \\
i\left(D_{\nu} \bar{u}_{\mathrm{R} s}^{a} D_{\mu} e_{\mathrm{L} r}\right)\left(\bar{e}_{\mathrm{R} p} \gamma^{\nu} D^{\mu} u_{\mathrm{R} t a}\right)
\end{array} \\
& \mathcal{O}_{d_{\mathrm{R}} \bar{e}_{\mathrm{R}} \nu_{\mathrm{L}} \bar{u}_{\mathrm{R}} D^{3}}^{(10,0)} \mid \begin{array}{l}
i\left(\bar{e}_{\mathrm{R} p} D_{\mu} D_{\nu} \nu_{\mathrm{L} s}\right)\left(\bar{u}_{\mathrm{R} r}^{a} \gamma^{\nu} D^{\mu} d_{\mathrm{R} t a}\right) \\
i\left(D_{\mu} \bar{u}_{\mathrm{R}}^{a} D_{\nu} \nu_{\mathrm{L} s}\right)\left(\bar{e}_{\mathrm{R} p} \gamma^{\nu} D^{\mu} d_{\mathrm{R} t a}\right)
\end{array} \\
& \mathcal{O}_{\nu_{\mathrm{L}}^{2} \bar{u}_{\mathrm{R}} u_{\mathrm{R}} D^{3}}^{(1,2,2)} \mid \begin{array}{l}
\mathcal{Y}\left[\begin{array}{l}
{[\underline{r}]} \\
\mathcal{s}
\end{array}\right]\left(\bar{u}_{\mathrm{R}}^{a} D_{\mu} D_{\nu} \nu_{\mathrm{L} s}\right)\left(\nu_{\mathrm{L} r} C \gamma^{\nu} D^{\mu} u_{\mathrm{R} t a}\right) \\
\mathcal{Y}[\underline{r r s}] i\left(\bar{u}_{\mathrm{R}}^{a} D_{\mu} D_{\nu} \nu_{\mathrm{L} s}\right)\left(\nu_{\mathrm{L} r} C \gamma^{\nu} D^{\mu} u_{\mathrm{R} t a}\right)
\end{array} \\
& \mathcal{O}_{d_{\mathrm{L}}^{3} \bar{e}_{\mathrm{L}} D^{3}}(1,-1) \mid \mathcal{Y}\left[\frac{p p r}{s s}\right] i \epsilon^{a b c}\left(d_{\mathrm{L} p a} C D_{\mu} D_{\nu} d_{\mathrm{L} s c}\right)\left(D^{\mu} \bar{e}_{\mathrm{L} t} \gamma^{\nu} d_{\mathrm{L} r b}\right)
\end{aligned}
$$

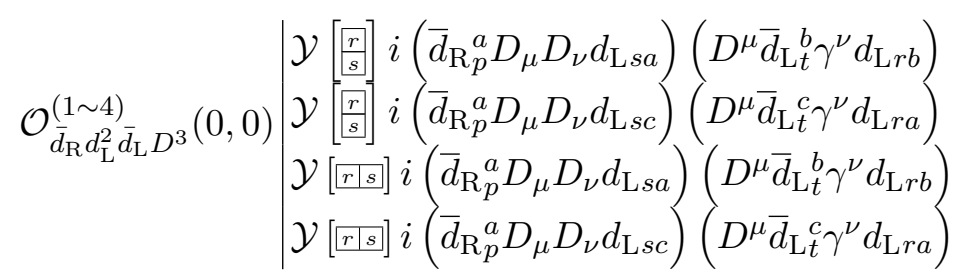

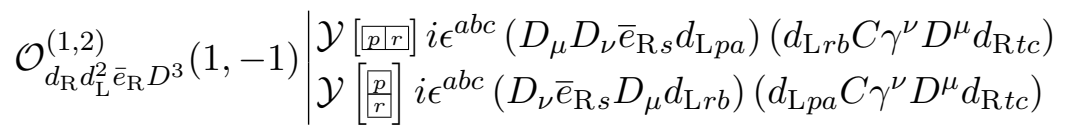

$$
\begin{aligned}
& \mathcal{O}_{d_{\mathrm{L}}^{2} \nu_{\mathrm{L}} u_{\mathrm{R}} D^{3}}^{(1,2)}(1,1) \mid \begin{array}{l}
\mathcal{Y}\left[\underline{[p r]} i \epsilon^{a b c}\left(d_{\mathrm{L} p a} C D_{\mu} D_{\nu} \nu_{\mathrm{L} s}\right)\left(d_{\mathrm{L} r b} C \gamma^{\nu} D^{\mu} u_{\mathrm{R} t c}\right)\right. \\
\mathcal{Y}\left[\begin{array}{l}
{[p]} \\
{[r]}
\end{array}\right] \epsilon^{a b c}\left(D_{\mu} d_{\mathrm{L} r b} C D_{\nu} \nu_{\mathrm{L} s}\right)\left(d_{\mathrm{L} p a} C \gamma^{\nu} D^{\mu} u_{\mathrm{R} t c}\right)
\end{array}
\end{aligned}
$$




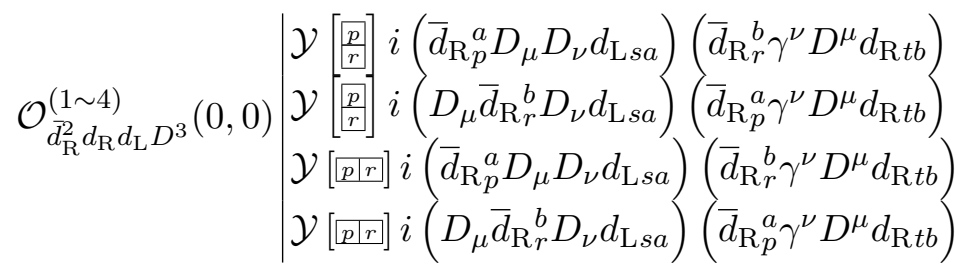

$$
\begin{aligned}
& \mathcal{O}_{\bar{d}_{\mathrm{R}} d_{\mathrm{L}} e_{\mathrm{L}} \bar{e}_{\mathrm{L}} D^{3}}^{(1,2,0)} \mid \begin{array}{l}
i\left(\bar{d}_{\mathrm{R}}^{a} D_{\mu} D_{\nu} e_{\mathrm{L} s}\right)\left(D^{\mu} \bar{e}_{\mathrm{L} t} \gamma^{\nu} d_{\mathrm{L} r a}\right) \\
i\left(D_{\mu} d_{\mathrm{L} r a} C D_{\nu} e_{\mathrm{L} s}\right)\left(\bar{d}_{\mathrm{R}}^{a} \gamma^{\nu} C D^{\mu} \bar{e}_{\mathrm{L} t}\right)
\end{array} \\
& \mathcal{O}_{\bar{d}_{\mathrm{R}} d_{\mathrm{L}} \bar{e}_{\mathrm{R}} e_{\mathrm{R}} D^{3}}^{(1,2,0)} \mid \begin{array}{l}
i\left(\bar{d}_{\mathrm{R}}^{a} C D_{\mu} D_{\nu} \bar{e}_{\mathrm{R} s}\right)\left(d_{\mathrm{L} r a} C \gamma^{\nu} D^{\mu} e_{\mathrm{R} t}\right) \\
i\left(D_{\nu} \bar{e}_{\mathrm{R} s} D_{\mu} d_{\mathrm{L} r a}\right)\left(\bar{d}_{\mathrm{R} p}^{a} \gamma^{\nu} D^{\mu} e_{\mathrm{R} t}\right)
\end{array} \\
& \mathcal{O}_{\bar{d}_{\mathrm{R}} d_{\mathrm{L}} \nu_{\mathrm{L}} \bar{\nu}_{\mathrm{L}} D^{3}}^{(1,2,0)} \mid \begin{array}{l}
i\left(\bar{d}_{\mathrm{R} p}^{a} D_{\mu} D_{\nu} \nu_{\mathrm{L} s}\right)\left(D^{\mu} \bar{\nu}_{\mathrm{L} t} \gamma^{\nu} d_{\mathrm{L} r a}\right) \\
i\left(D_{\mu} d_{\mathrm{L} r a} C D_{\nu} \nu_{\mathrm{L} s}\right)\left(\bar{d}_{\mathrm{R} p}^{a} \gamma^{\nu} C D^{\mu} \bar{\nu}_{\mathrm{L} t}\right)
\end{array} \\
& \mathcal{O}_{d_{\mathrm{L}} \overline{\mathrm{d}}_{\mathrm{L}} \bar{e}_{\mathrm{R}} e_{\mathrm{L}} D^{3}}^{(1,0,0)} \mid \begin{array}{l}
i\left(d_{\mathrm{L} p a} C D_{\mu} D_{\nu} e_{\mathrm{L} s}\right)\left(\bar{e}_{\mathrm{R} r} \gamma^{\nu} C D^{\mu} \bar{d}_{\mathrm{L} t}^{a}\right) \\
i\left(D_{\mu} \bar{e}_{\mathrm{R} r} D_{\nu} e_{\mathrm{L} s}\right)\left(D^{\mu} \bar{d}_{\mathrm{L} t}^{a} \gamma^{\nu} d_{\mathrm{L} p a}\right)
\end{array} \\
& \mathcal{O}_{d_{\mathrm{L}} \bar{e}_{\mathrm{R}} \nu_{\mathrm{L}} \bar{u}_{\mathrm{L}} D^{3}}^{(1,0,0)} \mid \begin{array}{l}
i\left(d_{\mathrm{L} p a} C D_{\mu} D_{\nu} \nu_{\mathrm{L} s}\right)\left(\bar{e}_{\mathrm{R} r} \gamma^{\nu} C D^{\mu} \bar{u}_{\mathrm{L} t}^{a}\right) \\
i\left(D_{\mu} \bar{e}_{\mathrm{R} r} D_{\nu} \nu_{\mathrm{L} s}\right)\left(D^{\mu} \bar{u}_{\mathrm{L}}^{a} \gamma^{\nu} d_{\mathrm{L} p a}\right)
\end{array} \\
& \mathcal{O}_{d_{\mathrm{L}} \bar{d}_{\mathrm{L}} \nu_{\mathrm{L}}^{2} D^{3}}^{(1,2)} \mid \begin{array}{l}
\mathcal{Y}\left[\begin{array}{l}
[r] s] \\
{[s}
\end{array}\right]\left(d_{\mathrm{L} p a} C D_{\mu} D_{\nu} \nu_{\mathrm{L} s}\right)\left(D^{\mu} \bar{d}_{\mathrm{L} t}^{a} \gamma^{\nu} \nu_{\mathrm{L} r}\right) \\
\mathcal{Y}[\underline{r r s}] i\left(d_{\mathrm{L} p a} C D_{\mu} D_{\nu} \nu_{\mathrm{L} s}\right)\left(D^{\mu} \bar{d}_{\mathrm{L} t}^{a} \gamma^{\nu} \nu_{\mathrm{L} r}\right)
\end{array} \\
& \mathcal{O}_{\bar{d}_{\mathrm{R}}^{3} e_{\mathrm{R}} D^{3}}(-1,1) \mid \mathcal{Y}\left[\frac{p p r}{s}\right] i \epsilon_{a b c}\left(\bar{d}_{\mathrm{R} p}^{a} C D_{\mu} D_{\nu} \bar{d}_{\mathrm{R}_{s}^{c}}^{c}\right)\left(\bar{d}_{\mathrm{R} r}^{b} \gamma^{\nu} D^{\mu} e_{\mathrm{R} t}\right) \\
& \mathcal{O}_{\bar{d}_{\mathrm{R}}^{2} \bar{d}_{\mathrm{L}} e_{\mathrm{L}} D^{3}}^{(1,-1,1)} \mid \begin{array}{l}
\mathcal{Y}[\overline{p r r}] i \epsilon_{a b c}\left(\bar{d}_{\mathrm{R}}^{a} D_{\mu} D_{\nu} e_{\mathrm{L} s}\right)\left(\bar{d}_{\mathrm{R} r}^{b} \gamma^{\nu} C D^{\mu} \bar{d}_{\mathrm{L} t}^{c}\right) \\
\mathcal{Y}\left[\frac{p]}{r}\right] i \epsilon_{a b c}\left(D_{\mu} \bar{d}_{\mathrm{R} r}^{b} D_{\nu} e_{\mathrm{L} s}\right)\left(\bar{d}_{\mathrm{R} p}^{a} \gamma^{\nu} C D^{\mu} \bar{d}_{\mathrm{L} t}^{c}\right)
\end{array}
\end{aligned}
$$

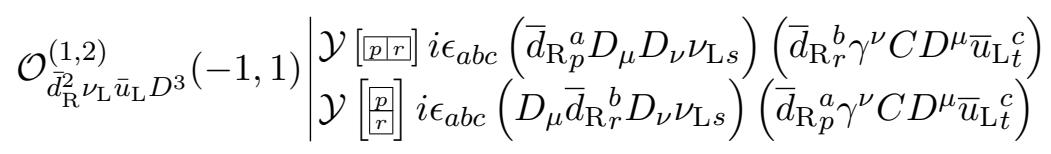

$$
\begin{aligned}
& \mathcal{O}_{\bar{d}_{\mathrm{R}} d_{\mathrm{R}} \bar{e}_{\mathrm{R}} e_{\mathrm{L}} D^{3}}^{(1,2)}(0,0) \mid \begin{array}{l}
i\left(\bar{d}_{\mathrm{R}}^{a} D_{\mu} D_{\nu} e_{\mathrm{L} s}\right)\left(\bar{e}_{\mathrm{R} r} \gamma^{\nu} D^{\mu} d_{\mathrm{R} t a}\right) \\
i\left(D_{\mu} \bar{e}_{\mathrm{R} r} D_{\nu} e_{\mathrm{L} s}\right)\left(\bar{d}_{\mathrm{R}}^{a}{ }_{p}^{\nu} \nu^{\nu} d_{\mathrm{R} t a}\right)
\end{array} \\
& \mathcal{O}_{\bar{d}_{\mathrm{R}} e_{\mathrm{L}} \nu_{\mathrm{L}} u_{\mathrm{R}} D^{3}}^{(1,2)}(0,2) \mid \begin{array}{l}
i\left(\bar{d}_{\mathrm{R}}^{a} D_{\mu} D_{\nu} \nu_{\mathrm{L} s}\right)\left(e_{\mathrm{L} r} C \gamma^{\nu} D^{\mu} u_{\mathrm{R} t a}\right) \\
i\left(D_{\mu} e_{\mathrm{L} r} C D_{\nu} \nu_{\mathrm{L} s}\right)\left(\bar{d}_{\mathrm{R} p}^{a} \gamma^{\nu} D^{\mu} u_{\mathrm{R} t a}\right)
\end{array}
\end{aligned}
$$




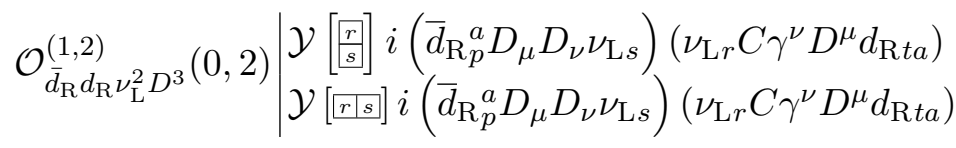

$$
\begin{aligned}
& \mathcal{O}_{\bar{e}_{\mathrm{R}} e_{\mathrm{L}}^{2} \bar{e}_{\mathrm{L}} D^{3}}^{(1,2,0)} \mid \begin{array}{l}
\mathcal{Y}\left[\begin{array}{l}
{[r]} \\
s]
\end{array}\right]\left(\bar{e}_{\mathrm{R} p} D_{\mu} D_{\nu} e_{\mathrm{L} s}\right)\left(D^{\mu} \bar{e}_{\mathrm{L} t} \gamma^{\nu} e_{\mathrm{L} r}\right) \\
\mathcal{Y}\left[\underline{r r s]} i\left(\bar{e}_{\mathrm{R} p} D_{\mu} D_{\nu} e_{\mathrm{L} s}\right)\left(D^{\mu} \bar{e}_{\mathrm{L} t} \gamma^{\nu} e_{\mathrm{L} r}\right)\right.
\end{array} \\
& \mathcal{O}_{\bar{e}_{\mathrm{R}}^{2} e_{\mathrm{R}} e_{\mathrm{L}} D^{3}}^{(1,2)}(0) \mid \begin{array}{l}
\mathcal{Y}\left[\left[\begin{array}{l}
p] \\
{[r]}
\end{array}\right] i\left(\bar{e}_{\mathrm{R} p} D_{\mu} D_{\nu} e_{\mathrm{L} s}\right)\left(\bar{e}_{\mathrm{R} r} \gamma^{\nu} D^{\mu} e_{\mathrm{R} t}\right)\right. \\
\mathcal{Y}\left[[\overline{p p r}] i\left(D_{\mu} \bar{e}_{\mathrm{R} r} D_{\nu} e_{\mathrm{L} s}\right)\left(\bar{e}_{\mathrm{R} p} \gamma^{\nu} D^{\mu} e_{\mathrm{R} t}\right)\right.
\end{array} \\
& \mathcal{O}_{\bar{e}_{\mathrm{R}} e_{\mathrm{L}} \nu_{\mathrm{L}} \bar{\nu}_{\mathrm{L}} D^{3}}^{(1,2,0)} \mid \begin{array}{l}
i\left(\bar{e}_{\mathrm{R} p} D_{\mu} D_{\nu} \nu_{\mathrm{L} s}\right)\left(D^{\mu} \bar{\nu}_{\mathrm{L} t} \gamma^{\nu} e_{\mathrm{L} r}\right) \\
i\left(D_{\mu} e_{\mathrm{L} r} C D_{\nu} \nu_{\mathrm{L} s}\right)\left(\bar{e}_{\mathrm{R} p} \gamma^{\nu} C D^{\mu} \bar{\nu}_{\mathrm{L} t}\right)
\end{array}
\end{aligned}
$$

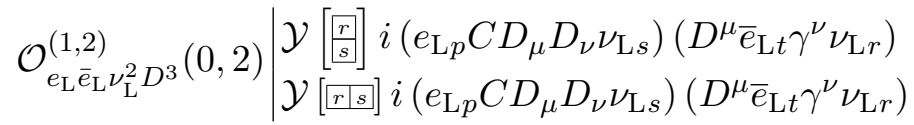

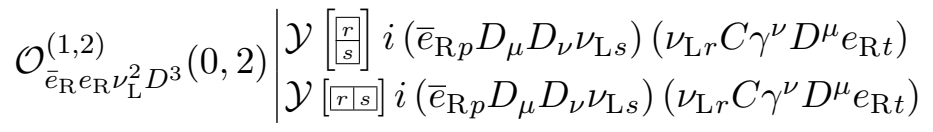

$$
\begin{aligned}
& \mathcal{O}_{\nu_{\mathrm{L}}^{3} \bar{\nu}_{\mathrm{L}} D^{3}}(0,2) \mid \mathcal{Y}\left[\frac{p r r}{s s}\right] i\left(\nu_{\mathrm{L} p} C D_{\mu} D_{\nu} \nu_{\mathrm{L} s}\right)\left(D^{\mu} \bar{\nu}_{\mathrm{L} t} \gamma^{\nu} \nu_{\mathrm{L} r}\right)
\end{aligned}
$$

\section{Class $F_{\mathrm{L}} \psi^{3} \psi^{\dagger} D: 98$ types}

$$
\begin{aligned}
& \mathcal{Y}\left[\begin{array}{l}
\frac{r}{s} \\
\frac{s}{n}
\end{array}\right] i\left(\lambda^{A}\right)_{d}^{b} G_{\mathrm{L}}^{A \mu}{ }_{\nu}\left(u_{\mathrm{L} r b} C D_{\mu} u_{\mathrm{L} s a}\right)\left(\bar{u}_{\mathrm{R} p}^{a} \gamma^{\nu} C \bar{u}_{\mathrm{L} t}^{d}\right) \\
& \mathcal{Y}\left[\frac{r}{s}\right] i\left(\lambda^{A}\right)_{a}^{b} G_{\mathrm{L}}^{A \mu}{ }_{\nu}\left(u_{\mathrm{L} r b} C D_{\mu} u_{\mathrm{L} s c}\right)\left(\bar{u}_{\mathrm{R}}^{a}{ }^{\nu} \gamma^{\nu} C \bar{u}_{\mathrm{L} t}^{c}\right) \\
& \mathcal{Y}\left[\begin{array}{l}
r \\
s
\end{array}\right] i\left(\lambda^{A}\right)_{d}^{c} G_{\mathrm{L}}^{A \mu}{ }_{\nu}\left(u_{\mathrm{L} r a} C D_{\mu} u_{\mathrm{L} s c}\right)\left(\bar{u}_{\mathrm{R} p}^{a} \gamma^{\nu} C \bar{u}_{\mathrm{L} t}^{d}\right) \\
& \mathcal{Y}\left[\frac{r}{s}\right] i\left(\lambda^{A}\right)_{a}^{c} G_{\mathrm{L}}^{A \mu}{ }_{\nu}\left(u_{\mathrm{L} r b} C D_{\mu} u_{\mathrm{L} s c}\right)\left(\bar{u}_{\mathrm{R}}^{a}{ }^{a} \gamma^{\nu} C \bar{u}_{\mathrm{L} t}^{b}\right) \\
& \mathcal{Y}\left[\frac{r}{s}\right] i\left(\lambda^{A}\right)_{d}^{b} G_{\mathrm{L}}^{A \mu}{ }_{\nu}\left(\bar{u}_{\mathrm{R} p}^{a} u_{\mathrm{L} s a}\right)\left(\bar{u}_{\mathrm{L} t}^{d} \gamma^{\nu} D_{\mu} u_{\mathrm{L} r b}\right) \\
& \mathcal{O}_{G_{\mathrm{L}} \bar{u}_{\mathrm{R}} u_{\mathrm{L}}^{2} \bar{u}_{\mathrm{L}} D}^{(1 \sim 16)}(0,0) \\
& \mathcal{Y}\left[\begin{array}{l}
r \\
s
\end{array}\right] i\left(\lambda^{A}\right)_{a}^{b} G_{\mathrm{L}}^{A \mu}{ }_{\nu}\left(\bar{u}_{\mathrm{R} p}^{a} u_{\mathrm{L} s c}\right)\left(\bar{u}_{\mathrm{L}}^{c} \gamma^{\nu} D_{\mu} u_{\mathrm{L} r b}\right) \\
& \mathcal{Y}\left[\begin{array}{l}
r \\
s]
\end{array} i\left(\lambda^{A}\right)_{d}^{q} G_{\mathrm{L}}^{A}{ }_{\nu}{ }_{\nu}\left(\bar{u}_{\mathrm{R}}{ }_{p}^{a} u_{\mathrm{L} s c}\right)\left(\bar{u}_{\mathrm{L} t}^{d} \gamma^{\nu} D_{\mu} u_{\mathrm{L} r a}\right)\right. \\
& \mathcal{Y}\left[\frac{r}{s}\right] i\left(\lambda^{A}\right)_{a}^{c} G_{\mathrm{L}}^{A \mu}{ }_{\nu}\left(\bar{u}_{\mathrm{R}}{ }^{a} u_{\mathrm{L} s c}\right)\left(\bar{u}_{\mathrm{L} t}^{b} \gamma^{\nu} D_{\mu} u_{\mathrm{L} r b}\right)
\end{aligned}
$$

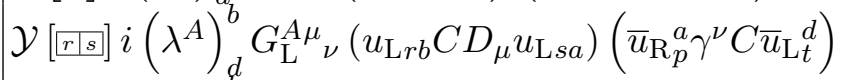

$$
\begin{aligned}
& \mathcal{Y}\left[[r s] i\left(\lambda^{A}\right)_{a}^{b} G_{\mathrm{L}}^{A \mu}{ }_{\nu}\left(u_{\mathrm{L} r b} C D_{\mu} u_{\mathrm{L} s c}\right)\left(\bar{u}_{\mathrm{R} p}^{a} \gamma^{\nu} C \bar{u}_{\mathrm{L} t}^{c}\right)\right.
\end{aligned}
$$

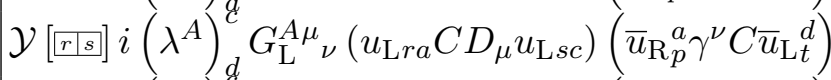

$$
\begin{aligned}
& \mathcal{Y}\left[[r s] i\left(\lambda^{A}\right)_{a}^{c} G_{\mathrm{L}}^{A \mu}{ }_{\nu}\left(u_{\mathrm{L} r b} C D_{\mu} u_{\mathrm{L} s c}\right)\left(\bar{u}_{\mathrm{R} p}^{a} \gamma^{\nu} C \bar{u}_{\mathrm{L} t}^{b}\right)\right. \\
& \mathcal{Y}[\underline{r r s}] i\left(\lambda^{A}\right)_{d}^{b} G_{\mathrm{L}}^{A \mu}{ }_{\nu}\left(\bar{u}_{\mathrm{R} p}^{a} u_{\mathrm{L} s a}\right)\left(\bar{u}_{\mathrm{L} t}{ }^{d} \gamma^{\nu} D_{\mu} u_{\mathrm{L} r b}\right) \\
& \mathcal{Y}[\overline{r r s}] i\left(\lambda^{A}\right)_{a}^{b} G_{\mathrm{L}}^{A \mu}{ }_{\nu}\left(\bar{u}_{\mathrm{R} p}^{a} u_{\mathrm{L} s c}\right)\left(\bar{u}_{\mathrm{L} t}^{c} \gamma^{\nu} D_{\mu} u_{\mathrm{L} r b}\right) \\
& \mathcal{Y}[\overline{r r s}] i\left(\lambda^{A}\right)_{d}^{a} G_{\mathrm{L}}^{A \mu}{ }_{\nu}\left(\bar{u}_{\mathrm{R} p}{ }_{p}^{a} u_{\mathrm{L} s c}\right)\left(\bar{u}_{\mathrm{L} t}{ }^{d} \gamma^{\nu} D_{\mu} u_{\mathrm{L} r a}\right) \\
& \mathcal{Y}[\overline{r r s}] i\left(\lambda^{A}\right)_{a}^{c} G_{\mathrm{L}}^{A \mu}{ }_{\nu}\left(\bar{u}_{\mathrm{R} p}^{a} u_{\mathrm{L} s c}\right)\left(\bar{u}_{\mathrm{L} t}^{b} \gamma^{\nu} D_{\mu} u_{\mathrm{L} r b}\right)
\end{aligned}
$$




$$
\begin{aligned}
& \mathcal{Y}\left[\begin{array}{l}
r \\
s
\end{array}\right] i\left(\lambda^{A}\right)_{d}^{b} \epsilon^{a c d} G_{\mathrm{L}}^{A \mu}{ }_{\nu}\left(d_{\mathrm{L} p a} C \gamma^{\nu} e_{\mathrm{R} t}\right)\left(u_{\mathrm{L} r b} C D_{\mu} u_{\mathrm{L} s c}\right) \\
& \mathcal{Y}\left[\begin{array}{l}
\frac{r}{s} \\
s
\end{array}\right] i\left(\lambda^{A}\right)_{d}^{d} \epsilon^{b c d} G_{\mathrm{L}}^{A \mu}{ }_{\nu}\left(d_{\mathrm{L} p a} C \gamma^{\nu} e_{\mathrm{R} t}\right)\left(u_{\mathrm{L} r b} C D_{\mu} u_{\mathrm{L} s c}\right)
\end{aligned}
$$

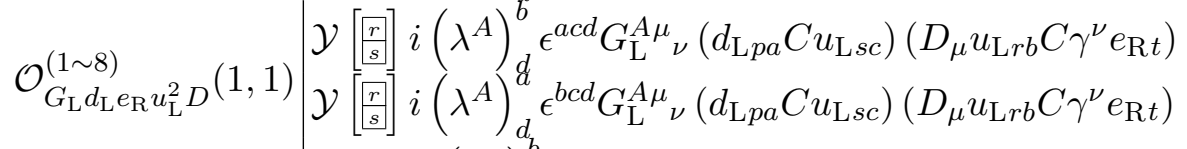

$$
\begin{aligned}
& \mathcal{Y}\left[r[s] i\left(\lambda^{A}\right)_{d}^{b} \epsilon^{a c d} G_{\mathrm{L}}^{A \mu}{ }_{\nu}\left(d_{\mathrm{L} p a} C \gamma^{\nu} e_{\mathrm{R} t}\right)\left(u_{\mathrm{L} r b} C D_{\mu} u_{\mathrm{L} s c}\right)\right. \\
& \mathcal{Y}\left[[r \mid s] i\left(\lambda^{A}\right)_{d}^{d} \epsilon^{b c d} G_{\mathrm{L}}^{A \mu}{ }_{\nu}\left(d_{\mathrm{L} p a} C \gamma^{\nu} e_{\mathrm{R} t}\right)\left(u_{\mathrm{L} r b} C D_{\mu} u_{\mathrm{L} s c}\right)\right. \\
& \mathcal{Y}[\overline{r r s}] i\left(\lambda^{A}\right)_{d}^{b} \epsilon^{a c d} G_{\mathrm{L}}^{A \mu}{ }_{\nu}\left(d_{\mathrm{L} p a} C u_{\mathrm{L} s c}\right)\left(D_{\mu} u_{\mathrm{L} r b} C \gamma^{\nu} e_{\mathrm{R} t}\right) \\
& \mathcal{Y}\left[[r \mid s] i\left(\lambda^{A}\right)_{d}^{d} \epsilon^{b c d} G_{\mathrm{L}}^{A}{ }_{\nu}{ }_{\nu}\left(d_{\mathrm{L} p a} C u_{\mathrm{L} s c}\right)\left(D_{\mu} u_{\mathrm{L} r b} C \gamma^{\nu} e_{\mathrm{R} t}\right)\right. \\
& \mathcal{Y}\left[\begin{array}{c}
r \\
s
\end{array}\right] i\left(\lambda^{A}\right)_{d}^{b} \epsilon^{a c d} G_{\mathrm{L}}^{A \mu}{ }_{\nu}\left(u_{\mathrm{L} r a} C D_{\mu} u_{\mathrm{L} s b}\right)\left(e_{\mathrm{L} p} C \gamma^{\nu} d_{\mathrm{R} t c}\right) \\
& \mathcal{Y}\left[\begin{array}{l}
\frac{r}{s} \\
s
\end{array}\right] i\left(\lambda^{A}\right)_{d}^{d} \epsilon^{b c d} G_{\mathrm{L}}^{A \mu}{ }_{\nu}\left(u_{\mathrm{L} r a} C D_{\mu} u_{\mathrm{L} s b}\right)\left(e_{\mathrm{L} p} C \gamma^{\nu} d_{\mathrm{R} t c}\right)
\end{aligned}
$$

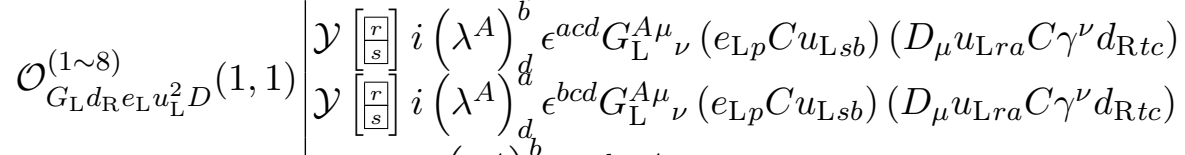

$$
\begin{aligned}
& \mathcal{Y}[\overline{r r s}] i\left(\lambda^{A}\right)_{d}^{b} \epsilon^{a c d} G_{\mathrm{L}}^{A \mu}{ }_{\nu}\left(u_{\mathrm{L} r a} C D_{\mu} u_{\mathrm{L} s b}\right)\left(e_{\mathrm{L} p} C \gamma^{\nu} d_{\mathrm{R} t c}\right) \\
& \mathcal{Y}\left[r[s] i\left(\lambda^{A}\right)_{d}^{d} \epsilon^{b c d} G_{\mathrm{L}}^{A \mu}{ }_{\nu}\left(u_{\mathrm{L} r a} C D_{\mu} u_{\mathrm{L} s b}\right)\left(e_{\mathrm{L} p} C \gamma^{\nu} d_{\mathrm{R} t c}\right)\right. \\
& \mathcal{Y}[\overline{r r s}] i\left(\lambda^{A}\right)_{d}^{b} \epsilon^{a c d} G_{\mathrm{L}}^{A \mu}{ }_{\nu}\left(e_{\mathrm{L} p} C u_{\mathrm{L} s b}\right)\left(D_{\mu} u_{\mathrm{L} r a} C \gamma^{\nu} d_{\mathrm{R} t c}\right) \\
& \mathcal{Y}\left[[r \mid s] i\left(\lambda^{A}\right)_{d}^{d} \epsilon^{b c d} G_{\mathrm{L}}^{A \mu}{ }_{\nu}\left(e_{\mathrm{L} p} C u_{\mathrm{L} s b}\right)\left(D_{\mu} u_{\mathrm{L} r a} C \gamma^{\nu} d_{\mathrm{R} t c}\right)\right. \\
& \mathcal{Y}\left[\frac{p}{r}\right] i\left(\lambda^{A}\right)_{b}^{d} G_{\mathrm{L}}^{A \mu}{ }_{\nu}\left(\bar{u}_{\mathrm{R}}{ }^{a} \gamma^{\nu} u_{\mathrm{R} t d}\right)\left(\bar{u}_{\mathrm{R} r}^{b} D_{\mu} u_{\mathrm{L} s a}\right) \\
& \mathcal{Y}\left[\frac{p}{r}\right] i\left(\lambda^{A}\right)_{a}^{d} G_{\mathrm{L}}^{A \mu}{ }_{\nu}\left(\bar{u}_{\mathrm{R}}^{a} \gamma^{\nu} u_{\mathrm{R} t d}\right)\left(\bar{u}_{\mathrm{R}}^{b} D_{\mu} u_{\mathrm{L} s b}\right) \\
& \mathcal{Y}\left[\frac{p}{r}\right] i\left(\lambda^{A}\right)_{a}^{q} G_{\mathrm{L}}^{A \mu} \nu\left(\bar{u}_{\mathrm{R}}^{a} \gamma^{\nu} u_{\mathrm{R} t b}\right)\left(\bar{u}_{\mathrm{R}}^{b} D_{\mu} u_{\mathrm{L} s c}\right) \\
& \mathcal{Y}\left[\frac{p}{r}\right] i\left(\lambda^{A}\right)_{b}^{a} G_{\mathrm{L}}^{A \mu}{ }_{\nu}\left(\bar{u}_{\mathrm{R}}^{a} \gamma^{\nu} u_{\mathrm{R} t a}\right)\left(\bar{u}_{\mathrm{R}}^{b} D_{\mu} u_{\mathrm{L} s c}\right) \\
& \mathcal{Y}\left[\begin{array}{l}
\left.\frac{p}{r}\right] \\
]
\end{array} i\left(\lambda^{A}\right)_{b}^{d} G_{\mathrm{L}}^{A \mu}{ }_{\nu}\left(\bar{u}_{\mathrm{R} p}^{a} \gamma^{\nu} u_{\mathrm{R} t d}\right)\left(D_{\mu} \bar{u}_{\mathrm{R}}^{b} u_{\mathrm{L} s a}\right)\right.
\end{aligned}
$$

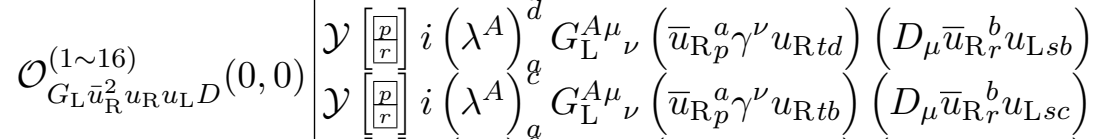

$$
\begin{aligned}
& \mathcal{Y}\left[\frac{p}{r}\right] i\left(\lambda^{A}\right)_{b}^{a} G_{\mathrm{L}}^{A} \nu_{\nu}\left(\bar{u}_{\mathrm{R}}^{a} \gamma^{\nu} u_{\mathrm{R} t a}\right)\left(D_{\mu} \bar{u}_{\mathrm{R}}^{b} u_{\mathrm{L} s c}\right) \\
& \mathcal{Y}[\overline{p p r}] i\left(\lambda^{A}\right)_{b}^{d} G_{\mathrm{L}}^{A \mu}{ }_{\nu}\left(\bar{u}_{\mathrm{R} p}^{a} \gamma^{\nu} u_{\mathrm{R} t d}\right)\left(\bar{u}_{\mathrm{R} r}^{b} D_{\mu} u_{\mathrm{L} s a}\right) \\
& \mathcal{Y}[\underline{p p r}] i\left(\lambda^{A}\right)_{a}^{d} G_{\mathrm{L}}^{A \mu}{ }_{\nu}\left(\bar{u}_{\mathrm{R}}^{a} \gamma^{\nu} u_{\mathrm{R} t d}\right)\left(\bar{u}_{\mathrm{R} r}^{b} D_{\mu} u_{\mathrm{L} s b}\right) \\
& \mathcal{Y}[\overline{p p r}] i\left(\lambda^{A}\right)_{a}^{q} G_{\mathrm{L}}^{A \mu}{ }_{\nu}\left(\bar{u}_{\mathrm{R} p}^{a} \gamma^{\nu} u_{\mathrm{R} t b}\right)\left(\bar{u}_{\mathrm{R} r}^{b} D_{\mu} u_{\mathrm{L} s c}\right) \\
& \mathcal{Y}[\overline{p \mid r}] i\left(\lambda^{A}\right)_{b}^{q} G_{\mathrm{L}}^{A \mu}{ }_{\nu}\left(\bar{u}_{\mathrm{R} p}^{a} \gamma^{\nu} u_{\mathrm{R} t a}\right)\left(\bar{u}_{\mathrm{R} r}^{b} D_{\mu} u_{\mathrm{L} s c}\right) \\
& \mathcal{Y}[\overline{p p r}] i\left(\lambda^{A}\right)_{b}^{d} G_{\mathrm{L}}^{A \mu}{ }_{\nu}\left(\bar{u}_{\mathrm{R}}^{a}{ }_{p} \gamma^{\nu} u_{\mathrm{R} t d}\right)\left(D_{\mu} \bar{u}_{\mathrm{R}}{ }_{r}^{b} u_{\mathrm{L} s a}\right) \\
& \mathcal{Y}[\overline{p p r}] i\left(\lambda^{A}\right)_{a}^{d} G_{\mathrm{L}}^{A \mu}{ }_{\nu}\left(\bar{u}_{\mathrm{R}}^{a}{ }^{a} \gamma^{\nu} u_{\mathrm{R} t d}\right)\left(D_{\mu} \bar{u}_{\mathrm{R} r}^{b} u_{\mathrm{L} s b}\right) \\
& \mathcal{Y}[\overline{p r}] i\left(\lambda^{A}\right)_{a}^{q} G_{\mathrm{L}}^{A \mu}{ }_{\nu}\left(\bar{u}_{\mathrm{R}}^{a}{ }_{p}^{\nu} u_{\mathrm{R} t b}\right)\left(D_{\mu} \bar{u}_{\mathrm{R} r}^{b} u_{\mathrm{L} s c}\right) \\
& \mathcal{Y}[\underline{p p r}] i\left(\lambda^{A}\right)_{b}^{c} G_{\mathrm{L}}^{A \mu}{ }_{\nu}\left(\bar{u}_{\mathrm{R}}{ }_{p}^{a} \gamma^{\nu} u_{\mathrm{R} t a}\right)\left(D_{\mu} \bar{u}_{\mathrm{R}}{ }_{r}^{b} u_{\mathrm{L} s c}\right)
\end{aligned}
$$




$$
\begin{aligned}
& i\left(\lambda^{A}\right)_{b}^{c} G_{\mathrm{L}}^{A \mu}{ }_{\nu}\left(\bar{d}_{\mathrm{L} t}^{a} \gamma^{\nu} d_{\mathrm{L} p a}\right)\left(\bar{u}_{\mathrm{R} r}^{b} D_{\mu} u_{\mathrm{L} s c}\right) \\
& i\left(\lambda^{A}\right)_{d}^{c} G_{\mathrm{L}}^{A \mu}{ }_{\nu}\left(\bar{d}_{\mathrm{L}}{ }_{t}^{d} \gamma^{\nu} d_{\mathrm{L} p a}\right)\left(\bar{u}_{\mathrm{R} r}^{a} D_{\mu} u_{\mathrm{L} s c}\right) \\
& i\left(\lambda^{A}\right)_{d}^{d} G_{\mathrm{L}}^{A \mu}{ }_{\nu}\left(\bar{d}_{\mathrm{L}}{ }_{t}^{d} \gamma^{\nu} d_{\mathrm{L} p a}\right)\left(\bar{u}_{\mathrm{R}}{ }_{r}^{b} D_{\mu} u_{\mathrm{L} s b}\right) \\
& i\left(\lambda^{A}\right)_{b}^{a} G_{\mathrm{L}}^{A \mu}{ }_{\nu}\left(\bar{d}_{\mathrm{L}}^{c} \gamma^{\nu} d_{\mathrm{L} p a}\right)\left(\bar{u}_{\mathrm{R} r}^{b} D_{\mu} u_{\mathrm{L} s c}\right) \\
& i\left(\lambda^{A}\right)_{b}^{c} G_{\mathrm{L}}^{A \mu}{ }_{\nu}\left(\bar{d}_{\mathrm{L} t}^{a} \gamma^{\nu} d_{\mathrm{L} p a}\right)\left(D_{\mu} \bar{u}_{\mathrm{R} r}^{b} u_{\mathrm{L} s c}\right) \\
& i\left(\lambda^{A}\right)_{d}^{c} G_{\mathrm{L}}^{A \mu}{ }_{\nu}\left(\bar{d}_{\mathrm{L}}{ }_{t} \gamma^{\nu} d_{\mathrm{L} p a}\right)\left(D_{\mu} \bar{u}_{\mathrm{R}}{ }_{r}^{a} u_{\mathrm{L} s c}\right) \\
& \mathcal{O}_{G_{\mathrm{L}} d_{\mathrm{L}} \bar{d}_{\mathrm{L}} \bar{u}_{\mathrm{R}} u_{\mathrm{L}} D}^{(1 \sim 16)}(0,0) i\left(\lambda^{A}\right)_{d}^{d} G_{\mathrm{L}}^{A \mu}{ }_{\nu}\left(\bar{d}_{\mathrm{L} t}^{d} \gamma^{\nu} d_{\mathrm{L} p a}\right)\left(D_{\mu} \bar{u}_{\mathrm{R}}{ }_{r}^{b} u_{\mathrm{L} s b}\right) \\
& i\left(\lambda^{A}\right)_{b}^{a} G_{\mathrm{L}}^{A \mu}{ }_{\nu}\left(\bar{d}_{\mathrm{L} t}^{c} \gamma^{\nu} d_{\mathrm{L} p a}\right)\left(D_{\mu} \bar{u}_{\mathrm{R} r}^{b} u_{\mathrm{L} s c}\right) \\
& i\left(\lambda^{A}\right)_{b}^{c} G_{\mathrm{L}}^{A \mu}{ }_{\nu}\left(d_{\mathrm{L} p a} C u_{\mathrm{L} s c}\right)\left(D_{\mu} \bar{u}_{\mathrm{R} r}^{b} \gamma^{\nu} C \bar{d}_{\mathrm{L} t}^{a}\right) \\
& i\left(\lambda^{A}\right)_{d}^{c} G_{\mathrm{L}}^{A \mu}{ }_{\nu}\left(d_{\mathrm{L} p a} C u_{\mathrm{L} s c}\right)\left(D_{\mu} \bar{u}_{\mathrm{R} r}^{a} \gamma^{\nu} C \bar{d}_{\mathrm{L} t}{ }^{d}\right) \\
& i\left(\lambda^{A}\right)_{d}^{d} G_{\mathrm{L}}^{A \mu}{ }_{\nu}\left(d_{\mathrm{L} p a} C u_{\mathrm{L} s b}\right)\left(D_{\mu} \bar{u}_{\mathrm{R} r}^{b} \gamma^{\nu} C \bar{d}_{\mathrm{L} t}^{d}\right) \\
& i\left(\lambda^{A}\right)_{b}^{d} G_{\mathrm{L}}^{A \mu}{ }_{\nu}\left(d_{\mathrm{L} p a} C u_{\mathrm{L} s c}\right)\left(D_{\mu} \bar{u}_{\mathrm{R} r}^{b} \gamma^{\nu} C \bar{d}_{\mathrm{L} t}^{c}\right) \\
& i\left(\lambda^{A}\right)_{b}^{c} G_{\mathrm{L}}^{A \mu}{ }_{\nu}\left(d_{\mathrm{L} p a} C D_{\mu} u_{\mathrm{L} s c}\right)\left(\bar{u}_{\mathrm{R} r}^{b} \gamma^{\nu} C \bar{d}_{\mathrm{L} t}^{a}\right) \\
& i\left(\lambda^{A}\right)_{d}^{c} G_{\mathrm{L}}^{A \mu}{ }_{\nu}\left(d_{\mathrm{L} p a} C D_{\mu} u_{\mathrm{L} s c}\right)\left(\bar{u}_{\mathrm{R} r}^{a} \gamma^{\nu} C \bar{d}_{\mathrm{L} t}{ }_{t}\right) \\
& i\left(\lambda^{A}\right)_{d}^{d} G_{\mathrm{L}}^{A \mu}{ }_{\nu}\left(d_{\mathrm{L} p a} C D_{\mu} u_{\mathrm{L} s b}\right)\left(\bar{u}_{\mathrm{R}}{ }_{r}^{b} \gamma^{\nu} C \bar{d}_{\mathrm{L} t}^{d}\right) \\
& i\left(\lambda^{A}\right)_{b}^{a} G_{\mathrm{L}}^{A \mu}{ }_{\nu}\left(d_{\mathrm{L} p a} C D_{\mu} u_{\mathrm{L} s c}\right)\left(\bar{u}_{\mathrm{R}}{ }_{r}^{b} \gamma^{\nu} C \bar{d}_{\mathrm{L} t}^{c}\right)
\end{aligned}
$$

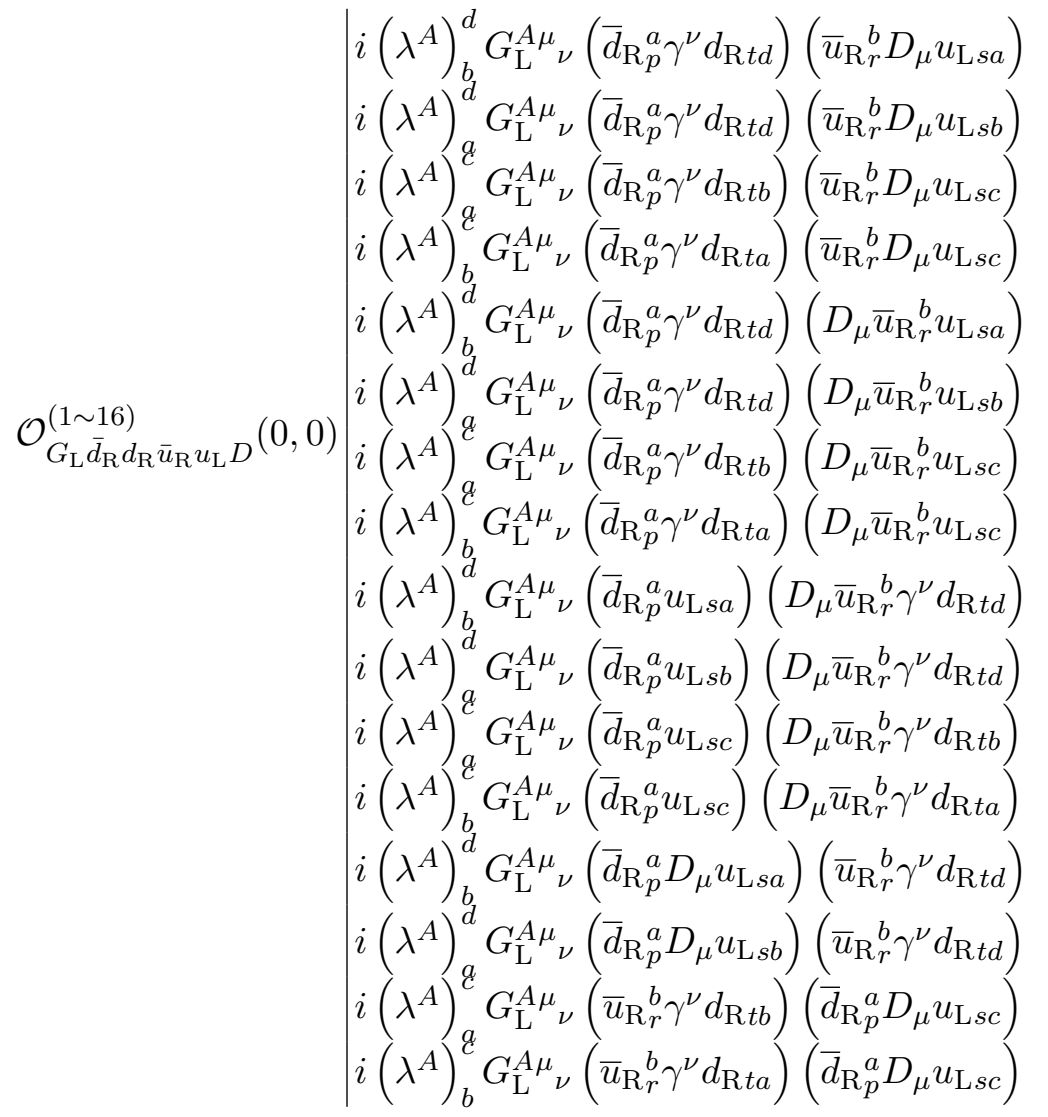




$$
\begin{aligned}
& \mid i\left(\lambda^{A}\right)_{a}^{b} G_{\mathrm{L}}^{A \mu}{ }_{\nu}\left(\bar{e}_{\mathrm{L} t} \gamma^{\nu} e_{\mathrm{L} p}\right)\left(\bar{u}_{\mathrm{R}}{ }_{r}^{a} D_{\mu} u_{\mathrm{L} s b}\right) \\
& \mathcal{O}_{G_{\mathrm{L}} e_{\mathrm{L}} \bar{e}_{\mathrm{L}} \bar{u}_{\mathrm{R}} u_{\mathrm{L}} D}^{(1 \sim 4)}(0,0) i\left(\lambda^{A}\right)_{a}^{b} G_{\mathrm{L}}^{A \mu}{ }_{\nu}\left(\bar{e}_{\mathrm{L} t} \gamma^{\nu} e_{\mathrm{L} p}\right)\left(D_{\mu} \bar{u}_{\mathrm{R} r}{ }^{a} u_{\mathrm{L} s b}\right) \\
& i\left(\lambda^{A}\right)_{a}^{b} G_{\mathrm{L}}^{A \mu}{ }_{\nu}\left(e_{\mathrm{L} p} C u_{\mathrm{L} s b}\right)\left(D_{\mu} \bar{u}_{\mathrm{R}}{ }_{r}^{a} \gamma^{\nu} C \bar{e}_{\mathrm{L} t}\right) \\
& i\left(\lambda^{A}\right)_{a}^{b} G_{\mathrm{L}}^{A \mu}{ }_{\nu}\left(e_{\mathrm{L} p} C D_{\mu} u_{\mathrm{L} s b}\right)\left(\bar{u}_{\mathrm{R}}{ }_{r}^{a} \gamma^{\nu} C \bar{e}_{\mathrm{L} t}\right) \\
& \mathcal{O}_{G_{\mathrm{L}} \bar{e}_{\mathrm{R}} e_{\mathrm{R}} \bar{u}_{\mathrm{R}} u_{\mathrm{L}} D}^{(1 \sim 0,0)} \mid \begin{array}{l}
i\left(\lambda^{A}\right)_{a}^{b} G_{\mathrm{L}}^{A \mu}{ }_{\nu}\left(\bar{e}_{\mathrm{R} p} \gamma^{\nu} e_{\mathrm{R} t}\right)\left(\bar{u}_{\mathrm{R}}{ }_{r}^{a} D_{\mu} u_{\mathrm{L} s b}\right) \\
i\left(\lambda^{A}\right)_{a}^{b} G_{\mathrm{L}}^{A \mu}{ }_{\nu}\left(\bar{e}_{\mathrm{R} p} \gamma^{\nu} e_{\mathrm{R} t}\right)\left(D_{\mu} \bar{u}_{\mathrm{R}}{ }_{r}^{a} u_{\mathrm{L} s b}\right)
\end{array} \\
& i\left(\lambda^{A}\right)_{a}^{b} G_{\mathrm{L}}^{A \mu}{ }_{\nu}\left(\bar{e}_{\mathrm{R} p} u_{\mathrm{L} s b}\right)\left(D_{\mu} \bar{u}_{\mathrm{R}}{ }_{r}^{a} \gamma^{\nu} e_{\mathrm{R} t}\right) \\
& i\left(\lambda^{A}\right)_{a}^{b} G_{\mathrm{L}}^{A \mu}{ }_{\nu}\left(\bar{e}_{\mathrm{R} p} D_{\mu} u_{\mathrm{L} s b}\right)\left(\bar{u}_{\mathrm{R}}{ }_{r}^{a} \gamma^{\nu} e_{\mathrm{R} t}\right) \\
& \mathcal{O}_{G_{\mathrm{L}} \nu_{\mathrm{L}} \bar{\nu}_{\mathrm{L}} \bar{u}_{\mathrm{R}} u_{\mathrm{L}} D}^{(1 \sim, 0)} \mid \begin{array}{l}
i\left(\lambda^{A}\right)_{a}^{b} G_{\mathrm{L}}^{A \mu}{ }_{\nu}\left(u_{\mathrm{L} r b} C D_{\mu} \nu_{\mathrm{L} s}\right)\left(\bar{u}_{\mathrm{R}}^{a} \gamma^{\nu} C \bar{\nu}_{\mathrm{L} t}\right) \\
i\left(\lambda^{A}\right)_{a}^{b} G_{\mathrm{L}}^{A \mu}{ }_{\nu}\left(D_{\mu} u_{\mathrm{L} r b} C \nu_{\mathrm{L} s}\right)\left(\bar{u}_{\mathrm{R}}^{a} \gamma^{\nu} C \bar{\nu}_{\mathrm{L} t}\right) \\
i\left(\lambda^{A}\right)_{a}^{b} G_{\mathrm{L}}^{A \mu}{ }_{\nu}\left(\bar{u}_{\mathrm{R} p}^{a} \nu_{\mathrm{L} s}\right)\left(\bar{\nu}_{\mathrm{L} t} \gamma^{\nu} D_{\mu} u_{\mathrm{L} r b}\right) \\
i\left(\lambda^{A}\right)_{a}^{b} G_{\mathrm{L}}^{A \mu}{ }_{\nu}\left(\bar{\nu}_{\mathrm{L} t} \gamma^{\nu} u_{\mathrm{L} r b}\right)\left(\bar{u}_{\mathrm{R} p}^{a} D_{\mu} \nu_{\mathrm{L} s}\right)
\end{array} \\
& \mathcal{Y}\left[\frac{p}{r}\right] i\left(\lambda^{A}\right)_{d}^{b} \epsilon^{a c d} G_{\mathrm{L}}^{A \mu}{ }_{\nu}\left(\bar{\nu}_{\mathrm{L} t} \gamma^{\nu} d_{\mathrm{L} p a}\right)\left(d_{\mathrm{L} r b} C D_{\mu} u_{\mathrm{L} s c}\right) \\
& \mathcal{Y}\left[\frac{p}{r}\right] i\left(\lambda^{A}\right)_{d}^{d} \epsilon^{b c d} G_{\mathrm{L}}^{A \mu}{ }_{\nu}\left(\bar{\nu}_{\mathrm{L} t} \gamma^{\nu} d_{\mathrm{L} p a}\right)\left(d_{\mathrm{L} r b} C D_{\mu} u_{\mathrm{L} s c}\right)
\end{aligned}
$$

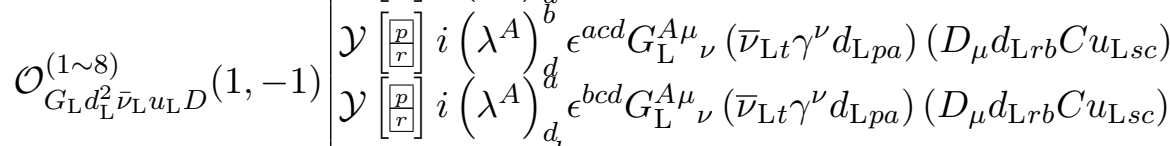

$$
\begin{aligned}
& \mathcal{Y}[\overline{p r r}] i\left(\lambda^{A}\right)_{d}^{b} \epsilon^{a c d} G_{\mathrm{L}}^{A \mu}{ }_{\nu}\left(\bar{\nu}_{\mathrm{L} t} \gamma^{\nu} d_{\mathrm{L} p a}\right)\left(d_{\mathrm{L} r b} C D_{\mu} u_{\mathrm{L} s c}\right) \\
& \mathcal{Y}[\overline{p r r}] i\left(\lambda^{A}\right)_{d}^{a} \epsilon^{b c d} G_{\mathrm{L}}^{A \mu}{ }_{\nu}\left(\bar{\nu}_{\mathrm{L} t} \gamma^{\nu} d_{\mathrm{L} p a}\right)\left(d_{\mathrm{L} r b} C D_{\mu} u_{\mathrm{L} s c}\right) \\
& \mathcal{Y}[\overline{p r r}] i\left(\lambda^{A}\right)_{d}^{b} \epsilon^{a c d} G_{\mathrm{L}}^{A \mu}{ }_{\nu}\left(\bar{\nu}_{\mathrm{L} t} \gamma^{\nu} d_{\mathrm{L} p a}\right)\left(D_{\mu} d_{\mathrm{L} r b} C u_{\mathrm{L} s c}\right) \\
& \mathcal{Y}[\overline{p p r}] i\left(\lambda^{A}\right)_{d}^{d} \epsilon^{b c d} G_{\mathrm{L}}^{A \mu}{ }_{\nu}\left(\bar{\nu}_{\mathrm{L} t} \gamma^{\nu} d_{\mathrm{L} p a}\right)\left(D_{\mu} d_{\mathrm{L} r b} C u_{\mathrm{L} s c}\right)
\end{aligned}
$$




$$
\begin{aligned}
& \mid i\left(\lambda^{A}\right)_{d}^{b} G_{\mathrm{L}}^{A \mu}{ }_{\nu}\left(d_{\mathrm{L} r b} C D_{\mu} u_{\mathrm{L} s a}\right)\left(\bar{d}_{\mathrm{R}}^{a} \gamma^{\nu} C \bar{u}_{\mathrm{L} t}^{d}\right) \\
& i\left(\lambda^{A}\right)_{a}^{b} G_{\mathrm{L}}^{A \mu}{ }_{\nu}\left(d_{\mathrm{L} r b} C D_{\mu} u_{\mathrm{L} s c}\right)\left(\bar{d}_{\mathrm{R} p}^{a} \gamma^{\nu} C \bar{u}_{\mathrm{L} t}^{c}\right) \\
& i\left(\lambda^{A}\right)_{d}^{q} G_{\mathrm{L}}^{A \mu}{ }_{\nu}\left(d_{\mathrm{L} r a} C D_{\mu} u_{\mathrm{L} s c}\right)\left(\bar{d}_{\mathrm{R} p}^{a} \gamma^{\nu} C \bar{u}_{\mathrm{L}}{ }_{t}^{d}\right) \\
& i\left(\lambda^{A}\right)_{a}^{c} G_{\mathrm{L}}^{A \mu}{ }_{\nu}\left(d_{\mathrm{L} r b} C D_{\mu} u_{\mathrm{L} s c}\right)\left(\bar{d}_{\mathrm{R} p}^{a} \gamma^{\nu} C \bar{u}_{\mathrm{L} t}^{b}\right) \\
& i\left(\lambda^{A}\right)_{d}^{b} G_{\mathrm{L}}^{A \mu}{ }_{\nu}\left(D_{\mu} d_{\mathrm{L} r b} C u_{\mathrm{L} s a}\right)\left(\bar{d}_{\mathrm{R}_{p}^{a}} \gamma^{\nu} C \bar{u}_{\mathrm{L}}{ }_{t}^{d}\right)
\end{aligned}
$$

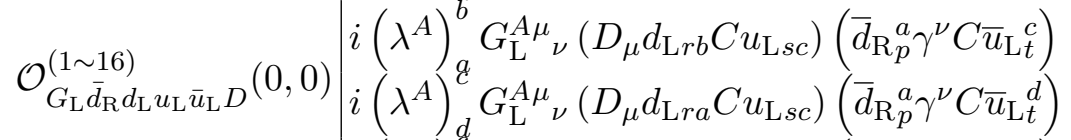

$$
\begin{aligned}
& i\left(\lambda^{A}\right)_{a}^{c} G_{\mathrm{L}}^{A \mu}{ }_{\nu}\left(D_{\mu} d_{\mathrm{L} r b} C u_{\mathrm{L} s c}\right)\left(\bar{d}_{\mathrm{R}_{p}^{a}} \gamma^{\nu} C \bar{u}_{\mathrm{L} t}^{b}\right) \\
& i\left(\lambda^{A}\right)_{d}^{b} G_{\mathrm{L}}^{A \mu}{ }_{\nu}\left(\bar{d}_{\mathrm{R} p}^{a} u_{\mathrm{L} s a}\right)\left(\bar{u}_{\mathrm{L} t}^{d} \gamma^{\nu} D_{\mu} d_{\mathrm{L} r b}\right) \\
& i\left(\lambda^{A}\right)_{a}^{b} G_{\mathrm{L}}^{A \mu}{ }_{\nu}\left(\bar{d}_{\mathrm{R} p}^{a} u_{\mathrm{L} s c}\right)\left(\bar{u}_{\mathrm{L} t}^{c} \gamma^{\nu} D_{\mu} d_{\mathrm{L} r b}\right) \\
& i\left(\lambda^{A}\right)_{d}^{q} G_{\mathrm{L}}^{A \mu}{ }_{\nu}\left(\bar{d}_{\mathrm{R} p}^{a} u_{\mathrm{L} s c}\right)\left(\bar{u}_{\mathrm{L} t}^{d} \gamma^{\nu} D_{\mu} d_{\mathrm{L} r a}\right) \\
& i\left(\lambda^{A}\right)_{a}^{c} G_{\mathrm{L}}^{A \mu}{ }_{\nu}\left(\bar{d}_{\mathrm{R} p}^{a} u_{\mathrm{L} s c}\right)\left(\bar{u}_{\mathrm{L} t}^{b} \gamma^{\nu} D_{\mu} d_{\mathrm{L} r b}\right) \\
& i\left(\lambda^{A}\right)_{d}^{b} G_{\mathrm{L}}^{A \mu}{ }_{\nu}\left(\bar{d}_{\mathrm{R} p}^{a} D_{\mu} u_{\mathrm{L} s a}\right)\left(\bar{u}_{\mathrm{L} t}^{d} \gamma^{\nu} d_{\mathrm{L} r b}\right) \\
& i\left(\lambda^{A}\right)_{a}^{b} G_{\mathrm{L}}^{A \mu}{ }_{\nu}\left(\bar{d}_{\mathrm{R} p}^{a} D_{\mu} u_{\mathrm{L} s c}\right)\left(\bar{u}_{\mathrm{L} t}^{c} \gamma^{\nu} d_{\mathrm{L} r b}\right) \\
& i\left(\lambda^{A}\right)_{d}^{c} G_{\mathrm{L}}^{A \mu}{ }_{\nu}\left(\bar{d}_{\mathrm{R} p}^{a} D_{\mu} u_{\mathrm{L} s c}\right)\left(\bar{u}_{\mathrm{L} t}^{d} \gamma^{\nu} d_{\mathrm{L} r a}\right) \\
& i\left(\lambda^{A}\right)_{a}^{c} G_{\mathrm{L}}^{A \mu}{ }_{\nu}\left(\bar{u}_{\mathrm{L} t}^{b} \gamma^{\nu} d_{\mathrm{L} r b}\right)\left(\bar{d}_{\left.\mathrm{R}_{p}^{a} D_{\mu} u_{\mathrm{L} s c}\right)}\right. \\
& i\left(\lambda^{A}\right)_{d}^{b} \epsilon^{a c d} G_{\mathrm{L}}^{A \mu}{ }_{\nu}\left(e_{\mathrm{L} r} C D_{\mu} u_{\mathrm{L} s b}\right)\left(d_{\mathrm{L} p a} C \gamma^{\nu} u_{\mathrm{R} t c}\right) \\
& i\left(\lambda^{A}\right)_{d}^{d} \epsilon^{b c d} G_{\mathrm{L}}^{A \mu}{ }_{\nu}\left(e_{\mathrm{L} r} C D_{\mu} u_{\mathrm{L} s b}\right)\left(d_{\mathrm{L} p a} C \gamma^{\nu} u_{\mathrm{R} t c}\right) \\
& \mathcal{O}_{G_{\mathrm{L}} d_{\mathrm{L}} e_{\mathrm{L}} u_{\mathrm{R}} u_{\mathrm{L}} D}^{(1 \sim 8)}(1,1) \mid \begin{array}{l}
i\left(\lambda^{A}\right)_{d}^{b} \epsilon^{a c d} G_{\mathrm{L}}^{A \mu}{ }_{\nu}\left(D_{\mu} e_{\mathrm{L} r} C u_{\mathrm{L} s b}\right)\left(d_{\mathrm{L} p a} C \gamma^{\nu} u_{\mathrm{R} t c}\right) \\
i\left(\lambda^{A}\right)_{d}^{d} \epsilon^{b c d} G_{\mathrm{L}}^{A \mu}{ }_{\nu}\left(D_{\mu} e_{\mathrm{L} r} C u_{\mathrm{L} s b}\right)\left(d_{\mathrm{L} p a} C \gamma^{\nu} u_{\mathrm{R} t c}\right)
\end{array} \\
& i\left(\lambda^{A}\right)_{d}^{b} \epsilon^{a c d} G_{\mathrm{L}}^{A \mu}{ }_{\nu}\left(d_{\mathrm{L} p a} C u_{\mathrm{L} s b}\right)\left(D_{\mu} e_{\mathrm{L} r} C \gamma^{\nu} u_{\mathrm{R} t c}\right) \\
& i\left(\lambda^{A}\right)_{d}^{d} \epsilon^{b c d} G_{\mathrm{L}}^{A \mu}{ }_{\nu}\left(d_{\mathrm{L} p a} C u_{\mathrm{L} s b}\right)\left(D_{\mu} e_{\mathrm{L} r} C \gamma^{\nu} u_{\mathrm{R} t c}\right) \\
& i\left(\lambda^{A}\right)_{d}^{b} \epsilon^{a c d} G_{\mathrm{L}}^{A \mu}{ }_{\nu}\left(e_{\mathrm{L} r} C \gamma^{\nu} u_{\mathrm{R} t c}\right)\left(d_{\mathrm{L} p a} C D_{\mu} u_{\mathrm{L} s b}\right) \\
& i\left(\lambda^{A}\right)_{d}^{d} \epsilon^{b c d} G_{\mathrm{L}}^{A \mu}{ }_{\nu}\left(e_{\mathrm{L} r} C \gamma^{\nu} u_{\mathrm{R} t c}\right)\left(d_{\mathrm{L} p a} C D_{\mu} u_{\mathrm{L} s b}\right)
\end{aligned}
$$




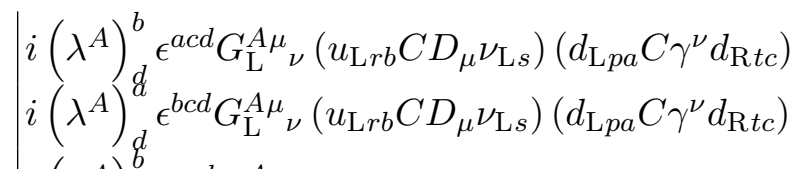

$$
\begin{aligned}
& \mathcal{O}_{G_{\mathrm{L}} d_{\mathrm{R}} d_{\mathrm{L}} \nu_{\mathrm{L}} u_{\mathrm{L}} D}^{(1,1)} \mid \begin{array}{l}
i\left(\lambda^{A}\right)_{d}^{b} \epsilon^{a c d} G_{\mathrm{L}}^{A \mu}{ }_{\nu}\left(D_{\mu} u_{\mathrm{L} r b} C \nu_{\mathrm{L} s}\right)\left(d_{\mathrm{L} p a} C \gamma^{\nu} d_{\mathrm{R} t c}\right) \\
i\left(\lambda^{A}\right)_{d}^{d} \epsilon^{b c d} G_{\mathrm{L}}^{A \mu}{ }_{\nu}\left(D_{\mu} u_{\mathrm{L} r b} C \nu_{\mathrm{L} s}\right)\left(d_{\mathrm{L} p a} C \gamma^{\nu} d_{\mathrm{R} t c}\right)
\end{array} \\
& i\left(\lambda^{A}\right)_{d}^{b} \epsilon^{a c d} G_{\mathrm{L}}^{A \mu}{ }_{\nu}\left(d_{\mathrm{L} p a} C \nu_{\mathrm{L} s}\right)\left(D_{\mu} u_{\mathrm{L} r b} C \gamma^{\nu} d_{\mathrm{R} t c}\right) \\
& i\left(\lambda^{A}\right)_{d}^{d} \epsilon^{b c d} G_{\mathrm{L}}^{A \mu}{ }_{\nu}\left(d_{\mathrm{L} p a} C \nu_{\mathrm{L} s}\right)\left(D_{\mu} u_{\mathrm{L} r b} C \gamma^{\nu} d_{\mathrm{R} t c}\right) \\
& i\left(\lambda^{A}\right)_{d}^{b} \epsilon^{a c d} G_{\mathrm{L}}^{A \mu}{ }_{\nu}\left(d_{\mathrm{L} p a} C D_{\mu} \nu_{\mathrm{L} s}\right)\left(u_{\mathrm{L} r b} C \gamma^{\nu} d_{\mathrm{R} t c}\right) \\
& i\left(\lambda^{A}\right)_{d}^{d} \epsilon^{b c d} G_{\mathrm{L}}^{A \mu}{ }_{\nu}\left(d_{\mathrm{L} p a} C D_{\mu} \nu_{\mathrm{L} s}\right)\left(u_{\mathrm{L} r b} C \gamma^{\nu} d_{\mathrm{R} t c}\right) \\
& \mathcal{O}_{G_{\mathrm{L}} \bar{d}_{\mathrm{R}} e_{\mathrm{L}} \bar{\nu}_{\mathrm{L}} u_{\mathrm{L}} D}^{(1 \sim 4)} \mid \begin{array}{l}
i\left(\lambda^{A}\right)_{a}^{b} G_{\mathrm{L}}^{A \mu}{ }_{\nu}\left(e_{\mathrm{L} r} C D_{\mu} u_{\mathrm{L} s b}\right)\left(\bar{d}_{\mathrm{R} p}^{a} \gamma^{\nu} C \bar{\nu}_{\mathrm{L} t}\right) \\
i\left(\lambda^{A}\right)_{a}^{b} G_{\mathrm{L}}^{A \mu}{ }_{\nu}\left(D_{\mu} e_{\mathrm{L} r} C u_{\mathrm{L} s b}\right)\left(\bar{d}_{\mathrm{R} p}^{a} \gamma^{\nu} C \bar{\nu}_{\mathrm{L} t}\right)
\end{array} \\
& \begin{array}{l}
i\left(\lambda^{A}\right)_{a}^{b} G_{\mathrm{L}}^{A \mu}{ }_{\nu}\left(\bar{d}_{\mathrm{R} p}^{a} u_{\mathrm{L} s b}\right)\left(\bar{\nu}_{\mathrm{L} t} \gamma^{\nu} D_{\mu} e_{\mathrm{L} r}\right) \\
i\left(\lambda^{A}\right)_{a}^{b} G_{\mathrm{L}}^{A \mu}{ }_{\nu}\left(\bar{\nu}_{\mathrm{L} t} \gamma^{\nu} e_{\mathrm{L} r}\right)\left(\bar{d}_{\mathrm{R}}{ }^{a} D_{\mu} u_{\mathrm{L} s b}\right)
\end{array} \\
& \mathcal{O}_{G_{\mathrm{L}} \bar{d}_{\mathrm{R}} e_{\mathrm{R}} \nu_{\mathrm{L}} u_{\mathrm{L}} D}^{(1 \sim 2)} \mid \begin{array}{l}
i\left(\lambda^{A}\right)_{a}^{b} G_{\mathrm{L}}^{A \mu}{ }_{\nu}\left(u_{\mathrm{L} r b} C D_{\mu} \nu_{\mathrm{L} s}\right)\left(\bar{d}_{\mathrm{R}}{ }_{p}^{a} \gamma^{\nu} e_{\mathrm{R} t}\right) \\
i\left(\lambda^{A}\right)_{a}^{b} G_{\mathrm{L}}^{A \mu}{ }_{\nu}\left(D_{\mu} u_{\mathrm{L} r b} C \nu_{\mathrm{L} s}\right)\left(\bar{d}_{\mathrm{R}}{ }_{p}^{a} \gamma^{\nu} e_{\mathrm{R} t}\right)
\end{array} \\
& i\left(\lambda^{A}\right)_{a}^{b} G_{\mathrm{L}}^{A \mu}{ }_{\nu}\left(\bar{d}_{\mathrm{R} p}^{a} \nu_{\mathrm{L} s}\right)\left(D_{\mu} u_{\mathrm{L} r b} C \gamma^{\nu} e_{\mathrm{R} t}\right) \\
& i\left(\lambda^{A}\right)_{a}^{b} G_{\mathrm{L}}^{A \mu}{ }_{\nu}\left(u_{\mathrm{L} r b} C \gamma^{\nu} e_{\mathrm{R} t}\right)\left(\bar{d}_{\mathrm{R}}^{a} D_{\mu} \nu_{\mathrm{L} s}\right) \\
& i\left(\lambda^{A}\right)_{b}^{a} G_{\mathrm{L}}^{A \mu}{ }_{\nu}\left(e_{\mathrm{L} r} C D_{\mu} u_{\mathrm{L} s a}\right)\left(\bar{e}_{\mathrm{R} p} \gamma^{\nu} C \bar{u}_{\mathrm{L} t}^{b}\right) \\
& \mathcal{O}_{G_{\mathrm{L}} \bar{e}_{\mathrm{R}} e_{\mathrm{L}} u_{\mathrm{L}} \bar{u}_{\mathrm{L}} D}^{(1 \sim 4)}(0,0) i\left(\lambda^{A}\right)_{b}^{b} G_{\mathrm{L}}^{A \mu}{ }_{\nu}\left(D_{\mu} e_{\mathrm{L} r} C u_{\mathrm{L} s a}\right)\left(\bar{e}_{\mathrm{R} p} \gamma^{\nu} C \bar{u}_{\mathrm{L} t}^{b}\right) \\
& i\left(\lambda^{A}\right)_{b}^{a} G_{\mathrm{L}}^{A \mu}{ }_{\nu}\left(\bar{e}_{\mathrm{R} p} u_{\mathrm{L} s a}\right)\left(\bar{u}_{\mathrm{L} t}^{b} \gamma^{\nu} D_{\mu} e_{\mathrm{L} r}\right) \\
& i\left(\lambda^{A}\right)_{b}^{a} G_{\mathrm{L}}^{A \mu}{ }_{\nu}\left(\bar{e}_{\mathrm{R} p} D_{\mu} u_{\mathrm{L} s a}\right)\left(\bar{u}_{\mathrm{L} t}^{b} \gamma^{\nu} e_{\mathrm{L} r}\right) \\
& \mathcal{O}_{G_{\mathrm{L}} \bar{d}_{\mathrm{L}} e_{\mathrm{L}} \nu_{\mathrm{L}} u_{\mathrm{L}} D}^{(1 \sim 2)} \mid \begin{array}{l}
i\left(\lambda^{A}\right)_{b}^{a} G_{\mathrm{L}}^{A \mu}{ }_{\nu}\left(u_{\mathrm{L} r a} C D_{\mu} \nu_{\mathrm{L} s}\right)\left(\bar{d}_{\mathrm{L} t}^{b} \gamma^{\nu} e_{\mathrm{L} p}\right) \\
i\left(\lambda^{A}\right)_{b}^{a} G_{\mathrm{L}}^{A \mu}{ }_{\nu}\left(D_{\mu} u_{\mathrm{L} r a} C \nu_{\mathrm{L} s}\right)\left(\bar{d}_{\mathrm{L} t}^{b} \gamma^{\nu} e_{\mathrm{L} p}\right) \\
i\left(\lambda^{A}\right)_{b}^{a} G_{\mathrm{L}}^{A \mu}{ }_{\nu}\left(e_{\mathrm{L} p} C \nu_{\mathrm{L} s}\right)\left(\bar{d}_{\mathrm{L} t}^{b} \gamma^{\nu} D_{\mu} u_{\mathrm{L} r a}\right) \\
i\left(\lambda^{A}\right)_{b}^{a} G_{\mathrm{L}}^{A \mu}{ }_{\nu}\left(e_{\mathrm{L} p} C D_{\mu} \nu_{\mathrm{L} s}\right)\left(\bar{d}_{\mathrm{L} t}^{b} \gamma^{\nu} u_{\mathrm{L} r a}\right)
\end{array}
\end{aligned}
$$

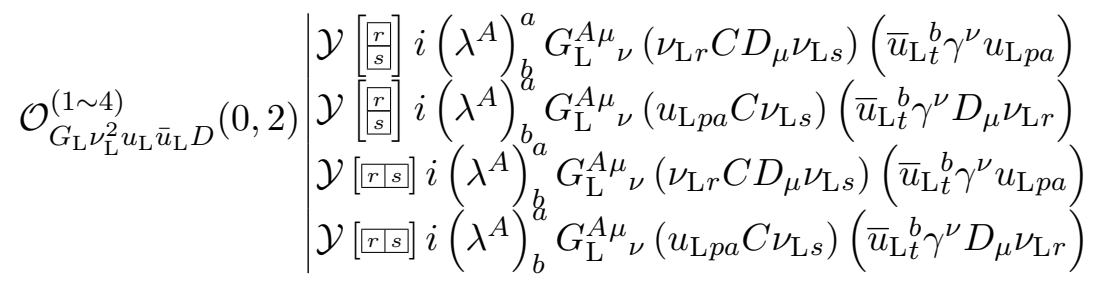




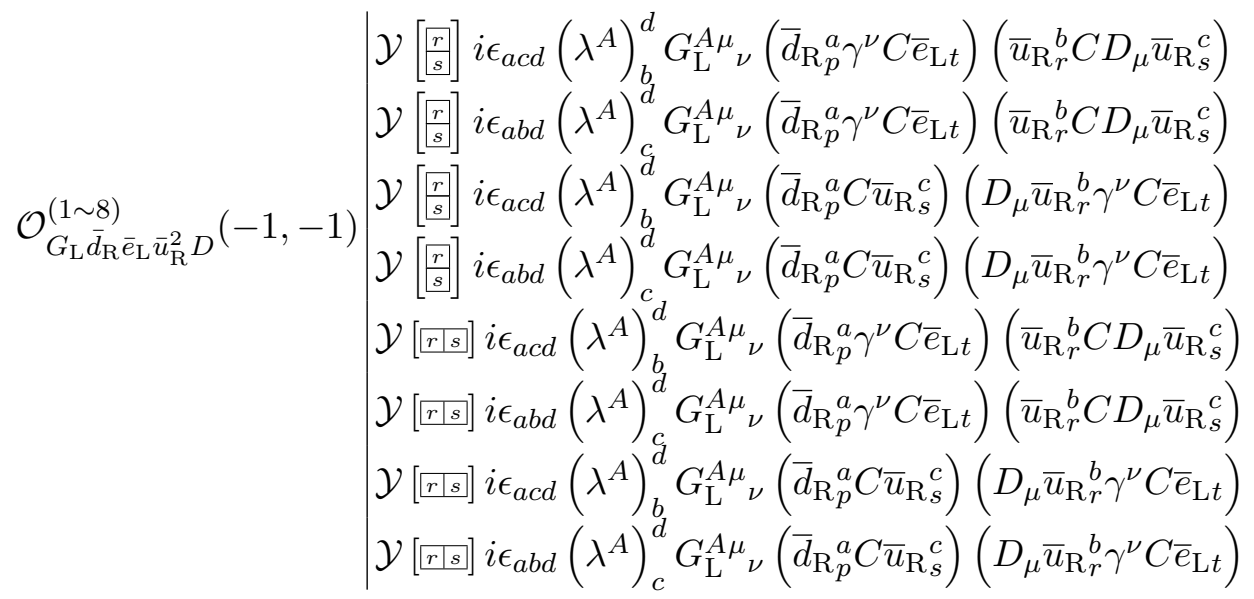

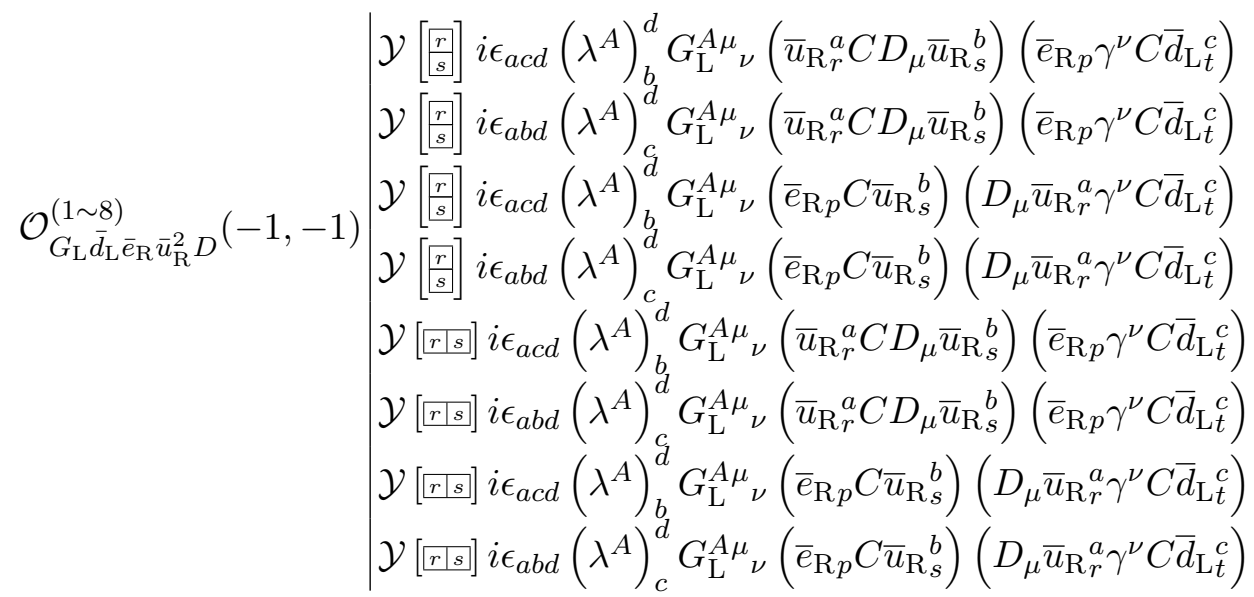




$$
\begin{aligned}
& \mid \begin{array}{l}
i\left(\lambda^{A}\right)_{c}^{b} G_{\mathrm{L}}^{A \mu}{ }_{\nu}\left(\bar{d}_{\mathrm{R} p}^{a} \gamma^{\nu} u_{\mathrm{R} t a}\right)\left(D_{\mu} \bar{u}_{\mathrm{R}}{ }_{s}^{c} d_{\mathrm{L} r b}\right) \\
i\left(\lambda^{A}\right)_{a}^{b} G_{\mathrm{L}}^{A \mu}{ }_{\nu}\left(\bar{d}_{\mathrm{R}}^{a} \gamma^{\nu} u_{\mathrm{R} t c}\right)\left(D_{\mu} \bar{u}_{\mathrm{R}}{ }_{s}^{c} d_{\mathrm{L} r b}\right) \\
i\left(\lambda^{A}\right)_{c}^{d} G_{\mathrm{L}}^{A \mu}{ }_{\nu}\left(D_{\mu} \bar{u}_{\mathrm{R}}^{c}{ }_{\mathrm{L} r a}\right)\left(\bar{d}_{\mathrm{R}}^{a}{ }_{p}^{\nu} \gamma_{\mathrm{R} t d}\right) \\
i\left(\lambda^{A}\right)_{a}^{d} G_{\mathrm{L}}^{A \mu}{ }_{\nu}\left(D_{\mu} \bar{u}_{\mathrm{R}}{ }^{b} d_{\mathrm{L} r b}\right)\left(\bar{d}_{\mathrm{R} p}^{a} \gamma^{\nu} u_{\mathrm{R} t d}\right) \\
i\left(\lambda^{A}\right)_{c}^{b} G_{\mathrm{L}}^{A \mu}{ }_{\nu}\left(\bar{d}_{\mathrm{R} p}^{a} \gamma^{\nu} u_{\mathrm{R} t a}\right)\left(\bar{u}_{\mathrm{R}}{ }_{s}^{c} D_{\mu} d_{\mathrm{L} r b}\right)
\end{array} \\
& \mathcal{O}_{G_{\mathrm{L}} \bar{d}_{\mathrm{R}} d_{\mathrm{L}} \bar{u}_{\mathrm{R}} u_{\mathrm{R}} D}^{(1 \sim 10)}(0,0) i\left(\lambda^{A}\right)_{a}^{b} G_{\mathrm{L}}^{A \mu}{ }_{\nu}\left(\bar{d}_{\mathrm{R}}{ }^{a} \gamma^{\nu} u_{\mathrm{R} t c}\right)\left(\bar{u}_{\mathrm{R}}^{c} D_{\mu} D_{\mu} d_{\mathrm{L} r b}\right) \\
& i\left(\lambda^{A}\right)_{c}^{d} G_{\mathrm{L}}^{A \mu}{ }_{\nu}\left(\bar{u}_{\mathrm{R}}^{c}{ }_{s}^{c} D_{\mu} d_{\mathrm{L} r a}\right)\left(\bar{d}_{\mathrm{R}}^{a} \gamma^{\nu} u_{\mathrm{R} t d}\right) \\
& i\left(\lambda^{A}\right)_{a}^{d} G_{\mathrm{L}}^{A \mu}{ }_{\nu}\left(\bar{u}_{\mathrm{R} s}^{b} D_{\mu} d_{\mathrm{L} r b}\right)\left(\bar{d}_{\mathrm{R} p}^{a} \gamma^{\nu} u_{\mathrm{R} t d}\right) \\
& i\left(\lambda^{A}\right)_{c}^{b} G_{\mathrm{L}}^{A \mu}{ }_{\nu}\left(\bar{d}_{\mathrm{R} p}^{a} C \bar{u}_{\mathrm{R}}{ }_{s}\right)\left(D_{\mu} d_{\mathrm{L} r b} C \gamma^{\nu} u_{\mathrm{R} t a}\right) \\
& i\left(\lambda^{A}\right)_{a}^{b} G_{\mathrm{L}}^{A \mu}{ }_{\nu}\left(\bar{d}_{\mathrm{R} p}^{a} C \bar{u}_{\mathrm{R}}{ }^{c}\right)\left(D_{\mu} d_{\mathrm{L} r b} C \gamma^{\nu} u_{\mathrm{R} t c}\right) \\
& i\left(\lambda^{A}\right)_{c}^{d} G_{\mathrm{L}}^{A \mu}{ }_{\nu}\left(\bar{d}_{\mathrm{R}_{p}^{a} C \bar{u}_{\mathrm{R}}{ }_{s}^{c}}\right)\left(D_{\mu} d_{\mathrm{L} r a} C \gamma^{\nu} u_{\mathrm{R} t d}\right) \\
& i\left(\lambda^{A}\right)_{a}^{d} G_{\mathrm{L}}^{A \mu}{ }_{\nu}\left(\bar{d}_{\mathrm{R} p}^{a} C \bar{u}_{\mathrm{R}}^{b}\right)\left(D_{\mu} d_{\mathrm{L} r b} C \gamma^{\nu} u_{\mathrm{R} t d}\right) \\
& i\left(\lambda^{A}\right)_{C}^{b} G_{\mathrm{L}}^{A \mu}{ }_{\nu}\left(d_{\mathrm{L} r b} C \gamma^{\nu} u_{\mathrm{R} t a}\right)\left(\bar{d}_{\mathrm{R} p}^{a} C D_{\mu} \bar{u}_{\mathrm{R}}^{c}{ }_{s}\right) \\
& i\left(\lambda^{A}\right)_{a}^{b} G_{\mathrm{L}}^{A \mu}{ }_{\nu}\left(d_{\mathrm{L} r b} C \gamma^{\nu} u_{\mathrm{R} t c}\right)\left(\bar{d}_{\mathrm{R} p}{ }^{a} C D_{\mu} \bar{u}_{\mathrm{R} s}^{c}\right) \\
& \mid \begin{array}{ll}
i\left(\lambda^{A}\right)_{c}^{d} G_{\mathrm{L}}^{A \mu}{ }_{\nu}\left(d_{\mathrm{L} r a} C \gamma^{\nu} u_{\mathrm{R} t d}\right) & \left(\bar{d}_{\mathrm{R}}{ }_{p} C D_{\mu} \bar{u}_{\mathrm{R}}^{c}\right) \\
i\left(\lambda^{A}\right)_{a}^{d} G_{\mathrm{L}}^{A \mu}{ }_{\nu}\left(d_{\mathrm{L} r b} C \gamma^{\nu} u_{\mathrm{R} t d}\right) & \left(\bar{d}_{\mathrm{R} p}{ }_{p}^{a} C D_{\mu} \bar{u}_{\mathrm{R}}^{b}\right)
\end{array} \\
& \mathcal{O}_{G_{\mathrm{L}} d_{\mathrm{L}} \bar{e}_{\mathrm{R}} \bar{\nu}_{\mathrm{L}} \bar{u}_{\mathrm{R}} D}^{(0,-2)} \mid \begin{array}{l}
i\left(\lambda^{A}\right)_{b}^{a} G_{\mathrm{L}}^{A \mu}{ }_{\nu}\left(\bar{\nu}_{\mathrm{L} t} \gamma^{\nu} d_{\mathrm{L} p a}\right)\left(\bar{e}_{\mathrm{R} r} C D_{\mu} \bar{u}_{\mathrm{R} s}^{b}\right) \\
i\left(\lambda^{A}\right)_{b}^{a} G_{\mathrm{L}}^{A \mu}{ }_{\nu}\left(\bar{\nu}_{\mathrm{L} t} \gamma^{\nu} d_{\mathrm{L} p a}\right)\left(D_{\mu} \bar{e}_{\mathrm{R} r} C \bar{u}_{\mathrm{R}_{s}}^{b}\right) \\
i\left(\lambda^{A}\right)_{b}^{a} G_{\mathrm{L}}^{A \mu}{ }_{\nu}\left(\bar{u}_{\mathrm{R} s}^{b} d_{\mathrm{L} p a}\right)\left(D_{\mu} \bar{e}_{\mathrm{R} r} \gamma^{\nu} C \bar{\nu}_{\mathrm{L} t}\right) \\
i\left(\lambda^{A}\right)_{b}^{a} G_{\mathrm{L}}^{A \mu}{ }_{\nu}\left(\bar{e}_{\mathrm{R} r} \gamma^{\nu} C \bar{\nu}_{\mathrm{L} t}\right)\left(D_{\mu} \bar{u}_{\mathrm{R} s}^{b} d_{\mathrm{L} p a}\right)
\end{array} \\
& \mathcal{O}_{G_{\mathrm{L}} d_{\mathrm{L}} \bar{e}_{\mathrm{L}} \nu_{\mathrm{L}} \bar{u}_{\mathrm{R}} D}(0,0) \mid \begin{array}{l}
i\left(\lambda^{A}\right)_{b}^{a} G_{\mathrm{L}}^{A \mu}{ }_{\nu}\left(\bar{e}_{\mathrm{L} t} \gamma^{\nu} d_{\mathrm{L} p a}\right)\left(\bar{u}_{\mathrm{R} r}^{b} D_{\mu} \nu_{\mathrm{L} s}\right) \\
i\left(\lambda^{A}\right)_{b}^{a} G_{\mathrm{L}}^{A \mu}{ }_{\nu}\left(\bar{e}_{\mathrm{L} t} \gamma^{\nu} d_{\mathrm{L} p a}\right)\left(D_{\mu} \bar{u}_{\mathrm{R}}^{b} \nu_{\mathrm{L} s}\right) \\
i\left(\lambda^{A}\right)_{b}^{a} G_{\mathrm{L}}^{A \mu}{ }_{\nu}\left(d_{\mathrm{L} p a} C \nu_{\mathrm{L} s}\right)\left(D_{\mu} \bar{u}_{\mathrm{R} r}^{b} \gamma^{\nu} C \bar{e}_{\mathrm{L} t}\right) \\
i\left(\lambda^{A}\right)_{b}^{a} G_{\mathrm{L}}^{A \mu}{ }_{\nu}\left(d_{\mathrm{L} p a} C D_{\mu} \nu_{\mathrm{L} s}\right)\left(\bar{u}_{\mathrm{R}}{ }_{r}^{b} \gamma^{\nu} C \bar{e}_{\mathrm{L} t}\right)
\end{array}
\end{aligned}
$$




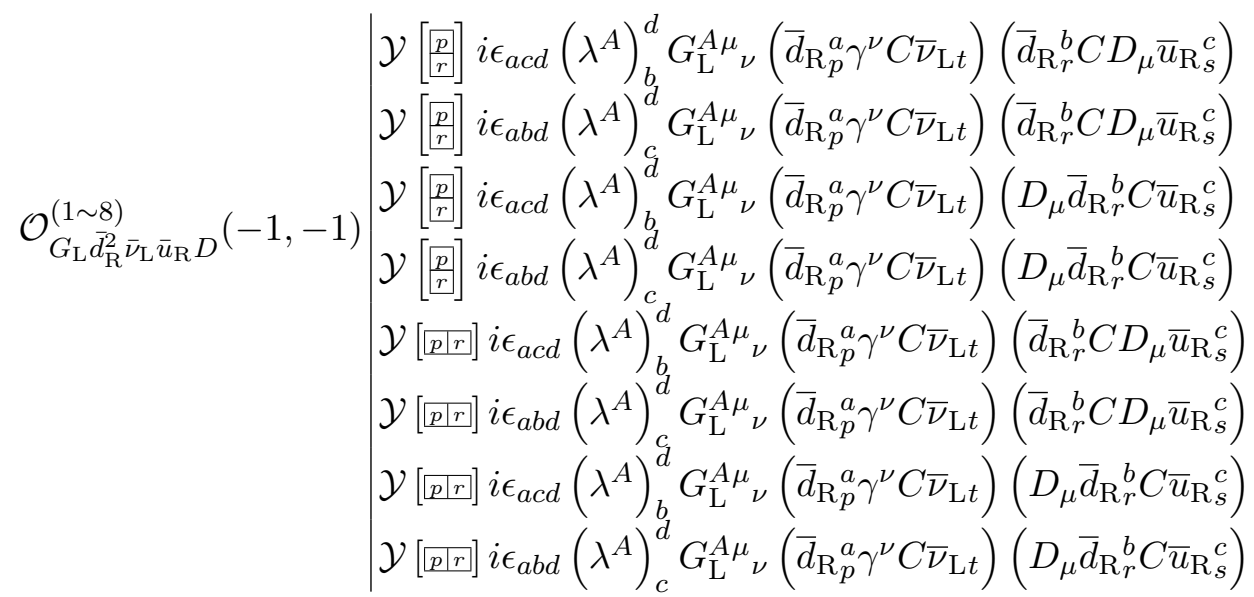

$$
\begin{aligned}
& \mid i \epsilon_{a c d}\left(\lambda^{A}\right)_{b}^{d} G_{\mathrm{L}}^{A \mu}{ }_{\nu}\left(\bar{e}_{\mathrm{R} r} C D_{\mu} \bar{u}_{\mathrm{R}}^{b}\right)\left(\bar{d}_{\mathrm{R} p}^{a} \gamma^{\nu} C \bar{u}_{\mathrm{L}}{ }_{t}^{c}\right)
\end{aligned}
$$

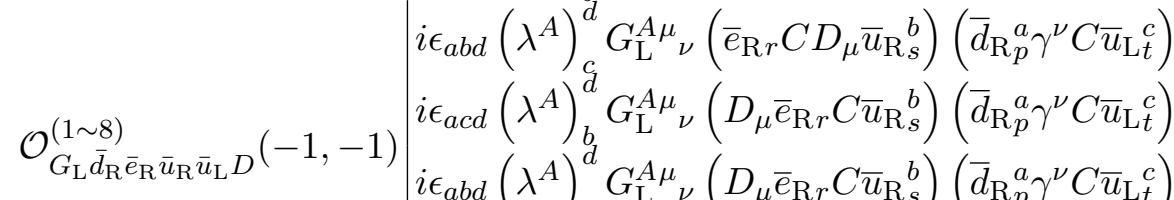

$$
\begin{aligned}
& i \epsilon_{a c d}\left(\lambda^{A}\right)_{b}^{d} G_{\mathrm{L}}^{A \mu}{ }_{\nu}\left(\bar{d}_{\mathrm{R}}^{a} C \bar{u}_{\mathrm{R}}^{b}\right)\left(D_{\mu} \bar{e}_{\mathrm{R} r} \gamma^{\nu} C \bar{u}_{\mathrm{L}}^{c}{ }_{t}^{c}\right) \\
& i \epsilon_{a b d}\left(\lambda^{A}\right)_{C}^{d} G_{\mathrm{L}}^{A \mu}{ }_{\nu}\left(\bar{d}_{\mathrm{R}}{ }_{p}^{a} C \bar{u}_{\mathrm{R} s}^{b}\right)\left(D_{\mu} \bar{e}_{\mathrm{R} r} \gamma^{\nu} C \bar{u}_{\mathrm{L} t}^{c}\right) \\
& i \epsilon_{a c d}\left(\lambda^{A}\right)_{b}^{d} G_{\mathrm{L}}^{A \mu}{ }_{\nu}\left(\bar{e}_{\mathrm{R} r} \gamma^{\nu} C \bar{u}_{\mathrm{L} t}^{c}\right)\left(\bar{d}_{\mathrm{R} p}^{a} C D_{\mu} \bar{u}_{\mathrm{R} s}^{b}\right) \\
& i \epsilon_{a b d}\left(\lambda^{A}\right)_{c}^{d} G_{\mathrm{L}}^{A \mu}{ }_{\nu}\left(\bar{e}_{\mathrm{R} r} \gamma^{\nu} C \bar{u}_{\mathrm{L} t}^{c}\right)\left(\bar{d}_{\left.\mathrm{R}_{p}^{a} C D_{\mu} \bar{u}_{\mathrm{R}}^{b}\right)}\right)
\end{aligned}
$$

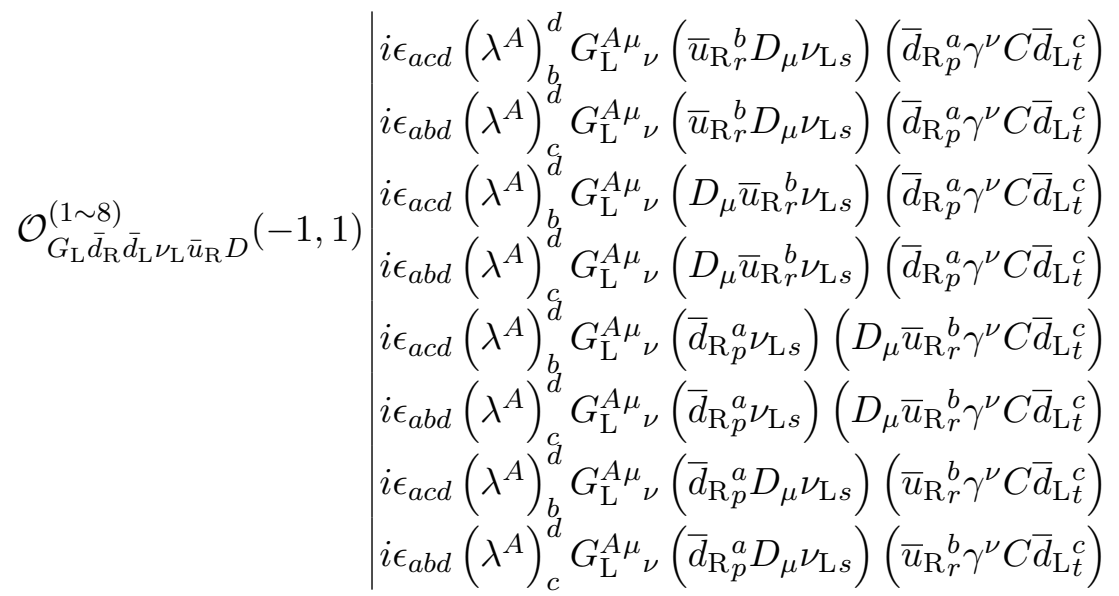

$$
\begin{aligned}
& \mathcal{O}_{G_{\mathrm{L}} \bar{e}_{\mathrm{R}} e_{\mathrm{L}} \bar{u}_{\mathrm{R}} u_{\mathrm{R}} D}^{(1 \sim 0)} \mid \begin{array}{l}
i\left(\lambda^{A}\right)_{a}^{b} G_{\mathrm{L}}^{A \mu}{ }_{\nu}\left(\bar{e}_{\mathrm{R} p} \gamma^{\nu} u_{\mathrm{R} t b}\right)\left(D_{\mu} \bar{u}_{\mathrm{R}}{ }_{s}^{a} e_{\mathrm{L} r}\right) \\
i\left(\lambda^{A}\right)_{a}^{b} G_{\mathrm{L}}^{A \mu}{ }_{\nu}\left(\bar{e}_{\mathrm{R} p} \gamma^{\nu} u_{\mathrm{R} t b}\right)\left(\bar{u}_{\mathrm{R}}{ }^{a} D_{\mu} e_{\mathrm{L} r}\right)
\end{array} \\
& \begin{array}{l}
i\left(\lambda^{A}\right)_{a}^{b} G_{\mathrm{L}}^{A \mu}{ }_{\nu}\left(\bar{e}_{\mathrm{R} p} C \bar{u}_{\mathrm{R}}^{a}{ }_{s}\right)\left(D_{\mu} e_{\mathrm{L} r} C \gamma^{\nu} u_{\mathrm{R} t b}\right) \\
i\left(\lambda^{A}\right)_{a}^{b} G_{\mathrm{L}}^{A \mu}{ }_{\nu}\left(e_{\mathrm{L} r} C \gamma^{\nu} u_{\mathrm{R} t b}\right)\left(\bar{e}_{\mathrm{R} p} C D_{\mu} \bar{u}_{\mathrm{R}}^{a}\right)
\end{array}
\end{aligned}
$$




$$
\begin{aligned}
& \mathcal{O}_{G_{\mathrm{L}} d_{\mathrm{R}} \bar{e}_{\mathrm{R}} \nu_{\mathrm{L}} \bar{u}_{\mathrm{R}} D}^{(0,0)} \mid \begin{array}{l}
i\left(\lambda^{A}\right)_{a}^{b} G_{\mathrm{L}}^{A \mu}{ }_{\nu}\left(\bar{e}_{\mathrm{R} p} \gamma^{\nu} d_{\mathrm{R} t b}\right)\left(\bar{u}_{\mathrm{R}}{ }_{r}^{a} D_{\mu} \nu_{\mathrm{L} s}\right) \\
i\left(\lambda^{A}\right)_{a}^{b} G_{\mathrm{L}}^{A \mu}{ }_{\nu}\left(\bar{e}_{\mathrm{R} p} \gamma^{\nu} d_{\mathrm{R} t b}\right)\left(D_{\mu} \bar{u}_{\mathrm{R} r}^{a} \nu_{\mathrm{L} s}\right) \\
i\left(\lambda^{A}\right)_{a}^{b}\left(\bar{e}_{\mathrm{R} p} \nu_{\mathrm{L} s}\right) G_{\mathrm{L}}^{A \mu}{ }_{\nu}\left(D_{\mu} \bar{u}_{\mathrm{R}}{ }_{r}^{a} \gamma^{\nu} d_{\mathrm{R} t b}\right) \\
i\left(\lambda^{A}\right)_{a}^{b} G_{\mathrm{L}}^{A \mu}{ }_{\nu}\left(\bar{e}_{\mathrm{R} p} D_{\mu} \nu_{\mathrm{L} s}\right)\left(\bar{u}_{\mathrm{R}}{ }_{r} \gamma^{\nu} d_{\mathrm{R} t b}\right)
\end{array}
\end{aligned}
$$

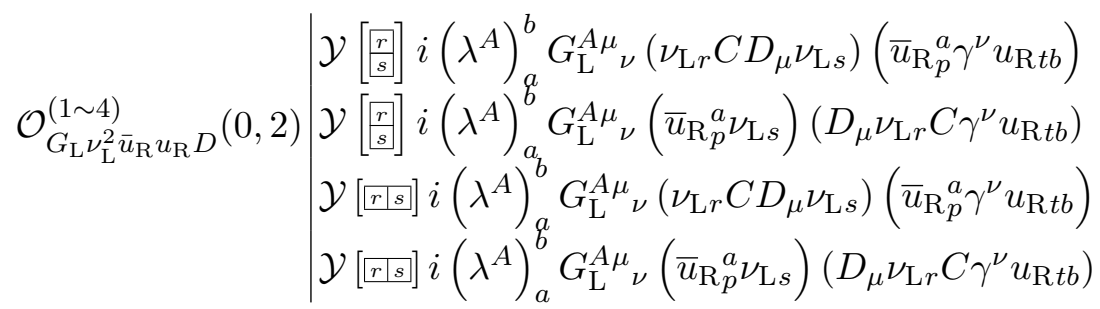

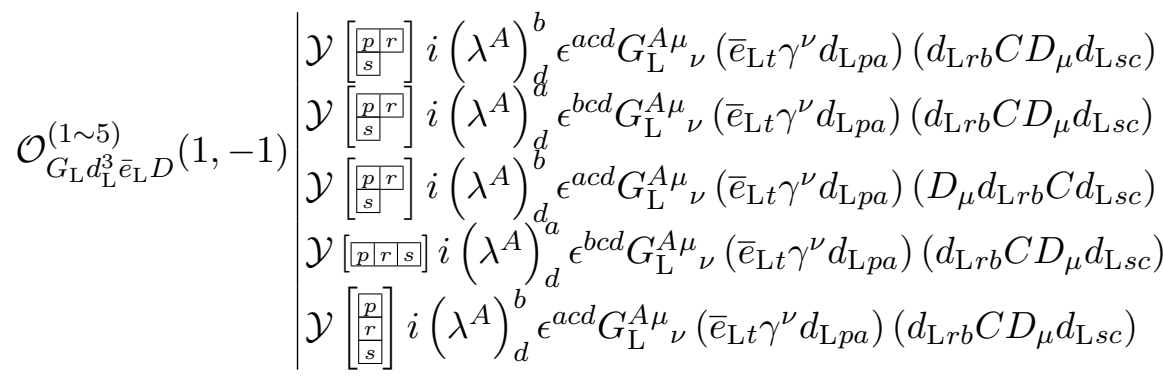

$$
\begin{aligned}
& \mathcal{Y}\left[\begin{array}{c}
r \\
s
\end{array}\right] i\left(\lambda^{A}\right)_{d}^{b} G_{\mathrm{L}}^{A \mu}{ }_{\nu}\left(d_{\mathrm{L} r b} C D_{\mu} d_{\mathrm{L} s a}\right)\left(\bar{d}_{\mathrm{R}}^{a} \gamma^{\nu} C \bar{d}_{\mathrm{L} t}^{d}\right) \\
& \mathcal{Y}\left[\begin{array}{l}
r \\
s
\end{array}\right] i\left(\lambda^{A}\right)_{a}^{b} G_{\mathrm{L}}^{A \mu}{ }_{\nu}\left(d_{\mathrm{L} r b} C D_{\mu} d_{\mathrm{L} s c}\right)\left(\bar{d}_{\mathrm{R}}^{a}{ }^{a} \gamma^{\nu} C \bar{d}_{\mathrm{L} t}^{c}\right) \\
& \mathcal{Y}\left[\frac{r}{s}\right] i\left(\lambda^{A}\right)_{d}^{c} G_{\mathrm{L}}^{A \mu}{ }_{\nu}\left(d_{\mathrm{L} r a} C D_{\mu} d_{\mathrm{L} s c}\right)\left(\bar{d}_{\mathrm{R}_{p}^{a}} \gamma^{\nu} C \bar{d}_{\mathrm{L} t}^{d}\right) \\
& \mathcal{Y}\left[\frac{r}{s}\right] i\left(\lambda^{A}\right)_{a}^{c} G_{\mathrm{L}}^{A \mu}{ }_{\nu}\left(d_{\mathrm{L} r b} C D_{\mu} d_{\mathrm{L} s c}\right)\left(\bar{d}_{\mathrm{R} p}^{a} \gamma^{\nu} C \bar{d}_{\mathrm{L} t}^{b}\right) \\
& \mathcal{Y}\left[\frac{r}{s}\right] i\left(\lambda^{A}\right)_{d}^{b} G_{\mathrm{L}}^{A \mu}{ }_{\nu}\left(\bar{d}_{\mathrm{R} p}^{a} d_{\mathrm{L} s a}\right)\left(\bar{d}_{\mathrm{L} t}{ }^{d} \gamma^{\nu} D_{\mu} d_{\mathrm{L} r b}\right)
\end{aligned}
$$

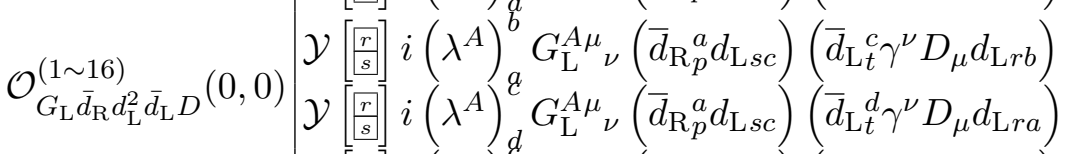

$$
\begin{aligned}
& \mathcal{Y}\left[\frac{r}{s}\right] i\left(\lambda^{A}\right)_{a}^{c} G_{\mathrm{L}}^{A \mu}{ }_{\nu}\left(\bar{d}_{\mathrm{R} p}^{a} d_{\mathrm{L} s c}\right)\left(\bar{d}_{\mathrm{L} t}^{b} \gamma^{\nu} D_{\mu} d_{\mathrm{L} r b}\right) \\
& \mathcal{Y}\left[[r s] i\left(\lambda^{A}\right)_{d}^{b} G_{\mathrm{L}}^{A \mu}{ }_{\nu}\left(d_{\mathrm{L} r b} C D_{\mu} d_{\mathrm{L} s a}\right)\left(\bar{d}_{\left.\mathrm{R}_{p}^{a} \gamma^{\nu} C \bar{d}_{\mathrm{L} t}^{d}\right)}\right)\right. \\
& \mathcal{Y}\left[[r s] i\left(\lambda^{A}\right)_{a}^{b} G_{\mathrm{L}}^{A \mu}{ }_{\nu}\left(d_{\mathrm{L} r b} C D_{\mu} d_{\mathrm{L} s c}\right)\left(\bar{d}_{\mathrm{R} p}^{a} \gamma^{\nu} C \bar{d}_{\mathrm{L} t}^{c}\right)\right. \\
& \mathcal{Y}\left[[r s] i\left(\lambda^{A}\right)_{d}^{c} G_{\mathrm{L}}^{A \mu}{ }_{\nu}\left(d_{\mathrm{L} r a} C D_{\mu} d_{\mathrm{L} s c}\right)\left(\bar{d}_{\mathrm{R} p}^{a} \gamma^{\nu} C \bar{d}_{\mathrm{L} t}^{d}\right)\right. \\
& \mathcal{Y}[\overline{r r s}] i\left(\lambda^{A}\right)_{a}^{c} G_{\mathrm{L}}^{A \mu}{ }_{\nu}\left(d_{\mathrm{L} r b} C D_{\mu} d_{\mathrm{L} s c}\right)\left(\bar{d}_{\mathrm{R} p}^{a} \gamma^{\nu} C \bar{d}_{\mathrm{L} t}^{b}\right) \\
& \mathcal{Y}[\overline{r r s}] i\left(\lambda^{A}\right)_{d}^{b} G_{\mathrm{L}}^{A \mu}{ }_{\nu}\left(\bar{d}_{\mathrm{R} p}^{a} d_{\mathrm{L} s a}\right)\left(\bar{d}_{\mathrm{L} t}{ }^{d} \gamma^{\nu} D_{\mu} d_{\mathrm{L} r b}\right) \\
& \mathcal{Y}\left[[r s] i\left(\lambda^{A}\right)_{a}^{b} G_{\mathrm{L}}^{A \mu}{ }_{\nu}\left(\bar{d}_{\mathrm{R} p}^{a} d_{\mathrm{L} s c}\right)\left(\bar{d}_{\mathrm{L} t}^{c} \gamma^{\nu} D_{\mu} d_{\mathrm{L} r b}\right)\right. \\
& \mathcal{Y}[\overline{r r s}] i\left(\lambda^{A}\right)_{d}^{c} G_{\mathrm{L}}^{A \mu}{ }_{\nu}\left(\bar{d}_{\mathrm{R} p}^{a} d_{\mathrm{L} s c}\right)\left(\bar{d}_{\mathrm{L} t}{ }^{d} \gamma^{\nu} D_{\mu} d_{\mathrm{L} r a}\right) \\
& \mathcal{Y}\left[[r \mid s] i\left(\lambda^{A}\right)_{a}^{c} G_{\mathrm{L}}^{A \mu}{ }_{\nu}\left(\bar{d}_{\mathrm{R} p}^{a} d_{\mathrm{L} s c}\right)\left(\bar{d}_{\mathrm{L} t}^{b} \gamma^{\nu} D_{\mu} d_{\mathrm{L} r b}\right)\right.
\end{aligned}
$$




$$
\begin{aligned}
& \mid \begin{array}{l}
\mathcal{Y}\left[\begin{array}{l}
{\left[\frac{p}{r}\right]} \\
]
\end{array} i\left(\lambda^{A}\right)_{d}^{b} \epsilon^{a c d} G_{\mathrm{L}}^{A \mu}{ }_{\nu}\left(D_{\mu} \bar{e}_{\mathrm{R} s} d_{\mathrm{L} r b}\right)\left(d_{\mathrm{L} p a} C \gamma^{\nu} d_{\mathrm{R} t c}\right)\right. \\
\mathcal{Y}\left[\begin{array}{l}
\frac{p}{r} \\
{[}
\end{array}\right] i\left(\lambda^{A}\right)_{d}^{d} \epsilon^{b c d} G_{\mathrm{L}}^{A \mu}{ }_{\nu}\left(D_{\mu} \bar{e}_{\mathrm{R} s} d_{\mathrm{L} r b}\right)\left(d_{\mathrm{L} p a} C \gamma^{\nu} d_{\mathrm{R} t c}\right)
\end{array}
\end{aligned}
$$

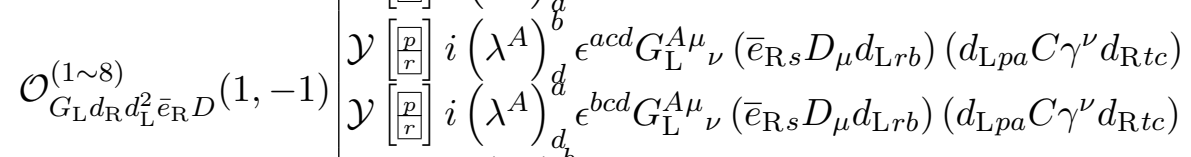

$$
\begin{aligned}
& \mathcal{Y}[\overline{p p r}] i\left(\lambda^{A}\right)_{d}^{b} \epsilon^{a c d} G_{\mathrm{L}}^{A \mu}{ }_{\nu}\left(D_{\mu} \bar{e}_{\mathrm{R} s} d_{\mathrm{L} r b}\right)\left(d_{\mathrm{L} p a} C \gamma^{\nu} d_{\mathrm{R} t c}\right) \\
& \mathcal{Y}[\overline{p p r}] i\left(\lambda^{A}\right)_{d}^{d} \epsilon^{b c d} G_{\mathrm{L}}^{A \mu}{ }_{\nu}\left(D_{\mu} \bar{e}_{\mathrm{R} s} d_{\mathrm{L} r b}\right)\left(d_{\mathrm{L} p a} C \gamma^{\nu} d_{\mathrm{R} t c}\right) \\
& \mathcal{Y}[\overline{p p r}] i\left(\lambda^{A}\right)_{d}^{b} \epsilon^{a c d} G_{\mathrm{L}}^{A \mu}{ }_{\nu}\left(\bar{e}_{\mathrm{R} s} D_{\mu} d_{\mathrm{L} r b}\right)\left(d_{\mathrm{L} p a} C \gamma^{\nu} d_{\mathrm{R} t c}\right) \\
& \mathcal{Y}[\overline{p p r}] i\left(\lambda^{A}\right)_{d}^{d} \epsilon^{b c d} G_{\mathrm{L}}^{A \mu}{ }_{\nu}\left(\bar{e}_{\mathrm{R} s} D_{\mu} d_{\mathrm{L} r b}\right)\left(d_{\mathrm{L} p a} C \gamma^{\nu} d_{\mathrm{R} t c}\right)
\end{aligned}
$$

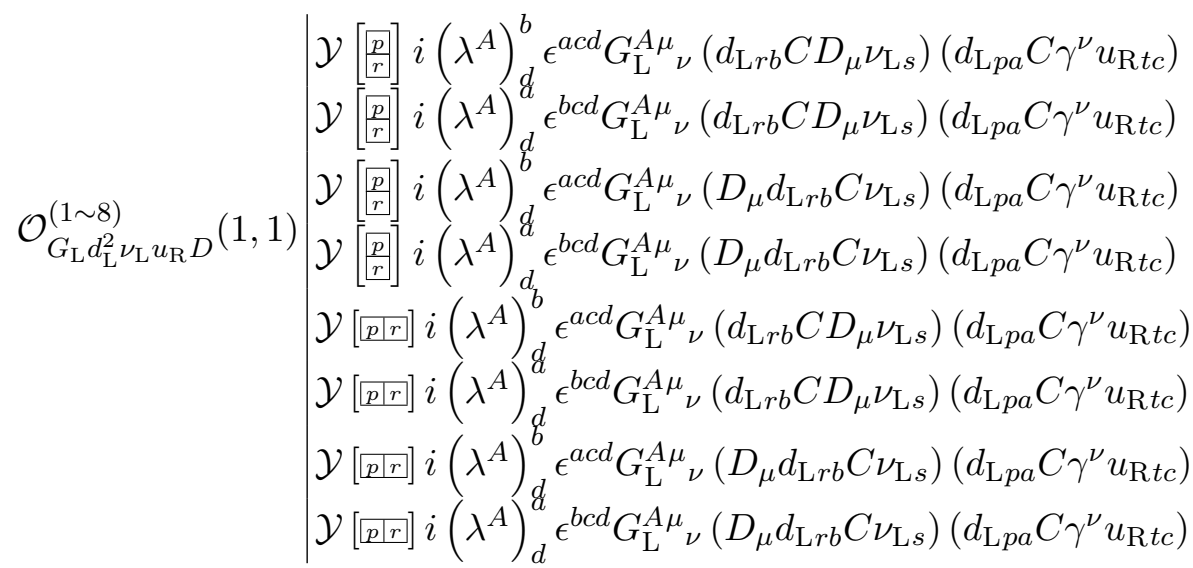




$$
\begin{aligned}
& \mid \mathcal{Y}\left[\frac{p}{r}\right] i\left(\lambda^{A}\right)_{b}^{d} G_{\mathrm{L}}^{A \mu}{ }_{\nu}\left(\bar{d}_{\mathrm{R}}^{a} \gamma^{\nu} d_{\mathrm{R} t d}\right)\left(\bar{d}_{\mathrm{R} r}^{b} D_{\mu} d_{\mathrm{L} s a}\right) \\
& \mathcal{Y}\left[\frac{p}{r}\right] i\left(\lambda^{A}\right)_{a}^{d} G_{\mathrm{L}}^{A \mu}{ }_{\nu}\left(\bar{d}_{\mathrm{R} p}^{a} \gamma^{\nu} d_{\mathrm{R} t d}\right)\left(\bar{d}_{\mathrm{R} r}^{b} D_{\mu} d_{\mathrm{L} s b}\right) \\
& \mathcal{Y}\left[\frac{p}{r}\right] i\left(\lambda^{A}\right)_{a}^{c} G_{\mathrm{L}}^{A \mu}{ }_{\nu}\left(\bar{d}_{\mathrm{R} p}^{a} \gamma^{\nu} d_{\mathrm{R} t b}\right)\left(\bar{d}_{\mathrm{R} r}^{b} D_{\mu} d_{\mathrm{L} s c}\right) \\
& \mathcal{Y}\left[\frac{p}{r}\right] i\left(\lambda^{A}\right)_{b}^{c} G_{\mathrm{L}}^{A \mu}{ }_{\nu}\left(\bar{d}_{\mathrm{R} p}^{a} \gamma^{\nu} d_{\mathrm{R} t a}\right)\left(\bar{d}_{\mathrm{R} r}^{b} D_{\mu} d_{\mathrm{L} s c}\right) \\
& \mathcal{Y}\left[\frac{p}{r}\right] i\left(\lambda^{A}\right)_{b}^{d} G_{\mathrm{L}}^{A \mu}{ }_{\nu}\left(\bar{d}_{\mathrm{R} p}^{a} \gamma^{\nu} d_{\mathrm{R} t d}\right)\left(D_{\mu} \bar{d}_{\mathrm{R}}{ }_{r}^{b} d_{\mathrm{L} s a}\right)
\end{aligned}
$$

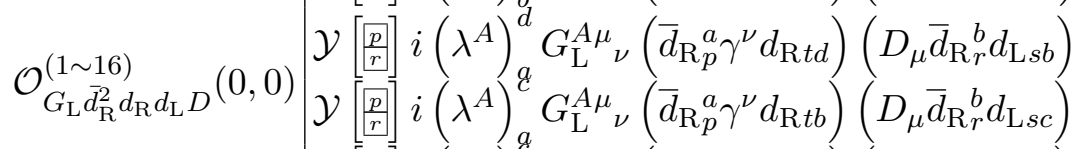

$$
\begin{aligned}
& \mathcal{Y}\left[\frac{p}{r}\right] i\left(\lambda^{A}\right)_{b}^{a} G_{\mathrm{L}}^{A \mu}{ }_{\nu}\left(\bar{d}_{\mathrm{R}}^{a} \gamma^{\nu} d_{\mathrm{R} t a}\right)\left(D_{\mu} \bar{d}_{\mathrm{R}}{ }_{r}^{b} d_{\mathrm{L} s c}\right) \\
& \mathcal{Y}[\overline{p r r}] i\left(\lambda^{A}\right)_{b}^{d} G_{\mathrm{L}}^{A \mu}{ }_{\nu}\left(\bar{d}_{\mathrm{R}}^{a} \gamma^{\nu} d_{\mathrm{R} t d}\right)\left(\bar{d}_{\mathrm{R} r}^{b} D_{\mu} d_{\mathrm{L} s a}\right) \\
& \mathcal{Y}[\overline{p p r}] i\left(\lambda^{A}\right)_{a}^{d} G_{\mathrm{L}}^{A \mu}{ }_{\nu}\left(\bar{d}_{\mathrm{R} p}^{a} \gamma^{\nu} d_{\mathrm{R} t d}\right)\left(\bar{d}_{\mathrm{R} r}^{b} D_{\mu} d_{\mathrm{L} s b}\right) \\
& \mathcal{Y}[\overline{p r r}] i\left(\lambda^{A}\right)_{a}^{a} G_{\mathrm{L}}^{A \mu}{ }_{\nu}\left(\bar{d}_{\mathrm{R}}^{a} \gamma^{\nu} d_{\mathrm{R} t b}\right)\left(\bar{d}_{\mathrm{R} r}^{b} D_{\mu} d_{\mathrm{L} s c}\right) \\
& \mathcal{Y}\left[\overline{p \mid r]} i\left(\lambda^{A}\right)_{b}^{a} G_{\mathrm{L}}^{A \mu}{ }_{\nu}\left(\bar{d}_{\mathrm{R} p}^{a} \gamma^{\nu} d_{\mathrm{R} t a}\right)\left(\bar{d}_{\mathrm{R} r}^{b} D_{\mu} d_{\mathrm{L} s c}\right)\right. \\
& \mathcal{Y}[\overline{p r r}] i\left(\lambda^{A}\right)_{b}^{d} G_{\mathrm{L}}^{A \mu}{ }_{\nu}\left(\bar{d}_{\mathrm{R}}^{a} \gamma^{\nu} d_{\mathrm{R} t d}\right)\left(D_{\mu} \bar{d}_{\mathrm{R} r}^{b} d_{\mathrm{L} s a}\right) \\
& \mathcal{Y}[\overline{p r r}] i\left(\lambda^{A}\right)_{a}^{d} G_{\mathrm{L}}^{A \mu}{ }_{\nu}\left(\bar{d}_{\mathrm{R}}^{a} \gamma^{\nu} d_{\mathrm{R} t d}\right)\left(D_{\mu} \bar{d}_{\mathrm{R}}{ }_{r}^{b} d_{\mathrm{L} s b}\right) \\
& \mathcal{Y}[\overline{p r r}] i\left(\lambda^{A}\right)_{q}^{q} G_{\mathrm{L}}^{A \mu}{ }_{\nu}\left(\bar{d}_{\mathrm{R} p}^{a} \gamma^{\nu} d_{\mathrm{R} t b}\right)\left(D_{\mu} \bar{d}_{\mathrm{R}}^{b}{ }_{\mathrm{L} s c}\right) \\
& \mathcal{Y}[\underline{p p r}] i\left(\lambda^{A}\right)_{b}^{q} G_{\mathrm{L}}^{A \mu}{ }_{\nu}\left(\bar{d}_{\mathrm{R}}^{a} \gamma^{\nu} d_{\mathrm{R} t a}\right)\left(D_{\mu} \bar{d}_{\mathrm{R} r}^{b} d_{\mathrm{L} s c}\right) \\
& \mid i\left(\lambda^{A}\right)_{a}^{b} G_{\mathrm{L}}^{A \mu}{ }_{\nu}\left(d_{\mathrm{L} r b} C D_{\mu} e_{\mathrm{L} s}\right)\left(\bar{d}_{\mathrm{R} p}^{a} \gamma^{\nu} C \bar{e}_{\mathrm{L} t}\right) \\
& \mathcal{O}_{G_{\mathrm{L}} \bar{d}_{\mathrm{R}} d_{\mathrm{L}} e_{\mathrm{L}} \bar{e}_{\mathrm{L}} D}^{(0,0)} i\left(\lambda^{A}\right)_{a}^{b} G_{\mathrm{L}}^{A \mu}{ }_{\nu}\left(D_{\mu} d_{\mathrm{L} r b} C e_{\mathrm{L} s}\right)\left(\bar{d}_{\mathrm{R}}^{a} \gamma^{\nu} C \bar{e}_{\mathrm{L} t}\right) \\
& i\left(\lambda^{A}\right)_{a}^{b} G_{\mathrm{L}}^{A \mu}{ }_{\nu}\left(\bar{d}_{\mathrm{R} p}^{a} e_{\mathrm{L} s}\right)\left(\bar{e}_{\mathrm{L} t} \gamma^{\nu} D_{\mu} d_{\mathrm{L} r b}\right) \\
& i\left(\lambda^{A}\right)_{a}^{b} G_{\mathrm{L}}^{A \mu}{ }_{\nu}\left(\bar{e}_{\mathrm{L} t} \gamma^{\nu} d_{\mathrm{L} r b}\right)\left(\bar{d}_{\mathrm{R} p}^{a} D_{\mu} e_{\mathrm{L} s}\right) \\
& i\left(\lambda^{A}\right)_{a}^{b} G_{\mathrm{L}}^{A \mu}{ }_{\nu}\left(D_{\mu} \bar{e}_{\mathrm{R} s} d_{\mathrm{L} r b}\right)\left(\bar{d}_{\mathrm{R} p}^{a} \gamma^{\nu} e_{\mathrm{R} t}\right) \\
& \mathcal{O}_{G_{\mathrm{L}}(1 \sim 4)}^{\left(\sim \bar{d}_{\mathrm{R}} d_{\mathrm{L}} \bar{e}_{\mathrm{R}} e_{\mathrm{R}} D\right.}(0,0) i\left(\lambda^{A}\right)_{a}^{b} G_{\mathrm{L}}^{A \mu}{ }_{\nu}\left(\bar{e}_{\mathrm{R} s} D_{\mu} d_{\mathrm{L} r b}\right)\left(\bar{d}_{\mathrm{R}}^{a} \gamma^{\nu} e_{\mathrm{R} t}\right) \\
& i\left(\lambda^{A}\right)_{a}^{b} G_{\mathrm{L}}^{A \mu}{ }_{\nu}\left(\bar{d}_{\mathrm{R} p}^{a} C \bar{e}_{\mathrm{R} s}\right)\left(D_{\mu} d_{\mathrm{L} r b} C \gamma^{\nu} e_{\mathrm{R} t}\right) \\
& i\left(\lambda^{A}\right)_{a}^{b} G_{\mathrm{L}}^{A \mu}{ }_{\nu}\left(d_{\mathrm{L} r b} C \gamma^{\nu} e_{\mathrm{R} t}\right)\left(\bar{d}_{\mathrm{R}}^{a} C D_{\mu} \bar{e}_{\mathrm{R} s}\right) \\
& \mathcal{O}_{G_{\mathrm{L}} \bar{d}_{\mathrm{R}} d_{\mathrm{L}} \nu_{\mathrm{L}} \bar{\nu}_{\mathrm{L}} D}^{(1 \sim, 0)} \mid \begin{array}{l}
i\left(\lambda^{A}\right)_{a}^{b} G_{\mathrm{L}}^{A \mu}{ }_{\nu}\left(d_{\mathrm{L} r b} C D_{\mu} \nu_{\mathrm{L} s}\right)\left(\bar{d}_{\mathrm{R}}{ }^{a} \gamma^{\nu} C \bar{\nu}_{\mathrm{L} t}\right) \\
i\left(\lambda^{A}\right)_{a}^{b} G_{\mathrm{L}}^{A \mu}{ }_{\nu}\left(D_{\mu} d_{\mathrm{L} r b} C \nu_{\mathrm{L} s}\right)\left(\bar{d}_{\mathrm{R} p}^{a} \gamma^{\nu} C \bar{\nu}_{\mathrm{L} t}\right) \\
i\left(\lambda^{A}\right)_{a}^{b} G_{\mathrm{L}}^{A \mu}{ }_{\nu}\left(\bar{d}_{\mathrm{R} p}^{a} \nu_{\mathrm{L} s}\right)\left(\bar{\nu}_{\mathrm{L} t} \gamma^{\nu} D_{\mu} d_{\mathrm{L} r b}\right) \\
i\left(\lambda^{A}\right)_{a}^{b} G_{\mathrm{L}}^{A \mu}{ }_{\nu}\left(\bar{\nu}_{\mathrm{L} t} \gamma^{\nu} d_{\mathrm{L} r b}\right)\left(\bar{d}_{\mathrm{R} p}^{a} D_{\mu} \nu_{\mathrm{L} s}\right)
\end{array}
\end{aligned}
$$




$$
\begin{aligned}
& \mathcal{O}_{G_{\mathrm{L}} d_{\mathrm{L}} \bar{d}_{\mathrm{L}} \bar{e}_{\mathrm{R}} e_{\mathrm{L}} D}^{(1 \sim, 0)} \mid \begin{array}{l}
i\left(\lambda^{A}\right)_{b}^{a} G_{\mathrm{L}}^{A \mu}{ }_{\nu}\left(\bar{e}_{\mathrm{R} r} D_{\mu} e_{\mathrm{L} s}\right)\left(\bar{d}_{\mathrm{L}}^{b} \gamma^{\nu} d_{\mathrm{L} p a}\right) \\
i\left(\lambda^{A}\right)_{b}^{a} G_{\mathrm{L}}^{A \mu}{ }_{\nu}\left(D_{\mu} \bar{e}_{\mathrm{R} r} e_{\mathrm{L} s}\right)\left(\bar{d}_{\mathrm{L} t}^{b} \gamma^{\nu} d_{\mathrm{L} p a}\right) \\
i\left(\lambda^{A}\right)_{b}^{a} G_{\mathrm{L}}^{A \mu}{ }_{\nu}\left(d_{\mathrm{L} p a} C e_{\mathrm{L} s}\right)\left(D_{\mu} \bar{e}_{\mathrm{R} r} \gamma^{\nu} C \bar{d}_{\mathrm{L} t}^{b}\right) \\
i\left(\lambda^{A}\right)_{b}^{b} G_{\mathrm{L}}^{A \mu}{ }_{\nu}\left(d_{\mathrm{L} p a} C D_{\mu} e_{\mathrm{L} s}\right)\left(\bar{e}_{\mathrm{R} r} \gamma^{\nu} C \bar{d}_{\mathrm{L} t}^{b}\right)
\end{array} \\
& \mathcal{O}_{G_{\mathrm{L}} d_{\mathrm{L}} \bar{e}_{\mathrm{R}} \nu_{\mathrm{L}} \bar{u}_{\mathrm{L}} D}(0,0) \mid \begin{array}{l}
i\left(\lambda^{A}\right)_{b}^{a} G_{\mathrm{L}}^{A \mu}{ }_{\nu}\left(\bar{e}_{\mathrm{R} r} D_{\mu} \nu_{\mathrm{L} s}\right)\left(\bar{u}_{\mathrm{L} t}^{b} \gamma^{\nu} d_{\mathrm{L} p a}\right) \\
i\left(\lambda^{A}\right)_{b}^{a} G_{\mathrm{L}}^{A \mu}{ }_{\nu}\left(D_{\mu} \bar{e}_{\mathrm{R} r} \nu_{\mathrm{L} s}\right)\left(\bar{u}_{\mathrm{L}}^{b} \gamma^{\nu} d_{\mathrm{L} p a}\right) \\
i\left(\lambda^{A}\right)_{b}^{a} G_{\mathrm{L}}^{A \mu}{ }_{\nu}\left(d_{\mathrm{L} p a} C \nu_{\mathrm{L} s}\right)\left(D_{\mu} \bar{e}_{\mathrm{R} r} \gamma^{\nu} C \bar{u}_{\mathrm{L} t}^{b}\right) \\
i\left(\lambda^{A}\right)_{b}^{a} G_{\mathrm{L}}^{A \mu}{ }_{\nu}\left(d_{\mathrm{L} p a} C D_{\mu} \nu_{\mathrm{L} s}\right)\left(\bar{e}_{\mathrm{R} r} \gamma^{\nu} C \bar{u}_{\mathrm{L} t}^{b}\right)
\end{array}
\end{aligned}
$$

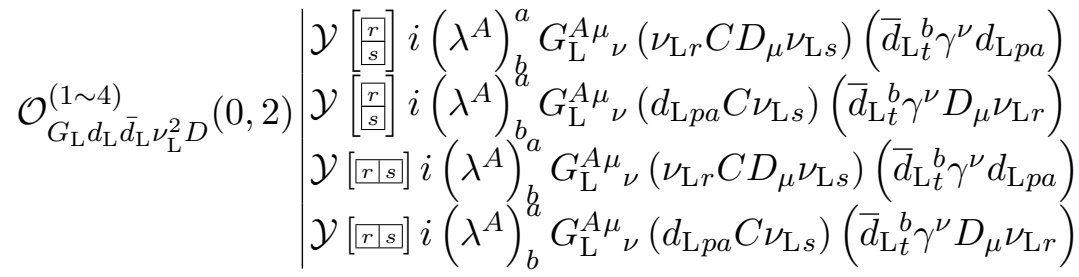

$$
\begin{aligned}
& \mathcal{Y}\left[\frac{p r r}{s}\right] i \epsilon_{a c d}\left(\lambda^{A}\right)_{b}^{d} G_{\mathrm{L}}^{A \mu}{ }_{\nu}\left(\bar{d}_{\mathrm{R}}^{a}{ }_{p}^{\nu} \gamma_{\mathrm{R} t}\right)\left(\bar{d}_{\mathrm{R}}{ }_{r}^{b} C D_{\mu} \bar{d}_{\mathrm{R}}^{c}\right)
\end{aligned}
$$

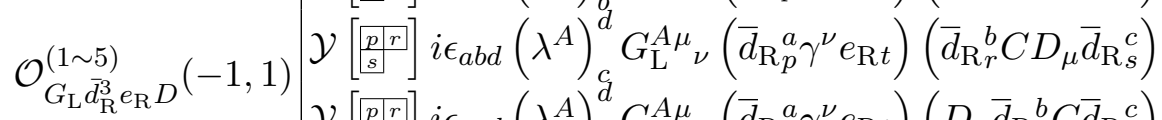

$$
\begin{aligned}
& \mathcal{Y}\left[\frac{p r r}{s}\right] i \epsilon_{a c d}\left(\lambda^{A}\right)_{b}^{d} G_{\mathrm{L}}^{A \mu}{ }_{\nu}\left(\bar{d}_{\mathrm{R}}^{a}{ }^{a} \gamma^{\nu} e_{\mathrm{R} t}\right)\left(D_{\mu} \bar{d}_{\mathrm{R}}{ }_{r}^{b} C \bar{d}_{\mathrm{R}}^{c}{ }_{s}^{c}\right) \\
& \mathcal{Y}\left[\overline{p|r| s]} i \epsilon_{a b d}\left(\lambda^{A}\right)_{c}^{d} G_{\mathrm{L}}^{A \mu}{ }_{\nu}\left(\bar{d}_{\mathrm{R} p}^{a} \gamma^{\nu} e_{\mathrm{R} t}\right)\left(\bar{d}_{\mathrm{R}}^{b} C D_{\mu} \bar{d}_{\mathrm{R} s}^{c}\right)\right. \\
& \mathcal{Y}\left[\begin{array}{c}
p \\
\frac{p}{s} \\
s
\end{array}\right] i \epsilon_{a c d}\left(\lambda^{A}\right)_{b}^{d} G_{\mathrm{L}}^{A \mu}{ }_{\nu}\left(\bar{d}_{\mathrm{R} p}^{a} \gamma^{\nu} e_{\mathrm{R} t}\right)\left(\bar{d}_{\mathrm{R} r}^{b} C D_{\mu} \bar{d}_{\mathrm{R} s}^{c}\right)
\end{aligned}
$$

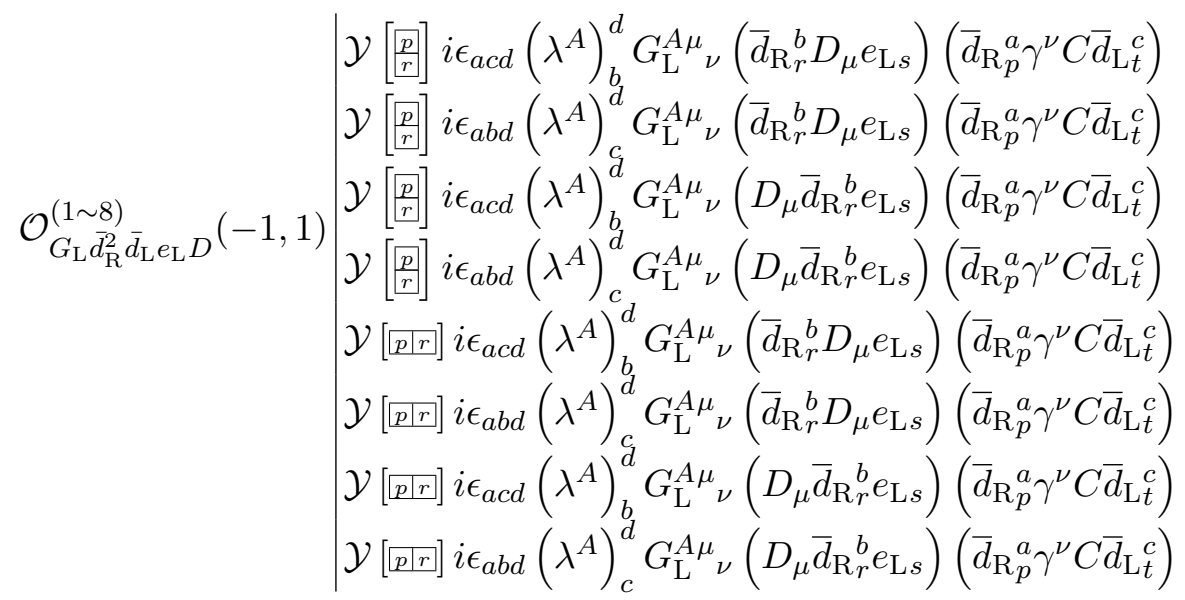




$$
\begin{aligned}
& \mathcal{Y}\left[\left[\begin{array}{l}
p \\
r
\end{array}\right] i \epsilon_{\text {acd }}\left(\lambda^{A}\right)_{b}^{d} G_{\mathrm{L}}^{A \mu}{ }_{\nu}\left(\bar{d}_{\mathrm{R}}{ }_{r}^{b} D_{\mu} \nu_{\mathrm{L} s}\right)\left(\bar{d}_{\mathrm{R}}^{a} \gamma^{\nu} C \bar{u}_{\mathrm{L} t}^{c}\right)\right. \\
& \mathcal{Y}\left[\begin{array}{l}
p \\
\vec{r}
\end{array}\right] i \epsilon_{a b d}\left(\lambda^{A}\right)_{c}^{d} G_{\mathrm{L}}^{A \mu}{ }_{\nu}\left(\bar{d}_{\mathrm{R}}{ }_{r}^{b} D_{\mu} \nu_{\mathrm{L} s}\right)\left(\bar{d}_{\mathrm{R}}^{a} \gamma^{a} C \bar{u}_{\mathrm{L} t}^{c}\right)
\end{aligned}
$$

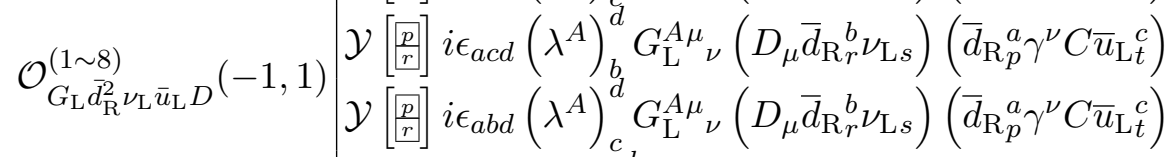

$$
\begin{aligned}
& \mathcal{Y}[\overline{p p r}] i \epsilon_{a c d}\left(\lambda^{A}\right)_{b}^{d} G_{\mathrm{L}}^{A \mu}{ }_{\nu}\left(\bar{d}_{\mathrm{R}}^{b} D_{\mu} \nu_{\mathrm{L} s}\right)\left(\bar{d}_{\mathrm{R} p}^{a} \gamma^{\nu} C \bar{u}_{\mathrm{L} t}^{c}\right) \\
& \mathcal{Y}[\overline{p p r}] i \epsilon_{a b d}\left(\lambda^{A}\right)_{c}^{d} G_{\mathrm{L}}^{A \mu}{ }_{\nu}\left(\bar{d}_{\mathrm{R} r}^{b} D_{\mu} \nu_{\mathrm{L} s}\right)\left(\bar{d}_{\mathrm{R}}^{a} \gamma^{\nu} C \bar{u}_{\mathrm{L} t}^{c}\right) \\
& \mathcal{Y}[\underline{p p r}] i \epsilon_{\text {acd }}\left(\lambda^{A}\right)_{b}^{d} G_{\mathrm{L}}^{A \mu}{ }_{\nu}\left(D_{\mu} \bar{d}_{\mathrm{R} r}^{b} \nu_{\mathrm{L} s}\right)\left(\bar{d}_{\left.\mathrm{R}_{p}^{a} \gamma^{\nu} C \bar{u}_{\mathrm{L}}^{c}\right)}\right) \\
& \mathcal{Y}[\overline{p p r}] i \epsilon_{a b d}\left(\lambda^{A}\right)_{c}^{d} G_{\mathrm{L}}^{A \mu}{ }_{\nu}\left(D_{\mu} \bar{d}_{\mathrm{R} r}^{b} \nu_{\mathrm{L} s}\right)\left(\bar{d}_{\mathrm{R}}^{a} \gamma^{\nu} C \bar{u}_{\mathrm{L} t}^{c}\right) \\
& \mid \begin{array}{l}
i\left(\lambda^{A}\right)_{a}^{b} G_{\mathrm{L}}^{A \mu}{ }_{\nu}\left(\bar{e}_{\mathrm{R} r} D_{\mu} e_{\mathrm{L} s}\right)\left(\bar{d}_{\mathrm{R}}^{a} \gamma^{\nu} d_{\mathrm{R} t b}\right) \\
\mathcal{O}_{G_{\mathrm{L}} \bar{d}_{\mathrm{R}} d_{\mathrm{R}} \bar{e}_{\mathrm{R}} e_{\mathrm{L}} D}^{(1 \sim 0,0)} i\left(\lambda^{A}\right)_{a}^{b} G_{\mathrm{L}}^{A \mu}{ }_{\nu}\left(D_{\mu} \bar{e}_{\mathrm{R} r} e_{\mathrm{L} s}\right)\left(\bar{d}_{\mathrm{R}}^{a} \gamma^{\nu} d_{\mathrm{R} t b}\right)
\end{array} \\
& i\left(\lambda^{A}\right)_{a}^{b} G_{\mathrm{L}}^{A \mu}{ }_{\nu}\left(\bar{d}_{\mathrm{R} p}{ }_{p}^{a} e_{\mathrm{L} s}\right)\left(D_{\mu} \bar{e}_{\mathrm{R} r} \gamma^{\nu} d_{\mathrm{R} t b}\right) \\
& i\left(\lambda^{A}\right)_{a}^{b} G_{\mathrm{L}}^{A \mu}{ }_{\nu}\left(\bar{e}_{\mathrm{R} r} \gamma^{\nu} d_{\mathrm{R} t b}\right)\left(\bar{d}_{\mathrm{R} p}^{a} D_{\mu} e_{\mathrm{L} s}\right) \\
& \mathcal{O}_{G_{\mathrm{L}} \bar{d}_{\mathrm{R}} e_{\mathrm{L}} \nu_{\mathrm{L}} u_{\mathrm{R}} D}^{(1 \sim 2)} \mid \begin{array}{l}
i\left(\lambda^{A}\right)_{a}^{b} G_{\mathrm{L}}^{A \mu}{ }_{\nu}\left(e_{\mathrm{L} r} C D_{\mu} \nu_{\mathrm{L} s}\right)\left(\bar{d}_{\mathrm{R}}^{a} \gamma^{\nu} u_{\mathrm{R} t b}\right) \\
i\left(\lambda^{A}\right)_{a}^{b} G_{\mathrm{L}}^{A \mu}{ }_{\nu}\left(D_{\mu} e_{\mathrm{L} r} C \nu_{\mathrm{L} s}\right)\left(\bar{d}_{\mathrm{R}}{ }_{p} \gamma^{\nu} u_{\mathrm{R} t b}\right) \\
i\left(\lambda^{A}\right)_{a}^{b} G_{\mathrm{L}}^{A \mu}{ }_{\nu}\left(\bar{d}_{\mathrm{R} p}^{a} \nu_{\mathrm{L} s}\right)\left(D_{\mu} e_{\mathrm{L} r} C \gamma^{\nu} u_{\mathrm{R} t b}\right) \\
i\left(\lambda^{A}\right)_{a}^{b} G_{\mathrm{L}}^{A \mu}{ }_{\nu}\left(e_{\mathrm{L} r} C \gamma^{\nu} u_{\mathrm{R} t b}\right)\left(\bar{d}_{\mathrm{R} p}^{a} D_{\mu} \nu_{\mathrm{L} s}\right)
\end{array}
\end{aligned}
$$

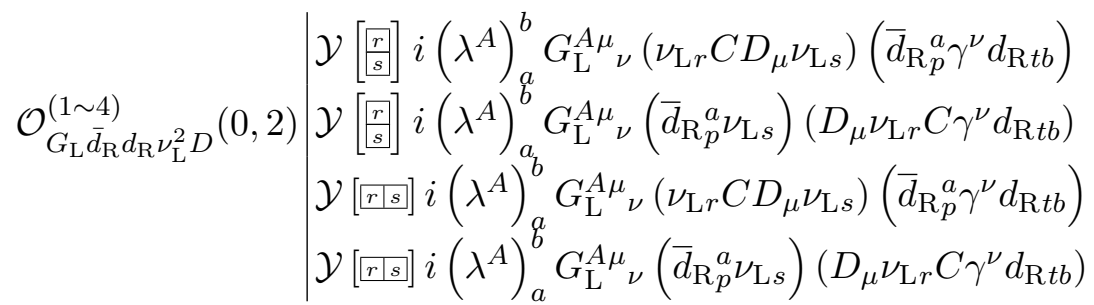

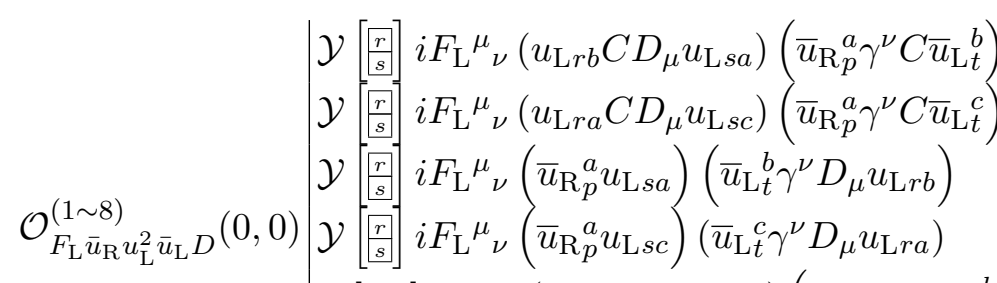

$$
\begin{aligned}
& \mathcal{Y}[\overline{r r s}] i F_{\mathrm{L}}{ }_{\nu}{ }_{\nu}\left(u_{\mathrm{L} r b} C D_{\mu} u_{\mathrm{L} s a}\right)\left(\bar{u}_{\mathrm{R}}{ }^{a} \gamma^{\nu} C \bar{u}_{\mathrm{L} t}^{b}\right) \\
& \mathcal{Y}\left[{ }_{r r s}\right] i F_{\mathrm{L}}{ }_{\nu}{ }_{\nu}\left(u_{\mathrm{L} r a} C D_{\mu} u_{\mathrm{L} s c}\right)\left(\bar{u}_{\mathrm{R}}{ }_{p}^{a} \gamma^{\nu} C \bar{u}_{\mathrm{L}}{ }_{t}^{c}\right) \\
& \mathcal{Y}\left[r[s] i F_{\mathrm{L}}{ }^{\mu}{ }_{\nu}\left(\bar{u}_{\mathrm{R} p}^{a} u_{\mathrm{L} s a}\right)\left(\bar{u}_{\mathrm{L} t}^{b} \gamma^{\nu} D_{\mu} u_{\mathrm{L} r b}\right)\right. \\
& \mathcal{Y}[\overline{r r s}] i F_{\mathrm{L}}{ }_{\nu}{ }_{\nu}\left(\bar{u}_{\mathrm{R} p}^{a} u_{\mathrm{L} s c}\right)\left(\bar{u}_{\mathrm{L} t}^{c} \gamma^{\nu} D_{\mu} u_{\mathrm{L} r a}\right)
\end{aligned}
$$




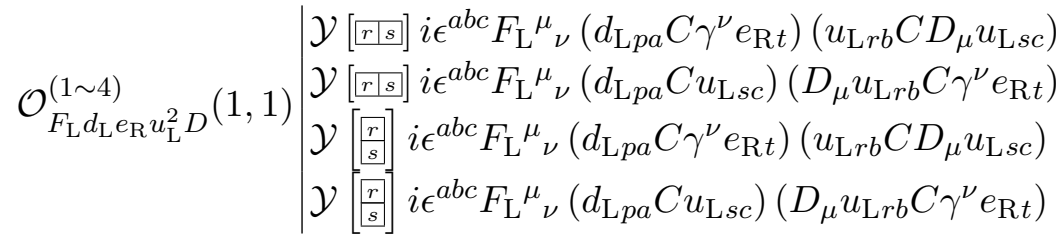

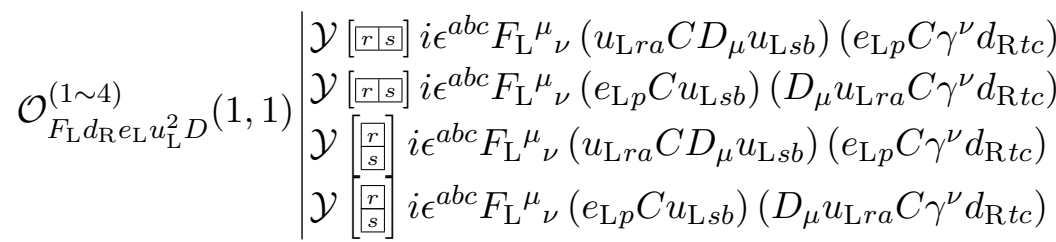

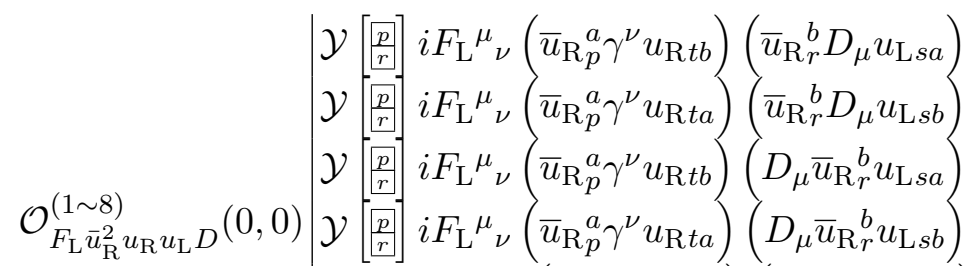

$$
\begin{aligned}
& \mathcal{Y}[\overline{p \mid r]}] i F_{\mathrm{L}}{ }^{\mu}{ }_{\nu}\left(\bar{u}_{\mathrm{R} p}{ }^{a} \gamma^{\nu} u_{\mathrm{R} t b}\right)\left(\bar{u}_{\mathrm{R}}{ }_{r}^{b} D_{\mu} u_{\mathrm{L} s a}\right) \\
& \mathcal{Y}\left[[p r r] i F_{\mathrm{L}}{ }_{\nu}{ }_{\nu}\left(\bar{u}_{\mathrm{R}}{ }^{a} \gamma^{\nu} u_{\mathrm{R} t a}\right)\left(\bar{u}_{\mathrm{R} r}^{b} D_{\mu} u_{\mathrm{L} s b}\right)\right. \\
& \mathcal{Y}\left[[p \mid r] i F_{\mathrm{L}}{ }^{\mu}{ }_{\nu}\left(\bar{u}_{\mathrm{R} p}{ }^{a} \gamma^{\nu} u_{\mathrm{R} t b}\right)\left(D_{\mu} \bar{u}_{\mathrm{R} r}{ }^{b} u_{\mathrm{L} s a}\right)\right. \\
& \mathcal{Y}\left[[\overline{p \mid r}] i F_{\mathrm{L}}{ }^{\mu}{ }_{\nu}\left(\bar{u}_{\mathrm{R} p}^{a} \gamma^{\nu} u_{\mathrm{R} t a}\right)\left(D_{\mu} \bar{u}_{\mathrm{R}}{ }_{r}^{b} u_{\mathrm{L} s b}\right)\right.
\end{aligned}
$$

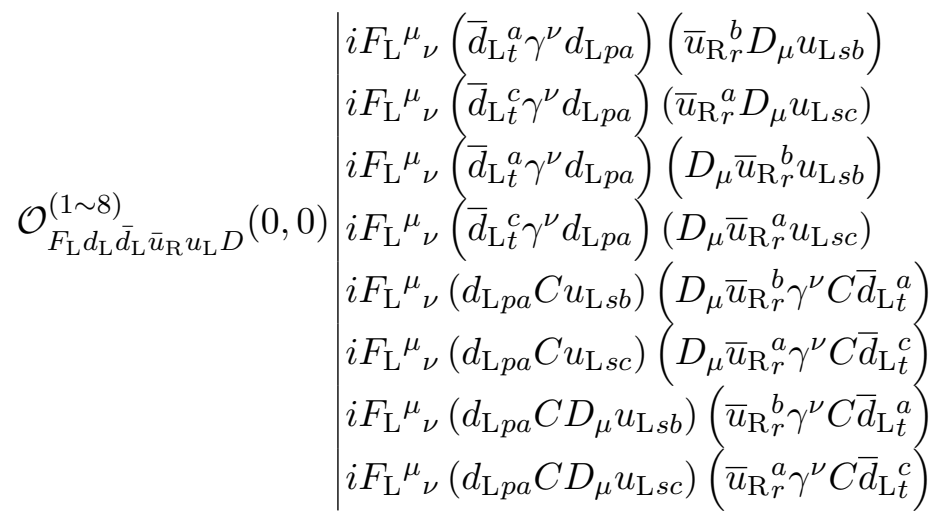

$$
\begin{aligned}
& \left.\mathcal{O}_{F_{\mathrm{L}} \bar{d}_{\mathrm{R}} d_{\mathrm{R}} \bar{u}_{\mathrm{R}} u_{\mathrm{L}} D}^{(0,0)} \mid \begin{array}{ll}
i F_{\mathrm{L}}{ }^{\mu}{ }_{\nu}\left(\bar{d}_{\mathrm{R}}{ }_{p}^{a} \gamma^{\nu} d_{\mathrm{R} t b}\right) & \left(\bar{u}_{\mathrm{R}}{ }_{r}^{b} D_{\mu} u_{\mathrm{L} s a}\right. \\
i F_{\mathrm{L}}{ }^{\mu}{ }_{\nu}\left(\bar{d}_{\mathrm{R}}{ }_{p}^{a} \gamma^{\nu} d_{\mathrm{R} t a}\right) & \left(\bar{u}_{\mathrm{R}}^{b} D_{\mu} D_{\mathrm{L} s b}\right. \\
i F_{\mathrm{L}}{ }^{\mu}{ }_{\nu}\left(\bar{d}_{\mathrm{R}}{ }_{p}^{a} \gamma^{\nu} d_{\mathrm{R} t b}\right) & \left(D_{\mu} \bar{u}_{\mathrm{R}}{ }_{r}^{b} u_{\mathrm{L} s a}\right. \\
i F_{\mathrm{L}}{ }^{\mu}{ }_{\nu}\left(\bar{d}_{\mathrm{R}}{ }_{p}^{a} \gamma^{\nu} d_{\mathrm{R} t a}\right) & \left(D_{\mu} \bar{u}_{\mathrm{R}}^{b} u_{\mathrm{L} s b}\right.
\end{array}\right) \\
& i F_{\mathrm{L}}{ }_{\nu}{ }_{\nu}\left(\bar{d}_{\mathrm{R}}{ }_{p}^{a} u_{\mathrm{L} s a}\right)\left(D_{\mu} \bar{u}_{\mathrm{R}}^{b} \gamma^{\nu} d_{\mathrm{R} t b}\right) \\
& i F_{\mathrm{L}}^{\mu}{ }_{\nu}\left(\bar{d}_{\mathrm{R} p}^{a} u_{\mathrm{L} s b}\right)\left(D_{\mu} \bar{u}_{\mathrm{R}}^{b} \gamma^{\nu} d_{\mathrm{R} t a}\right) \\
& i F_{\mathrm{L}}{ }_{\nu}{ }_{\nu}\left(\bar{d}_{\mathrm{R} p}^{a} D_{\mu} u_{\mathrm{L} s a}\right)\left(\bar{u}_{\mathrm{R} r}^{b} \gamma^{\nu} d_{\mathrm{R} t b}\right) \\
& i F_{\mathrm{L}}{ }_{\nu}{ }_{\nu}\left(\bar{d}_{\mathrm{R} p}^{a} D_{\mu} u_{\mathrm{L} s b}\right)\left(\bar{u}_{\mathrm{R} r}^{b} \gamma^{\nu} d_{\mathrm{R} t a}\right)
\end{aligned}
$$




$$
\begin{aligned}
& \mathcal{O}_{F_{\mathrm{L}} e_{\mathrm{L}} \bar{e}_{\mathrm{L}} \bar{u}_{\mathrm{R}} u_{\mathrm{L}} D}(0,0) \mid \begin{array}{l}
i F_{\mathrm{L}}{ }^{\mu}{ }_{\nu}\left(\bar{e}_{\mathrm{L} t} \gamma^{\nu} e_{\mathrm{L} p}\right)\left(\bar{u}_{\mathrm{R} r}{ }_{r}^{a} D_{\mu} u_{\mathrm{L} s a}\right) \\
i F_{\mathrm{L}}{ }^{\mu}{ }_{\nu}\left(\bar{e}_{\mathrm{L} t} \gamma^{\nu} e_{\mathrm{L} p}\right)\left(D_{\mu} \bar{u}_{\mathrm{R}}{ }^{a} u_{\mathrm{L} s a}\right) \\
i F_{\mathrm{L}}{ }^{\mu}{ }_{\nu}\left(e_{\mathrm{L} p} C u_{\mathrm{L} s a}\right)\left(D_{\mu} \bar{u}_{\mathrm{R}}{ }_{r} \gamma^{\nu} C \bar{e}_{\mathrm{L} t}\right) \\
i F_{\mathrm{L}}{ }^{\mu}{ }_{\nu}\left(e_{\mathrm{L} p} C D_{\mu} u_{\mathrm{L} s a}\right)\left(\bar{u}_{\mathrm{R}}{ }_{r} \gamma^{\nu} C \bar{e}_{\mathrm{L} t}\right)
\end{array} \\
& \mathcal{O}_{F_{\mathrm{L}} \bar{e}_{\mathrm{R}} e_{\mathrm{R}} \bar{u}_{\mathrm{R}} u_{\mathrm{L}} D}(0,0) \mid \begin{array}{l}
i F_{\mathrm{L}}{ }^{\mu}{ }_{\nu}\left(\bar{e}_{\mathrm{R} p} \gamma^{\nu} e_{\mathrm{R} t}\right)\left(\bar{u}_{\mathrm{R} r}^{a} D_{\mu} u_{\mathrm{L} s a}\right) \\
i F_{\mathrm{L}}{ }^{\mu}{ }_{\nu}\left(\bar{e}_{\mathrm{R} p} \gamma^{\nu} e_{\mathrm{R} t}\right)\left(D_{\mu} \bar{u}_{\mathrm{R} r}{ }^{a} u_{\mathrm{L} s a}\right) \\
i F_{\mathrm{L}}{ }^{\mu}{ }_{\nu}\left(\bar{e}_{\mathrm{R} p} u_{\mathrm{L} s a}\right)\left(D_{\mu} \bar{u}_{\mathrm{R}}{ }_{r} \gamma^{\nu} e_{\mathrm{R} t}\right) \\
i F_{\mathrm{L}}{ }^{\mu}{ }_{\nu}\left(\bar{e}_{\mathrm{R} p} D_{\mu} u_{\mathrm{L} s a}\right)\left(\bar{u}_{\mathrm{R}}{ }_{r} \gamma^{\nu} e_{\mathrm{R} t}\right)
\end{array}
\end{aligned}
$$

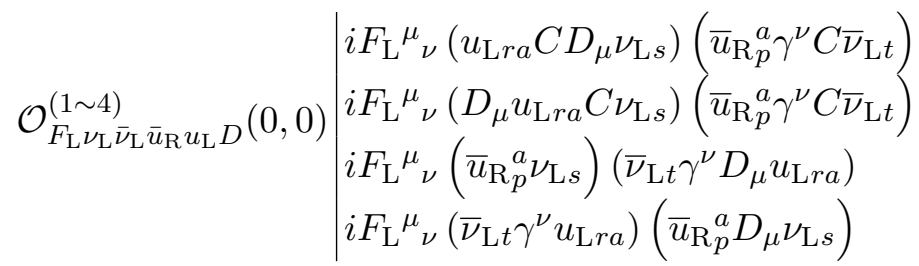

$$
\begin{aligned}
& \mathcal{Y}\left[[p \mid r] i \epsilon^{a b c} F_{\mathrm{L}}{ }_{\nu}{ }_{\nu}\left(\bar{\nu}_{\mathrm{L} t} \gamma^{\nu} d_{\mathrm{L} p a}\right)\left(d_{\mathrm{L} r b} C D_{\mu} u_{\mathrm{L} s c}\right)\right. \\
& \mathcal{O}_{F_{\mathrm{L}} d_{\mathrm{L}}^{2} \bar{\nu}_{\mathrm{L}} u_{\mathrm{L}} D}^{(1,-1)}\left[\begin{array}{l}
\mathcal{Y}[\overline{p r r}] i \epsilon^{a b c} F_{\mathrm{L}}{ }^{\mu}{ }_{\nu}\left(\bar{\nu}_{\mathrm{L} t} \gamma^{\nu} d_{\mathrm{L} p a}\right)\left(D_{\mu} d_{\mathrm{L} r b} C u_{\mathrm{L} s c}\right) \\
\mathcal{Y}\left[\begin{array}{l}
p \\
{[r]}
\end{array}\right] \epsilon^{a b c} F_{\mathrm{L}}{ }^{\mu}{ }_{\nu}\left(\bar{\nu}_{\mathrm{L} t} \gamma^{\nu} d_{\mathrm{L} p a}\right)\left(d_{\mathrm{L} r b} C D_{\mu} u_{\mathrm{L} s c}\right)
\end{array}\right. \\
& \mathcal{Y}\left[\frac{p}{r}\right] i \epsilon^{a b c} F_{\mathrm{L}}{ }^{\mu}{ }_{\nu}\left(\bar{\nu}_{\mathrm{L} t} \gamma^{\nu} d_{\mathrm{L} p a}\right)\left(D_{\mu} d_{\mathrm{L} r b} C u_{\mathrm{L} s c}\right)
\end{aligned}
$$

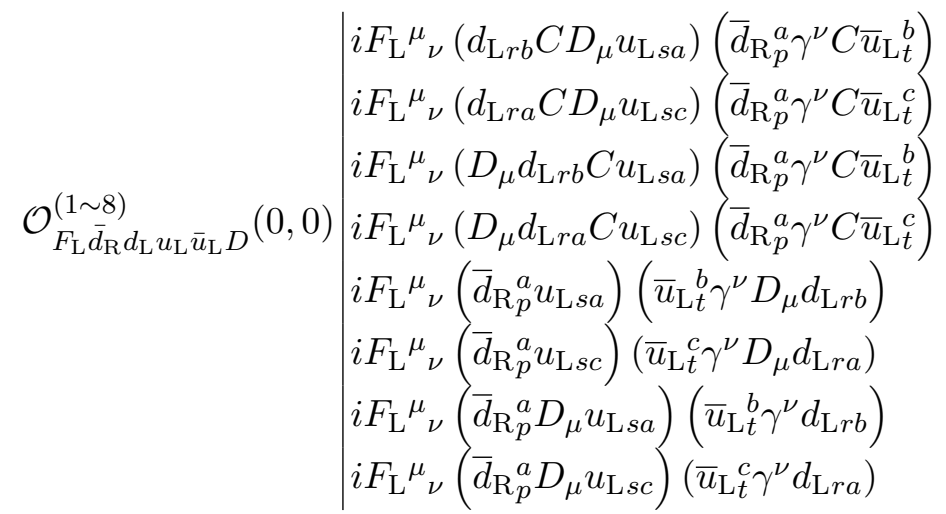

$$
\begin{aligned}
& \left.\mathcal{O}_{F_{\mathrm{L}} d_{\mathrm{L}} e_{\mathrm{L}} u_{\mathrm{R}} u_{\mathrm{L}} D}(1,1) \mid \begin{array}{l}
i \epsilon^{a b c} F_{\mathrm{L}}{ }^{\mu}{ }_{\nu}\left(e_{\mathrm{L} r} C D_{\mu} u_{\mathrm{L} s b}\right)\left(d_{\mathrm{L} p a} C \gamma^{\nu} u_{\mathrm{R} t c}\right) \\
i \epsilon^{a b c} F_{\mathrm{L}}{ }^{\mu}{ }_{\nu}\left(D_{\mu} e_{\mathrm{L} r} C u_{\mathrm{L} s b}\right)\left(d_{\mathrm{L} p a} C \gamma^{\nu} u_{\mathrm{R} t c}\right) \\
i \epsilon^{a b c} F_{\mathrm{L}}{ }^{\mu}{ }_{\nu}\left(d_{\mathrm{L} p a} C u_{\mathrm{L} s b}\right)\left(D_{\mu} e_{\mathrm{L} r} C \gamma^{\nu} u_{\mathrm{R} t c}\right) \\
i \epsilon^{a b c} F_{\mathrm{L}}{ }^{\mu}{ }_{\nu}\left(e_{\mathrm{L} r} C \gamma^{\nu} u_{\mathrm{R} t c}\right)\left(d_{\mathrm{L} p a} C D_{\mu} u_{\mathrm{L} s b}\right)
\end{array}\right) \\
& \mathcal{O}_{F_{\mathrm{L}} d_{\mathrm{R}} d_{\mathrm{L}} \nu_{\mathrm{L}} u_{\mathrm{L}} D}(1,1) \mid \begin{array}{l}
i \epsilon^{a b c} F_{\mathrm{L}}{ }^{\mu}{ }_{\nu}\left(u_{\mathrm{L} r b} C D_{\mu} \nu_{\mathrm{L} s}\right)\left(d_{\mathrm{L} p a} C \gamma^{\nu} d_{\mathrm{R} t c}\right) \\
i \epsilon^{a b c} F_{\mathrm{L}}{ }^{\mu}{ }_{\nu}\left(D_{\mu} u_{\mathrm{L} r b} C \nu_{\mathrm{L} s}\right)\left(d_{\mathrm{L} p a} C \gamma^{\nu} d_{\mathrm{R} t c}\right) \\
i \epsilon^{a b c} F_{\mathrm{L}}{ }^{\mu}{ }_{\nu}\left(d_{\mathrm{L} p a} C \nu_{\mathrm{L} s}\right)\left(D_{\mu} u_{\mathrm{L} r b} C \gamma^{\nu} d_{\mathrm{R} t c}\right) \\
i \epsilon^{a b c} F_{\mathrm{L}}{ }^{\mu}{ }_{\nu}\left(d_{\mathrm{L} p a} C D_{\mu} \nu_{\mathrm{L} s}\right)\left(u_{\mathrm{L} r b} C \gamma^{\nu} d_{\mathrm{R} t c}\right)
\end{array} \\
& \left.\mathcal{O}_{F_{\mathrm{L}} \bar{d}_{\mathrm{R}} e_{\mathrm{L}} \bar{\nu}_{\mathrm{L}} u_{\mathrm{L}} D}^{(1 \sim 0)} \mid \begin{array}{l}
i F_{\mathrm{L}}{ }^{\mu}{ }_{\nu}\left(e_{\mathrm{L} r} C D_{\mu} u_{\mathrm{L} s a}\right)\left(\bar{d}_{\mathrm{R}}^{a} \gamma^{\nu} C \bar{\nu}_{\mathrm{L} t}\right) \\
i F_{\mathrm{L}}{ }^{\mu}{ }_{\nu}\left(D_{\mu} e_{\mathrm{L} r} C u_{\mathrm{L} s a}\right)\left(\bar{d}_{\mathrm{R}}{ }_{p} \gamma^{\nu} C \bar{\nu}_{\mathrm{L} t}\right. \\
i F_{\mathrm{L}}{ }^{\mu}{ }_{\nu}\left(\bar{d}_{\mathrm{R}}{ }_{p}^{a} u_{\mathrm{L} s a}\right)\left(\bar{\nu}_{\mathrm{L} t} \gamma^{\nu} D_{\mu} e_{\mathrm{L} r}\right) \\
i F_{\mathrm{L}}{ }^{\mu}{ }_{\nu}\left(\bar{\nu}_{\mathrm{L} t} \gamma^{\nu} e_{\mathrm{L} r}\right)\left(\bar{d}_{\mathrm{R}}{ }_{p}^{a} D_{\mu} u_{\mathrm{L} s a}\right)
\end{array}\right)
\end{aligned}
$$




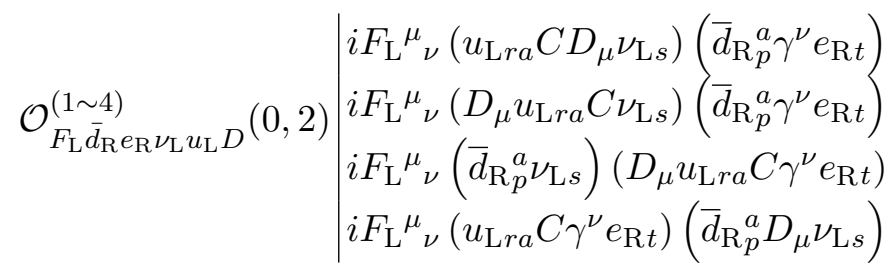

$$
\begin{aligned}
& \mathcal{O}_{F_{\mathrm{L}} \bar{e}_{\mathrm{R}} e_{\mathrm{L}} u_{\mathrm{L}} \bar{u}_{\mathrm{L}} D}(0,0) \mid \begin{array}{l|l}
i F_{\mathrm{L}}{ }^{\mu}{ }_{\nu}\left(e_{\mathrm{L} r} C D_{\mu} u_{\mathrm{L} s a}\right)\left(\bar{e}_{\mathrm{R} p} \gamma^{\nu} C \bar{u}_{\mathrm{L} t}^{a}\right) \\
i F_{\mathrm{L}}{ }^{\mu}{ }_{\nu}\left(D_{\mu} e_{\mathrm{L} r} C u_{\mathrm{L} s a}\right)\left(\bar{e}_{\mathrm{R} p} \gamma^{\nu} C \bar{u}_{\mathrm{L} t}^{a}\right) \\
i F_{\mathrm{L}}{ }^{\mu}{ }_{\nu}\left(\bar{e}_{\mathrm{R} p} u_{\mathrm{L} s a}\right)\left(\bar{u}_{\mathrm{L} t}{ }^{a} \gamma^{\nu} D_{\mu} e_{\mathrm{L} r}\right) \\
i F_{\mathrm{L}}{ }^{\mu}{ }_{\nu}\left(\bar{e}_{\mathrm{R} p} D_{\mu} u_{\mathrm{L} s a}\right)\left(\bar{u}_{\mathrm{L} t}{ }^{a} \gamma^{\nu} e_{\mathrm{L} r}\right)
\end{array} \\
& \mathcal{O}_{F_{\mathrm{L}} \bar{d}_{\mathrm{L}} e_{\mathrm{L}} \nu_{\mathrm{L}} u_{\mathrm{L}} D}^{(1 \sim, 2)}\left(\begin{array}{l}
i F_{\mathrm{L}}{ }^{\mu}{ }_{\nu}\left(u_{\mathrm{L} r a} C D_{\mu} \nu_{\mathrm{L} s}\right)\left(\bar{d}_{\mathrm{L} t}{ }^{a} \gamma^{\nu} e_{\mathrm{L} p}\right. \\
i F_{\mathrm{L}}{ }^{\mu}{ }_{\nu}\left(D_{\mu} u_{\mathrm{L} r a} C \nu_{\mathrm{L} s}\right)\left(\bar{d}_{\mathrm{L} t}^{a} \gamma^{\nu} e_{\mathrm{L} p}\right. \\
i F_{\mathrm{L}}{ }^{\mu}{ }_{\nu}\left(e_{\mathrm{L} p} C \nu_{\mathrm{L} s}\right)\left(\bar{d}_{\mathrm{L} t}{ }^{a} \gamma^{\nu} D_{\mu} u_{\mathrm{L} r a}\right. \\
i F_{\mathrm{L}}{ }^{\mu}{ }_{\nu}\left(e_{\mathrm{L} p} C D_{\mu} \nu_{\mathrm{L} s}\right)\left(\bar{d}_{\mathrm{L} t}{ }^{a} \gamma^{\nu} u_{\mathrm{L} r a}\right.
\end{array}\right)
\end{aligned}
$$

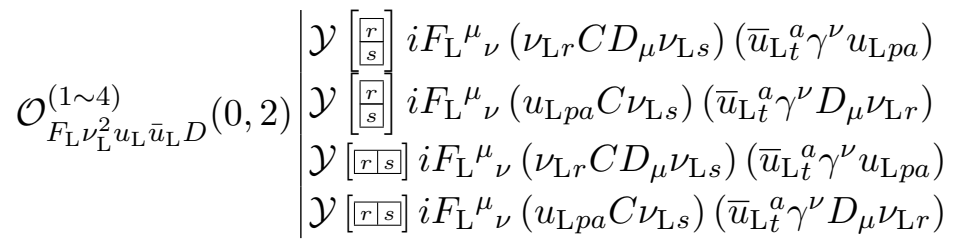

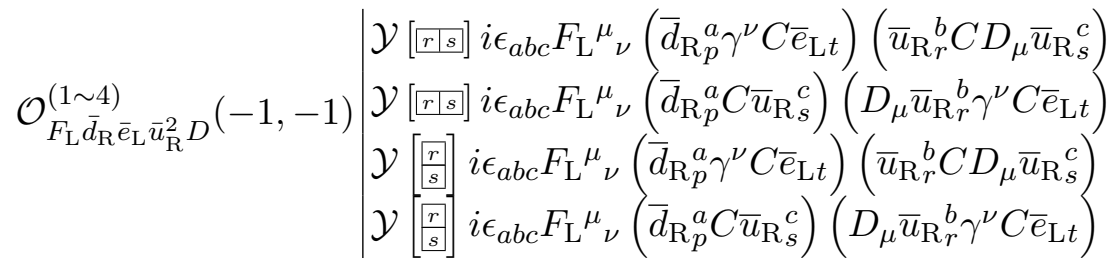

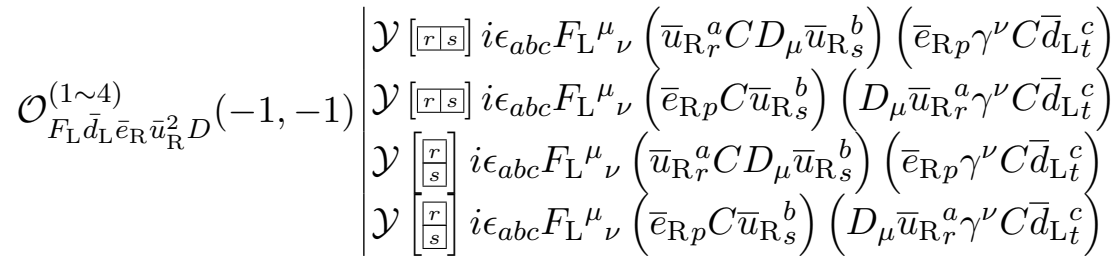

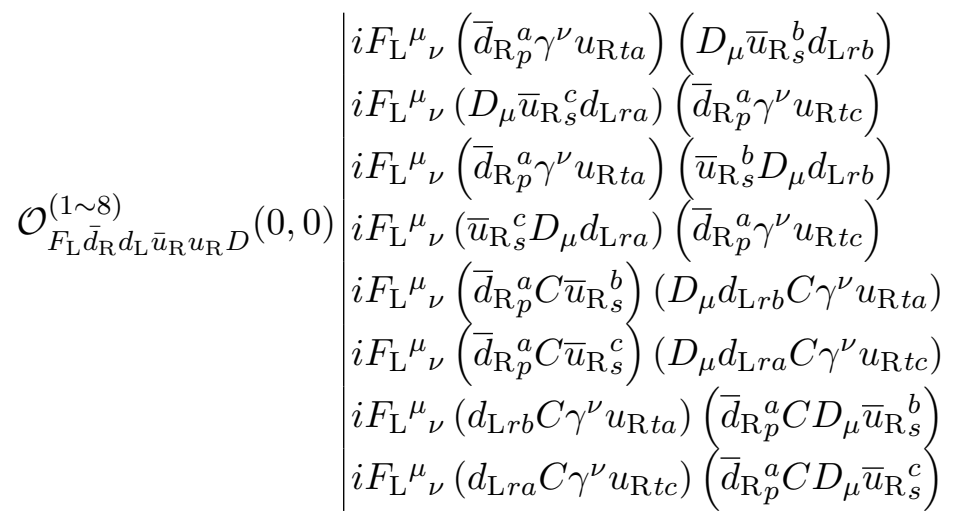




$$
\begin{aligned}
& \mathcal{O}_{F_{\mathrm{L}} d_{\mathrm{L}} \bar{e}_{\mathrm{R}} \bar{\nu}_{\mathrm{L}} \bar{u}_{\mathrm{R}} D}(0,-2) \mid \begin{array}{l}
i F_{\mathrm{L}}{ }_{\nu}{ }_{\nu}\left(\bar{\nu}_{\mathrm{L} t} \gamma^{\nu} d_{\mathrm{L} p a}\right)\left(\bar{e}_{\mathrm{R} r} C D_{\mu} \bar{u}_{\mathrm{R}}{ }^{a}\right) \\
i F_{\mathrm{L}}{ }^{\mu}{ }_{\nu}\left(\bar{\nu}_{\mathrm{L} t} \gamma^{\nu} d_{\mathrm{L} p a}\right)\left(D_{\mu} \bar{e}_{\mathrm{R} r} C \bar{u}_{\mathrm{R}}{ }^{a}\right) \\
i F_{\mathrm{L}}{ }^{\mu}{ }_{\nu}\left(\bar{u}_{\mathrm{R} s}{ }^{a} d_{\mathrm{L} p a}\right)\left(D_{\mu} \bar{e}_{\mathrm{R} r} \gamma^{\nu} C \bar{\nu}_{\mathrm{L} t}\right) \\
i F_{\mathrm{L}}{ }^{\mu}{ }_{\nu}\left(\bar{e}_{\mathrm{R} r} \gamma^{\nu} C \bar{\nu}_{\mathrm{L} t}\right)\left(D_{\mu} \bar{u}_{\mathrm{R}}{ }^{a} d_{\mathrm{L} p a}\right)
\end{array} \\
& \mathcal{O}_{F_{\mathrm{L}} d_{\mathrm{L}} \bar{e}_{\mathrm{L}} \nu_{\mathrm{L}} \bar{u}_{\mathrm{R}} D}(0,0) \mid \begin{array}{l}
i F_{\mathrm{L}}{ }^{\mu}{ }_{\nu}\left(\bar{e}_{\mathrm{L} t} \gamma^{\nu} d_{\mathrm{L} p a}\right)\left(\bar{u}_{\mathrm{R} r}{ }^{a} D_{\mu} \nu_{\mathrm{L} s}\right) \\
i F_{\mathrm{L}}{ }^{\mu}{ }_{\nu}\left(\bar{e}_{\mathrm{L} t} \gamma^{\nu} d_{\mathrm{L} p a}\right)\left(D_{\mu} \bar{u}_{\mathrm{R}}{ }_{r}^{a} \nu_{\mathrm{L} s}\right) \\
i F_{\mathrm{L}}{ }^{\mu}{ }_{\nu}\left(d_{\mathrm{L} p a} C \nu_{\mathrm{L} s}\right)\left(D_{\mu} \bar{u}_{\mathrm{R}}{ }_{r} \gamma^{\nu} C \bar{e}_{\mathrm{L} t}\right) \\
i F_{\mathrm{L}}{ }^{\mu}{ }_{\nu}\left(d_{\mathrm{L} p a} C D_{\mu} \nu_{\mathrm{L} s}\right)\left(\bar{u}_{\mathrm{R}}{ }_{r} \gamma^{\nu} C \bar{e}_{\mathrm{L} t}\right)
\end{array}
\end{aligned}
$$

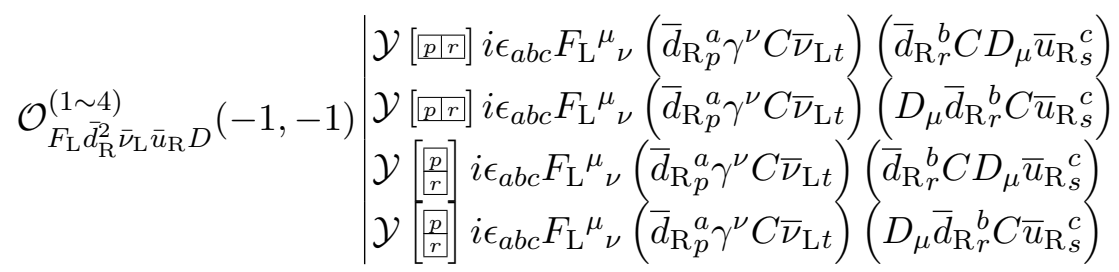

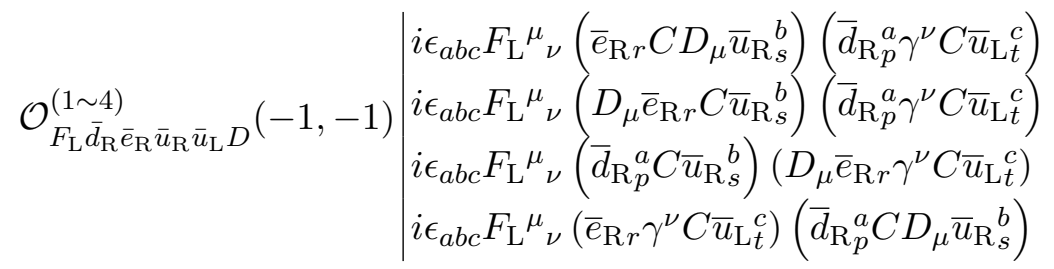

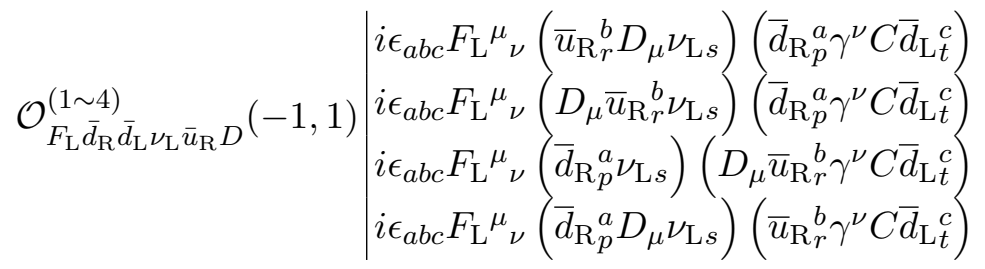

$$
\begin{aligned}
& \mathcal{O}_{F_{\mathrm{L}} \bar{e}_{\mathrm{R}} e_{\mathrm{L}} \bar{u}_{\mathrm{R}} u_{\mathrm{R}} D}(0,0) \mid \begin{array}{l}
i F_{\mathrm{L}}{ }^{\mu}{ }_{\nu}\left(\bar{e}_{\mathrm{R} p} \gamma^{\nu} u_{\mathrm{R} t a}\right)\left(D_{\mu} \bar{u}_{\mathrm{R}}{ }^{a} e_{\mathrm{L} r}\right) \\
i F_{\mathrm{L}}{ }^{\mu}{ }_{\nu}\left(\bar{e}_{\mathrm{R} p} \gamma^{\nu} u_{\mathrm{R} t a}\right)\left(\bar{u}_{\mathrm{R}}^{a} D_{\mu} D_{\mu} e_{\mathrm{L} r}\right) \\
i F_{\mathrm{L}}{ }^{\mu}{ }_{\nu}\left(\bar{e}_{\mathrm{R} p} C \bar{u}_{\mathrm{R} s}{ }^{a}\right)\left(D_{\mu} e_{\mathrm{L} r} C \gamma^{\nu} u_{\mathrm{R} t a}\right) \\
i F_{\mathrm{L}}{ }^{\mu}{ }_{\nu}\left(e_{\mathrm{L} r} C \gamma^{\nu} u_{\mathrm{R} t a}\right)\left(\bar{e}_{\mathrm{R} p} C D_{\mu} \bar{u}_{\mathrm{R}}{ }^{a}\right)
\end{array} \\
& \mathcal{O}_{F_{\mathrm{L}} d_{\mathrm{R}} \bar{e}_{\mathrm{R}} \nu_{\mathrm{L}} \bar{u}_{\mathrm{R}} D}(0,0) \mid \begin{array}{l}
i F_{\mathrm{L}}{ }_{\nu}{ }_{\nu}\left(\bar{e}_{\mathrm{R} p} \gamma^{\nu} d_{\mathrm{R} t a}\right)\left(\bar{u}_{\mathrm{R} r}^{a} D_{\mu} \nu_{\mathrm{L} s}\right) \\
i F_{\mathrm{L}}{ }^{\mu}{ }_{\nu}\left(\bar{e}_{\mathrm{R} p} \gamma^{\nu} d_{\mathrm{R} t a}\right)\left(D_{\mu} \bar{u}_{\mathrm{R}}{ }_{\mathrm{R}} \nu_{\mathrm{L} s}\right) \\
i F_{\mathrm{L}}{ }^{\mu}{ }_{\nu}\left(\bar{e}_{\mathrm{R} p} \nu_{\mathrm{L} s}\right)\left(D_{\mu} \bar{u}_{\mathrm{R}}{ }_{r}^{a} \gamma^{\nu} d_{\mathrm{R} t a}\right) \\
i F_{\mathrm{L}}{ }^{\mu}{ }_{\nu}\left(\bar{e}_{\mathrm{R} p} D_{\mu} \nu_{\mathrm{L} s}\right)\left(\bar{u}_{\mathrm{R}}{ }_{r}^{a} \gamma^{\nu} d_{\mathrm{R} t a}\right)
\end{array}
\end{aligned}
$$

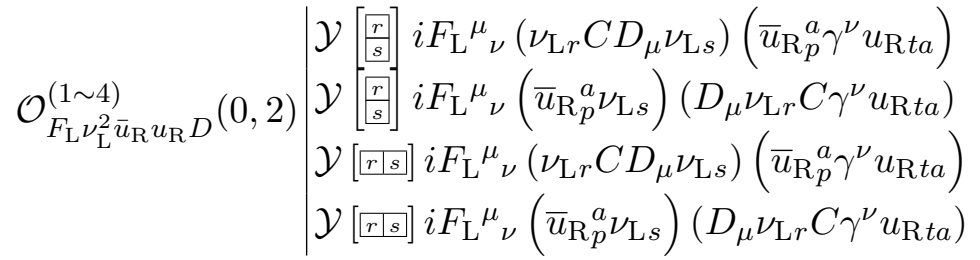

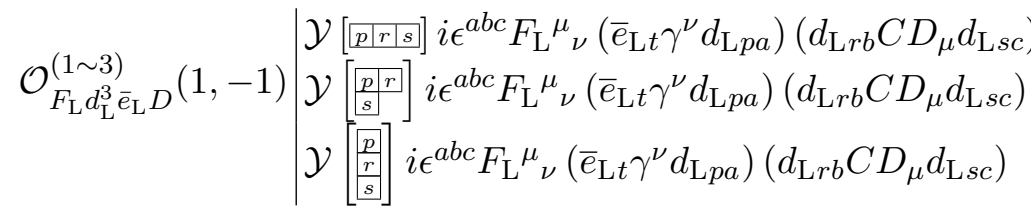




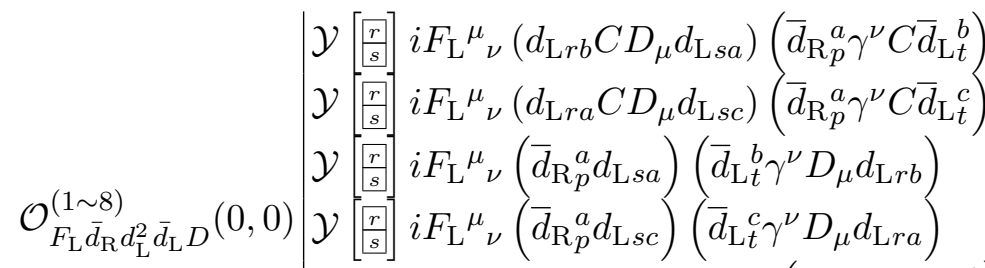

$$
\begin{aligned}
& \mathcal{Y}[\overline{r r s}] i F_{\mathrm{L}}^{\mu}{ }_{\nu}\left(d_{\mathrm{L} r b} C D_{\mu} d_{\mathrm{L} s a}\right)\left(\bar{d}_{\mathrm{R} p}^{a} \gamma^{\nu} C \bar{d}_{\mathrm{L} t}^{b}\right) \\
& \mathcal{Y}[r r s] i F_{\mathrm{L}}{ }_{\nu}{ }_{\nu}\left(d_{\mathrm{L} r a} C D_{\mu} d_{\mathrm{L} s c}\right)\left(\bar{d}_{\mathrm{R} p}^{a} \gamma^{\nu} C \bar{d}_{\mathrm{L} t}^{c}\right) \\
& \mathcal{Y}[r r s] i F_{\mathrm{L}}{ }^{\mu}{ }_{\nu}\left(\bar{d}_{\mathrm{R} p}^{a} d_{\mathrm{L} s a}\right)\left(\bar{d}_{\mathrm{L} t}^{b} \gamma^{\nu} D_{\mu} d_{\mathrm{L} r b}\right) \\
& \mathcal{Y}\left[[r s] i F_{\mathrm{L}}{ }^{\mu}{ }_{\nu}\left(\bar{d}_{\mathrm{R} p}{ }^{a} d_{\mathrm{L} s c}\right)\left(\bar{d}_{\mathrm{L} t}{ }^{c} \gamma^{\nu} D_{\mu} d_{\mathrm{L} r a}\right)\right.
\end{aligned}
$$

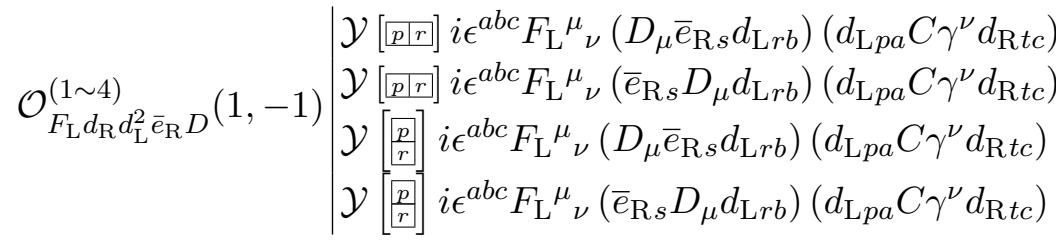

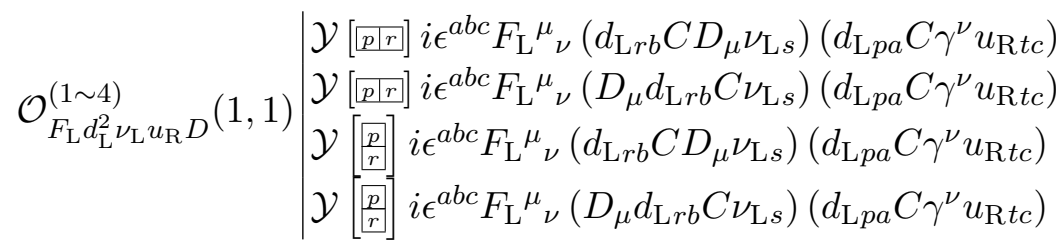

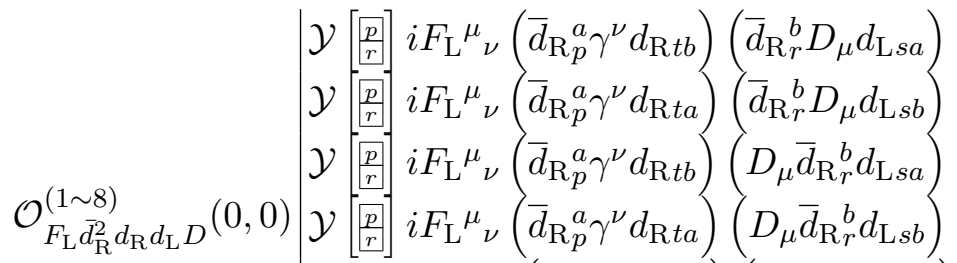

$$
\begin{aligned}
& \mathcal{Y}[\overline{p \mid r}] i F_{\mathrm{L}}{ }_{\nu}{ }_{\nu}\left(\bar{d}_{\mathrm{R} p}^{a} \gamma^{\nu} d_{\mathrm{R} t b}\right)\left(\bar{d}_{\mathrm{R} r}^{b} D_{\mu} d_{\mathrm{L} s a}\right) \\
& \mathcal{Y}[\overline{p \mid r]}] i F_{\mathrm{L}}{ }^{\mu}{ }_{\nu}\left(\bar{d}_{\mathrm{R} p}{ }^{a} \gamma^{\nu} d_{\mathrm{R} t a}\right)\left(\bar{d}_{\mathrm{R} r}^{b} D_{\mu} d_{\mathrm{L} s b}\right) \\
& \mathcal{Y}\left[\underline{p \mid r]} i F_{\mathrm{L}}{ }^{\mu}{ }_{\nu}\left(\bar{d}_{\mathrm{R}}{ }^{a} \gamma^{\nu} d_{\mathrm{R} t b}\right)\left(D_{\mu} \bar{d}_{\mathrm{R} r}^{b} d_{\mathrm{L} s a}\right)\right. \\
& \mathcal{Y}\left[\overline{p \mid r]} i F_{\mathrm{L}}{ }_{\nu}{ }_{\nu}\left(\bar{d}_{\mathrm{R}}{ }^{a} \gamma^{\nu} d_{\mathrm{R} t a}\right)\left(D_{\mu} \bar{d}_{\mathrm{R} r}^{b} d_{\mathrm{L} s b}\right)\right.
\end{aligned}
$$

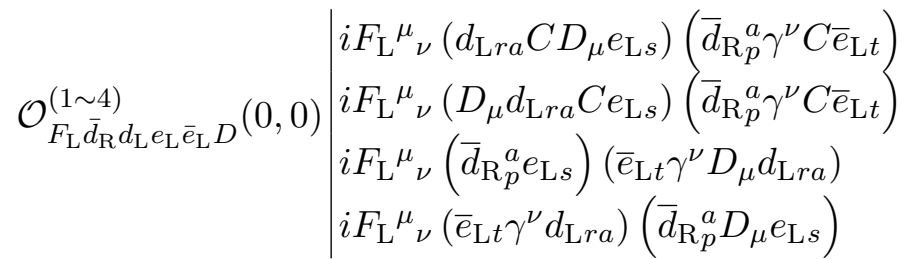

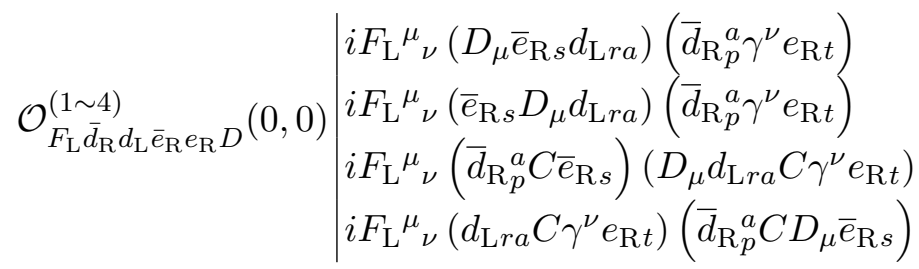




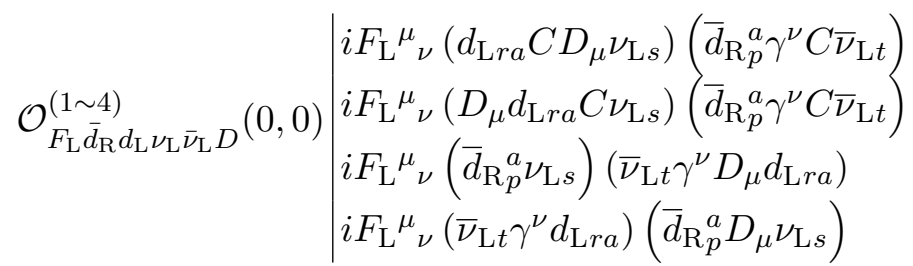

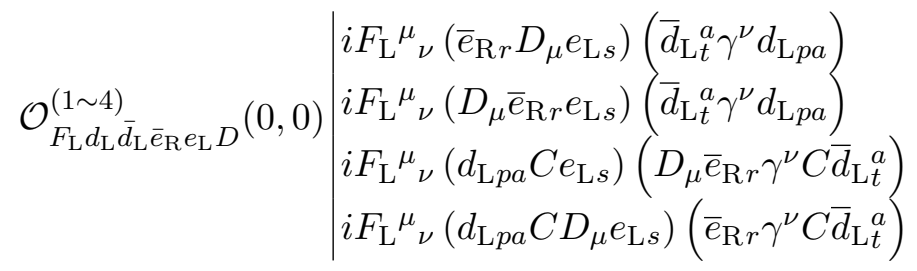

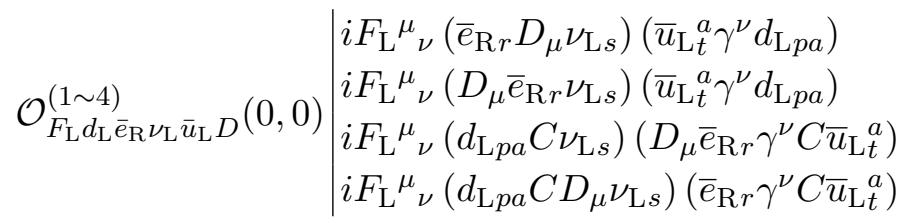

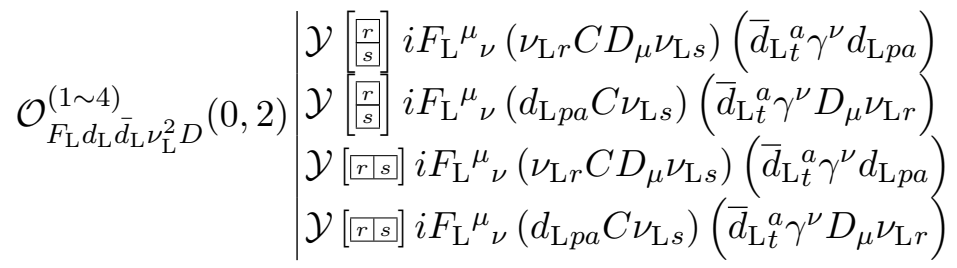

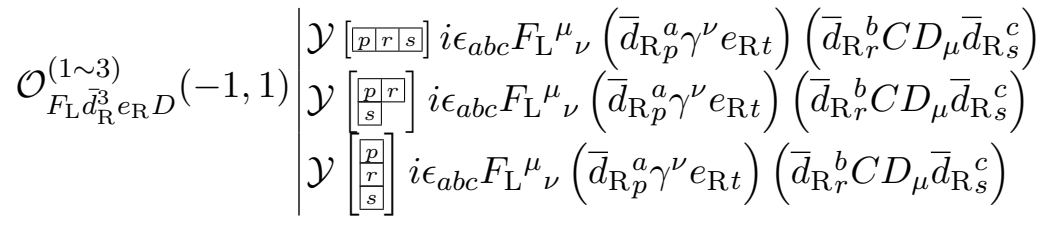

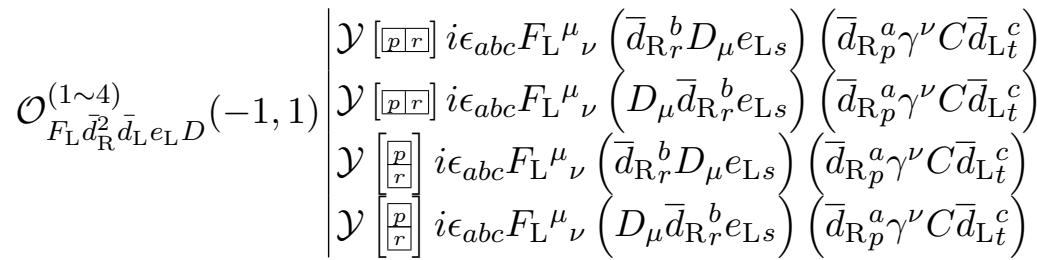

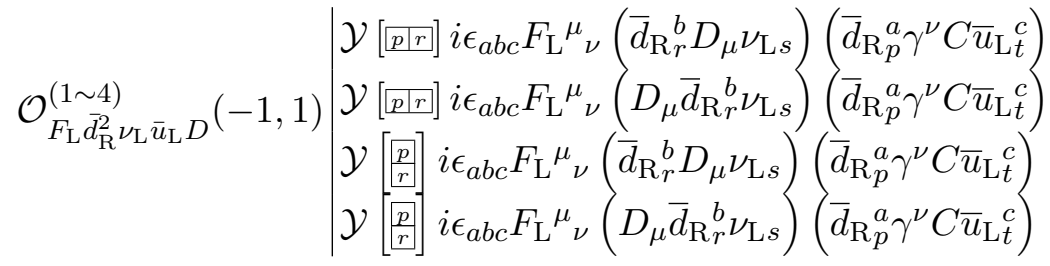

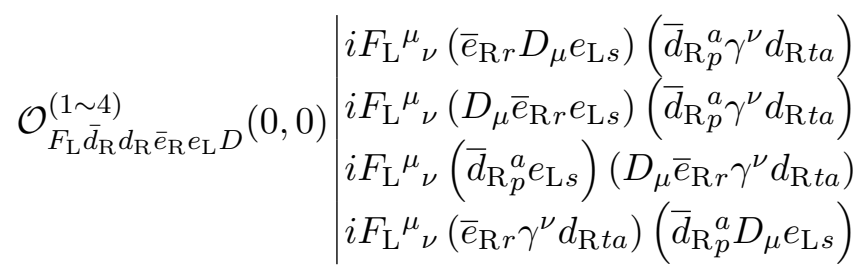




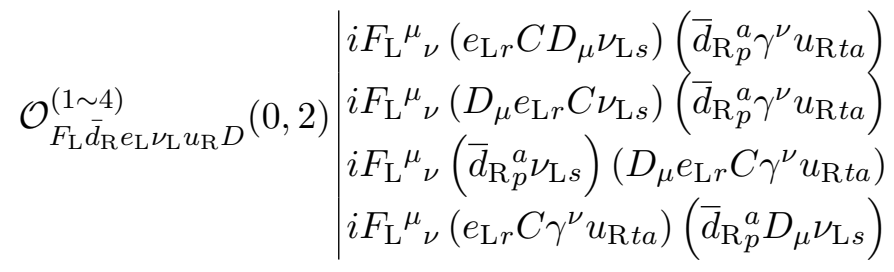

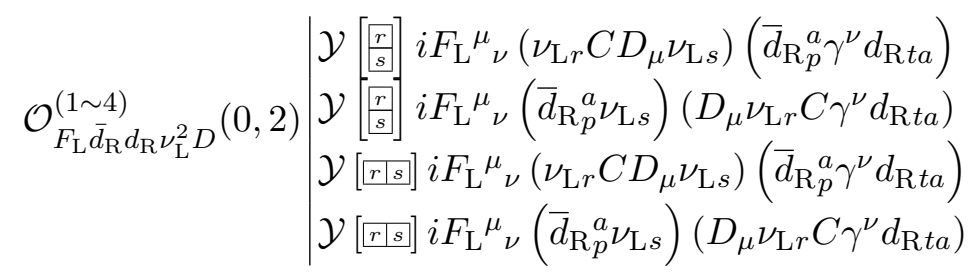

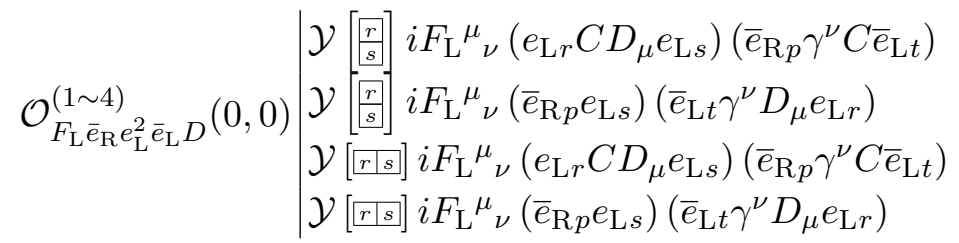

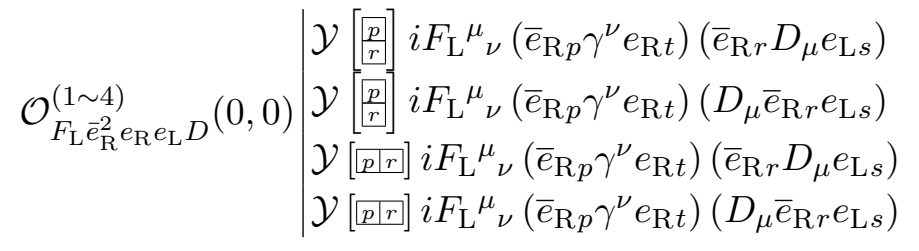

$$
\begin{aligned}
& \mathcal{O}_{F_{\mathrm{L}} \bar{e}_{\mathrm{R}} e_{\mathrm{L}} \nu_{\mathrm{L}} \bar{\nu}_{\mathrm{L}} D}(0,0) \mid \begin{array}{l}
i F_{\mathrm{L}}{ }^{\mu}{ }_{\nu}\left(e_{\mathrm{L} r} C D_{\mu} \nu_{\mathrm{L} s}\right)\left(\bar{e}_{\mathrm{R} p} \gamma^{\nu} C \bar{\nu}_{\mathrm{L} t}\right) \\
i F_{\mathrm{L}}{ }^{\mu}{ }_{\nu}\left(D_{\mu} e_{\mathrm{L} r} C \nu_{\mathrm{L} s}\right)\left(\bar{e}_{\mathrm{R} p} \gamma^{\nu} C \bar{\nu}_{\mathrm{L} t}\right) \\
i F_{\mathrm{L}}{ }^{\mu}{ }_{\nu}\left(\bar{e}_{\mathrm{R} p} \nu_{\mathrm{L} s}\right)\left(\bar{\nu}_{\mathrm{L} t} \gamma^{\nu} D_{\mu} e_{\mathrm{L} r}\right) \\
i F_{\mathrm{L}}{ }^{\mu}{ }_{\nu}\left(\bar{e}_{\mathrm{R} p} D_{\mu} \nu_{\mathrm{L} s}\right)\left(\bar{\nu}_{\mathrm{L} t} \gamma^{\nu} e_{\mathrm{L} r}\right)
\end{array}
\end{aligned}
$$

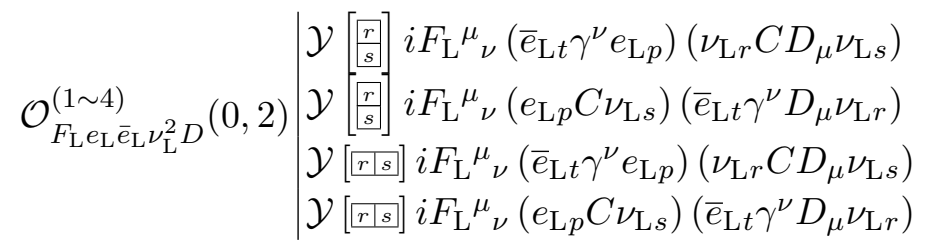

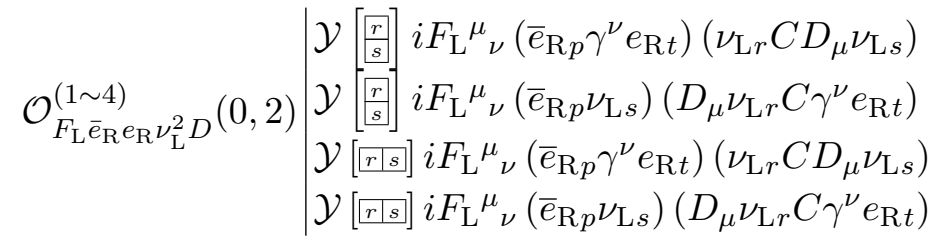

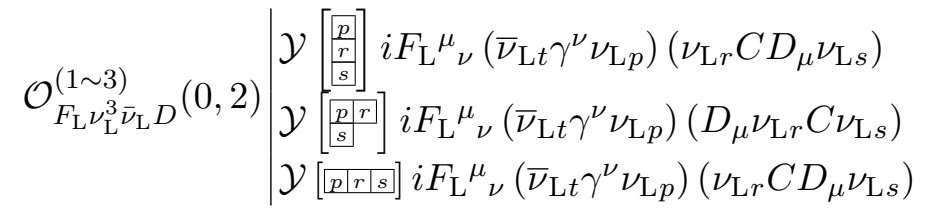




\section{Class $F_{\mathrm{R}} \psi^{3} \psi^{\dagger} D: 98$ types}

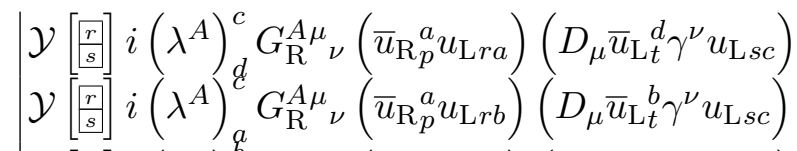

$$
\begin{aligned}
& \mathcal{Y}\left[\frac{r}{s}\right] i\left(\lambda^{A}\right)_{d}^{b} G_{\mathrm{R}}^{A}{ }_{\nu}{ }_{\nu}\left(\bar{u}_{\mathrm{R} p}{ }_{p}^{a} u_{\mathrm{L} r b}\right)\left(D_{\mu} \bar{u}_{\mathrm{L} t}^{d} \gamma^{\nu} u_{\mathrm{L} s a}\right) \\
& \mathcal{Y}\left[\frac{r}{s}\right] i\left(\lambda^{A}\right)_{a}^{b} G_{\mathrm{R}}^{A \mu}{ }_{\nu}\left(\bar{u}_{\mathrm{R} p}^{a} u_{\mathrm{L} r b}\right)\left(D_{\mu} \bar{u}_{\mathrm{L} t}{ }^{c} \gamma^{\nu} u_{\mathrm{L} s c}\right) \\
& \mathcal{O}_{G_{\mathrm{R}} \bar{u}_{\mathrm{R}} u_{\mathrm{L}}^{2} \bar{u}_{\mathrm{L}} D}^{(1 \sim 12)}(0,0) \mathcal{Y}\left[\frac{r}{s}\right] i\left(\lambda^{A}\right)_{d}^{q} G_{\mathrm{R}}^{A \mu}{ }_{\nu}\left(u_{\mathrm{L} r a} C D_{\mu} u_{\mathrm{L} s c}\right)\left(\bar{u}_{\mathrm{R}}{ }_{p}^{a} \gamma^{\nu} C \bar{u}_{\mathrm{L} t}^{d}\right) \\
& \mathcal{Y}\left[\begin{array}{l}
\left.\frac{r}{s}\right] \\
s
\end{array}\right]\left(\lambda^{A}\right)_{a c}^{c} G_{\mathrm{R}}^{A \mu}{ }_{\nu}\left(u_{\mathrm{L} r b} C D_{\mu} u_{\mathrm{L} s c}\right)\left(\bar{u}_{\mathrm{R} p}^{a} \gamma^{\nu} C \bar{u}_{\mathrm{L} t}^{b}\right) \\
& \mathcal{Y}[\overline{r r s}] i\left(\lambda^{A}\right)_{d}^{a_{c}^{c}} G_{\mathrm{R}}^{A \mu}{ }_{\nu}\left(\bar{u}_{\mathrm{R} p}{ }^{a} u_{\mathrm{L} r a}\right)\left(D_{\mu} \bar{u}_{\mathrm{L} t}{ }^{d} \gamma^{\nu} u_{\mathrm{L} s c}\right) \\
& \mathcal{Y}[\overline{r r s}] i\left(\lambda^{A}\right)_{a}^{c} G_{\mathrm{R}}^{A \mu}{ }_{\nu}\left(\bar{u}_{\mathrm{R}}{ }_{p}^{a} u_{\mathrm{L} r b}\right)\left(D_{\mu} \bar{u}_{\mathrm{L} t}^{b} \gamma^{\nu} u_{\mathrm{L} s c}\right) \\
& \mathcal{Y}[\overline{r r s}] i\left(\lambda^{A}\right)_{d}^{b} G_{\mathrm{R}}^{A \mu}{ }_{\nu}\left(\bar{u}_{\mathrm{R}}^{a}{ }_{p} u_{\mathrm{L} r b}\right)\left(D_{\mu} \bar{u}_{\mathrm{L} t}{ }^{d} \gamma^{\nu} u_{\mathrm{L} s a}\right) \\
& \mathcal{Y}[\overline{r s s}] i\left(\lambda^{A}\right)_{a}^{b} G_{\mathrm{R}}^{A \mu}{ }_{\nu}\left(\bar{u}_{\mathrm{R}}{ }_{p}^{a} u_{\mathrm{L} r b}\right)\left(D_{\mu} \bar{u}_{\mathrm{L} t}^{c} \gamma^{\nu} u_{\mathrm{L} s c}\right) \\
& \mathcal{Y}[\overline{r r s}] i\left(\lambda^{A}\right)_{d}^{q} G_{\mathrm{R}}^{A}{ }_{\nu}\left(u_{\mathrm{L} r a} C D_{\mu} u_{\mathrm{L} s c}\right)\left(\bar{u}_{\mathrm{R}}^{a} \gamma^{\nu} C \bar{u}_{\mathrm{L} t}^{d}\right) \\
& \mathcal{Y}\left[[r s] i\left(\lambda^{A}\right)_{a}^{c} G_{\mathrm{R}}^{A \mu}{ }_{\nu}\left(u_{\mathrm{L} r b} C D_{\mu} u_{\mathrm{L} s c}\right)\left(\bar{u}_{\mathrm{R}}^{a} \gamma^{\nu} C \bar{u}_{\mathrm{L} t}^{b}\right)\right. \\
& \mathcal{Y}\left[\frac{r}{s}\right] i F_{\mathrm{R}}{ }_{\nu}{ }_{\nu}\left(\bar{u}_{\mathrm{R} p}^{a} u_{\mathrm{L} r b}\right)\left(D_{\mu} \bar{u}_{\mathrm{L} t}^{b} \gamma^{\nu} u_{\mathrm{L} s a}\right) \\
& \mathcal{Y}\left[\begin{array}{lll}
\hline s \\
\hdashline
\end{array}\right] F_{\mathrm{R}}{ }_{\nu}{ }_{\nu}\left(\bar{u}_{\mathrm{R} p}^{a} u_{\mathrm{L} r a}\right)\left(D_{\mu} \bar{u}_{\mathrm{L}}^{c} \gamma^{\nu} u_{\mathrm{L} s c}\right) \\
& \mathcal{O}_{F_{\mathrm{R}} \bar{u}_{\mathrm{R}} u_{\mathrm{L}}^{2} \bar{u}_{\mathrm{L}} D}^{(1 \sim 6)}(0,0) \mathcal{Y}\left[\overline{\frac{r}{s}}\right] i F_{\mathrm{R}}{ }^{\mu}{ }_{\nu}\left(u_{\mathrm{L} r b} C D_{\mu} u_{\mathrm{L} s a}\right)\left(\bar{u}_{\mathrm{R} p}^{a} \gamma^{\nu} C \bar{u}_{\mathrm{L} t}^{b}\right) \\
& \mathcal{Y}[\underline{[r] s}] i F_{\mathrm{R}}{ }_{\nu}{ }_{\nu}\left(\bar{u}_{\mathrm{R} p}^{a} u_{\mathrm{L} r b}\right)\left(D_{\mu} \bar{u}_{\mathrm{L} t}^{b} \gamma^{\nu} u_{\mathrm{L} s a}\right) \\
& \mathcal{Y}\left[\overline{r r s]} i F_{\mathrm{R}}{ }_{\nu}{ }_{\nu}\left(\bar{u}_{\mathrm{R} p}^{a} u_{\mathrm{L} r a}\right)\left(D_{\mu} \bar{u}_{\mathrm{L} t}^{c} \gamma^{\nu} u_{\mathrm{L} s c}\right)\right. \\
& \mathcal{Y}[r] s] i F_{\mathrm{R}}^{\mu}{ }_{\nu}\left(u_{\mathrm{L} r b} C D_{\mu} u_{\mathrm{L} s a}\right)\left(\bar{u}_{\mathrm{R}}^{a} \gamma^{\nu} C \bar{u}_{\mathrm{L} t}^{b}\right) \\
& \mathcal{Y}\left[\frac{r}{s}\right] i\left(\lambda^{A}\right)_{d}^{a} \epsilon^{b c d} G_{\mathrm{R}}^{A \mu}{ }_{\nu}\left(d_{\mathrm{L} p a} C u_{\mathrm{L} r b}\right)\left(u_{\mathrm{L} s c} C \gamma^{\nu} D_{\mu} e_{\mathrm{R} t}\right) \\
& \mathcal{Y}\left[\begin{array}{l}
r \\
s
\end{array}\right] i\left(\lambda^{A}\right)_{d}^{c} \epsilon^{a b d} G_{\mathrm{R}}^{A \mu}{ }_{\nu}\left(d_{\mathrm{L} p a} C u_{\mathrm{L} r b}\right)\left(u_{\mathrm{L} s c} C \gamma^{\nu} D_{\mu} e_{\mathrm{R} t}\right)
\end{aligned}
$$

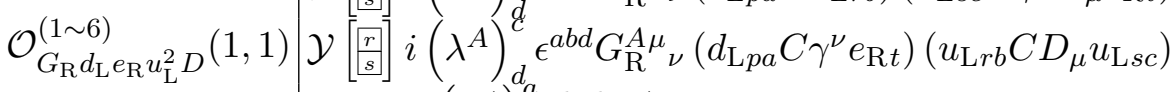

$$
\begin{aligned}
& \mathcal{Y}\left[[r s s] i\left(\lambda^{A}\right)_{d}^{a} \epsilon^{b c d} G_{\mathrm{R}}^{A \mu}{ }_{\nu}\left(d_{\mathrm{L} p a} C u_{\mathrm{L} r b}\right)\left(u_{\mathrm{L} s c} C \gamma^{\nu} D_{\mu} e_{\mathrm{R} t}\right)\right. \\
& \mathcal{Y}\left[r[s] i\left(\lambda^{A}\right)_{d}^{c} \epsilon^{a b d} G_{\mathrm{R}}^{A \mu}{ }_{\nu}\left(d_{\mathrm{L} p a} C u_{\mathrm{L} r b}\right)\left(u_{\mathrm{L} s c} C \gamma^{\nu} D_{\mu} e_{\mathrm{R} t}\right)\right. \\
& \mathcal{Y}\left[[r s] i\left(\lambda^{A}\right)_{d}^{d} \epsilon^{b c d} G_{\mathrm{R}}^{A \mu}{ }_{\nu}\left(d_{\mathrm{L} p a} C \gamma^{\nu} e_{\mathrm{R} t}\right)\left(u_{\mathrm{L} r b} C D_{\mu} u_{\mathrm{L} s c}\right)\right. \\
& \mathcal{Y}\left[[r s] i \epsilon^{a b c} F_{\mathrm{R}}{ }_{\nu}{ }_{\nu}\left(d_{\mathrm{L} p a} C u_{\mathrm{L} r b}\right)\left(u_{\mathrm{L} s c} C \gamma^{\nu} D_{\mu} e_{\mathrm{R} t}\right)\right. \\
& \mathcal{O}_{F_{\mathrm{R}} d_{\mathrm{L}} e_{\mathrm{R}} u_{\mathrm{L}}^{2} D}^{(\sim 3)}(1,1) \mathcal{Y}[[r]] i \epsilon^{a b c} F_{\mathrm{R}}{ }^{\mu}{ }_{\nu}\left(d_{\mathrm{L} p a} C \gamma^{\nu} e_{\mathrm{R} t}\right)\left(u_{\mathrm{L} r b} C D_{\mu} u_{\mathrm{L} s c}\right) \\
& \mathcal{Y}\left[\frac{r}{s}\right] i \epsilon^{a b c} F_{\mathrm{R}}{ }^{\mu}{ }_{\nu}\left(d_{\mathrm{L} p a} C u_{\mathrm{L} r b}\right)\left(u_{\mathrm{L} s c} C \gamma^{\nu} D_{\mu} e_{\mathrm{R} t}\right) \\
& \mathcal{Y}\left[\begin{array}{l}
r \\
s
\end{array}\right] i\left(\lambda^{A}\right)_{d}^{a} \epsilon^{b c d} G_{\mathrm{R}}^{A \mu}{ }_{\nu}\left(e_{\mathrm{L} p} C u_{\mathrm{L} r a}\right)\left(u_{\mathrm{L} s b} C \gamma^{\nu} D_{\mu} d_{\mathrm{R} t c}\right) \\
& \mathcal{Y}\left[\frac{r}{s}\right] i\left(\lambda^{A}\right)_{d}^{c} \epsilon^{a b d} G_{\mathrm{R}}^{A \mu}{ }_{\nu}\left(e_{\mathrm{L} p} C u_{\mathrm{L} r a}\right)\left(u_{\mathrm{L} s b} C \gamma^{\nu} D_{\mu} d_{\mathrm{R} t c}\right) \\
& \mathcal{O}_{G_{\mathrm{R}} d_{\mathrm{R}} e_{\mathrm{L}} u_{\mathrm{L}}^{2} D}^{(1 \sim 6)}(1,1) \mathcal{Y}\left[\begin{array}{l}
\left.\frac{r}{s}\right] \\
s
\end{array}\right] i\left(\lambda^{A}\right)_{d}^{a} \epsilon^{b c d} G_{\mathrm{R}}^{A \mu}{ }_{\nu}\left(u_{\mathrm{L} r a} C D_{\mu} u_{\mathrm{L} s b}\right)\left(e_{\mathrm{L} p} C \gamma^{\nu} d_{\mathrm{R} t c}\right) \\
& \mathcal{Y}[[r] s] i\left(\lambda^{A}\right)_{d}^{a} \epsilon^{b c d} G_{\mathrm{R}}^{A \mu}{ }_{\nu}\left(e_{\mathrm{L} p} C u_{\mathrm{L} r a}\right)\left(u_{\mathrm{L} s b} C \gamma^{\nu} D_{\mu} d_{\mathrm{R} t c}\right) \\
& \mathcal{Y}[\overline{r r s}] i\left(\lambda^{A}\right)_{d}^{c} \epsilon^{a b d} G_{\mathrm{R}}^{A \mu}{ }_{\nu}\left(e_{\mathrm{L} p} C u_{\mathrm{L} r a}\right)\left(u_{\mathrm{L} s b} C \gamma^{\nu} D_{\mu} d_{\mathrm{R} t c}\right) \\
& \mathcal{Y}[\overline{r r s}] i\left(\lambda^{A}\right)_{d}^{d} \epsilon^{b c d} G_{\mathrm{R}}^{A \mu}{ }_{\nu}\left(u_{\mathrm{L} r a} C D_{\mu} u_{\mathrm{L} s b}\right)\left(e_{\mathrm{L} p} C \gamma^{\nu} d_{\mathrm{R} t c}\right)
\end{aligned}
$$




$$
\begin{aligned}
& \mathcal{Y}[\overline{r r s}] i \epsilon^{a b c} F_{\mathrm{R}^{\mu}}{ }_{\nu}\left(e_{\mathrm{L} p} C u_{\mathrm{L} r a}\right)\left(u_{\mathrm{L} s b} C \gamma^{\nu} D_{\mu} d_{\mathrm{R} t c}\right) \\
& \mathcal{O}_{F_{\mathrm{R}} d_{\mathrm{R}} e_{\mathrm{L}} u_{\mathrm{L}}^{2} D}^{(1 \sim 3)^{2}}(1,1) \mathcal{Y}\left[[r \mid s] i \epsilon^{a b c} F_{\mathrm{R}}{ }^{\mu}{ }_{\nu}\left(u_{\mathrm{L} r a} C D_{\mu} u_{\mathrm{L} s b}\right)\left(e_{\mathrm{L} p} C \gamma^{\nu} d_{\mathrm{R} t c}\right)\right. \\
& \mathcal{Y}\left[\begin{array}{l}
\frac{r}{s} \\
s
\end{array}\right] i \epsilon^{a b c} F_{\mathrm{R}}{ }_{\nu}{ }_{\nu}\left(e_{\mathrm{L} p} C u_{\mathrm{L} r a}\right)\left(u_{\mathrm{L} s b} C \gamma^{\nu} D_{\mu} d_{\mathrm{R} t c}\right)
\end{aligned}
$$

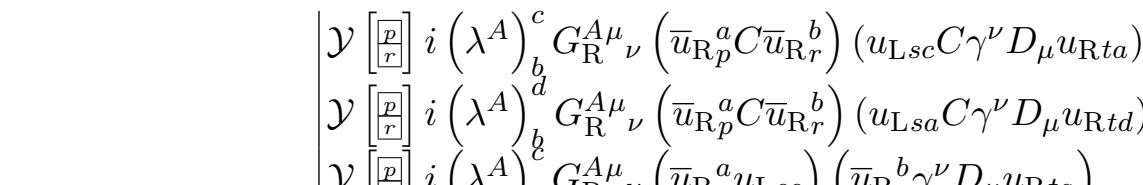

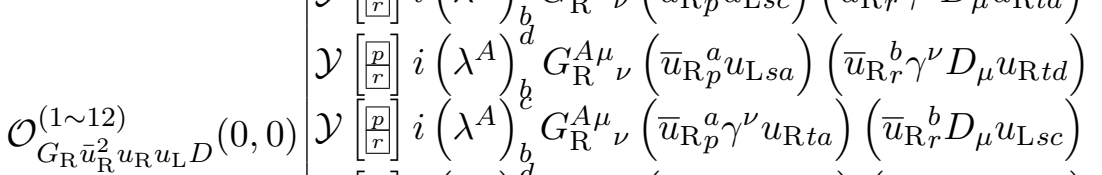

$$
\begin{aligned}
& \mathcal{Y}\left[\frac{p}{r}\right] i\left(\lambda^{A}\right)_{b}^{d} G_{\mathrm{R}}^{A}{ }_{\nu}{ }_{\nu}\left(\bar{u}_{\mathrm{R}}^{a} \gamma^{\nu} u_{\mathrm{R} t d}\right)\left(\bar{u}_{\mathrm{R} r}^{b} D_{\mu} u_{\mathrm{L} s a}\right) \\
& \mathcal{Y}[\overline{p p r}] i\left(\lambda^{A}\right)_{b}^{c} G_{\mathrm{R}}^{A \mu}{ }_{\nu}\left(\bar{u}_{\mathrm{R}}{ }_{p}^{a} C \bar{u}_{\mathrm{R} r}^{b}\right)\left(u_{\mathrm{L} s c} C \gamma^{\nu} D_{\mu} u_{\mathrm{R} t a}\right) \\
& \mathcal{Y}[\overline{p p r}] i\left(\lambda^{A}\right)_{b}^{d} G_{\mathrm{R}}^{A \mu}{ }_{\nu}\left(\bar{u}_{\mathrm{R}}^{a} C \bar{u}_{\mathrm{R}}{ }_{r}^{b}\right)\left(u_{\mathrm{L} s a} C \gamma^{\nu} D_{\mu} u_{\mathrm{R} t d}\right) \\
& \mathcal{Y}[\overline{p p r}] i\left(\lambda^{A}\right)_{b}^{c} G_{\mathrm{R}}^{A \mu}{ }_{\nu}\left(\bar{u}_{\mathrm{R} p}^{a} u_{\mathrm{L} s c}\right)\left(\bar{u}_{\mathrm{R} r}^{b} \gamma^{\nu} D_{\mu} u_{\mathrm{R} t a}\right) \\
& \mathcal{Y}[\overline{p p r}] i\left(\lambda^{A}\right)_{b}^{d} G_{\mathrm{R}}^{A \mu}{ }_{\nu}\left(\bar{u}_{\mathrm{R} p}^{a} u_{\mathrm{L} s a}\right)\left(\bar{u}_{\mathrm{R}}^{b} \gamma^{\nu} D_{\mu} u_{\mathrm{R} t d}\right) \\
& \mathcal{Y}[\overline{p \mid r]}] i\left(\lambda^{A}\right)_{b}^{c} G_{\mathrm{R}}^{A \mu}{ }_{\nu}\left(\bar{u}_{\mathrm{R} p}^{a} \gamma^{\nu} u_{\mathrm{R} t a}\right)\left(\bar{u}_{\mathrm{R}}{ }_{r}^{b} D_{\mu} u_{\mathrm{L} s c}\right) \\
& \mathcal{Y}\left[[p \mid r] i\left(\lambda^{A}\right)_{b}^{d} G_{\mathrm{R}}^{A \mu}{ }_{\nu}\left(\bar{u}_{\mathrm{R}}^{a} \gamma^{\nu} u_{\mathrm{R} t d}\right)\left(\bar{u}_{\mathrm{R}}{ }_{r}^{b} D_{\mu} u_{\mathrm{L} s a}\right)\right. \\
& \mathcal{Y}\left[\frac{p}{r}\right] i F_{\mathrm{R}}{ }_{\nu}{ }_{\nu}\left(\bar{u}_{\mathrm{R} p}^{a} C \bar{u}_{\mathrm{R}}{ }_{r}^{b}\right)\left(u_{\mathrm{L} s a} C \gamma^{\nu} D_{\mu} u_{\mathrm{R} t b}\right) \\
& \mathcal{Y}\left[\frac{p}{r}\right] i F_{\mathrm{R}}{ }_{\nu}{ }_{\nu}\left(\bar{u}_{\mathrm{R} p}^{a} u_{\mathrm{L} s a}\right)\left(\bar{u}_{\mathrm{R}}{ }_{r}^{b} \gamma^{\nu} D_{\mu} u_{\mathrm{R} t b}\right) \\
& \mathcal{O}_{F_{\mathrm{R}} \bar{u}_{\mathrm{R}}^{2} u_{\mathrm{R}} u_{\mathrm{L}} D}^{(1 \sim 6)}(0,0) \mathcal{Y}\left[\begin{array}{l}
\underline{p} \\
\vec{r}
\end{array}\right] i F_{\mathrm{R}}{ }_{\nu}{ }_{\nu}\left(\bar{u}_{\mathrm{R} p}^{a} \gamma^{\nu} u_{\mathrm{R} t b}\right)\left(\bar{u}_{\mathrm{R}}{ }_{r}^{b} D_{\mu} u_{\mathrm{L} s a}\right) \\
& \mathcal{Y}[\overline{p p r}] i F_{\mathrm{R}}^{\mu}{ }_{\nu}\left(\bar{u}_{\mathrm{R} p}^{a} C \bar{u}_{\mathrm{R} r}^{b}\right)\left(u_{\mathrm{L} s a} C \gamma^{\nu} D_{\mu} u_{\mathrm{R} t b}\right) \\
& \mathcal{Y}\left[\underline{p n r]} i F_{\mathrm{R}}{ }_{\nu}{ }_{\nu}\left(\bar{u}_{\mathrm{R} p}^{a} u_{\mathrm{L} s a}\right)\left(\bar{u}_{\mathrm{R} r}^{b} \gamma^{\nu} D_{\mu} u_{\mathrm{R} t b}\right)\right. \\
& \mathcal{Y}[\overline{p p r}] i F_{\mathrm{R}}{ }_{\nu}{ }_{\nu}\left(\bar{u}_{\mathrm{R} p}^{a} \gamma^{\nu} u_{\mathrm{R} t b}\right)\left(\bar{u}_{\mathrm{R}}^{b} D_{\mu} u_{\mathrm{L} s a}\right)
\end{aligned}
$$

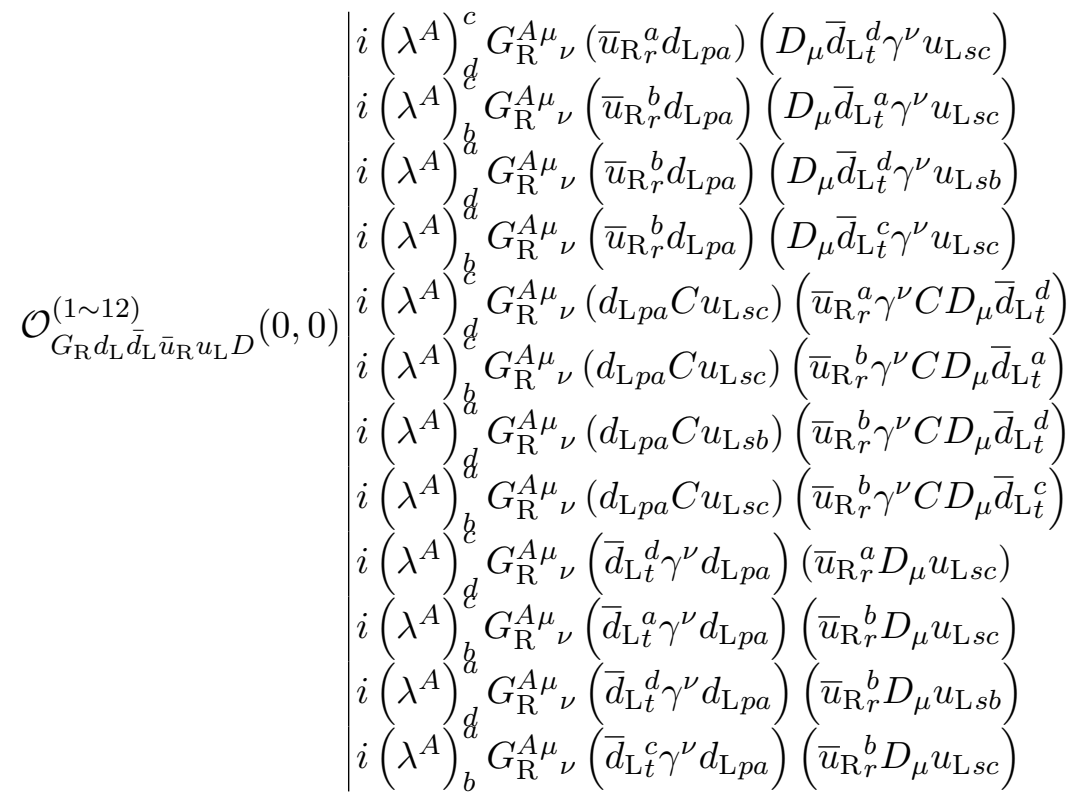




$$
\begin{aligned}
& i F_{\mathrm{R}}^{\mu}{ }_{\nu}\left(\bar{u}_{\mathrm{R} r}^{b} d_{\mathrm{L} p a}\right)\left(D_{\mu} \bar{d}_{\mathrm{L} t}^{a} \gamma^{\nu} u_{\mathrm{L} s b}\right) \\
& i F_{\mathrm{R}}{ }_{\nu}{ }_{\nu}\left(\bar{u}_{\mathrm{R} r}^{a} d_{\mathrm{L} p a}\right)\left(D_{\mu} \bar{d}_{\mathrm{L}}^{c} \gamma^{\nu} u_{\mathrm{L} s c}\right) \\
& \mathcal{O}_{F_{\mathrm{R}} d_{\mathrm{L}} \bar{d}_{\mathrm{L}} \bar{u}_{\mathrm{R}} u_{\mathrm{L}} D}^{\left(1 \sim{ }^{\prime}\right.}(0,0) i F_{\mathrm{R}}{ }_{\nu}{ }_{\nu}\left(d_{\mathrm{L} p a} C u_{\mathrm{L} s b}\right)\left(\bar{u}_{\mathrm{R}}{ }_{r}^{b} \gamma^{\nu} C D_{\mu} \bar{d}_{\mathrm{L} t}^{a}\right) \\
& i F_{\mathrm{R}}^{\mu}{ }_{\nu}\left(d_{\mathrm{L} p a} C u_{\mathrm{L} s c}\right)\left(\bar{u}_{\mathrm{R}}{ }_{r} \gamma^{\nu} C D_{\mu} \bar{d}_{\mathrm{L} t}^{c}\right) \\
& i F_{\mathrm{R}}{ }_{\nu}{ }_{\nu}\left(\bar{d}_{\mathrm{L} t}^{a} \gamma^{\nu} d_{\mathrm{L} p a}\right)\left(\bar{u}_{\mathrm{R} r}^{b} D_{\mu} u_{\mathrm{L} s b}\right) \\
& i F_{\mathrm{R}}{ }_{\nu}{ }_{\nu}\left(\bar{d}_{\mathrm{L} t}^{c} \gamma^{\nu} d_{\mathrm{L} p a}\right)\left(\bar{u}_{\mathrm{R} r}^{a} D_{\mu} u_{\mathrm{L} s c}\right)
\end{aligned}
$$

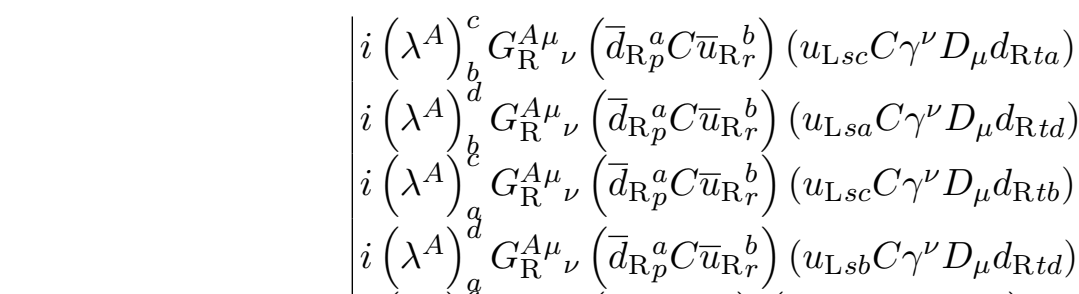

$$
\begin{aligned}
& \mathcal{O}_{G_{\mathrm{R}} \bar{d}_{\mathrm{R}} d_{\mathrm{R}} \bar{u}_{\mathrm{R}} u_{\mathrm{L}} D}^{(1 \sim 12)}(0,0) i\left(\lambda^{A}\right)_{b}^{c} G_{\mathrm{R}}^{A \mu}{ }_{\nu}\left(\bar{d}_{\mathrm{R} p}^{a} u_{\mathrm{L} s c}\right)\left(\bar{u}_{\mathrm{R} r}^{b} \gamma^{\nu} D_{\mu} d_{\mathrm{R} t a}\right) \\
& i\left(\lambda^{A}\right)_{b}^{d} G_{\mathrm{R}}^{A \mu}{ }_{\nu}\left(\bar{d}_{\mathrm{R} p}{ }_{p}^{a} u_{\mathrm{L} s a}\right)\left(\bar{u}_{\mathrm{R}}{ }_{r}^{b} \gamma^{\nu} D_{\mu} d_{\mathrm{R} t d}\right) \\
& i\left(\lambda^{A}\right)_{a}^{c} G_{\mathrm{R}}^{A \mu}{ }_{\nu}\left(\bar{d}_{\mathrm{R} p}^{a} u_{\mathrm{L} s c}\right)\left(\bar{u}_{\mathrm{R} r}^{b} \gamma^{\nu} D_{\mu} d_{\mathrm{R} t b}\right) \\
& i\left(\lambda^{A}\right)_{a}^{d} G_{\mathrm{R}}^{A \mu}{ }_{\nu}\left(\bar{d}_{\mathrm{R} p}^{a} u_{\mathrm{L} s b}\right)\left(\bar{u}_{\mathrm{R}}^{b} \gamma^{\nu} D_{\mu} d_{\mathrm{R} t d}\right) \\
& i\left(\lambda^{A}\right)_{b}^{c} G_{\mathrm{R}}^{A \mu}{ }_{\nu}\left(\bar{d}_{\mathrm{R} p}^{a} \gamma^{\nu} d_{\mathrm{R} t a}\right)\left(\bar{u}_{\mathrm{R} r}^{b} D_{\mu} u_{\mathrm{L} s c}\right) \\
& i\left(\lambda^{A}\right)_{b}^{d} G_{\mathrm{R}}^{A \mu}{ }_{\nu}\left(\bar{d}_{\mathrm{R} p}^{a} \gamma^{\nu} d_{\mathrm{R} t d}\right)\left(\bar{u}_{\mathrm{R} r}^{b} D_{\mu} u_{\mathrm{L} s a}\right) \\
& i\left(\lambda^{A}\right)_{a}^{c} G_{\mathrm{R}}^{A \mu}{ }_{\nu}\left(\bar{d}_{\mathrm{R}}^{a} \gamma^{\nu} d_{\mathrm{R} t b}\right)\left(\bar{u}_{\mathrm{R} r}^{b} D_{\mu} u_{\mathrm{L} s c}\right) \\
& i\left(\lambda^{A}\right)_{a}^{d} G_{\mathrm{R}}^{A \mu}{ }_{\nu}\left(\bar{d}_{\mathrm{R}}^{a} \gamma^{\nu} d_{\mathrm{R} t d}\right)\left(\bar{u}_{\mathrm{R} r}^{b} D_{\mu} u_{\mathrm{L} s b}\right)
\end{aligned}
$$

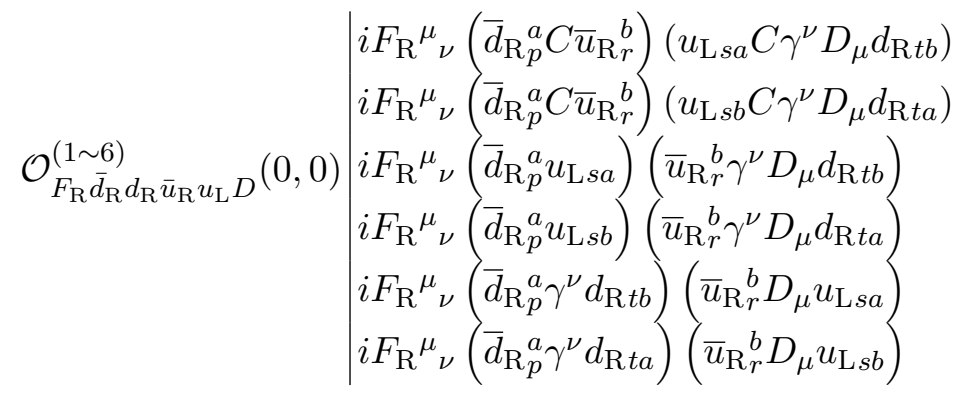

$$
\begin{aligned}
& \mathcal{O}_{G_{\mathrm{R}} e_{\mathrm{L}} \bar{e}_{\mathrm{L}} \bar{u}_{\mathrm{R}} u_{\mathrm{L}} D}(0,0) \mid \begin{array}{l}
i\left(\lambda^{A}\right)_{a}^{b} G_{\mathrm{R}}^{A \mu}{ }_{\nu}\left(\bar{u}_{\mathrm{R}}{ }_{r}^{a} e_{\mathrm{L} p}\right)\left(D_{\mu} \bar{e}_{\mathrm{L} t} \gamma^{\nu} u_{\mathrm{L} s b}\right) \\
i\left(\lambda^{A}\right)_{a}^{b} G_{\mathrm{R}}^{A \mu}{ }_{\nu}\left(e_{\mathrm{L} p} C u_{\mathrm{L} s b}\right)\left(\bar{u}_{\mathrm{R}}{ }_{r}^{a} \gamma^{\nu} C D_{\mu} \bar{e}_{\mathrm{L} t}\right) \\
i\left(\lambda^{A}\right)_{a}^{b} G_{\mathrm{R}}^{A \mu}{ }_{\nu}\left(\bar{e}_{\mathrm{L} t} \gamma^{\nu} e_{\mathrm{L} p}\right)\left(\bar{u}_{\mathrm{R}}{ }_{r}^{a} D_{\mu} u_{\mathrm{L} s b}\right)
\end{array} \\
& \mathcal{O}_{F_{\mathrm{R}} e_{\mathrm{L}} \bar{e}_{\mathrm{L}} \bar{u}_{\mathrm{R}} u_{\mathrm{L}} D}^{(1 \sim 3,0)} \mid \begin{array}{l}
i F_{\mathrm{R}}{ }^{\mu}{ }_{\nu}\left(\bar{u}_{\mathrm{R} r}{ }^{a} e_{\mathrm{L} p}\right)\left(D_{\mu} \bar{e}_{\mathrm{L} t} \gamma^{\nu} u_{\mathrm{L} s a}\right) \\
i F_{\mathrm{R}}{ }^{\mu}{ }_{\nu}\left(e_{\mathrm{L} p} C u_{\mathrm{L} s a}\right)\left(\bar{u}_{\mathrm{R}}{ }_{r}^{a} \gamma^{\nu} C D_{\mu} \bar{e}_{\mathrm{L} t}\right)
\end{array} \\
& i F_{\mathrm{R}}{ }_{\nu}{ }_{\nu}\left(\bar{e}_{\mathrm{L} t} \gamma^{\nu} e_{\mathrm{L} p}\right)\left(\bar{u}_{\mathrm{R}}{ }_{r}^{a} D_{\mu} u_{\mathrm{L} s a}\right)
\end{aligned}
$$




$$
\begin{aligned}
& \mathcal{O}_{G_{\mathrm{R}} \bar{e}_{\mathrm{R}} e_{\mathrm{R}} \bar{u}_{\mathrm{R}} u_{\mathrm{L}} D}^{(1 \sim 0)} \mid \begin{array}{l}
i\left(\lambda^{A}\right)_{a}^{b} G_{\mathrm{R}}^{A \mu}{ }_{\nu}\left(\bar{e}_{\mathrm{R} p} C \bar{u}_{\mathrm{R}}{ }_{r}^{a}\right)\left(u_{\mathrm{L} s b} C \gamma^{\nu} D_{\mu} e_{\mathrm{R} t}\right) \\
i\left(\lambda^{A}\right)_{a}^{b} G_{\mathrm{R}}^{A \mu}{ }_{\nu}\left(\bar{e}_{\mathrm{R} p} u_{\mathrm{L} s b}\right)\left(\bar{u}_{\mathrm{R}}{ }_{r} \gamma^{\nu} D_{\mu} e_{\mathrm{R} t}\right) \\
i\left(\lambda^{A}\right)_{a}^{b} G_{\mathrm{R}}^{A \mu}{ }_{\nu}\left(\bar{e}_{\mathrm{R} p} \gamma^{\nu} e_{\mathrm{R} t}\right)\left(\bar{u}_{\mathrm{R}}{ }_{r}^{a} D_{\mu} u_{\mathrm{L} s b}\right)
\end{array} \\
& \mathcal{O}_{F_{\mathrm{R}} \bar{e}_{\mathrm{R}} e_{\mathrm{R}} \bar{u}_{\mathrm{R}} u_{\mathrm{L}} D}(0,0) \mid \begin{array}{l}
i F_{\mathrm{R}}{ }^{\mu}{ }_{\nu}\left(\bar{e}_{\mathrm{R} p} C \bar{u}_{\mathrm{R}}{ }_{r}^{a}\right)\left(u_{\mathrm{L} s a} C \gamma^{\nu} D_{\mu} e_{\mathrm{R} t}\right) \\
i F_{\mathrm{R}}{ }_{\nu}{ }_{\nu}\left(\bar{e}_{\mathrm{R} p} u_{\mathrm{L} s a}\right)\left(\bar{u}_{\mathrm{R} r}^{a} \gamma^{\nu} D_{\mu} e_{\mathrm{R} t}\right) \\
i F_{\mathrm{R}}{ }^{\mu}{ }_{\nu}\left(\bar{e}_{\mathrm{R} p} \gamma^{\nu} e_{\mathrm{R} t}\right)\left(\bar{u}_{\mathrm{R} r}{ }^{a} D_{\mu} u_{\mathrm{L} s a}\right)
\end{array} \\
& \mathcal{O}_{G_{\mathrm{R}} \nu_{\mathrm{L}} \bar{\nu}_{\mathrm{L}} \bar{u}_{\mathrm{R}} u_{\mathrm{L}} D}^{(1 \sim 3)}\left(\begin{array}{l}
i\left(\lambda^{A}\right)_{a}^{b} G_{\mathrm{R}}^{A \mu}{ }_{\nu}\left(\bar{u}_{\mathrm{R}}{ }_{p}^{a} u_{\mathrm{L} r b}\right)\left(D_{\mu} \bar{\nu}_{\mathrm{L} t} \gamma^{\nu} \nu_{\mathrm{L} s}\right) \\
i\left(\lambda^{A}\right)_{a}^{b} G_{\mathrm{R}}^{A \mu}{ }_{\nu}\left(\bar{u}_{\mathrm{R}}{ }_{p}^{a} \nu_{\mathrm{L} s}\right)\left(D_{\mu} \bar{\nu}_{\mathrm{L} t} \gamma^{\nu} u_{\mathrm{L} r b}\right) \\
i\left(\lambda^{A}\right)_{a}^{b} G_{\mathrm{R}}^{A \mu}{ }_{\nu}\left(u_{\mathrm{L} r b} C D_{\mu} \nu_{\mathrm{L} s}\right)\left(\bar{u}_{\mathrm{R}}^{a} \gamma^{\nu} C \bar{\nu}_{\mathrm{L} t}\right)
\end{array}\right. \\
& \mathcal{O}_{F_{\mathrm{R}} \nu_{\mathrm{L}} \bar{\nu}_{\mathrm{L}} \bar{u}_{\mathrm{R}} u_{\mathrm{L}} D}^{(1 \sim, 0)} \mid \begin{array}{l}
i F_{\mathrm{R}}{ }^{\mu}{ }_{\nu}\left(\bar{u}_{\mathrm{R}}{ }_{p}^{a} u_{\mathrm{L} r a}\right)\left(D_{\mu} \bar{\nu}_{\mathrm{L} t} \gamma^{\nu} \nu_{\mathrm{L} s}\right) \\
i F_{\mathrm{R}}{ }^{\mu}{ }_{\nu}\left(\bar{u}_{\mathrm{R}}{ }_{p}^{a} \nu_{\mathrm{L} s}\right)\left(D_{\mu} \bar{\nu}_{\mathrm{L} t} \gamma^{\nu} u_{\mathrm{L} r a}\right) \\
i F_{\mathrm{R}}{ }^{\mu}{ }_{\nu}\left(u_{\mathrm{L} r a} C D_{\mu} \nu_{\mathrm{L} s}\right)\left(\bar{u}_{\mathrm{R}}{ }_{p}^{a} \gamma^{\nu} C \bar{\nu}_{\mathrm{L} t}\right)
\end{array}
\end{aligned}
$$

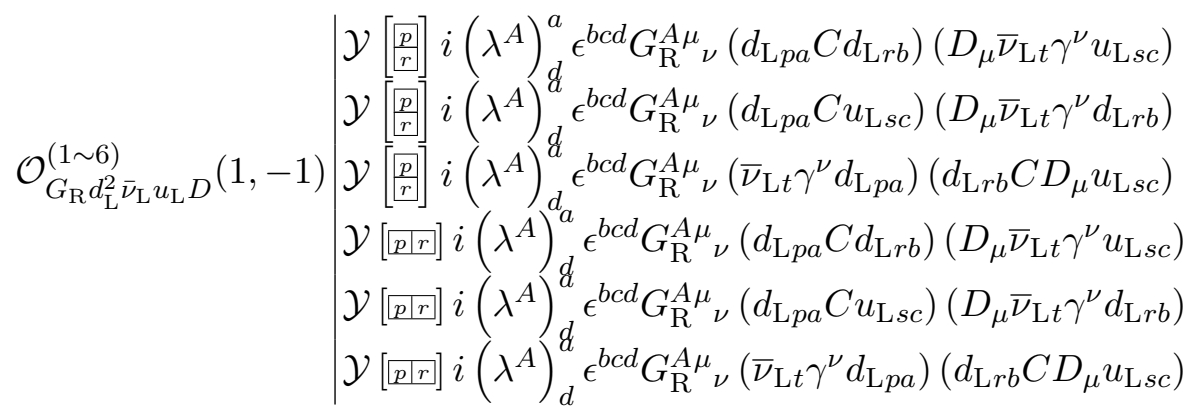

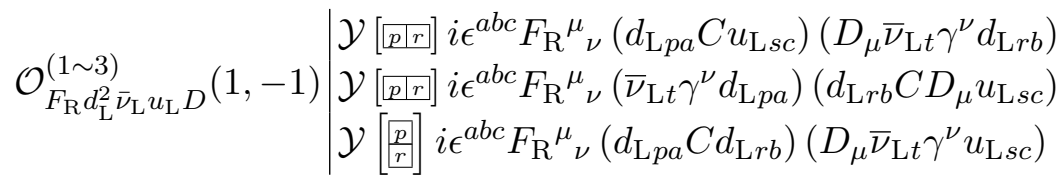




$$
\begin{aligned}
& \mid \begin{array}{l}
i\left(\lambda^{A}\right)_{d}^{c} G_{\mathrm{R}}^{A \mu}{ }_{\nu}\left(\bar{d}_{\mathrm{R} p}^{a} d_{\mathrm{L} r a}\right)\left(D_{\mu} \bar{u}_{\mathrm{L}}^{d} \gamma^{\nu} u_{\mathrm{L} s c}\right) \\
i\left(\lambda^{A}\right)^{c} G_{\mathrm{R}}^{A \mu}{ }_{\nu}\left(\bar{d}_{\mathrm{R} p}{ }_{p} d_{\mathrm{L} r b}\right)\left(D_{\mu} \bar{u}_{\mathrm{L}}^{b} \gamma^{\nu} u_{\mathrm{L} s c}\right)
\end{array} \\
& i\left(\lambda^{A}\right)_{d}^{b} G_{\mathrm{R}}^{A \mu}{ }_{\nu}\left(\bar{d}_{\mathrm{R} p}^{a} d_{\mathrm{L} r b}\right)\left(D_{\mu} \bar{u}_{\mathrm{L} t}^{d} \gamma^{\nu} u_{\mathrm{L} s a}\right) \\
& i\left(\lambda^{A}\right)_{a}^{b} G_{\mathrm{R}}^{A \mu}{ }_{\nu}\left(\bar{d}_{\mathrm{R} p}^{a} d_{\mathrm{L} r b}\right)\left(D_{\mu} \bar{u}_{\mathrm{L} t}^{c} \gamma^{\nu} u_{\mathrm{L} s c}\right) \\
& \mathcal{O}_{G_{\mathrm{R}} \bar{d}_{\mathrm{R}} d_{\mathrm{L}} u_{\mathrm{L}} \bar{u}_{\mathrm{L}} D}^{(1 \sim 12)}(0,0) i\left(\lambda^{A}\right)_{d}^{q} G_{\mathrm{R}}^{A \mu}{ }_{\nu}\left(\bar{d}_{\mathrm{R} p}^{a} u_{\mathrm{L} s c}\right)\left(D_{\mu} \bar{u}_{\mathrm{L} t}^{d} \gamma^{\nu} d_{\mathrm{L} r a}\right) \\
& i\left(\lambda^{A}\right)_{a}^{c} G_{\mathrm{R}}^{A \mu}{ }_{\nu}\left(\bar{d}_{\mathrm{R} p}^{a} u_{\mathrm{L} s c}\right)\left(D_{\mu} \bar{u}_{\mathrm{L} t}^{b} \gamma^{\nu} d_{\mathrm{L} r b}\right) \\
& i\left(\lambda^{A}\right)_{d}^{b} G_{\mathrm{R}}^{A \mu}{ }_{\nu}\left(\bar{d}_{\mathrm{R} p}{ }_{p}^{a} u_{\mathrm{L} s a}\right)\left(D_{\mu} \bar{u}_{\mathrm{L} t}{ }^{d} \gamma^{\nu} d_{\mathrm{L} r b}\right) \\
& i\left(\lambda^{A}\right)_{a}^{b} G_{\mathrm{R}}^{A \mu}{ }_{\nu}\left(\bar{d}_{\mathrm{R}}{ }_{p}^{a} u_{\mathrm{L} s c}\right)\left(D_{\mu} \bar{u}_{\mathrm{L}}^{c} \gamma^{\nu} d_{\mathrm{L} r b}\right)
\end{aligned}
$$

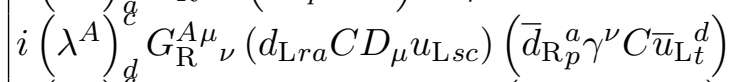

$$
\begin{aligned}
& i\left(\lambda^{A}\right)_{a}^{c} G_{\mathrm{R}}^{A \mu}{ }_{\nu}\left(d_{\mathrm{L} r b} C D_{\mu} u_{\mathrm{L} s c}\right)\left(\bar{d}_{\mathrm{R} p}^{a} \gamma^{\nu} C \bar{u}_{\mathrm{L}}{ }_{t}^{b}\right) \\
& i\left(\lambda^{A}\right)_{d}^{b} G_{\mathrm{R}}^{A \mu}{ }_{\nu}\left(d_{\mathrm{L} r b} C D_{\mu} u_{\mathrm{L} s a}\right)\left(\bar{d}_{\mathrm{R} p}^{a} \gamma^{\nu} C \bar{u}_{\mathrm{L} t}^{d}\right) \\
& i\left(\lambda^{A}\right)_{a}^{b} G_{\mathrm{R}}^{A \mu}{ }_{\nu}\left(d_{\mathrm{L} r b} C D_{\mu} u_{\mathrm{L} s c}\right)\left(\bar{d}_{\mathrm{R}}^{a} \gamma^{\nu} C \bar{u}_{\mathrm{L} t}^{c}\right)
\end{aligned}
$$

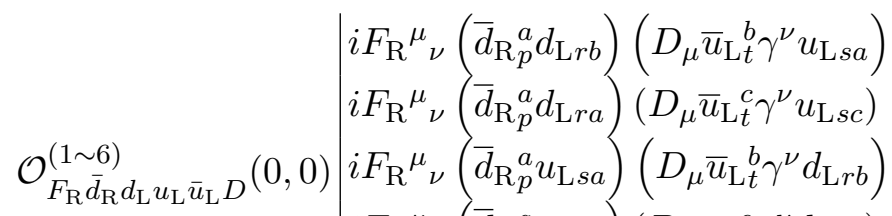

$$
\begin{aligned}
& i F_{\mathrm{R}}{ }_{\nu}{ }_{\nu}\left(\bar{d}_{\mathrm{R} p}{ }^{a} u_{\mathrm{L} s c}\right)\left(D_{\mu} \bar{u}_{\mathrm{L} t}{ }^{c} \gamma^{\nu} d_{\mathrm{L} r a}\right) \\
& i F_{\mathrm{R}}{ }_{\nu}{ }_{\nu}\left(d_{\mathrm{L} r b} C D_{\mu} u_{\mathrm{L} s a}\right)\left(\bar{d}_{\mathrm{R} p}^{a} \gamma^{\nu} C \bar{u}_{\mathrm{L} t}^{b}\right) \\
& i F_{\mathrm{R}}{ }_{\nu}{ }_{\nu}\left(d_{\mathrm{L} r a} C D_{\mu} u_{\mathrm{L} s c}\right)\left(\bar{d}_{\mathrm{R} p}^{a} \gamma^{\nu} C \bar{u}_{\mathrm{L} t}^{c}\right) \\
& \mathcal{O}_{G_{\mathrm{R}} d_{\mathrm{L}} e_{\mathrm{L}} u_{\mathrm{R}} u_{\mathrm{L}} D}^{(1 \sim 1)} \mid \begin{array}{l}
i\left(\lambda^{A}\right)_{d}^{a} \epsilon^{b c d} G_{\mathrm{R}}^{A \mu}{ }_{\nu}\left(d_{\mathrm{L} p a} C e_{\mathrm{L} r}\right)\left(u_{\mathrm{L} s b} C \gamma^{\nu} D_{\mu} u_{\mathrm{R} t c}\right) \\
i\left(\lambda^{A}\right)_{d}^{c} \epsilon^{a b d} G_{\mathrm{R}}^{A \mu}{ }_{\nu}\left(d_{\mathrm{L} p a} C e_{\mathrm{L} r}\right)\left(u_{\mathrm{L} s b} C \gamma^{\nu} D_{\mu} u_{\mathrm{R} t c}\right) \\
i\left(\lambda^{A}\right)_{d}^{d} \epsilon^{b c d} G_{\mathrm{R}}^{A \mu}{ }_{\nu}\left(d_{\mathrm{L} p a} C u_{\mathrm{L} s b}\right)\left(e_{\mathrm{L} r} C \gamma^{\nu} D_{\mu} u_{\mathrm{R} t c}\right) \\
i\left(\lambda^{A}\right)_{d}^{c} \epsilon^{a b d} G_{\mathrm{R}}^{A \mu}{ }_{\nu}\left(d_{\mathrm{L} p a} C u_{\mathrm{L} s b}\right)\left(e_{\mathrm{L} r} C \gamma^{\nu} D_{\mu} u_{\mathrm{R} t c}\right) \\
i\left(\lambda^{A}\right)_{d}^{d} \epsilon^{b c d} G_{\mathrm{R}}^{A \mu}{ }_{\nu}\left(e_{\mathrm{L} r} C D_{\mu} u_{\mathrm{L} s b}\right)\left(d_{\mathrm{L} p a} C \gamma^{\nu} u_{\mathrm{R} t c}\right) \\
i\left(\lambda^{A}\right)_{d}^{d} \epsilon^{a b d} G_{\mathrm{R}}^{A \mu}{ }_{\nu}\left(e_{\mathrm{L} r} C D_{\mu} u_{\mathrm{L} s b}\right)\left(d_{\mathrm{L} p a} C \gamma^{\nu} u_{\mathrm{R} t c}\right)
\end{array} \\
& \mathcal{O}_{F_{\mathrm{R}} d_{\mathrm{L}} e_{\mathrm{L}} u_{\mathrm{R}} u_{\mathrm{L}} D}^{(1,1)} \mid \begin{array}{l}
i \epsilon^{a b c} F_{\mathrm{R}}{ }^{\mu}{ }_{\nu}\left(d_{\mathrm{L} p a} C e_{\mathrm{L} r}\right)\left(u_{\mathrm{L} s b} C \gamma^{\nu} D_{\mu} u_{\mathrm{R} t c}\right) \\
i \epsilon^{a b c} F_{\mathrm{R}}{ }^{\mu}{ }_{\nu}\left(d_{\mathrm{L} p a} C u_{\mathrm{L} s b}\right)\left(e_{\mathrm{L} r} C \gamma^{\nu} D_{\mu} u_{\mathrm{R} t c}\right) \\
i \epsilon^{a b c} F_{\mathrm{R}}{ }^{\mu}{ }_{\nu}\left(e_{\mathrm{L} r} C D_{\mu} u_{\mathrm{L} s b}\right)\left(d_{\mathrm{L} p a} C \gamma^{\nu} u_{\mathrm{R} t c}\right)
\end{array}
\end{aligned}
$$




$$
\begin{aligned}
& \mathcal{O}_{G_{\mathrm{R}} d_{\mathrm{R}} d_{\mathrm{L}} \nu_{\mathrm{L}} u_{\mathrm{L}} D}^{(1 \sim 1)} \mid \begin{array}{l}
i\left(\lambda^{A}\right)_{d}^{a} \epsilon^{b c d} G_{\mathrm{R}}^{A \mu}{ }_{\nu}\left(d_{\mathrm{L} p a} C u_{\mathrm{L} r b}\right)\left(\nu_{\mathrm{L} s} C \gamma^{\nu} D_{\mu} d_{\mathrm{R} t c}\right) \\
i\left(\lambda^{A}\right)_{d}^{c} \epsilon^{a b d} G_{\mathrm{R}}^{A \mu}{ }_{\nu}\left(d_{\mathrm{L} p a} C u_{\mathrm{L} r b}\right)\left(\nu_{\mathrm{L} s} C \gamma^{\nu} D_{\mu} d_{\mathrm{R} t c}\right) \\
i\left(\lambda^{A}\right)_{d}^{d} \epsilon^{b c d} G_{\mathrm{R}}^{A \mu}{ }_{\nu}\left(d_{\mathrm{L} p a} C \nu_{\mathrm{L} s}\right)\left(u_{\mathrm{L} r b} C \gamma^{\nu} D_{\mu} d_{\mathrm{R} t c}\right) \\
i\left(\lambda^{A}\right)_{d}^{c} \epsilon^{a b d} G_{\mathrm{R}}^{A \mu}{ }_{\nu}\left(d_{\mathrm{L} p a} C \nu_{\mathrm{L} s}\right)\left(u_{\mathrm{L} r b} C \gamma^{\nu} D_{\mu} d_{\mathrm{R} t c}\right) \\
i\left(\lambda^{A}\right)_{d}^{a} \epsilon^{b c d} G_{\mathrm{R}}^{A \mu}{ }_{\nu}\left(u_{\mathrm{L} r b} C D_{\mu} \nu_{\mathrm{L} s}\right)\left(d_{\mathrm{L} p a} C \gamma^{\nu} d_{\mathrm{R} t c}\right) \\
i\left(\lambda^{A}\right)_{d}^{c} \epsilon^{a b d} G_{\mathrm{R}}^{A \mu}{ }_{\nu}\left(u_{\mathrm{L} r b} C D_{\mu} \nu_{\mathrm{L} s}\right)\left(d_{\mathrm{L} p a} C \gamma^{\nu} d_{\mathrm{R} t c}\right)
\end{array} \\
& \mathcal{O}_{F_{\mathrm{R}} d_{\mathrm{R}} d_{\mathrm{L}} \nu_{\mathrm{L}} u_{\mathrm{L}} D}(1,1)\left|\begin{array}{l}
i \epsilon^{a b c} F_{\mathrm{R}}{ }^{\mu}{ }_{\nu}\left(d_{\mathrm{L} p a} C u_{\mathrm{L} r b}\right)\left(\nu_{\mathrm{L} s} C \gamma^{\nu} D_{\mu} d_{\mathrm{R} t c}\right) \\
i \epsilon^{a b c} F_{\mathrm{R}}{ }^{\mu}{ }_{\nu}\left(d_{\mathrm{L} p a} C \nu_{\mathrm{L} s}\right)\left(u_{\mathrm{L} r b} C \gamma^{\nu} D_{\mu} d_{\mathrm{R} t c}\right) \\
i \epsilon^{a b c} F_{\mathrm{R}}{ }^{\mu}{ }_{\nu}\left(u_{\mathrm{L} r b} C D_{\mu} \nu_{\mathrm{L} s}\right)\left(d_{\mathrm{L} p a} C \gamma^{\nu} d_{\mathrm{R} t c}\right)
\end{array}\right| \\
& \mathcal{O}_{G_{\mathrm{R}} \bar{d}_{\mathrm{R}} e_{\mathrm{L}} \bar{\nu}_{\mathrm{L}} u_{\mathrm{L}} D}^{(1 \sim 3)}(0,0) \mid \begin{array}{l}
i\left(\lambda^{A}\right)_{a}^{b} G_{\mathrm{R}}^{A \mu}{ }_{\nu}\left(\bar{d}_{\mathrm{R}}^{a} e_{\mathrm{L} r}\right)\left(D_{\mu} \bar{\nu}_{\mathrm{L} t} \gamma^{\nu} u_{\mathrm{L} s b}\right) \\
i\left(\lambda^{A}\right)_{a}^{b} G_{\mathrm{R}}^{A \mu}{ }_{\nu}\left(\bar{d}_{\mathrm{R}}^{a}{ }_{p} u_{\mathrm{L} s b}\right)\left(D_{\mu} \bar{\nu}_{\mathrm{L} t} \gamma^{\nu} e_{\mathrm{L} r}\right)
\end{array} \\
& i\left(\lambda^{A}\right)_{a}^{b} G_{\mathrm{R}}^{A \mu}{ }_{\nu}\left(e_{\mathrm{L} r} C D_{\mu} u_{\mathrm{L} s b}\right)\left(\bar{d}_{\mathrm{R}}{ }^{a} \gamma^{\nu} C \bar{\nu}_{\mathrm{L} t}\right) \\
& \mathcal{O}_{F_{\mathrm{R}} \bar{d}_{\mathrm{R}} e_{\mathrm{L}} \bar{\nu}_{\mathrm{L}} u_{\mathrm{L}} D}^{(0,0)} \mid \begin{array}{l}
i F_{\mathrm{R}}{ }^{\mu}{ }_{\nu}\left(\bar{d}_{\mathrm{R}}{ }_{p}^{a} e_{\mathrm{L} r}\right)\left(D_{\mu} \bar{\nu}_{\mathrm{L} t} \gamma^{\nu} u_{\mathrm{L} s a}\right) \\
i F_{\mathrm{R}}{ }^{\mu}{ }_{\nu}\left(\bar{d}_{\mathrm{R}}{ }_{\mathrm{R}}^{a} u_{\mathrm{L} s a}\right)\left(D_{\mu} \bar{\nu}_{\mathrm{L} t} \gamma^{\nu} e_{\mathrm{L} r}\right) \\
i F_{\mathrm{R}}{ }^{\mu}{ }_{\nu}\left(e_{\mathrm{L} r} C D_{\mu} u_{\mathrm{L} s a}\right)\left(\bar{d}_{\mathrm{R}}{ }_{p} \gamma^{\nu} C \bar{\nu}_{\mathrm{L} t}\right)
\end{array} \\
& \mathcal{O}_{G_{\mathrm{R}} \bar{d}_{\mathrm{R}} e_{\mathrm{R}} \nu_{\mathrm{L}} u_{\mathrm{L}} D}^{(1 \sim 3)} \mid \begin{array}{l}
i\left(\lambda^{A}\right)_{a}^{b} G_{\mathrm{R}}^{A \mu}{ }_{\nu}\left(\bar{d}_{\mathrm{R}}^{a} u_{\mathrm{L} r b}\right)\left(\nu_{\mathrm{L} s} C \gamma^{\nu} D_{\mu} e_{\mathrm{R} t}\right) \\
i\left(\lambda^{A}\right)_{a}^{b} G_{\mathrm{R}}^{A \mu}{ }_{\nu}\left(\bar{d}_{\mathrm{R} p}^{a} \nu_{\mathrm{L} s}\right)\left(u_{\mathrm{L} r b} C \gamma^{\nu} D_{\mu} e_{\mathrm{R} t}\right) \\
i\left(\lambda^{A}\right)_{a}^{b} G_{\mathrm{R}}^{A \mu}{ }_{\nu}\left(u_{\mathrm{L} r b} C D_{\mu} \nu_{\mathrm{L} s}\right)\left(\bar{d}_{\mathrm{R} p}^{a} \gamma^{\nu} e_{\mathrm{R} t}\right)
\end{array} \\
& \mathcal{O}_{F_{\mathrm{R}} \bar{d}_{\mathrm{R}} e_{\mathrm{R}} \nu_{\mathrm{L}} u_{\mathrm{L}} D}^{(1 \sim 2)} \mid \begin{array}{l}
i F_{\mathrm{R}}{ }_{\nu}{ }_{\nu}\left(\bar{d}_{\mathrm{R} p}{ }_{p} u_{\mathrm{L} r a}\right)\left(\nu_{\mathrm{L} s} C \gamma^{\nu} D_{\mu} e_{\mathrm{R} t}\right) \\
i F_{\mathrm{R}}{ }^{\mu}{ }_{\nu}\left(\bar{d}_{\mathrm{R} p}{ }^{a} \nu_{\mathrm{L} s}\right)\left(u_{\mathrm{L} r a} C \gamma^{\nu} D_{\mu} e_{\mathrm{R} t}\right) \\
i F_{\mathrm{R}}{ }^{\mu}{ }_{\nu}\left(u_{\mathrm{L} r a} C D_{\mu} \nu_{\mathrm{L} s}\right)\left(\bar{d}_{\mathrm{R} p}{ }^{a} \gamma^{\nu} e_{\mathrm{R} t}\right)
\end{array} \\
& \mathcal{O}_{G_{\mathrm{R}} \bar{e}_{\mathrm{R}} e_{\mathrm{L}} u_{\mathrm{L}} \bar{u}_{\mathrm{L}} D}^{(1 \sim 3)} \mid \begin{array}{l}
i\left(\lambda^{A}\right)_{b}^{a}\left(\bar{e}_{\mathrm{R} p} e_{\mathrm{L} r}\right) G_{\mathrm{R}}^{A \mu}{ }_{\nu}\left(D_{\mu} \bar{u}_{\mathrm{L} t}^{b} \gamma^{\nu} u_{\mathrm{L} s a}\right) \\
i\left(\lambda^{A}\right)_{b}^{a} G_{\mathrm{R}}^{A \mu}{ }_{\nu}\left(\bar{e}_{\mathrm{R} p} u_{\mathrm{L} s a}\right)\left(D_{\mu} \bar{u}_{\mathrm{L} t}^{b} \gamma^{\nu} e_{\mathrm{L} r}\right) \\
i\left(\lambda^{A}\right)_{b}^{a} G_{\mathrm{R}}^{A \mu}{ }_{\nu}\left(e_{\mathrm{L} r} C D_{\mu} u_{\mathrm{L} s a}\right)\left(\bar{e}_{\mathrm{R} p} \gamma^{\nu} C \bar{u}_{\mathrm{L} t}^{b}\right)
\end{array} \\
& \mathcal{O}_{F_{\mathrm{R}} \bar{e}_{\mathrm{R}} e_{\mathrm{L}} u_{\mathrm{L}} \bar{u}_{\mathrm{L}} D}^{(1 \sim 3,0)} \mid \begin{array}{l}
i F_{\mathrm{R}}{ }^{\mu}{ }_{\nu}\left(\bar{e}_{\mathrm{R} p} e_{\mathrm{L} r}\right)\left(D_{\mu} \bar{u}_{\mathrm{L} t}^{a} \gamma^{\nu} u_{\mathrm{L} s a}\right) \\
i F_{\mathrm{R}}{ }^{\mu}{ }_{\nu}\left(\bar{e}_{\mathrm{R} p} u_{\mathrm{L} s a}\right)\left(D_{\mu} \bar{u}_{\mathrm{L}}{ }^{a} \gamma^{\nu} e_{\mathrm{L} r}\right)
\end{array} \\
& i F_{\mathrm{R}}{ }^{\mu}{ }_{\nu}\left(e_{\mathrm{L} r} C D_{\mu} u_{\mathrm{L} s a}\right)\left(\bar{e}_{\mathrm{R} p} \gamma^{\nu} C \bar{u}_{\mathrm{L} t}^{a}\right)
\end{aligned}
$$




$$
\begin{aligned}
& \mathcal{O}_{G_{\mathrm{R}} \bar{d}_{\mathrm{L}} e_{\mathrm{L}} \nu_{\mathrm{L}} u_{\mathrm{L}} D}(0,2) \mid \begin{array}{l}
i\left(\lambda^{A}\right)_{b}^{a} G_{\mathrm{R}}^{A \mu}{ }_{\nu}\left(e_{\mathrm{L} p} C u_{\mathrm{L} r a}\right)\left(D_{\mu} \bar{d}_{\mathrm{L} t}^{b} \gamma^{\nu} \nu_{\mathrm{L} s}\right) \\
i\left(\lambda^{A}\right)_{b}^{a} G_{\mathrm{R}}^{A \mu}{ }_{\nu}\left(e_{\mathrm{L} p} C \nu_{\mathrm{L} s}\right)\left(D_{\mu} \bar{d}_{\mathrm{L} t}^{b} \gamma^{\nu} u_{\mathrm{L} r a}\right) \\
i\left(\lambda^{A}\right)_{b}^{a} G_{\mathrm{R}}^{A \mu}{ }_{\nu}\left(u_{\mathrm{L} r a} C D_{\mu} \nu_{\mathrm{L} s}\right)\left(\bar{d}_{\mathrm{L} t}^{b} \gamma^{\nu} e_{\mathrm{L} p}\right)
\end{array} \\
& \left.\mathcal{O}_{F_{\mathrm{R}} \bar{d}_{\mathrm{L}} e_{\mathrm{L}} \nu_{\mathrm{L}} u_{\mathrm{L}} D}^{(0,2)} \mid \begin{array}{l}
i F_{\mathrm{R}}{ }^{\mu}{ }_{\nu}\left(e_{\mathrm{L} p} C u_{\mathrm{L} r a}\right)\left(D_{\mu} \bar{d}_{\mathrm{L} t}^{a} \gamma^{\nu} \nu_{\mathrm{L} s}\right. \\
i F_{\mathrm{R}}{ }^{\mu}{ }_{\nu}\left(e_{\mathrm{L} p} C \nu_{\mathrm{L} s}\right)\left(D_{\mu} \bar{d}_{\mathrm{L} t}^{a} \gamma^{\nu} u_{\mathrm{L} r a}\right. \\
i F_{\mathrm{R}}{ }^{\mu}{ }_{\nu}\left(u_{\mathrm{L} r a} C D_{\mu} \nu_{\mathrm{L} s}\right)\left(\bar{d}_{\mathrm{L} t}^{a} \gamma^{\nu} e_{\mathrm{L} p}\right.
\end{array}\right)
\end{aligned}
$$

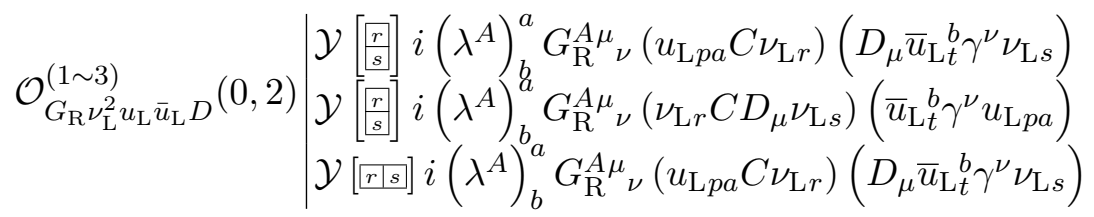

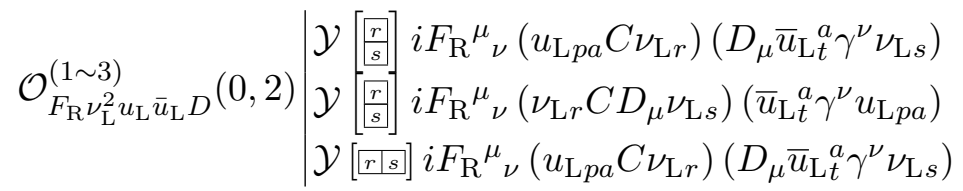

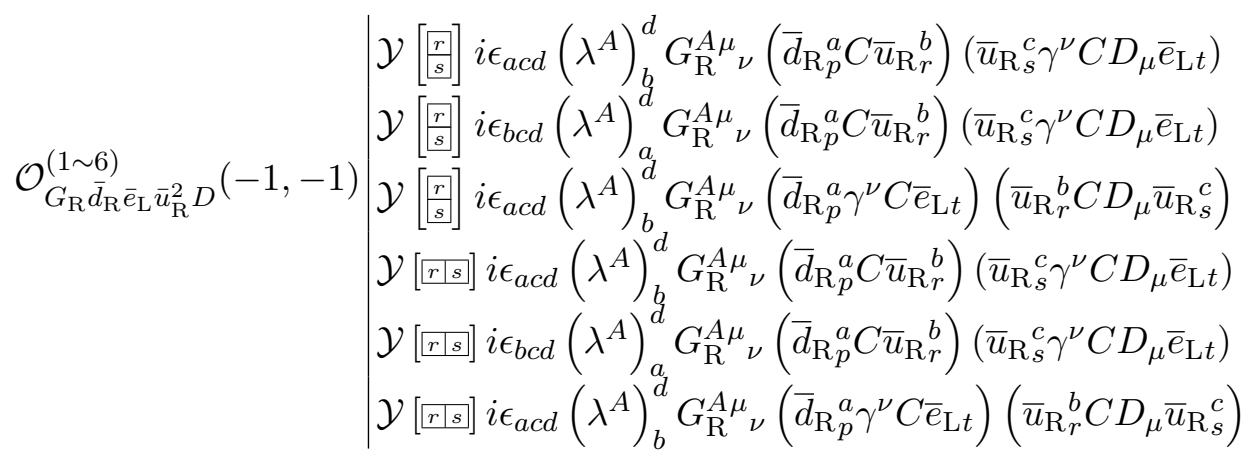

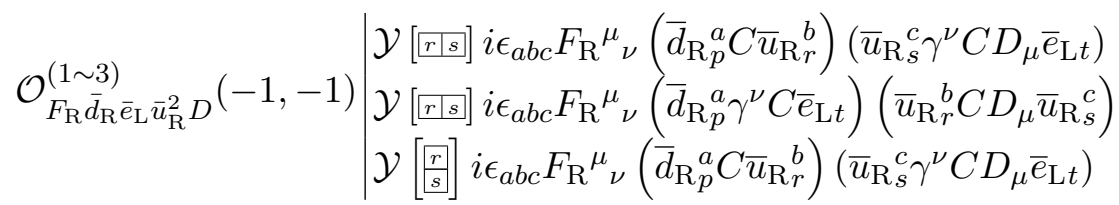

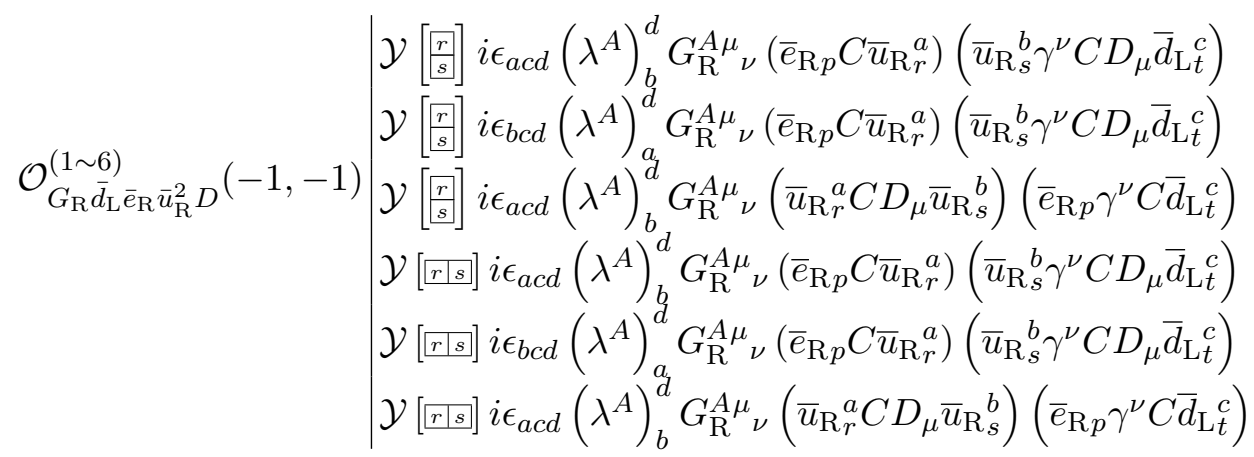




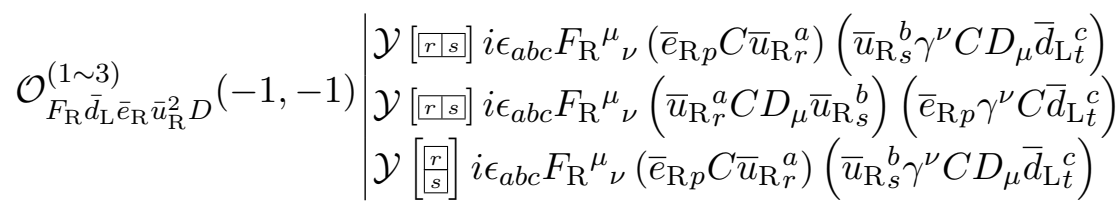

$$
\begin{aligned}
& \mathcal{O}_{G_{\mathrm{R}} \bar{d}_{\mathrm{R}} d_{\mathrm{L}} \bar{u}_{\mathrm{R}} u_{\mathrm{R}} D}^{(1 \sim 12}(0,0) i\left(\lambda^{A}\right)_{c}^{b} G_{\mathrm{R}}^{A \mu}{ }_{\nu}\left(\bar{d}_{\mathrm{R}}{ }_{p}^{a} C \bar{u}_{\mathrm{R}}{ }_{s}^{c}\right)\left(d_{\mathrm{L} r b} C \gamma^{\nu} D_{\mu} u_{\mathrm{R} t a}\right) \\
& i\left(\lambda^{A}\right)_{c}^{c} G_{\mathrm{R}}^{A \mu}{ }_{\nu}\left(\bar{d}_{\mathrm{R}}{ }_{p}^{a} C \bar{u}_{\mathrm{R}}{ }_{s}^{c}\right)\left(d_{\mathrm{L} r a} C \gamma^{\nu} D_{\mu} u_{\mathrm{R} t d}\right) \\
& i\left(\lambda^{A}\right)_{a}^{d} G_{\mathrm{R}}^{A \mu}{ }_{\nu}\left(\bar{d}_{\mathrm{R}}^{a} C \bar{u}_{\mathrm{R} s}^{b}\right)\left(d_{\mathrm{L} r b} C \gamma^{\nu} D_{\mu} u_{\mathrm{R} t d}\right) \\
& i\left(\lambda^{A}\right)_{a}^{b} G_{\mathrm{R}}^{A \mu}{ }_{\nu}\left(\bar{d}_{\mathrm{R} p}^{a} C \bar{u}_{\mathrm{R}}^{c}\right)\left(d_{\mathrm{L} r b} C \gamma^{\nu} D_{\mu} u_{\mathrm{R} t c}\right) \\
& i\left(\lambda^{A}\right)_{c}^{b} G_{\mathrm{R}}^{A \mu}{ }_{\nu}\left(\bar{d}_{\mathrm{R} p}^{a} \gamma^{\nu} u_{\mathrm{R} t a}\right)\left(D_{\mu} \bar{u}_{\mathrm{R}}^{c}{ }_{s} d_{\mathrm{L} r b}\right) \\
& i\left(\lambda^{A}\right)_{c}^{c} G_{\mathrm{R}}^{A \mu}{ }_{\nu}\left(D_{\mu} \bar{u}_{\mathrm{R}}{ }_{s}^{c} d_{\mathrm{L} r a}\right)\left(\bar{d}_{\mathrm{R} p}^{a} \gamma^{\nu} u_{\mathrm{R} t d}\right) \\
& \begin{array}{l}
i\left(\lambda^{A}\right)_{a}^{d} G_{\mathrm{R}}^{A \mu}{ }_{\nu}\left(D_{\mu} \bar{u}_{\mathrm{R}}^{b} d_{\mathrm{L} r b}\right)\left(\bar{d}_{\mathrm{R}}^{a} \gamma^{\nu} u_{\mathrm{R} t d}\right) \\
i\left(\lambda^{A}\right)_{a}^{b} G_{\mathrm{R}}^{A \mu}{ }_{\nu}\left(\bar{d}_{\mathrm{R}}^{a}{ }^{\nu} \gamma^{\nu} u_{\mathrm{R} t c}\right)\left(D_{\mu} \bar{u}_{\mathrm{R}}^{c}{ }_{s}^{c} d_{\mathrm{L} r b}\right)
\end{array}
\end{aligned}
$$

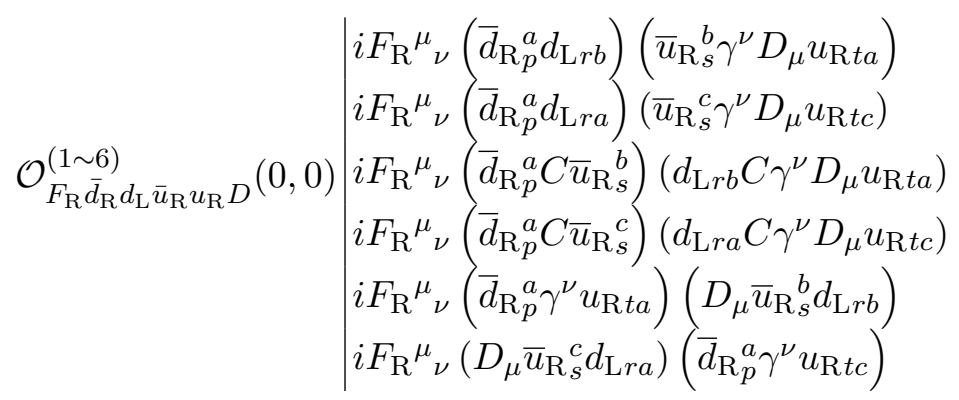

$$
\begin{aligned}
& \mathcal{O}_{G_{\mathrm{R}} d_{\mathrm{L}} \bar{e}_{\mathrm{R}} \bar{\nu}_{\mathrm{L}} \bar{u}_{\mathrm{R}} D}(0,-2) \mid \begin{array}{l}
i\left(\lambda^{A}\right)_{b}^{a} G_{\mathrm{R}}^{A \mu}{ }_{\nu}\left(\bar{e}_{\mathrm{R} r} d_{\mathrm{L} p a}\right)\left(\bar{u}_{\mathrm{R} s}^{b} \gamma^{\nu} C D_{\mu} \bar{\nu}_{\mathrm{L} t}\right) \\
i\left(\lambda^{A}\right)_{b}^{a} G_{\mathrm{R}}^{A \mu}{ }_{\nu}\left(\bar{u}_{\mathrm{R}}^{b}{ }_{s} d_{\mathrm{L} p a}\right)\left(\bar{e}_{\mathrm{R} r} \gamma^{\nu} C D_{\mu} \bar{\nu}_{\mathrm{L} t}\right) \\
i\left(\lambda^{A}\right)_{b}^{a} G_{\mathrm{R}}^{A \mu}{ }_{\nu}\left(\bar{\nu}_{\mathrm{L} t} \gamma^{\nu} d_{\mathrm{L} p a}\right)\left(\bar{e}_{\mathrm{R} r} C D_{\mu} \bar{u}_{\mathrm{R}}{ }_{\mathrm{R}}^{b}\right)
\end{array} \\
& \mathcal{O}_{F_{\mathrm{R}} d_{\mathrm{L}} \bar{e}_{\mathrm{R}} \overline{\mathrm{L}}_{\mathrm{L}} \bar{u}_{\mathrm{R}} D}(0,-2) \mid \begin{array}{l}
i F_{\mathrm{R}}{ }^{\mu}{ }_{\nu}\left(\bar{e}_{\mathrm{R} r} d_{\mathrm{L} p a}\right)\left(\bar{u}_{\mathrm{R}}{ }_{s}^{a} \gamma^{\nu} C D_{\mu} \bar{\nu}_{\mathrm{L} t}\right) \\
i F_{\mathrm{R}}{ }^{\mu}{ }_{\nu}\left(\bar{u}_{\mathrm{R}}{ }^{a} d_{\mathrm{L} p a}\right)\left(\bar{e}_{\mathrm{R} r} \gamma^{\nu} C D_{\mu} \bar{\nu}_{\mathrm{L} t}\right) \\
i F_{\mathrm{R}}{ }^{\mu}{ }_{\nu}\left(\bar{\nu}_{\mathrm{L} t} \gamma^{\nu} d_{\mathrm{L} p a}\right)\left(\bar{e}_{\mathrm{R} r} C D_{\mu} \bar{u}_{\mathrm{R}}{ }^{a}\right)
\end{array}
\end{aligned}
$$




$$
\begin{aligned}
& \mathcal{O}_{G_{\mathrm{R}} d_{\mathrm{L}} \bar{e}_{\mathrm{L}} \nu_{\mathrm{L}} \bar{u}_{\mathrm{R}} D}(0,0) \mid \begin{array}{l}
i\left(\lambda^{A}\right)_{b}^{a} G_{\mathrm{R}}^{A \mu}{ }_{\nu}\left(\bar{u}_{\mathrm{R} r}^{b} d_{\mathrm{L} p a}\right)\left(D_{\mu} \bar{e}_{\mathrm{L} t} \gamma^{\nu} \nu_{\mathrm{L} s}\right) \\
i\left(\lambda^{A}\right)_{b}^{a} G_{\mathrm{R}}^{A \mu}{ }_{\nu}\left(d_{\mathrm{L} p a} C \nu_{\mathrm{L} s}\right)\left(\bar{u}_{\mathrm{R}}^{b} \gamma^{\nu} C D_{\mu} \bar{e}_{\mathrm{L} t}\right) \\
i\left(\lambda^{A}\right)_{b}^{a} G_{\mathrm{R}}^{A \mu}{ }_{\nu}\left(\bar{e}_{\mathrm{L} t} \gamma^{\nu} d_{\mathrm{L} p a}\right)\left(\bar{u}_{\mathrm{R}}^{b} D_{\mu} \nu_{\mathrm{L} s}\right)
\end{array} \\
& \mathcal{O}_{F_{\mathrm{R}} d_{\mathrm{L}} \bar{e}_{\mathrm{L}} \nu_{\mathrm{L}} \bar{u}_{\mathrm{R}} D}(0,0) \mid \begin{array}{l}
i F_{\mathrm{R}}{ }^{\mu}{ }_{\nu}\left(\bar{u}_{\mathrm{R}}{ }_{r}^{a} d_{\mathrm{L} p a}\right)\left(D_{\mu} \bar{e}_{\mathrm{L} t} \gamma^{\nu} \nu_{\mathrm{L} s}\right) \\
i F_{\mathrm{R}}{ }_{\nu}{ }_{\nu}\left(d_{\mathrm{L} p a} C \nu_{\mathrm{L} s}\right)\left(\bar{u}_{\mathrm{R}}{ }_{r}^{a} \gamma^{\nu} C D_{\mu} \bar{e}_{\mathrm{L} t}\right) \\
i F_{\mathrm{R}}{ }^{\mu}{ }_{\nu}\left(\bar{e}_{\mathrm{L} t} \gamma^{\nu} d_{\mathrm{L} p a}\right)\left(\bar{u}_{\mathrm{R}}{ }_{r} D_{\mu} D_{\mu} \nu_{\mathrm{L} s}\right)
\end{array}
\end{aligned}
$$

$$
\begin{aligned}
& \mathcal{Y}\left[\frac{p}{r}\right] i \epsilon_{a c d}\left(\lambda^{A}\right)_{b}^{d} G_{\mathrm{R}}^{A \mu}{ }_{\nu}\left(\bar{d}_{\mathrm{R}}{ }_{p}^{a} C \bar{d}_{\mathrm{R}}^{b}\right)\left(\bar{u}_{\mathrm{R}}^{c}{ }_{s} \gamma^{\nu} C D_{\mu} \bar{\nu}_{\mathrm{L} t}\right) \\
& \mathcal{Y}\left[\frac{p}{r}\right] i \epsilon_{a c d}\left(\lambda^{A}\right)_{b}^{d} G_{\mathrm{R}}^{A \mu}{ }_{\nu}\left(\bar{d}_{\mathrm{R}}^{a} C \bar{u}_{\mathrm{R}}^{c}\right)\left(\bar{d}_{\mathrm{R}}^{b} \gamma^{\nu} C D_{\mu} \bar{\nu}_{\mathrm{L} t}\right) \\
& \mathcal{O}_{G_{\mathrm{R}} \bar{d}_{\mathrm{R}}^{2} \bar{\nu}_{\mathrm{L}} \bar{u}_{\mathrm{R}} D}^{(-1,-1)} \mathcal{Y}\left[\begin{array}{l}
\underline{p} \\
r
\end{array}\right] i \epsilon_{a c d}\left(\lambda^{A}\right)_{b}^{d} G_{\mathrm{R}}^{A \mu}{ }_{\nu}\left(\bar{d}_{\mathrm{R} p}^{a} \gamma^{\nu} C \bar{\nu}_{\mathrm{L} t}\right)\left(\bar{d}_{\mathrm{R}}{ }_{r}^{b} C D_{\mu} \bar{u}_{\mathrm{R}}{ }^{c}\right) \\
& \mathcal{Y}\left[\underline{p \mid r]} i \epsilon_{a c d}\left(\lambda^{A}\right)_{b}^{d} G_{\mathrm{R}}^{A \mu}{ }_{\nu}\left(\bar{d}_{\mathrm{R}}^{a} C \bar{d}_{\mathrm{R}}^{b}\right)\left(\bar{u}_{\mathrm{R}}^{c}{ }_{s} \gamma^{\nu} C D_{\mu} \bar{\nu}_{\mathrm{L} t}\right)\right. \\
& \mathcal{Y}[\overline{p p r}] i \epsilon_{a c d}\left(\lambda^{A}\right)_{b}^{d} G_{\mathrm{R}}^{A \mu}{ }_{\nu}\left(\bar{d}_{\mathrm{R} p}^{a} C \bar{u}_{\mathrm{R} s}^{c}\right)\left(\bar{d}_{\mathrm{R} r}^{b} \gamma^{\nu} C D_{\mu} \bar{\nu}_{\mathrm{L} t}\right) \\
& \mathcal{Y}[\overline{p p r}] i \epsilon_{a c d}\left(\lambda^{A}\right)_{b}^{d} G_{\mathrm{R}}^{A \mu}{ }_{\nu}\left(\bar{d}_{\mathrm{R}}{ }_{p}^{a} \gamma^{\nu} C \bar{\nu}_{\mathrm{L} t}\right)\left(\bar{d}_{\mathrm{R} r}^{b} C D_{\mu} \bar{u}_{\mathrm{R}}^{c}{ }_{s}\right)
\end{aligned}
$$

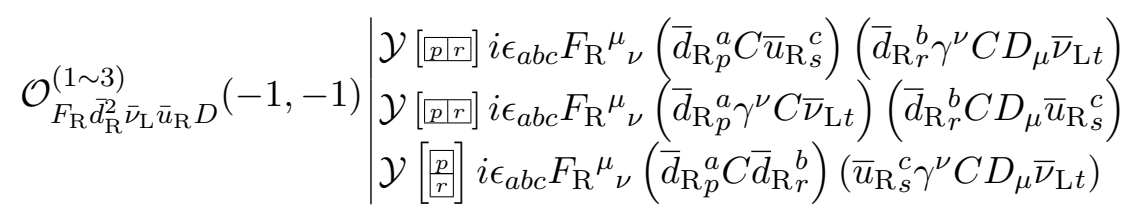

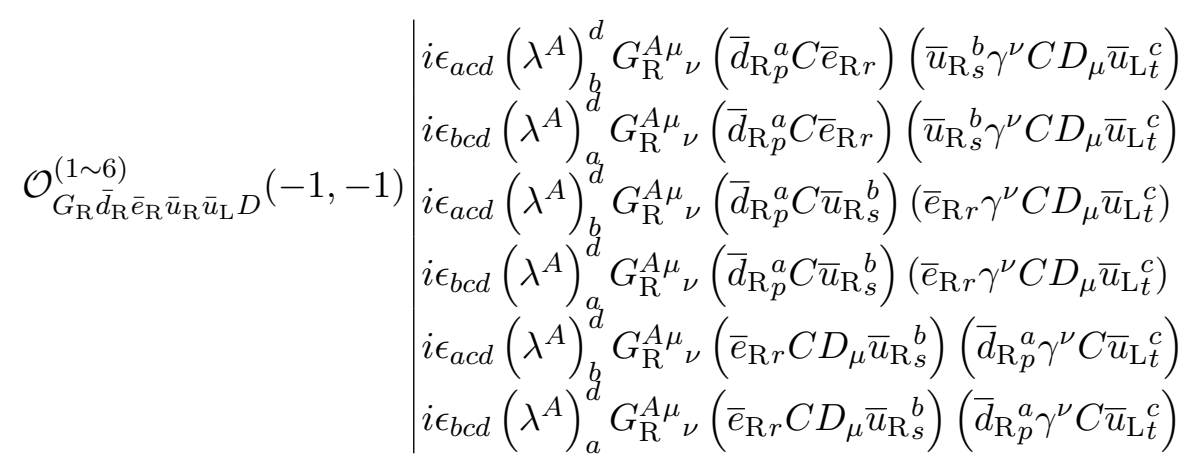

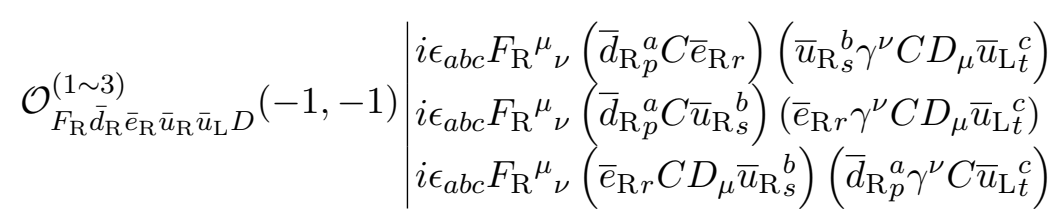




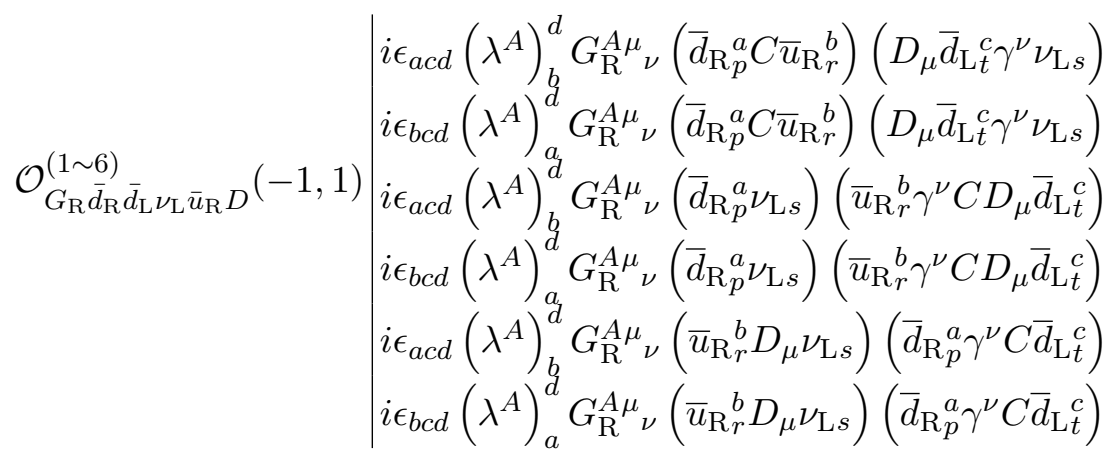

$$
\begin{aligned}
& \mathcal{O}_{F_{\mathrm{R}} \bar{d}_{\mathrm{R}} \bar{d}_{\mathrm{L}} \nu_{\mathrm{L}} \bar{u}_{\mathrm{R}} D}^{(-1,1)} \mid \begin{array}{l}
i \epsilon_{a b c} F_{\mathrm{R}}^{\mu}{ }_{\nu}\left(\bar{d}_{\mathrm{R}}^{a} C \bar{u}_{\mathrm{R}}^{b}\right)\left(D_{\mu} \bar{d}_{\mathrm{L}}^{c} \gamma^{\nu} \nu_{\mathrm{L} s}\right) \\
i \epsilon_{a b c} F_{\mathrm{R}}{ }_{\nu}{ }_{\nu}\left(\bar{d}_{\mathrm{R} p}^{a} \nu_{\mathrm{L} s}\right)\left(\bar{u}_{\mathrm{R} r}^{b} \gamma^{\nu} C D_{\mu} \bar{d}_{\mathrm{L} t}^{c}\right) \\
i \epsilon_{a b c} F_{\mathrm{R}}{ }_{\nu}{ }_{\nu}\left(\bar{u}_{\mathrm{R}}^{b} D_{\mu} D_{\mathrm{L} s}\right)\left(\bar{d}_{\mathrm{R}}{ }_{p} \gamma^{\nu} C \bar{d}_{\mathrm{L} t}^{c}\right)
\end{array} \\
& \mathcal{O}_{G_{\mathrm{R}} \bar{e}_{\mathrm{R}} e_{\mathrm{L}} \bar{u}_{\mathrm{R}} u_{\mathrm{R}} D}^{(1 \sim 3)}(0,0) \mid \begin{array}{l}
i\left(\lambda^{A}\right)_{a}^{b}\left(\bar{e}_{\mathrm{R} p} e_{\mathrm{L} r}\right) G_{\mathrm{R}}^{A \mu}{ }_{\nu}\left(\bar{u}_{\mathrm{R}}{ }_{s}^{a} \gamma^{\nu} D_{\mu} u_{\mathrm{R} t b}\right) \\
i\left(\lambda^{A}\right)_{a}^{b} G_{\mathrm{R}}^{A \mu}{ }_{\nu}\left(\bar{e}_{\mathrm{R} p} C \bar{u}_{\mathrm{R}}{ }_{s}^{a}\right)\left(e_{\mathrm{L} r} C \gamma^{\nu} D_{\mu} u_{\mathrm{R} t b}\right) \\
i\left(\lambda^{A}\right)_{a}^{b} G_{\mathrm{R}}^{A \mu}{ }_{\nu}\left(\bar{e}_{\mathrm{R} p} \gamma^{\nu} u_{\mathrm{R} t b}\right)\left(D_{\mu} \bar{u}_{\mathrm{R}}{ }_{s}^{a} e_{\mathrm{L} r}\right)
\end{array} \\
& \mathcal{O}_{F_{\mathrm{R}} \bar{e}_{\mathrm{R}} e_{\mathrm{L}} \bar{u}_{\mathrm{R}} u_{\mathrm{R}} D}^{(1 \sim, 0)} \mid \begin{array}{l}
i F_{\mathrm{R}}{ }^{\mu}{ }_{\nu}\left(\bar{e}_{\mathrm{R} p} e_{\mathrm{L} r}\right)\left(\bar{u}_{\mathrm{R}}{ }_{s} \gamma^{\nu} D_{\mu} u_{\mathrm{R} t a}\right) \\
i F_{\mathrm{R}}{ }_{\nu}{ }_{\nu}\left(\bar{e}_{\mathrm{R} p} C \bar{u}_{\mathrm{R}}{ }_{\mathrm{R}}^{a}\right)\left(e_{\mathrm{L} r} C \gamma^{\nu} D_{\mu} u_{\mathrm{R} t a}\right) \\
i F_{\mathrm{R}}{ }^{\mu}{ }_{\nu}\left(\bar{e}_{\mathrm{R} p} \gamma^{\nu} u_{\mathrm{R} t a}\right)\left(D_{\mu} \bar{u}_{\mathrm{R} s}^{a} e_{\mathrm{L} r}\right)
\end{array} \\
& \mathcal{O}_{G_{\mathrm{R}} d_{\mathrm{R}} \bar{e}_{\mathrm{R}} \nu_{\mathrm{L}} \bar{u}_{\mathrm{R}} D}^{(1 \sim 0)} \mid \begin{array}{l}
i\left(\lambda^{A}\right)_{a}^{b} G_{\mathrm{R}}^{A \mu}{ }_{\nu}\left(\bar{e}_{\mathrm{R} p} C \bar{u}_{\mathrm{R}}{ }_{r}^{a}\right)\left(\nu_{\mathrm{L} s} C \gamma^{\nu} D_{\mu} d_{\mathrm{R} t b}\right) \\
i\left(\lambda^{A}\right)_{a}^{b}\left(\bar{e}_{\mathrm{R} p} \nu_{\mathrm{L} s}\right) G_{\mathrm{R}}^{A \mu}{ }_{\nu}\left(\bar{u}_{\mathrm{R} r}^{a} \gamma^{\nu} D_{\mu} d_{\mathrm{R} t b}\right) \\
i\left(\lambda^{A}\right)_{a}^{b} G_{\mathrm{R}}^{A \mu}{ }_{\nu}\left(\bar{e}_{\mathrm{R} p} \gamma^{\nu} d_{\mathrm{R} t b}\right)\left(\bar{u}_{\mathrm{R} r}^{a} D_{\mu} \nu_{\mathrm{L} s}\right)
\end{array} \\
& \mathcal{O}_{F_{\mathrm{R}} d_{\mathrm{R}} \bar{e}_{\mathrm{R}} \nu_{\mathrm{L}} \bar{u}_{\mathrm{R}} D}(0,0) \mid \begin{array}{l}
i F_{\mathrm{R}}{ }^{\mu}{ }_{\nu}\left(\bar{e}_{\mathrm{R} p} C \bar{u}_{\mathrm{R} r}{ }_{r}^{a}\right)\left(\nu_{\mathrm{L} s} C \gamma^{\nu} D_{\mu} d_{\mathrm{R} t a}\right) \\
i F_{\mathrm{R}}{ }^{\mu}{ }_{\nu}\left(\bar{e}_{\mathrm{R} p} \nu_{\mathrm{L} s}\right)\left(\bar{u}_{\mathrm{R}}^{a} \gamma^{\nu} D_{\mu} d_{\mathrm{R} t a}\right) \\
i F_{\mathrm{R}}{ }_{\nu}{ }_{\nu}\left(\bar{e}_{\mathrm{R} p} \gamma^{\nu} d_{\mathrm{R} t a}\right)\left(\bar{u}_{\mathrm{R} r}{ }^{a} D_{\mu} \nu_{\mathrm{L} s}\right)
\end{array} \\
& \mathcal{O}_{G_{\mathrm{R}} \nu_{\mathrm{L}}^{2} \bar{u}_{\mathrm{R}} u_{\mathrm{R}} D}^{(1 \sim 2)} \mid \begin{array}{l}
\mathcal{Y}\left[\begin{array}{l}
r \\
s
\end{array}\right] i\left(\lambda^{A}\right)_{a}^{b} G_{\mathrm{R}}^{A \mu}{ }_{\nu}\left(\bar{u}_{\mathrm{R}}{ }_{p} \nu_{\mathrm{L} r}\right)\left(\nu_{\mathrm{L} s} C \gamma^{\nu} D_{\mu} u_{\mathrm{R} t b}\right) \\
\mathcal{Y}\left[\begin{array}{l}
r \\
s
\end{array}\right] i\left(\lambda^{A}\right)_{a}^{b} G_{\mathrm{R}}^{A \mu}{ }_{\nu}\left(\nu_{\mathrm{L} r} C D_{\mu} \nu_{\mathrm{L} s}\right)\left(\bar{u}_{\mathrm{R}}{ }_{p}^{a} \gamma^{\nu} u_{\mathrm{R} t b}\right) \\
\mathcal{Y}\left[[r s] i\left(\lambda^{A}\right)_{a}^{b} G_{\mathrm{R}}^{A \mu}{ }_{\nu}\left(\bar{u}_{\mathrm{R}}{ }_{p}^{a} \nu_{\mathrm{L} r}\right)\left(\nu_{\mathrm{L} s} C \gamma^{\nu} D_{\mu} u_{\mathrm{R} t b}\right)\right.
\end{array}
\end{aligned}
$$

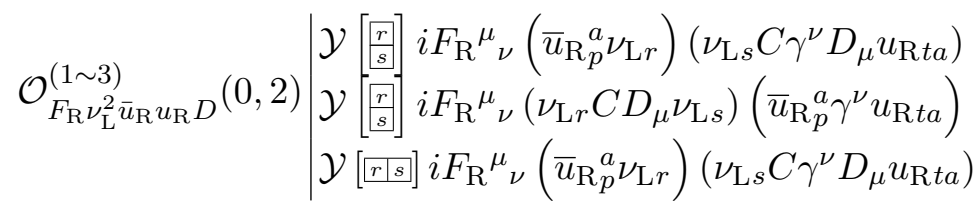




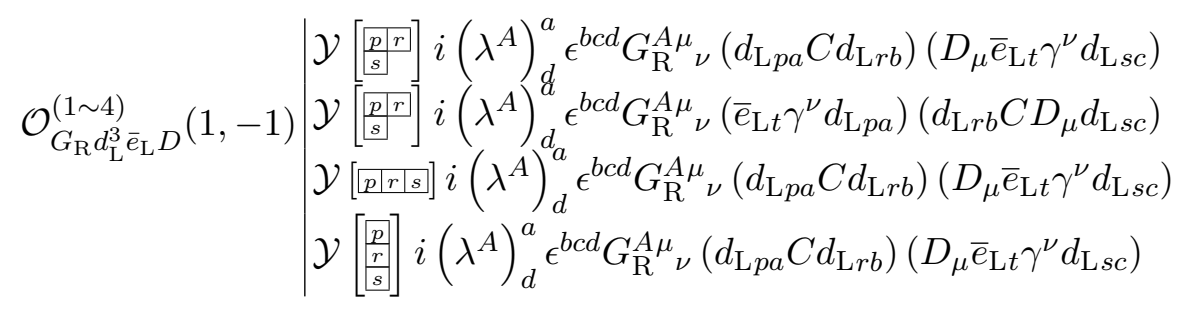

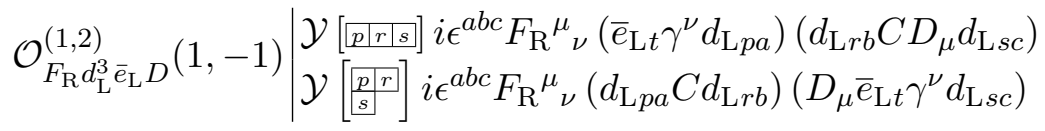

$$
\begin{aligned}
& \mathcal{Y}\left[\frac{r}{s}\right] i\left(\lambda^{A}\right)_{d}^{c} G_{\mathrm{R}}^{A \mu}{ }_{\nu}\left(\bar{d}_{\mathrm{R} p}^{a} d_{\mathrm{L} r a}\right)\left(D_{\mu} \bar{d}_{\mathrm{L} t}{ }^{d} \gamma^{\nu} d_{\mathrm{L} s c}\right) \\
& \mathcal{Y}\left[\frac{r}{s}\right] i\left(\lambda^{A}\right)_{a}^{c} G_{\mathrm{R}}^{A \mu}{ }_{\nu}\left(\bar{d}_{\mathrm{R} p}^{a} d_{\mathrm{L} r b}\right)\left(D_{\mu} \bar{d}_{\mathrm{L} t}^{b} \gamma^{\nu} d_{\mathrm{L} s c}\right) \\
& \mathcal{Y}\left[\frac{r}{s}\right] i\left(\lambda^{A}\right)_{d}^{b} G_{\mathrm{R}}^{A \mu}{ }_{\nu}\left(\bar{d}_{\mathrm{R} p}^{a} d_{\mathrm{L} r b}\right)\left(D_{\mu} \bar{d}_{\mathrm{L} t}^{d} \gamma^{\nu} d_{\mathrm{L} s a}\right) \\
& \mathcal{Y}\left[\begin{array}{l}
\left.\frac{r}{s}\right] \\
]
\end{array}\left(\lambda^{A}\right)_{a}^{b} G_{\mathrm{R}}^{A \mu}{ }_{\nu}\left(\bar{d}_{\mathrm{R} p}^{a} d_{\mathrm{L} r b}\right)\left(D_{\mu} \bar{d}_{\mathrm{L} t}^{c} \gamma^{\nu} d_{\mathrm{L} s c}\right)\right. \\
& \mathcal{O}_{G_{\mathrm{R}} \bar{d}_{\mathrm{R}} d_{\mathrm{L}}^{2} \bar{d}_{\mathrm{L}} D}^{(1 \sim 12)}(0,0) \mathcal{Y}\left[\frac{r}{s}\right] i\left(\lambda^{A}\right)_{d}^{a} G_{\mathrm{R}}^{A \mu}{ }_{\nu}\left(d_{\mathrm{L} r a} C D_{\mu} d_{\mathrm{L} s c}\right)\left(\bar{d}_{\left.\mathrm{R}_{p}^{a} \gamma^{\nu} C \bar{d}_{\mathrm{L} t}^{d}\right)}\right) \\
& \mathcal{Y}\left[\frac{r}{s}\right] i\left(\lambda^{A}\right)_{a c}^{c} G_{\mathrm{R}}^{A \mu}{ }_{\nu}\left(d_{\mathrm{L} r b} C D_{\mu} d_{\mathrm{L} s c}\right)\left(\bar{d}_{\mathrm{R}_{p}^{a}}^{a} \gamma^{\nu} C \bar{d}_{\mathrm{L} t}^{b}\right) \\
& \mathcal{Y}\left[[r \mid s] i\left(\lambda^{A}\right)_{d}^{c} G_{\mathrm{R}}^{A \mu}{ }_{\nu}\left(\bar{d}_{\mathrm{R} p}^{a} d_{\mathrm{L} r a}\right)\left(D_{\mu} \bar{d}_{\mathrm{L} t}^{d} \gamma^{\nu} d_{\mathrm{L} s c}\right)\right. \\
& \mathcal{Y}\left[\overline{r r s]} i\left(\lambda^{A}\right)_{a}^{c} G_{\mathrm{R}}^{A \mu}{ }_{\nu}\left(\bar{d}_{\mathrm{R} p}^{a} d_{\mathrm{L} r b}\right)\left(D_{\mu} \bar{d}_{\mathrm{L} t}^{b} \gamma^{\nu} d_{\mathrm{L} s c}\right)\right. \\
& \mathcal{Y}[\overline{r r s}] i\left(\lambda^{A}\right)_{d}^{b} G_{\mathrm{R}}^{A \mu}{ }_{\nu}\left(\bar{d}_{\mathrm{R} p}^{a} d_{\mathrm{L} r b}\right)\left(D_{\mu} \bar{d}_{\mathrm{L} t}^{d} \gamma^{\nu} d_{\mathrm{L} s a}\right) \\
& \mathcal{Y}\left[\overline{[r s]} i\left(\lambda^{A}\right)_{a}^{b} G_{\mathrm{R}}^{A \mu}{ }_{\nu}\left(\bar{d}_{\mathrm{R} p}^{a} d_{\mathrm{L} r b}\right)\left(D_{\mu} \bar{d}_{\mathrm{L} t}^{c} \gamma^{\nu} d_{\mathrm{L} s c}\right)\right. \\
& \mathcal{Y}[\overline{r r s}] i\left(\lambda^{A}\right)_{d}^{q} G_{\mathrm{R}}^{A \mu}{ }_{\nu}\left(d_{\mathrm{L} r a} C D_{\mu} d_{\mathrm{L} s c}\right)\left(\bar{d}_{\mathrm{R}}^{a} \gamma^{\nu} C \bar{d}_{\mathrm{L} t}^{d}\right) \\
& \mathcal{Y}\left[r[s] i\left(\lambda^{A}\right)_{a}^{c} G_{\mathrm{R}}^{A \mu}{ }_{\nu}\left(d_{\mathrm{L} r b} C D_{\mu} d_{\mathrm{L} s c}\right)\left(\bar{d}_{\mathrm{R} p}^{a} \gamma^{\nu} C \bar{d}_{\mathrm{L} t}^{b}\right)\right.
\end{aligned}
$$

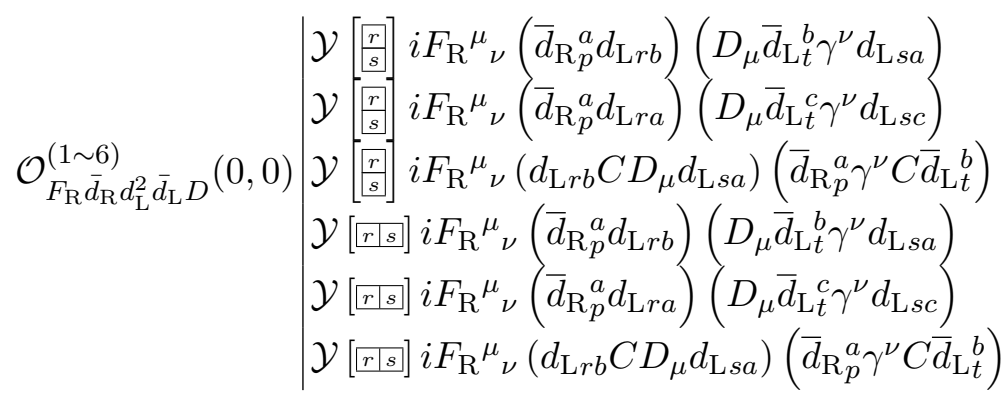

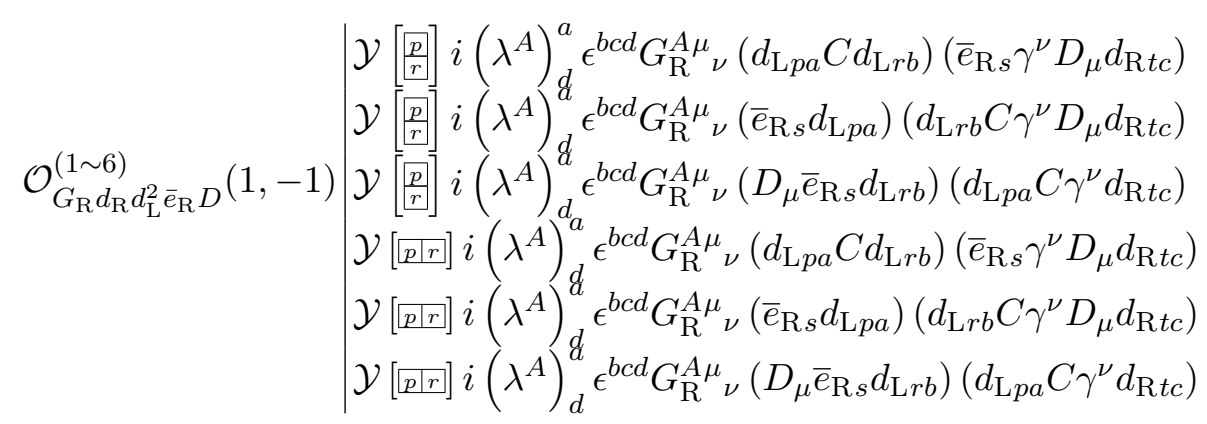




$$
\begin{aligned}
& \mathcal{Y}\left[[p r r] i \epsilon^{a b c} F_{\mathrm{R}}{ }^{\mu}{ }_{\nu}\left(\bar{e}_{\mathrm{R} s} d_{\mathrm{L} p a}\right)\left(d_{\mathrm{L} r b} C \gamma^{\nu} D_{\mu} d_{\mathrm{R} t c}\right)\right. \\
& \mathcal{O}_{F_{\mathrm{R}} d_{\mathrm{R}} d_{\mathrm{L}}^{2} \bar{e}_{\mathrm{R}} D}^{(1 \sim 3)}(1,-1) \mathcal{Y}[[p r]] i \epsilon^{a b c} F_{\mathrm{R}}{ }^{\mu}{ }_{\nu}\left(D_{\mu} \bar{e}_{\mathrm{R} s} d_{\mathrm{L} r b}\right)\left(d_{\mathrm{L} p a} C \gamma^{\nu} d_{\mathrm{R} t c}\right) \\
& \mathcal{Y}\left[\frac{p}{r}\right] i \epsilon^{a b c} F_{\mathrm{R}}{ }^{\mu}{ }_{\nu}\left(d_{\mathrm{L} p a} C d_{\mathrm{L} r b}\right)\left(\bar{e}_{\mathrm{R} s} \gamma^{\nu} D_{\mu} d_{\mathrm{R} t c}\right)
\end{aligned}
$$

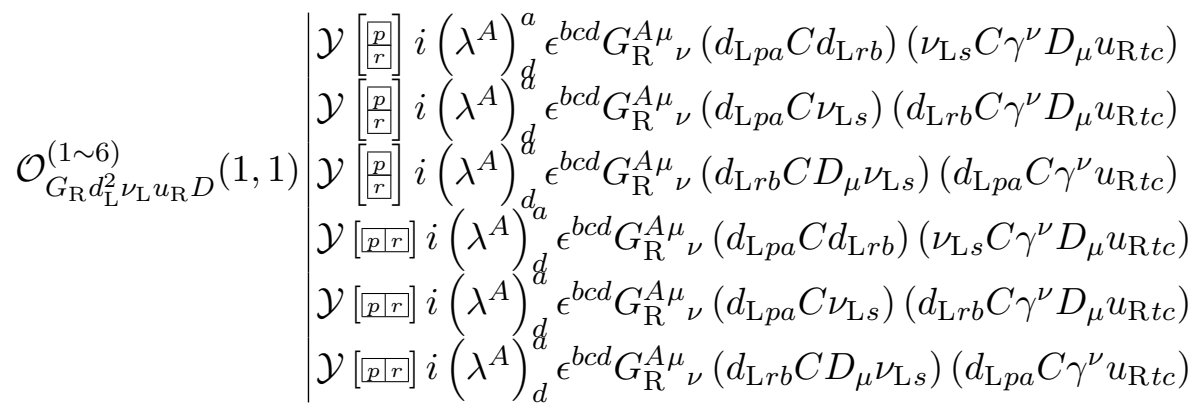

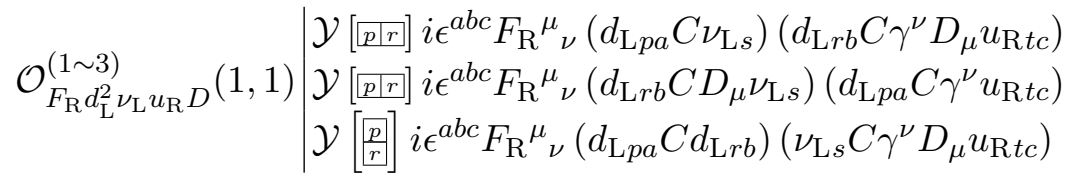

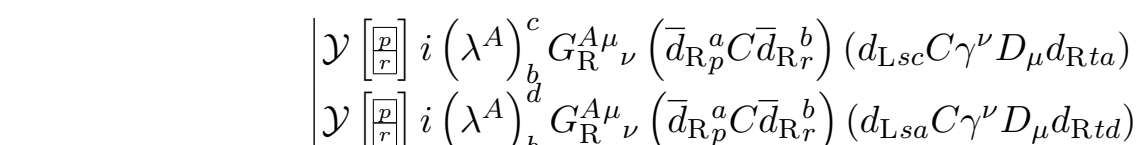

$$
\begin{aligned}
& \mathcal{Y}\left[\begin{array}{l}
p \\
r
\end{array}\right] i\left(\lambda^{A}\right)_{b}^{c} G_{\mathrm{R}}^{A \mu}{ }_{\nu}\left(\bar{d}_{\mathrm{R} p}^{a} d_{\mathrm{L} s c}\right)\left(\bar{d}_{\mathrm{R}}{ }_{r}^{b} \gamma^{\nu} D_{\mu} d_{\mathrm{R} t a}\right) \\
& \mathcal{Y}\left[\frac{p}{r}\right] i\left(\lambda^{A}\right)_{b}^{d} G_{\mathrm{R}}^{A \mu}{ }_{\nu}\left(\bar{d}_{\mathrm{R} p}^{a} d_{\mathrm{L} s a}\right)\left(\bar{d}_{\mathrm{R}_{r}^{b}} \gamma^{\nu} D_{\mu} d_{\mathrm{R} t d}\right) \\
& \mathcal{O}_{G_{\mathrm{R}} \bar{d}_{\mathrm{R}}^{2} d_{\mathrm{R}} d_{\mathrm{L}} D}^{(112)}(0,0) \mathcal{Y}\left[\frac{p}{r}\right] i\left(\lambda^{A}\right)_{b}^{c} G_{\mathrm{R}}^{A \mu}{ }_{\nu}\left(\bar{d}_{\mathrm{R}}{ }_{p}^{a} \gamma^{\nu} d_{\mathrm{R} t a}\right)\left(\bar{d}_{\mathrm{R}}{ }_{r}^{b} D_{\mu} d_{\mathrm{L} s c}\right) \\
& \mathcal{Y}\left[\frac{p}{r}\right] i\left(\lambda^{A}\right)_{b}^{d} G_{\mathrm{R}}^{A \mu}{ }_{\nu}\left(\bar{d}_{\mathrm{R}}^{a} \gamma^{\nu} d_{\mathrm{R} t d}\right)\left(\bar{d}_{\mathrm{R} r}^{b} D_{\mu} d_{\mathrm{L} s a}\right) \\
& \mathcal{Y}[\overline{p p r}] i\left(\lambda^{A}\right)_{b}^{c} G_{\mathrm{R}}^{A \mu}{ }_{\nu}\left(\bar{d}_{\mathrm{R} p}^{a} C \bar{d}_{\mathrm{R}}{ }_{r}^{b}\right)\left(d_{\mathrm{L} s c} C \gamma^{\nu} D_{\mu} d_{\mathrm{R} t a}\right) \\
& \mathcal{Y}[\overline{p \mid r}] i\left(\lambda^{A}\right)_{b}^{d} G_{\mathrm{R}}^{A \mu}{ }_{\nu}\left(\bar{d}_{\mathrm{R} p}^{a} C \bar{d}_{\mathrm{R}}{ }_{r}^{b}\right)\left(d_{\mathrm{L} s a} C \gamma^{\nu} D_{\mu} d_{\mathrm{R} t d}\right) \\
& \mathcal{Y}[\underline{p \eta r}] i\left(\lambda^{A}\right)_{b}^{c} G_{\mathrm{R}}^{A \mu}{ }_{\nu}\left(\bar{d}_{\mathrm{R}}^{a}{ }_{p} d_{\mathrm{L} s c}\right)\left(\bar{d}_{\mathrm{R}}{ }_{r}^{b} \gamma^{\nu} D_{\mu} d_{\mathrm{R} t a}\right) \\
& \mathcal{Y}[\overline{p \mid r}] i\left(\lambda^{A}\right)_{b}^{d} G_{\mathrm{R}}^{A \mu}{ }_{\nu}\left(\bar{d}_{\mathrm{R} p}^{a} d_{\mathrm{L} s a}\right)\left(\bar{d}_{\mathrm{R} r}^{b} \gamma^{\nu} D_{\mu} d_{\mathrm{R} t d}\right) \\
& \mathcal{Y}[\overline{p r r}] i\left(\lambda^{A}\right)_{b}^{c} G_{\mathrm{R}}^{A \mu}{ }_{\nu}\left(\bar{d}_{\mathrm{R}}^{a} \gamma^{\nu} d_{\mathrm{R} t a}\right)\left(\bar{d}_{\mathrm{R} r}^{b} D_{\mu} d_{\mathrm{L} s c}\right) \\
& \mathcal{Y}[\overline{p p r}] i\left(\lambda^{A}\right)_{b}^{d} G_{\mathrm{R}}^{A \mu}{ }_{\nu}\left(\bar{d}_{\mathrm{R}}^{a} \gamma^{\nu} d_{\mathrm{R} t d}\right)\left(\bar{d}_{\mathrm{R}}{ }_{r}^{b} D_{\mu} d_{\mathrm{L} s a}\right)
\end{aligned}
$$

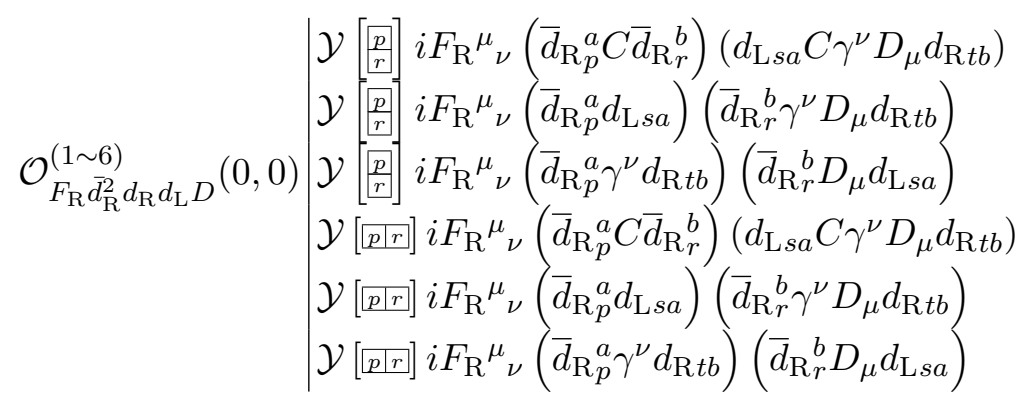




$$
\begin{aligned}
& \mathcal{O}_{G_{\mathrm{R}} \bar{d}_{\mathrm{R}} d_{\mathrm{L}} e_{\mathrm{L}} \bar{e}_{\mathrm{L}} D}(0,0) \mid \begin{array}{l}
i\left(\lambda^{A}\right)_{a}^{b} G_{\mathrm{R}}^{A \mu}{ }_{\nu}\left(\bar{d}_{\mathrm{R}}^{a}{ }_{p} d_{\mathrm{L} r b}\right)\left(D_{\mu} \bar{e}_{\mathrm{L} t} \gamma^{\nu} e_{\mathrm{L} s}\right) \\
i\left(\lambda^{A}\right)_{a}^{b} G_{\mathrm{R}}^{A \mu}{ }_{\nu}\left(\bar{d}_{\mathrm{R}}{ }_{p}^{a} e_{\mathrm{L} s}\right)\left(D_{\mu} \bar{e}_{\mathrm{L} t} \gamma^{\nu} d_{\mathrm{L} r b}\right)
\end{array} \\
& i\left(\lambda^{A}\right)_{a}^{b} G_{\mathrm{R}}^{A \mu}{ }_{\nu}\left(d_{\mathrm{L} r b} C D_{\mu} e_{\mathrm{L} s}\right)\left(\bar{d}_{\mathrm{R}}{ }_{p}^{a} \gamma^{\nu} C \bar{e}_{\mathrm{L} t}\right) \\
& \mathcal{O}_{F_{\mathrm{R}} \bar{d}_{\mathrm{R}} d_{\mathrm{L}} e_{\mathrm{L}} \bar{e}_{\mathrm{L}} D}^{(1 \sim 3)}(0) \mid \begin{array}{l}
i F_{\mathrm{R}}{ }_{\nu}{ }_{\nu}\left(\bar{d}_{\mathrm{R}}{ }_{p}^{a} d_{\mathrm{L} r a}\right)\left(D_{\mu} \bar{e}_{\mathrm{L} t} \gamma^{\nu} e_{\mathrm{L} s}\right) \\
i F_{\mathrm{R}}{ }_{\nu}{ }_{\nu}\left(\bar{d}_{\mathrm{R}}{ }_{p}^{a} e_{\mathrm{L} s}\right)\left(D_{\mu} \bar{e}_{\mathrm{L} t} \gamma^{\nu} d_{\mathrm{L} r a}\right) \\
i F_{\mathrm{R}}{ }^{\mu}{ }_{\nu}\left(d_{\mathrm{L} r a} C D_{\mu} e_{\mathrm{L} s}\right)\left(\bar{d}_{\mathrm{R} p}^{a} \gamma^{\nu} C \bar{e}_{\mathrm{L} t}\right)
\end{array} \\
& \mathcal{O}_{G_{\mathrm{R}} \bar{d}_{\mathrm{R}} d_{\mathrm{L}} \bar{e}_{\mathrm{R}} e_{\mathrm{R}} D}^{(1 \sim, 0)} \mid \begin{array}{l}
i\left(\lambda^{A}\right)_{a}^{b} G_{\mathrm{R}}^{A \mu}{ }_{\nu}\left(\bar{d}_{\mathrm{R} p}^{a} d_{\mathrm{L} r b}\right)\left(\bar{e}_{\mathrm{R} s} \gamma^{\nu} D_{\mu} e_{\mathrm{R} t}\right) \\
i\left(\lambda^{A}\right)_{a}^{b} G_{\mathrm{R}}^{A \mu}{ }_{\nu}\left(\bar{d}_{\mathrm{R}}^{a} C \bar{e}_{\mathrm{R} s}\right)\left(d_{\mathrm{L} r b} C \gamma^{\nu} D_{\mu} e_{\mathrm{R} t}\right)
\end{array} \\
& i\left(\lambda^{A}\right)_{a}^{b} G_{\mathrm{R}}^{A \mu}{ }_{\nu}\left(D_{\mu} \bar{e}_{\mathrm{R} s} d_{\mathrm{L} r b}\right)\left(\bar{d}_{\mathrm{R} p}^{a} \gamma^{\nu} e_{\mathrm{R} t}\right) \\
& \mathcal{O}_{F_{\mathrm{R}} \bar{d}_{\mathrm{R}} d_{\mathrm{L}} \bar{e}_{\mathrm{R}} e_{\mathrm{R}} D}(0,0) \mid \begin{array}{l}
i F_{\mathrm{R}}{ }^{\mu}{ }_{\nu}\left(\bar{d}_{\mathrm{R} p}{ }_{p}^{a} d_{\mathrm{L} r a}\right)\left(\bar{e}_{\mathrm{R} s} \gamma^{\nu} D_{\mu} e_{\mathrm{R} t}\right) \\
i F_{\mathrm{R}}{ }^{\mu}{ }_{\nu}\left(\bar{d}_{\mathrm{R}}{ }_{p}^{a} C \bar{e}_{\mathrm{R} s}\right)\left(d_{\mathrm{L} r a} C \gamma^{\nu} D_{\mu} e_{\mathrm{R} t}\right)
\end{array} \\
& i F_{\mathrm{R}}^{\mu}{ }_{\nu}\left(D_{\mu} \bar{e}_{\mathrm{R} s} d_{\mathrm{L} r a}\right)\left(\bar{d}_{\mathrm{R} p}^{a} \gamma^{\nu} e_{\mathrm{R} t}\right) \\
& \mathcal{O}_{G_{\mathrm{R}} \bar{d}_{\mathrm{R}} d_{\mathrm{L}} \nu_{\mathrm{L}} \bar{\nu}_{\mathrm{L}} D}^{(1 \sim 3)}(0,0) \mid \begin{array}{l}
i\left(\lambda^{A}\right)_{a}^{b} G_{\mathrm{R}}^{A \mu}{ }_{\nu}\left(\bar{d}_{\mathrm{R}}{ }_{p}^{a} d_{\mathrm{L} r b}\right)\left(D_{\mu} \bar{\nu}_{\mathrm{L} t} \gamma^{\nu} \nu_{\mathrm{L} s}\right) \\
i\left(\lambda^{A}\right)_{a}^{b} G_{\mathrm{R}}^{A \mu}{ }_{\nu}\left(\bar{d}_{\mathrm{R}}{ }_{p}^{a} \nu_{\mathrm{L} s}\right)\left(D_{\mu} \bar{\nu}_{\mathrm{L} t} \gamma^{\nu} d_{\mathrm{L} r b}\right) \\
i\left(\lambda^{A}\right)_{a}^{b} G_{\mathrm{R}}^{A \mu}{ }_{\nu}\left(d_{\mathrm{L} r b} C D_{\mu} \nu_{\mathrm{L} s}\right)\left(\bar{d}_{\mathrm{R}}{ }_{p} \gamma^{\nu} C \bar{\nu}_{\mathrm{L} t}\right)
\end{array} \\
& \mathcal{O}_{F_{\mathrm{R}} \bar{d}_{\mathrm{R}} d_{\mathrm{L} \nu_{\mathrm{L}} \bar{\nu}_{\mathrm{L}} D}^{(1 \sim 3)}(0,0)} \mid \begin{array}{l}
i F_{\mathrm{R}}{ }^{\mu}{ }_{\nu}\left(\bar{d}_{\mathrm{R} p}{ }_{p}^{a} d_{\mathrm{L} r a}\right)\left(D_{\mu} \bar{\nu}_{\mathrm{L} t} \gamma^{\nu} \nu_{\mathrm{L} s}\right) \\
i F_{\mathrm{R}}{ }^{\mu}{ }_{\nu}\left(\bar{d}_{\mathrm{R}}{ }_{p}^{a} \nu_{\mathrm{L} s}\right)\left(D_{\mu} \bar{\nu}_{\mathrm{L} t} \gamma^{\nu} d_{\mathrm{L} r a}\right) \\
i F_{\mathrm{R}}{ }_{\nu}{ }_{\nu}\left(d_{\mathrm{L} r a} C D_{\mu} \nu_{\mathrm{L} s}\right)\left(\bar{d}_{\mathrm{R} p}^{a} \gamma^{\nu} C \bar{\nu}_{\mathrm{L} t}\right)
\end{array} \\
& \mathcal{O}_{G_{\mathrm{R}} d_{\mathrm{L}} \bar{d}_{\mathrm{L}} \bar{e}_{\mathrm{R}} e_{\mathrm{L}} D}(0,0) \mid \begin{array}{l}
i\left(\lambda^{A}\right)_{b}^{a} G_{\mathrm{R}}^{A \mu}{ }_{\nu}\left(\bar{e}_{\mathrm{R} r} d_{\mathrm{L} p a}\right)\left(D_{\mu} \bar{d}_{\mathrm{L} t}{ }^{b} \gamma^{\nu} e_{\mathrm{L} s}\right) \\
i\left(\lambda^{A}\right)_{b}^{a} G_{\mathrm{R}}^{A \mu}{ }_{\nu}\left(d_{\mathrm{L} p a} C e_{\mathrm{L} s}\right)\left(\bar{e}_{\mathrm{R} r} \gamma^{\nu} C D_{\mu} \bar{d}_{\mathrm{L} t}^{b}\right)
\end{array} \\
& i\left(\lambda^{A}\right)_{b}^{a} G_{\mathrm{R}}^{A \mu}{ }_{\nu}\left(\bar{e}_{\mathrm{R} r} D_{\mu} e_{\mathrm{L} s}\right)\left(\bar{d}_{\mathrm{L} t}^{b} \gamma^{\nu} d_{\mathrm{L} p a}\right) \\
& \mathcal{O}_{F_{\mathrm{R}} d_{\mathrm{L}} \bar{d}_{\mathrm{L}} \bar{e}_{\mathrm{R}} e_{\mathrm{L}} D}^{(1 \sim 3)}(0) \mid \begin{array}{l}
i F_{\mathrm{R}}^{\mu}{ }_{\nu}\left(\bar{e}_{\mathrm{R} r} d_{\mathrm{L} p a}\right)\left(D_{\mu} \bar{d}_{\mathrm{L} t}^{a} \gamma^{\nu} e_{\mathrm{L} s}\right) \\
i F_{\mathrm{R}}{ }_{\nu}{ }_{\nu}\left(d_{\mathrm{L} p a} C e_{\mathrm{L} s}\right)\left(\bar{e}_{\mathrm{R} r} \gamma^{\nu} C D_{\mu} \bar{d}_{\mathrm{L} t}^{a}\right) \\
i F_{\mathrm{R}}{ }^{\mu}{ }_{\nu}\left(\bar{e}_{\mathrm{R} r} D_{\mu} e_{\mathrm{L} s}\right)\left(\bar{d}_{\mathrm{L} t}^{a} \gamma^{\nu} d_{\mathrm{L} p a}\right)
\end{array} \\
& \mathcal{O}_{G_{\mathrm{R}} d_{\mathrm{L}} \bar{e}_{\mathrm{R}} \nu_{\mathrm{L}} \bar{u}_{\mathrm{L}} D}^{(1 \sim, 0)} \mid \begin{array}{l}
i\left(\lambda^{A}\right)_{b}^{a} G_{\mathrm{R}}^{A \mu}{ }_{\nu}\left(\bar{e}_{\mathrm{R} r} d_{\mathrm{L} p a}\right)\left(D_{\mu} \bar{u}_{\mathrm{L}}{ }^{b} \gamma^{\nu} \nu_{\mathrm{L} s}\right) \\
i\left(\lambda^{A}\right)_{b}^{a} G_{\mathrm{R}}^{A \mu}{ }_{\nu}\left(d_{\mathrm{L} p a} C \nu_{\mathrm{L} s}\right)\left(\bar{e}_{\mathrm{R} r} \gamma^{\nu} C D_{\mu} \bar{u}_{\mathrm{L} t}^{b}\right) \\
i\left(\lambda^{A}\right)_{b}^{a} G_{\mathrm{R}}^{A \mu}{ }_{\nu}\left(\bar{e}_{\mathrm{R} r} D_{\mu} \nu_{\mathrm{L} s}\right)\left(\bar{u}_{\mathrm{L}}{ }_{t}^{b} \gamma^{\nu} d_{\mathrm{L} p a}\right)
\end{array}
\end{aligned}
$$




$$
\begin{aligned}
& \mathcal{O}_{F_{\mathrm{R}} d_{\mathrm{L}} \bar{e}_{\mathrm{R}} \nu_{\mathrm{L}} \bar{u}_{\mathrm{L}} D}(0,0) \mid \begin{array}{l}
i F_{\mathrm{R}}{ }^{\mu}{ }_{\nu}\left(\bar{e}_{\mathrm{R} r} d_{\mathrm{L} p a}\right)\left(D_{\mu} \bar{u}_{\mathrm{L} t}{ }^{a} \gamma^{\nu} \nu_{\mathrm{L} s}\right) \\
i F_{\mathrm{R}}{ }^{\mu}{ }_{\nu}\left(d_{\mathrm{L} p a} C \nu_{\mathrm{L} s}\right)\left(\bar{e}_{\mathrm{R} r} \gamma^{\nu} C D_{\mu} \bar{u}_{\mathrm{L} t}^{a}\right) \\
i F_{\mathrm{R}}{ }^{\mu}{ }_{\nu}\left(\bar{e}_{\mathrm{R} r} D_{\mu} \nu_{\mathrm{L} s}\right)\left(\bar{u}_{\mathrm{L} t}{ }^{a} \gamma^{\nu} d_{\mathrm{L} p a}\right)
\end{array} \\
& \mathcal{O}_{G_{\mathrm{R}} d_{\mathrm{L}} \bar{d}_{\mathrm{L}} \nu_{\mathrm{L}}^{2} D}(0,2) \mid \begin{array}{l}
\mathcal{Y}\left[\begin{array}{l}
r \\
s]
\end{array}\right] i\left(\lambda^{A}\right)_{b}^{a} G_{\mathrm{R}}^{A \mu}{ }_{\nu}\left(d_{\mathrm{L} p a} C \nu_{\mathrm{L} r}\right)\left(D_{\mu} \bar{d}_{\mathrm{L} t}^{b} \gamma^{\nu} \nu_{\mathrm{L} s}\right) \\
\mathcal{Y}\left[\begin{array}{l}
r \\
\underline{s}]
\end{array}\right] i\left(\lambda^{A}\right)_{b}^{a} G_{\mathrm{R}}^{A \mu}{ }_{\nu}\left(\nu_{\mathrm{L} r} C D_{\mu} \nu_{\mathrm{L} s}\right)\left(\bar{d}_{\mathrm{L}}{ }^{b} \gamma^{\nu} d_{\mathrm{L} p a}\right) \\
\mathcal{Y}\left[\frac{r r s}{r}\right] i\left(\lambda^{A}\right)_{b}^{a} G_{\mathrm{R}}^{A \mu}{ }_{\nu}\left(d_{\mathrm{L} p a} C \nu_{\mathrm{L} r}\right)\left(D_{\mu} \bar{d}_{\mathrm{L} t}^{b} \gamma^{\nu} \nu_{\mathrm{L} s}\right)
\end{array}
\end{aligned}
$$

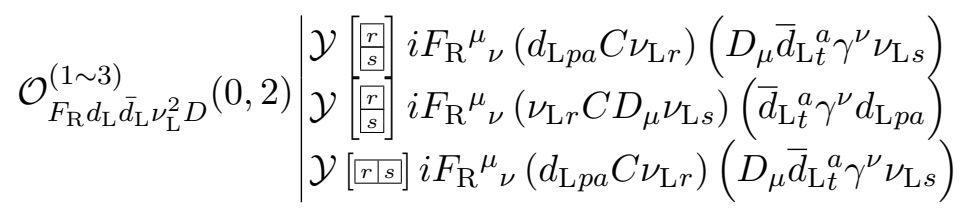

$$
\begin{aligned}
& \mathcal{Y}\left[\frac{p r r}{s s}\right] i \epsilon_{a c d}\left(\lambda^{A}\right)_{b}^{d} G_{\mathrm{R}}^{A \mu}{ }_{\nu}\left(\bar{d}_{\mathrm{R}}^{a} C \bar{d}_{\mathrm{R}}^{b}\right)\left(\bar{d}_{\mathrm{R}}^{c}{ }_{s}^{c} \gamma^{\nu} D_{\mu} e_{\mathrm{R} t}\right) \\
& \mathcal{O}_{G_{\mathrm{R}} \bar{d}_{\mathrm{R}}^{3} e_{\mathrm{R}} D}^{(1 \sim 4)}(-1,1) \mathcal{Y}\left[\frac{p r}{s s}\right] i \epsilon_{b c d}\left(\lambda^{A}\right)_{a}^{d} G_{\mathrm{R}}^{A \mu}{ }_{\nu}\left(\bar{d}_{\mathrm{R} p}^{a} \gamma^{\nu} e_{\mathrm{R} t}\right)\left(\bar{d}_{\mathrm{R} r}^{b} C D_{\mu} \bar{d}_{\mathrm{R}}{ }_{s}^{c}\right) \\
& \mathcal{Y}\left[\overline{p|r| s]} i \epsilon_{a c d}\left(\lambda^{A}\right)_{b}^{d} G_{\mathrm{R}}^{A \mu}{ }_{\nu}\left(\bar{d}_{\mathrm{R} p}^{a} C \bar{d}_{\mathrm{R}}{ }_{r}^{b}\right)\left(\bar{d}_{\mathrm{R}_{s}^{c}} \gamma^{\nu} D_{\mu} e_{\mathrm{R} t}\right)\right. \\
& \mathcal{Y}\left[\begin{array}{l}
\frac{p}{r} \\
s
\end{array}\right] i \epsilon_{a c d}\left(\lambda^{A}\right)_{b}^{d} G_{\mathrm{R}}^{A \mu}{ }_{\nu}\left(\bar{d}_{\mathrm{R} p}^{a} C \bar{d}_{\mathrm{R} r}^{b}\right)\left(\bar{d}_{\mathrm{R} s}^{c} \gamma^{\nu} D_{\mu} e_{\mathrm{R} t}\right)
\end{aligned}
$$

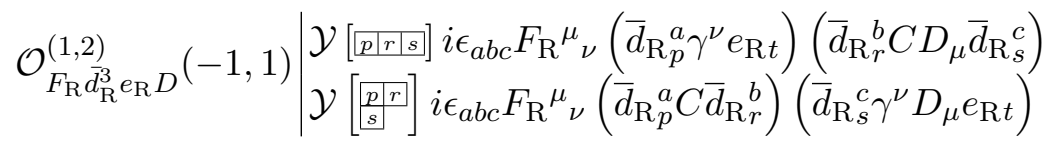

$$
\begin{aligned}
& \mathcal{Y}\left[\begin{array}{l}
\left.\frac{p}{r}\right] \\
{[}
\end{array}\right] \epsilon_{a c d}\left(\lambda^{A}\right)_{b}^{d} G_{\mathrm{R}}^{A \mu}{ }_{\nu}\left(\bar{d}_{\mathrm{R} p}^{a} C \bar{d}_{\mathrm{R}}{ }_{r}^{b}\right)\left(D_{\mu} \bar{d}_{\mathrm{L} t}^{c} \gamma^{\nu} e_{\mathrm{L} s}\right) \\
& \mathcal{Y}\left[\frac{p}{r}\right] i \epsilon_{a c d}\left(\lambda^{A}\right)_{b}^{d} G_{\mathrm{R}}^{A \mu}{ }_{\nu}\left(\bar{d}_{\mathrm{R} p}^{a} e_{\mathrm{L} s}\right)\left(\bar{d}_{\mathrm{R}}{ }^{b} \gamma^{\nu} C D_{\mu} \bar{d}_{\mathrm{L} t}^{c}\right)
\end{aligned}
$$

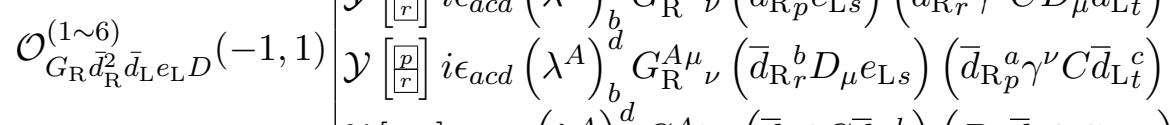

$$
\begin{aligned}
& \mathcal{Y}[\overline{p p r}] i \epsilon_{a c d}\left(\lambda^{A}\right)_{b}^{d} G_{\mathrm{R}}^{A \mu}{ }_{\nu}\left(\bar{d}_{\mathrm{R} p}^{a} C \bar{d}_{\mathrm{R}}{ }_{r}^{b}\right)\left(D_{\mu} \bar{d}_{\mathrm{L} t}^{c} \gamma^{\nu} e_{\mathrm{L} s}\right) \\
& \mathcal{Y}[\underline{p \eta r}] i \epsilon_{\text {acd }}\left(\lambda^{A}\right)_{b}^{d} G_{\mathrm{R}}^{A \mu}{ }_{\nu}\left(\bar{d}_{\mathrm{R}}{ }_{p}^{a} e_{\mathrm{L} s}\right)\left(\bar{d}_{\mathrm{R} r}^{b} \gamma^{\nu} C D_{\mu} \bar{d}_{\mathrm{L} t}^{c}\right) \\
& \mathcal{Y}[\underline{p p r}] i \epsilon_{a c d}\left(\lambda^{A}\right)_{b}^{d} G_{\mathrm{R}}^{A \mu}{ }_{\nu}\left(\bar{d}_{\mathrm{R} r}^{b} D_{\mu} e_{\mathrm{L} s}\right)\left(\bar{d}_{\mathrm{R}}^{a} \gamma^{\nu} C \bar{d}_{\mathrm{L} t}^{c}\right)
\end{aligned}
$$

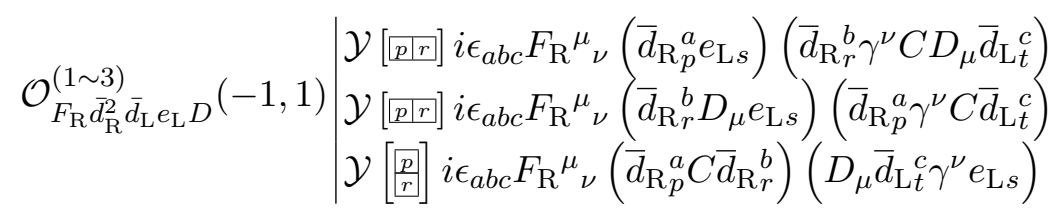




$$
\begin{aligned}
& \mathcal{Y}\left[\frac{p}{r}\right] i \epsilon_{a c d}\left(\lambda^{A}\right)_{b}^{d} G_{\mathrm{R}}^{A \mu}{ }_{\nu}\left(\bar{d}_{\mathrm{R}}{ }_{p}^{a} C \bar{d}_{\mathrm{R}}{ }_{r}^{b}\right)\left(D_{\mu} \bar{u}_{\mathrm{L} t}^{c} \gamma^{\nu} \nu_{\mathrm{L} s}\right) \\
& \mathcal{Y}\left[\frac{p}{r}\right] i \epsilon_{a c d}\left(\lambda^{A}\right)_{b}^{d} G_{\mathrm{R}}^{A \mu}{ }_{\nu}\left(\bar{d}_{\mathrm{R} p}^{a} \nu_{\mathrm{L} s}\right)\left(\bar{d}_{\mathrm{R}}^{b} \gamma^{\nu} C D_{\mu} \bar{u}_{\mathrm{L} t}^{c}\right) \\
& \mathcal{O}_{G_{\mathrm{R}} \bar{d}_{\mathrm{R}}^{2} \nu_{\mathrm{L}} \bar{u}_{\mathrm{L}} D}^{(1 \sim 1,1)} \mathcal{Y}\left[\begin{array}{l}
{\left[\frac{p}{r}\right]} \\
]
\end{array} i \epsilon_{a c d}\left(\lambda^{A}\right)_{b}^{d} G_{\mathrm{R}}^{A \mu}{ }_{\nu}\left(\bar{d}_{\mathrm{R} r}^{b} D_{\mu} \nu_{\mathrm{L} s}\right)\left(\bar{d}_{\mathrm{R}}{ }_{p}^{a} \gamma^{\nu} C \bar{u}_{\mathrm{L}}{ }^{c}\right)\right. \\
& \mathcal{Y}[\overline{p p r}] i \epsilon_{a c d}\left(\lambda^{A}\right)_{b}^{d} G_{\mathrm{R}}^{A \mu}{ }_{\nu}\left(\bar{d}_{\mathrm{R} p}^{a} C \bar{d}_{\mathrm{R} r}^{b}\right)\left(D_{\mu} \bar{u}_{\mathrm{L} t}^{c} \gamma^{\nu} \nu_{\mathrm{L} s}\right) \\
& \mathcal{Y}[\overline{p p r}] i \epsilon_{a c d}\left(\lambda^{A}\right)_{b}^{d} G_{\mathrm{R}}^{A \mu}{ }_{\nu}\left(\bar{d}_{\mathrm{R} p}{ }_{p}^{a} \nu_{\mathrm{L} s}\right)\left(\bar{d}_{\mathrm{R} r}^{b} \gamma^{\nu} C D_{\mu} \bar{u}_{\mathrm{L} t}^{c}\right) \\
& \mathcal{Y}[[p r]] i \epsilon_{a c d}\left(\lambda^{A}\right)_{b}^{d} G_{\mathrm{R}}^{A \mu}{ }_{\nu}\left(\bar{d}_{\mathrm{R} r}^{b} D_{\mu} \nu_{\mathrm{L} s}\right)\left(\bar{d}_{\mathrm{R}}^{a} \gamma^{\nu} C \bar{u}_{\mathrm{L} t}^{c}\right)
\end{aligned}
$$

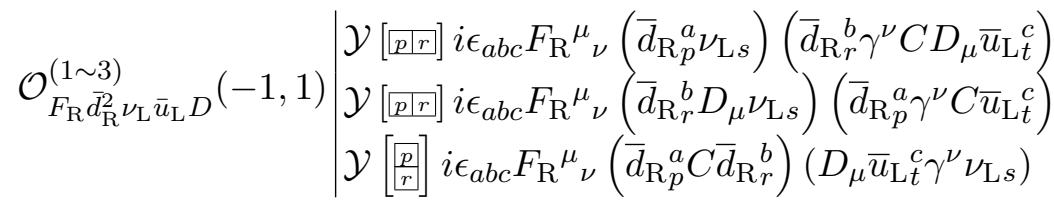

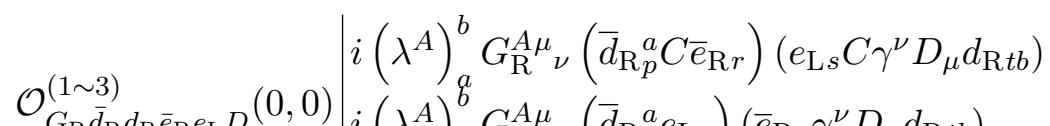

$$
\begin{aligned}
& \begin{array}{l}
i\left(\lambda^{A}\right)_{a}^{b} G_{\mathrm{R}}^{A \mu}{ }_{\nu}\left(\bar{d}_{\mathrm{R} p}^{a} e_{\mathrm{L} s}\right)\left(\bar{e}_{\mathrm{R} r} \gamma^{\nu} D_{\mu} d_{\mathrm{R} t b}\right) \\
i\left(\lambda^{A}\right)_{a}^{b} G_{\mathrm{R}}^{A \mu}{ }_{\nu}\left(\bar{e}_{\mathrm{R} r} D_{\mu} e_{\mathrm{L} s}\right)\left(\bar{d}_{\mathrm{R} p}^{a} \gamma^{\nu} d_{\mathrm{R} t b}\right)
\end{array} \\
& \mathcal{O}_{F_{\mathrm{R}} \bar{d}_{\mathrm{R}} d_{\mathrm{R}} \bar{e}_{\mathrm{R}} e_{\mathrm{L}} D}^{(1 \sim, 0)} \mid \begin{array}{l}
i F_{\mathrm{R}}{ }^{\mu}{ }_{\nu}\left(\bar{d}_{\mathrm{R} p}^{a} C \bar{e}_{\mathrm{R} r}\right)\left(e_{\mathrm{L} s} C \gamma^{\nu} D_{\mu} d_{\mathrm{R} t a}\right) \\
i F_{\mathrm{R}}{ }^{\mu}{ }_{\nu}\left(\bar{d}_{\mathrm{R} p}{ }^{a} e_{\mathrm{L} s}\right)\left(\bar{e}_{\mathrm{R} r} \gamma^{\nu} D_{\mu} d_{\mathrm{R} t a}\right) \\
i F_{\mathrm{R}}{ }^{\mu}{ }_{\nu}\left(\bar{e}_{\mathrm{R} r} D_{\mu} e_{\mathrm{L} s}\right)\left(\bar{d}_{\mathrm{R}}{ }_{p}^{a} \gamma^{\nu} d_{\mathrm{R} t a}\right)
\end{array} \\
& \mathcal{O}_{G_{\mathrm{R}} \bar{d}_{\mathrm{R}} e_{\mathrm{L}} \nu_{\mathrm{L}} u_{\mathrm{R}} D}^{(1 \sim 2)} \mid \begin{array}{l}
i\left(\lambda^{A}\right)_{a}^{b} G_{\mathrm{R}}^{A \mu}{ }_{\nu}\left(\bar{d}_{\mathrm{R}}^{a} e_{\mathrm{L} r}\right)\left(\nu_{\mathrm{L} s} C \gamma^{\nu} D_{\mu} u_{\mathrm{R} t b}\right) \\
i\left(\lambda^{A}\right)_{a}^{b} G_{\mathrm{R}}^{A \mu}{ }_{\nu}\left(\bar{d}_{\mathrm{R} p}^{a} \nu_{\mathrm{L} s}\right)\left(e_{\mathrm{L} r} C \gamma^{\nu} D_{\mu} u_{\mathrm{R} t b}\right) \\
i\left(\lambda^{A}\right)_{a}^{b} G_{\mathrm{R}}^{A \mu}{ }_{\nu}\left(e_{\mathrm{L} r} C D_{\mu} \nu_{\mathrm{L} s}\right)\left(\bar{d}_{\mathrm{R}}{ }^{a} \gamma^{\nu} u_{\mathrm{R} t b}\right)
\end{array} \\
& \mathcal{O}_{F_{\mathrm{R}} \bar{d}_{\mathrm{R}} e_{\mathrm{L}} \nu_{\mathrm{L}} u_{\mathrm{R}} D}^{(1 \sim 2)} \mid \begin{array}{l}
i F_{\mathrm{R}}{ }_{\nu}{ }_{\nu}\left(\bar{d}_{\mathrm{R}}{ }_{p}^{a} e_{\mathrm{L} r}\right)\left(\begin{array}{l}
\left.\nu_{\mathrm{L} s} C \gamma^{\nu} D_{\mu} u_{\mathrm{R} t a}\right) \\
i F_{\mathrm{R}}{ }^{\mu}{ }_{\nu}\left(\bar{d}_{\mathrm{R}}{ }_{\mathrm{R}}^{a} \nu_{\mathrm{L} s}\right) \\
\left(e_{\mathrm{L} r} C \gamma^{\nu} D_{\mu} u_{\mathrm{R} t a}\right) \\
i F_{\mathrm{R}}{ }^{\mu}{ }_{\nu}\left(e_{\mathrm{L} r} C D_{\mu} \nu_{\mathrm{L} s}\right)\left(\bar{d}_{\mathrm{R}}{ }_{p}^{a} \gamma^{\nu} u_{\mathrm{R} t a}\right)
\end{array}\right.
\end{array}
\end{aligned}
$$

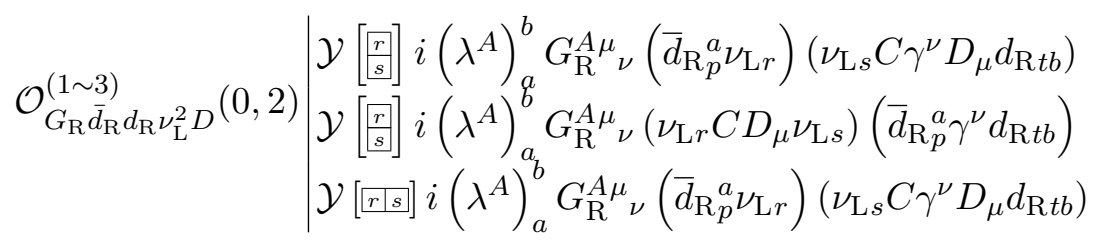

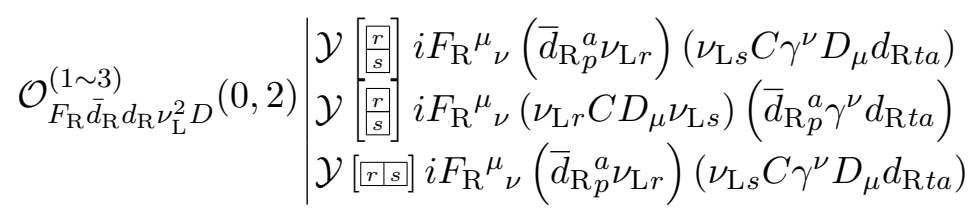




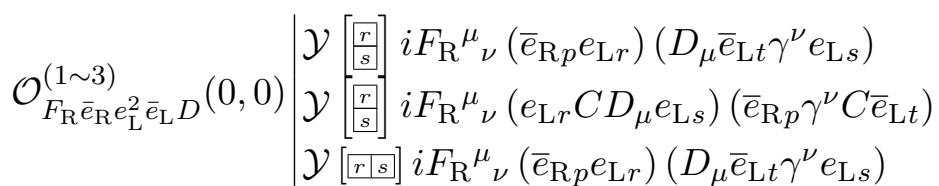

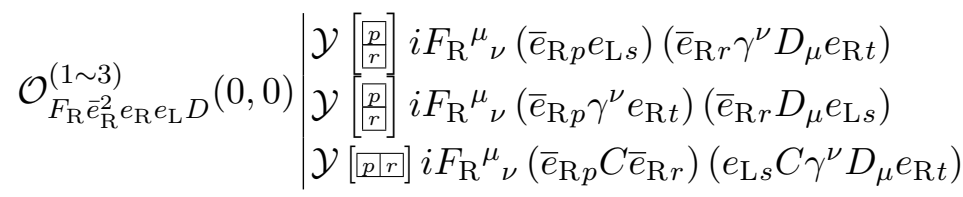

$$
\begin{aligned}
& \mathcal{O}_{F_{\mathrm{R}} \bar{e}_{\mathrm{R}} e_{\mathrm{L}} \nu_{\mathrm{L}} \bar{\nu}_{\mathrm{L}} D}(0,0) \mid \begin{array}{l}
i F_{\mathrm{R}}{ }^{\mu}{ }_{\nu}\left(\bar{e}_{\mathrm{R} p} e_{\mathrm{L} r}\right)\left(D_{\mu} \bar{\nu}_{\mathrm{L} t} \gamma^{\nu} \nu_{\mathrm{L} s}\right) \\
i F_{\mathrm{R}}{ }^{\mu}{ }_{\nu}\left(\bar{e}_{\mathrm{R} p} \nu_{\mathrm{L} s}\right)\left(D_{\mu} \bar{\nu}_{\mathrm{L} t} \gamma^{\nu} e_{\mathrm{L} r}\right) \\
i F_{\mathrm{R}}{ }^{\mu}{ }_{\nu}\left(e_{\mathrm{L} r} C D_{\mu} \nu_{\mathrm{L} s}\right)\left(\bar{e}_{\mathrm{R} p} \gamma^{\nu} C \bar{\nu}_{\mathrm{L} t}\right)
\end{array}
\end{aligned}
$$

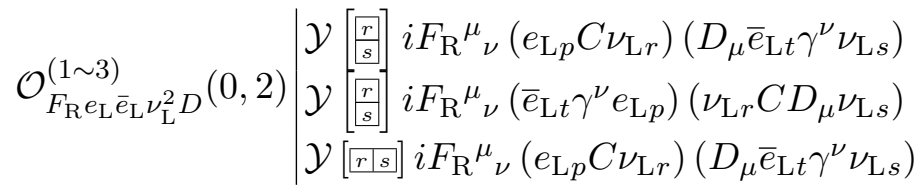

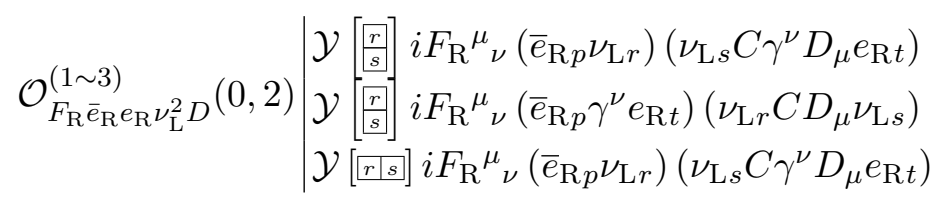

$$
\begin{aligned}
& \mathcal{O}_{F_{\mathrm{R}} \nu_{\mathrm{L}}^{3} \bar{\nu}_{\mathrm{L}} D}^{(1,2,2)} \mid \begin{array}{l}
\mathcal{Y}\left[\begin{array}{l}
\frac{p}{r} \\
\frac{r}{s}
\end{array}\right] i F_{\mathrm{R}}{ }^{\mu}{ }_{\nu}\left(\bar{\nu}_{\mathrm{L} t} \gamma^{\nu} \nu_{\mathrm{L} p}\right)\left(\nu_{\mathrm{L} r} C D_{\mu} \nu_{\mathrm{L} s}\right) \\
\mathcal{Y}\left[\begin{array}{l}
\frac{p r}{s} \\
\frac{s}{2}
\end{array}\right] i\left(\nu_{\mathrm{L} p} C \nu_{\mathrm{L} r}\right) F_{\mathrm{R}}{ }^{\mu}{ }_{\nu}\left(D_{\mu} \bar{\nu}_{\mathrm{L} t} \gamma^{\nu} \nu_{\mathrm{L} s}\right)
\end{array}
\end{aligned}
$$

\subsubsection{Classes involving six-fermions: $\psi^{6}$}

There are 58 complex types in this class.

$$
(\Delta B, \Delta L)=(0,0)
$$

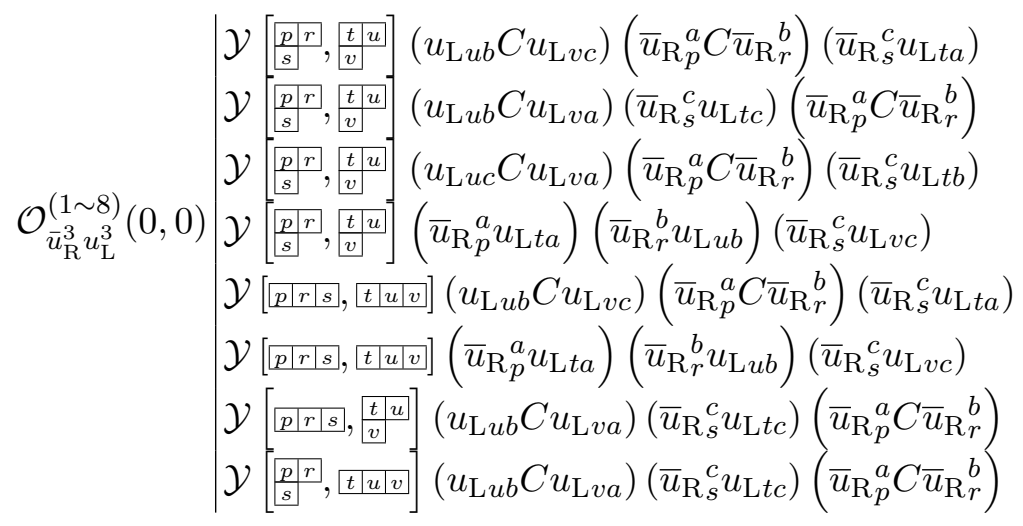




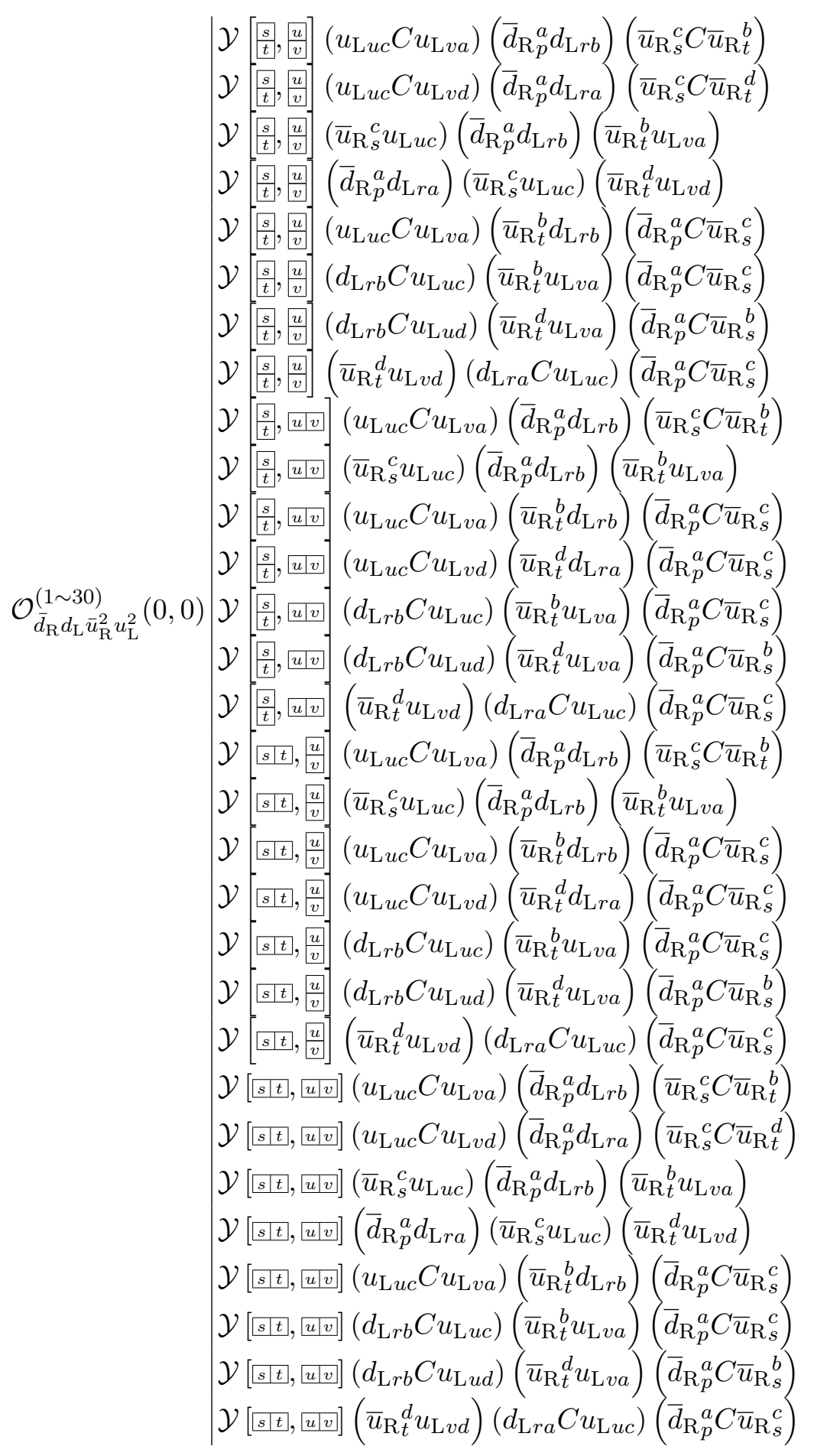




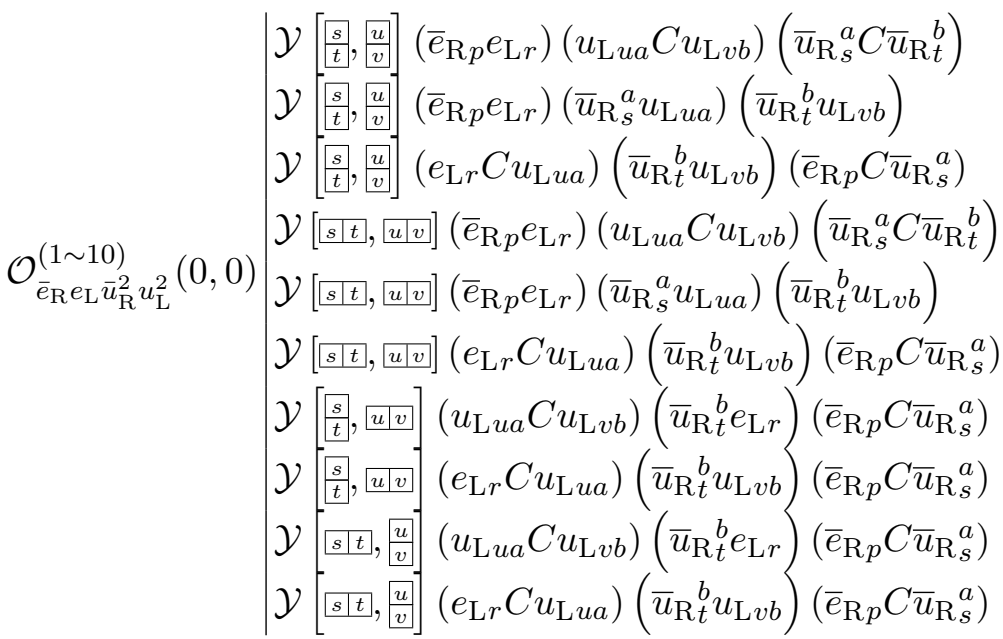

$$
\begin{aligned}
& \mathcal{Y}\left[\begin{array}{l}
s \\
\frac{s}{t}
\end{array}\right]\left(\bar{e}_{\mathrm{R} r} d_{\mathrm{L} p a}\right)\left(u_{\mathrm{L} u b} C \nu_{\mathrm{L} v}\right)\left(\bar{u}_{\mathrm{R}}^{b} C \bar{u}_{\mathrm{R} t}^{a}\right) \\
& \mathcal{Y}\left[\begin{array}{c}
s \\
t
\end{array}\right]\left(\bar{e}_{\mathrm{R} r} d_{\mathrm{L} p a}\right)\left(\bar{u}_{\mathrm{R} t}^{a} \nu_{\mathrm{L} v}\right)\left(\bar{u}_{\mathrm{R} s}^{b} u_{\mathrm{L} u b}\right) \\
& \mathcal{Y}\left[\begin{array}{c}
s \\
\frac{s}{t}
\end{array}\right]\left(u_{\mathrm{L} u b} C \nu_{\mathrm{L} v}\right)\left(\bar{u}_{\mathrm{R} s}^{b} d_{\mathrm{L} p a}\right)\left(\bar{e}_{\mathrm{R} r} C \bar{u}_{\mathrm{R} t}^{a}\right)
\end{aligned}
$$

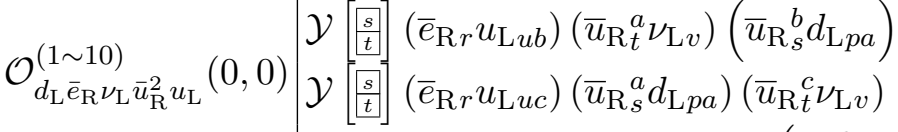

$$
\begin{aligned}
& \mathcal{Y}[s \mid t]\left(\bar{e}_{\mathrm{R} r} d_{\mathrm{L} p a}\right)\left(u_{\mathrm{L} u b} C \nu_{\mathrm{L} v}\right)\left(\bar{u}_{\mathrm{R} s}^{b} C \bar{u}_{\mathrm{R} t}^{a}\right) \\
& \mathcal{Y}[s \mid t]\left(\bar{e}_{\mathrm{R} r} d_{\mathrm{L} p a}\right)\left(\bar{u}_{\mathrm{R} t}^{a} \nu_{\mathrm{L} v}\right)\left(\bar{u}_{\mathrm{R} s}^{b} u_{\mathrm{L} u b}\right) \\
& \mathcal{Y}[s \mid t]\left(u_{\mathrm{L} u b} C \nu_{\mathrm{L} v}\right)\left(\bar{u}_{\mathrm{R}}^{b} d_{\mathrm{L} p a}\right)\left(\bar{e}_{\mathrm{R} r} C \bar{u}_{\mathrm{R} t}^{a}\right) \\
& \mathcal{Y}[s \mid t]\left(\bar{e}_{\mathrm{R} r} u_{\mathrm{L} u b}\right)\left(\bar{u}_{\mathrm{R} t}^{a} \nu_{\mathrm{L} v}\right)\left(\bar{u}_{\mathrm{R} s}^{b} d_{\mathrm{L} p a}\right) \\
& \mathcal{Y}[s \mid t]\left(\bar{e}_{\mathrm{R} r} u_{\mathrm{L} u c}\right)\left(\bar{u}_{\mathrm{R}}^{a} d_{\mathrm{L} p a}\right)\left(\bar{u}_{\mathrm{R}}^{c} \nu_{\mathrm{L} v}\right)
\end{aligned}
$$




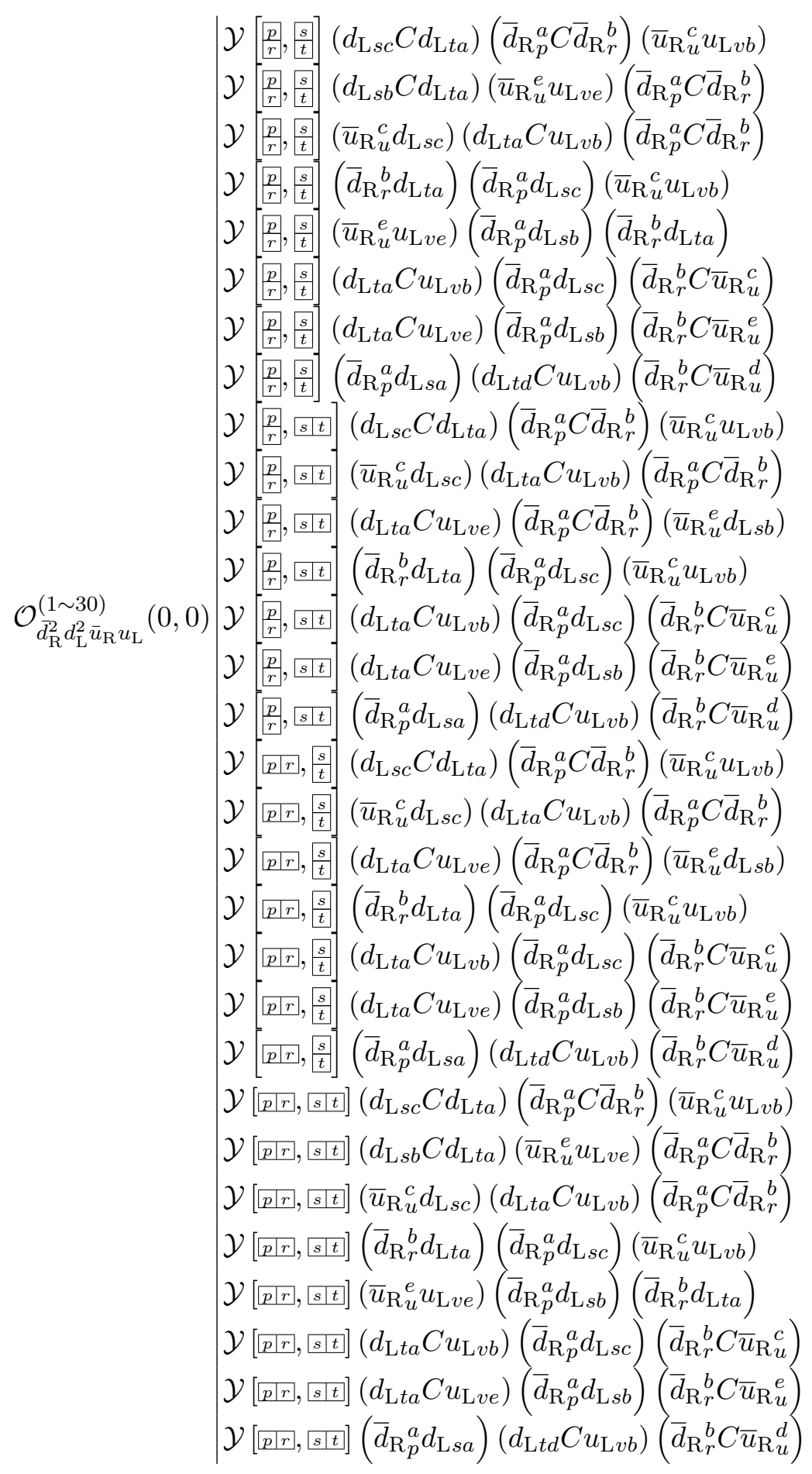




$$
\begin{aligned}
& \mathcal{O}_{\bar{d}_{\mathrm{R}} d_{\mathrm{L}} \bar{e}_{\mathrm{R}} e_{\mathrm{L}} \bar{u}_{\mathrm{R}} u_{\mathrm{L}}}(0,0) \mid \begin{array}{l}
\left(\bar{e}_{\mathrm{R} s} e_{\mathrm{L} t}\right)\left(\bar{d}_{\mathrm{R} p}^{a} d_{\mathrm{L} r b}\right)\left(\bar{u}_{\mathrm{R} u}^{b} u_{\mathrm{L} v a}\right) \\
\left(\bar{e}_{\mathrm{R} s} e_{\mathrm{L} t}\right)\left(\bar{d}_{\mathrm{R} p}^{a} d_{\mathrm{L} r a}\right)\left(\bar{u}_{\mathrm{R} u}^{c} u_{\mathrm{L} v c}\right) \\
\left(e_{\mathrm{L} t} C u_{\mathrm{L} v a}\right)\left(\bar{d}_{\mathrm{R} p}^{a} d_{\mathrm{L} r b}\right)\left(\bar{e}_{\mathrm{R} s} C \bar{u}_{\mathrm{R} u}^{b}\right) \\
\left(e_{\mathrm{L} t} C u_{\mathrm{L} v c}\right)\left(\bar{d}_{\mathrm{R} p}^{a} d_{\mathrm{L} r a}\right)\left(\bar{e}_{\mathrm{R} s} C \bar{u}_{\mathrm{R}}^{c}\right) \\
\left(d_{\mathrm{L} r b} C e_{\mathrm{L} t}\right)\left(\bar{u}_{\mathrm{R} u}^{b} u_{\mathrm{L} v a}\right)\left(\bar{d}_{\mathrm{R} p}^{a} C \bar{e}_{\mathrm{R} s}\right) \\
\left(d_{\mathrm{L} r a} C e_{\mathrm{L} t}\right)\left(\bar{u}_{\mathrm{R} u}^{c} u_{\mathrm{L} v c}\right)\left(\bar{d}_{\mathrm{R} p}^{a} C \bar{e}_{\mathrm{R} s}\right) \\
\left(e_{\mathrm{L} t} C u_{\mathrm{L} v a}\right)\left(\bar{u}_{\mathrm{R} u}^{b} d_{\mathrm{L} r b}\right)\left(\bar{d}_{\mathrm{R} p}^{a} C \bar{e}_{\mathrm{R} s}\right) \\
\left(e_{\mathrm{L} t} C u_{\mathrm{L} v c}\right)\left(\bar{u}_{\mathrm{R}}^{c} d_{\mathrm{L} r a}\right)\left(\bar{d}_{\mathrm{R} p}^{a} C \bar{e}_{\mathrm{R} s}\right) \\
\left(\bar{e}_{\mathrm{R} s} u_{\mathrm{L} v a}\right)\left(\bar{d}_{\mathrm{R} p}^{a} e_{\mathrm{L} t}\right)\left(\bar{u}_{\mathrm{R} u}^{b} d_{\mathrm{L} r b}\right) \\
\left(\bar{e}_{\mathrm{R} s} u_{\mathrm{L} v c}\right)\left(\bar{d}_{\mathrm{R} p}^{a} e_{\mathrm{L} t}\right)\left(\bar{u}_{\mathrm{R} u}^{c} d_{\mathrm{L} r a}\right)
\end{array}
\end{aligned}
$$

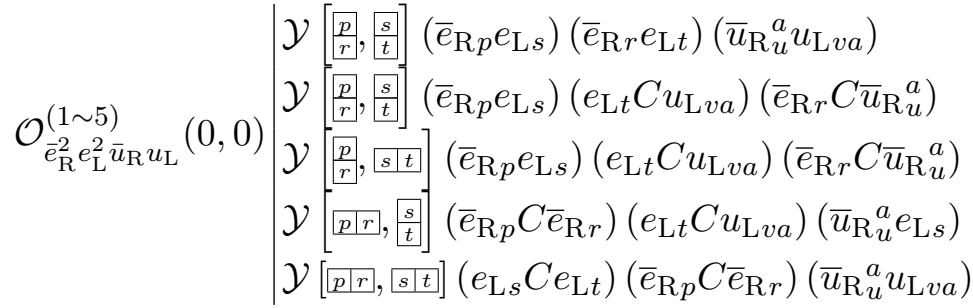

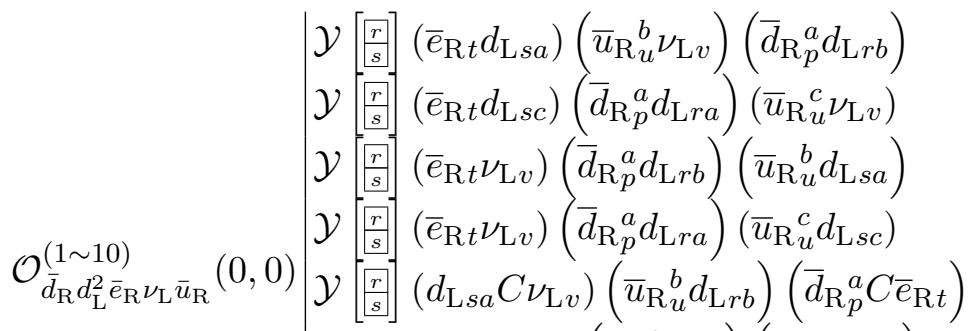

$$
\begin{aligned}
& \mathcal{Y}\left[[r s]\left(\bar{e}_{\mathrm{R} t} d_{\mathrm{L} s a}\right)\left(\bar{u}_{\mathrm{R} u}^{b} \nu_{\mathrm{L} v}\right)\left(\bar{d}_{\mathrm{R} p}^{a} d_{\mathrm{Lrb}}\right)\right. \\
& \mathcal{Y}[\overline{r r s}]\left(\bar{e}_{\mathrm{R} t} d_{\mathrm{L} s c}\right)\left(\bar{d}_{\mathrm{R} p}^{a} d_{\mathrm{L} r a}\right)\left(\bar{u}_{\mathrm{R} u}^{c} \nu_{\mathrm{L} v}\right) \\
& \mathcal{Y}\left[{ }_{r r s]}\right]\left(\bar{e}_{\mathrm{R} t} \nu_{\mathrm{L} v}\right)\left(\bar{d}_{\mathrm{R} p}^{a} d_{\mathrm{L} r b}\right)\left(\bar{u}_{\mathrm{R} u}^{b} d_{\mathrm{L} s a}\right) \\
& \mathcal{Y}[\overline{r r s}]\left(\bar{e}_{\mathrm{R} t} \nu_{\mathrm{L} v}\right)\left(\bar{d}_{\mathrm{R} p}^{a} d_{\mathrm{L} r a}\right)\left(\bar{u}_{\mathrm{R} u}^{c} d_{\mathrm{L} s c}\right) \\
& \mathcal{Y}[\underline{r r s}]\left(d_{\mathrm{L} s a} C \nu_{\mathrm{L} v}\right)\left(\bar{u}_{\mathrm{R} u}^{b} d_{\mathrm{L} r b}\right)\left(\bar{d}_{\mathrm{R} p}^{a} C \bar{e}_{\mathrm{R} t}\right)
\end{aligned}
$$

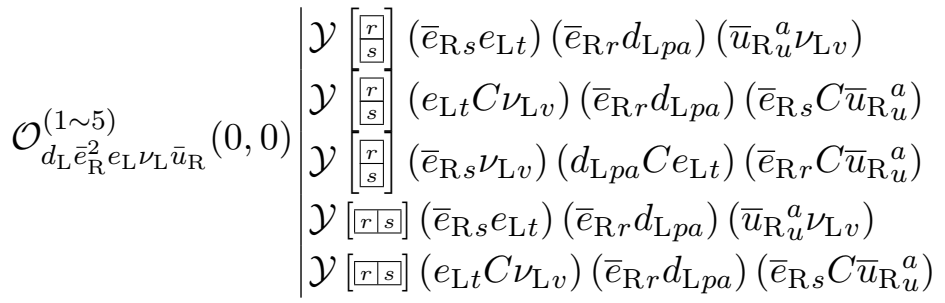




$$
\begin{aligned}
& \mathcal{Y}\left[\begin{array}{c}
\frac{p}{r}, \frac{t}{s}, \frac{t}{v} \\
\hdashline
\end{array}\right]\left(d_{\mathrm{L} u b} C d_{\mathrm{L} v c}\right)\left(\bar{d}_{\mathrm{R} p}^{a} C \bar{d}_{\mathrm{R}}^{b}\right)\left(\bar{d}_{\mathrm{R}}^{c} d_{\mathrm{L} t a}\right) \\
& \mathcal{Y}\left[\begin{array}{c}
\frac{p}{\frac{p}{s}}, \frac{t}{v} \\
\frac{u}{v}
\end{array}\right]\left(\bar{d}_{\mathrm{R} p}^{a} d_{\mathrm{L} t a}\right)\left(\bar{d}_{\mathrm{R} r}^{b} d_{\mathrm{L} u b}\right)\left(\bar{d}_{\mathrm{R}}^{c}{ }^{c} d_{\mathrm{L} v c}\right) \\
& \mathcal{Y}\left[\frac{p r r}{\underline{s}}, \frac{t \mid u}{v}\right]\left(d_{\mathrm{L} u b} C d_{\mathrm{L} v c}\right)\left(\bar{d}_{\mathrm{R} p}^{a} C \bar{d}_{\mathrm{R} r}^{b}\right)\left(\bar{d}_{\mathrm{R} s}^{c} d_{\mathrm{L} t a}\right) \\
& \mathcal{Y}\left[\frac{p r r}{s}, \frac{t u u}{v}\right]\left(d_{\mathrm{L} u b} C d_{\mathrm{L} v a}\right)\left(\bar{d}_{\mathrm{R} s}^{c} d_{\mathrm{L} t c}\right)\left(\bar{d}_{\mathrm{R} p}^{a} C \bar{d}_{\mathrm{R} r}^{b}\right) \\
& \mathcal{Y}\left[\frac{p r r}{s s}, \frac{t u}{v}\right]\left(d_{\mathrm{L} u c} C d_{\mathrm{L} v a}\right)\left(\bar{d}_{\mathrm{R} p}^{a} C \bar{d}_{\mathrm{R}}^{b}\right)\left(\bar{d}_{\mathrm{R}}^{c} d_{\mathrm{L} t b}\right)
\end{aligned}
$$

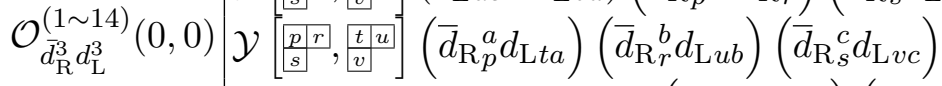

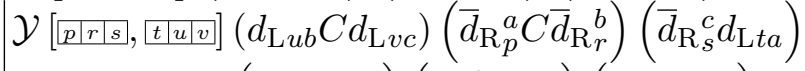

$$
\begin{aligned}
& \mathcal{Y}\left[\overline{p p r \mid s}, \underline{t|u| v]}\left(\bar{d}_{\mathrm{R} p}^{a} d_{\mathrm{L} t a}\right)\left(\bar{d}_{\mathrm{R}}^{b} d_{\mathrm{L} u b}\right)\left(\bar{d}_{\mathrm{R}}^{c}{ }_{s} d_{\mathrm{L} v c}\right)\right. \\
& \mathcal{Y}\left[\overline{p p r \mid s}, \frac{t \mid u}{v}\right]\left(d_{\mathrm{L} u b} C d_{\mathrm{L} v a}\right)\left(\bar{d}_{\mathrm{R}}^{c} d_{\mathrm{L} t c}\right)\left(\bar{d}_{\mathrm{R} p}^{a} C \bar{d}_{\mathrm{R}}^{b}\right)
\end{aligned}
$$

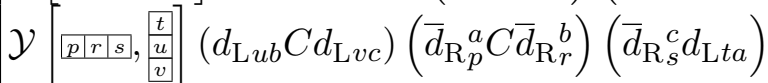

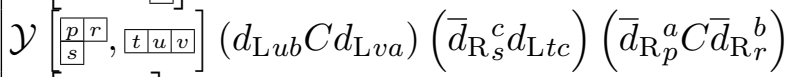

$$
\begin{aligned}
& \mathcal{Y}\left[\frac{p r r}{s}, \frac{t}{\underline{v}}\right]\left(d_{\mathrm{L} u b} C d_{\mathrm{L} v c}\right)\left(\bar{d}_{\mathrm{R} p}^{a} C \bar{d}_{\mathrm{R}}{ }_{r}^{b}\right)\left(\bar{d}_{\mathrm{R}_{s}^{c}}^{c} d_{\mathrm{L} t a}\right)
\end{aligned}
$$

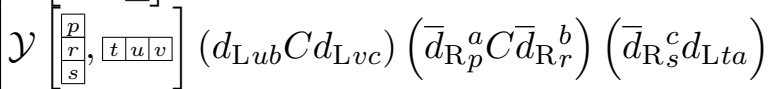

$$
\begin{aligned}
& \mathcal{Y}\left[\frac{p}{\frac{r}{s},}, \frac{t u}{v}\right]\left(d_{\mathrm{L} u b} C d_{\mathrm{L} v c}\right)\left(\bar{d}_{\mathrm{R} p}^{a} C \bar{d}_{\mathrm{R} r}^{b}\right)\left(\bar{d}_{\mathrm{R}}^{c}{ }_{s} d_{\mathrm{L} t a}\right) \\
& \mathcal{Y}\left[\begin{array}{c}
p \\
r
\end{array}, \frac{s}{t}\right] .\left(\bar{e}_{\mathrm{R} u} e_{\mathrm{L} v}\right)\left(d_{\mathrm{L} s a} C d_{\mathrm{L} t b}\right)\left(\bar{d}_{\mathrm{R}_{p}^{a}} C \bar{d}_{\mathrm{R}}^{b}\right) \\
& \mathcal{Y}\left[\frac{p}{r}, \frac{s}{t}\right],\left(\bar{e}_{\mathrm{R} u} e_{\mathrm{L} v}\right)\left(\bar{d}_{\mathrm{R} p}^{a} d_{\mathrm{L} s a}\right)\left(\bar{d}_{\mathrm{R} r}^{b} d_{\mathrm{L} t b}\right) \\
& \mathcal{Y}\left[\frac{p}{r}, \frac{s}{t}\right]\left(d_{\mathrm{L} t b} C e_{\mathrm{L} v}\right)\left(\bar{d}_{\mathrm{R} p}^{a} d_{\mathrm{L} s a}\right)\left(\bar{d}_{\mathrm{R} r}^{b} C \bar{e}_{\mathrm{R} u}\right) \\
& \mathcal{Y}[\overline{p p r}, s s t]\left(\bar{e}_{\mathrm{R} u} e_{\mathrm{L} v}\right)\left(d_{\mathrm{L} s a} C d_{\mathrm{L} t b}\right)\left(\bar{d}_{\mathrm{R}_{p}^{a}} C \bar{d}_{\mathrm{R} r}^{b}\right) \\
& \mathcal{O}_{\bar{d}_{\mathrm{R}}^{2} d_{\mathrm{L}}^{2} \bar{e}_{\mathrm{R}} e_{\mathrm{L}}}^{(10,0)}(0) \mathcal{Y}[\overline{p p r}, s \mid t]\left(\bar{e}_{\mathrm{R} u} e_{\mathrm{L} v}\right)\left(\bar{d}_{\mathrm{R} p}^{a} d_{\mathrm{L} s a}\right)\left(\bar{d}_{\mathrm{R} r}^{b} d_{\mathrm{L} t b}\right) \\
& \mathcal{Y}[\underline{p \mid r}, \underline{s \mid t]}]\left(d_{\mathrm{L} t b} C e_{\mathrm{L} v}\right)\left(\bar{d}_{\mathrm{R} p}^{a} d_{\mathrm{L} s a}\right)\left(\bar{d}_{\mathrm{R}}{ }_{r}^{b} C \bar{e}_{\mathrm{R} u}\right) \\
& \left.\mathcal{Y}\left[\frac{p}{r}, s \mid t\right]\right]\left(\bar{e}_{\mathrm{R} u} d_{\mathrm{L} s a}\right)\left(d_{\mathrm{L} t b} C e_{\mathrm{L} v}\right)\left(\bar{d}_{\mathrm{R} p}^{a} C \bar{d}_{\mathrm{R}}{ }_{r}^{b}\right) \\
& \mathcal{Y}\left[\frac{p}{r}, s \mid t\right]\left(d_{\mathrm{L} t b} C e_{\mathrm{L} v}\right)\left(\bar{d}_{\mathrm{R} p}^{a} d_{\mathrm{L} s a}\right)\left(\bar{d}_{\mathrm{R}}^{b} C \bar{e}_{\mathrm{R} u}\right) \\
& \mathcal{Y}\left[\overline{p p r}, \frac{s}{t}\right]\left(\bar{e}_{\mathrm{R} u} d_{\mathrm{L} s a}\right)\left(d_{\mathrm{L} t b} C e_{\mathrm{L} v}\right)\left(\bar{d}_{\mathrm{R} p}^{a} C \bar{d}_{\mathrm{R} r}^{b}\right) \\
& \mathcal{Y}\left[p, r, \frac{s}{t}\right]\left(d_{\mathrm{L} t b} C e_{\mathrm{L} v}\right)\left(\bar{d}_{\mathrm{R} p}^{a} d_{\mathrm{L} s a}\right)\left(\bar{d}_{\mathrm{R} r}^{b} C \bar{e}_{\mathrm{R} u}\right)
\end{aligned}
$$

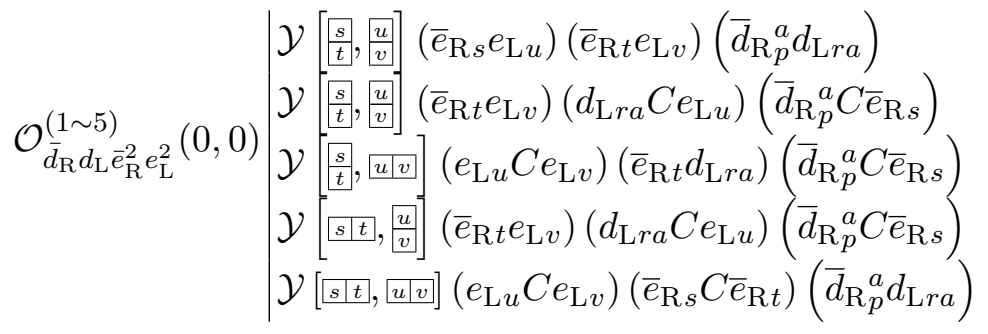

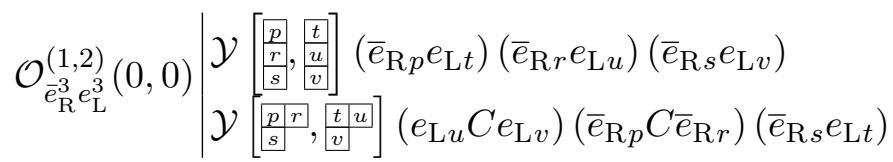


$(\Delta B, \Delta L)=(0, \pm 2)$

$$
\begin{aligned}
& \mathcal{Y}\left[\frac{p}{r}, \frac{s}{t}, \frac{u}{v}\right]\left(u_{\mathrm{L} t b} C \nu_{\mathrm{L} v}\right)\left(\bar{u}_{\mathrm{R} p}^{a} u_{\mathrm{L} s a}\right)\left(\bar{u}_{\mathrm{R} r}^{b} \nu_{\mathrm{L} u}\right) \\
& \mathcal{Y}\left[\frac{p \mid r}{[-s \mid t,}, \underline{u} v\right]\left(u_{\mathrm{L} t b} C \nu_{\mathrm{L} v}\right)\left(\bar{u}_{\mathrm{R}}{ }^{a} u_{\mathrm{L} s a}\right)\left(\bar{u}_{\mathrm{R}}{ }_{r}^{b} \nu_{\mathrm{L} u}\right) \\
& \mathcal{Y}\left[\frac{p}{r}, \frac{s}{t}, u \mid v\right] .\left(\nu_{\mathrm{L} u} C \nu_{\mathrm{L} v}\right)\left(u_{\mathrm{L} s a} C u_{\mathrm{L} t b}\right)\left(\bar{u}_{\mathrm{R} p}^{a} C \bar{u}_{\mathrm{R}}^{b}\right)
\end{aligned}
$$

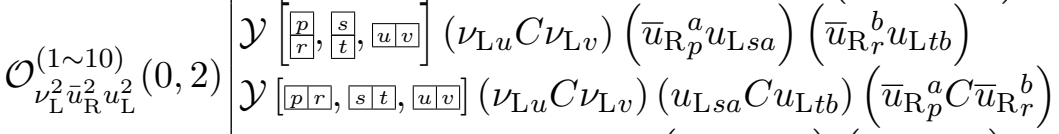

$$
\begin{aligned}
& \mathcal{Y}[\overline{p p r}, \overline{s \mid t},, u v]]\left(\nu_{\mathrm{L} u} C \nu_{\mathrm{L} v}\right)\left(\bar{u}_{\mathrm{R}}{ }_{p}^{a} u_{\mathrm{L} s a}\right)\left(\bar{u}_{\mathrm{R}}^{b} u_{\mathrm{L} t b}\right) \\
& \mathcal{Y}\left[\frac{p}{r}, s \mid t, \frac{u}{v}\right]\left(u_{\mathrm{L} s a} C \nu_{\mathrm{L} u}\right)\left(u_{\mathrm{L} t b} C \nu_{\mathrm{L} v}\right)\left(\bar{u}_{\mathrm{R} p}^{a} C \bar{u}_{\mathrm{R}}{ }_{r}^{b}\right) \\
& \mathcal{Y}\left[\frac{p}{r}, s \mid t, \frac{u}{v}\right]\left(u_{\mathrm{L} t b} C \nu_{\mathrm{L} v}\right)\left(\bar{u}_{\mathrm{R} p}^{a} u_{\mathrm{L} s a}\right)\left(\bar{u}_{\mathrm{R} r}^{b} \nu_{\mathrm{L} u}\right) \\
& \mathcal{Y}\left[\frac{p p r}{,}, \frac{s}{t}, \frac{u}{v}\right]\left(u_{\mathrm{L} s a} C \nu_{\mathrm{L} u}\right)\left(u_{\mathrm{L} t b} C \nu_{\mathrm{L} v}\right)\left(\bar{u}_{\mathrm{R} p}^{a} C \bar{u}_{\mathrm{R} r}{ }^{b}\right)
\end{aligned}
$$

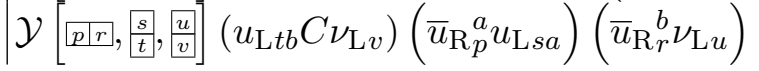

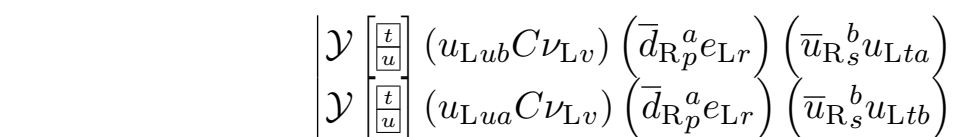

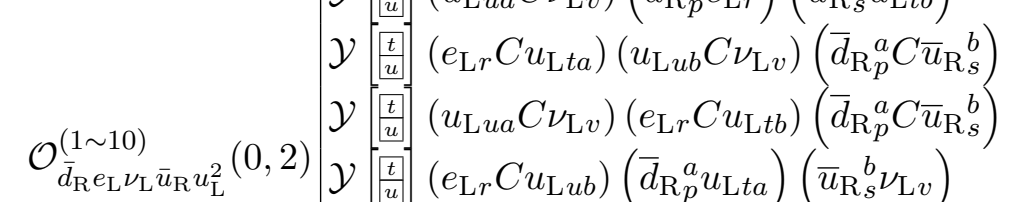

$$
\begin{aligned}
& \mathcal{Y}\left[\overline{t] u]}\left(u_{\mathrm{L} u b} C \nu_{\mathrm{L} v}\right)\left(\bar{d}_{\mathrm{R} p}^{a} e_{\mathrm{L} r}\right)\left(\bar{u}_{\mathrm{R}}^{b} u_{\mathrm{L} t a}\right)\right. \\
& \mathcal{Y}[[t] u]\left(u_{\mathrm{L} u a} C \nu_{\mathrm{L} v}\right)\left(\bar{d}_{\mathrm{R} p}^{a} e_{\mathrm{L} r}\right)\left(\bar{u}_{\mathrm{R}}^{b} u_{\mathrm{L} t b}\right) \\
& \mathcal{Y}[\overline{t l u}]\left(e_{\mathrm{L} r} C u_{\mathrm{L} t a}\right)\left(u_{\mathrm{L} u b} C \nu_{\mathrm{L} v}\right)\left(\bar{d}_{\mathrm{R} p}^{a} C \bar{u}_{\mathrm{R} s}^{b}\right) \\
& \mathcal{Y}\left[\left[_{t \mid u]}\left(u_{\mathrm{L} u a} C \nu_{\mathrm{L} v}\right)\left(e_{\mathrm{L} r} C u_{\mathrm{L} t b}\right)\left(\bar{d}_{\mathrm{R}_{p}^{a} C \bar{u}_{\mathrm{R}}^{b}}\right)\right.\right. \\
& \mathcal{Y}\left[\underline{t \mid u]}\left(e_{\mathrm{L} r} C u_{\mathrm{L} u b}\right)\left(\bar{d}_{\mathrm{R} p}^{a} u_{\mathrm{L} t a}\right)\left(\bar{u}_{\mathrm{R}}^{b} \nu_{\mathrm{L} v}\right)\right. \\
& \mathcal{Y}\left[\frac{p}{r}, \frac{s}{t}, \frac{u}{v}\right]\left(e_{\mathrm{L} t} C u_{\mathrm{L} v b}\right)\left(\bar{d}_{\mathrm{R}}^{a} e_{\mathrm{L} s}\right)\left(\bar{d}_{\mathrm{R}}{ }_{r}^{b} u_{\mathrm{L} u a}\right) \\
& \mathcal{Y}\left[\frac{p p r}{,}, \frac{s}{t},,_{u \mid v}\right]\left(e_{\mathrm{L} t} C u_{\mathrm{L} v b}\right)\left(\bar{d}_{\mathrm{R}}{ }_{p}^{a} e_{\mathrm{L} s}\right)\left(\bar{d}_{\mathrm{R} r}^{b} u_{\mathrm{L} u a}\right) \\
& \mathcal{Y}\left[\frac{p}{r}, \frac{s}{t}, u[v],\left(e_{\mathrm{L} s} C u_{\mathrm{L} u a}\right)\left(e_{\mathrm{L} t} C u_{\mathrm{L} v b}\right)\left(\bar{d}_{\mathrm{R}}^{a} C \bar{d}_{\mathrm{R}}^{b}\right)\right.
\end{aligned}
$$

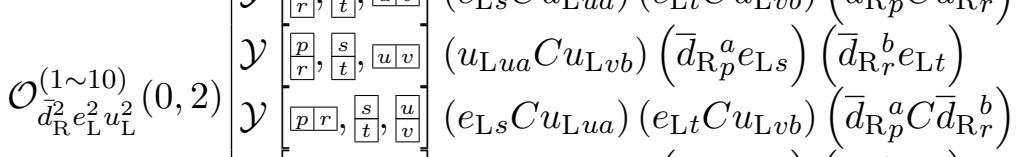

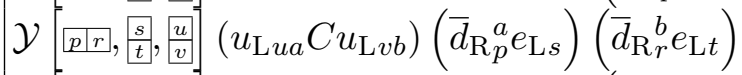

$$
\begin{aligned}
& \mathcal{Y}\left[\frac{p}{r}, s \mid t, \frac{u}{v}\right]\left(e_{\mathrm{L} s} C e_{\mathrm{L} t}\right)\left(u_{\mathrm{L} u a} C u_{\mathrm{L} v b}\right)\left(\bar{d}_{\mathrm{R} p}^{a} C \bar{d}_{\mathrm{R}}^{b}\right) \\
& \mathcal{Y}\left[\frac{p}{r}, s \mid t, \frac{u}{v}\right]\left(e_{\mathrm{L} t} C u_{\mathrm{L} v b}\right)\left(\bar{d}_{\mathrm{R} p}^{a} e_{\mathrm{L} s}\right)\left(\bar{d}_{\mathrm{R}}{ }_{r}^{b} u_{\mathrm{L} u a}\right) \\
& \mathcal{Y}[\underline{p \mid r}, s \mid t, u, u]]\left(e_{\mathrm{L} s} C e_{\mathrm{L} t}\right)\left(u_{\mathrm{L} u a} C u_{\mathrm{L} v b}\right)\left(\bar{d}_{\mathrm{R} p}^{a} C \bar{d}_{\mathrm{R}}^{b}\right) \\
& \mathcal{Y}\left[\overline{p \mid r}, \underline{s \mid t}, \overline{u \mid v]}\left(e_{\mathrm{L} t} C u_{\mathrm{L} v b}\right)\left(\bar{d}_{\mathrm{R} p}^{a} e_{\mathrm{L} s}\right)\left(\bar{d}_{\mathrm{R} r}^{b} u_{\mathrm{L} u a}\right)\right.
\end{aligned}
$$




$$
\begin{aligned}
& \mathcal{Y}\left[\frac{u}{v}\right]\left(u_{\mathrm{L} t a} C \nu_{\mathrm{L} v}\right)\left(\bar{u}_{\mathrm{R}}^{b} \nu_{\mathrm{L} u}\right)\left(\bar{d}_{\mathrm{R} p}^{a} d_{\mathrm{L} r b}\right) \\
& \mathcal{Y}\left[\begin{array}{ll}
a \\
v
\end{array}\left(u_{\mathrm{L} t c} C \nu_{\mathrm{L} v}\right)\left(\bar{d}_{\mathrm{R} p}^{a} d_{\mathrm{L} r a}\right)\left(\bar{u}_{\mathrm{R}}^{c} \nu_{\mathrm{L} u}\right)\right. \\
& \mathcal{Y}\left[\begin{array}{ll}
u \\
v
\end{array}\right]\left(u_{\mathrm{L} t a} C \nu_{\mathrm{L} v}\right)\left(d_{\mathrm{L} r b} C \nu_{\mathrm{L} u}\right)\left(\bar{d}_{\mathrm{R} p}^{a} C \bar{u}_{\mathrm{R} s}^{b}\right)
\end{aligned}
$$

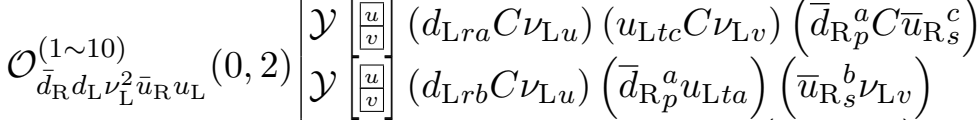

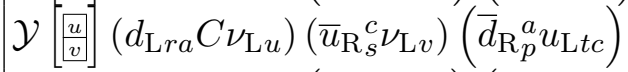

$$
\begin{aligned}
& \mathcal{Y}[\overline{u v v}]\left(\nu_{\mathrm{L} u} C \nu_{\mathrm{L} v}\right)\left(\bar{d}_{\mathrm{R} p}^{a} d_{\mathrm{L} r b}\right)\left(\bar{u}_{\mathrm{R} s}^{b} u_{\mathrm{L} t a}\right) \\
& \mathcal{Y}[\overline{u v v}]\left(\nu_{\mathrm{L} u} C \nu_{\mathrm{L} v}\right)\left(\bar{d}_{\mathrm{R} p}^{a} d_{\mathrm{L} r a}\right)\left(\bar{u}_{\mathrm{R}}^{c}{ }_{s} u_{\mathrm{L} t c}\right) \\
& \mathcal{Y}[u v v]\left(\nu_{\mathrm{L} u} C \nu_{\mathrm{L} v}\right)\left(d_{\mathrm{L} r b} C u_{\mathrm{L} t a}\right)\left(\bar{d}_{\mathrm{R} p}^{a} C \bar{u}_{\mathrm{R}}^{b}\right) \\
& \mathcal{Y}[\underline{u v}]\left(\nu_{\mathrm{L} u} C \nu_{\mathrm{L} v}\right)\left(d_{\mathrm{L} r a} C u_{\mathrm{L} t c}\right)\left(\bar{d}_{\mathrm{R} p}^{a} C \bar{u}_{\mathrm{R} s}^{c}\right)
\end{aligned}
$$

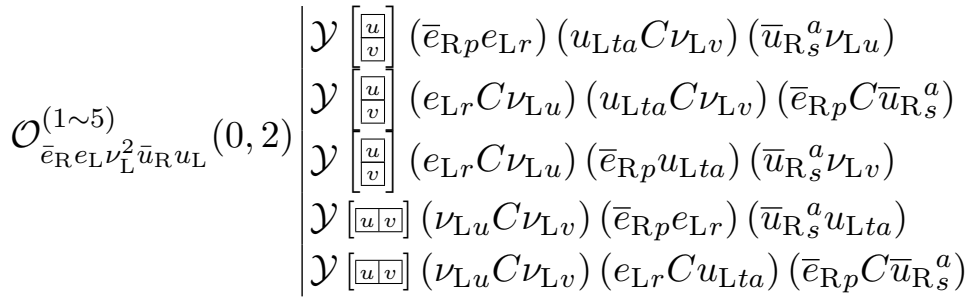

$$
\begin{aligned}
& \mathcal{Y}\left[\frac{p}{r}\right]\left(d_{\mathrm{L} s a} C e_{\mathrm{L} t}\right)\left(u_{\mathrm{L} u b} C \nu_{\mathrm{L} v}\right)\left(\bar{d}_{\mathrm{R}}^{a} C \bar{d}_{\mathrm{R}}^{b}\right)
\end{aligned}
$$

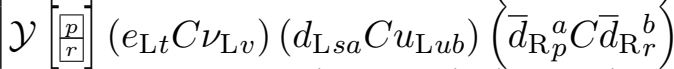

$$
\begin{aligned}
& \mathcal{Y}\left[\begin{array}{ll}
r \\
r
\end{array}\left(u_{\mathrm{L} u b} C \nu_{\mathrm{L} v}\right)\left(\bar{d}_{\mathrm{R} p}^{a} d_{\mathrm{L} s a}\right)\left(\bar{d}_{\mathrm{R} r}^{b} e_{\mathrm{L} t}\right)\right. \\
& \mathcal{Y}\left[\frac{p}{r}\right]\left(e_{\mathrm{L} t} C \nu_{\mathrm{L} v}\right)\left(\bar{d}_{\mathrm{R} p}^{a} d_{\mathrm{L} s a}\right)\left(\bar{d}_{\mathrm{R} r}^{b} u_{\mathrm{L} u b}\right)
\end{aligned}
$$

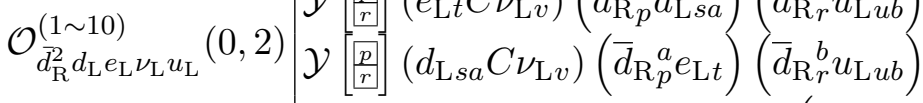

$$
\begin{aligned}
& \mathcal{Y}[\underline{p \mid r}]\left(d_{\mathrm{L} s a} C e_{\mathrm{L} t}\right)\left(u_{\mathrm{L} u b} C \nu_{\mathrm{L} v}\right)\left(\bar{d}_{\mathrm{R}_{p}^{a}} C \bar{d}_{\mathrm{R}_{r}^{b}}\right) \\
& \mathcal{Y}[\underline{p p r}]\left(e_{\mathrm{L} t} C \nu_{\mathrm{L} v}\right)\left(d_{\mathrm{L} s a} C u_{\mathrm{L} u b}\right)\left(\bar{d}_{\mathrm{R} p}^{a} C \bar{d}_{\mathrm{R} r}^{b}\right) \\
& \mathcal{Y}[\overline{p r r}]\left(u_{\mathrm{L} u b} C \nu_{\mathrm{L} v}\right)\left(\bar{d}_{\mathrm{R} p}^{a} d_{\mathrm{L} s a}\right)\left(\bar{d}_{\mathrm{R} r}^{b} e_{\mathrm{L} t}\right) \\
& \mathcal{Y}[\overline{p p r}]\left(e_{\mathrm{L} t} C \nu_{\mathrm{L} v}\right)\left(\bar{d}_{\mathrm{R} p}^{a} d_{\mathrm{L} s a}\right)\left(\bar{d}_{\mathrm{R} r}^{b} u_{\mathrm{L} u b}\right) \\
& \mathcal{Y}[\overline{p p r}]\left(d_{\mathrm{L} s a} C \nu_{\mathrm{L} v}\right)\left(\bar{d}_{\mathrm{R} p}^{a} e_{\mathrm{L} t}\right)\left(\bar{d}_{\mathrm{R}}{ }_{r}^{b} u_{\mathrm{L} u b}\right)
\end{aligned}
$$

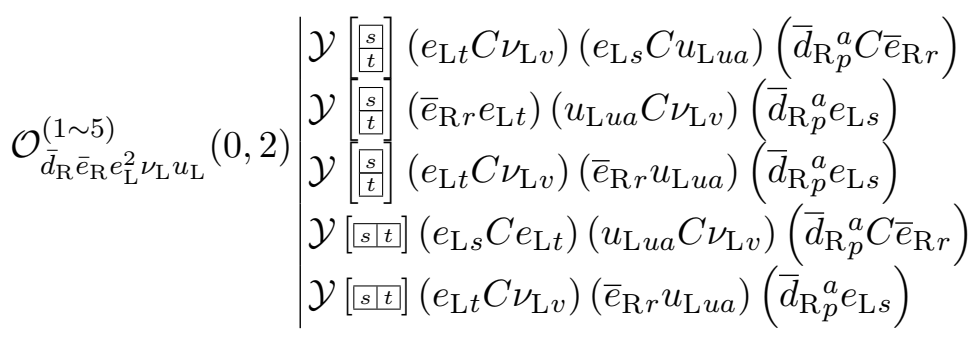




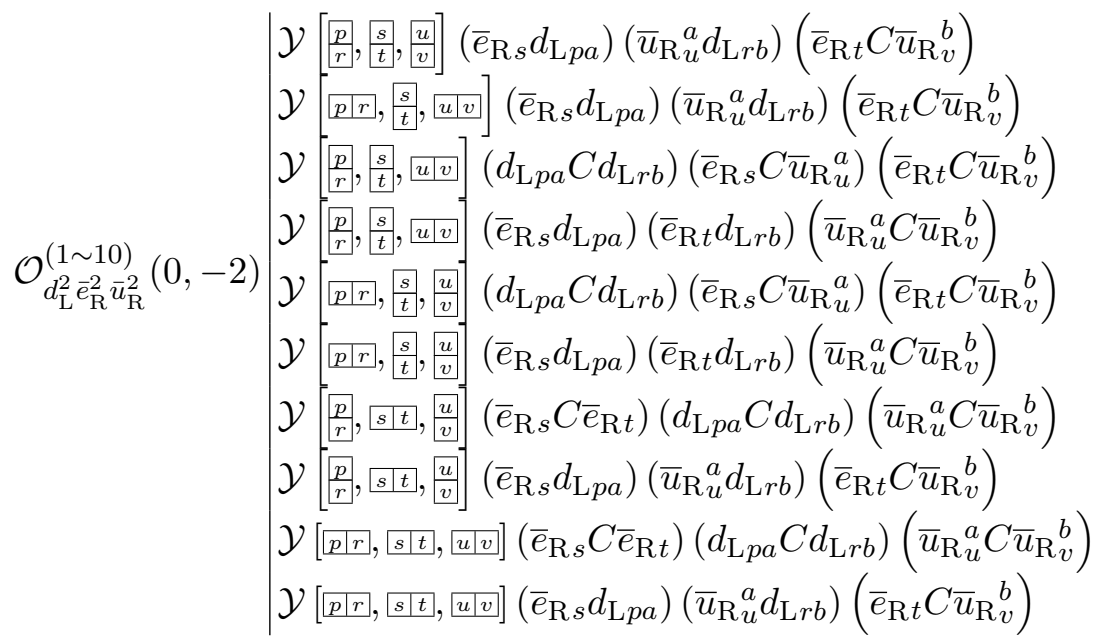

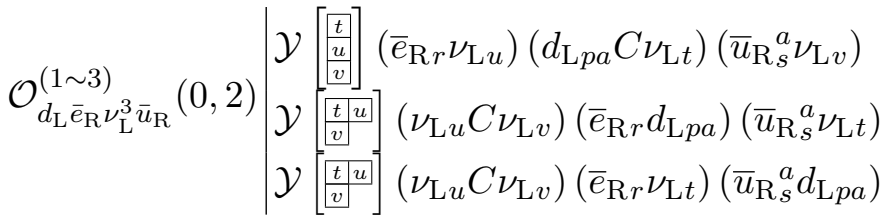

$$
\begin{aligned}
& \mathcal{Y}\left[\begin{array}{l}
\frac{p}{r}, \frac{s}{t}, \frac{u}{v} \\
]
\end{array}\right]\left(d_{\mathrm{L} t b} C \nu_{\mathrm{L} v}\right)\left(\bar{d}_{\mathrm{R}}^{a} d_{\mathrm{L} s a}\right)\left(\bar{d}_{\mathrm{R}}^{b} \nu_{\mathrm{L} u}\right) \\
& \mathcal{Y}\left[p|r, s| t, \frac{u}{v]}\right]\left(d_{\mathrm{L} t b} C \nu_{\mathrm{L} v}\right)\left(\bar{d}_{\mathrm{R} p}^{a} d_{\mathrm{L} s a}\right)\left(\bar{d}_{\mathrm{R}}{ }_{r}^{b} \nu_{\mathrm{L} u}\right) \\
& \mathcal{Y}\left[\frac{p}{r}, \frac{s}{t}, u v v\right]\left(\nu_{\mathrm{L} u} C \nu_{\mathrm{L} v}\right)\left(d_{\mathrm{L} s a} C d_{\mathrm{L} t b}\right)\left(\bar{d}_{\mathrm{R} p}^{a} C \bar{d}_{\mathrm{R}}^{b}\right) \\
& \mathcal{Y}\left[\frac{p}{r}, \frac{s}{t}, \underline{u \mid v}\right]\left(\nu_{\mathrm{L} u} C \nu_{\mathrm{L} v}\right)\left(\bar{d}_{\mathrm{R} p}^{a} d_{\mathrm{L} s a}\right)\left(\bar{d}_{\mathrm{R} r}^{b} d_{\mathrm{L} t b}\right) \\
& \mathcal{O}_{\bar{d}_{\mathrm{R}}^{2} d_{\mathrm{L}}^{2} \nu_{\mathrm{L}}^{2}}^{(10)}(0,2) \mathcal{Y}\left[[p|r,, s| t, u \mid v]\left(\nu_{\mathrm{L} u} C \nu_{\mathrm{L} v}\right)\left(d_{\mathrm{L} s a} C d_{\mathrm{L} t b}\right)\left(\bar{d}_{\mathrm{R} p}^{a} C \bar{d}_{\mathrm{R}}{ }_{\mathrm{R}}\right)\right. \\
& \mathcal{Y}\left[\left[p|r,, s| t,[u \mid v]\left(\nu_{\mathrm{L} u} C \nu_{\mathrm{L} v}\right)\left(\bar{d}_{\mathrm{R} p}^{a} d_{\mathrm{L} s a}\right)\left(\bar{d}_{\mathrm{R} r}^{b} d_{\mathrm{L} t b}\right)\right.\right. \\
& \mathcal{Y}\left[\frac{p}{r}, s \mid t,, \frac{u}{v}\right]\left(d_{\mathrm{L} s a} C \nu_{\mathrm{L} u}\right)\left(d_{\mathrm{L} t b} C \nu_{\mathrm{L} v}\right)\left(\bar{d}_{\mathrm{R}}^{a} C \bar{d}_{\mathrm{R}}^{b}\right) \\
& \mathcal{Y}\left[\frac{p}{r}, s \mid t, \frac{u}{v}\right]\left(d_{\mathrm{L} t b} C \nu_{\mathrm{L} v}\right)\left(\bar{d}_{\mathrm{R} p}^{a} d_{\mathrm{L} s a}\right)\left(\bar{d}_{\mathrm{R}}{ }_{r}^{b} \nu_{\mathrm{L} u}\right) \\
& \mathcal{Y}\left[\frac{p p r}{[-, ~}, \frac{s}{t}, \frac{w}{v}\right]\left(d_{\mathrm{L} s a} C \nu_{\mathrm{L} u}\right)\left(d_{\mathrm{L} t b} C \nu_{\mathrm{L} v}\right)\left(\bar{d}_{\mathrm{R}_{p}^{a}}^{a} C \bar{d}_{\mathrm{R}_{r}^{b}}^{b}\right) \\
& \mathcal{Y}\left[\underline{\underline{p \mid r},}, \frac{s}{t}, \underline{u}, \frac{u}{v}\right]\left(d_{\mathrm{L} t b} C \nu_{\mathrm{L} v}\right)\left(\bar{d}_{\mathrm{R} p}^{a} d_{\mathrm{L} s a}\right)\left(\bar{d}_{\mathrm{R}}{ }_{r}^{b} \nu_{\mathrm{L} u}\right) \\
& \mathcal{Y}\left[\frac{u}{v}\right]\left(\bar{e}_{\mathrm{R} s} \nu_{\mathrm{L} u}\right)\left(e_{\mathrm{L} t} C \nu_{\mathrm{L} v}\right)\left(\bar{d}_{\mathrm{R} p}^{a} d_{\mathrm{L} r a}\right)
\end{aligned}
$$

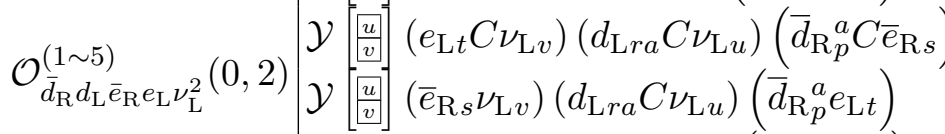

$$
\begin{aligned}
& \mathcal{Y}\left[u[v]\left(\nu_{\mathrm{L} u} C \nu_{\mathrm{L} v}\right)\left(\bar{e}_{\mathrm{R} s} e_{\mathrm{L} t}\right)\left(\bar{d}_{\mathrm{R} p}^{a} d_{\mathrm{L} r a}\right)\right. \\
& \mathcal{Y}\left[\underline{u v v]}\left(\nu_{\mathrm{L} u} C \nu_{\mathrm{L} v}\right)\left(d_{\mathrm{L} r a} C e_{\mathrm{L} t}\right)\left(\bar{d}_{\mathrm{R} p}^{a} C \bar{e}_{\mathrm{R} s}\right)\right. \\
& \mathcal{Y}\left[\frac{p}{r}, \frac{s}{t},, \frac{u}{v}\right]\left(\bar{e}_{\mathrm{R} p} e_{\mathrm{L} s}\right)\left(\bar{e}_{\mathrm{R} r} \nu_{\mathrm{L} u}\right)\left(e_{\mathrm{L} t} C \nu_{\mathrm{L} v}\right)
\end{aligned}
$$

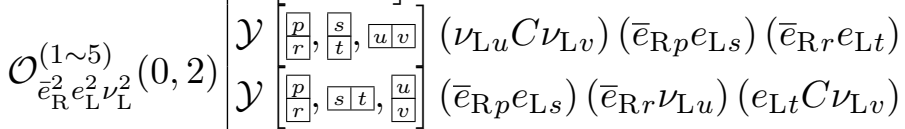

$$
\begin{aligned}
& \mathcal{Y}\left[\overline{p r r}, \frac{s}{t},, \frac{u}{v}\right]\left(\bar{e}_{\mathrm{R} p} C \bar{e}_{\mathrm{R} r}\right)\left(e_{\mathrm{L} s} C \nu_{\mathrm{L} u}\right)\left(e_{\mathrm{L} t} C \nu_{\mathrm{L} v}\right) \\
& \mathcal{Y}[[p r r, s \mid t,, u v]]\left(e_{\mathrm{L} s} C e_{\mathrm{L} t}\right)\left(\nu_{\mathrm{L} u} C \nu_{\mathrm{L} v}\right)\left(\bar{e}_{\mathrm{R} p} C \bar{e}_{\mathrm{R} r}\right)
\end{aligned}
$$


$(\Delta B, \Delta L)=(0, \pm 4)$

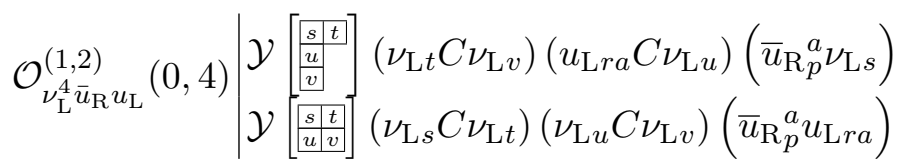

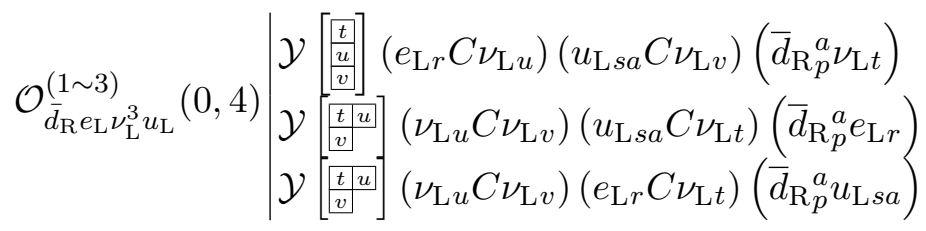

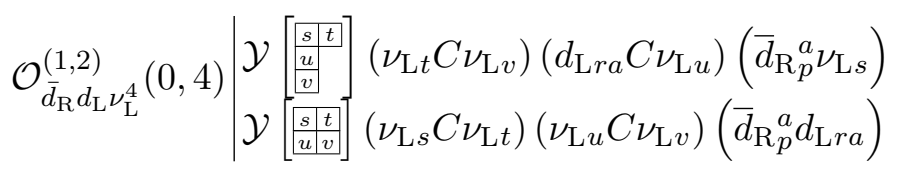

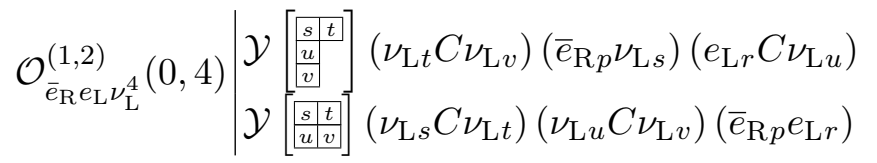

$(\Delta B, \Delta L)=(0, \pm 6)$

$$
\mathcal{O}_{\nu_{\mathrm{L}}^{6}}(0,6) \mid \mathcal{Y}\left[\begin{array}{l}
\frac{p r}{s t} \\
\underline{s} t \\
u v
\end{array}\right]\left(\nu_{\mathrm{L} p} C \nu_{\mathrm{L} r}\right)\left(\nu_{\mathrm{L} s} C \nu_{\mathrm{L} t}\right)\left(\nu_{\mathrm{L} u} C \nu_{\mathrm{L} v}\right)
$$

$(\Delta B, \Delta L)=( \pm 1, \pm 1)$

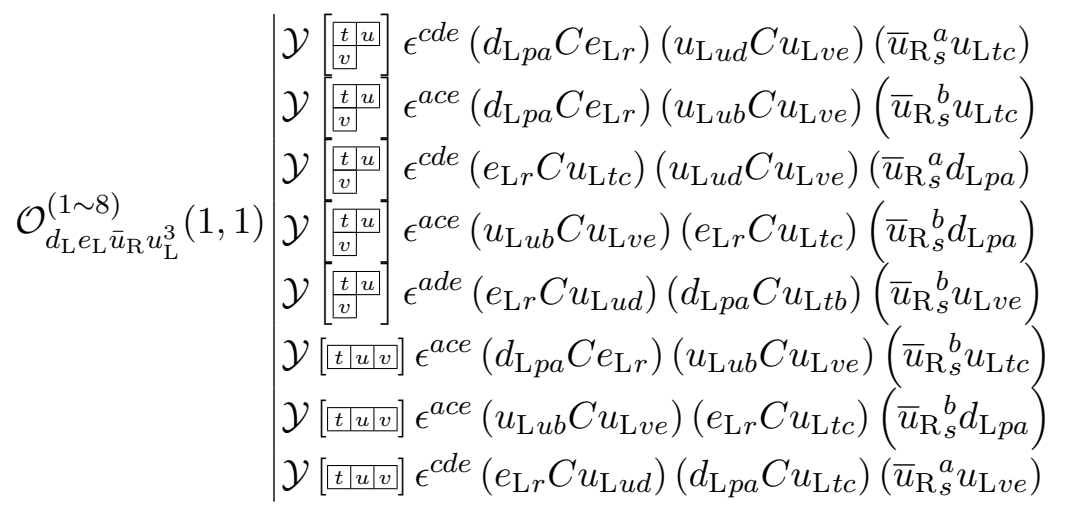




$$
\begin{aligned}
& \mathcal{Y}\left[\frac{p}{r}, t \mid w\right] \epsilon^{b d e}\left(d_{\mathrm{L} p a} C d_{\mathrm{L} r b}\right)\left(u_{\mathrm{L} u e} C \nu_{\mathrm{L} v}\right)\left(\bar{u}_{\mathrm{R}}{ }_{s}^{a} u_{\mathrm{L} t d}\right) \\
& \mathcal{Y}\left[\frac{p}{r}, t \mid w\right] \epsilon^{a b e}\left(d_{\mathrm{L} p a} C d_{\mathrm{L} r b}\right)\left(u_{\mathrm{L} u e} C \nu_{\mathrm{L} v}\right)\left(\bar{u}_{\mathrm{R} s}^{c} u_{\mathrm{L} t c}\right) \\
& \left.\mathcal{Y}\left[\frac{p}{r}, t\right] \mid u\right] \epsilon^{b d e}\left(u_{\mathrm{L} u e} C \nu_{\mathrm{L} v}\right)\left(\bar{u}_{\mathrm{R}}^{a} d_{\mathrm{L} p a}\right)\left(d_{\mathrm{L} r b} C u_{\mathrm{L} t d}\right) \\
& \mathcal{Y}\left[\frac{p}{r}, t|t| u \mid \epsilon^{b d e}\left(\bar{u}_{\mathrm{R} s}^{a} \nu_{\mathrm{L} v}\right)\left(d_{\mathrm{L} p a} C u_{\mathrm{L} t d}\right)\left(d_{\mathrm{L} r b} C u_{\mathrm{L} u e}\right)\right. \\
& \mathcal{Y}\left[p \mid r, \frac{t}{u}\right] \epsilon^{b d e}\left(d_{\mathrm{L} p a} C d_{\mathrm{L} r b}\right)\left(u_{\mathrm{L} u e} C \nu_{\mathrm{L} v}\right)\left(\bar{u}_{\mathrm{R}}{ }_{s}^{a} u_{\mathrm{L} t d}\right) \\
& \mathcal{Y}\left[\overline{p p r}, \frac{t}{u}\right] \epsilon^{b d e}\left(u_{\mathrm{L} u e} C \nu_{\mathrm{L} v}\right)\left(\bar{u}_{\mathrm{R}}^{a} d_{\mathrm{L} p a}\right)\left(d_{\mathrm{L} r b} C u_{\mathrm{L} t d}\right) \\
& \mathcal{O}_{d_{\mathrm{L}}^{2} \nu_{\mathrm{L}} \bar{u}_{\mathrm{R}} u_{\mathrm{L}}^{2}}^{(1 \sim 15)} \mathcal{Y}\left[\overline{p p r}, \frac{\bar{t}}{u}\right] \epsilon^{a b e}\left(u_{\mathrm{L} u e} C \nu_{\mathrm{L} v}\right)\left(d_{\mathrm{L} r b} C u_{\mathrm{L} t c}\right)\left(\bar{u}_{\mathrm{R}}{ }^{c} d_{\mathrm{L} p a}\right) \\
& \mathcal{Y}\left[\overline{p p r}, \frac{t}{u}\right] \epsilon^{a b e}\left(\bar{u}_{\mathrm{R}_{s}^{c} \nu_{\mathrm{L} v}}\right)\left(d_{\mathrm{L} p a} C u_{\mathrm{L} t c}\right)\left(d_{\mathrm{L} r b} C u_{\mathrm{L} u e}\right) \\
& \mathcal{Y}[\overline{p p r}, t] u] \epsilon^{b d e}\left(d_{\mathrm{L} p a} C d_{\mathrm{L} r b}\right)\left(u_{\mathrm{L} u e} C \nu_{\mathrm{L} v}\right)\left(\bar{u}_{\mathrm{R}}{ }_{s}^{a} u_{\mathrm{L} t d}\right) \\
& \mathcal{Y}\left[{ }_{[p \mid r},{ }_{t \mid u]}\right] \epsilon^{b d e}\left(u_{\mathrm{L} u e} C \nu_{\mathrm{L} v}\right)\left(\bar{u}_{\mathrm{R} s}^{a} d_{\mathrm{L} p a}\right)\left(d_{\mathrm{L} r b} C u_{\mathrm{L} t d}\right) \\
& \mathcal{Y}[\overline{p p r}, \overline{t \mid u]}] \epsilon^{a b e}\left(u_{\mathrm{L} u e} C \nu_{\mathrm{L} v}\right)\left(d_{\mathrm{L} r b} C u_{\mathrm{L} t c}\right)\left(\bar{u}_{\mathrm{R}}^{c} d_{\mathrm{L} p a}\right) \\
& \mathcal{Y}\left[[p|r, t| t \mid u] \epsilon^{b d e}\left(\bar{u}_{\mathrm{R}}{ }_{s}^{a} \nu_{\mathrm{L} v}\right)\left(d_{\mathrm{L} p a} C u_{\mathrm{L} t d}\right)\left(d_{\mathrm{L} r b} C u_{\mathrm{L} u e}\right)\right. \\
& \mathcal{Y}\left[\frac{p}{r}, \frac{t}{u}\right] \epsilon^{b d e}\left(d_{\mathrm{L} p a} C d_{\mathrm{L} r b}\right)\left(u_{\mathrm{L} u e} C \nu_{\mathrm{L} v}\right)\left(\bar{u}_{\mathrm{R}}^{a}{ }_{s} u_{\mathrm{L} t d}\right) \\
& \mathcal{Y}\left[\frac{p}{r}, \frac{t}{u}\right] \epsilon^{a b e}\left(d_{\mathrm{L} p a} C d_{\mathrm{L} r b}\right)\left(u_{\mathrm{L} u e} C \nu_{\mathrm{L} v}\right)\left(\bar{u}_{\mathrm{R}}^{c}{ }_{s}^{c} u_{\mathrm{L} t c}\right) \\
& \left.\mathcal{Y}\left[\frac{p}{r}, \frac{t}{u}\right]\right] \epsilon^{b d e}\left(u_{\mathrm{L} u e} C \nu_{\mathrm{L} v}\right)\left(\bar{u}_{\mathrm{R} s}^{a} d_{\mathrm{L} p a}\right)\left(d_{\mathrm{L} r b} C u_{\mathrm{L} t d}\right) \\
& \mathcal{Y}\left[\frac{r}{s}, \underline{u v}\right] \epsilon^{b c d}\left(u_{\mathrm{L} u d} C u_{\mathrm{L} v a}\right)\left(d_{\mathrm{L} s c} C e_{\mathrm{L} t}\right)\left(\bar{d}_{\mathrm{R}}{ }_{p}^{a} d_{\mathrm{L} r b}\right) \\
& \mathcal{Y}, \frac{r}{s}, u v v \mid \epsilon^{c d e}\left(e_{\mathrm{L} t} C u_{\mathrm{L} v e}\right)\left(\bar{d}_{\mathrm{R}}{ }_{p}^{a} d_{\mathrm{L} r a}\right)\left(d_{\mathrm{L} s c} C u_{\mathrm{L} u d}\right) \\
& \mathcal{Y}\left[\frac{r}{s}, u \mid v\right] \epsilon^{b d e}\left(e_{\mathrm{L} t} C u_{\mathrm{L} v e}\right)\left(d_{\mathrm{L} s a} C u_{\mathrm{L} u d}\right)\left(\bar{d}_{\mathrm{R} p}^{a} d_{\mathrm{L} r b}\right) \\
& \mathcal{Y}\left[\frac{r}{s}, u v\right] \epsilon^{c d e}\left(\bar{d}_{\mathrm{R} p}^{a} e_{\mathrm{L} t}\right)\left(d_{\mathrm{L} r a} C u_{\mathrm{L} u d}\right)\left(d_{\mathrm{L} s c} C u_{\mathrm{L} v e}\right) \\
& \mathcal{Y}\left[\frac{r r s}{\underline{v}}, \frac{u}{v}\right] \epsilon^{c d e}\left(d_{\mathrm{L} s c} C e_{\mathrm{L} t}\right)\left(u_{\mathrm{L} u d} C u_{\mathrm{L} v e}\right)\left(\bar{d}_{\mathrm{R} p}^{a} d_{\mathrm{L} r a}\right) \\
& \mathcal{Y}\left[\overline{r r s}, \frac{u}{v}\right] \epsilon^{b d e}\left(d_{\mathrm{L} s a} C e_{\mathrm{L} t}\right)\left(u_{\mathrm{L} u d} C u_{\mathrm{L} v e}\right)\left(\bar{d}_{\mathrm{R} p}^{a} d_{\mathrm{L} r b}\right)
\end{aligned}
$$

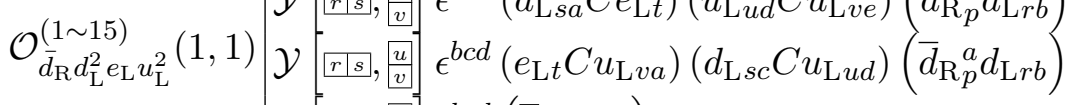

$$
\begin{aligned}
& \mathcal{Y}\left[\overline{r r s}, \frac{u}{v}\right] \epsilon^{b c d}\left(\bar{d}_{\mathrm{R} p}^{a} e_{\mathrm{L} t}\right)\left(d_{\mathrm{L} s c} C u_{\mathrm{L} v a}\right)\left(d_{\mathrm{L} r b} C u_{\mathrm{L} u d}\right) \\
& \mathcal{Y}\left[[r s,, u v v] \epsilon^{b c d}\left(u_{\mathrm{L} u d} C u_{\mathrm{L} v a}\right)\left(d_{\mathrm{L} s c} C e_{\mathrm{L} t}\right)\left(\bar{d}_{\mathrm{R} p}^{a} d_{\mathrm{L} r b}\right)\right. \\
& \mathcal{Y}\left[[r s s, u v v] \epsilon^{c d e}\left(e_{\mathrm{L} t} C u_{\mathrm{L} v e}\right)\left(\bar{d}_{\mathrm{R} p}^{a} d_{\mathrm{L} r a}\right)\left(d_{\mathrm{L} s c} C u_{\mathrm{L} u d}\right)\right. \\
& \mathcal{Y}\left[\left[r \mid s, \underline{u v v]} \epsilon^{b d e}\left(e_{\mathrm{L} t} C u_{\mathrm{L} v e}\right)\left(d_{\mathrm{L} s a} C u_{\mathrm{L} u d}\right)\left(\bar{d}_{\mathrm{R} p}^{a} d_{\mathrm{L} r b}\right)\right.\right. \\
& \mathcal{Y}\left[[r s,, u v v] \epsilon^{c d e}\left(\bar{d}_{\mathrm{R} p}^{a} e_{\mathrm{L} t}\right)\left(d_{\mathrm{L} r a} C u_{\mathrm{L} u d}\right)\left(d_{\mathrm{L} s c} C u_{\mathrm{L} v e}\right)\right. \\
& \mathcal{Y}\left[\frac{r}{s}, \frac{u}{v}\right] \epsilon^{c d e}\left(d_{\mathrm{L} s c} C e_{\mathrm{L} t}\right)\left(u_{\mathrm{L} u d} C u_{\mathrm{L} v e}\right)\left(\bar{d}_{\mathrm{R} p}^{a} d_{\mathrm{L} r a}\right) \\
& \mathcal{Y}\left[\frac{r}{s}, \frac{u}{v}\right] \epsilon^{b d e}\left(d_{\mathrm{L} s a} C e_{\mathrm{L} t}\right)\left(u_{\mathrm{L} u d} C u_{\mathrm{L} v e}\right)\left(\bar{d}_{\mathrm{R} p}^{a} d_{\mathrm{L} r b}\right) \\
& \mathcal{Y}\left[\frac{r}{s}, \frac{u}{v}\right] \epsilon^{b c d}\left(e_{\mathrm{L} t} C u_{\mathrm{L} v a}\right)\left(d_{\mathrm{L} s c} C u_{\mathrm{L} u d}\right)\left(\bar{d}_{\mathrm{R} p}^{a} d_{\mathrm{L} r b}\right)
\end{aligned}
$$

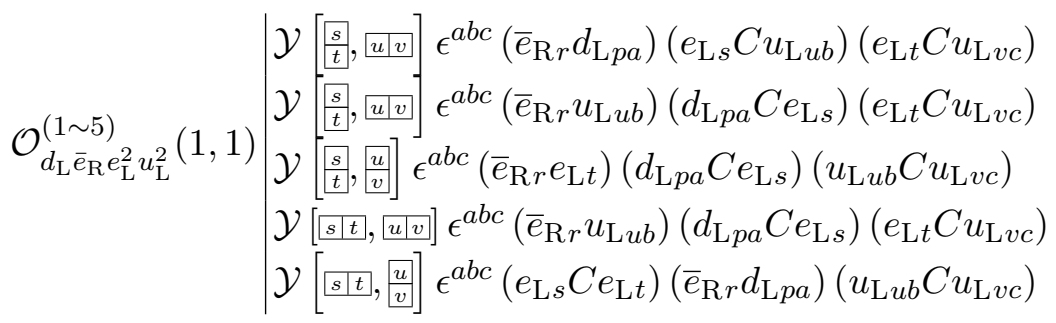




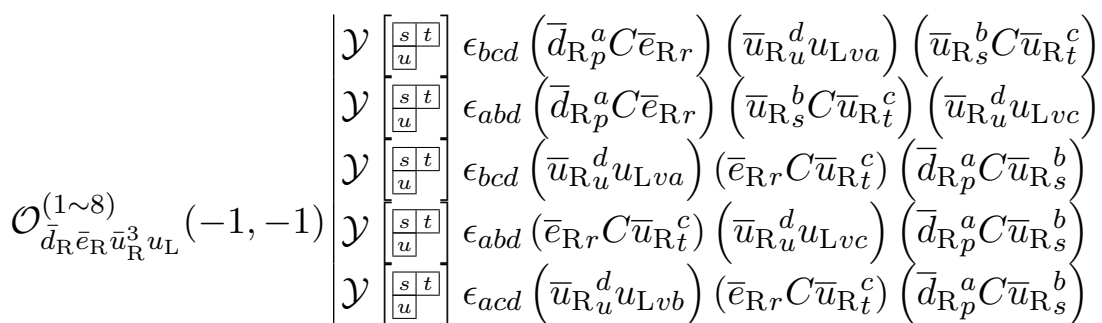

$$
\begin{aligned}
& \mathcal{Y}[s|t| u] \epsilon_{a b d}\left(\bar{d}_{\mathrm{R} p}^{a} C \bar{e}_{\mathrm{R} r}\right)\left(\bar{u}_{\mathrm{R} s}^{b} C \bar{u}_{\mathrm{R} t}^{c}\right)\left(\bar{u}_{\mathrm{R} u}^{d} u_{\mathrm{L} v c}\right) \\
& \mathcal{Y}[\overline{s|t| u]}] \epsilon_{b c d}\left(\bar{u}_{\mathrm{R} u}^{d} u_{\mathrm{L} v a}\right)\left(\bar{e}_{\mathrm{R} r} C \bar{u}_{\mathrm{R} t}^{c}\right)\left(\bar{d}_{\mathrm{R} p}^{a} C \bar{u}_{\mathrm{R} s}^{b}\right) \\
& \mathcal{Y}[\overline{s|t| u]}] \epsilon_{a b d}\left(\bar{e}_{\mathrm{R} r} C \bar{u}_{\mathrm{R} t}^{c}\right)\left(\bar{u}_{\mathrm{R} u}^{d} u_{\mathrm{L} v c}\right)\left(\bar{d}_{\mathrm{R}_{p}^{a}} C \bar{u}_{\mathrm{R}_{s}^{b}}^{b}\right) \\
& \mathcal{Y}\left[\begin{array}{l}
{[r s} \\
t
\end{array}\right] \epsilon^{c d e}\left(d_{\mathrm{L} s c} C d_{\mathrm{L} t d}\right)\left(u_{\mathrm{L} u e} C \nu_{\mathrm{L} v}\right)\left(\bar{d}_{\mathrm{R} p}^{a} d_{\mathrm{L} r a}\right)
\end{aligned}
$$

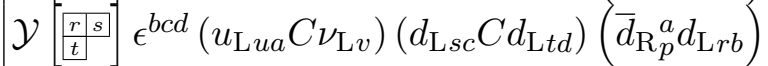

$$
\begin{aligned}
& \mathcal{Y}\left[\begin{array}{cc}
r s s \\
t s
\end{array} \epsilon^{c d e}\left(d_{\mathrm{L} t d} C \nu_{\mathrm{L} v}\right)\left(\bar{d}_{\mathrm{R} p}^{a} d_{\mathrm{L} r a}\right)\left(d_{\mathrm{L} s c} C u_{\mathrm{L} u e}\right)\right. \\
& \mathcal{Y}\left[\frac{r r s}{t-s}\right] \epsilon^{b d e}\left(d_{\mathrm{L} t d} C \nu_{\mathrm{L} v}\right)\left(d_{\mathrm{L} s a} C u_{\mathrm{L} u e}\right)\left(\bar{d}_{\mathrm{R} p}^{a} d_{\mathrm{L} r b}\right)
\end{aligned}
$$

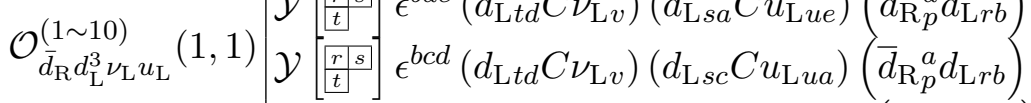

$$
\begin{aligned}
& \mathcal{Y}[[r|s| t]] \epsilon^{b d e}\left(d_{\mathrm{L} s a} C d_{\mathrm{L} t d}\right)\left(u_{\mathrm{L} u e} C \nu_{\mathrm{L} v}\right)\left(\bar{d}_{\mathrm{R} p}^{a} d_{\mathrm{L} r b}\right) \\
& \mathcal{Y}\left[\overline{r|s| t]} \epsilon^{c d e}\left(d_{\mathrm{L} t d} C \nu_{\mathrm{L} v}\right)\left(\bar{d}_{\mathrm{R} p}^{a} d_{\mathrm{L} r a}\right)\left(d_{\mathrm{L} s c} C u_{\mathrm{L} u e}\right)\right. \\
& \mathcal{Y}[\overline{r|s| t]}] \epsilon^{b d e}\left(d_{\mathrm{L} t d} C \nu_{\mathrm{L} v}\right)\left(d_{\mathrm{L} s a} C u_{\mathrm{L} u e}\right)\left(\bar{d}_{\mathrm{R} p}^{a} d_{\mathrm{L} r b}\right) \\
& \mathcal{Y}\left[\begin{array}{c}
r \\
s \\
t
\end{array}\right] \epsilon^{c d e}\left(d_{\mathrm{L} s c} C d_{\mathrm{L} t d}\right)\left(u_{\mathrm{L} u e} C \nu_{\mathrm{L} v}\right)\left(\bar{d}_{\mathrm{R} p}^{a} d_{\mathrm{L} r a}\right) \\
& \mathcal{Y}\left[\begin{array}{c}
r \\
\frac{s}{t}
\end{array}\right] \epsilon^{b d e}\left(d_{\mathrm{L} t d} C \nu_{\mathrm{L} v}\right)\left(d_{\mathrm{L} s a} C u_{\mathrm{L} u e}\right)\left(\bar{d}_{\mathrm{R} p}^{a} d_{\mathrm{L} r b}\right) \\
& \mathcal{Y}[\overline{p p r}] \epsilon^{a b c}\left(\bar{e}_{\mathrm{R} s} d_{\mathrm{L} p a}\right)\left(d_{\mathrm{L} r b} C e_{\mathrm{L} t}\right)\left(u_{\mathrm{L} u c} C \nu_{\mathrm{L} v}\right) \\
& \mathcal{Y}[[p r]] \epsilon^{a b c}\left(e_{\mathrm{L} t} C \nu_{\mathrm{L} v}\right)\left(\bar{e}_{\mathrm{R} s} d_{\mathrm{L} p a}\right)\left(d_{\mathrm{L} r b} C u_{\mathrm{L} u c}\right) \\
& \mathcal{O}_{d_{\mathrm{L}}^{2} \bar{e}_{\mathrm{R}} e_{\mathrm{L}} \nu_{\mathrm{L}} u_{\mathrm{L}}}^{(1 \sim \bar{s}}(1,1) \mathcal{Y}\left[[p \mid r] \epsilon^{a b c}\left(\bar{e}_{\mathrm{R} s} \nu_{\mathrm{L} v}\right)\left(d_{\mathrm{L} p a} C e_{\mathrm{L} t}\right)\left(d_{\mathrm{L} r b} C u_{\mathrm{L} u c}\right)\right. \\
& \mathcal{Y}\left[\frac{p}{r}\right] \epsilon^{a b c}\left(\bar{e}_{\mathrm{R} s} e_{\mathrm{L} t}\right)\left(d_{\mathrm{L} p a} C d_{\mathrm{L} r b}\right)\left(u_{\mathrm{L} u c} C \nu_{\mathrm{L} v}\right) \\
& \mathcal{Y}\left[\frac{p}{r}\right] \epsilon^{a b c}\left(e_{\mathrm{L} t} C \nu_{\mathrm{L} v}\right)\left(\bar{e}_{\mathrm{R} s} u_{\mathrm{L} u c}\right)\left(d_{\mathrm{L} p a} C d_{\mathrm{L} r b}\right)
\end{aligned}
$$

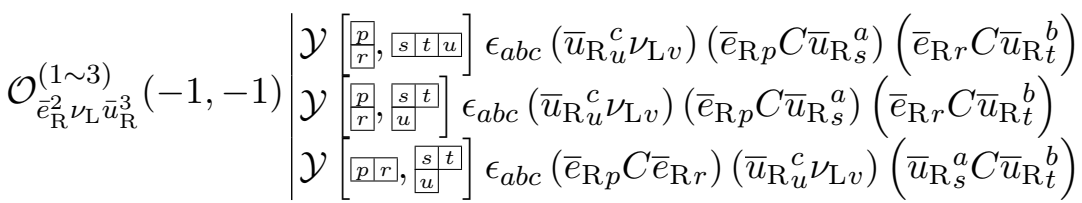




$$
\begin{aligned}
& \mathcal{Y}\left[\frac{p}{r}, u w v\right] \epsilon_{a b e}\left(\bar{e}_{\mathrm{R} t} d_{\mathrm{L} s c}\right)\left(\bar{d}_{\mathrm{R}}^{a} C \bar{d}_{\mathrm{R}}^{b}\right)\left(\bar{u}_{\mathrm{R}}^{c} C \bar{u}_{\mathrm{R}}^{e}\right) \\
& \mathcal{Y}, \frac{p}{r}, u v v \mid \epsilon_{b d e}\left(\bar{d}_{\mathrm{R} p}^{a} C \bar{d}_{\mathrm{R} r}^{b}\right)\left(\bar{u}_{\mathrm{R} u}^{d} d_{\mathrm{L} s a}\right)\left(\bar{e}_{\mathrm{R} t} C \bar{u}_{\mathrm{R} v}^{e}\right) \\
& \mathcal{Y}\left[\frac{p}{r}, u v v \mid \epsilon_{b d e}\left(\bar{d}_{\mathrm{R} p}^{a} d_{\mathrm{L} s a}\right)\left(\bar{e}_{\mathrm{R} t} C \bar{u}_{\mathrm{R} v}^{e}\right)\left(\bar{d}_{\mathrm{R} r}^{b} C \bar{u}_{\mathrm{R} u}^{d}\right)\right. \\
& \mathcal{Y}, \frac{p}{r}, u v v \mid \epsilon_{b d e}\left(\bar{d}_{\mathrm{R} p}^{a} C \bar{e}_{\mathrm{R} t}\right)\left(\bar{u}_{\mathrm{R} v}^{e} d_{\mathrm{L} s a}\right)\left(\bar{d}_{\mathrm{R} r}^{b} C \bar{u}_{\mathrm{R}}^{d}\right) \\
& \mathcal{Y}\left[p \mid r, \frac{u}{v}\right] \epsilon_{b d e}\left(\bar{e}_{\mathrm{R} t} d_{\mathrm{L} s a}\right)\left(\bar{d}_{\mathrm{R} p}^{a} C \bar{d}_{\mathrm{R}}{ }_{r}^{b}\right)\left(\bar{u}_{\mathrm{R} u}^{d} C \bar{u}_{\mathrm{R}}{ }_{v}^{e}\right)
\end{aligned}
$$

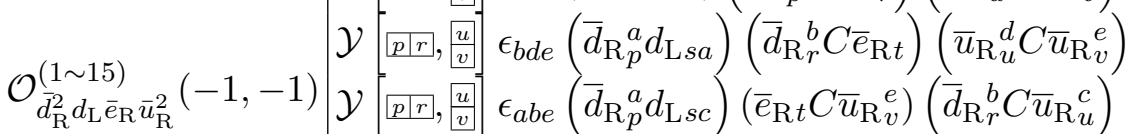

$$
\begin{aligned}
& \mathcal{Y}\left[\overline{p p r}, \frac{u}{v}\right] \epsilon_{a b e}\left(\bar{d}_{\mathrm{R} p}^{a} C \bar{e}_{\mathrm{R} t}\right)\left(\bar{u}_{\mathrm{R} v}^{e} d_{\mathrm{L} s c}\right)\left(\bar{d}_{\mathrm{R} r}^{b} C \bar{u}_{\mathrm{R}}^{c}{ }_{u}^{c}\right) \\
& \mathcal{Y}\left[\overline{p r r}, \underline{u v v]} \epsilon_{b d e}\left(\bar{d}_{\mathrm{R} p}^{a} C \bar{d}_{\mathrm{R} r}^{b}\right)\left(\bar{u}_{\mathrm{R} u}^{d} d_{\mathrm{L} s a}\right)\left(\bar{e}_{\mathrm{R} t} C \bar{u}_{\mathrm{R} v}^{e}\right)\right. \\
& \mathcal{Y}\left[[p, r, u \mid v] \epsilon_{a b e}\left(\bar{d}_{\mathrm{R}}^{a} d_{\mathrm{L} s c}\right)\left(\bar{d}_{\mathrm{R} r}^{b} C \bar{e}_{\mathrm{R} t}\right)\left(\bar{u}_{\mathrm{R} u}^{c} C \bar{u}_{\mathrm{R} v}^{e}\right)\right. \\
& \mathcal{Y}\left[\left[p \mid r, \underline{u v v]} \epsilon_{b d e}\left(\bar{d}_{\mathrm{R} p}^{a} d_{\mathrm{L} s a}\right)\left(\bar{e}_{\mathrm{R} t} C \bar{u}_{\mathrm{R} v}^{e}\right)\left(\bar{d}_{\mathrm{R} r}^{b} C \bar{u}_{\mathrm{R} u}^{d}\right)\right.\right. \\
& \mathcal{Y}[\overline{p \mid r}, \overline{u v}] \epsilon_{b d e}\left(\bar{d}_{\mathrm{R} p}^{a} C \bar{e}_{\mathrm{R} t}\right)\left(\bar{u}_{\mathrm{R} v}^{e} d_{\mathrm{L} s a}\right)\left(\bar{d}_{\mathrm{R}}{ }_{r}^{b} C \bar{u}_{\mathrm{R} u}^{d}\right) \\
& \mathcal{Y}\left[\frac{p}{r}, \frac{u}{v}\right] \epsilon_{b d e}\left(\bar{e}_{\mathrm{R} t} d_{\mathrm{L} s a}\right)\left(\bar{d}_{\mathrm{R} p}^{a} C \bar{d}_{\mathrm{R} r}^{b}\right)\left(\bar{u}_{\mathrm{R} u}^{d} C \bar{u}_{\mathrm{R} v}^{e}\right)
\end{aligned}
$$

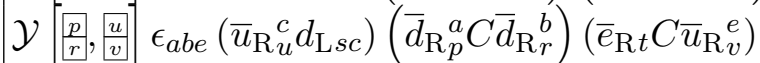

$$
\begin{aligned}
& \mathcal{Y}\left[\frac{p}{r}, \underline{v}, \underline{v}\right] \epsilon_{b d e}\left(\bar{d}_{\mathrm{R}}^{a}{ }_{p} d_{\mathrm{L} s a}\right)\left(\bar{d}_{\mathrm{R}}{ }_{r}^{b} C \bar{e}_{\mathrm{R} t}\right)\left(\bar{u}_{\mathrm{R}}{ }_{u}^{d} C \bar{u}_{\mathrm{R} v}^{e}\right)
\end{aligned}
$$

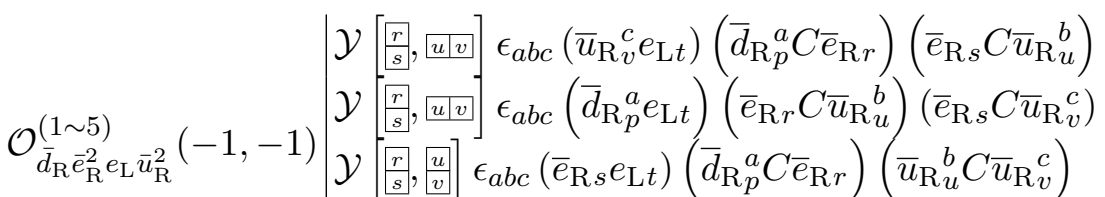

$$
\begin{aligned}
& \mathcal{Y}[\overline{r r s}, u \underline{u v}] \epsilon_{a b c}\left(\bar{u}_{\mathrm{R} v}^{c} e_{\mathrm{L} t}\right)\left(\bar{d}_{\mathrm{R} p}^{a} C \bar{e}_{\mathrm{R} r}\right)\left(\bar{e}_{\mathrm{R} s} C \bar{u}_{\mathrm{R}}^{b}\right) \\
& \mathcal{Y}\left[\overline{r r s}, \frac{u}{v}\right] \epsilon_{a b c}\left(\bar{e}_{\mathrm{R} s} e_{\mathrm{L} t}\right)\left(\bar{d}_{\mathrm{R}_{p}^{a}} C \bar{e}_{\mathrm{R} r}\right)\left(\bar{u}_{\mathrm{R} u}^{b} C \bar{u}_{\mathrm{R} v}^{c}\right)
\end{aligned}
$$

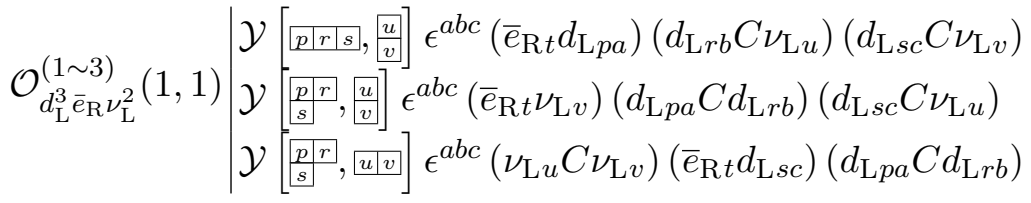


$(\Delta B, \Delta L)=( \pm 1, \mp 1)$

$$
\begin{aligned}
& \mathcal{Y}\left[\frac{p}{r}, s \mid t\right] \epsilon_{a b d}\left(u_{\mathrm{L} u c} C \nu_{\mathrm{L} v}\right)\left(\bar{d}_{\mathrm{R} p}^{a} C \bar{d}_{\mathrm{R} r}^{b}\right)\left(\bar{u}_{\mathrm{R}}^{c} C \bar{u}_{\mathrm{R} t}^{d}\right) \\
& \mathcal{Y}\left[\frac{p}{r}, s \mid t\right] \epsilon_{b c d}\left(\bar{u}_{\mathrm{R} t}^{d} \nu_{\mathrm{L} v}\right)\left(\bar{d}_{\mathrm{R}}^{a} C \bar{d}_{\mathrm{R}}^{b}\right)\left(\bar{u}_{\mathrm{R}}^{c} u_{\mathrm{L} u a}\right) \\
& \mathcal{Y}\left[\frac{p}{r}, s \mid t\right] \epsilon_{b c d}\left(u_{\mathrm{L} u a} C \nu_{\mathrm{L} v}\right)\left(\bar{d}_{\mathrm{R} p}^{a} C \bar{u}_{\mathrm{R}}^{c}\right)\left(\bar{d}_{\mathrm{R} r}^{b} C \bar{u}_{\mathrm{R} t}^{d}\right) \\
& \mathcal{Y}\left[\frac{p}{r}, s[t] \epsilon_{b c d}\left(\bar{u}_{\mathrm{R} t}^{d} \nu_{\mathrm{L} v}\right)\left(\bar{d}_{\mathrm{R} r}^{b} u_{\mathrm{L} u a}\right)\left(\bar{d}_{\mathrm{R} p}^{a} C \bar{u}_{\mathrm{R} s}^{c}\right)\right. \\
& \mathcal{Y}\left[p \mid r, \frac{s}{t}\right] \epsilon_{b c d}\left(u_{\mathrm{L} u a} C \nu_{\mathrm{L} v}\right)\left(\bar{d}_{\mathrm{R} p}^{a} C \bar{d}_{\mathrm{R} r}^{b}\right)\left(\bar{u}_{\mathrm{R}}^{c} C \bar{u}_{\mathrm{R} t}^{d}\right) \\
& \mathcal{Y}\left[\frac{p p r}{,}, \frac{s}{t}\right] \epsilon_{a b d}\left(u_{\mathrm{L} u c} C \nu_{\mathrm{L} v}\right)\left(\bar{d}_{\mathrm{R} p}^{a} C \bar{u}_{\mathrm{R} s}^{c}\right)\left(\bar{d}_{\mathrm{R} r}^{b} C \bar{u}_{\mathrm{R} t}^{d}\right)
\end{aligned}
$$

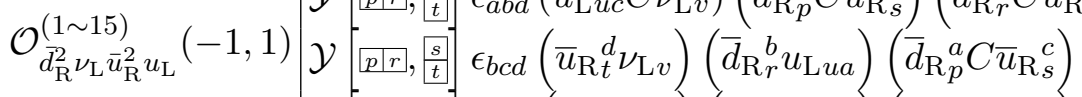

$$
\begin{aligned}
& \mathcal{Y}\left[\overline{p p r}, \frac{s}{t}\right] \epsilon_{a b d}\left(\bar{u}_{\mathrm{R} t}^{d} \nu_{\mathrm{L} v}\right)\left(\bar{d}_{\mathrm{R} r}^{b} u_{\mathrm{L} u c}\right)\left(\bar{d}_{\mathrm{R} p}^{a} C \bar{u}_{\mathrm{R} s}^{c}\right) \\
& \mathcal{Y}[\overline{p p r}, s] t] \epsilon_{b c d}\left(\bar{u}_{\mathrm{R} t}^{d} \nu_{\mathrm{L} v}\right)\left(\bar{d}_{\mathrm{R}}^{a}{ }_{p}^{a} \bar{d}_{\mathrm{R}}{ }_{r}^{b}\right)\left(\bar{u}_{\mathrm{R}}^{c} u_{\mathrm{L} u a}\right) \\
& \mathcal{Y}[\overline{p p r}, s \mid t]] \epsilon_{b c d}\left(u_{\mathrm{L} u a} C \nu_{\mathrm{L} v}\right)\left(\bar{d}_{\mathrm{R} p}^{a} C \bar{u}_{\mathrm{R} s}^{c}\right)\left(\bar{d}_{\mathrm{R} r}^{b} C \bar{u}_{\mathrm{R} t}^{d}\right) \\
& \mathcal{Y}[\overline{p \mid r}, s \mid t]] \epsilon_{b c d}\left(\bar{u}_{\mathrm{R} t}^{d} \nu_{\mathrm{L} v}\right)\left(\bar{d}_{\mathrm{R} r}^{b} u_{\mathrm{L} u a}\right)\left(\bar{d}_{\mathrm{R}_{p}^{a}} C \bar{u}_{\mathrm{R}}^{c}\right) \\
& \mathcal{Y}[[p|r, s| t]] \epsilon_{a b d}\left(\bar{u}_{\mathrm{R} t}^{d} \nu_{\mathrm{L} v}\right)\left(\bar{d}_{\mathrm{R}}{ }_{r}^{b} u_{\mathrm{L} u c}\right)\left(\bar{d}_{\mathrm{R}}^{a} C \bar{u}_{\mathrm{R}}^{c}{ }_{s}^{c}\right)
\end{aligned}
$$

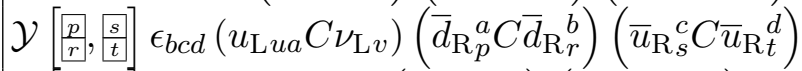

$$
\begin{aligned}
& \mathcal{Y}\left[\frac{p}{r}, \frac{s}{t}\right] \epsilon_{a b d}\left(\bar{u}_{\mathrm{R}}^{c} u_{\mathrm{L} u c}\right)\left(\bar{u}_{\mathrm{R} t}^{d} \nu_{\mathrm{L} v}\right)\left(\bar{d}_{\mathrm{R}}^{a} C \bar{d}_{\mathrm{R}_{r}^{b}}\right) \\
& \mathcal{Y}\left[\frac{p}{r}, \frac{s}{t}\right] \epsilon_{b c d}\left(\bar{u}_{\mathrm{R}}^{d} \nu_{\mathrm{L} v}\right)\left(\bar{d}_{\mathrm{R}}{ }_{r}^{b} u_{\mathrm{L} u a}\right)\left(\bar{d}_{\mathrm{R}_{p}^{a}} C \bar{u}_{\mathrm{R}_{s}^{c}}\right)
\end{aligned}
$$

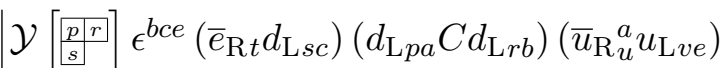

$$
\begin{aligned}
& \mathcal{Y}\left[\frac{p r r}{s}\right] \epsilon^{a b e}\left(\bar{e}_{\mathrm{R} t} d_{\mathrm{L} s c}\right)\left(d_{\mathrm{L} p a} C d_{\mathrm{L} r b}\right)\left(\bar{u}_{\mathrm{R}}^{c} u_{\mathrm{L} v e}\right) \\
& \mathcal{Y}\left[\frac{p r}{s}\right] \epsilon^{b c e}\left(\bar{e}_{\mathrm{R} t} u_{\mathrm{L} v e}\right)\left(d_{\mathrm{L} p a} C d_{\mathrm{L} r b}\right)\left(\bar{u}_{\mathrm{R} u}^{a} d_{\mathrm{L} s c}\right) \\
& \mathcal{Y}\left[\begin{array}{ll}
p r \\
s
\end{array} \epsilon^{a b e}\left(\bar{e}_{\mathrm{R} t} u_{\mathrm{L} v e}\right)\left(d_{\mathrm{L} p a} C d_{\mathrm{L} r b}\right)\left(\bar{u}_{\mathrm{R}}{ }_{u}^{c} d_{\mathrm{L} s c}\right)\right. \\
& \mathcal{O}_{d_{\mathrm{L}}^{3} \bar{e}_{\mathrm{R}} \bar{u}_{\mathrm{R}} u_{\mathrm{L}}}^{(1 \sim 10)}(1,-1) \\
& \mathcal{Y}\left[\frac{p r r}{s}\right] \epsilon^{b c e}\left(\bar{e}_{\mathrm{R} t} d_{\mathrm{L} p a}\right)\left(d_{\mathrm{L} s c} C u_{\mathrm{L} v e}\right)\left(\bar{u}_{\mathrm{R} u}^{a} d_{\mathrm{L} r b}\right) \\
& \mathcal{Y}\left[\overline{p|r| s]} \epsilon^{b c e}\left(\bar{e}_{\mathrm{R} t} d_{\mathrm{L} s c}\right)\left(d_{\mathrm{L} p a} C d_{\mathrm{L} r b}\right)\left(\bar{u}_{\mathrm{R}}{ }_{u}^{a} u_{\mathrm{L} v e}\right)\right. \\
& \mathcal{Y}\left[\overline{p|r| s]} \epsilon^{b c e}\left(\bar{e}_{\mathrm{R} t} u_{\mathrm{L} v e}\right)\left(d_{\mathrm{L} p a} C d_{\mathrm{L} r b}\right)\left(\bar{u}_{\mathrm{R}}{ }_{u}^{a} d_{\mathrm{L} s c}\right)\right. \\
& \mathcal{Y}\left[\overline{p|r| s]} \epsilon^{b c e}\left(\bar{e}_{\mathrm{R} t} d_{\mathrm{L} p a}\right)\left(d_{\mathrm{L} s c} C u_{\mathrm{L} v e}\right)\left(\bar{u}_{\mathrm{R} u}^{a} d_{\mathrm{L} r b}\right)\right. \\
& \mathcal{Y}\left[\begin{array}{c}
\frac{p}{r} \\
s
\end{array}\right] \epsilon^{b c e}\left(\bar{e}_{\mathrm{R} t} d_{\mathrm{L} s c}\right)\left(d_{\mathrm{L} p a} C d_{\mathrm{L} r b}\right)\left(\bar{u}_{\mathrm{R} u}{ }_{u} u_{\mathrm{L} v e}\right) \\
& \mathcal{Y}\left[\begin{array}{c}
\frac{p}{r} \\
\frac{r}{s}
\end{array}\right] \epsilon^{b c e}\left(\bar{e}_{\mathrm{R} t} u_{\mathrm{L} v e}\right)\left(d_{\mathrm{L} p a} C d_{\mathrm{L} r b}\right)\left(\bar{u}_{\mathrm{R}}^{a} d_{\mathrm{L} s c}\right)
\end{aligned}
$$




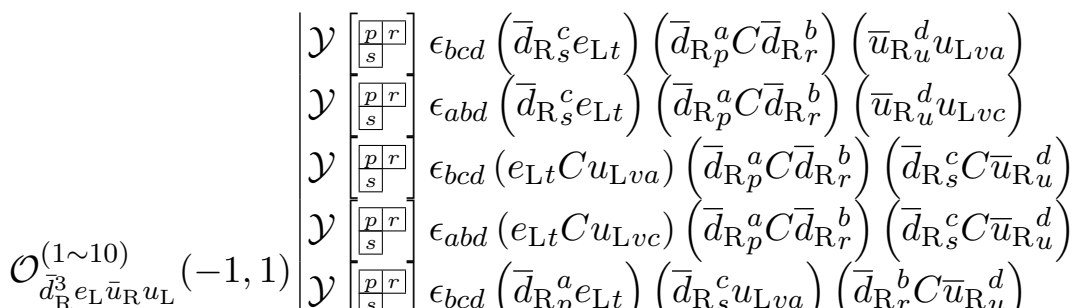

$$
\begin{aligned}
& \mathcal{Y}\left[\overline{p|r| s]} \epsilon_{b c d}\left(\bar{d}_{\mathrm{R}}^{c} e_{\mathrm{L} t}\right)\left(\bar{d}_{\mathrm{R}}^{a} C \bar{d}_{\mathrm{R} r}^{b}\right)\left(\bar{u}_{\mathrm{R} u}^{d} u_{\mathrm{L} v a}\right)\right. \\
& \mathcal{Y}[\overline{p|r| s}] \epsilon_{b c d}\left(e_{\mathrm{L} t} C u_{\mathrm{L} v a}\right)\left(\bar{d}_{\mathrm{R} p}^{a} C \bar{d}_{\mathrm{R} r}^{b}\right)\left(\bar{d}_{\mathrm{R}}^{c} C \bar{u}_{\mathrm{R} u}^{d}\right) \\
& \mathcal{Y}\left[\overline{p|r| s]} \epsilon_{b c d}\left(\bar{d}_{\mathrm{R} p}^{a} e_{\mathrm{L} t}\right)\left(\bar{d}_{\mathrm{R}}^{c} u_{\mathrm{L} v a}\right)\left(\bar{d}_{\mathrm{R}}{ }_{r}^{b} C \bar{u}_{\mathrm{R} u}^{d}\right)\right. \\
& \mathcal{Y}\left[\begin{array}{c}
\frac{p}{r} \\
\frac{r}{s}
\end{array}\right] \epsilon_{b c d}\left(\bar{d}_{\mathrm{R} s}^{c} e_{\mathrm{L} t}\right)\left(\bar{d}_{\mathrm{R} p}^{a} C \bar{d}_{\mathrm{R} r}^{b}\right)\left(\bar{u}_{\mathrm{R} u}^{d} u_{\mathrm{L} v a}\right) \\
& \mathcal{Y}\left[\begin{array}{c}
\frac{p}{r} \\
s
\end{array}\right] \epsilon_{b c d}\left(e_{\mathrm{L} t} C u_{\mathrm{L} v a}\right)\left(\bar{d}_{\mathrm{R} p}^{a} C \bar{d}_{\mathrm{R}}^{b}\right)\left(\bar{d}_{\mathrm{R}}^{c} C \bar{u}_{\mathrm{R} u}^{d}\right) \\
& \mathcal{Y}\left[s \mid t,, \frac{u}{v}\right] \epsilon_{a b c}\left(\bar{u}_{\mathrm{R}}^{b} \nu_{\mathrm{L} u}\right)\left(\bar{u}_{\mathrm{R}}^{c} \nu_{\mathrm{L} v}\right)\left(\bar{d}_{\mathrm{R}}^{a} C \bar{e}_{\mathrm{R} r}\right)
\end{aligned}
$$

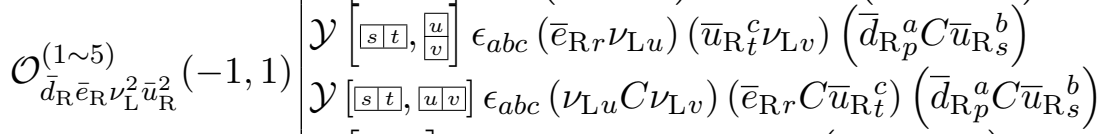

$$
\begin{aligned}
& \mathcal{Y}\left[\begin{array}{l}
s \\
t
\end{array},\left[\begin{array}{l}
u \\
v
\end{array}\right] \epsilon_{a b c}\left(\bar{e}_{\mathrm{R} r} \nu_{\mathrm{L} u}\right)\left(\bar{u}_{\mathrm{R} t}^{c} \nu_{\mathrm{L} v}\right)\left(\bar{d}_{\left.\mathrm{R}_{p}^{a} C \bar{u}_{\mathrm{R}}^{b}\right)}^{b}\right.\right. \\
& \mathcal{Y}\left[\frac{s}{t}, u v v\right] \epsilon_{a b c}\left(\nu_{\mathrm{L} u} C \nu_{\mathrm{L} v}\right)\left(\bar{d}_{\mathrm{R} p}^{a} C \bar{e}_{\mathrm{R} r}\right)\left(\bar{u}_{\mathrm{R}}^{b} C \bar{u}_{\mathrm{R}}^{c}\right) \\
& \mathcal{Y}\left[\frac{p r r}{s s}\right] \epsilon_{\text {ace }}\left(\bar{u}_{\mathrm{R}}{ }_{u}^{e} \nu_{\mathrm{L} v}\right)\left(\bar{d}_{\mathrm{R}_{p}^{a} C} C \bar{d}_{\mathrm{R}_{r}^{b}}\right)\left(\bar{d}_{\left.\mathrm{R}_{s}^{c} d_{\mathrm{L} t b}\right)}\right. \\
& \mathcal{Y}\left[\begin{array}{ll}
p r \\
s
\end{array} \epsilon_{b c e}\left(\bar{u}_{\mathrm{R} u}^{e} \nu_{\mathrm{L} v}\right)\left(\bar{d}_{\mathrm{R} p}^{a} C \bar{d}_{\mathrm{R} r}^{b}\right)\left(\bar{d}_{\mathrm{R}_{s}^{c}} d_{\mathrm{L} t a}\right)\right. \\
& \mathcal{Y}\left[\begin{array}{l}
p r \\
s
\end{array} \epsilon_{a c e}\left(d_{\mathrm{L} t b} C \nu_{\mathrm{L} v}\right)\left(\bar{d}_{\mathrm{R} p}^{a} C \bar{d}_{\mathrm{R} r}^{b}\right)\left(\bar{d}_{\mathrm{R} s}^{c} C \bar{u}_{\mathrm{R} u}^{e}\right)\right.
\end{aligned}
$$

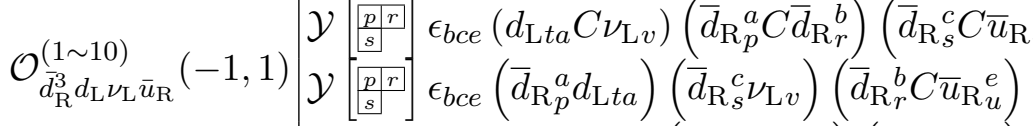

$$
\begin{aligned}
& \mathcal{Y}\left[\overline{p|r| s]} \epsilon_{a c e}\left(\bar{u}_{\mathrm{R}}{ }_{u}^{e} \nu_{\mathrm{L} v}\right)\left(\bar{d}_{\mathrm{R} p}^{a} C \bar{d}_{\mathrm{R} r}^{b}\right)\left(\bar{d}_{\mathrm{R}}^{c} d_{\mathrm{L} t b}\right)\right. \\
& \mathcal{Y}\left[\overline{p|r| s]} \epsilon_{a c e}\left(d_{\mathrm{L} t b} C \nu_{\mathrm{L} v}\right)\left(\bar{d}_{\mathrm{R} p}^{a} C \bar{d}_{\mathrm{R} r}^{b}\right)\left(\bar{d}_{\mathrm{R}}^{c} C \bar{u}_{\mathrm{R}}^{e}\right)\right. \\
& \mathcal{Y}\left[\overline{p|r| s]} \epsilon_{\text {ace }}\left(\bar{d}_{\mathrm{R}}^{c} \nu_{\mathrm{L} v}\right)\left(\bar{d}_{\mathrm{R} p}^{a} d_{\mathrm{L} t b}\right)\left(\bar{d}_{\mathrm{R}}^{b} C \bar{u}_{\mathrm{R}}^{e}\right)\right. \\
& \mathcal{Y}\left[\begin{array}{l}
p \\
\frac{p}{r} \\
s
\end{array}\right] \epsilon_{\text {ace }}\left(\bar{u}_{\mathrm{R}}{ }_{u}^{e} \nu_{\mathrm{L} v}\right)\left(\bar{d}_{\mathrm{R} p}^{a} C \bar{d}_{\mathrm{R}}^{b}\right)\left(\bar{d}_{\mathrm{R}}^{c} d_{\mathrm{L} t b}\right)
\end{aligned}
$$

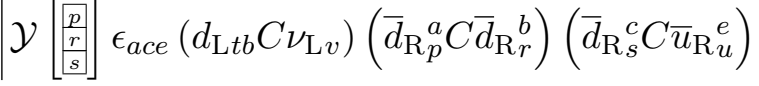

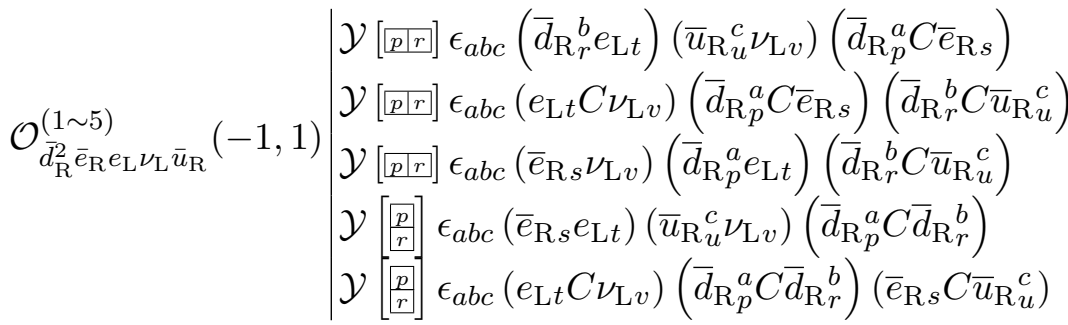




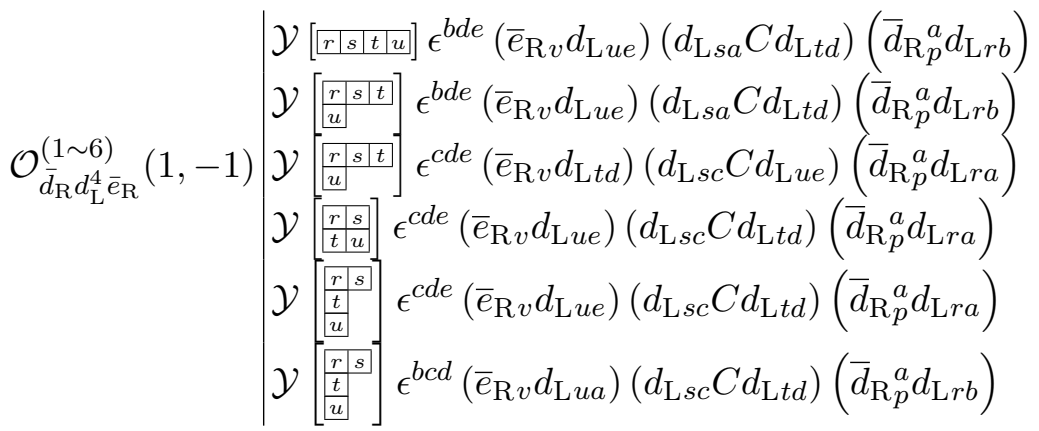

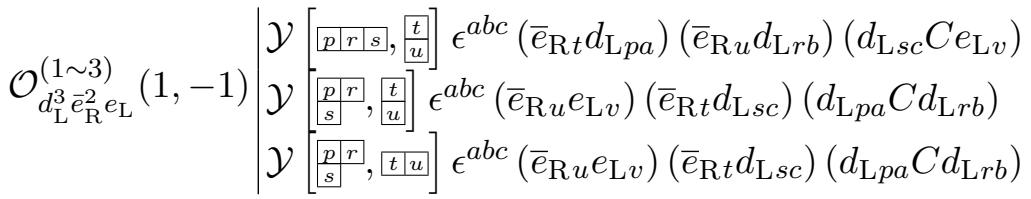

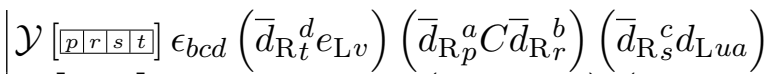

$$
\begin{aligned}
& \mathcal{Y}\left[\frac{p p r \mid s]}{t}\right] \epsilon_{b c d}\left(d_{\mathrm{L} u a} C e_{\mathrm{L} v}\right)\left(\bar{d}_{\mathrm{R}}^{a} C \bar{d}_{\mathrm{R}}{ }_{r}^{b}\right)\left(\bar{d}_{\mathrm{R}_{s}^{c}} C \bar{d}_{\mathrm{R} t}^{d}\right) \\
& \mathcal{O}_{\bar{d}_{\mathrm{R}}^{4} d_{\mathrm{L}} e_{\mathrm{L}}}^{(1 \sim 6)}(-1,1) \mathcal{Y}\left[\frac{p r r s}{t}\right] \epsilon_{b c d}\left(\bar{d}_{\mathrm{R} t}^{d} e_{\mathrm{L} v}\right)\left(\bar{d}_{\mathrm{R}}^{a}{ }_{p}^{a} C \bar{d}_{\mathrm{R}}^{b}\right)\left(\bar{d}_{\mathrm{R}}^{c} d_{\mathrm{L} u a}\right) \\
& \mathcal{Y}\left[\begin{array}{l}
p r r \\
s i t
\end{array}\right] \epsilon_{b c d}\left(\bar{d}_{\mathrm{R} t}^{d} e_{\mathrm{L} v}\right)\left(\bar{d}_{\mathrm{R}}^{a} C \bar{d}_{\mathrm{R}}^{b}\right)\left(\bar{d}_{\mathrm{R}}^{c} d_{\mathrm{L} u a}\right)
\end{aligned}
$$

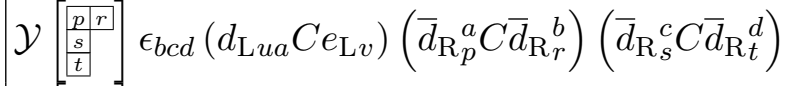

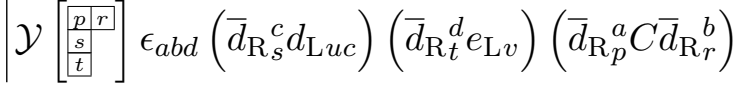

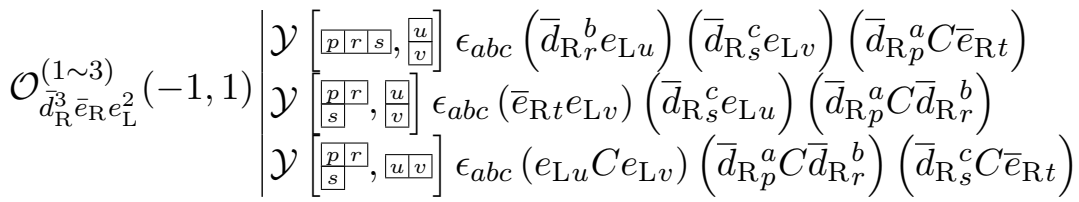

$(\Delta B, \Delta L)=( \pm 1, \pm 3)$

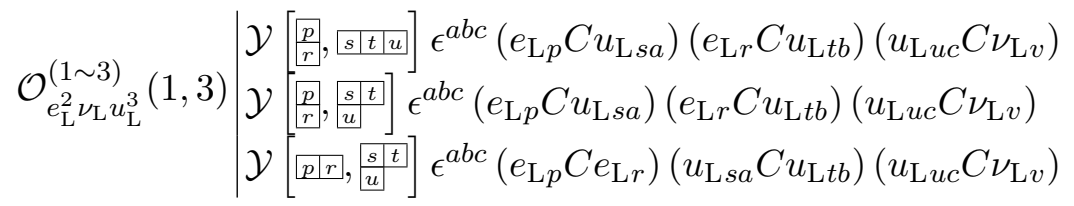

$$
\begin{aligned}
& \mathcal{Y}\left[s \mid t, \frac{u}{v}\right] \epsilon^{a b c}\left(d_{\mathrm{L} p a} C e_{\mathrm{L} r}\right)\left(u_{\mathrm{L} s b} C \nu_{\mathrm{L} u}\right)\left(u_{\mathrm{L} t c} C \nu_{\mathrm{L} v}\right)
\end{aligned}
$$

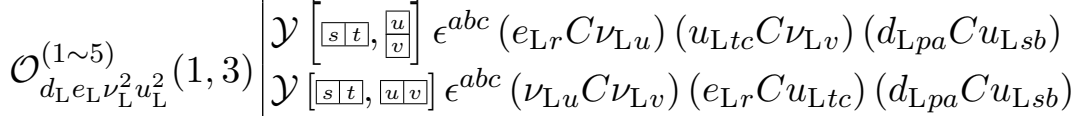

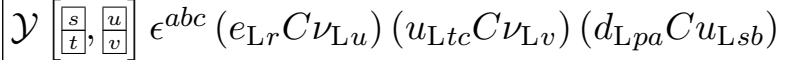

$$
\begin{aligned}
& \mathcal{Y}\left[\frac{s}{t}, u[v] \epsilon^{a b c}\left(\nu_{\mathrm{L} u} C \nu_{\mathrm{L} v}\right)\left(d_{\mathrm{L} p a} C e_{\mathrm{L} r}\right)\left(u_{\mathrm{L} s b} C u_{\mathrm{L} t c}\right)\right.
\end{aligned}
$$

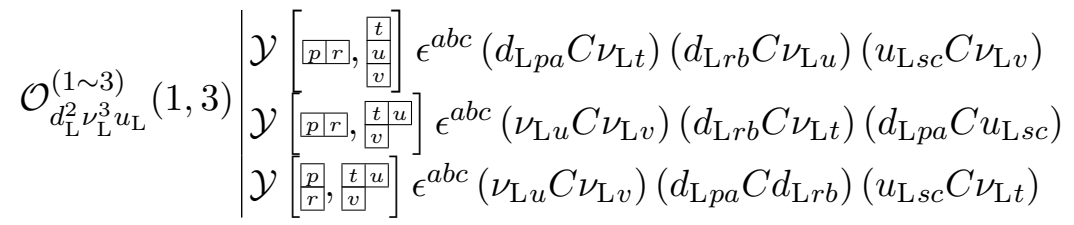


$(\Delta B, \Delta L)=( \pm 1, \mp 3)$

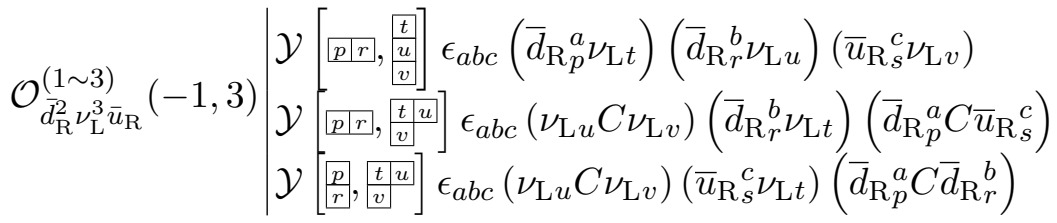

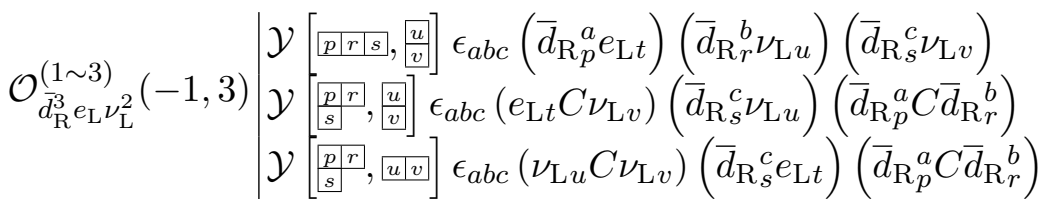

$(\Delta B, \Delta L)=( \pm 2,0)$

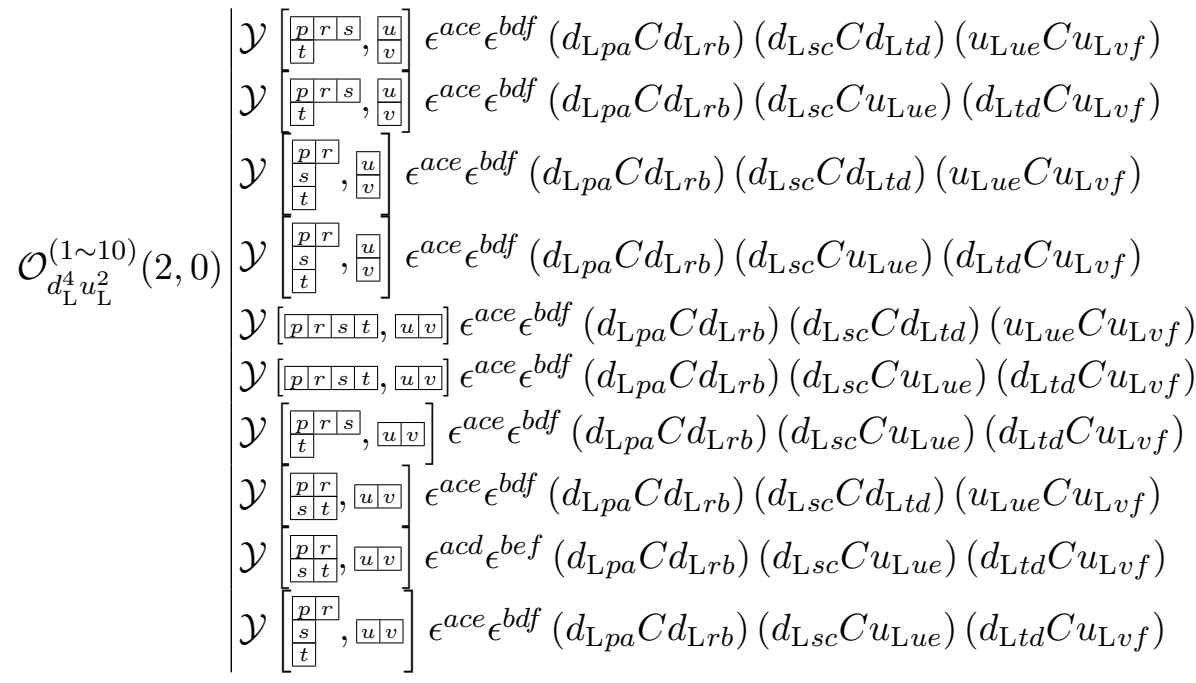

$$
\begin{aligned}
& \mathcal{Y}\left[\frac{p r r \mid s}{t}, \underline{u} v\right] \epsilon_{\text {ace }} \epsilon_{b d f}\left(\bar{d}_{\mathrm{R}}^{a} C \bar{d}_{\mathrm{R}}^{b}\right)\left(\bar{d}_{\mathrm{R}}^{c} C \bar{d}_{\mathrm{R} t}^{d}\right)\left(\bar{u}_{\mathrm{R}}^{e} C \bar{u}_{\mathrm{R} v}^{f}\right) \\
& \mathcal{Y}\left[\frac{p|r| s}{t r}, \underline{u} v\right] \epsilon_{a c e} \epsilon_{b d f}\left(\bar{d}_{\mathrm{R} p}^{a} C \bar{d}_{\mathrm{R}}^{b}\right)\left(\bar{d}_{\mathrm{R}}^{c} C \bar{u}_{\mathrm{R}}{ }^{e}\right)\left(\bar{d}_{\mathrm{R} t}^{d} C \bar{u}_{\mathrm{R} v}^{f}\right)
\end{aligned}
$$

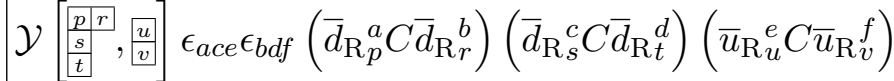

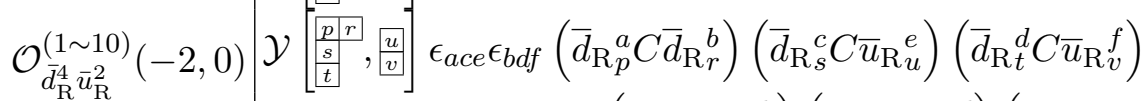

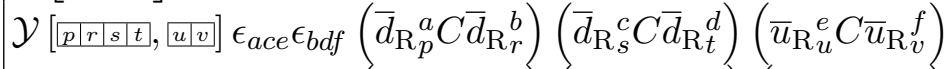

$$
\begin{aligned}
& \mathcal{Y}\left[[p|r| s|t, u| u \mid v] \epsilon_{a c e} \epsilon_{b d f}\left(\bar{d}_{\mathrm{R} p}^{a} C \bar{d}_{\mathrm{R}}{ }_{r}^{b}\right)\left(\bar{d}_{\mathrm{R}}^{c} C \bar{u}_{\mathrm{R} u}^{e}\right)\left(\bar{d}_{\mathrm{R} t}^{d} C \bar{u}_{\mathrm{R}}{ }_{v}^{f}\right)\right.
\end{aligned}
$$

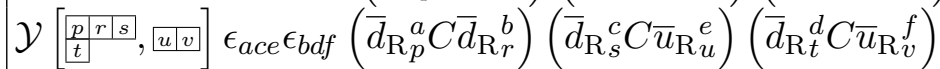

$$
\begin{aligned}
& \mathcal{Y}\left[\frac{p r r}{s t}, u|v| \epsilon_{a c e} \epsilon_{b d f}\left(\bar{d}_{\mathrm{R}}{ }_{p}^{a} C \bar{d}_{\mathrm{R}}{ }_{r}^{b}\right)\left(\bar{d}_{\mathrm{R}}{ }_{s}^{c} C \bar{d}_{\mathrm{R} t}^{d}\right)\left(\bar{u}_{\mathrm{R}}{ }_{u}^{e} C \bar{u}_{\mathrm{R}}^{f}\right)\right.
\end{aligned}
$$

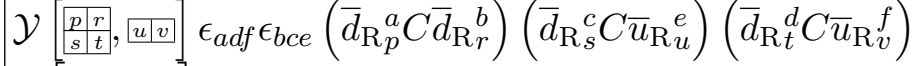

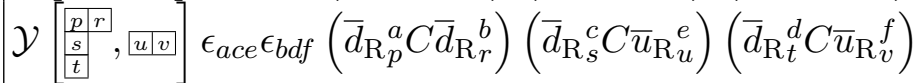




\subsubsection{Classes involving six-fermions: $\psi^{4} \psi^{\dagger 2}$}

There are 410 complex types in this class.

$(\Delta B, \Delta L)=(0,0)$

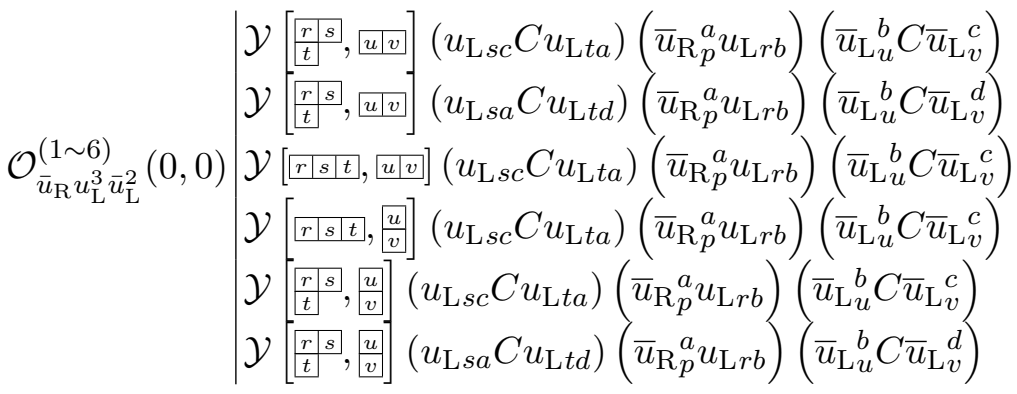

$$
\begin{aligned}
& \mathcal{Y}\left[\begin{array}{l}
\left.\frac{p}{r}, \frac{s}{t}\right] \\
{[}
\end{array}\right]\left(u_{\mathrm{L} s c} C u_{\mathrm{L} t a}\right)\left(\bar{u}_{\mathrm{R} p}^{a} C \bar{u}_{\mathrm{R} r}^{b}\right)\left(\bar{u}_{\mathrm{L} v}^{c} u_{\mathrm{R} u b}\right)
\end{aligned}
$$

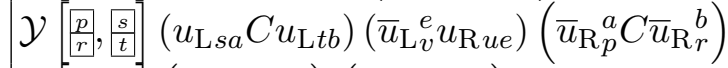

$$
\begin{aligned}
& \mathcal{Y}\left[\begin{array}{c}
\left.\frac{p}{r}, \frac{s}{t}\right] \\
0
\end{array}\left(\bar{u}_{\mathrm{R} r}^{b} u_{\mathrm{L} t a}\right)\left(\bar{u}_{\mathrm{R} p}^{a} u_{\mathrm{L} s c}\right)\left(\bar{u}_{\mathrm{L} v}^{c} u_{\mathrm{R} u b}\right)\right. \\
& \mathcal{Y}\left[\frac{p}{r}, \frac{s}{t}\right],\left(\bar{u}_{\mathrm{R} p}^{a} u_{\mathrm{L} s a}\right)\left(\bar{u}_{\mathrm{R}}{ }_{r}^{b} u_{\mathrm{L} t b}\right)\left(\bar{u}_{\mathrm{L} v}{ }^{e} u_{\mathrm{R} u e}\right)
\end{aligned}
$$

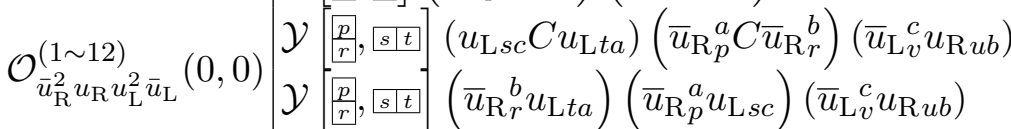

$$
\begin{aligned}
& \mathcal{Y}\left[p \mid r, \frac{s}{t}\right]\left(u_{\mathrm{L} s c} C u_{\mathrm{L} t a}\right)\left(\bar{u}_{\mathrm{R} p}^{a} C \bar{u}_{\mathrm{R} r}^{b}\right)\left(\bar{u}_{\mathrm{L} v}^{c} u_{\mathrm{R} u b}\right) \\
& \mathcal{Y}\left[p \mid r, \frac{s}{t}\right]\left(\bar{u}_{\mathrm{R}}{ }_{r}^{b} u_{\mathrm{L} t a}\right)\left(\bar{u}_{\mathrm{R} p}^{a} u_{\mathrm{L} s c}\right)\left(\bar{u}_{\mathrm{L} v}^{c} u_{\mathrm{R} u b}\right) \\
& \mathcal{Y}[\overline{p \mid r}, s \mid t]\left(u_{\mathrm{L} s c} C u_{\mathrm{L} t a}\right)\left(\bar{u}_{\mathrm{R} p}^{a} C \bar{u}_{\mathrm{R} r}^{b}\right)\left(\bar{u}_{\mathrm{L} v}^{c} u_{\mathrm{R} u b}\right) \\
& \mathcal{Y}[\overline{p \mid r}, \overline{s \mid t}]\left(u_{\mathrm{L} s a} C u_{\mathrm{L} t b}\right)\left(\bar{u}_{\mathrm{L}} e_{v}^{e} u_{\mathrm{R} u e}\right)\left(\bar{u}_{\mathrm{R} p}^{a} C \bar{u}_{\mathrm{R}}^{b}\right) \\
& \mathcal{Y}[\underline{p \mid r}, \underline{s \mid t]}]\left(\bar{u}_{\mathrm{R} r}^{b} u_{\mathrm{L} t a}\right)\left(\bar{u}_{\mathrm{R} p}^{a} u_{\mathrm{L} s c}\right)\left(\bar{u}_{\mathrm{L} v}^{c} u_{\mathrm{R} u b}\right) \\
& \mathcal{Y}\left[\overline{p \mid r}, s^{s \mid t}\right]\left(\bar{u}_{\mathrm{R} p}^{a} u_{\mathrm{L} s a}\right)\left(\bar{u}_{\mathrm{R}}^{b} u_{\mathrm{L} t b}\right)\left(\bar{u}_{\mathrm{L} v}^{e} u_{\mathrm{R} u e}\right) \\
& \mathcal{Y}\left[\begin{array}{l}
\frac{p}{r}, \\
,
\end{array}, \frac{s}{t}\right]\left(u_{\mathrm{L} s c} C u_{\mathrm{L} t a}\right)\left(\bar{u}_{\mathrm{R} p}^{a} C \bar{u}_{\mathrm{R} r}^{b}\right)\left(\bar{d}_{\mathrm{L} v}^{c} d_{\mathrm{R} u b}\right) \\
& \mathcal{Y}\left[\frac{p}{r}, \frac{s}{t}\right]\left(u_{\mathrm{L} s a} C u_{\mathrm{L} t b}\right)\left(\bar{d}_{\mathrm{L} v}^{e} d_{\mathrm{R} u e}\right)\left(\bar{u}_{\mathrm{R} p}^{a} C \bar{u}_{\mathrm{R}}^{b}\right) \\
& \mathcal{Y}\left[\frac{p}{r}, \frac{s}{t}\right]\left(\bar{u}_{\mathrm{R}}^{b} u_{\mathrm{L} t a}\right)\left(\bar{u}_{\mathrm{R}}^{a} u_{\mathrm{L} s c}\right)\left(\bar{d}_{\mathrm{L} v}^{c} d_{\mathrm{R} u b}\right) \\
& \mathcal{Y}\left[\frac{p}{r}, \frac{s}{t}\right]\left(\bar{u}_{\mathrm{R} p}^{a} u_{\mathrm{L} s a}\right)\left(\bar{u}_{\mathrm{R} r}^{b} u_{\mathrm{L} t b}\right)\left(\bar{d}_{\mathrm{L} v}^{e} d_{\mathrm{R} u e}\right)
\end{aligned}
$$

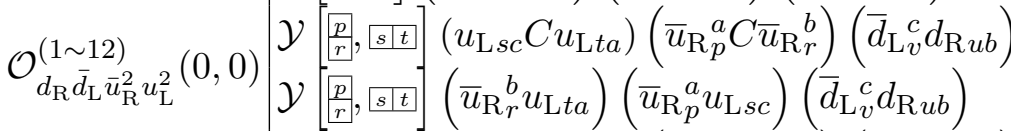

$$
\begin{aligned}
& \mathcal{Y}\left[p \mid r, \frac{s}{t}\right]\left(u_{\mathrm{L} s c} C u_{\mathrm{L} t a}\right)\left(\bar{u}_{\mathrm{R} p}^{a} C \bar{u}_{\mathrm{R} r}^{b}\right)\left(\bar{d}_{\mathrm{L} v}^{c} d_{\mathrm{R} u b}\right) \\
& \mathcal{Y}\left[p, r,\left[\begin{array}{l}
s \\
t
\end{array}\right]\left(\bar{u}_{\mathrm{R} r}^{b} u_{\mathrm{L} t a}\right)\left(\bar{u}_{\mathrm{R} p}^{a} u_{\mathrm{L} s c}\right)\left(\bar{d}_{\mathrm{L} v}^{c} d_{\mathrm{R} u b}\right)\right. \\
& \mathcal{Y}\left[[p|r, s| t]\left(u_{\mathrm{L} s c} C u_{\mathrm{L} t a}\right)\left(\bar{u}_{\mathrm{R}}^{a} C \bar{u}_{\mathrm{R} r}^{b}\right)\left(\bar{d}_{\mathrm{L} v}^{c} d_{\mathrm{R} u b}\right)\right. \\
& \mathcal{Y}\left[\overline{p \mid r}, s_{\mid c t}\right]\left(u_{\mathrm{L} s a} C u_{\mathrm{L} t b}\right)\left(\bar{d}_{\mathrm{L}}^{e} d_{\mathrm{R} u e}\right)\left(\bar{u}_{\mathrm{R} p}^{a} C \bar{u}_{\mathrm{R} r}^{b}\right) \\
& \mathcal{Y}\left[[p|r, s| t]\left(\bar{u}_{\mathrm{R}}^{b} u_{\mathrm{L} t a}\right)\left(\bar{u}_{\mathrm{R}}^{a} u_{\mathrm{L} s c}\right)\left(\bar{d}_{\mathrm{L} v}^{c} d_{\mathrm{R} u b}\right)\right. \\
& \mathcal{Y}[\overline{p \mid r}, s \mid t]]\left(\bar{u}_{\mathrm{R} p}^{a} u_{\mathrm{L} s a}\right)\left(\bar{u}_{\mathrm{R} r}^{b} u_{\mathrm{L} t b}\right)\left(\bar{d}_{\mathrm{L} v}^{e} d_{\mathrm{R} u e}\right)
\end{aligned}
$$




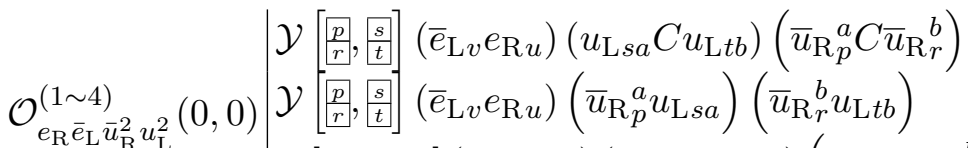

$$
\begin{aligned}
& \mathcal{Y}\left[\overline{p \mid r}, s^{s \mid t}\right]\left(\bar{e}_{\mathrm{L} v} e_{\mathrm{R} u}\right)\left(u_{\mathrm{L} s a} C u_{\mathrm{L} t b}\right)\left(\bar{u}_{\mathrm{R} p}^{a} C \bar{u}_{\mathrm{R} r}^{b}\right) \\
& \mathcal{Y}[\overline{p \mid r}, \overline{s \mid t}]\left(\bar{e}_{\mathrm{L} v} e_{\mathrm{R} u}\right)\left(\bar{u}_{\mathrm{R} p}^{a} u_{\mathrm{L} s a}\right)\left(\bar{u}_{\mathrm{R}}^{b} u_{\mathrm{L} t b}\right) \\
& \mathcal{Y}\left[\begin{array}{c}
s \\
\underline{t}
\end{array}\right]\left(u_{\mathrm{L} s c} C u_{\mathrm{L} t b}\right)\left(\bar{u}_{\mathrm{R} r}^{b} d_{\mathrm{L} p a}\right)\left(\bar{d}_{\mathrm{L} u}^{a} C \bar{u}_{\mathrm{L} v}^{c}\right)
\end{aligned}
$$

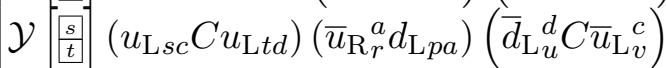

$$
\begin{aligned}
& \mathcal{Y}\left[\begin{array}{c}
s \\
t
\end{array}\right]\left(u_{\mathrm{L} s c} C u_{\mathrm{L} t b}\right)\left(\bar{u}_{\mathrm{R} r}^{b} d_{\mathrm{L} p a}\right)\left(\bar{d}_{\mathrm{L} u}^{c} C \bar{u}_{\mathrm{L} v}^{a}\right) \\
& \mathcal{Y}\left[\begin{array}{c}
s \\
\frac{s}{t}
\end{array}\right]\left(\bar{u}_{\mathrm{R} r}^{b} u_{\mathrm{L} t b}\right)\left(d_{\mathrm{L} p a} C u_{\mathrm{L} s c}\right)\left(\bar{d}_{\mathrm{L} u}^{a} C \bar{u}_{\mathrm{L} v}^{c}\right)
\end{aligned}
$$

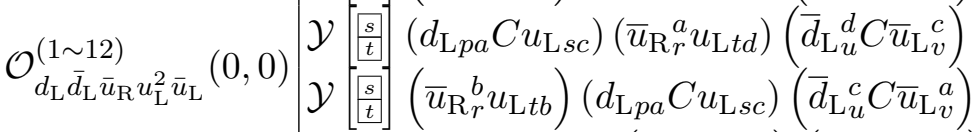

$$
\begin{aligned}
& \mathcal{Y}[s \mid t]\left(u_{\mathrm{L} s c} C u_{\mathrm{L} t b}\right)\left(\bar{u}_{\mathrm{R} r}^{b} d_{\mathrm{L} p a}\right)\left(\bar{d}_{\mathrm{L} u}^{a} C \bar{u}_{\mathrm{L} v}^{c}\right) \\
& \mathcal{Y}[s \mid t]\left(u_{\mathrm{L} s c} C u_{\mathrm{L} t d}\right)\left(\bar{u}_{\mathrm{R} r}{ }^{a} d_{\mathrm{L} p a}\right)\left(\bar{d}_{\mathrm{L} u}^{d} C \bar{u}_{\mathrm{L} v}^{c}\right) \\
& \mathcal{Y}[s \mid t]\left(u_{\mathrm{L} s c} C u_{\mathrm{L} t b}\right)\left(\bar{u}_{\mathrm{R} r}^{b} d_{\mathrm{L} p a}\right)\left(\bar{d}_{\mathrm{L} u}^{c} C \bar{u}_{\mathrm{L} v}^{a}\right) \\
& \mathcal{Y}[s \mid t]\left(\bar{u}_{\mathrm{R} r}^{b} u_{\mathrm{L} t b}\right)\left(d_{\mathrm{L} p a} C u_{\mathrm{L} s c}\right)\left(\bar{d}_{\mathrm{L} u}^{a} C \bar{u}_{\mathrm{L} v}^{c}\right) \\
& \mathcal{Y}[s \mid t]\left(d_{\mathrm{L} p a} C u_{\mathrm{L} s c}\right)\left(\bar{u}_{\mathrm{R} r}^{a} u_{\mathrm{L} t d}\right)\left(\bar{d}_{\mathrm{L} u}^{d} C \bar{u}_{\mathrm{L} v}^{c}\right) \\
& \mathcal{Y}[s \mid t]\left(\bar{u}_{\mathrm{R} r}^{b} u_{\mathrm{L} t b}\right)\left(d_{\mathrm{L} p a} C u_{\mathrm{L} s c}\right)\left(\bar{d}_{\mathrm{L} u}^{c} C \bar{u}_{\mathrm{L} v}^{a}\right) \\
& \mathcal{Y}\left[\begin{array}{c}
s \\
t
\end{array}\right]\left(u_{\mathrm{L} s c} C u_{\mathrm{L} t a}\right)\left(\bar{u}_{\mathrm{L} v}^{c} d_{\mathrm{R} u b}\right)\left(\bar{d}_{\mathrm{R}_{p}^{a} C \bar{u}_{\mathrm{R}}^{b}}^{b}\right)
\end{aligned}
$$

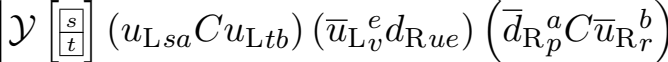

$$
\begin{aligned}
& \mathcal{Y}\left[\begin{array}{ll}
s \\
t
\end{array}\right]\left(u_{\mathrm{L} s c} C u_{\mathrm{L} t b}\right)\left(\bar{u}_{\mathrm{L} v}^{c} d_{\mathrm{R} u a}\right)\left(\bar{d}_{\mathrm{R} p}^{a} C \bar{u}_{\mathrm{R} r}^{b}\right) \\
& \mathcal{Y}\left[\begin{array}{c}
s \\
\hline t \\
\hline
\end{array}\right]\left(\bar{u}_{\mathrm{R}}^{b} u_{\mathrm{L} t a}\right)\left(\bar{d}_{\mathrm{R} p}^{a} u_{\mathrm{L} s c}\right)\left(\bar{u}_{\mathrm{L} v}^{c} d_{\mathrm{R} u b}\right)
\end{aligned}
$$

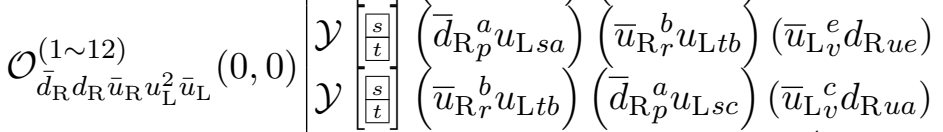

$$
\begin{aligned}
& \mathcal{Y}[s \mid t]\left(u_{\mathrm{L} s c} C u_{\mathrm{L} t a}\right)\left(\bar{u}_{\mathrm{L} v}^{c} d_{\mathrm{R} u b}\right)\left(\bar{d}_{\left.\mathrm{R}_{p}^{a} C \bar{u}_{\mathrm{R}}^{b}\right)}\right) \\
& \mathcal{Y}[s \mid t]\left(u_{\mathrm{L} s a} C u_{\mathrm{L} t b}\right)\left(\bar{u}_{\mathrm{L}}^{e} d_{\mathrm{R} u e}\right)\left(\bar{d}_{\mathrm{R} p}^{a} C \bar{u}_{\mathrm{R}}^{b}\right) \\
& \mathcal{Y}[s \mid t]\left(u_{\mathrm{L} s c} C u_{\mathrm{L} t b}\right)\left(\bar{u}_{\mathrm{L} v}^{c} d_{\mathrm{R} u a}\right)\left(\bar{d}_{\mathrm{R}_{p}^{a} C \bar{u}_{\mathrm{R}}^{b}}\right) \\
& \mathcal{Y}[s \mid t]\left(\bar{u}_{\mathrm{R}}^{b} u_{\mathrm{L} t a}\right)\left(\bar{d}_{\mathrm{R} p}^{a} u_{\mathrm{L} s c}\right)\left(\bar{u}_{\mathrm{L} v}^{c} d_{\mathrm{R} u b}\right) \\
& \mathcal{Y}[s \mid t]\left(\bar{d}_{\mathrm{R}}^{a} u_{\mathrm{L} s a}\right)\left(\bar{u}_{\mathrm{R}}^{b} u_{\mathrm{L} t b}\right)\left(\bar{u}_{\mathrm{L} v}^{e} d_{\mathrm{R} u e}\right) \\
& \mathcal{Y}[s \mid t]\left(\bar{u}_{\mathrm{R}}^{b} u_{\mathrm{L} t b}\right)\left(\bar{d}_{\mathrm{R} p}^{a} u_{\mathrm{L} s c}\right)\left(\bar{u}_{\mathrm{L} v}^{c} d_{\mathrm{R} u a}\right) \\
& \mathcal{Y}\left[\begin{array}{l}
s \\
t
\end{array}\right]\left(\bar{\nu}_{\mathrm{L} v} e_{\mathrm{R} u}\right)\left(u_{\mathrm{L} s a} C u_{\mathrm{L} t b}\right)\left(\bar{d}_{\mathrm{R} p}^{a} C \bar{u}_{\mathrm{R}}^{b}\right) \\
& \left.\mathcal{O}_{\bar{d}_{\mathrm{R}} e_{\mathrm{R}} \bar{\nu}_{\mathrm{L}} \bar{u}_{\mathrm{R}} u_{\mathrm{L}}^{2}}^{(\sim, 0)}\right) \mathcal{Y}\left[\begin{array}{l}
\bar{s} \\
\bar{t}
\end{array}\right]\left(\bar{\nu}_{\mathrm{L} v} e_{\mathrm{R} u}\right)\left(\bar{d}_{\mathrm{R}}^{a} u_{\mathrm{L} s a}\right)\left(\bar{u}_{\mathrm{R}}^{b} u_{\mathrm{L} t b}\right) \\
& \mathcal{Y}[s \mid t]\left(\bar{\nu}_{\mathrm{L} v} e_{\mathrm{R} u}\right)\left(u_{\mathrm{L} s a} C u_{\mathrm{L} t b}\right)\left(\bar{d}_{\mathrm{R} p}^{a} C \bar{u}_{\mathrm{R}}^{b}\right) \\
& \mathcal{Y}[s \mid t]\left(\bar{\nu}_{\mathrm{L} v} e_{\mathrm{R} u}\right)\left(\bar{d}_{\mathrm{R} p}^{a} u_{\mathrm{L} s a}\right)\left(\bar{u}_{\mathrm{R} r}^{b} u_{\mathrm{L} t b}\right)
\end{aligned}
$$




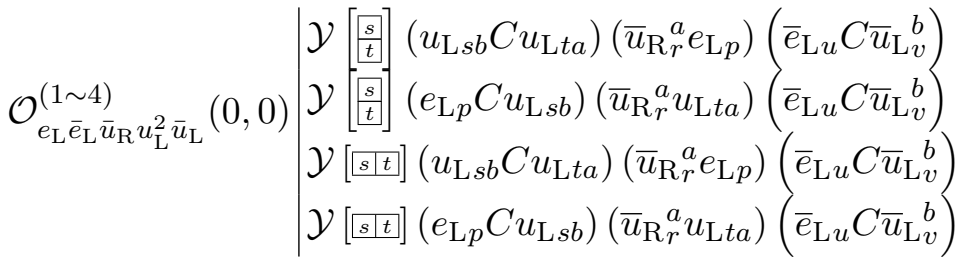

$$
\begin{aligned}
& \mathcal{Y}\left[\begin{array}{l}
s \\
t
\end{array}\right]\left(u_{\mathrm{L} s b} C u_{\mathrm{L} t a}\right)\left(\bar{u}_{\mathrm{R} r}^{a} e_{\mathrm{L} p}\right)\left(\bar{d}_{\mathrm{L} u}^{b} C \bar{\nu}_{\mathrm{L} v}\right)
\end{aligned}
$$

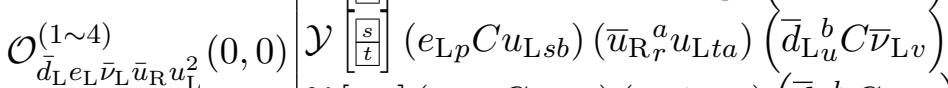

$$
\begin{aligned}
& \mathcal{Y}[s \mid t]]\left(u_{\mathrm{L} s b} C u_{\mathrm{L} t a}\right)\left(\bar{u}_{\mathrm{R} r}^{a} e_{\mathrm{L} p}\right)\left(\bar{d}_{\mathrm{L} u}^{b} C \bar{\nu}_{\mathrm{L} v}\right) \\
& \mathcal{Y}[s \mid t]\left(e_{\mathrm{L} p} C u_{\mathrm{L} s b}\right)\left(\bar{u}_{\mathrm{R} r}^{a} u_{\mathrm{L} t a}\right)\left(\bar{d}_{\mathrm{L} u}^{b} C \bar{\nu}_{\mathrm{L} v}\right) \\
& \mathcal{Y}\left[\begin{array}{c}
{[s} \\
t
\end{array}\right]\left(u_{\mathrm{L} s b} C u_{\mathrm{L} t a}\right)\left(\bar{u}_{\mathrm{L} v}^{b} e_{\mathrm{R} u}\right)\left(\bar{e}_{\mathrm{R} p} C \bar{u}_{\mathrm{R}}^{a}\right)
\end{aligned}
$$

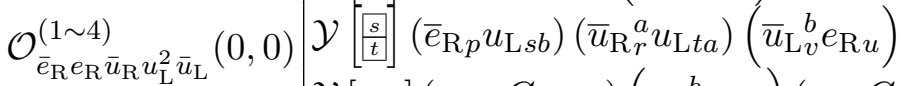

$$
\begin{aligned}
& \mathcal{Y}[s \mid t]\left(u_{\mathrm{L} s b} C u_{\mathrm{L} t a}\right)\left(\bar{u}_{\mathrm{L} v}^{b} e_{\mathrm{R} u}\right)\left(\bar{e}_{\mathrm{R} p} C \bar{u}_{\mathrm{R} r}^{a}\right) \\
& \mathcal{Y}[s \mid t]\left(\bar{e}_{\mathrm{R} p} u_{\mathrm{L} s b}\right)\left(\bar{u}_{\mathrm{R} r}^{a} u_{\mathrm{L} t a}\right)\left(\bar{u}_{\mathrm{L} v}^{b} e_{\mathrm{R} u}\right)
\end{aligned}
$$

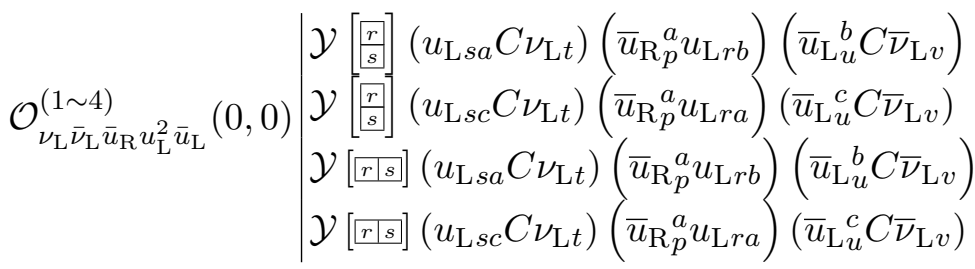

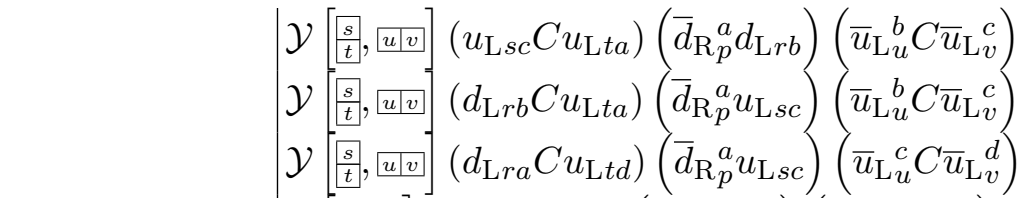

$$
\begin{aligned}
& \mathcal{Y}\left[\begin{array}{c}
\frac{s}{t}, \\
\frac{u}{v} \\
v
\end{array}\right]\left(u_{\mathrm{L} s c} C u_{\mathrm{L} t a}\right)\left(\bar{d}_{\mathrm{R} p}^{a} d_{\mathrm{L} r b}\right)\left(\bar{u}_{\mathrm{L} u}^{b} C \bar{u}_{\mathrm{L} v}^{c}\right)
\end{aligned}
$$

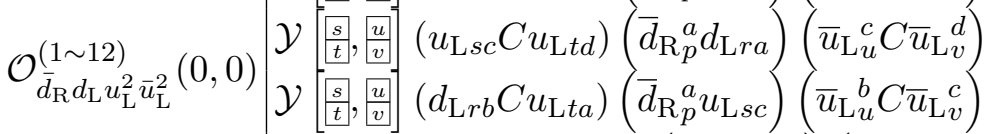

$$
\begin{aligned}
& \mathcal{Y}[\overline{s \mid t}, \text { u } u v]\left(u_{\mathrm{L} s c} C u_{\mathrm{L} t a}\right)\left(\bar{d}_{\mathrm{R} p}^{a} d_{\mathrm{L} r b}\right)\left(\bar{u}_{\mathrm{L} u}^{b} C \bar{u}_{\mathrm{L} v}^{c}\right) \\
& \mathcal{Y}[s \mid t, u, v]\left(u_{\mathrm{L} s c} C u_{\mathrm{L} t d}\right)\left(\bar{d}_{\mathrm{R} p}^{a} d_{\mathrm{L} r a}\right)\left(\bar{u}_{\mathrm{L} u}^{c} C \bar{u}_{\mathrm{L} v}^{d}\right) \\
& \mathcal{Y}\left[s \underline{s \mid t, u v v]}\left(d_{\mathrm{L} r b} C u_{\mathrm{L} t a}\right)\left(\bar{d}_{\mathrm{R} p}^{a} u_{\mathrm{L} s c}\right)\left(\bar{u}_{\mathrm{L} u}^{b} C \bar{u}_{\mathrm{L} v}^{c}\right)\right. \\
& \mathcal{Y}\left[s \mid t, \frac{u}{v}\right]\left(u_{\mathrm{L} s c} C u_{\mathrm{L} t a}\right)\left(\bar{d}_{\mathrm{R} p}^{a} d_{\mathrm{L} r b}\right)\left(\bar{u}_{\mathrm{L} u}^{b} C \bar{u}_{\mathrm{L} v}^{c}\right) \\
& \mathcal{Y}\left[s \mid t, \frac{u}{v}\right]\left(d_{\mathrm{L} r b} C u_{\mathrm{L} t a}\right)\left(\bar{d}_{\mathrm{R} p}^{a} u_{\mathrm{L} s c}\right)\left(\bar{u}_{\mathrm{L} u}^{b} C \bar{u}_{\mathrm{L} v}^{c}\right) \\
& \mathcal{Y}[s \mid t, \underline{u} v]\left(d_{\mathrm{L} r a} C u_{\mathrm{L} t d}\right)\left(\bar{d}_{\mathrm{R} p}^{a} u_{\mathrm{L} s c}\right)\left(\bar{u}_{\mathrm{L} u}^{c} C \bar{u}_{\mathrm{L} v}^{d}\right) \\
& \mathcal{Y}\left[\begin{array}{c}
s \\
t
\end{array}\right]\left(u_{\mathrm{L} s b} C u_{\mathrm{L} t a}\right)\left(\bar{d}_{\mathrm{R} p}^{a} e_{\mathrm{L} r}\right)\left(\bar{u}_{\mathrm{L} u}^{b} C \bar{\nu}_{\mathrm{L} v}\right)
\end{aligned}
$$

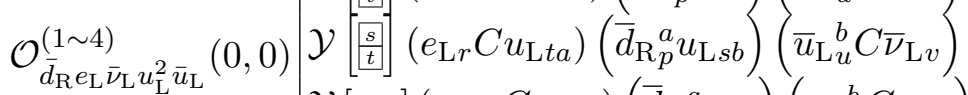

$$
\begin{aligned}
& \mathcal{Y}[s \mid t]\left(u_{\mathrm{L} s b} C u_{\mathrm{L} t a}\right)\left(\bar{d}_{\mathrm{R} p}^{a} e_{\mathrm{L} r}\right)\left(\bar{u}_{\mathrm{L} u}^{b} C \bar{\nu}_{\mathrm{L} v}\right) \\
& \mathcal{Y}[s \mid t]]\left(e_{\mathrm{L} r} C u_{\mathrm{L} t a}\right)\left(\bar{d}_{\mathrm{R} p}^{a} u_{\mathrm{L} s b}\right)\left(\bar{u}_{\mathrm{L} u}^{b} C \bar{\nu}_{\mathrm{L} v}\right)
\end{aligned}
$$




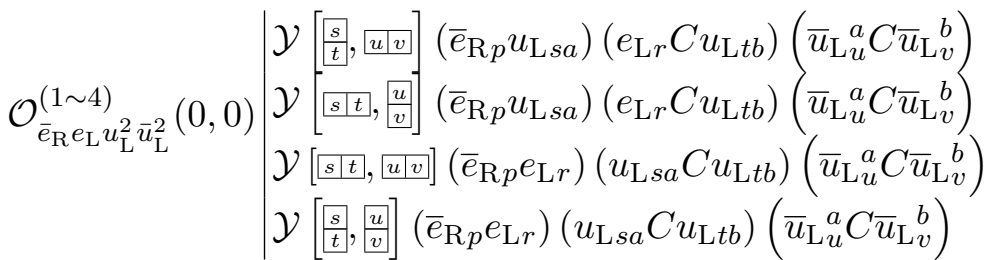

$$
\begin{aligned}
& \mid \mathcal{Y}\left[\frac{p r r}{s}, u v\right] .\left(u_{\mathrm{R} u b} C u_{\mathrm{R} v c}\right)\left(\bar{u}_{\mathrm{R} p}^{a} C \bar{u}_{\mathrm{R} r}^{b}\right)\left(\bar{u}_{\mathrm{R}}^{c}{ }_{s}^{c} u_{\mathrm{L} t a}\right) \\
& \mathcal{Y}\left[\frac{p r r}{s}, u \mid v\right]=\left(u_{\mathrm{R} u b} C u_{\mathrm{R} v a}\right)\left(\bar{u}_{\mathrm{R}}^{c}{ }_{s}^{c} u_{\mathrm{L} t c}\right)\left(\bar{u}_{\mathrm{R}}^{a} C \bar{u}_{\mathrm{R} r}^{b}\right) \\
& \mathcal{O}_{\bar{u}_{\mathrm{R}}^{3} u_{\mathrm{R}}^{2} u_{\mathrm{L}}}^{(1 \sim 6)}(0,0) \mathcal{Y}[\overline{p|r| s}, \overline{u \mid v}]\left(u_{\mathrm{R} u b} C u_{\mathrm{R} v c}\right)\left(\bar{u}_{\mathrm{R} p}^{a} C \bar{u}_{\mathrm{R} r}^{b}\right)\left(\bar{u}_{\mathrm{R}}^{c} u_{\mathrm{L} t a}\right) \\
& \mathcal{Y}\left[\frac{[p|r| s,}{v}, \frac{u}{v}\right]\left(u_{\mathrm{R} u b} C u_{\mathrm{R} v c}\right)\left(\bar{u}_{\mathrm{R} p}^{a} C \bar{u}_{\mathrm{R} r}^{b}\right)\left(\bar{u}_{\mathrm{R}}^{c} u_{\mathrm{L} t a}\right) \\
& \left.\mathcal{Y} \frac{p r r}{s,}, \frac{u}{v}\right]\left(u_{\mathrm{R} u b} C u_{\mathrm{R} v c}\right)\left(\bar{u}_{\mathrm{R}}^{a} C \bar{u}_{\mathrm{R}}^{b}\right)\left(\bar{u}_{\mathrm{R}}^{c} u_{\mathrm{L} t a}\right) \\
& \mathcal{Y}\left[\frac{p r r}{s}, \underline{u} v\right]\left(u_{\mathrm{R} u b} C u_{\mathrm{R} v a}\right)\left(\bar{u}_{\mathrm{R}}^{c} u_{\mathrm{L} t c}\right)\left(\bar{u}_{\mathrm{R} p}^{a} C \bar{u}_{\mathrm{R} r}^{b}\right) \\
& \mathcal{Y}\left[\frac{r}{s}\right]\left(\bar{u}_{\mathrm{R} r}^{b} d_{\mathrm{L} p a}\right)\left(\bar{d}_{\mathrm{L} u}^{a} u_{\mathrm{R} v c}\right)\left(\bar{u}_{\mathrm{R}}^{c} u_{\mathrm{L} t b}\right) \\
& \mathcal{Y}\left[\frac{p}{s}\right]\left(\bar{u}_{\mathrm{R} r}^{a} d_{\mathrm{L} p a}\right)\left(\bar{d}_{\mathrm{L} u}^{d} u_{\mathrm{R} v c}\right)\left(\bar{u}_{\mathrm{R}}^{c}{ }_{s} u_{\mathrm{L} t d}\right) \\
& \mathcal{Y}\left[\begin{array}{l}
\frac{r}{s} \\
\hline
\end{array}\left(\bar{d}_{\mathrm{L} u}^{e} u_{\mathrm{R} v e}\right)\left(\bar{u}_{\mathrm{R} r}^{b} d_{\mathrm{L} p a}\right)\left(\bar{u}_{\mathrm{R}}^{a} u_{\mathrm{L} t b}\right)\right.
\end{aligned}
$$

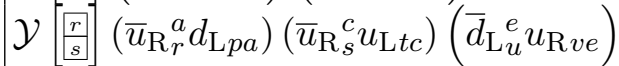

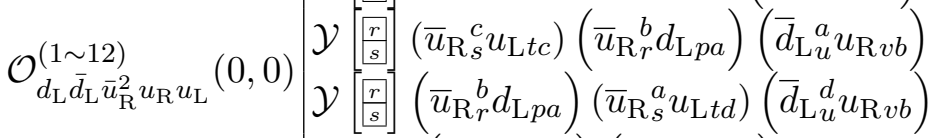

$$
\begin{aligned}
& \mathcal{Y}[r r s]\left(\bar{u}_{\mathrm{R}}^{b} d_{\mathrm{L} p a}\right)\left(\bar{d}_{\mathrm{L} u}^{a} u_{\mathrm{R} v c}\right)\left(\bar{u}_{\left.\mathrm{R}_{s}^{c} u_{\mathrm{L} t b}\right)}\right. \\
& \mathcal{Y}[r r s]\left(\bar{u}_{\mathrm{R} r}^{a} d_{\mathrm{L} p a}\right)\left(\bar{d}_{\mathrm{L} u}^{d} u_{\mathrm{R} v c}\right)\left(\bar{u}_{\mathrm{R} s}^{c} u_{\mathrm{L} t d}\right) \\
& \mathcal{Y}[\overline{r r s}]\left(\bar{d}_{\mathrm{L} u}^{e} u_{\mathrm{R} v e}\right)\left(\bar{u}_{\mathrm{R} r}^{b} d_{\mathrm{L} p a}\right)\left(\bar{u}_{\mathrm{R} s}^{a} u_{\mathrm{L} t b}\right) \\
& \mathcal{Y}[\overline{r r s}]\left(\bar{u}_{\mathrm{R}}{ }_{r}^{a} d_{\mathrm{L} p a}\right)\left(\bar{u}_{\mathrm{R}}^{c}{ }_{s} u_{\mathrm{L} t c}\right)\left(\bar{d}_{\mathrm{L} u}^{e} u_{\mathrm{R} v e}\right) \\
& \mathcal{Y}[r r s]\left(\bar{u}_{\mathrm{R} s}^{c} u_{\mathrm{L} t c}\right)\left(\bar{u}_{\mathrm{R}}{ }_{r}^{b} d_{\mathrm{L} p a}\right)\left(\bar{d}_{\mathrm{L} u}^{a} u_{\mathrm{R} v b}\right) \\
& \mathcal{Y}[\overline{r r s}]\left(\bar{u}_{\mathrm{R} r}^{b} d_{\mathrm{L} p a}\right)\left(\bar{u}_{\mathrm{R} s}^{a} u_{\mathrm{L} t d}\right)\left(\bar{d}_{\mathrm{L} u}^{d} u_{\mathrm{R} v b}\right)
\end{aligned}
$$

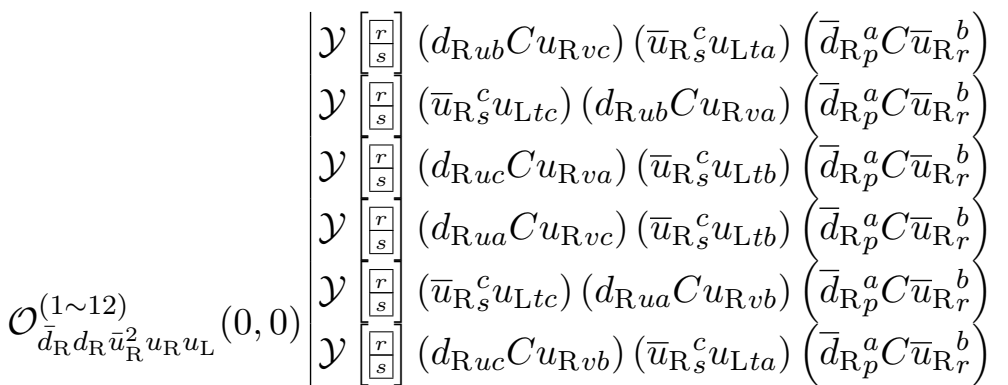

$$
\begin{aligned}
& \mathcal{Y}[r r s]\left(d_{\mathrm{R} u b} C u_{\mathrm{R} v c}\right)\left(\bar{u}_{\mathrm{R}}{ }_{s}^{c} u_{\mathrm{L} t a}\right)\left(\bar{d}_{\mathrm{R}_{p}^{a}}^{a} C \bar{u}_{\mathrm{R}}^{b}\right) \\
& \mathcal{Y}[\overline{r r s}]\left(\bar{u}_{\mathrm{R}}^{c} u_{\mathrm{L} t c}\right)\left(d_{\mathrm{R} u b} C u_{\mathrm{R} v a}\right)\left(\bar{d}_{\mathrm{R}_{p}^{a} C \bar{u}_{\mathrm{R}}^{b}}^{b}\right) \\
& \mathcal{Y}\left[\overline{r s s]}\left(d_{\mathrm{R} u c} C u_{\mathrm{R} v a}\right)\left(\bar{u}_{\mathrm{R}}^{c}{ }_{s} u_{\mathrm{L} t b}\right)\left(\bar{d}_{\mathrm{R} p}^{a} C \bar{u}_{\mathrm{R}}^{b}\right)\right. \\
& \mathcal{Y}[\overline{r r s}]\left(d_{\mathrm{R} u a} C u_{\mathrm{R} v c}\right)\left(\bar{u}_{\mathrm{R}}{ }_{s}^{c} u_{\mathrm{L} t b}\right)\left(\bar{d}_{\mathrm{R} p}^{a} C \bar{u}_{\mathrm{R}}^{b}\right) \\
& \mathcal{Y}[r \mid s]\left(\bar{u}_{\mathrm{R}}^{c} u_{\mathrm{L} t c}\right)\left(d_{\mathrm{R} u a} C u_{\mathrm{R} v b}\right)\left(\bar{d}_{\mathrm{R}_{p}^{a}} C \bar{u}_{\mathrm{R}}^{b}\right) \\
& \mathcal{Y}\left[\overline{r r s]}\left(d_{\mathrm{R} u c} C u_{\mathrm{R} v b}\right)\left(\bar{u}_{\mathrm{R}}^{c} u_{\mathrm{L} t a}\right)\left(\bar{d}_{\mathrm{R}}^{a} C \bar{u}_{\mathrm{R}}^{b}\right)\right.
\end{aligned}
$$




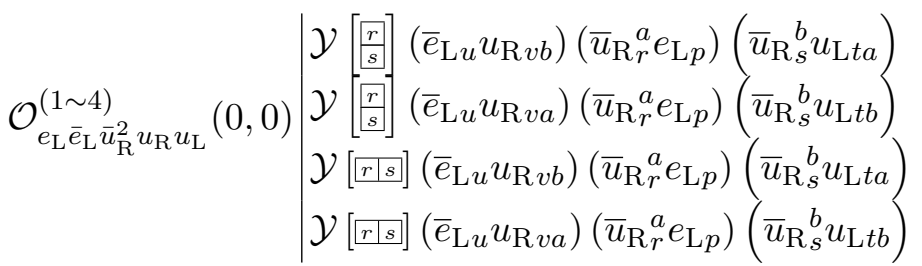

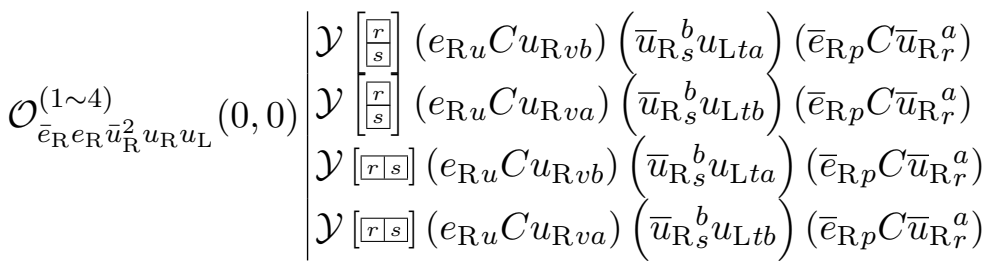

$$
\begin{aligned}
& \mathcal{Y}\left[\begin{array}{l}
p \\
r
\end{array}\right]\left(\bar{\nu}_{\mathrm{L} v} u_{\mathrm{R} u b}\right)\left(u_{\mathrm{L} s a} C \nu_{\mathrm{L} t}\right)\left(\bar{u}_{\mathrm{R} p}^{a} C \bar{u}_{\mathrm{R} r}^{b}\right) \\
& \mathcal{O}_{\nu_{\mathrm{L}} \bar{\nu}_{\mathrm{L}} \bar{u}_{\mathrm{R}}^{2} u_{\mathrm{R}} u_{\mathrm{L}}}^{(1 \sim, 0)} \mathcal{Y}\left[\begin{array}{l}
{\left[\frac{p}{r}\right.} \\
\frac{\pi}{r}
\end{array}\right]\left(\bar{\nu}_{\mathrm{L} v} u_{\mathrm{R} u b}\right)\left(\bar{u}_{\mathrm{R}}^{a} u_{\mathrm{L} s a}\right)\left(\bar{u}_{\mathrm{R}}^{b} \nu_{\mathrm{L} t}\right) \\
& \mathcal{Y}[\overline{p r r}]\left(\bar{\nu}_{\mathrm{L} v} u_{\mathrm{R} u b}\right)\left(u_{\mathrm{L} s a} C \nu_{\mathrm{L} t}\right)\left(\bar{u}_{\mathrm{R} p}^{a} C \bar{u}_{\mathrm{R} r}^{b}\right) \\
& \mathcal{Y}[\overline{p p r}]\left(\bar{\nu}_{\mathrm{L} v} u_{\mathrm{R} u b}\right)\left(\bar{u}_{\mathrm{R} p}^{a} u_{\mathrm{L} s a}\right)\left(\bar{u}_{\mathrm{R}}^{b} \nu_{\mathrm{L} t}\right) \\
& \mathcal{Y}\left[\frac{p}{r}\right]\left(\bar{e}_{\mathrm{L} v} d_{\mathrm{R} u b}\right)\left(u_{\mathrm{L} s a} C \nu_{\mathrm{L} t}\right)\left(\bar{u}_{\mathrm{R} p}^{a} C \bar{u}_{\mathrm{R} r}^{b}\right) \\
& \mathcal{O}_{d_{\mathrm{R}} \bar{e}_{\mathrm{L}} \nu_{\mathrm{L}} \bar{u}_{\mathrm{R}}^{2} u_{\mathrm{L}}}^{(1 \sim 4)^{\prime}}(0,0) \mathcal{Y}\left[\frac{p}{r}\right]\left(\bar{e}_{\mathrm{L} v} d_{\mathrm{R} u b}\right)\left(\bar{u}_{\mathrm{R} p}^{a} u_{\mathrm{L} s a}\right)\left(\bar{u}_{\mathrm{R} r}^{b} \nu_{\mathrm{L} t}\right) \\
& \mathcal{Y}[\overline{p \mid r]}]\left(\bar{e}_{\mathrm{L} v} d_{\mathrm{R} u b}\right)\left(u_{\mathrm{L} s a} C \nu_{\mathrm{L} t}\right)\left(\bar{u}_{\mathrm{R} p}^{a} C \bar{u}_{\mathrm{R} r}^{b}\right) \\
& \mathcal{Y}[\overline{p p r}]\left(\bar{e}_{\mathrm{L} v} d_{\mathrm{R} u b}\right)\left(\bar{u}_{\mathrm{R} p}^{a} u_{\mathrm{L} s a}\right)\left(\bar{u}_{\mathrm{R} r}^{b} \nu_{\mathrm{L} t}\right) \\
& \mid \mathcal{Y}\left[\frac{p}{r}, u v v\right]\left(d_{\mathrm{L} p a} C d_{\mathrm{L} r b}\right)\left(\bar{u}_{\mathrm{R} s}^{a} u_{\mathrm{L} t d}\right)\left(\bar{d}_{\mathrm{L} u}^{b} C \bar{d}_{\mathrm{L} v}^{d}\right) \\
& \mathcal{Y}\left[\frac{p}{r}, u|v|\left(d_{\mathrm{L} r b} C u_{\mathrm{L} t c}\right)\left(\bar{d}_{\mathrm{L} u}^{b} C \bar{d}_{\mathrm{L} v}^{a}\right)\left(\bar{u}_{\mathrm{R} s}^{c} d_{\mathrm{L} p a}\right)\right. \\
& \mathcal{Y}\left[\frac{p}{r}, u v v\right]\left(\bar{u}_{\mathrm{R}}^{a} d_{\mathrm{L} p a}\right)\left(d_{\mathrm{L} r b} C u_{\mathrm{L} t d}\right)\left(\bar{d}_{\mathrm{L} u}^{b} C \bar{d}_{\mathrm{L} v}^{d}\right) \\
& \mathcal{Y}\left[\frac{p}{r}, \frac{u}{v}\right]\left(d_{\mathrm{L} p a} C d_{\mathrm{L} r b}\right)\left(\bar{u}_{\mathrm{R} s}^{c} u_{\mathrm{L} t c}\right)\left(\bar{d}_{\mathrm{L} u}^{b} C \bar{d}_{\mathrm{L} v}^{a}\right)
\end{aligned}
$$

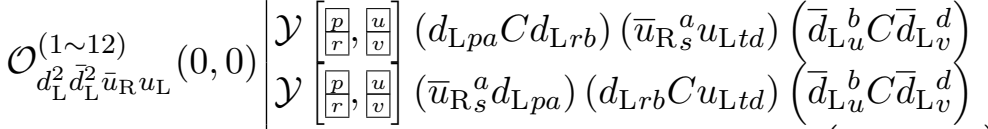

$$
\begin{aligned}
& \mathcal{Y}\left[\underline{p \mid r}, \underline{u v v]}\left(d_{\mathrm{L} p a} C d_{\mathrm{L} r b}\right)\left(\bar{u}_{\mathrm{R}}^{c}{ }_{s} u_{\mathrm{L} t c}\right)\left(\bar{d}_{\mathrm{L} u}^{b} C \bar{d}_{\mathrm{L} v}^{a}\right)\right. \\
& \mathcal{Y}[\overline{p r r}, u, v]\left(d_{\mathrm{L} p a} C d_{\mathrm{L} r b}\right)\left(\bar{u}_{\mathrm{R} s}^{a} u_{\mathrm{L} t d}\right)\left(\bar{d}_{\mathrm{L} u}^{b} C \bar{d}_{\mathrm{L} v}^{d}\right) \\
& \mathcal{Y}\left[\overline{p r r}, \underline{u v v]}\left(\bar{u}_{\mathrm{R} s}^{a} d_{\mathrm{L} p a}\right)\left(d_{\mathrm{L} r b} C u_{\mathrm{L} t d}\right)\left(\bar{d}_{\mathrm{L} u}^{b} C \bar{d}_{\mathrm{L} v}^{d}\right)\right. \\
& \mathcal{Y}\left[p \mid r,, \frac{u}{v}\right]\left(d_{\mathrm{L} p a} C d_{\mathrm{L} r b}\right)\left(\bar{u}_{\mathrm{R}}{ }_{s} u_{\mathrm{L} t d}\right)\left(\bar{d}_{\mathrm{L} u}^{b} C \bar{d}_{\mathrm{L} v}{ }^{d}\right) \\
& \mathcal{Y}\left[\overline{p p r}, \frac{u}{v}\right]\left(d_{\mathrm{L} r b} C u_{\mathrm{L} t c}\right)\left(\bar{d}_{\mathrm{L} u}^{b} C \bar{d}_{\mathrm{L} v}^{a}\right)\left(\bar{u}_{\mathrm{R} s}^{c} d_{\mathrm{L} p a}\right) \\
& \mathcal{Y}\left[\underline{p p r}, \underline{u}, \frac{u}{v}\right]\left(\bar{u}_{\mathrm{R} s}^{a} d_{\mathrm{L} p a}\right)\left(d_{\mathrm{L} r b} C u_{\mathrm{L} t d}\right)\left(\bar{d}_{\mathrm{L} u}^{b} C \bar{d}_{\mathrm{L} v}^{d}\right)
\end{aligned}
$$




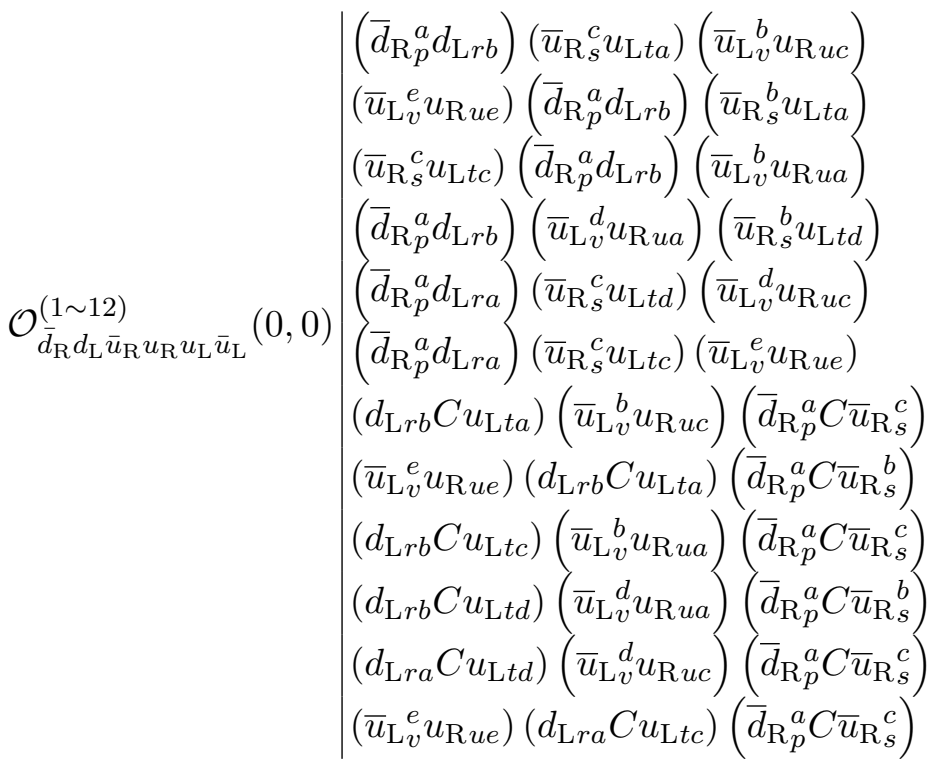

$$
\begin{aligned}
& \mid \begin{array}{l}
\left(\bar{d}_{\mathrm{R}}^{a} d_{\mathrm{L} r b}\right)\left(\bar{u}_{\mathrm{R}}^{c}{ }_{s}^{c} u_{\mathrm{L} t a}\right)\left(\bar{d}_{\mathrm{L} v}^{b} d_{\mathrm{R} u c}\right) \\
\left(\bar{d}_{\mathrm{L}}^{e} d_{\mathrm{R} u e}\right)\left(\bar{d}_{\mathrm{R} p}^{a} d_{\mathrm{L} r b}\right)\left(\bar{u}_{\mathrm{R} s}^{b} u_{\mathrm{L} t a}\right)
\end{array} \\
& \left(\bar{u}_{\mathrm{R} s}^{c} u_{\mathrm{L} t c}\right)\left(\bar{d}_{\mathrm{R} p}^{a} d_{\mathrm{L} r b}\right)\left(\bar{d}_{\mathrm{L} v}^{b} d_{\mathrm{R} u a}\right) \\
& \left(\bar{d}_{\mathrm{R} p}^{a} d_{\mathrm{L} r b}\right)\left(\bar{d}_{\mathrm{L} v}^{d} d_{\mathrm{R} u a}\right)\left(\bar{u}_{\mathrm{R} s}^{b} u_{\mathrm{L} t d}\right) \\
& \mathcal{O}_{\bar{d}_{\mathrm{R}} d_{\mathrm{R}} d_{\mathrm{L}} \bar{d}_{\mathrm{L}} \bar{u}_{\mathrm{R}} u_{\mathrm{L}}}^{(12,0)}(0)\left(\begin{array}{ll}
\left(\bar{d}_{\mathrm{R} p}^{a} d_{\mathrm{L} r a}\right) & \left(\bar{d}_{\mathrm{L} v}^{d} d_{\mathrm{R} u c}\right)\left(\bar{u}_{\mathrm{R}}^{c}{ }_{s} u_{\mathrm{L} t d}\right) \\
\left(\bar{d}_{\mathrm{R} p}^{a} d_{\mathrm{L} r a}\right) & \left(\bar{u}_{\mathrm{R}}{ }^{c} u_{\mathrm{L} t c}\right)\left(\bar{d}_{\mathrm{L} v}^{e} d_{\mathrm{R} u e}\right)
\end{array}\right. \\
& \left(d_{\mathrm{L} r b} C u_{\mathrm{L} t a}\right)\left(\bar{d}_{\mathrm{L} v}^{b} d_{\mathrm{R} u c}\right)\left(\bar{d}_{\mathrm{R} p}^{a} C \bar{u}_{\mathrm{R} s}^{c}\right) \\
& \left(\bar{d}_{\mathrm{L} v}^{e} d_{\mathrm{R} u e}\right)\left(d_{\mathrm{L} r b} C u_{\mathrm{L} t a}\right)\left(\bar{d}_{\mathrm{R} p}^{a} C \bar{u}_{\mathrm{R} s}^{b}\right) \\
& \left(d_{\mathrm{L} r b} C u_{\mathrm{L} t c}\right)\left(\bar{d}_{\mathrm{L} v}^{b} d_{\mathrm{R} u a}\right)\left(\bar{d}_{\mathrm{R} p}^{a} C \bar{u}_{\mathrm{R}}^{c}\right) \\
& \left(d_{\mathrm{Lrb}} C u_{\mathrm{L} t d}\right)\left(\bar{d}_{\mathrm{L} v}^{d} d_{\mathrm{R} u a}\right)\left(\bar{d}_{\mathrm{R} p}^{a} C \bar{u}_{\mathrm{R} s}^{b}\right) \\
& \left(d_{\mathrm{L} r a} C u_{\mathrm{L} t d}\right)\left(\bar{d}_{\mathrm{L} v}^{d} d_{\mathrm{R} u c}\right)\left(\bar{d}_{\mathrm{R} p}^{a} C \bar{u}_{\mathrm{R} s}^{c}\right) \\
& \left(\bar{d}_{\mathrm{L} v}^{e} d_{\mathrm{R} u e}\right)\left(d_{\mathrm{L} r a} C u_{\mathrm{L} t c}\right)\left(\bar{d}_{\mathrm{R} p}^{a} C \bar{u}_{\mathrm{R}}^{c}\right) \\
& \left.\mathcal{O}_{\bar{d}_{\mathrm{R}} d_{\mathrm{L}} e_{\mathrm{R}} \bar{e}_{\mathrm{L}} \bar{u}_{\mathrm{R}} u_{\mathrm{L}}}^{(1 \sim, 0)} \mid \begin{array}{l}
\left(\bar{e}_{\mathrm{L} v} e_{\mathrm{R} u}\right)\left(\bar{d}_{\mathrm{R} p}^{a} d_{\mathrm{L} r b}\right)\left(\bar{u}_{\mathrm{R}}^{b} u_{\mathrm{L} t a}\right) \\
\left(\bar{e}_{\mathrm{L} v} e_{\mathrm{R} u}\right)\left(\bar{d}_{\mathrm{R}}^{a} d_{\mathrm{L} r a}\right)\left(\bar{u}_{\mathrm{R}}^{c}{ }_{s} u_{\mathrm{L} t c}\right) \\
\left(\bar{e}_{\mathrm{L} v} e_{\mathrm{R} u}\right)\left(d_{\mathrm{L} r b} C u_{\mathrm{L} t a}\right)\left(\bar{d}_{\mathrm{R} p}^{a} C \bar{u}_{\mathrm{R} s}^{b}\right) \\
\left(\bar{e}_{\mathrm{L} v} e_{\mathrm{R} u}\right)\left(d_{\mathrm{L} r a} C u_{\mathrm{L} t c}\right)\left(\bar{d}_{\mathrm{R}}^{a} C \bar{u}_{\mathrm{R}}^{c}{ }_{s}^{c}\right.
\end{array}\right)
\end{aligned}
$$

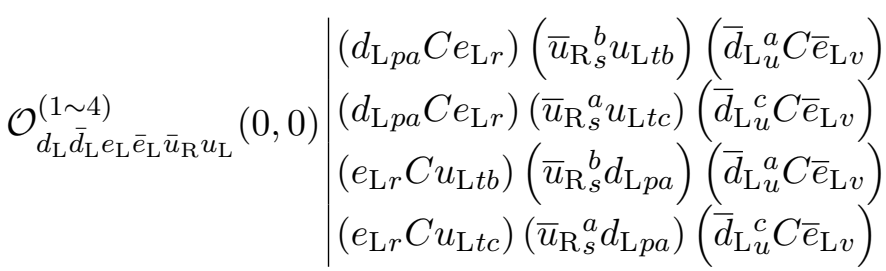




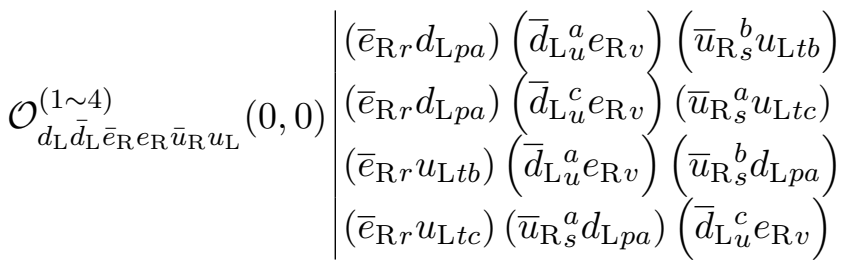

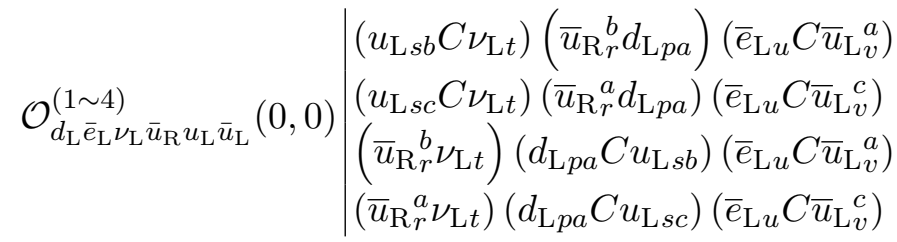

$$
\begin{aligned}
& \mathcal{O}_{d_{\mathrm{L}} \bar{d}_{\mathrm{L}} \nu_{\mathrm{L}} \bar{\nu}_{\mathrm{L}} \bar{u}_{\mathrm{R}} u_{\mathrm{L}}}^{(10,0)} \mid \begin{array}{l}
\left(u_{\mathrm{L} s b} C \nu_{\mathrm{L} t}\right)\left(\bar{u}_{\mathrm{R} r}^{b} d_{\mathrm{L} p a}\right)\left(\bar{d}_{\mathrm{L} u}^{a} C \bar{\nu}_{\mathrm{L} v}\right) \\
\left(u_{\mathrm{L} s c} C \nu_{\mathrm{L} t}\right)\left(\bar{u}_{\mathrm{R} r}^{a} d_{\mathrm{L} p a}\right)\left(\bar{d}_{\mathrm{L} u}^{c} C \bar{\nu}_{\mathrm{L} v}\right) \\
\left(\bar{u}_{\mathrm{R} r}^{b} \nu_{\mathrm{L} t}\right)\left(d_{\mathrm{L} p a} C u_{\mathrm{L} s b}\right)\left(\bar{d}_{\mathrm{L} u}^{a} C \bar{\nu}_{\mathrm{L} v}\right) \\
\left(\bar{u}_{\mathrm{R} r}^{a} \nu_{\mathrm{L} t}\right)\left(d_{\mathrm{L} p a} C u_{\mathrm{L} s c}\right)\left(\bar{d}_{\mathrm{L} u}^{c} C \bar{\nu}_{\mathrm{L} v}\right)
\end{array} \\
& \mathcal{Y}\left[\frac{p}{r}, \overline{u v}\right]\left(d_{\mathrm{R} u b} C d_{\mathrm{R} v c}\right)\left(\bar{d}_{\mathrm{R}}^{a} C \bar{d}_{\mathrm{R}}{ }_{r}^{b}\right)\left(\bar{u}_{\mathrm{R}}^{c}{ }_{s}^{c} u_{\mathrm{L} t a}\right) \\
& \mathcal{Y}\left[\frac{p}{r}, u v v \mid\left(d_{\mathrm{R} u b} C d_{\mathrm{R} v c}\right)\left(\bar{d}_{\mathrm{R}}^{b} u_{\mathrm{L} t a}\right)\left(\bar{d}_{\mathrm{R}}^{a} C \bar{u}_{\mathrm{R}}^{c}{ }_{s}\right)\right. \\
& \mathcal{Y}\left[\frac{p}{r}, u \mid v\right]\left(d_{\mathrm{R} u b} C d_{\mathrm{R} v a}\right)\left(\bar{d}_{\mathrm{R} r}^{b} u_{\mathrm{L} t c}\right)\left(\bar{d}_{\mathrm{R}}^{a} C \bar{u}_{\mathrm{R}}^{c}\right) \\
& \mathcal{Y}\left[\frac{p}{r}, \underline{v}, \frac{a}{v}\right]\left(d_{\mathrm{R} u b} C d_{\mathrm{R} v c}\right)\left(\bar{d}_{\mathrm{R} p}^{a} C \bar{d}_{\mathrm{R} r}^{b}\right)\left(\bar{u}_{\mathrm{R}}^{c} u_{\mathrm{L} t a}\right)
\end{aligned}
$$

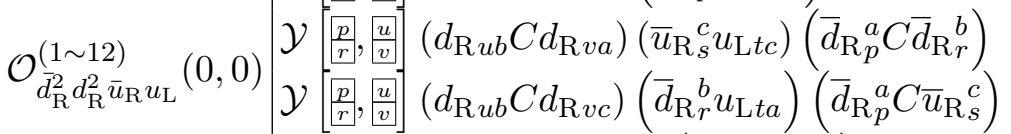

$$
\begin{aligned}
& \mathcal{Y}[\overline{p p r}, \overline{u v}]\left(d_{\mathrm{R} u b} C d_{\mathrm{R} v c}\right)\left(\bar{d}_{\mathrm{R} p}^{a} C \bar{d}_{\mathrm{R} r}^{b}\right)\left(\bar{u}_{\mathrm{R}}^{c} u_{\mathrm{L} t a}\right) \\
& \mathcal{Y}[\overline{p p r}, u \underline{u v}]\left(d_{\mathrm{R} u b} C d_{\mathrm{R} v a}\right)\left(\bar{u}_{\mathrm{R}}^{c}{ }_{s} u_{\mathrm{L} t c}\right)\left(\bar{d}_{\mathrm{R} p}^{a} C \bar{d}_{\mathrm{R} r}^{b}\right) \\
& \mathcal{Y}\left[\overline{p \mid r}, \overline{u \mid v]}\left(d_{\mathrm{R} u b} C d_{\mathrm{R} v c}\right)\left(\bar{d}_{\mathrm{R}}{ }_{r}^{b} u_{\mathrm{L} t a}\right)\left(\bar{d}_{\mathrm{R} p}^{a} C \bar{u}_{\mathrm{R}}^{c}{ }_{s}^{c}\right)\right.
\end{aligned}
$$

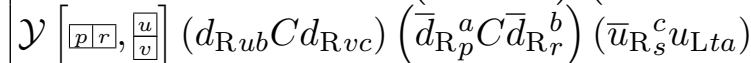

$$
\begin{aligned}
& \mathcal{Y}\left[\underline{p \mid r,}, \frac{u}{v}\right]\left(d_{\mathrm{R} u b} C d_{\mathrm{R} v c}\right)\left(\bar{d}_{\mathrm{R} r}^{b} u_{\mathrm{L} t a}\right)\left(\bar{d}_{\mathrm{R} p}^{a} C \bar{u}_{\mathrm{R}}^{c}{ }_{s}\right) \\
& \mathcal{Y}[\overline{p p r}, \underline{u} v]\left(d_{\mathrm{R} u b} C d_{\mathrm{R} v a}\right)\left(\bar{d}_{\mathrm{R} r}^{b} u_{\mathrm{L} t c}\right)\left(\bar{d}_{\mathrm{R} p}^{a} C \bar{u}_{\mathrm{R} s}^{c}\right)
\end{aligned}
$$

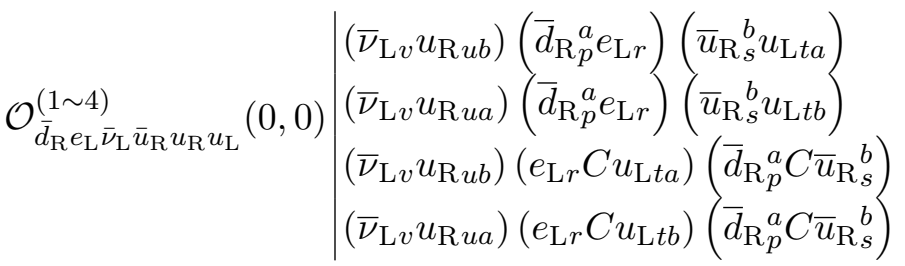

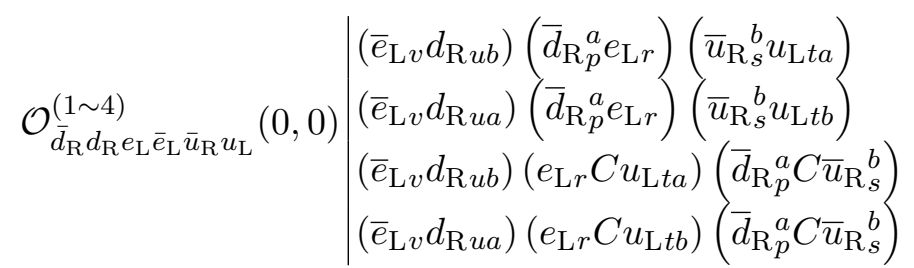




$$
\begin{aligned}
& \left.\mathcal{O}_{\bar{d}_{\mathrm{R}} d_{\mathrm{R}} \bar{e}_{\mathrm{R}} e_{\mathrm{R}} \bar{u}_{\mathrm{R}} u_{\mathrm{L}}}^{(1 \sim, 0)} \mid \begin{array}{l}
\left(d_{\mathrm{R} u b} C e_{\mathrm{R} v}\right)\left(\bar{u}_{\mathrm{R} s}^{b} u_{\mathrm{L} t a}\right)\left(\bar{d}_{\mathrm{R} p}^{a} C \bar{e}_{\mathrm{R} r}\right) \\
\left(d_{\mathrm{R} u a} C e_{\mathrm{R} v}\right)\left(\bar{u}_{\mathrm{R}}^{b} u_{\mathrm{L} t b}\right)\left(\bar{d}_{\mathrm{R} p}^{a} C \bar{e}_{\mathrm{R} r}\right. \\
\left(\bar{e}_{\mathrm{R} r} u_{\mathrm{L} t a}\right)\left(d_{\mathrm{R} u b} C e_{\mathrm{R} v}\right)\left(\bar{d}_{\mathrm{R} p}^{a} C \bar{u}_{\mathrm{R}}^{b}\right) \\
\left(\bar{e}_{\mathrm{R} r} u_{\mathrm{L} t b}\right)\left(d_{\mathrm{R} u a} C e_{\mathrm{R} v}\right)\left(\bar{d}_{\mathrm{R}_{p}^{a} C \bar{u}_{\mathrm{R} s}^{b}}^{b}\right.
\end{array}\right)
\end{aligned}
$$

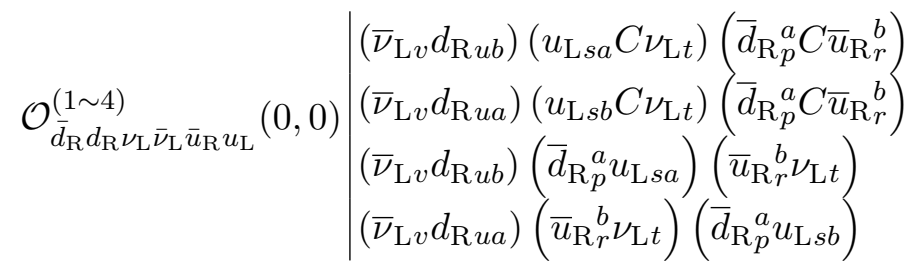

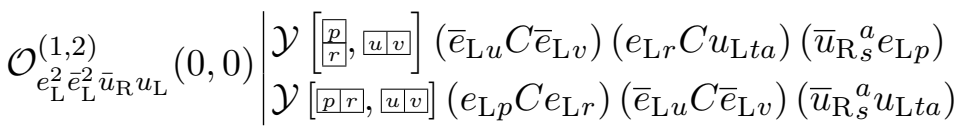

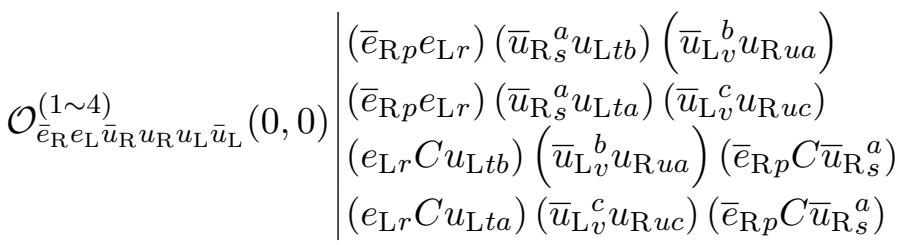

$$
\begin{aligned}
& \mathcal{O}_{d_{\mathrm{R}} \bar{d}_{\mathrm{L}} \bar{e}_{\mathrm{R}} e_{\mathrm{L}} \bar{u}_{\mathrm{R}} u_{\mathrm{L}}}^{(\sim, 0)} \mid \begin{array}{l}
\left(\bar{e}_{\mathrm{R} p} e_{\mathrm{L} r}\right)\left(\bar{d}_{\mathrm{L} v}^{b} d_{\mathrm{R} u a}\right)\left(\bar{u}_{\mathrm{R} s}^{a} u_{\mathrm{L} t b}\right) \\
\left(\bar{e}_{\mathrm{R} p} e_{\mathrm{L} r}\right)\left(\bar{u}_{\mathrm{R}}^{a} u_{\mathrm{L} t a}\right)\left(\bar{d}_{\mathrm{L}}^{c} d_{\mathrm{R} u c}\right) \\
\left(e_{\mathrm{L} r} C u_{\mathrm{L} t b}\right)\left(\bar{d}_{\mathrm{L}}^{b} d_{\mathrm{R} u a}\right)\left(\bar{e}_{\mathrm{R} p} C \bar{u}_{\mathrm{R} s}^{a}\right) \\
\left(e_{\mathrm{L} r} C u_{\mathrm{L} t a}\right)\left(\bar{d}_{\mathrm{L}}^{c} d_{\mathrm{R} u c}\right)\left(\bar{e}_{\mathrm{R} p} C \bar{u}_{\mathrm{R}}^{a}\right)
\end{array}
\end{aligned}
$$

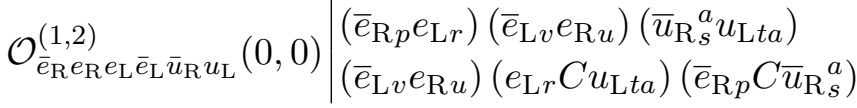

$$
\begin{aligned}
& \mathcal{O}_{e_{\mathrm{L}} \bar{e}_{\mathrm{L}} \nu_{\mathrm{L}} \bar{\nu}_{\mathrm{L}} \bar{u}_{\mathrm{R}} u_{\mathrm{L}}}^{(0,0)} \mid \begin{array}{l}
\left(\bar{e}_{\mathrm{L} u} C \bar{\nu}_{\mathrm{L} v}\right)\left(u_{\mathrm{L} s a} C \nu_{\mathrm{L} t}\right)\left(\bar{u}_{\mathrm{R}}^{a} e_{\mathrm{L} p}\right) \\
\left(\bar{e}_{\mathrm{L} u} C \bar{\nu}_{\mathrm{L} v}\right)\left(e_{\mathrm{L} p} C u_{\mathrm{L} s a}\right)\left(\bar{u}_{\mathrm{R}}{ }_{r}^{a} \nu_{\mathrm{L} t}\right)
\end{array}
\end{aligned}
$$

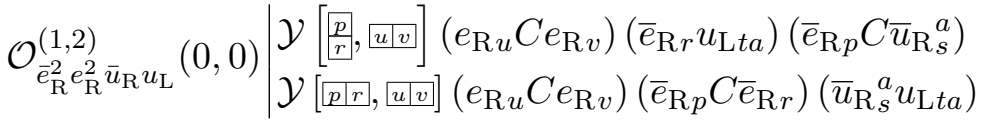

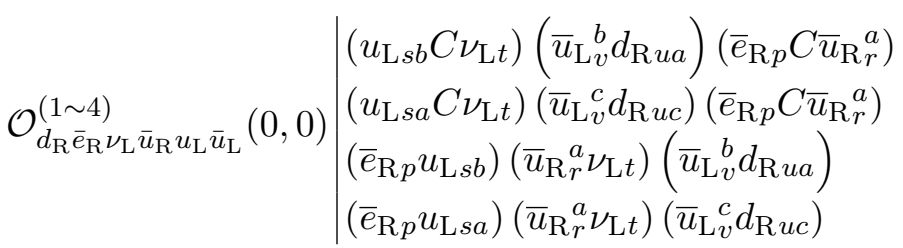

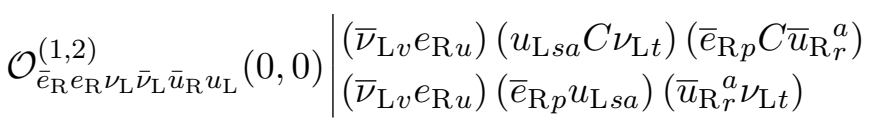

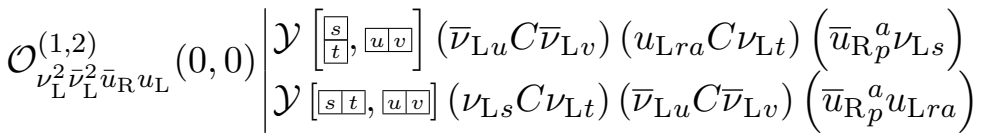




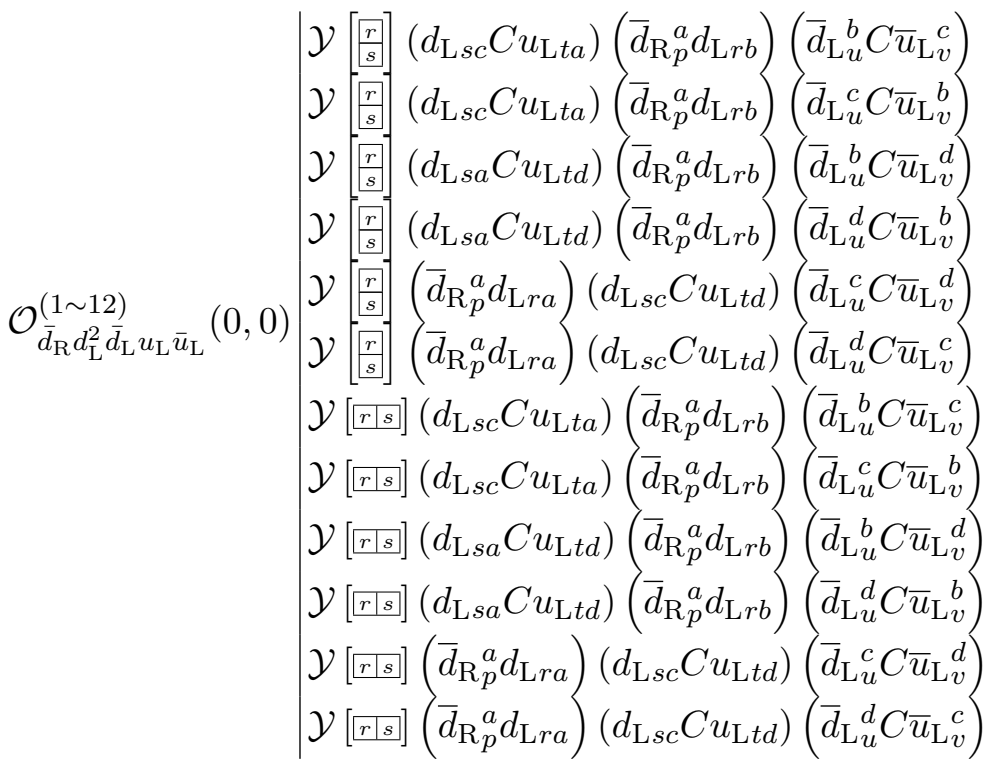

$$
\begin{aligned}
& \mathcal{Y}\left[\begin{array}{l}
\frac{p}{r} \\
r
\end{array}\left(d_{\mathrm{L} s c} C u_{\mathrm{L} t a}\right)\left(\bar{d}_{\mathrm{R}}^{a} C \bar{d}_{\mathrm{R}}^{b}\right)\left(\bar{u}_{\mathrm{L}}^{c} d_{\mathrm{R} u b}\right)\right.
\end{aligned}
$$

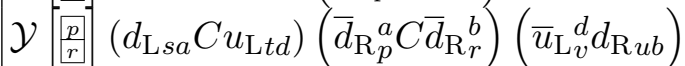

$$
\begin{aligned}
& \mathcal{Y}\left[\frac{p}{r}\right]\left(\bar{u}_{\mathrm{L} v}^{e} d_{\mathrm{R} u e}\right)\left(d_{\mathrm{L} s a} C u_{\mathrm{L} t b}\right)\left(\bar{d}_{\mathrm{R} p}^{a} C \bar{d}_{\mathrm{R} r}^{b}\right) \\
& \mathcal{Y}\left[\begin{array}{l}
\underline{p} \\
r
\end{array}\left(\bar{d}_{\mathrm{R} r}^{b} u_{\mathrm{L} t a}\right)\left(\bar{d}_{\mathrm{R}}^{a} d_{\mathrm{L} s c}\right)\left(\bar{u}_{\mathrm{L} v}^{c} d_{\mathrm{R} u b}\right)\right.
\end{aligned}
$$

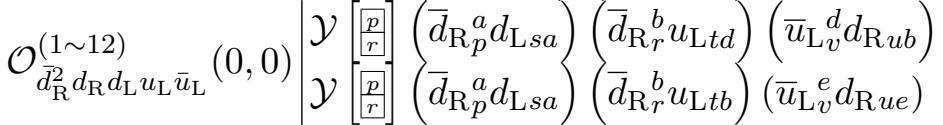

$$
\begin{aligned}
& \mathcal{Y}[\overline{p p r}]\left(d_{\mathrm{L} s c} C u_{\mathrm{L} t a}\right)\left(\bar{d}_{\mathrm{R} p}^{a} C \bar{d}_{\mathrm{R} r}^{b}\right)\left(\bar{u}_{\mathrm{L} v}^{c} d_{\mathrm{R} u b}\right) \\
& \mathcal{Y}\left[\underline{p p r]}\left(d_{\mathrm{L} s a} C u_{\mathrm{L} t d}\right)\left(\bar{d}_{\mathrm{R}_{p}^{a}} C \bar{d}_{\mathrm{R}}^{b}\right)\left(\bar{u}_{\mathrm{L} v}^{d} d_{\mathrm{R} u b}\right)\right. \\
& \mathcal{Y}[\overline{p p r}]\left(\bar{u}_{\mathrm{L} v}^{e} d_{\mathrm{R} u e}\right)\left(d_{\mathrm{L} s a} C u_{\mathrm{L} t b}\right)\left(\bar{d}_{\mathrm{R} p}^{a} C \bar{d}_{\mathrm{R} r}^{b}\right) \\
& \mathcal{Y}[\overline{p p r}]\left(\bar{d}_{\mathrm{R} r}^{b} u_{\mathrm{L} t a}\right)\left(\bar{d}_{\mathrm{R} p}^{a} d_{\mathrm{L} s c}\right)\left(\bar{u}_{\mathrm{L} v}^{c} d_{\mathrm{R} u b}\right) \\
& \mathcal{Y}[\overline{p p r}]\left(\bar{d}_{\mathrm{R} p}^{a} d_{\mathrm{L} s a}\right)\left(\bar{d}_{\mathrm{R} r}^{b} u_{\mathrm{L} t d}\right)\left(\bar{u}_{\mathrm{L} v}^{d} d_{\mathrm{R} u b}\right)
\end{aligned}
$$

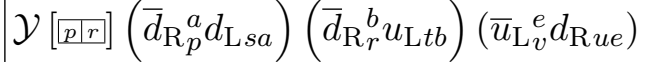

$$
\begin{aligned}
& \mathcal{Y}\left[\begin{array}{l}
\frac{p}{r} \\
r
\end{array}\right]\left(\bar{\nu}_{\mathrm{L} v} e_{\mathrm{R} u}\right)\left(d_{\mathrm{L} s a} C u_{\mathrm{L} t b}\right)\left(\bar{d}_{\mathrm{R} p}^{a} C \bar{d}_{\mathrm{R} r}^{b}\right) \\
& \mathcal{O}_{\bar{d}_{\mathrm{R}}^{2} d_{\mathrm{L}} e_{\mathrm{R}} \bar{\nu}_{\mathrm{L}} u_{\mathrm{L}}}^{(\sim, 0)} \mathcal{Y}\left[\begin{array}{l}
\bar{p} \\
r
\end{array}\right]\left(\bar{\nu}_{\mathrm{L} v} e_{\mathrm{R} u}\right)\left(\bar{d}_{\mathrm{R} p}^{a} d_{\mathrm{L} s a}\right)\left(\bar{d}_{\mathrm{R} r}^{b} u_{\mathrm{L} t b}\right) \\
& \mathcal{Y}[\underline{p \mid r}]\left(\bar{\nu}_{\mathrm{L} v} e_{\mathrm{R} u}\right)\left(d_{\mathrm{L} s a} C u_{\mathrm{L} t b}\right)\left(\bar{d}_{\mathrm{R} p}^{a} C \bar{d}_{\mathrm{R} r}^{b}\right) \\
& \mathcal{Y}[\overline{p p r}]\left(\bar{\nu}_{\mathrm{L} v} e_{\mathrm{R} u}\right)\left(\bar{d}_{\mathrm{R} p}^{a} d_{\mathrm{L} s a}\right)\left(\bar{d}_{\mathrm{R}}^{b} u_{\mathrm{L} t b}\right) \\
& \mathcal{O}_{\bar{d}_{\mathrm{R}} d_{\mathrm{L} e} e_{\mathrm{L}} \bar{e}_{\mathrm{L}} u_{\mathrm{L}} \bar{u}_{\mathrm{L}}}^{(1 \sim 0)} \mid \begin{array}{l}
\left(e_{\mathrm{L} s} C u_{\mathrm{L} t a}\right)\left(\bar{d}_{\mathrm{R} p}^{a} d_{\mathrm{L} r b}\right)\left(\bar{e}_{\mathrm{L} u} C \bar{u}_{\mathrm{L} v}^{b}\right) \\
\left(e_{\mathrm{L} s} C u_{\mathrm{L} t c}\right)\left(\bar{d}_{\mathrm{R} p}^{a} d_{\mathrm{L} r a}\right)\left(\bar{e}_{\mathrm{L} u} C \bar{u}_{\mathrm{L}}^{c}\right) \\
\left(\bar{d}_{\mathrm{R} p}^{a} e_{\mathrm{L} s}\right)\left(d_{\mathrm{L} r b} C u_{\mathrm{L} t a}\right)\left(\bar{e}_{\mathrm{L} u} C \bar{u}_{\mathrm{L} v}^{b}\right) \\
\left(\bar{d}_{\mathrm{R} p}^{a} e_{\mathrm{L} s}\right)\left(d_{\mathrm{L} r a} C u_{\mathrm{L} t c}\right)\left(\bar{e}_{\mathrm{L} u} C \bar{u}_{\mathrm{L} v}^{c}\right)
\end{array}
\end{aligned}
$$




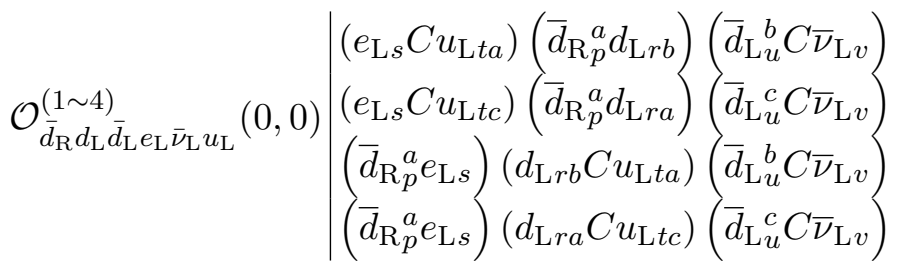

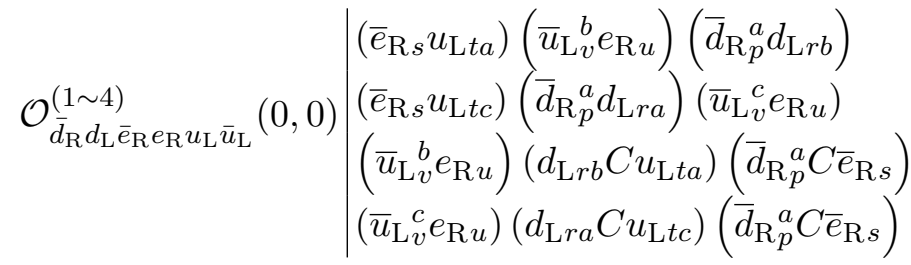

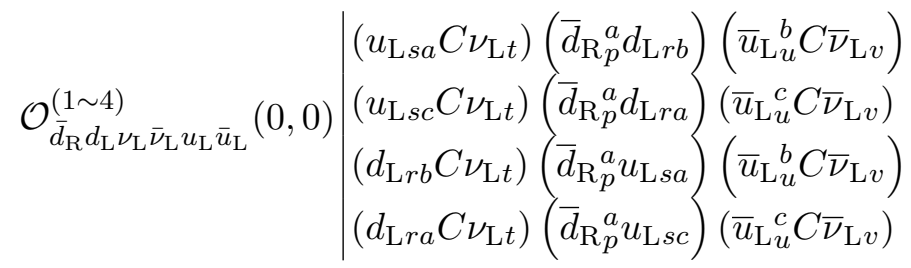

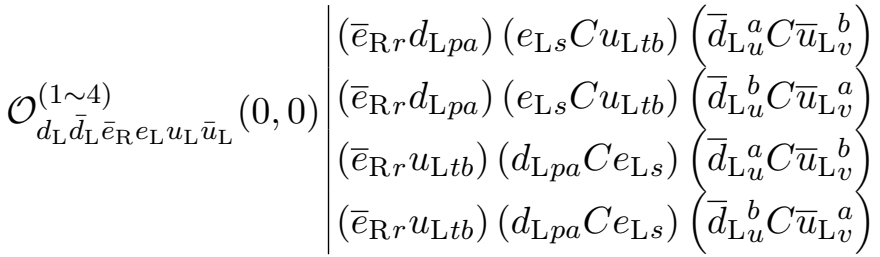

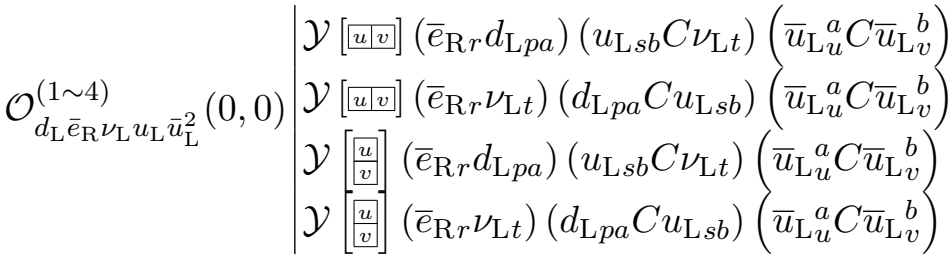

$$
\begin{aligned}
& \mathcal{Y}\left[\frac{p}{r}\right]\left(\bar{\nu}_{\mathrm{L} v} d_{\mathrm{R} u b}\right)\left(e_{\mathrm{L} s} C u_{\mathrm{L} t a}\right)\left(\bar{d}_{\mathrm{R} p}^{a} C \bar{d}_{\mathrm{R} r}^{b}\right)
\end{aligned}
$$

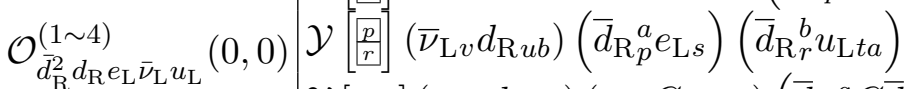

$$
\begin{aligned}
& \mathcal{Y}\left[\overline{p \mid r]}\left(\bar{\nu}_{\mathrm{L} v} d_{\mathrm{R} u b}\right)\left(e_{\mathrm{L} s} C u_{\mathrm{L} t a}\right)\left(\bar{d}_{\mathrm{R} p}^{a} C \bar{d}_{\mathrm{R} r}^{b}\right)\right. \\
& \mathcal{Y}[\overline{p p r}]\left(\bar{\nu}_{\mathrm{L} v} d_{\mathrm{R} u b}\right)\left(\bar{d}_{\mathrm{R} p}^{a} e_{\mathrm{L} s}\right)\left(\bar{d}_{\mathrm{R} r}^{b} u_{\mathrm{L} t a}\right)
\end{aligned}
$$

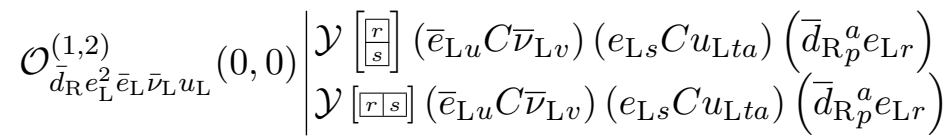

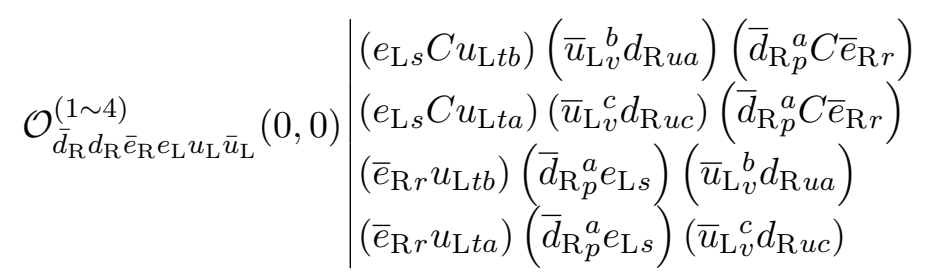

$$
\begin{aligned}
& \mathcal{O}_{\bar{d}_{\mathrm{R}} \bar{e}_{\mathrm{R}} e_{\mathrm{R}} e_{\mathrm{L}} \bar{\nu}_{\mathrm{L}} u_{\mathrm{L}}}^{(1,2,0)}\left(0 \begin{array}{l}
\left(\bar{\nu}_{\mathrm{L} v} e_{\mathrm{R} u}\right)\left(e_{\mathrm{L} s} C u_{\mathrm{L} t a}\right)\left(\bar{d}_{\mathrm{R} p}^{a} C \bar{e}_{\mathrm{R} r}\right) \\
\left(\bar{\nu}_{\mathrm{L} v} e_{\mathrm{R} u}\right)\left(\bar{e}_{\mathrm{R} r} u_{\mathrm{L} t a}\right)\left(\bar{d}_{\mathrm{R} p}^{a} e_{\mathrm{L} s}\right)
\end{array}\right.
\end{aligned}
$$




$$
\begin{aligned}
& \left.\mathcal{O}_{\bar{d}_{\mathrm{R}} e_{\mathrm{L}} \nu_{\mathrm{L}} \bar{\nu}_{\mathrm{L}}^{2} u_{\mathrm{L}}}^{(0,0)} \mid \begin{array}{l}
\mathcal{Y}[u v]\left(\bar{\nu}_{\mathrm{L} u} C \bar{\nu}_{\mathrm{L} v}\right)\left(u_{\mathrm{L} s a} C \nu_{\mathrm{L} t}\right)\left(\bar{d}_{\mathrm{R}}{ }_{p}^{a} e_{\mathrm{L} r}\right) \\
\mathcal{Y}[\overline{u v}]\left(\bar{\nu}_{\mathrm{L} u} C \bar{\nu}_{\mathrm{L} v}\right)\left(e_{\mathrm{L} r} C \nu_{\mathrm{L} t}\right)\left(\bar{d}_{\mathrm{R} p}^{a} u_{\mathrm{L} s a}\right.
\end{array}\right)
\end{aligned}
$$

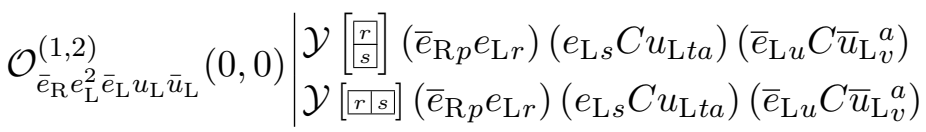

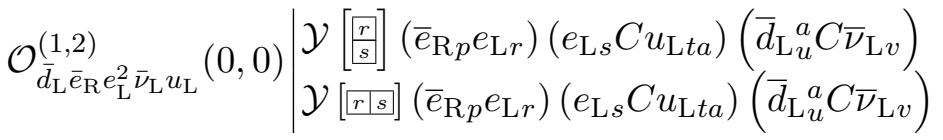

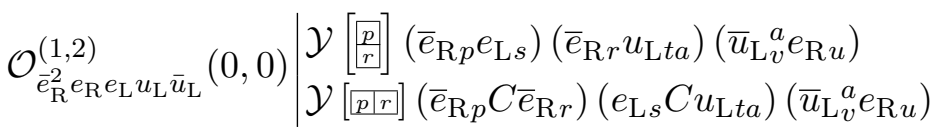

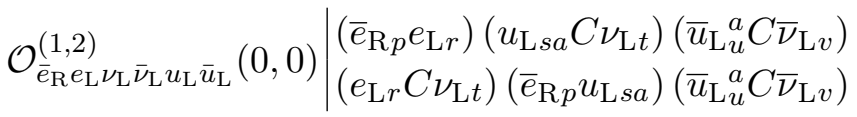

$$
\begin{aligned}
& \mathcal{Y}\left[\frac{s}{t}, \overline{u v}\right] .\left(u_{\mathrm{R} u c} C u_{\mathrm{R} v a}\right)\left(\bar{d}_{\mathrm{R} p}^{a} d_{\mathrm{L} r b}\right)\left(\bar{u}_{\mathrm{R}}^{c} C \bar{u}_{\mathrm{R} t}^{b}\right)
\end{aligned}
$$

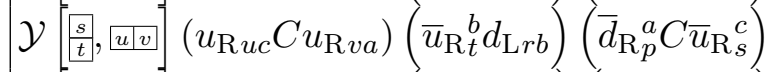

$$
\begin{aligned}
& \mathcal{Y}\left[\frac{s}{t}, u[v]\left(u_{\mathrm{R} u c} C u_{\mathrm{R} v d}\right)\left(\bar{u}_{\mathrm{R} t}^{d} d_{\mathrm{L} r a}\right)\left(\bar{d}_{\mathrm{R} p}^{a} C \bar{u}_{\mathrm{R}}^{c}\right)\right. \\
& \mathcal{Y}\left[\begin{array}{c}
s \\
\frac{s}{t},
\end{array}, \frac{u}{v}\right],\left(u_{\mathrm{R} u c} C u_{\mathrm{R} v a}\right)\left(\bar{d}_{\mathrm{R} p}^{a} d_{\mathrm{L} r b}\right)\left(\bar{u}_{\mathrm{R}}^{c} C \bar{u}_{\mathrm{R} t}^{b}\right)
\end{aligned}
$$

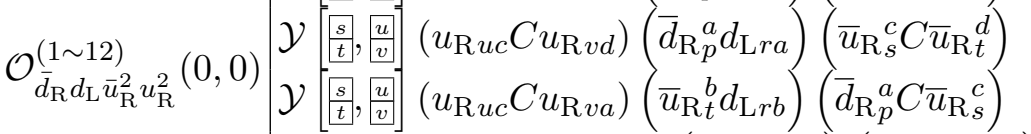

$$
\begin{aligned}
& \mathcal{Y}\left[s \mid t,[u v]\left(u_{\mathrm{R} u c} C u_{\mathrm{R} v a}\right)\left(\bar{d}_{\mathrm{R} p}^{a} d_{\mathrm{L} r b}\right)\left(\bar{u}_{\mathrm{R}}^{c} C \bar{u}_{\mathrm{R}}^{b}\right)\right. \\
& \mathcal{Y}[s|t, u| v],\left(u_{\mathrm{R} u c} C u_{\mathrm{R} v d}\right)\left(\bar{d}_{\mathrm{R} p}^{a} d_{\mathrm{L} r a}\right)\left(\bar{u}_{\mathrm{R}}^{c} C \bar{u}_{\mathrm{R} t}^{d}\right) \\
& \mathcal{Y}[s|t, u| v]]\left(u_{\mathrm{R} u c} C u_{\mathrm{R} v a}\right)\left(\bar{u}_{\mathrm{R} t}^{b} d_{\mathrm{L} r b}\right)\left(\bar{d}_{\mathrm{R} p}^{a} C \bar{u}_{\mathrm{R} s}^{c}\right) \\
& \mathcal{Y}\left[s \mid t, \frac{u}{v}\right]\left(u_{\mathrm{R} u c} C u_{\mathrm{R} v a}\right)\left(\bar{d}_{\mathrm{R} p}^{a} d_{\mathrm{L} r b}\right)\left(\bar{u}_{\mathrm{R}}^{c} C \bar{u}_{\mathrm{R} t}^{b}\right)
\end{aligned}
$$

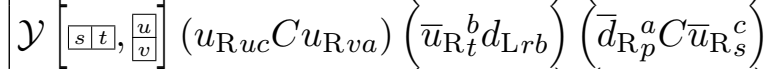

$$
\begin{aligned}
& \mathcal{Y}\left[s \mid t, \frac{u}{v}\right]\left(u_{\mathrm{R} u c} C u_{\mathrm{R} v d}\right)\left(\bar{u}_{\mathrm{R} t}^{d} d_{\mathrm{L} r a}\right)\left(\bar{d}_{\mathrm{R} p}^{a} C \bar{u}_{\mathrm{R} s}^{c}\right)
\end{aligned}
$$

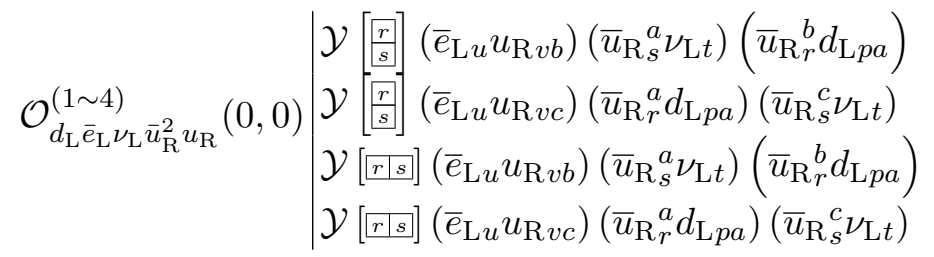

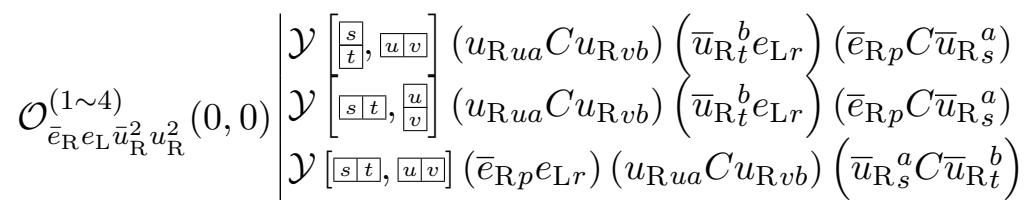

$$
\begin{aligned}
& \mathcal{Y}\left[\begin{array}{l}
s \\
t
\end{array},\left[\begin{array}{l}
u \\
v
\end{array}\right]\left(\bar{e}_{\mathrm{R} p} e_{\mathrm{L} r}\right)\left(u_{\mathrm{R} u a} C u_{\mathrm{R} v b}\right)\left(\bar{u}_{\mathrm{R} s}^{a} C \bar{u}_{\mathrm{R} t}^{b}\right)\right.
\end{aligned}
$$

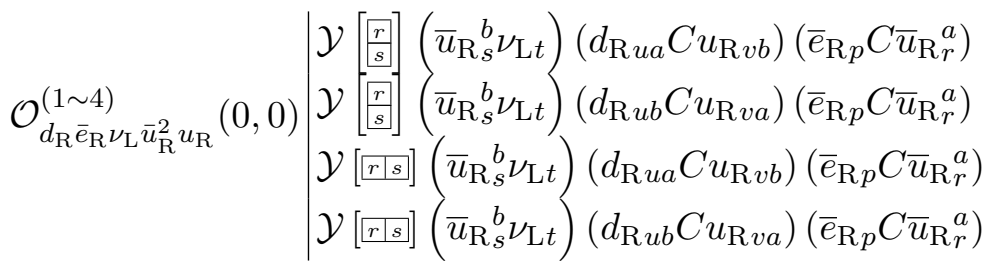




$$
\begin{aligned}
& \mathcal{Y}\left[\frac{r}{s}\right]\left(\bar{d}_{\mathrm{R} p}^{a} d_{\mathrm{L} r b}\right)\left(\bar{d}_{\mathrm{L} u}^{c} u_{\mathrm{R} v a}\right)\left(\bar{u}_{\mathrm{R} t}^{b} d_{\mathrm{L} s c}\right) \\
& \mathcal{Y}\left[\begin{array}{l}
r s \\
s]
\end{array}\left(\bar{d}_{\mathrm{R} p}^{a} d_{\mathrm{L} r a}\right)\left(\bar{u}_{\mathrm{R} t}^{d} d_{\mathrm{L} s c}\right)\left(\bar{d}_{\mathrm{L} u}^{c} u_{\mathrm{R} v d}\right)\right.
\end{aligned}
$$

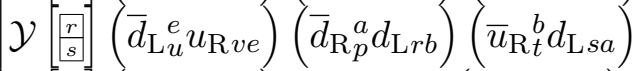

$$
\begin{aligned}
& \mathcal{Y}\left[\begin{array}{l}
r s \\
s]
\end{array}\left(\bar{d}_{\mathrm{R} p}^{a} d_{\mathrm{L} r a}\right)\left(\bar{u}_{\mathrm{R} t}^{c} d_{\mathrm{L} s c}\right)\left(\bar{d}_{\mathrm{L} u}^{e} u_{\mathrm{R} v e}\right)\right.
\end{aligned}
$$

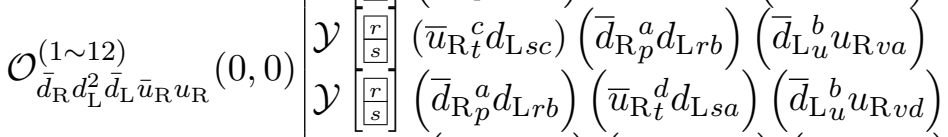

$$
\begin{aligned}
& \mathcal{Y}[\overline{r r s}]\left(\bar{d}_{\mathrm{R} p}^{a} d_{\mathrm{L} r b}\right)\left(\bar{d}_{\mathrm{L} u}^{c} u_{\mathrm{R} v a}\right)\left(\bar{u}_{\mathrm{R} t}^{b} d_{\mathrm{L} s c}\right) \\
& \mathcal{Y}[r r s]\left(\bar{d}_{\mathrm{R} p}^{a} d_{\mathrm{L} r a}\right)\left(\bar{u}_{\mathrm{R} t}^{d} d_{\mathrm{L} s c}\right)\left(\bar{d}_{\mathrm{L} u}^{c} u_{\mathrm{R} v d}\right) \\
& \mathcal{Y}[r r s]\left(\bar{d}_{\mathrm{L} u}^{e} u_{\mathrm{R} v e}\right)\left(\bar{d}_{\mathrm{R} p}^{a} d_{\mathrm{L} r b}\right)\left(\bar{u}_{\mathrm{R} t}^{b} d_{\mathrm{L} s a}\right) \\
& \mathcal{Y}\left[\underline{r r s]}\left(\bar{d}_{\mathrm{R} p}^{a} d_{\mathrm{L} r a}\right)\left(\bar{u}_{\mathrm{R} t}^{c} d_{\mathrm{L} s c}\right)\left(\bar{d}_{\mathrm{L} u}^{e} u_{\mathrm{R} v e}\right)\right. \\
& \mathcal{Y}\left[\overline{r r s]}\left(\bar{u}_{\mathrm{R} t}^{c} d_{\mathrm{L} s c}\right)\left(\bar{d}_{\mathrm{R} p}^{a} d_{\mathrm{L} r b}\right)\left(\bar{d}_{\mathrm{L} u}^{b} u_{\mathrm{R} v a}\right)\right. \\
& \mathcal{Y}\left[{ }_{r r s]}\right]\left(\bar{d}_{\mathrm{R} p}^{a} d_{\mathrm{L} r b}\right)\left(\bar{u}_{\mathrm{R} t}^{d} d_{\mathrm{L} s a}\right)\left(\bar{d}_{\mathrm{L} u}^{b} u_{\mathrm{R} v d}\right)
\end{aligned}
$$

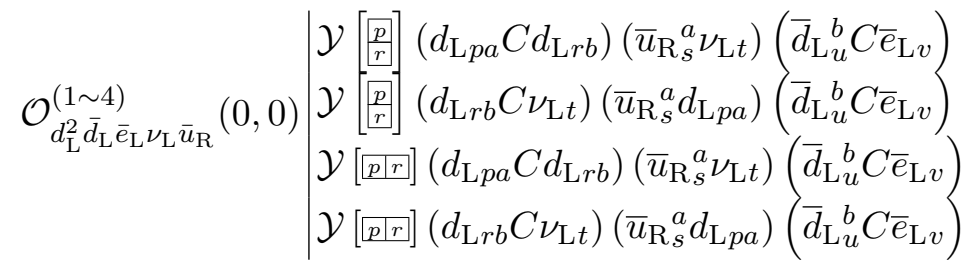

$$
\begin{aligned}
& \mathcal{Y}\left[\begin{array}{l}
\frac{p}{r} \\
{[}
\end{array}\right]\left(\bar{u}_{\mathrm{R}}^{c} d_{\mathrm{L} s c}\right)\left(d_{\mathrm{R} u b} C u_{\mathrm{R} v a}\right)\left(\bar{d}_{\mathrm{R}_{p}^{a}} C \bar{d}_{\mathrm{R}}^{b}\right) \\
& \mathcal{Y}\left[\frac{p}{r}\left(d_{\mathrm{R} u b} C u_{\mathrm{R} v d}\right)\left(\bar{d}_{\mathrm{R} p}^{a} C \bar{d}_{\mathrm{R} r}^{b}\right)\left(\bar{u}_{\mathrm{R} t}^{d} d_{\mathrm{L} s a}\right)\right.
\end{aligned}
$$

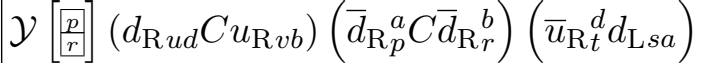

$$
\begin{aligned}
& \mathcal{Y}\left[\begin{array}{ll}
p \\
r
\end{array}\left(d_{\mathrm{R} u b} C u_{\mathrm{R} v a}\right)\left(\bar{d}_{\mathrm{R} p}^{a} d_{\mathrm{L} s c}\right)\left(\bar{d}_{\mathrm{R} r}^{b} C \bar{u}_{\mathrm{R} t}^{c}\right)\right.
\end{aligned}
$$

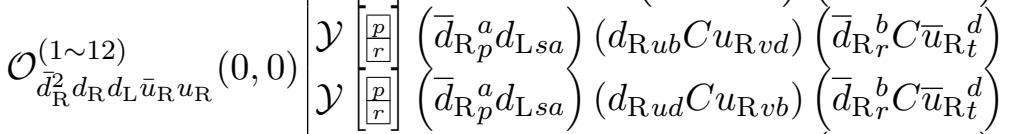

$$
\begin{aligned}
& \mathcal{Y}[\overline{p p r}]\left(\bar{u}_{\mathrm{R} t}^{c} d_{\mathrm{L} s c}\right)\left(d_{\mathrm{R} u b} C u_{\mathrm{R} v a}\right)\left(\bar{d}_{\mathrm{R} p}^{a} C \bar{d}_{\mathrm{R} r}^{b}\right) \\
& \mathcal{Y}[\underline{p p r}]\left(d_{\mathrm{R} u b} C u_{\mathrm{R} v d}\right)\left(\bar{d}_{\mathrm{R}}^{a} C \bar{d}_{\mathrm{R}}^{b}\right)\left(\bar{u}_{\mathrm{R} t}^{d} d_{\mathrm{L} s a}\right) \\
& \mathcal{Y}[\overline{p p r}]\left(d_{\mathrm{R} u d} C u_{\mathrm{R} v b}\right)\left(\bar{d}_{\mathrm{R}}^{a} C \bar{d}_{\mathrm{R}}^{b}\right)\left(\bar{u}_{\mathrm{R}}{ }_{t}^{d} d_{\mathrm{L} s a}\right) \\
& \mathcal{Y}[\overline{p p r}]\left(d_{\mathrm{R} u b} C u_{\mathrm{R} v a}\right)\left(\bar{d}_{\mathrm{R} p}^{a} d_{\mathrm{L} s c}\right)\left(\bar{d}_{\mathrm{R} r}^{b} C \bar{u}_{\mathrm{R} t}^{c}\right) \\
& \mathcal{Y}[\overline{p p r}]\left(\bar{d}_{\mathrm{R} p}^{a} d_{\mathrm{L} s a}\right)\left(d_{\mathrm{R} u b} C u_{\mathrm{R} v d}\right)\left(\bar{d}_{\mathrm{R} r}^{b} C \bar{u}_{\mathrm{R} t}^{d}\right) \\
& \mathcal{Y}[\overline{p p r}]\left(\bar{d}_{\mathrm{R} p}^{a} d_{\mathrm{L} s a}\right)\left(d_{\mathrm{R} u d} C u_{\mathrm{R} v b}\right)\left(\bar{d}_{\mathrm{R} r}^{b} C \bar{u}_{\mathrm{R} t}^{d}\right)
\end{aligned}
$$

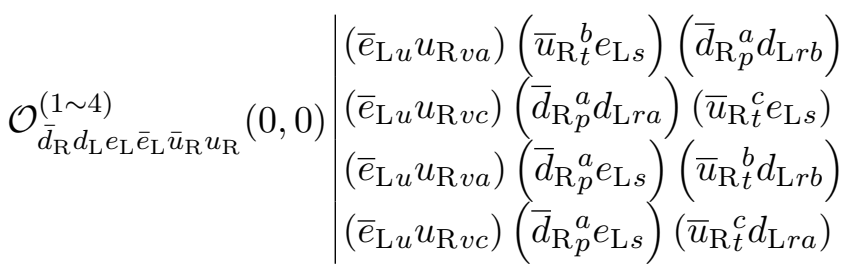




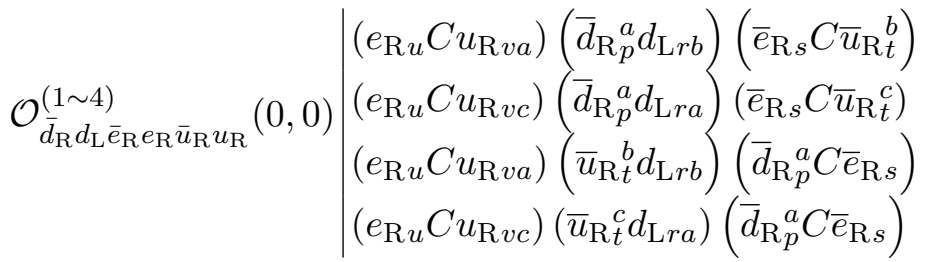

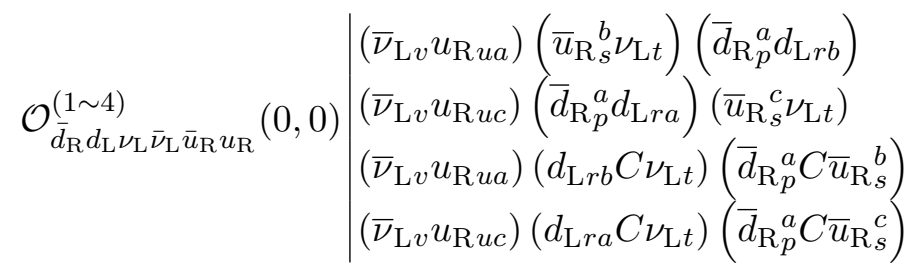

$$
\begin{aligned}
& \mathcal{O}_{\bar{d}_{\mathrm{R}} d_{\mathrm{R}} d_{\mathrm{L}} \bar{e}_{\mathrm{L}} \nu_{\mathrm{L}} \bar{u}_{\mathrm{R}}}^{(1 \sim 0)} \mid \begin{array}{l}
\left(\bar{e}_{\mathrm{L} v} d_{\mathrm{R} u a}\right)\left(\bar{u}_{\mathrm{R} s}^{b} \nu_{\mathrm{L} t}\right)\left(\bar{d}_{\mathrm{R} p}^{a} d_{\mathrm{L} r b}\right) \\
\left(\bar{e}_{\mathrm{L} v} d_{\mathrm{R} u c}\right)\left(\bar{d}_{\mathrm{R}}^{a} d_{\mathrm{L} r a}\right)\left(\bar{u}_{\mathrm{R}}^{c} \nu_{\mathrm{L} t}\right) \\
\left(\bar{e}_{\mathrm{L} v} d_{\mathrm{R} u a}\right)\left(d_{\mathrm{L} r b} C \nu_{\mathrm{L} t}\right)\left(\bar{d}_{\mathrm{R} p}^{a} C \bar{u}_{\mathrm{R}}^{b}\right) \\
\left(\bar{e}_{\mathrm{L} v} d_{\mathrm{R} u c}\right)\left(d_{\mathrm{L} r a} C \nu_{\mathrm{L} t}\right)\left(\bar{d}_{\mathrm{R} p}^{a} C \bar{u}_{\mathrm{R}}^{c}\right)
\end{array} \\
& \mathcal{O}_{d_{\mathrm{L}} \bar{d}_{\mathrm{L}} \bar{e}_{\mathrm{R}} e_{\mathrm{L}} \bar{u}_{\mathrm{R} u} u_{\mathrm{R}}}^{(\sim, 0)} \mid \begin{array}{l}
\left(\bar{e}_{\mathrm{R} r} d_{\mathrm{L} p a}\right)\left(\bar{u}_{\mathrm{R} t}^{b} e_{\mathrm{L} s}\right)\left(\bar{d}_{\mathrm{L} u}^{a} u_{\mathrm{R} v b}\right) \\
\left(\bar{e}_{\mathrm{R} r} d_{\mathrm{L} p a}\right)\left(\bar{u}_{\mathrm{R} t}^{a} e_{\mathrm{L} s}\right)\left(\bar{d}_{\mathrm{L} u}^{c} u_{\mathrm{R} v c}\right) \\
\left(d_{\mathrm{L} p a} C e_{\mathrm{L} s}\right)\left(\bar{d}_{\mathrm{L} u}^{a} u_{\mathrm{R} v b}\right)\left(\bar{e}_{\mathrm{R} r} C \bar{u}_{\mathrm{R} t}^{b}\right) \\
\left(d_{\mathrm{L} p a} C e_{\mathrm{L} s}\right)\left(\bar{d}_{\mathrm{L} u}^{c} u_{\mathrm{R} v c}\right)\left(\bar{e}_{\mathrm{R} r} C \bar{u}_{\mathrm{R} t}^{a}\right)
\end{array} \\
& \mathcal{O}_{d_{\mathrm{L}} e_{\mathrm{L}} \bar{e}_{\mathrm{L}}^{2} \nu_{\mathrm{L}} \bar{u}_{\mathrm{R}}}^{(1,0,0)} \mid \begin{array}{l}
\mathcal{Y}[\overline{u v}]\left(\bar{e}_{\mathrm{L} u} C \bar{e}_{\mathrm{L} v}\right)\left(d_{\mathrm{L} p a} C e_{\mathrm{L} r}\right)\left(\bar{u}_{\mathrm{R} s}^{a} \nu_{\mathrm{L} t}\right) \\
\mathcal{Y}[u v]]\left(\bar{e}_{\mathrm{L} u} C \bar{e}_{\mathrm{L} v}\right)\left(e_{\mathrm{L} r} C \nu_{\mathrm{L} t}\right)\left(\bar{u}_{\mathrm{R} s}^{a} d_{\mathrm{L} p a}\right)
\end{array}
\end{aligned}
$$

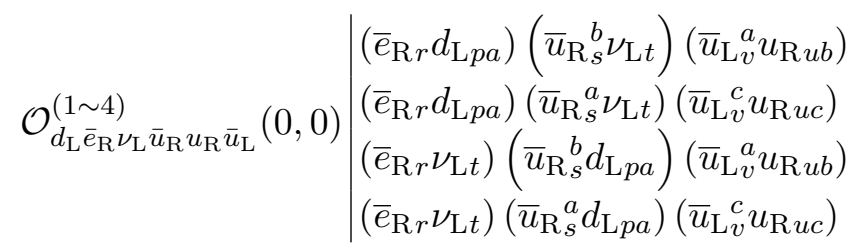

$$
\begin{aligned}
& \mathcal{O}_{d_{\mathrm{R}} d_{\mathrm{L}} \bar{d}_{\mathrm{L}} \bar{e}_{\mathrm{R}} \nu_{\mathrm{L}} \bar{u}_{\mathrm{R}}}(0,0) \mid \begin{array}{l}
\left(\bar{e}_{\mathrm{R} r} d_{\mathrm{L} p a}\right)\left(\bar{u}_{\mathrm{R}}^{b} \nu_{\mathrm{L} t}\right)\left(\bar{d}_{\mathrm{L} v}^{a} d_{\mathrm{R} u b}\right) \\
\left(\bar{e}_{\mathrm{R} r} d_{\mathrm{L} p a}\right)\left(\bar{u}_{\mathrm{R}}{ }_{s}^{a} \nu_{\mathrm{L} t}\right)\left(\bar{d}_{\mathrm{L} v}^{c} d_{\mathrm{R} u c}\right) \\
\left(\bar{e}_{\mathrm{R} r} \nu_{\mathrm{L} t}\right)\left(\bar{d}_{\mathrm{L} v}^{a} d_{\mathrm{R} u b}\right)\left(\bar{u}_{\mathrm{R} s}^{b} d_{\mathrm{L} p a}\right) \\
\left(\bar{e}_{\mathrm{R} r} \nu_{\mathrm{L} t}\right)\left(\bar{u}_{\mathrm{R}}^{a} d_{\mathrm{L} p a}\right)\left(\bar{d}_{\mathrm{L} v}^{c} d_{\mathrm{R} u c}\right)
\end{array} \\
& \mathcal{O}_{d_{\mathrm{L}} \bar{e}_{\mathrm{R}} e_{\mathrm{R}} \bar{e}_{\mathrm{L}} \nu_{\mathrm{L}} \bar{u}_{\mathrm{R}}}^{(1,2,0)} \mid \begin{array}{l}
\left(\bar{e}_{\mathrm{L} v} e_{\mathrm{R} u}\right)\left(\bar{e}_{\mathrm{R} r} d_{\mathrm{L} p a}\right)\left(\bar{u}_{\mathrm{R}}{ }_{s}^{a} \nu_{\mathrm{L} t}\right) \\
\left(\bar{e}_{\mathrm{L} v} e_{\mathrm{R} u}\right)\left(\bar{e}_{\mathrm{R} r} \nu_{\mathrm{L} t}\right)\left(\bar{u}_{\mathrm{R}}^{a}{ }_{s} d_{\mathrm{L} p a}\right)
\end{array}
\end{aligned}
$$

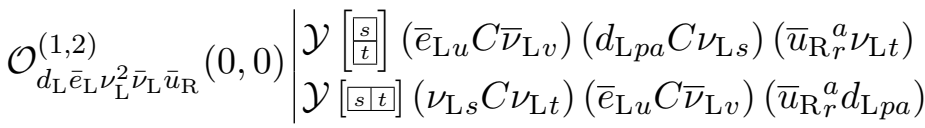

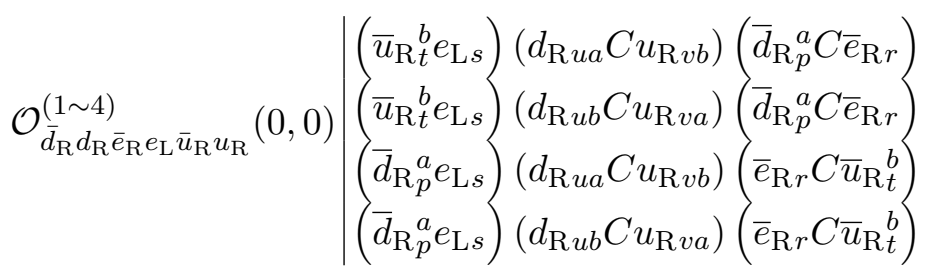




$$
\begin{aligned}
& \mathcal{Y}\left[\underline{u v v]}\left(d_{\mathrm{R} u a} C d_{\mathrm{R} v b}\right)\left(\bar{u}_{\mathrm{R}}^{b} \nu_{\mathrm{L} t}\right)\left(\bar{d}_{\mathrm{R} p}^{a} C \bar{e}_{\mathrm{R} r}\right)\right. \\
& \mathcal{O}_{\bar{d}_{\mathrm{R}} d_{\mathrm{R}}^{2} \bar{e}_{\mathrm{R}} \nu_{\mathrm{L}} \bar{u}_{\mathrm{R}}(0,0)}\left(\begin{array}{l}
\mathcal{Y}[u \mid v]\left(\bar{e}_{\mathrm{R} r} \nu_{\mathrm{L} t}\right)\left(d_{\mathrm{R} u a} C d_{\mathrm{R} v b}\right)\left(\bar{d}_{\mathrm{R}}^{a}{ }_{p}^{a} C \bar{u}_{\mathrm{R} s}^{b}\right) \\
\mathcal{Y}[u]\left(d_{\mathrm{R}}\right)
\end{array}\right. \\
& \mathcal{Y}[\underline{u} \underline{v}]\left(d_{\mathrm{R} u a} C d_{\mathrm{R} v b}\right)\left(\bar{u}_{\mathrm{R} s}^{b} \nu_{\mathrm{L} t}\right)\left(\bar{d}_{\mathrm{R} p}^{a} C \bar{e}_{\mathrm{R} r}\right) \\
& \mathcal{Y}\left[\begin{array}{l}
\frac{u}{v} \\
]
\end{array}\right]\left(\bar{e}_{\mathrm{R} r} \nu_{\mathrm{L} t}\right)\left(d_{\mathrm{R} u a} C d_{\mathrm{R} v b}\right)\left(\bar{d}_{\mathrm{R} p}^{a} C \bar{u}_{\mathrm{R}}^{b}\right)
\end{aligned}
$$

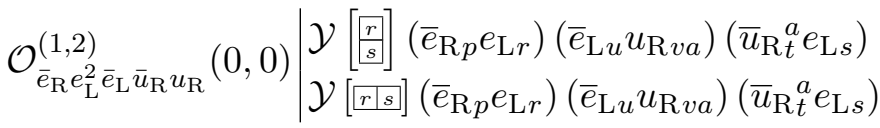

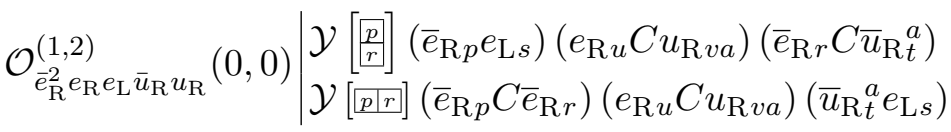

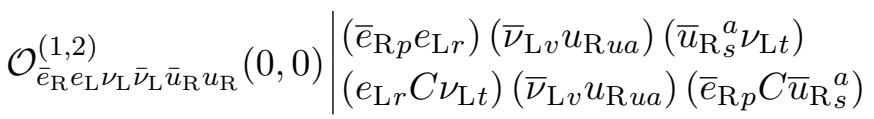

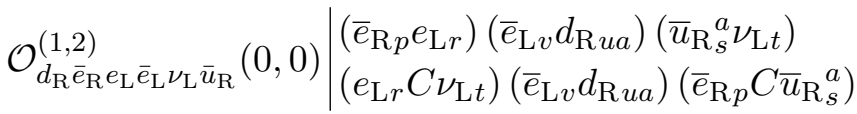

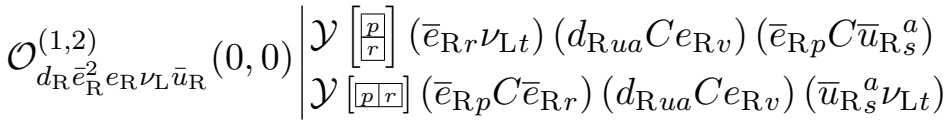

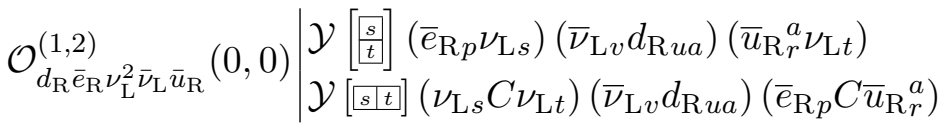

$$
\begin{aligned}
& \mathcal{Y}\left[\frac{r s s}{t \underline{t}, u v]},\left(d_{\mathrm{L} s c} C d_{\mathrm{L} t a}\right)\left(\bar{d}_{\mathrm{R} p}^{a} d_{\mathrm{L} r b}\right)\left(\bar{d}_{\mathrm{L} u}^{b} C \bar{d}_{\mathrm{L} v}^{c}\right)\right. \\
& \mathcal{Y}\left[\frac{r s s}{t}, u[v]\left(d_{\mathrm{L} s a} C d_{\mathrm{L} t d}\right)\left(\bar{d}_{\mathrm{R} p}^{a} d_{\mathrm{L} r b}\right)\left(\bar{d}_{\mathrm{L} u}^{b} C \bar{d}_{\mathrm{L} v}^{d}\right)\right. \\
& \mathcal{Y}\left[\overline{r|s| t,, u \mid v]}\left(d_{\mathrm{L} s c} C d_{\mathrm{L} t a}\right)\left(\bar{d}_{\mathrm{R}_{p}^{a}} d_{\mathrm{L} r b}\right)\left(\bar{d}_{\mathrm{L} u}^{b} C \bar{d}_{\mathrm{L} v}^{c}\right)\right.
\end{aligned}
$$

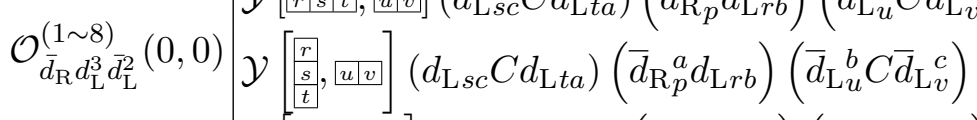

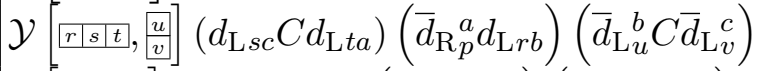

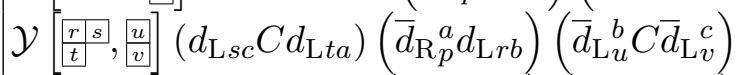

$$
\begin{aligned}
& \mathcal{Y}\left[\frac{r r s}{\left[\frac{u}{t},\right.}, \frac{u}{v}\right]\left(d_{\mathrm{L} s a} C d_{\mathrm{L} t d}\right)\left(\bar{d}_{\mathrm{R} p}^{a} d_{\mathrm{L} r b}\right)\left(\bar{d}_{\mathrm{L} u}^{b} C \bar{d}_{\mathrm{L} v}^{d}\right) \\
& \mathcal{Y}\left[\frac{r}{\frac{s}{t}}, \frac{u}{v}\right]\left(d_{\mathrm{L} s c} C d_{\mathrm{L} t a}\right)\left(\bar{d}_{\mathrm{R} p}^{a} d_{\mathrm{L} r b}\right)\left(\bar{d}_{\mathrm{L} u}^{b} C \bar{d}_{\mathrm{L} v}^{c}\right)
\end{aligned}
$$




$$
\begin{aligned}
& \mathcal{Y}\left[\frac{p}{r}, \frac{s}{t}\right] .\left(d_{\mathrm{L} s c} C d_{\mathrm{L} t a}\right)\left(\bar{d}_{\mathrm{R}_{p}^{a}} C \bar{d}_{\mathrm{R} r}^{b}\right)\left(\bar{u}_{\mathrm{L} v}^{c} u_{\mathrm{R} u b}\right) \\
& \mathcal{Y}\left[\begin{array}{c}
\frac{p}{r}, \frac{s}{t} \\
t
\end{array}\right]\left(d_{\mathrm{L} s a} C d_{\mathrm{L} t b}\right)\left(\bar{u}_{\mathrm{L}}{ }_{v}^{e} u_{\mathrm{R} u e}\right)\left(\bar{d}_{\mathrm{R} p}^{a} C \bar{d}_{\mathrm{R}}^{b}\right) \\
& \mathcal{Y}\left[\frac{p}{r}, \frac{s}{t}\right]\left(\bar{d}_{\mathrm{R} r}^{b} d_{\mathrm{L} t a}\right)\left(\bar{d}_{\mathrm{R} p}^{a} d_{\mathrm{L} s c}\right)\left(\bar{u}_{\mathrm{L} v}{ }^{c} u_{\mathrm{R} u b}\right) \\
& \mathcal{Y}\left[\frac{p}{r}, \frac{s}{t}\right] .\left(\bar{d}_{\mathrm{R} p}^{a} d_{\mathrm{L} s a}\right)\left(\bar{d}_{\mathrm{R} r}^{b} d_{\mathrm{L} t b}\right)\left(\bar{u}_{\mathrm{L} v}{ }^{e} u_{\mathrm{R} u e}\right)
\end{aligned}
$$

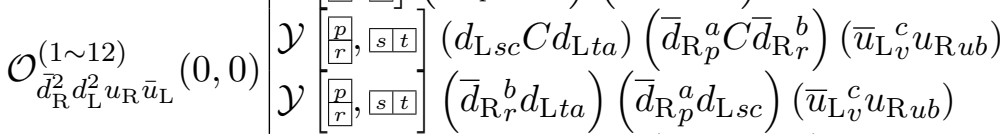

$$
\begin{aligned}
& \mathcal{Y}\left[p \mid r, \frac{s}{t}\right]\left(d_{\mathrm{L} s c} C d_{\mathrm{L} t a}\right)\left(\bar{d}_{\mathrm{R} p}^{a} C \bar{d}_{\mathrm{R} r}^{b}\right)\left(\bar{u}_{\mathrm{L} v}^{c} u_{\mathrm{R} u b}\right) \\
& \mathcal{Y}\left[p \mid r, \frac{s}{t}\right]\left(\bar{d}_{\mathrm{R}}^{b} d_{\mathrm{L} t a}\right)\left(\bar{d}_{\mathrm{R} p}^{a} d_{\mathrm{L} s c}\right)\left(\bar{u}_{\mathrm{L} v}^{c} u_{\mathrm{R} u b}\right) \\
& \mathcal{Y}[[p|r, s| t]]\left(d_{\mathrm{L} s c} C d_{\mathrm{L} t a}\right)\left(\bar{d}_{\mathrm{R} p}^{a} C \bar{d}_{\mathrm{R}}^{b}\right)\left(\bar{u}_{\mathrm{L}}^{c} u_{\mathrm{R} u b}\right) \\
& \mathcal{Y}\left[\overline{p r r}, s^{s \mid t}\right]\left(d_{\mathrm{L} s a} C d_{\mathrm{L} t b}\right)\left(\bar{u}_{\mathrm{L} v}^{e} u_{\mathrm{R} u e}\right)\left(\bar{d}_{\mathrm{R} p}^{a} C \bar{d}_{\mathrm{R} r}^{b}\right) \\
& \mathcal{Y}[\overline{p p r}, s \mid t]]\left(\bar{d}_{\mathrm{R} r}^{b} d_{\mathrm{L} t a}\right)\left(\bar{d}_{\mathrm{R} p}^{a} d_{\mathrm{L} s c}\right)\left(\bar{u}_{\mathrm{L} v}^{c} u_{\mathrm{R} u b}\right) \\
& \mathcal{Y}[\underline{p \mid r}, \underline{s \mid t}]\left(\bar{d}_{\mathrm{R} p}^{a} d_{\mathrm{L} s a}\right)\left(\bar{d}_{\mathrm{R} r}^{b} d_{\mathrm{L} t b}\right)\left(\bar{u}_{\mathrm{L} v}^{e} u_{\mathrm{R} u e}\right) \\
& \mathcal{Y}\left[\begin{array}{l}
\frac{p}{r}, \underline{s} \\
\frac{s}{t}
\end{array}\right]\left(d_{\mathrm{L} s c} C d_{\mathrm{L} t a}\right)\left(\bar{d}_{\mathrm{R}_{p}^{a}} C \bar{d}_{\mathrm{R}}^{b}\right)\left(\bar{d}_{\mathrm{L}}^{c} d_{\mathrm{R} u b}\right) \\
& \mathcal{Y}\left[\frac{p}{r}, \frac{s}{t}\right] \quad\left(d_{\mathrm{L} s a} C d_{\mathrm{L} t b}\right)\left(\bar{d}_{\mathrm{L} v}^{e} d_{\mathrm{R} u e}\right)\left(\bar{d}_{\mathrm{R} p}^{a} C \bar{d}_{\mathrm{R} r}^{b}\right) \\
& \mathcal{Y}\left[\frac{p}{r}, \frac{s}{t}\right]\left(\bar{d}_{\mathrm{R} r}{ }_{r}^{b} d_{\mathrm{L} t a}\right)\left(\bar{d}_{\mathrm{R} p}^{a} d_{\mathrm{L} s c}\right)\left(\bar{d}_{\mathrm{L} v}^{c} d_{\mathrm{R} u b}\right) \\
& \mathcal{Y}\left[\begin{array}{c}
\left.\frac{p}{r}, \frac{s}{t}\right] \\
t
\end{array}\right]\left(\bar{d}_{\mathrm{R} p}^{a} d_{\mathrm{L} s a}\right)\left(\bar{d}_{\mathrm{R} r}^{b} d_{\mathrm{L} t b}\right)\left(\bar{d}_{\mathrm{L} v}^{e} d_{\mathrm{R} u e}\right)
\end{aligned}
$$

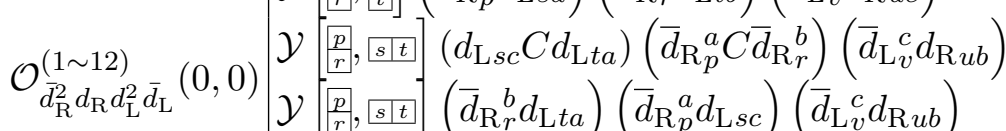

$$
\begin{aligned}
& \mathcal{Y}[r, s \mid t]\left(\bar{d}_{\mathrm{R} r} d_{\mathrm{L} t a}\right)\left(\bar{d}_{\mathrm{R} p}^{a} d_{\mathrm{L} s c}\right)\left(\bar{d}_{\mathrm{L} v}^{c} d_{\mathrm{R} u b}\right) \\
& \mathcal{Y}\left[\frac{p p r}{,}, \underline{s} .\left(d_{\mathrm{L} s c} C d_{\mathrm{L} t a}\right)\left(\bar{d}_{\mathrm{R} p}^{a} C \bar{d}_{\mathrm{R} r}^{b}\right)\left(\bar{d}_{\mathrm{L} v}^{c} d_{\mathrm{R} u b}\right)\right. \\
& \mathcal{Y}\left[\overline{p p r}, \frac{s}{t}\right]=\left(\bar{d}_{\mathrm{R}}{ }_{r}^{b} d_{\mathrm{L} t a}\right)\left(\bar{d}_{\mathrm{R} p}^{a} d_{\mathrm{L} s c}\right)\left(\bar{d}_{\mathrm{L} v}^{c} d_{\mathrm{R} u b}\right) \\
& \mathcal{Y}[p|r, s| t]\left(d_{\mathrm{L} s c} C d_{\mathrm{L} t a}\right)\left(\bar{d}_{\mathrm{R} p}^{a} C \bar{d}_{\mathrm{R} r}^{b}\right)\left(\bar{d}_{\mathrm{L} v}^{c} d_{\mathrm{R} u b}\right) \\
& \mathcal{Y}[\overline{p r r}, \underline{s \mid t}]\left(d_{\mathrm{L} s a} C d_{\mathrm{L} t b}\right)\left(\bar{d}_{\mathrm{L} v}^{e} d_{\mathrm{R} u e}\right)\left(\bar{d}_{\mathrm{R} p}^{a} C \bar{d}_{\mathrm{R} r}^{b}\right) \\
& \mathcal{Y}\left[\left[p \mid r,[s \mid t]\left(\bar{d}_{\mathrm{R} r}^{b} d_{\mathrm{L} t a}\right)\left(\bar{d}_{\mathrm{R} p}^{a} d_{\mathrm{L} s c}\right)\left(\bar{d}_{\mathrm{L} v}^{c} d_{\mathrm{R} u b}\right)\right.\right. \\
& \mathcal{Y}\left[\overline{p|r, s| t \mid t]}\left(\bar{d}_{\mathrm{R} p}^{a} d_{\mathrm{L} s a}\right)\left(\bar{d}_{\mathrm{R} r}^{b} d_{\mathrm{L} t b}\right)\left(\bar{d}_{\mathrm{L} v}^{e} d_{\mathrm{R} u e}\right)\right. \\
& \mathcal{Y}\left[\frac{p}{r}, \frac{s}{t}\right] .\left(\bar{e}_{\mathrm{L} v} e_{\mathrm{R} u}\right)\left(d_{\mathrm{L} s a} C d_{\mathrm{L} t b}\right)\left(\bar{d}_{\mathrm{R}_{p}^{a}} C \bar{d}_{\mathrm{R}_{r}^{b}}^{b}\right)
\end{aligned}
$$

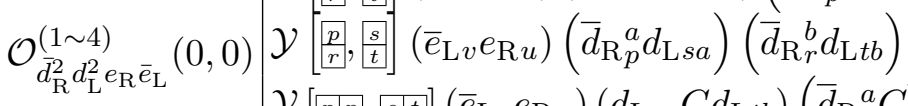

$$
\begin{aligned}
& \mathcal{Y}[\overline{p \mid r,}, s \mid t]]\left(\bar{e}_{\mathrm{L} v} e_{\mathrm{R} u}\right)\left(d_{\mathrm{L} s a} C d_{\mathrm{L} t b}\right)\left(\bar{d}_{\mathrm{R} p}^{a} C \bar{d}_{\mathrm{R} r}^{b}\right) \\
& \mathcal{Y}[\overline{p p r}, \overline{s \mid t}]\left(\bar{e}_{\mathrm{L} v} e_{\mathrm{R} u}\right)\left(\bar{d}_{\mathrm{R} p}^{a} d_{\mathrm{L} s a}\right)\left(\bar{d}_{\mathrm{R}}^{b} d_{\mathrm{L} t b}\right)
\end{aligned}
$$

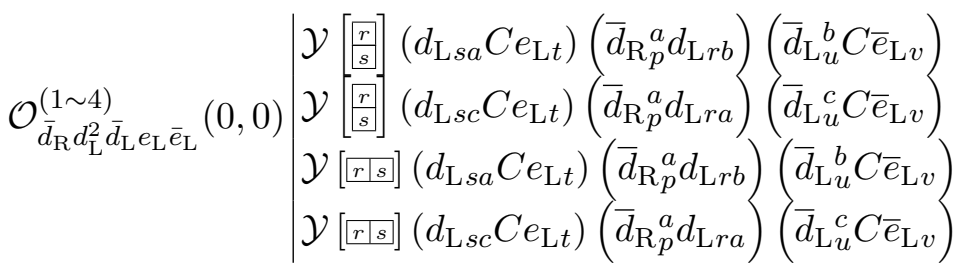




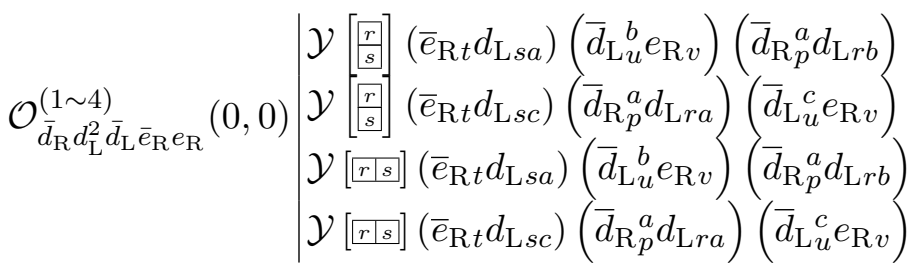

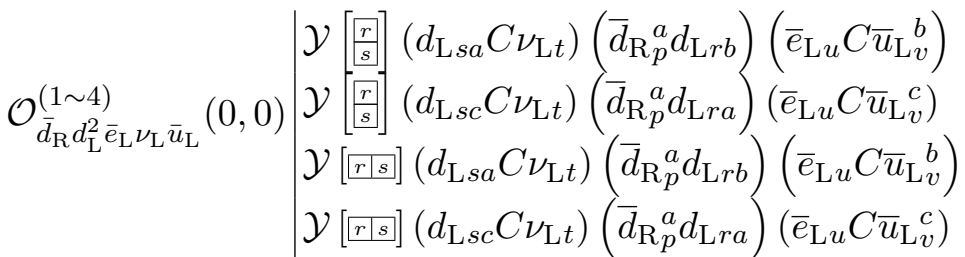

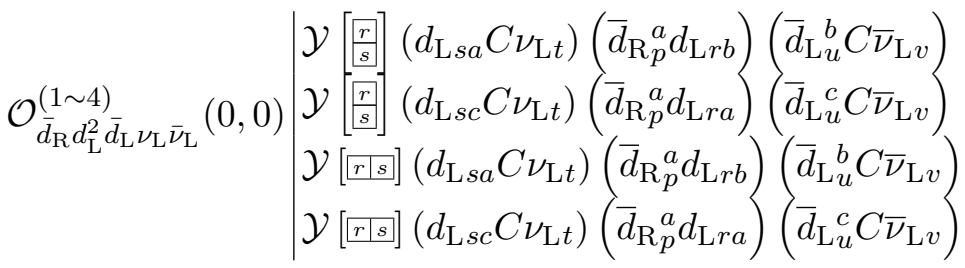

$$
\begin{aligned}
& \mathcal{Y}\left[\frac{p}{r}, u \mid v\right]\left(\bar{e}_{\mathrm{R} s} d_{\mathrm{L} p a}\right)\left(d_{\mathrm{L} r b} C e_{\mathrm{L} t}\right)\left(\bar{d}_{\mathrm{L} u}^{a} C \bar{d}_{\mathrm{L} v}^{b}\right) \\
& \mathcal{O}_{d_{\mathrm{L}}^{2} \bar{d}_{\mathrm{L}}^{2} \bar{e}_{\mathrm{R}} e_{\mathrm{L}}}^{(1 \sim 2)}(0,0) \mathcal{Y}\left[\overline{p r r}, \frac{u}{v}\right]\left(\bar{e}_{\mathrm{R} s} d_{\mathrm{L} p a}\right)\left(d_{\mathrm{L} r b} C e_{\mathrm{L} t}\right)\left(\bar{d}_{\mathrm{L} u}^{a} C \bar{d}_{\mathrm{L} v}^{b}\right) \\
& \mathcal{Y}\left[\overline{p p r}, u[v]\left(\bar{e}_{\mathrm{R} s} e_{\mathrm{L} t}\right)\left(d_{\mathrm{L} p a} C d_{\mathrm{L} r b}\right)\left(\bar{d}_{\mathrm{L} u}^{a} C \bar{d}_{\mathrm{L} v}^{b}\right)\right. \\
& \mathcal{Y}\left[\frac{p}{r}, \frac{u}{v}\right]\left(\bar{e}_{\mathrm{R} s} e_{\mathrm{L} t}\right)\left(d_{\mathrm{L} p a} C d_{\mathrm{L} r b}\right)\left(\bar{d}_{\mathrm{L} u}^{a} C \bar{d}_{\mathrm{L} v}^{b}\right)
\end{aligned}
$$

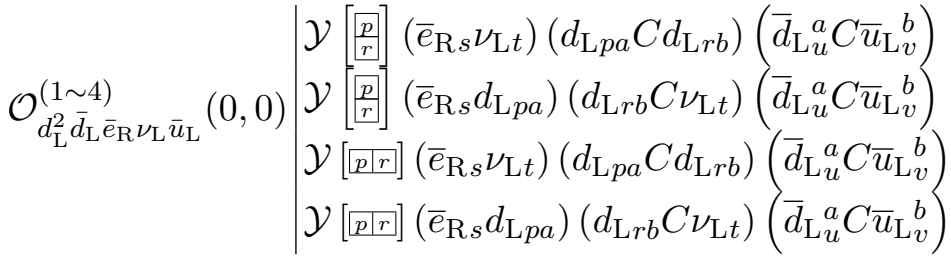

$$
\begin{aligned}
& \mathcal{Y}\left[\frac{p p r}{s p}, u v v\right]\left(d_{\mathrm{R} u b} C d_{\mathrm{R} v c}\right)\left(\bar{d}_{\mathrm{R} p}^{a} C \bar{d}_{\mathrm{R} r}^{b}\right)\left(\bar{d}_{\mathrm{R}}^{c} d_{\mathrm{L} t a}\right) \\
& \left.\mathcal{Y}\left[\frac{p r r}{s}, u v\right]\right]\left(d_{\mathrm{R} u b} C d_{\mathrm{R} v a}\right)\left(\bar{d}_{\mathrm{R} s}^{c} d_{\mathrm{L} t c}\right)\left(\bar{d}_{\mathrm{R}_{p}^{a}}^{a} C \bar{d}_{\mathrm{R} r}^{b}\right) \\
& \mathcal{Y}\left[\underline{p|r| s, u \mid v]},\left(d_{\mathrm{R} u b} C d_{\mathrm{R} v c}\right)\left(\bar{d}_{\mathrm{R} p}^{a} C \bar{d}_{\mathrm{R} r}^{b}\right)\left(\bar{d}_{\mathrm{R}_{s}^{c}} d_{\mathrm{L} t a}\right)\right. \\
& \mathcal{O}_{\bar{d}_{\mathrm{R}}^{3} d_{\mathrm{R}}^{2} d_{\mathrm{L}}}^{(\sim 8)}(0,0)\left[\mathcal{Y}\left[\begin{array}{l}
\left.\left.\frac{p}{r}, u\right] v\right] \\
\left.\frac{s}{s}\right]
\end{array}\right]\left(d_{\mathrm{R} u b} C d_{\mathrm{R} v c}\right)\left(\bar{d}_{\mathrm{R} p}^{a} C \bar{d}_{\mathrm{R}}^{b}\right)\left(\bar{d}_{\mathrm{R}}^{c} d_{\mathrm{L} t a}\right)\right. \\
& \mathcal{Y}\left[\overline{p|r| s,}, \frac{u}{v]}\right]\left(d_{\mathrm{R} u b} C d_{\mathrm{R} v c}\right)\left(\bar{d}_{\mathrm{R}}^{a} C \bar{d}_{\mathrm{R}}^{b}\right)\left(\bar{d}_{\mathrm{R}}^{c} d_{\mathrm{L} t a}\right) \\
& \mathcal{Y}\left[\frac{p r r}{s}, \frac{u}{v}\right]\left(d_{\mathrm{R} u b} C d_{\mathrm{R} v c}\right)\left(\bar{d}_{\mathrm{R}}^{a} C \bar{d}_{\mathrm{R} r}^{b}\right)\left(\bar{d}_{\mathrm{R}}^{c} d_{\mathrm{L} t a}\right) \\
& \mathcal{Y}\left[\frac{p r r}{\underline{s},}, \frac{u}{v]}\right]\left(d_{\mathrm{R} u b} C d_{\mathrm{R} v a}\right)\left(\bar{d}_{\mathrm{R} s}^{c} d_{\mathrm{L} t c}\right)\left(\bar{d}_{\mathrm{R} p}^{a} C \bar{d}_{\mathrm{R}}^{b}\right) \\
& \mathcal{Y}\left[\frac{p}{\frac{r}{s}}, \frac{u}{v}\right]\left(d_{\mathrm{R} u b} C d_{\mathrm{R} v c}\right)\left(\bar{d}_{\mathrm{R} p}^{a} C \bar{d}_{\mathrm{R}}^{b}\right)\left(\bar{d}_{\mathrm{R}}^{c} d_{\mathrm{L} t a}\right) \\
& \mathcal{Y}\left[\frac{p}{r}\right]\left(\bar{\nu}_{\mathrm{L} v} u_{\mathrm{R} u b}\right)\left(d_{\mathrm{L} s a} C e_{\mathrm{L} t}\right)\left(\bar{d}_{\mathrm{R} p}^{a} C \bar{d}_{\mathrm{R} r}^{b}\right)
\end{aligned}
$$

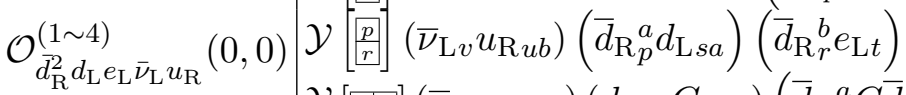

$$
\begin{aligned}
& \mathcal{Y}[\overline{p p r}]\left(\bar{\nu}_{\mathrm{L} v} u_{\mathrm{R} u b}\right)\left(d_{\mathrm{L} s a} C e_{\mathrm{L} t}\right)\left(\bar{d}_{\mathrm{R} p}^{a} C \bar{d}_{\mathrm{R} r}^{b}\right) \\
& \mathcal{Y}[\overline{p r r}]\left(\bar{\nu}_{\mathrm{L} v} u_{\mathrm{R} u b}\right)\left(\bar{d}_{\mathrm{R} p}^{a} d_{\mathrm{L} s a}\right)\left(\bar{d}_{\mathrm{R} r}^{b} e_{\mathrm{L} t}\right)
\end{aligned}
$$




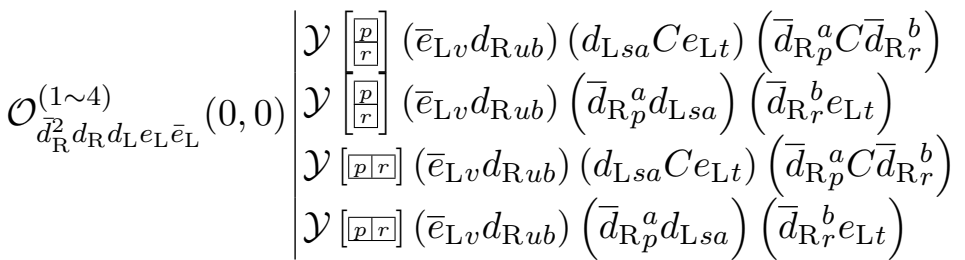

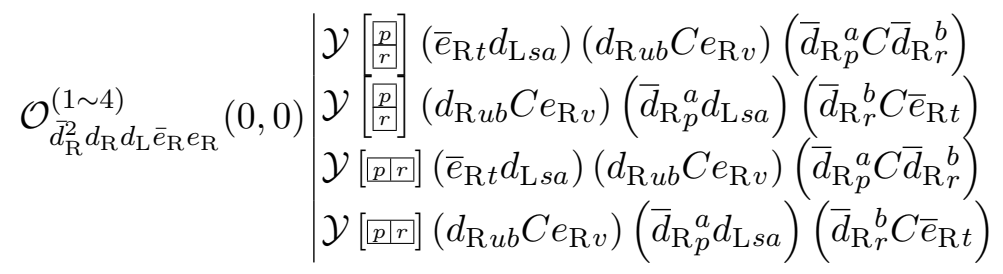

$$
\begin{aligned}
& \mathcal{Y}\left[\frac{p}{r}\right]\left(\bar{\nu}_{\mathrm{L} v} d_{\mathrm{R} u b}\right)\left(d_{\mathrm{L} s a} C \nu_{\mathrm{L} t}\right)\left(\bar{d}_{\mathrm{R}}^{a} C \bar{d}_{\mathrm{R} r}^{b}\right)
\end{aligned}
$$

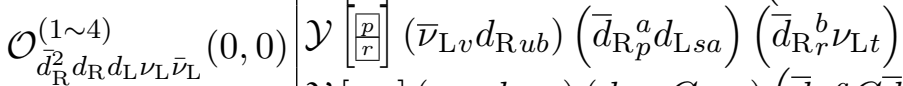

$$
\begin{aligned}
& \mathcal{Y}[\underline{p r r}]\left(\bar{\nu}_{\mathrm{L} v} d_{\mathrm{R} u b}\right)\left(d_{\mathrm{L} s a} C \nu_{\mathrm{L} t}\right)\left(\bar{d}_{\mathrm{R} p}^{a} C \bar{d}_{\mathrm{R}}^{b}\right) \\
& \mathcal{Y}[\overline{p r r}]\left(\bar{\nu}_{\mathrm{L} v} d_{\mathrm{R} u b}\right)\left(\bar{d}_{\mathrm{R} p}^{a} d_{\mathrm{L} s a}\right)\left(\bar{d}_{\mathrm{R} r}^{b} \nu_{\mathrm{L} t}\right)
\end{aligned}
$$

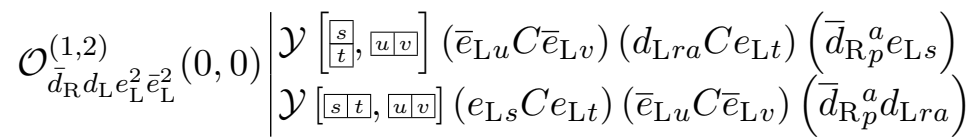

$$
\begin{aligned}
& \mathcal{O}_{\bar{d}_{\mathrm{R}} d_{\mathrm{L}} \bar{e}_{\mathrm{R}} e_{\mathrm{L}} u_{\mathrm{R}} \bar{u}_{\mathrm{L}}(0,0)} \mid \begin{array}{l}
\left(\bar{e}_{\mathrm{R} s} e_{\mathrm{L} t}\right)\left(\bar{d}_{\mathrm{R} p}^{a} d_{\mathrm{L} r b}\right)\left(\bar{u}_{\mathrm{L} v}^{b} u_{\mathrm{R} u a}\right) \\
\left(\bar{e}_{\mathrm{R} s} e_{\mathrm{L} t}\right)\left(\bar{d}_{\mathrm{R}}^{a} d_{\mathrm{L} r a}\right)\left(\bar{u}_{\mathrm{L}}^{c} u_{\mathrm{R} u c}\right) \\
\left(d_{\mathrm{L} r b} C e_{\mathrm{L} t}\right)\left(\bar{u}_{\mathrm{L} v}^{b} u_{\mathrm{R} u a}\right)\left(\bar{d}_{\mathrm{R} p}^{a} C \bar{e}_{\mathrm{R} s}\right) \\
\left(d_{\mathrm{L} r a} C e_{\mathrm{L} t}\right)\left(\bar{u}_{\mathrm{L} v}^{c} u_{\mathrm{R} u c}\right)\left(\bar{d}_{\mathrm{R} p}^{a} C \bar{e}_{\mathrm{R} s}\right)
\end{array}
\end{aligned}
$$

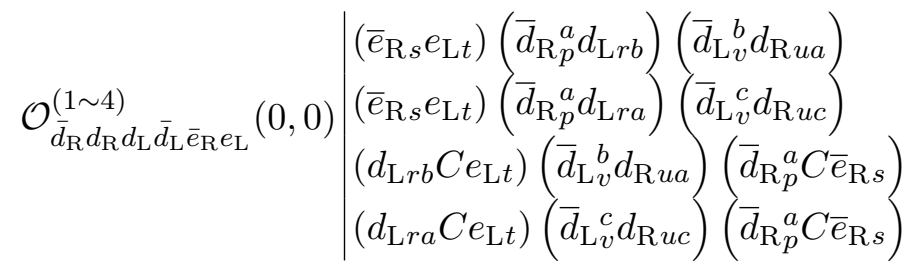

$$
\begin{aligned}
& \mathcal{O}_{\bar{d}_{\mathrm{R}} d_{\mathrm{L}} \bar{e}_{\mathrm{R}} e_{\mathrm{R}} e_{\mathrm{L}} \bar{e}_{\mathrm{L}}}^{(1,2,0)} \mid \begin{array}{l}
\left(\bar{e}_{\mathrm{R} s} e_{\mathrm{L} t}\right)\left(\bar{e}_{\mathrm{L} v} e_{\mathrm{R} u}\right)\left(\bar{d}_{\mathrm{R}}{ }^{a} d_{\mathrm{L} r a}\right) \\
\left(\bar{e}_{\mathrm{L} v} e_{\mathrm{R} u}\right)\left(d_{\mathrm{L} r a} C e_{\mathrm{L} t}\right)\left(\bar{d}_{\mathrm{R}}^{a} C \bar{e}_{\mathrm{R} s}\right)
\end{array} \\
& \left.\mathcal{O}_{\bar{d}_{\mathrm{R}} d_{\mathrm{L}} e_{\mathrm{L}} \bar{e}_{\mathrm{L}} \nu_{\mathrm{L}} \bar{\nu}_{\mathrm{L}}}^{(1,2,0)} \mid \begin{array}{l}
\left(e_{\mathrm{L} s} C \nu_{\mathrm{L} t}\right)\left(\bar{e}_{\mathrm{L} u} C \bar{\nu}_{\mathrm{L} v}\right)\left(\bar{d}_{\mathrm{R}}^{a} d_{\mathrm{L} r a}\right. \\
\left(\bar{e}_{\mathrm{L} u} C \bar{\nu}_{\mathrm{L} v}\right)\left(d_{\mathrm{L} r a} C \nu_{\mathrm{L} t}\right)\left(\bar{d}_{\mathrm{R} p}^{a} e_{\mathrm{L} s}\right.
\end{array}\right)
\end{aligned}
$$

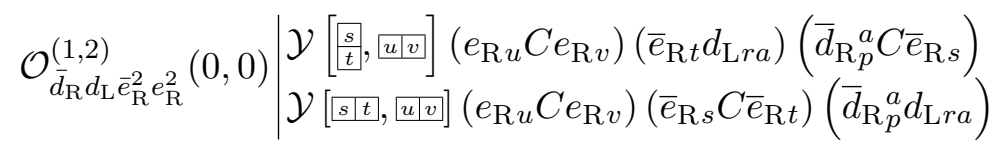

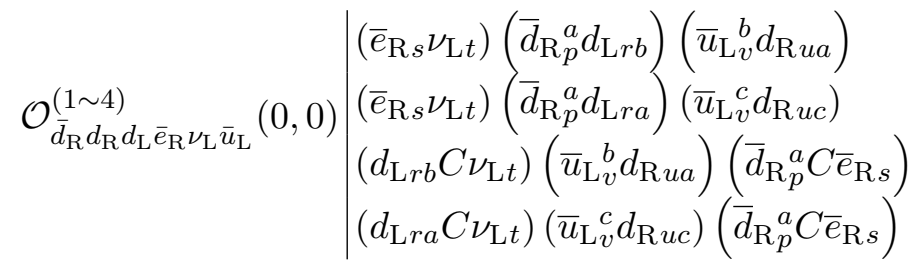




$$
\begin{aligned}
& \mathcal{O}_{\bar{d}_{\mathrm{R}} d_{\mathrm{L}} \bar{e}_{\mathrm{R}} e_{\mathrm{R}} \nu_{\mathrm{L}} \bar{\nu}_{\mathrm{L}}}^{(1,2,0)} \mid \begin{array}{l}
\left(\bar{e}_{\mathrm{R} s} \nu_{\mathrm{L} t}\right)\left(\bar{\nu}_{\mathrm{L} v} e_{\mathrm{R} u}\right)\left(\bar{d}_{\mathrm{R}}^{a} d_{\mathrm{L} r a}\right) \\
\left(\bar{\nu}_{\mathrm{L} v} e_{\mathrm{R} u}\right)\left(d_{\mathrm{L} r a} C \nu_{\mathrm{L} t}\right)\left(\bar{d}_{\mathrm{R} p}^{a} C \bar{e}_{\mathrm{R} s}\right)
\end{array}
\end{aligned}
$$

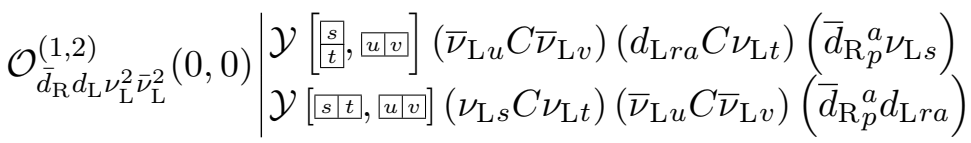

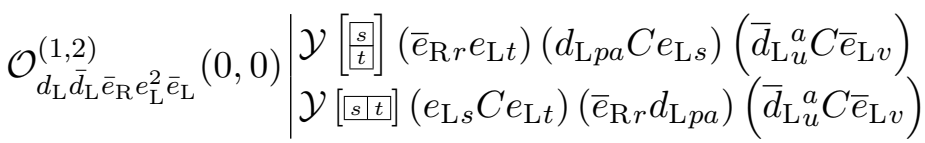

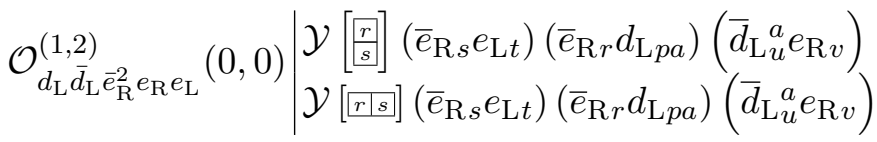

$$
\begin{aligned}
& \mathcal{O}_{d_{\mathrm{L}} \bar{e}_{\mathrm{R}} e_{\mathrm{L}} \bar{e}_{\mathrm{L}} \nu_{\mathrm{L}} \bar{u}_{\mathrm{L}}}^{(1,0,0)} \mid \begin{array}{l}
\left(e_{\mathrm{L} s} C \nu_{\mathrm{L} t}\right)\left(\bar{e}_{\mathrm{R} r} d_{\mathrm{L} p a}\right)\left(\bar{e}_{\mathrm{L} u} C \bar{u}_{\mathrm{L}}^{a}\right) \\
\left(\bar{e}_{\mathrm{R} r} \nu_{\mathrm{L} t}\right)\left(d_{\mathrm{L} p a} C e_{\mathrm{L} s}\right)\left(\bar{e}_{\mathrm{L} u} C \bar{u}_{\mathrm{L}}^{a}\right)
\end{array} \\
& \left.\mathcal{O}_{d_{\mathrm{L}} \bar{d}_{\mathrm{L}} \bar{e}_{\mathrm{R}} e_{\mathrm{L}} \nu_{\mathrm{L}} \bar{\nu}_{\mathrm{L}}}^{(1,0,0)} \mid \begin{array}{l}
\left(e_{\mathrm{L} s} C \nu_{\mathrm{L} t}\right)\left(\bar{e}_{\mathrm{R} r} d_{\mathrm{L} p a}\right)\left(\bar{d}_{\mathrm{L} u}^{a} C \bar{\nu}_{\mathrm{L} v}\right) \\
\left(\bar{e}_{\mathrm{R} r} \nu_{\mathrm{L} t}\right)\left(d_{\mathrm{L} p a} C e_{\mathrm{L} s}\right)\left(\bar{d}_{\mathrm{L} u}^{a} C \bar{\nu}_{\mathrm{L} v}\right.
\end{array}\right)
\end{aligned}
$$

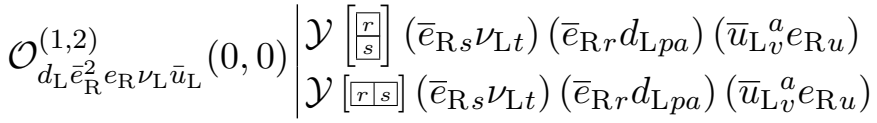

$$
\begin{aligned}
& \mathcal{O}_{d_{\mathrm{L}} \bar{e}_{\mathrm{R}} \nu_{\mathrm{L}}^{2} \bar{\nu}_{\mathrm{L}} \bar{u}_{\mathrm{L}}}^{(1,0,0)} \mid \begin{array}{l}
\mathcal{Y}\left[\begin{array}{l}
{[s]} \\
\underline{t}
\end{array}\right]\left(\bar{e}_{\mathrm{R} r} \nu_{\mathrm{L} t}\right)\left(d_{\mathrm{L} p a} C \nu_{\mathrm{L} s}\right)\left(\bar{u}_{\mathrm{L} u}^{a} C \bar{\nu}_{\mathrm{L} v}\right) \\
\mathcal{Y}\left[\begin{array}{l}
{[s \mid t]} \\
{[s}
\end{array}\right]\left(\nu_{\mathrm{L} s} C \nu_{\mathrm{L} t}\right)\left(\bar{e}_{\mathrm{R} r} d_{\mathrm{L} p a}\right)\left(\bar{u}_{\mathrm{L} u}^{a} C \bar{\nu}_{\mathrm{L} v}\right)
\end{array}
\end{aligned}
$$

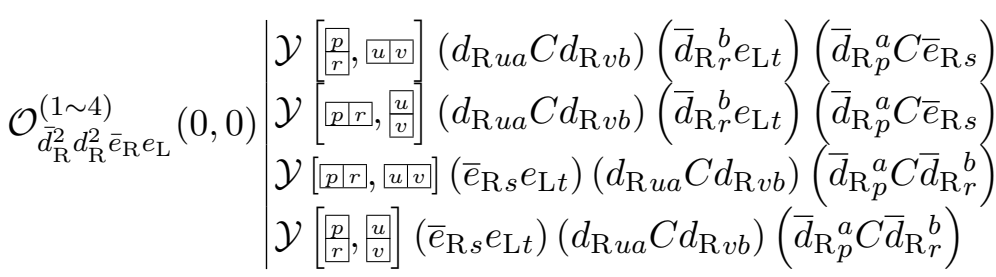

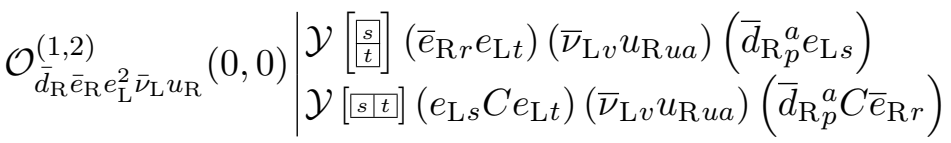

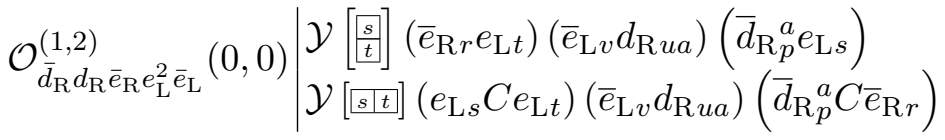

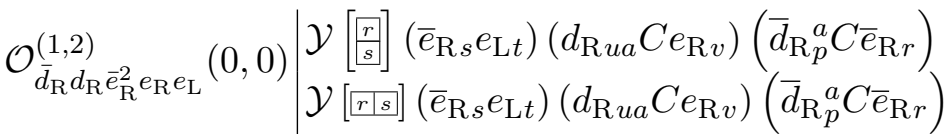

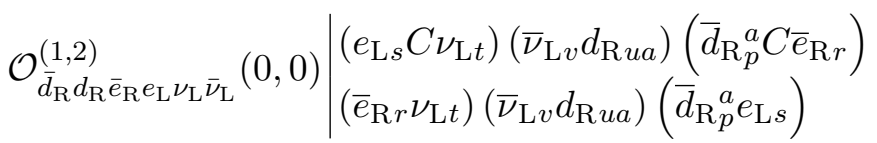

$$
\begin{aligned}
& \mathcal{O}_{\bar{e}_{\mathrm{R}} e_{\mathrm{L}}^{3} \bar{e}_{\mathrm{L}}^{2}}(0,0) \mid \mathcal{Y}\left[\frac{r r s}{t r}, u \underline{u v}\right]\left(e_{\mathrm{L} s} C e_{\mathrm{L} t}\right)\left(\bar{e}_{\mathrm{L} u} C \bar{e}_{\mathrm{L} v}\right)\left(\bar{e}_{\mathrm{R} p} e_{\mathrm{L} r}\right)
\end{aligned}
$$

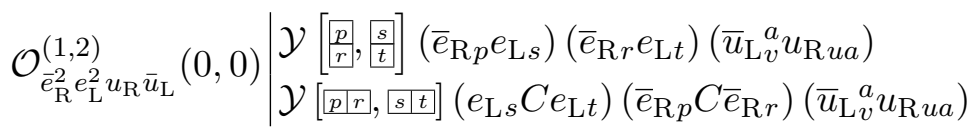




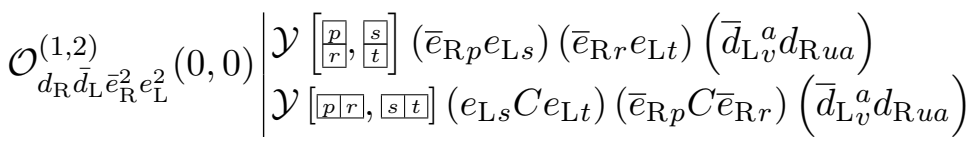

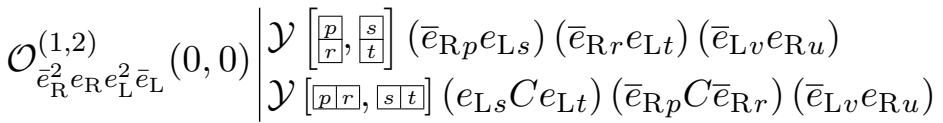

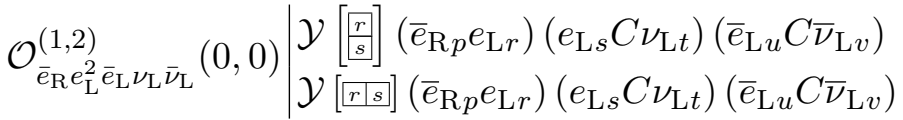

$$
\begin{aligned}
& \mathcal{O}_{\bar{e}_{\mathrm{R}}^{3} e_{\mathrm{R}}^{2} e_{\mathrm{L}}}(0,0) \mid \mathcal{Y}\left[\frac{p r r}{\underline{s}}, u \underline{u v}\right]\left(e_{\mathrm{R} u} C e_{\mathrm{R} v}\right)\left(\bar{e}_{\mathrm{R} p} C \bar{e}_{\mathrm{R} r}\right)\left(\bar{e}_{\mathrm{R} s} e_{\mathrm{L} t}\right)
\end{aligned}
$$

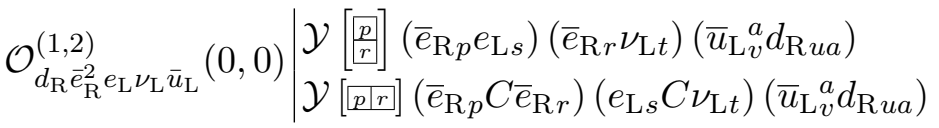

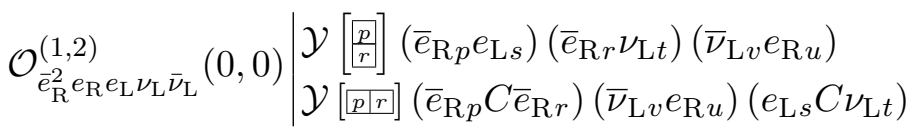

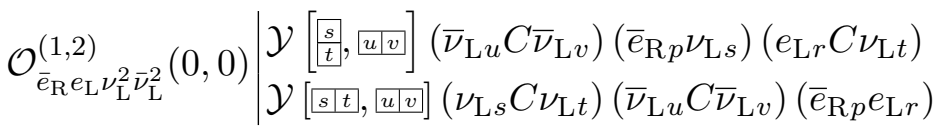

$(\Delta B, \Delta L)=(0, \pm 2)$

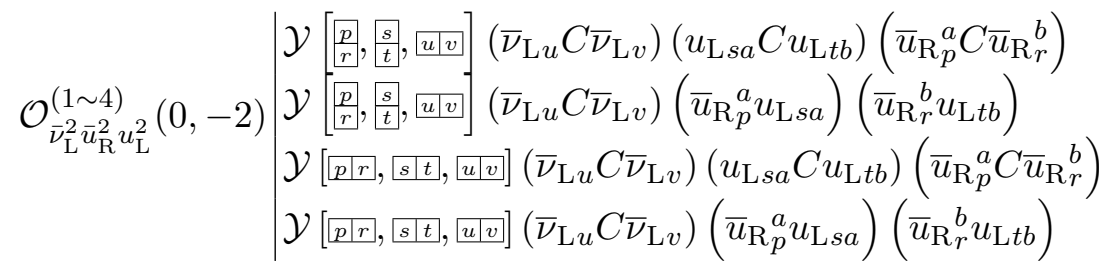

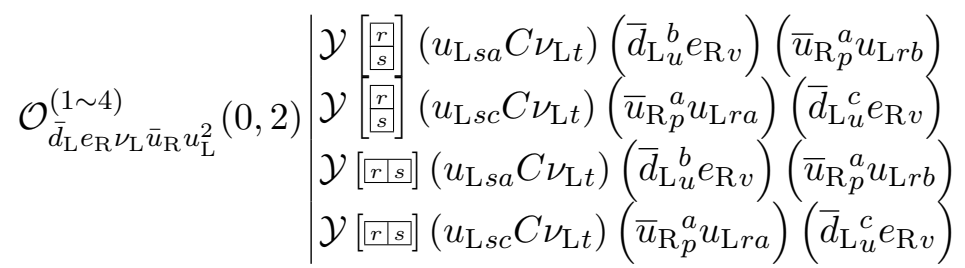

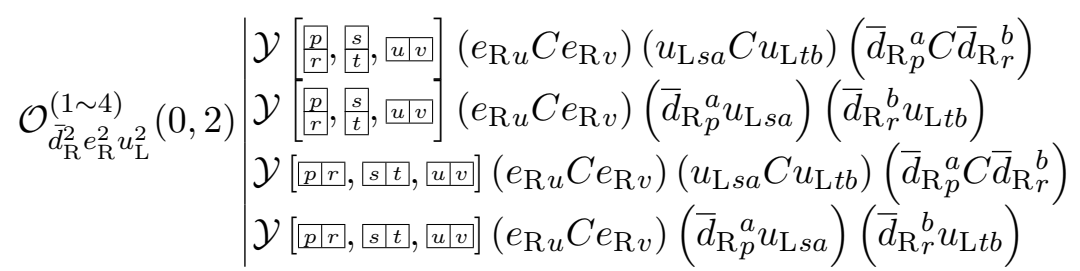

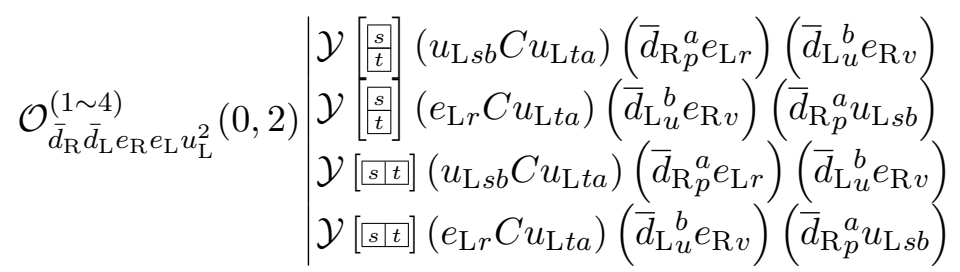




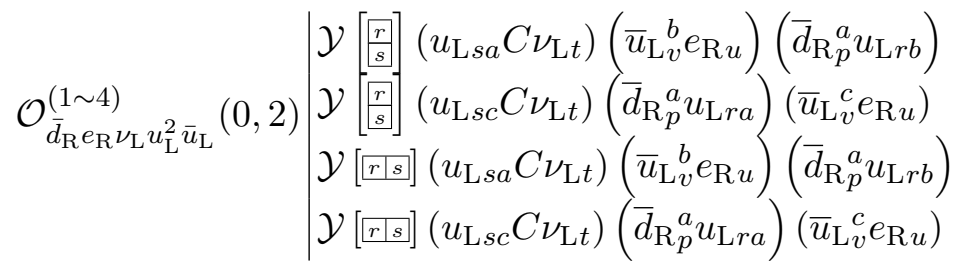

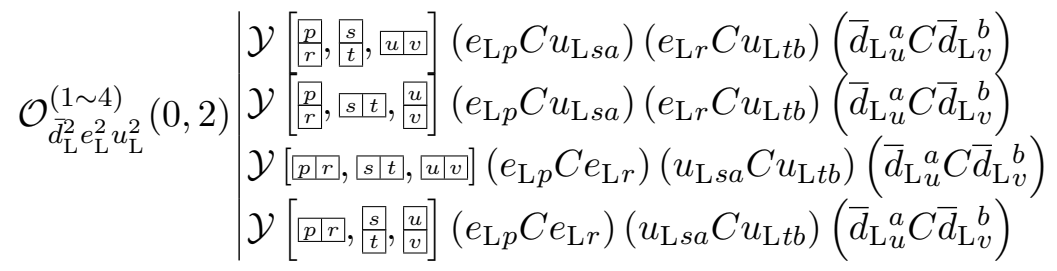

$$
\begin{aligned}
& \mathcal{Y}\left[\begin{array}{l}
\frac{r}{s} \\
]
\end{array}\left(e_{\mathrm{L} p} C u_{\mathrm{L} r a}\right)\left(u_{\mathrm{L} s b} C \nu_{\mathrm{L} t}\right)\left(\bar{d}_{\mathrm{L} u}^{a} C \bar{u}_{\mathrm{L} v}^{b}\right)\right.
\end{aligned}
$$

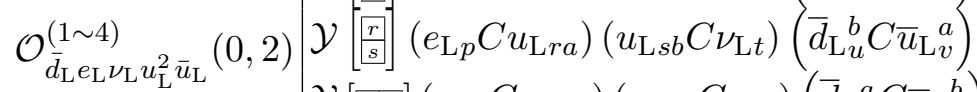

$$
\begin{aligned}
& \mathcal{Y}\left[r[s]\left(e_{\mathrm{L} p} C u_{\mathrm{L} r a}\right)\left(u_{\mathrm{L} s b} C \nu_{\mathrm{L} t}\right)\left(\bar{d}_{\mathrm{L} u}^{a} C \bar{u}_{\mathrm{L} v}^{b}\right)\right. \\
& \mathcal{Y}[r r s]\left(e_{\mathrm{L} p} C u_{\mathrm{L} r a}\right)\left(u_{\mathrm{L} s b} C \nu_{\mathrm{L} t}\right)\left(\bar{d}_{\mathrm{L} u}^{b} C \bar{u}_{\mathrm{L} v}^{a}\right)
\end{aligned}
$$

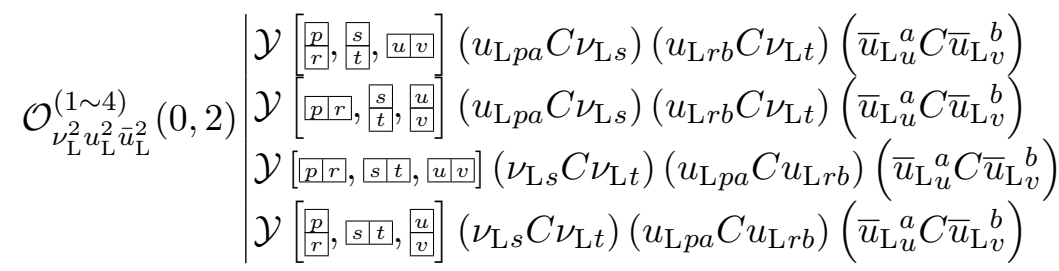

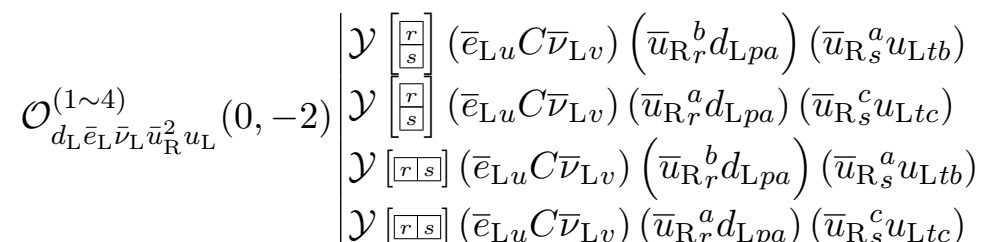

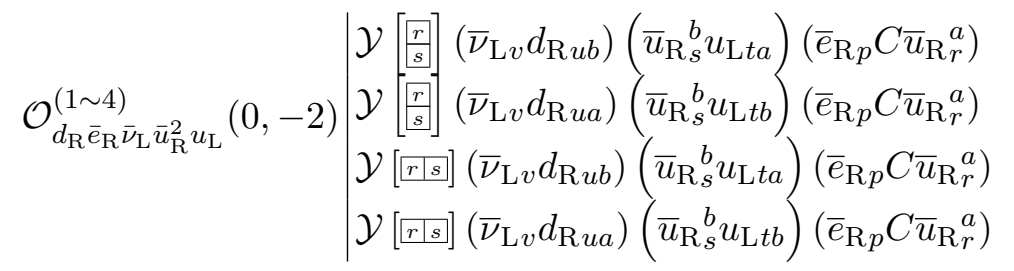

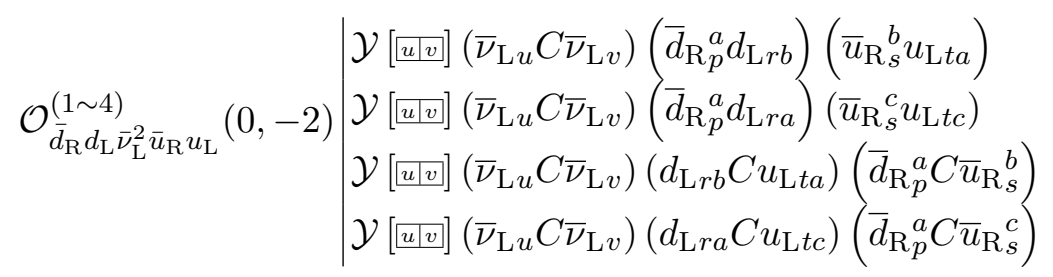

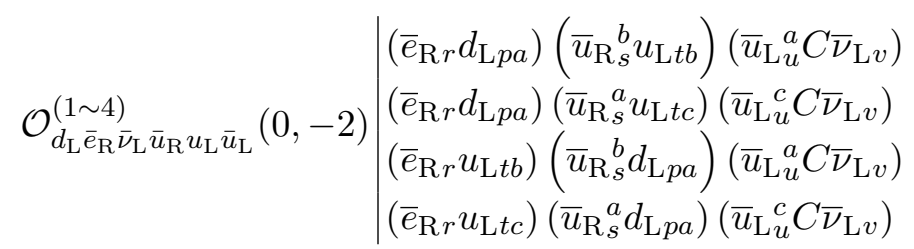




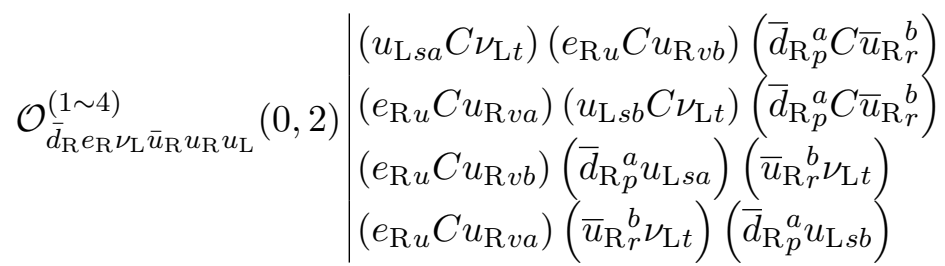

$$
\begin{aligned}
& \mathcal{O}_{\bar{e}_{\mathrm{R}} e_{\mathrm{L}} \bar{\nu}_{\mathrm{L}}^{2} \bar{u}_{\mathrm{R}} u_{\mathrm{L}}}^{(10,-2)} \mid \begin{array}{l}
\mathcal{Y}[\overline{u v}]\left(\bar{\nu}_{\mathrm{L} u} C \bar{\nu}_{\mathrm{L} v}\right)\left(\bar{e}_{\mathrm{R} p} e_{\mathrm{L} r}\right)\left(\bar{u}_{\mathrm{R} s}^{a} u_{\mathrm{L} t a}\right) \\
\mathcal{Y}\left[\overline{u v v}\left(\bar{\nu}_{\mathrm{L} u} C \bar{\nu}_{\mathrm{L} v}\right)\left(e_{\mathrm{L} r} C u_{\mathrm{L} t a}\right)\left(\bar{e}_{\mathrm{R} p} C \bar{u}_{\mathrm{R} s}^{a}\right)\right.
\end{array} \\
& \left.\mathcal{O}_{\bar{d}_{\mathrm{L}} e_{\mathrm{L}} \nu_{\mathrm{L}} \bar{u}_{\mathrm{R}} u_{\mathrm{R}} u_{\mathrm{L}}}^{(10,2)} \mid \begin{array}{l}
\left(u_{\mathrm{L} s b} C \nu_{\mathrm{L} t}\right)\left(\bar{u}_{\mathrm{R} r}^{a} e_{\mathrm{L} p}\right)\left(\bar{d}_{\mathrm{L} u}^{b} u_{\mathrm{R} v a}\right. \\
\left(u_{\mathrm{L} s a} C \nu_{\mathrm{L} t}\right)\left(\bar{u}_{\mathrm{R} r}^{a} e_{\mathrm{L} p}\right)\left(\bar{d}_{\mathrm{L} u}^{c} u_{\mathrm{R} v c}\right. \\
\left(e_{\mathrm{L} p} C u_{\mathrm{L} s b}\right)\left(\bar{u}_{\mathrm{R} r}{ }_{r}^{a} \nu_{\mathrm{L} t}\right)\left(\bar{d}_{\mathrm{L} u}^{b} u_{\mathrm{R} v a}\right. \\
\left(e_{\mathrm{L} p} C u_{\mathrm{L} s a}\right)\left(\bar{u}_{\mathrm{R} r}^{a} \nu_{\mathrm{L} t}\right)\left(\bar{d}_{\mathrm{L} u}^{c} u_{\mathrm{R} v c}\right.
\end{array}\right)
\end{aligned}
$$

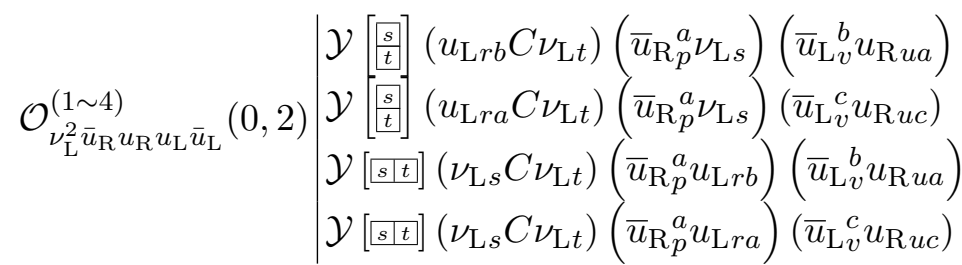

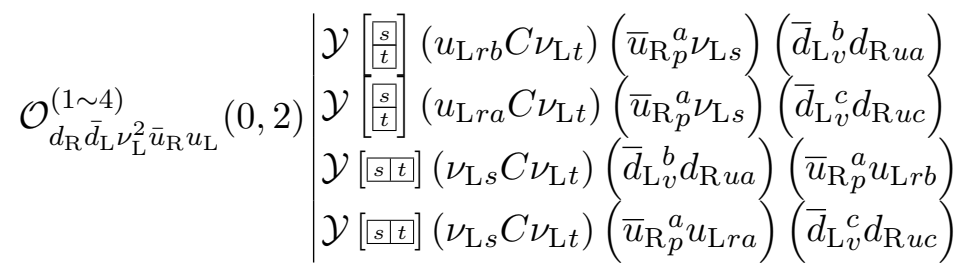

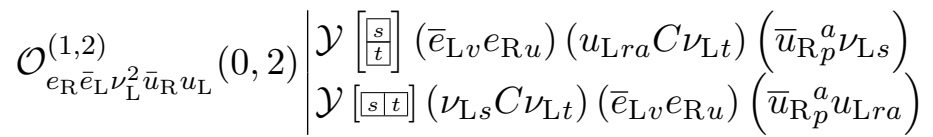

$$
\begin{aligned}
& \left.\mathcal{O}_{\bar{d}_{\mathrm{R}} d_{\mathrm{L}} \bar{d}_{\mathrm{L}} e_{\mathrm{R}} \nu_{\mathrm{L}} u_{\mathrm{L}}}(0,2) \mid \begin{array}{l}
\left(u_{\mathrm{L} s a} C \nu_{\mathrm{L} t}\right)\left(\bar{d}_{\mathrm{L} u}^{b} e_{\mathrm{R} v}\right)\left(\bar{d}_{\mathrm{R} p}^{a} d_{\mathrm{L} r b}\right) \\
\left(u_{\mathrm{L} s c} C \nu_{\mathrm{L} t}\right)\left(\bar{d}_{\mathrm{R}}^{a} d_{\mathrm{L} r a}\right)\left(\bar{d}_{\mathrm{L} u}^{c} e_{\mathrm{R} v}\right. \\
\left(d_{\mathrm{L} r b} C \nu_{\mathrm{L} t}\right)\left(\bar{d}_{\mathrm{R}}{ }^{a} u_{\mathrm{L} s a}\right)\left(\bar{d}_{\mathrm{L} u}^{b} e_{\mathrm{R} v}\right. \\
\left(d_{\mathrm{L} r a} C \nu_{\mathrm{L} t}\right)\left(\bar{d}_{\mathrm{L} u}^{c} e_{\mathrm{R} v}\right)\left(\bar{d}_{\mathrm{R} p}^{a} u_{\mathrm{L} s c}\right.
\end{array}\right)
\end{aligned}
$$

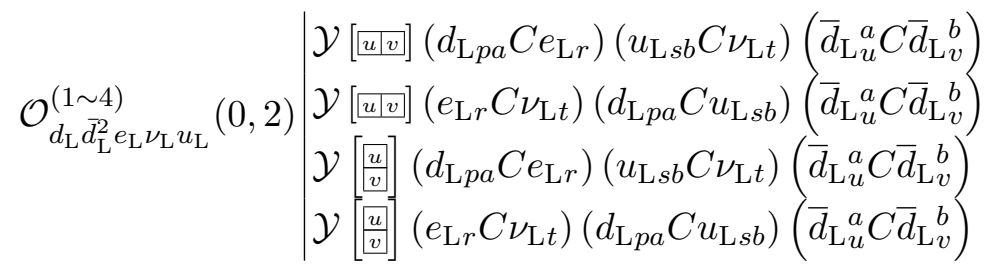

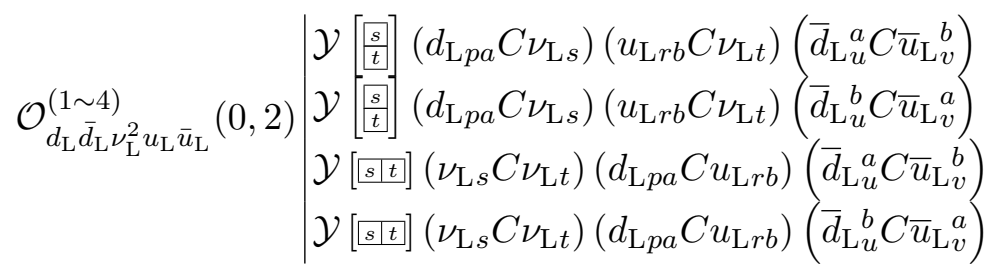




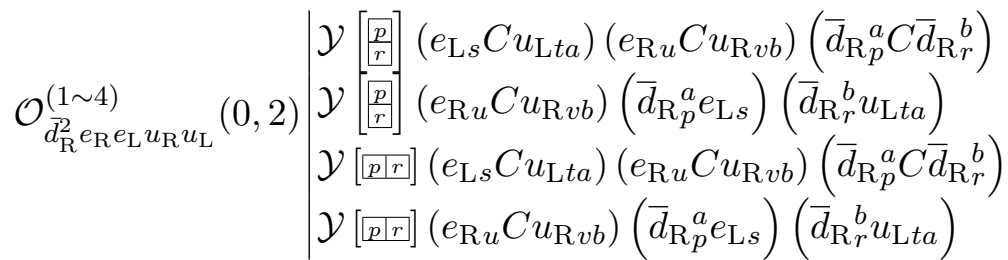

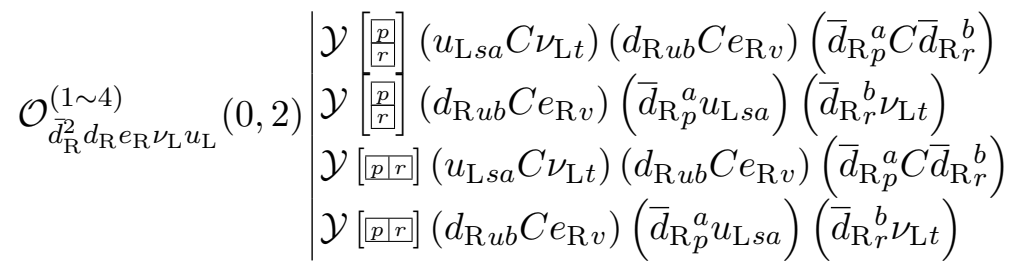

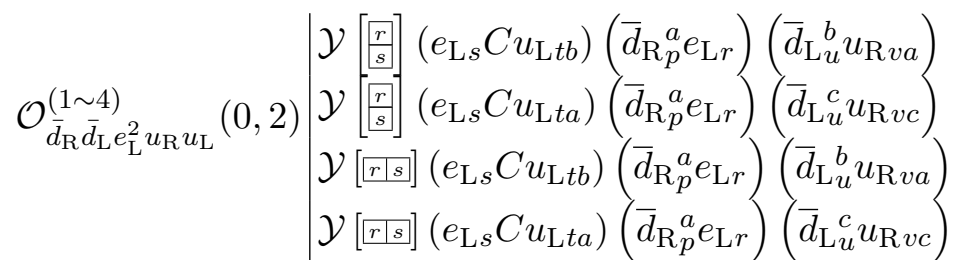

$$
\begin{aligned}
& \mathcal{O}_{\bar{d}_{\mathrm{R}} e_{\mathrm{L}} \nu_{\mathrm{L}} u_{\mathrm{R}} u_{\mathrm{L}} \bar{u}_{\mathrm{L}}}^{(1 \sim 2)} \mid \begin{array}{l}
\left(u_{\mathrm{L} s b} C \nu_{\mathrm{L} t}\right)\left(\bar{d}_{\mathrm{R} p}^{a} e_{\mathrm{L} r}\right)\left(\bar{u}_{\mathrm{L} v}^{b} u_{\mathrm{R} u a}\right) \\
\left(u_{\mathrm{L} s a} C \nu_{\mathrm{L} t}\right)\left(\bar{d}_{\mathrm{R}}^{a} e_{\mathrm{L} r}\right)\left(\bar{u}_{\mathrm{L}}^{c} u_{v} u_{\mathrm{R} u c}\right) \\
\left(e_{\mathrm{L} r} C \nu_{\mathrm{L} t}\right)\left(\bar{d}_{\mathrm{R}}^{a} u_{\mathrm{L} s b}\right)\left(\bar{u}_{\mathrm{L} v}^{b} u_{\mathrm{R} u a}\right) \\
\left(e_{\mathrm{L} r} C \nu_{\mathrm{L} t}\right)\left(\bar{d}_{\mathrm{R}}^{a} u_{\mathrm{L} s a}\right)\left(\bar{u}_{\mathrm{L}}^{c} u_{\mathrm{R} u c}\right)
\end{array} \\
& \left.\mathcal{O}_{\bar{d}_{\mathrm{R}} d_{\mathrm{R}} \bar{d}_{\mathrm{L}} e_{\mathrm{L}} \nu_{\mathrm{L}} u_{\mathrm{L}}}(0,2) \mid \begin{array}{l}
\left(u_{\mathrm{L} s b} C \nu_{\mathrm{L} t}\right)\left(\bar{d}_{\mathrm{R} p}^{a} e_{\mathrm{L} r}\right)\left(\bar{d}_{\mathrm{L} v}^{b} d_{\mathrm{R} u a}\right. \\
\left(u_{\mathrm{L} s a} C \nu_{\mathrm{L} t}\right)\left(\bar{d}_{\mathrm{R} p}^{a} e_{\mathrm{L} r}\right)\left(\bar{d}_{\mathrm{L}}^{c} d_{\mathrm{R} u c}\right. \\
\left(e_{\mathrm{L} r} C \nu_{\mathrm{L} t}\right)\left(\bar{d}_{\mathrm{L} v}^{b} d_{\mathrm{R} u a}\right)\left(\bar{d}_{\mathrm{R}}{ }^{a} u_{\mathrm{L} s b}\right. \\
\left(e_{\mathrm{L} r} C \nu_{\mathrm{L} t}\right)\left(\bar{d}_{\mathrm{R} p}^{a} u_{\mathrm{L} s a}\right)\left(\bar{d}_{\mathrm{L} v}^{c} d_{\mathrm{R} u c}\right.
\end{array}\right) \\
& \left.\mathcal{O}_{\bar{d}_{\mathrm{R}} e_{\mathrm{R}} e_{\mathrm{L}} \bar{e}_{\mathrm{L}} \nu_{\mathrm{L}} u_{\mathrm{L}}}^{(1,2)}(0,2) \mid \begin{array}{l}
\left(\bar{e}_{\mathrm{L} v} e_{\mathrm{R} u}\right)\left(u_{\mathrm{L} s a} C \nu_{\mathrm{L} t}\right)\left(\bar{d}_{\mathrm{R} p}^{a} e_{\mathrm{L} r}\right) \\
\left(\bar{e}_{\mathrm{L} v} e_{\mathrm{R} u}\right)\left(e_{\mathrm{L} r} C \nu_{\mathrm{L} t}\right)\left(\bar{d}_{\mathrm{R} p}^{a} u_{\mathrm{L} s a}\right.
\end{array}\right) \\
& \mathcal{O}_{\bar{d}_{\mathrm{R}} \bar{e}_{\mathrm{R}} e_{\mathrm{R}}^{2} \nu_{\mathrm{L}} u_{\mathrm{L}}}^{(1,2,2)} \mid \begin{array}{l}
\mathcal{Y}[\underline{[u v}]\left(e_{\mathrm{R} u} C e_{\mathrm{R} v}\right)\left(u_{\mathrm{L} s a} C \nu_{\mathrm{L} t}\right)\left(\bar{d}_{\mathrm{R} p}^{a} C \bar{e}_{\mathrm{R} r}\right) \\
\mathcal{Y}[\underline{u v}]\left(e_{\mathrm{R} u} C e_{\mathrm{R} v}\right)\left(\bar{e}_{\mathrm{R} r} \nu_{\mathrm{L} t}\right)\left(\bar{d}_{\mathrm{R} p}^{a} u_{\mathrm{L} s a}\right)
\end{array}
\end{aligned}
$$

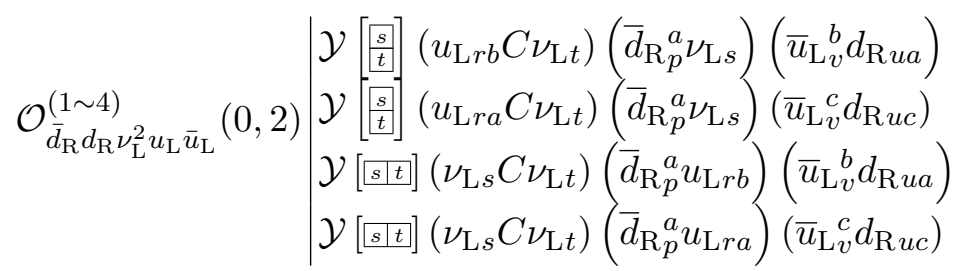

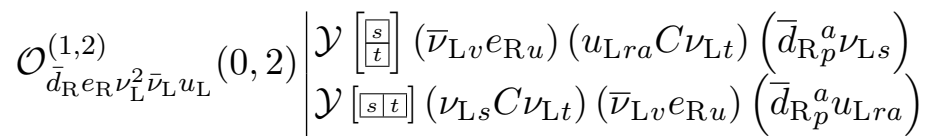

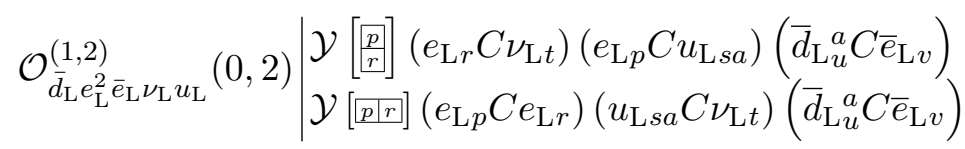




$$
\begin{aligned}
& \mathcal{O}_{\bar{d}_{\mathrm{L}} \bar{e}_{\mathrm{R}} e_{\mathrm{R}} e_{\mathrm{L}} \nu_{\mathrm{L}} u_{\mathrm{L}}}^{(0,2)}\left(\begin{array}{l}
\left(\bar{e}_{\mathrm{R} p} e_{\mathrm{L} r}\right)\left(u_{\mathrm{L} s a} C \nu_{\mathrm{L} t}\right)\left(\bar{d}_{\mathrm{L}}{ }_{u}^{a} e_{\mathrm{R} v}\right. \\
\left(e_{\mathrm{L} r} C \nu_{\mathrm{L} t}\right)\left(\bar{e}_{\mathrm{R} p} u_{\mathrm{L} s a}\right)\left(\bar{d}_{\mathrm{L} u}^{a} e_{\mathrm{R} v}\right.
\end{array}\right)
\end{aligned}
$$

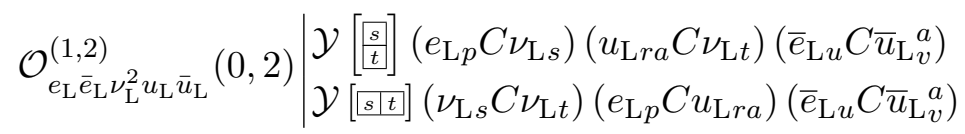

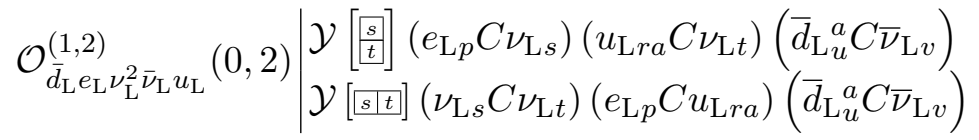

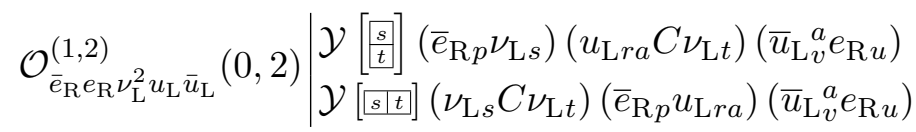

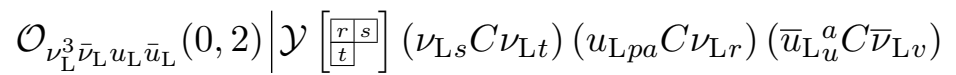

$$
\begin{aligned}
& \mid \mathcal{Y}\left[\frac{p}{r}, \frac{s}{t}, u v v\right]\left(\bar{e}_{\mathrm{L} u} C \bar{e}_{\mathrm{L} v}\right)\left(d_{\mathrm{L} p a} C d_{\mathrm{L} r b}\right)\left(\bar{u}_{\mathrm{R} s}^{a} C \bar{u}_{\mathrm{R} t}^{b}\right) \\
& \mathcal{O}_{d_{\mathrm{L}}^{2} \bar{e}_{\mathrm{L}}^{2} \bar{u}_{\mathrm{R}}^{2}}^{(1 \sim 4)}(0,-2) \mathcal{Y}\left[\frac{p}{r}, \frac{s}{t},,_{u v}\right]\left(\bar{e}_{\mathrm{L} u} C \bar{e}_{\mathrm{L} v}\right)\left(\bar{u}_{\mathrm{R}}^{a}{ }_{s} d_{\mathrm{L} p a}\right)\left(\bar{u}_{\mathrm{R}}^{b} d_{\mathrm{L} r b}\right)
\end{aligned}
$$

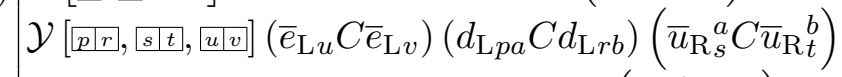

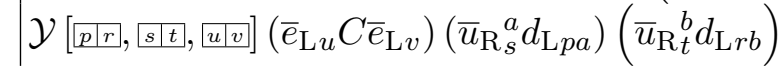

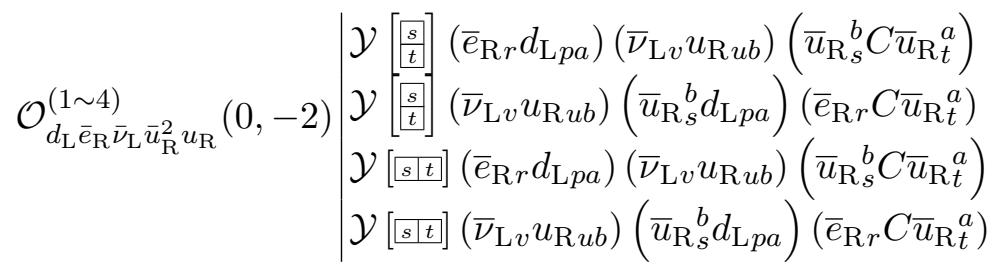

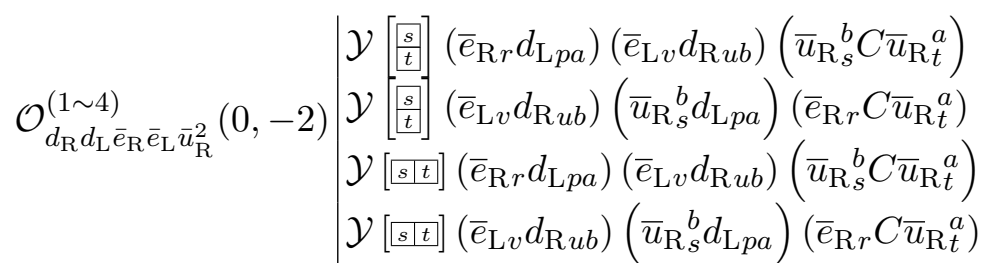

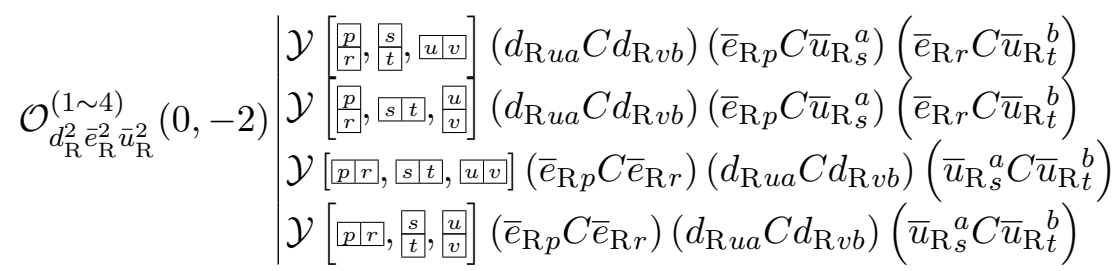

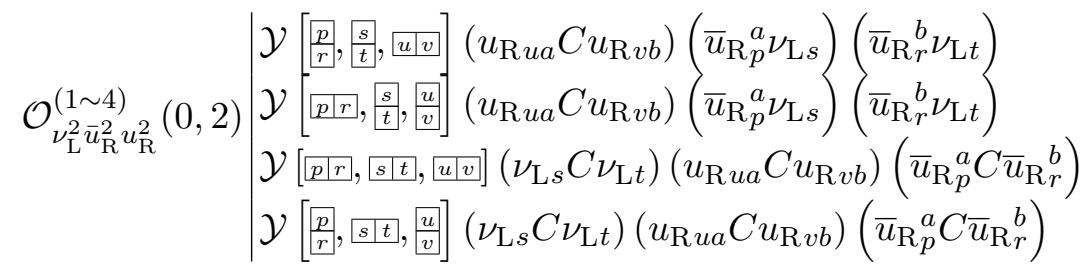




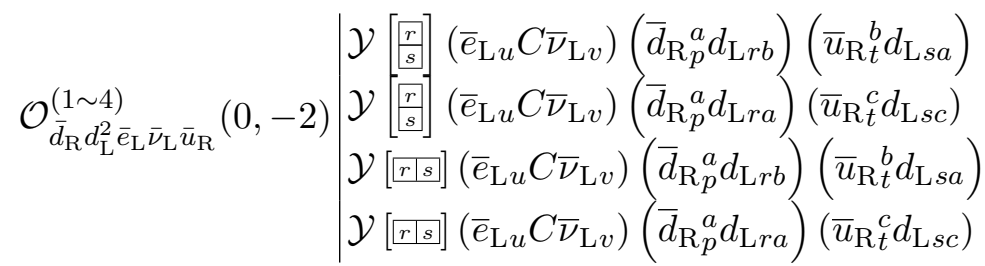

$$
\begin{aligned}
& \mathcal{Y}\left[\frac{p}{r}\right] .\left(d_{\mathrm{L} p a} C d_{\mathrm{L} r b}\right)\left(\bar{e}_{\mathrm{R} s} C \bar{u}_{\mathrm{R}}{ }_{t}^{a}\right)\left(\bar{e}_{\mathrm{L} u} C \bar{u}_{\mathrm{L} v}^{b}\right) \\
& \mathcal{O}_{d_{\mathrm{L}}^{2} \bar{e}_{\mathrm{R}} \bar{e}_{\mathrm{L}} \bar{u}_{\mathrm{R}} \bar{u}_{\mathrm{L}}}^{(0,-2)} \mathcal{Y}\left[\begin{array}{l}
\bar{p} \\
\underline{r}
\end{array}\right]\left(\bar{e}_{\mathrm{R} s} d_{\mathrm{L} p a}\right)\left(\bar{u}_{\mathrm{R} t}^{a} d_{\mathrm{L} r b}\right)\left(\bar{e}_{\mathrm{L} u} C \bar{u}_{\mathrm{L} v}^{b}\right) \\
& \mathcal{Y}[\overline{p r r}]\left(d_{\mathrm{L} p a} C d_{\mathrm{L} r b}\right)\left(\bar{e}_{\mathrm{R} s} C \bar{u}_{\mathrm{R} t}^{a}\right)\left(\bar{e}_{\mathrm{L} u} C \bar{u}_{\mathrm{L} v}^{b}\right) \\
& \mathcal{Y}\left[{ }_{p r r}\right]\left(\bar{e}_{\mathrm{R} s} d_{\mathrm{L} p a}\right)\left(\bar{u}_{\mathrm{R} t}^{a} d_{\mathrm{L} r b}\right)\left(\bar{e}_{\mathrm{L} u} C \bar{u}_{\mathrm{L} v}^{b}\right) \\
& \mathcal{Y}\left[\begin{array}{l}
\underline{p} \\
r
\end{array}\right]\left(d_{\mathrm{L} p a} C d_{\mathrm{L} r b}\right)\left(\bar{e}_{\mathrm{R} s} C \bar{u}_{\mathrm{R} t}^{a}\right)\left(\bar{d}_{\mathrm{L} u}^{b} C \bar{\nu}_{\mathrm{L} v}\right) \\
& \mathcal{O}_{d_{\mathrm{L}}^{2} \bar{d}_{\mathrm{L}} \bar{e}_{\mathrm{R}} \bar{\nu}_{\mathrm{L}} \bar{u}_{\mathrm{R}}}^{(0,-2)} \mathcal{Y}\left[\begin{array}{l}
\underline{p} \\
r
\end{array}\right]\left(\bar{e}_{\mathrm{R} s} d_{\mathrm{L} p a}\right)\left(\bar{u}_{\mathrm{R} t}^{a} d_{\mathrm{L} r b}\right)\left(\bar{d}_{\mathrm{L} u}^{b} C \bar{\nu}_{\mathrm{L} v}\right) \\
& \mathcal{Y}[\underline{p r r}]\left(d_{\mathrm{L} p a} C d_{\mathrm{L} r b}\right)\left(\bar{e}_{\mathrm{R} s} C \bar{u}_{\mathrm{R} t}^{a}\right)\left(\bar{d}_{\mathrm{L} u}^{b} C \bar{\nu}_{\mathrm{L} v}\right) \\
& \mathcal{Y}[\overline{p r r}]\left(\bar{e}_{\mathrm{R} s} d_{\mathrm{L} p a}\right)\left(\bar{u}_{\mathrm{R} t}^{a} d_{\mathrm{L} r b}\right)\left(\bar{d}_{\mathrm{L} u}^{b} C \bar{\nu}_{\mathrm{L} v}\right)
\end{aligned}
$$

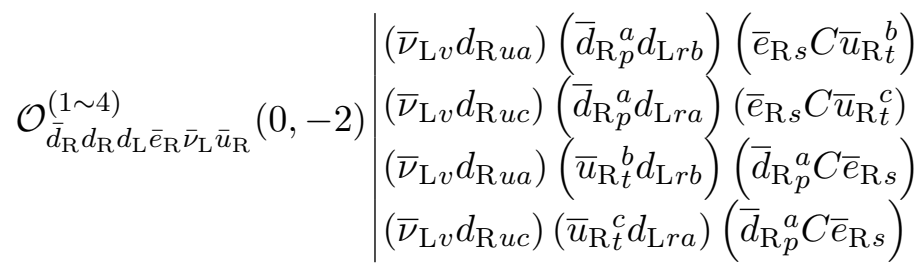

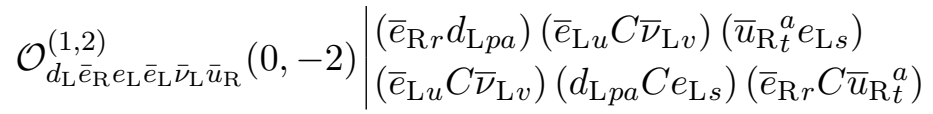

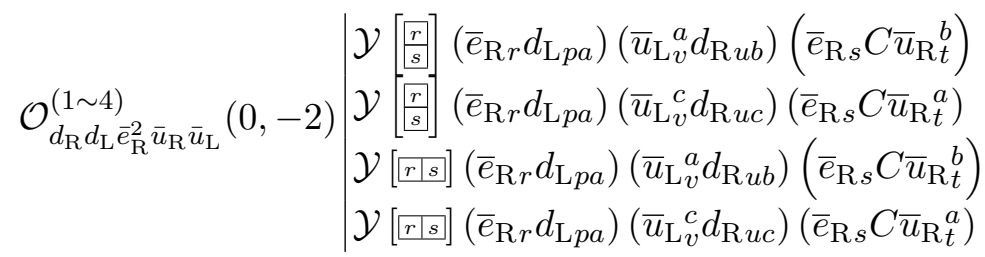

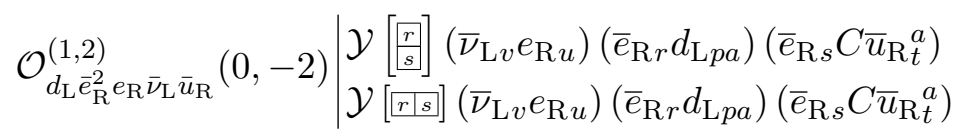

$$
\begin{aligned}
& \mathcal{O}_{d_{\mathrm{L}} \overline{\mathrm{e}}_{\mathrm{R}} \nu_{\mathrm{L}} \bar{\nu}_{\mathrm{L}}^{2} \bar{u}_{\mathrm{R}}}^{(10,-2)} \mid \begin{array}{l}
\mathcal{Y}[u v]]\left(\bar{\nu}_{\mathrm{L} u} C \bar{\nu}_{\mathrm{L} v}\right)\left(\bar{e}_{\mathrm{R} r} d_{\mathrm{L} p a}\right)\left(\bar{u}_{\mathrm{R} s}^{a} \nu_{\mathrm{L} t}\right) \\
\mathcal{Y}[\overline{u v}]\left(\bar{\nu}_{\mathrm{L} u} C \bar{\nu}_{\mathrm{L} v}\right)\left(\bar{e}_{\mathrm{R} r} \nu_{\mathrm{L} t}\right)\left(\bar{u}_{\mathrm{R} s}^{a} d_{\mathrm{L} p a}\right)
\end{array}
\end{aligned}
$$

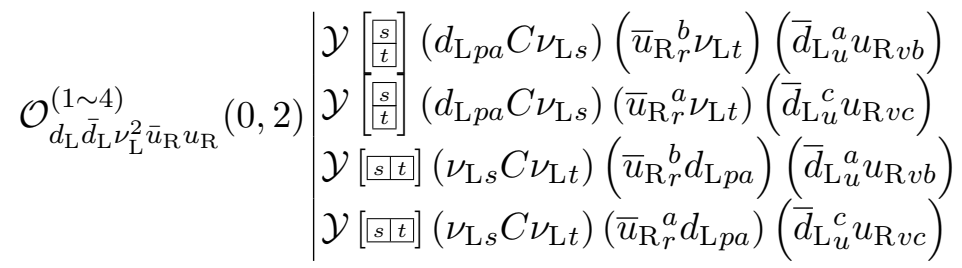




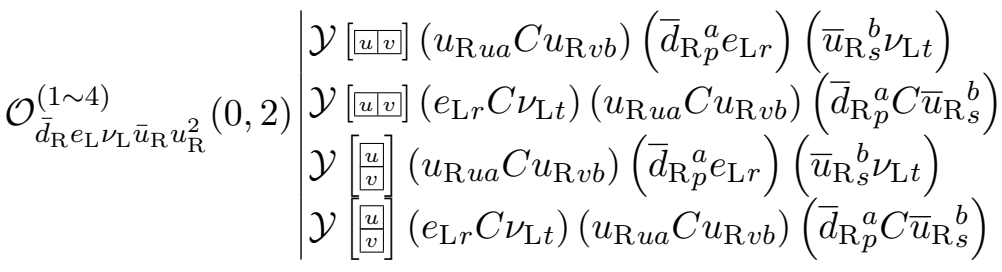

$$
\begin{aligned}
& \mathcal{Y}\left[\begin{array}{c}
{[s} \\
t
\end{array}\right]\left(\bar{d}_{\mathrm{R} p}^{a} \nu_{\mathrm{L} s}\right)\left(\bar{u}_{\mathrm{R}}{ }_{r}^{b} \nu_{\mathrm{L} t}\right)\left(d_{\mathrm{R} u a} C u_{\mathrm{R} v b}\right) \\
& \mathcal{O}_{\bar{d}_{\mathrm{R}} d_{\mathrm{R}} \nu_{\mathrm{L}}^{2} \bar{u}_{\mathrm{R}} u_{\mathrm{R}}}^{(0,2)} \mathcal{Y}\left[\begin{array}{l}
s \\
t
\end{array}\right]\left(\bar{d}_{\mathrm{R} p}^{a} \nu_{\mathrm{L} s}\right)\left(\bar{u}_{\mathrm{R} r}^{b} \nu_{\mathrm{L} t}\right)\left(d_{\mathrm{R} u b} C u_{\mathrm{R} v a}\right) \\
& \mathcal{Y}[s \mid t]\left(\nu_{\mathrm{L} s} C \nu_{\mathrm{L} t}\right)\left(d_{\mathrm{R} u a} C u_{\mathrm{R} v b}\right)\left(\bar{d}_{\mathrm{R} p}^{a} C \bar{u}_{\mathrm{R} r}^{b}\right) \\
& \mathcal{Y}[s \mid t]\left(\nu_{\mathrm{L} s} C \nu_{\mathrm{L} t}\right)\left(d_{\mathrm{R} u b} C u_{\mathrm{R} v a}\right)\left(\bar{d}_{\mathrm{R} p}^{a} C \bar{u}_{\mathrm{R} r}^{b}\right)
\end{aligned}
$$

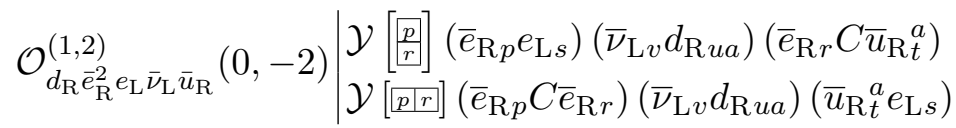

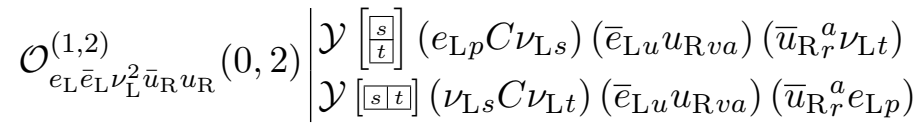

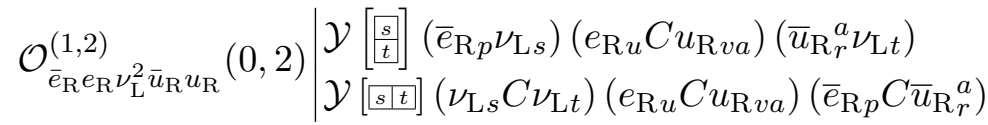

$$
\begin{aligned}
& \mathcal{O}_{\nu_{\mathrm{L}}^{3} \bar{\nu}_{\mathrm{L}} \bar{u}_{\mathrm{R}} u_{\mathrm{R}}}(0,2) \mid \mathcal{Y}\left[\frac{r s s}{t}\right]\left(\nu_{\mathrm{L} s} C \nu_{\mathrm{L} t}\right)\left(\bar{\nu}_{\mathrm{L} v} u_{\mathrm{R} u a}\right)\left(\bar{u}_{\mathrm{R}}{ }_{p}^{a} \nu_{\mathrm{L} r}\right)
\end{aligned}
$$

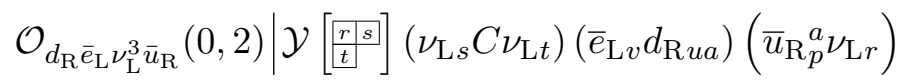

$$
\begin{aligned}
& \mathcal{Y}\left[\frac{p}{r}, \frac{s}{t}, u \underline{, u}\right]\left(\bar{\nu}_{\mathrm{L} u} C \bar{\nu}_{\mathrm{L} v}\right)\left(d_{\mathrm{L} s a} C d_{\mathrm{L} t b}\right)\left(\bar{d}_{\mathrm{R}_{p}^{a}} C \bar{d}_{\mathrm{R}_{r}^{b}}\right) \\
& \mathcal{O}_{\bar{d}_{\mathrm{R}}^{2} d_{\mathrm{L}}^{2} \bar{\nu}_{\mathrm{L}}^{2}}(0,-2) \mathcal{Y}\left[\frac{p}{r}, \frac{s}{t}, \overline{w v}\right]\left(\bar{\nu}_{\mathrm{L} u} C \bar{\nu}_{\mathrm{L} v}\right)\left(\bar{d}_{\mathrm{R} p}^{a} d_{\mathrm{L} s a}\right)\left(\bar{d}_{\mathrm{R} r}^{b} d_{\mathrm{L} t b}\right) \\
& \mathcal{Y}\left[\overline{p \mid r}, \underline{s \mid t}, \underline{u v v]}\left(\bar{\nu}_{\mathrm{L} u} C \bar{\nu}_{\mathrm{L} v}\right)\left(d_{\mathrm{L} s a} C d_{\mathrm{L} t b}\right)\left(\bar{d}_{\mathrm{R} p}^{a} C \bar{d}_{\mathrm{R} r}^{b}\right)\right. \\
& \mathcal{Y}\left[\underline{p \mid r}, \underline{s \mid t}, \underline{u \mid v]}\left(\bar{\nu}_{\mathrm{L} u} C \bar{\nu}_{\mathrm{L} v}\right)\left(\bar{d}_{\mathrm{R} p}^{a} d_{\mathrm{L} s a}\right)\left(\bar{d}_{\mathrm{R}}^{b} d_{\mathrm{L} t b}\right)\right.
\end{aligned}
$$

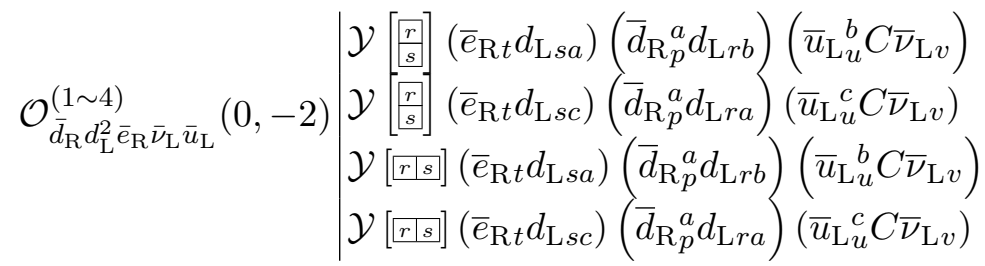

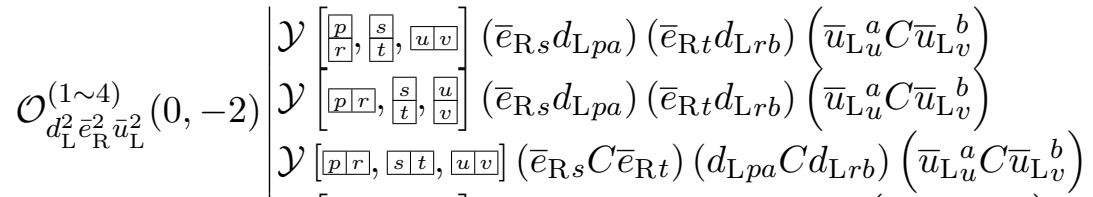

$$
\begin{aligned}
& \mathcal{Y}\left[\frac{p}{r}, s \mid t, \frac{u}{v}\right]\left(\bar{e}_{\mathrm{R} s} C \bar{e}_{\mathrm{R} t}\right)\left(d_{\mathrm{L} p a} C d_{\mathrm{L} r b}\right)\left(\bar{u}_{\mathrm{L} u}^{a} C \bar{u}_{\mathrm{L} v}^{b}\right)
\end{aligned}
$$

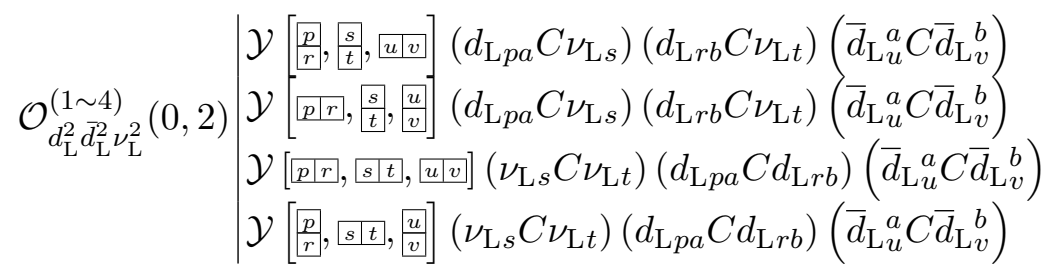




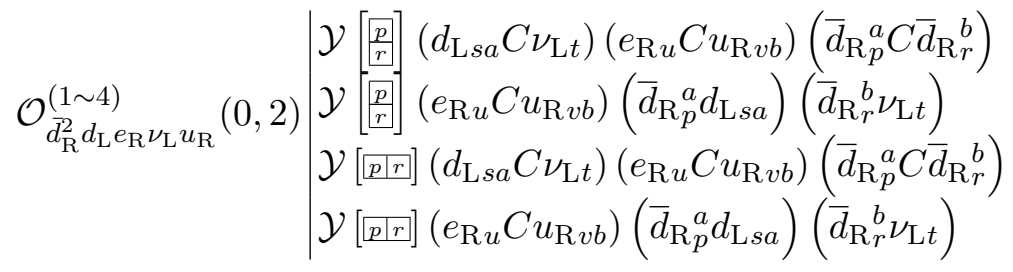

$$
\begin{aligned}
& \mathcal{O}_{\bar{d}_{\mathrm{R}} d_{\mathrm{L}} \bar{e}_{\mathrm{R}} e_{\mathrm{L}} \bar{\nu}_{\mathrm{L}}^{2}(0,-2)} \mid \begin{array}{l}
\mathcal{Y}[u v]\left(\bar{\nu}_{\mathrm{L} u} C \bar{\nu}_{\mathrm{L} v}\right)\left(\bar{e}_{\mathrm{R} s} e_{\mathrm{L} t}\right)\left(\bar{d}_{\mathrm{R}}^{a} d_{\mathrm{L} r a}\right) \\
\mathcal{Y}[\overline{u v}]\left(\bar{\nu}_{\mathrm{L} u} C \bar{\nu}_{\mathrm{L} v}\right)\left(d_{\mathrm{L} r a} C e_{\mathrm{L} t}\right)\left(\bar{d}_{\mathrm{R} p}^{a} C \bar{e}_{\mathrm{R} s}\right)
\end{array} \\
& \left.\mathcal{O}_{\bar{d}_{\mathrm{R}} d_{\mathrm{L}} \overline{\mathrm{L}}_{\mathrm{L}} e_{\mathrm{L}} \nu_{\mathrm{L}} u_{\mathrm{R}}}(0,2) \mid \begin{array}{l}
\left(e_{\mathrm{L} s} C \nu_{\mathrm{L} t}\right)\left(\bar{d}_{\mathrm{R} p}^{a} d_{\mathrm{L} r b}\right)\left(\bar{d}_{\mathrm{L} u}^{b} u_{\mathrm{R} v a}\right. \\
\left(e_{\mathrm{L} s} C \nu_{\mathrm{L} t}\right)\left(\bar{d}_{\mathrm{R}}^{a} d_{\mathrm{L} r a}\right)\left(\bar{d}_{\mathrm{L} u}^{c} u_{\mathrm{R} v c}\right. \\
\left(d_{\mathrm{L} r b} C \nu_{\mathrm{L} t}\right)\left(\bar{d}_{\mathrm{R} p}^{a} e_{\mathrm{L} s}\right)\left(\bar{d}_{\mathrm{L} u}^{b} u_{\mathrm{R} v a}\right. \\
\left(d_{\mathrm{L} r a} C \nu_{\mathrm{L} t}\right)\left(\bar{d}_{\mathrm{R}}^{a} e_{\mathrm{L} s}\right)\left(\bar{d}_{\mathrm{L} u}^{c} u_{\mathrm{R} v c}\right.
\end{array}\right)
\end{aligned}
$$

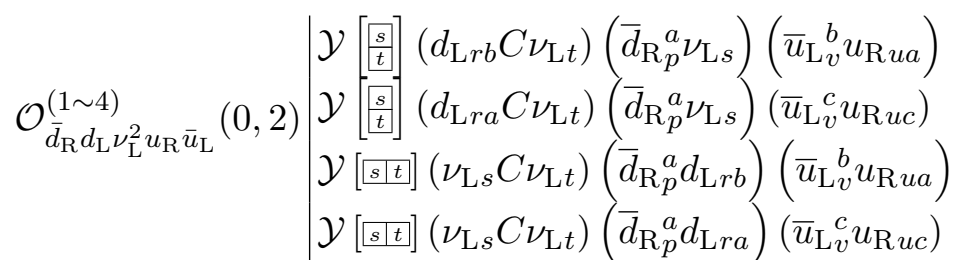

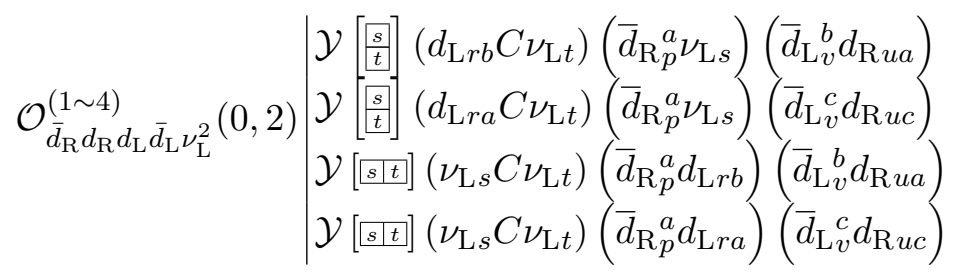

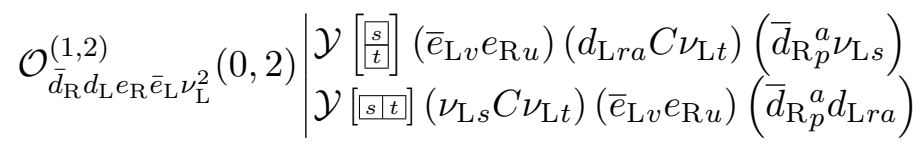

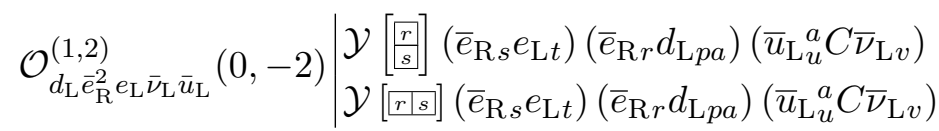

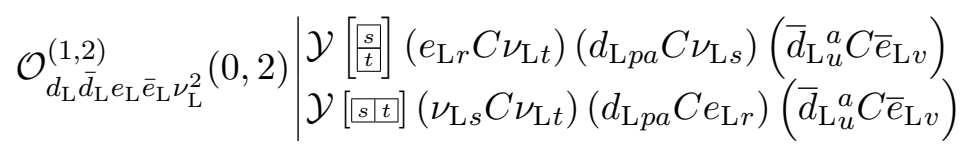

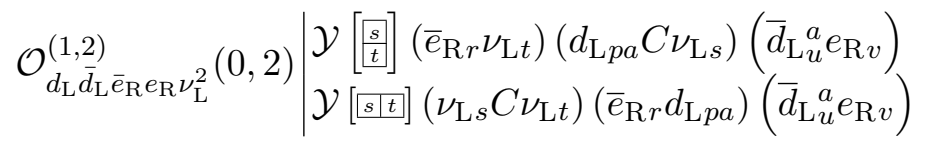

$$
\begin{aligned}
& \mathcal{O}_{d_{\mathrm{L}} \overline{\mathrm{e}}_{\mathrm{L}} \nu_{\mathrm{L}}^{3} \bar{u}_{\mathrm{L}}}(0,2) \mid \mathcal{Y}\left[\begin{array}{l}
r r s \\
\underline{t}]^{\prime}
\end{array}\right]\left(\nu_{\mathrm{L} s} C \nu_{\mathrm{L} t}\right)\left(d_{\mathrm{L} p a} C \nu_{\mathrm{L} r}\right)\left(\bar{e}_{\mathrm{L} u} C \bar{u}_{\mathrm{L} v}^{a}\right) \\
& \mathcal{O}_{d_{\mathrm{L}} \bar{d}_{\mathrm{L}} \nu_{\mathrm{L}}^{3} \bar{\nu}_{\mathrm{L}}}(0,2) \mid \mathcal{Y}\left[\frac{r s s}{t r}\right]\left(\nu_{\mathrm{L} s} C \nu_{\mathrm{L} t}\right)\left(d_{\mathrm{L} p a} C \nu_{\mathrm{L} r}\right)\left(\bar{d}_{\mathrm{L} u}^{a} C \bar{\nu}_{\mathrm{L} v}\right)
\end{aligned}
$$

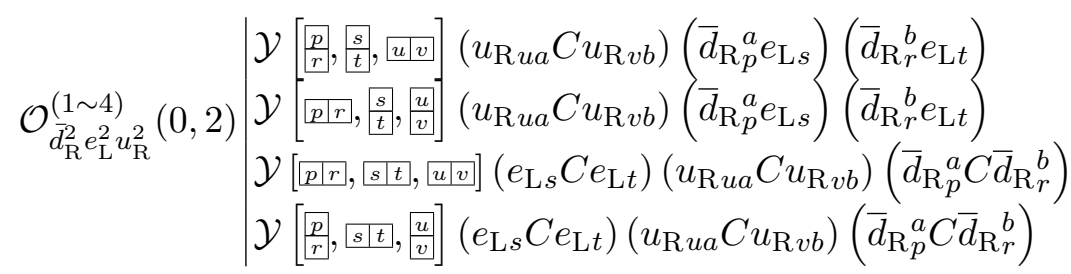




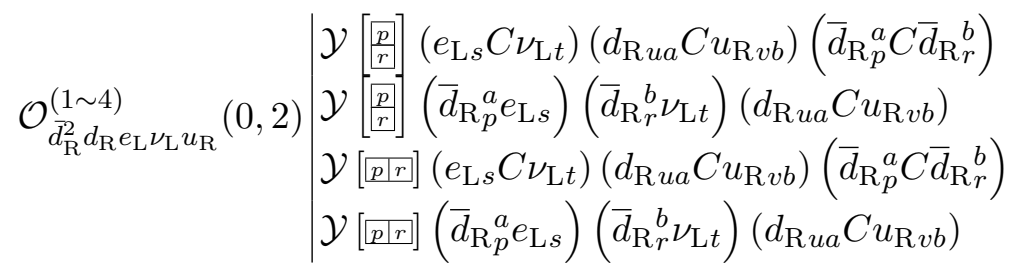

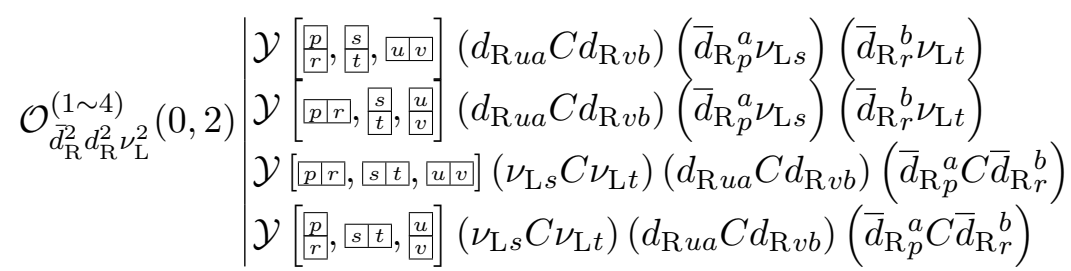

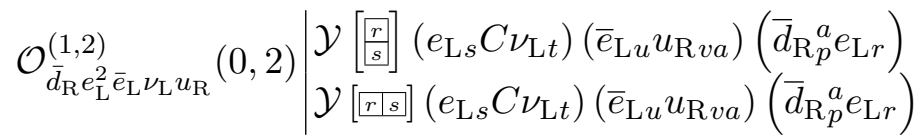

$$
\begin{aligned}
& \mathcal{O}_{\bar{d}_{\mathrm{R}} \bar{e}_{\mathrm{R}} e_{\mathrm{R}} e_{\mathrm{L}} \nu_{\mathrm{L}} u_{\mathrm{R}}}^{(1,2,2)} \mid \begin{array}{l}
\left(e_{\mathrm{L} s} C \nu_{\mathrm{L} t}\right)\left(e_{\mathrm{R} u} C u_{\mathrm{R} v a}\right)\left(\bar{d}_{\mathrm{R} p}^{a} C \bar{e}_{\mathrm{R} r}\right) \\
\left(\bar{e}_{\mathrm{R} r} \nu_{\mathrm{L} t}\right)\left(e_{\mathrm{R} u} C u_{\mathrm{R} v a}\right)\left(\bar{d}_{\mathrm{R} p}^{a} e_{\mathrm{L} s}\right)
\end{array}
\end{aligned}
$$

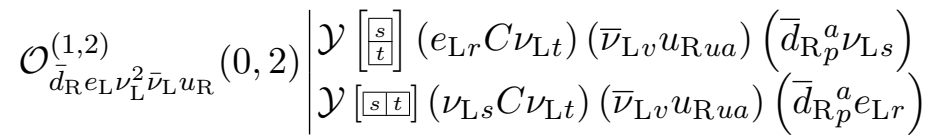

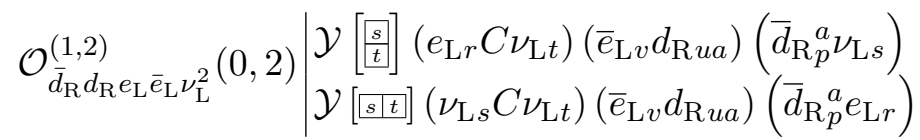

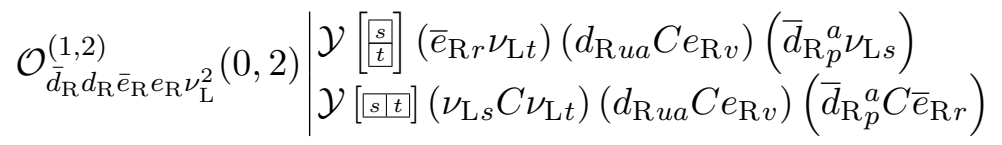

$$
\begin{aligned}
& \mathcal{O}_{\bar{d}_{\mathrm{R}} d_{\mathrm{R}} \nu_{\mathrm{L}}^{3} \bar{\nu}_{\mathrm{L}}}(0,2) \mid \mathcal{Y}\left[\frac{r s s}{\left.t \frac{t}{t}\right]}\left(\nu_{\mathrm{L} s} C \nu_{\mathrm{L} t}\right)\left(\bar{\nu}_{\mathrm{L} v} d_{\mathrm{R} u a}\right)\left(\bar{d}_{\mathrm{R}}{ }_{p}^{a} \nu_{\mathrm{L} r}\right)\right.
\end{aligned}
$$

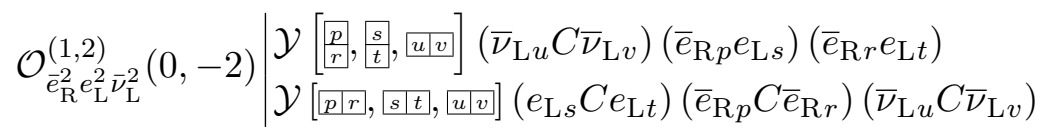

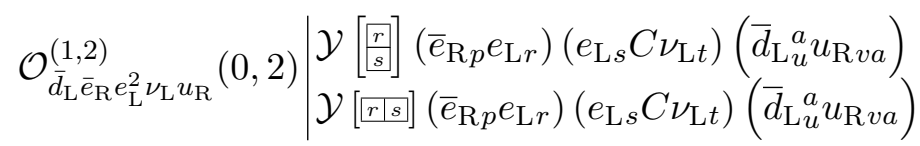

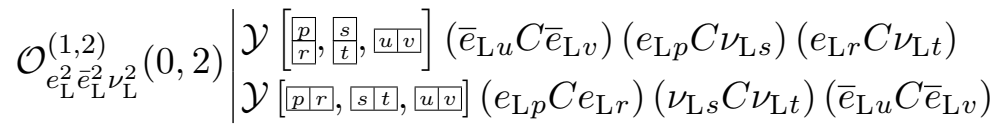

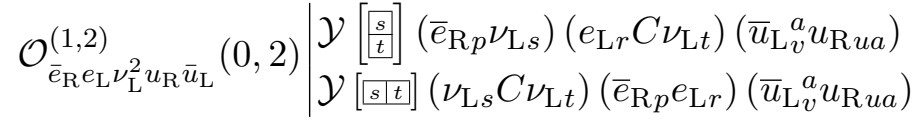

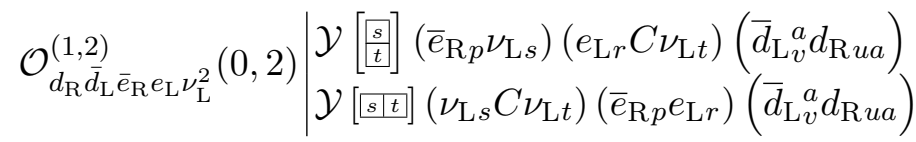

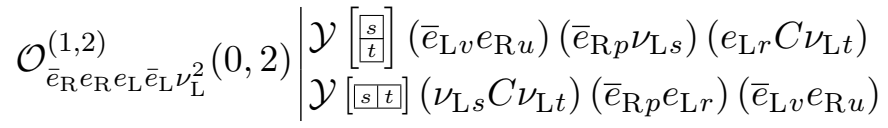




$$
\begin{aligned}
& \mathcal{O}_{e_{\mathrm{L}} \bar{e}_{\mathrm{L}} \nu_{\mathrm{L}}^{3} \bar{\nu}_{\mathrm{L}}}(0,2) \mid \mathcal{Y}\left[\frac{r s s}{t}\right]\left(\nu_{\mathrm{L} s} C \nu_{\mathrm{L} t}\right)\left(e_{\mathrm{L} p} C \nu_{\mathrm{L} r}\right)\left(\bar{e}_{\mathrm{L} u} C \bar{\nu}_{\mathrm{L} v}\right)
\end{aligned}
$$

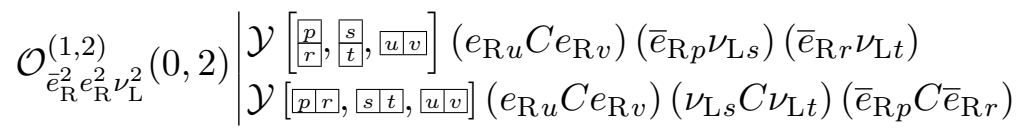

$$
\begin{aligned}
& \mathcal{O}_{d_{\mathrm{R}} \bar{e}_{\mathrm{R}} \nu_{\mathrm{L}}^{3} \bar{u}_{\mathrm{L}}}(0,2) \mid \mathcal{Y}\left[\frac{r s}{t t}\right]\left(\nu_{\mathrm{L} s} C \nu_{\mathrm{L} t}\right)\left(\bar{e}_{\mathrm{R} p} \nu_{\mathrm{L} r}\right)\left(\bar{u}_{\mathrm{L} v}^{a} d_{\mathrm{R} u a}\right) \\
& \mathcal{O}_{\bar{e}_{\mathrm{R}} e_{\mathrm{R}} \nu_{\mathrm{L}}^{3} \bar{\nu}_{\mathrm{L}}}(0,2) \mid \mathcal{Y}\left[\frac{[r s}{\underline{t}}\right]\left(\nu_{\mathrm{L} s} C \nu_{\mathrm{L} t}\right)\left(\bar{e}_{\mathrm{R} p} \nu_{\mathrm{L} r}\right)\left(\bar{\nu}_{\mathrm{L} v} e_{\mathrm{R} u}\right) \\
& \left.\mathcal{O}_{\nu_{\mathrm{L}}^{4} \bar{\nu}_{\mathrm{L}}^{2}}(0,2) \mid \mathcal{Y}\left[\frac{p r r}{s t t}, \overline{u v}\right]\right]\left(\nu_{\mathrm{L} p} C \nu_{\mathrm{L} r}\right)\left(\nu_{\mathrm{L} s} C \nu_{\mathrm{L} t}\right)\left(\bar{\nu}_{\mathrm{L} u} C \bar{\nu}_{\mathrm{L} v}\right)
\end{aligned}
$$

$(\Delta B, \Delta L)=(0, \pm 4)$

$$
\begin{aligned}
& \mathcal{O}_{\bar{d}_{\mathrm{L}} e_{\mathrm{R}} \nu_{\mathrm{L}}^{3} u_{\mathrm{L}}}(0,4) \mid \mathcal{Y}\left[\frac{r s s}{[t}\right]\left(\nu_{\mathrm{L} s} C \nu_{\mathrm{L} t}\right)\left(u_{\mathrm{L} p a} C \nu_{\mathrm{L} r}\right)\left(\bar{d}_{\mathrm{L} u}^{a} e_{\mathrm{R} v}\right) \\
& \mathcal{O}_{\bar{d}_{\mathrm{R}} e_{\mathrm{R}} \nu_{\mathrm{L}}^{3} u_{\mathrm{R}}}(0,4) \mid \mathcal{Y}\left[\begin{array}{c}
{[r s} \\
\left.\frac{r}{t}\right]
\end{array}\right]\left(\nu_{\mathrm{L} s} C \nu_{\mathrm{L} t}\right)\left(e_{\mathrm{R} u} C u_{\mathrm{R} v a}\right)\left(\bar{d}_{\mathrm{R}}{ }_{p}^{a} \nu_{\mathrm{L} r}\right) \\
& \mathcal{O}_{\bar{d}_{\mathrm{L}} e_{\mathrm{L}} \nu_{\mathrm{L}}^{3} u_{\mathrm{R}}}(0,4) \mid \mathcal{Y}\left[\begin{array}{l}
r s s \\
t s
\end{array}\right]\left(\nu_{\mathrm{L} s} C \nu_{\mathrm{L} t}\right)\left(e_{\mathrm{L} p} C \nu_{\mathrm{L} r}\right)\left(\bar{d}_{\mathrm{L} u}^{a} u_{\mathrm{R} v a}\right) \\
& \mathcal{O}_{\nu_{\mathrm{L}}^{4} u_{\mathrm{R}} \bar{u}_{\mathrm{L}}}(0,4) \mid \mathcal{Y}\left[\frac{p r r}{s \mid t}\right]\left(\nu_{\mathrm{L} p} C \nu_{\mathrm{L} r}\right)\left(\nu_{\mathrm{L} s} C \nu_{\mathrm{L} t}\right)\left(\bar{u}_{\mathrm{L}}{ }_{v}^{a} u_{\mathrm{R} u a}\right) \\
& \mathcal{O}_{d_{\mathrm{R}} \bar{d}_{\mathrm{L}} \nu_{\mathrm{L}}^{4}}(0,4) \mid \mathcal{Y}\left[\frac{p r r}{s t}\right]\left(\nu_{\mathrm{L} p} C \nu_{\mathrm{L} r}\right)\left(\nu_{\mathrm{L} s} C \nu_{\mathrm{L} t}\right)\left(\bar{d}_{\mathrm{L} v}^{a} d_{\mathrm{R} u a}\right) \\
& \mathcal{O}_{e_{\mathrm{R}} \bar{e}_{\mathrm{L}} \nu_{\mathrm{L}}^{4}}(0,4) \mid \mathcal{Y}\left[\frac{p r r}{s t}\right]\left(\nu_{\mathrm{L} p} C \nu_{\mathrm{L} r}\right)\left(\nu_{\mathrm{L} s} C \nu_{\mathrm{L} t}\right)\left(\bar{e}_{\mathrm{L} v} e_{\mathrm{R} u}\right)
\end{aligned}
$$

$(\Delta B, \Delta L)=( \pm 1, \pm 1)$

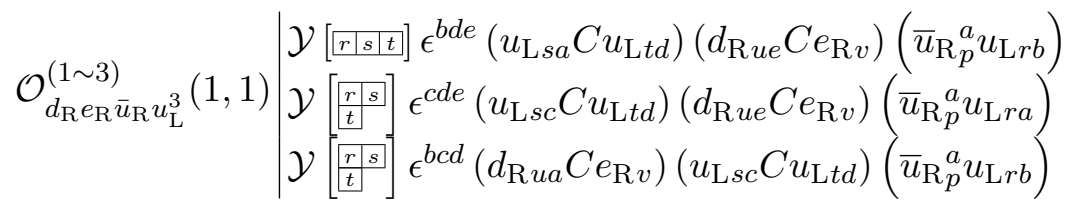

$$
\begin{aligned}
& \mathcal{Y}[[r|s| t]] \epsilon^{a b d}\left(u_{\mathrm{L} s c} C u_{\mathrm{L} t d}\right)\left(\bar{u}_{\mathrm{L}}^{c} e_{\mathrm{R} u}\right)\left(d_{\mathrm{L} p a} C u_{\mathrm{L} r b}\right) \\
& \mathcal{O}_{d_{\mathrm{L}} e_{\mathrm{R}} u_{\mathrm{L}}^{3} \bar{u}_{\mathrm{L}}}^{(1 \sim 3)}(1,1) \mathcal{Y}\left[\frac{r s s}{t s}\right] \epsilon^{b c d}\left(u_{\mathrm{L} s c} C u_{\mathrm{L} t d}\right)\left(\bar{u}_{\mathrm{L} v}^{a} e_{\mathrm{R} u}\right)\left(d_{\mathrm{L} p a} C u_{\mathrm{L} r b}\right) \\
& \mathcal{Y}\left[\begin{array}{c}
{[r s} \\
t t
\end{array}\right] \epsilon^{a b d}\left(u_{\mathrm{L} s c} C u_{\mathrm{L} t d}\right)\left(\bar{u}_{\mathrm{L} v}^{c} e_{\mathrm{R} u}\right)\left(d_{\mathrm{L} p a} C u_{\mathrm{L} r b}\right)
\end{aligned}
$$

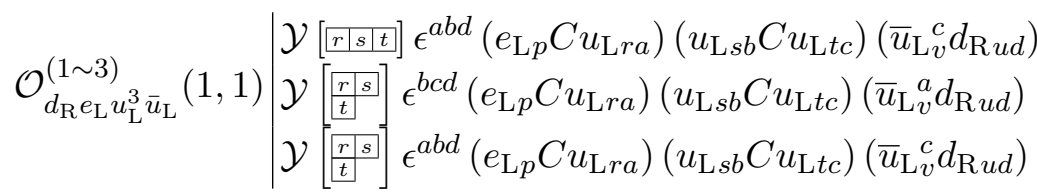

$$
\begin{aligned}
& \mathcal{O}_{e_{\mathrm{R}} e_{\mathrm{L}} \bar{\nu}_{\mathrm{L}} u_{\mathrm{L}}^{3}}(1,1) \mid \mathcal{Y}\left[\frac{r s s}{\left[t s^{s}\right.}\right] \epsilon^{a b c}\left(\bar{\nu}_{\mathrm{L} v} e_{\mathrm{R} u}\right)\left(e_{\mathrm{L} p} C u_{\mathrm{L} r a}\right)\left(u_{\mathrm{L} s b} C u_{\mathrm{L} t c}\right)
\end{aligned}
$$

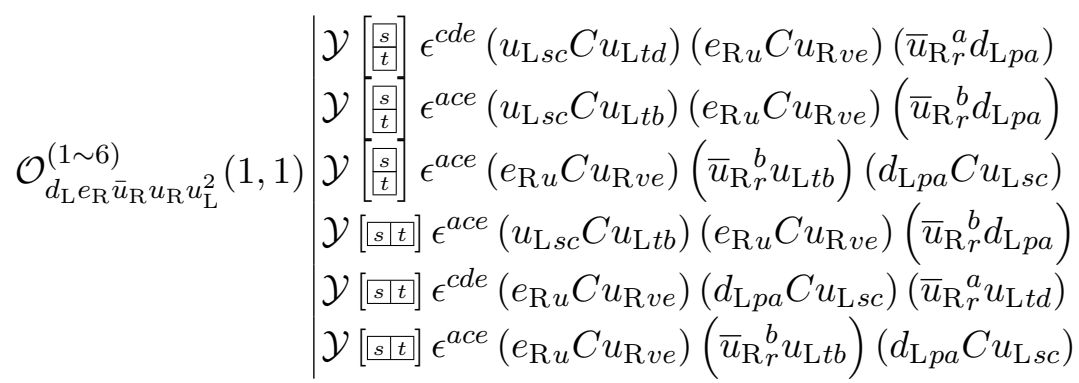




$$
\begin{aligned}
& \mathcal{Y}\left[\begin{array}{c}
s \\
t
\end{array}\right] \epsilon^{c d e}\left(u_{\mathrm{L} s a} C u_{\mathrm{L} t c}\right)\left(\bar{u}_{\mathrm{R} r}^{a} e_{\mathrm{L} p}\right)\left(d_{\mathrm{R} u d} C u_{\mathrm{R} v e}\right) \\
& \mathcal{Y}\left[\begin{array}{c}
s \\
t
\end{array}\right] \epsilon^{b c d}\left(u_{\mathrm{L} s b} C u_{\mathrm{L} t c}\right)\left(\bar{u}_{\mathrm{R}}{ }_{r}^{a} e_{\mathrm{L} p}\right)\left(d_{\mathrm{R} u d} C u_{\mathrm{R} v a}\right)
\end{aligned}
$$

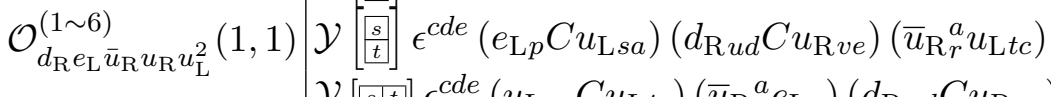

$$
\begin{aligned}
& \mathcal{Y}[s \mid t] \epsilon^{c d e}\left(u_{\mathrm{L} s a} C u_{\mathrm{Ltc}}\right)\left(\bar{u}_{\mathrm{R}}{ }_{r}^{a} e_{\mathrm{L} p}\right)\left(d_{\mathrm{R} u d} C u_{\mathrm{R} v e}\right) \\
& \mathcal{Y}[s] t] \epsilon^{c d e}\left(e_{\mathrm{L} p} C u_{\mathrm{L} s a}\right)\left(d_{\mathrm{R} u d} C u_{\mathrm{R} v e}\right)\left(\bar{u}_{\mathrm{R} r}{ }^{a} u_{\mathrm{L} t c}\right) \\
& \mathcal{Y}[s \mid t]] \epsilon^{b c d}\left(e_{\mathrm{L} p} C u_{\mathrm{L} s b}\right)\left(d_{\mathrm{R} u d} C u_{\mathrm{R} v a}\right)\left(\bar{u}_{\mathrm{R} r}^{a} u_{\mathrm{L} t c}\right) \\
& \mathcal{Y}\left[\begin{array}{l}
\frac{r}{s}, \frac{u}{v} \\
v
\end{array}\right] \epsilon^{c d e}\left(u_{\mathrm{L} s c} C \nu_{\mathrm{L} t}\right)\left(d_{\mathrm{R} u d} C d_{\mathrm{R} v e}\right)\left(\bar{u}_{\mathrm{R}}^{a} u_{\mathrm{L} r a}\right) \\
& \mathcal{Y}\left[\frac{r}{s},, \frac{u}{v}\right] \epsilon^{b d e}\left(u_{\mathrm{L} s a} C \nu_{\mathrm{L} t}\right)\left(d_{\mathrm{R} u d} C d_{\mathrm{R} v e}\right)\left(\bar{u}_{\mathrm{R} p}^{a} u_{\mathrm{L} r b}\right)
\end{aligned}
$$

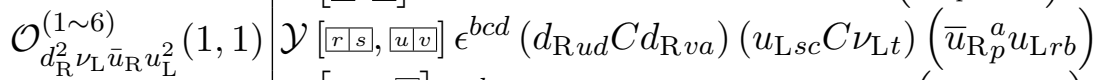

$$
\begin{aligned}
& \mathcal{Y}\left[\overline{r r s}, \frac{u}{v}\right] \epsilon^{c d e}\left(u_{\mathrm{L} s c} C \nu_{\mathrm{L} t}\right)\left(d_{\mathrm{R} u d} C d_{\mathrm{R} v e}\right)\left(\bar{u}_{\mathrm{R} p}^{a} u_{\mathrm{L} r a}\right) \\
& \mathcal{Y}\left[r \vec{r}, \frac{u}{v}\right] \epsilon^{b d e}\left(u_{\mathrm{L} s a} C \nu_{\mathrm{L} t}\right)\left(d_{\mathrm{R} u d} C d_{\mathrm{R} v e}\right)\left(\bar{u}_{\mathrm{R} p}^{a} u_{\mathrm{L} r b}\right) \\
& \mathcal{Y}\left[\frac{r}{s}, u v v\right] \epsilon^{b c d}\left(d_{\mathrm{R} u d} C d_{\mathrm{R} v a}\right)\left(u_{\mathrm{L} s c} C \nu_{\mathrm{L} t}\right)\left(\bar{u}_{\mathrm{R} p}^{a} u_{\mathrm{L} r b}\right) \\
& \left.\mathcal{Y}\left[\frac{p}{r}, s \mid t\right]\right] \epsilon^{a b d}\left(d_{\mathrm{L} p a} C d_{\mathrm{L} r b}\right)\left(u_{\mathrm{L} s c} C u_{\mathrm{L} t d}\right)\left(\bar{d}_{\mathrm{L} u}^{c} e_{\mathrm{R} v}\right) \\
& \mathcal{Y}\left[\frac{p}{r}, s \mid t\right] \epsilon^{b c d}\left(\bar{d}_{\mathrm{L} u}^{a} e_{\mathrm{R} v}\right)\left(d_{\mathrm{L} p a} C u_{\mathrm{L} s c}\right)\left(d_{\mathrm{L} r b} C u_{\mathrm{L} t d}\right) \\
& \mathcal{O}_{d_{\mathrm{L}}^{2} \bar{d}_{\mathrm{L}} e_{\mathrm{R}} u_{\mathrm{L}}^{2}}^{(\sim 6)}(1,1) \mathcal{Y}\left[\frac{p r r}{,}, \frac{s}{t}\right] \epsilon^{b c d}\left(d_{\mathrm{L} p a} C d_{\mathrm{L} r b}\right)\left(u_{\mathrm{L} s c} C u_{\mathrm{L} t d}\right)\left(\bar{d}_{\mathrm{L} u}^{a} e_{\mathrm{R} v}\right) \\
& \mathcal{Y}\left[\overline{p p r}, \frac{s}{t}\right] \epsilon^{a b d}\left(\bar{d}_{\mathrm{L} u}^{c} e_{\mathrm{R} v}\right)\left(d_{\mathrm{L} p a} C u_{\mathrm{L} s c}\right)\left(d_{\mathrm{L} r b} C u_{\mathrm{L} t d}\right) \\
& \mathcal{Y}[p p r,|s| t] \epsilon^{b c d}\left(\bar{d}_{\mathrm{L} u}^{a} e_{\mathrm{R} v}\right)\left(d_{\mathrm{L} p a} C u_{\mathrm{L} s c}\right)\left(d_{\mathrm{L} r b} C u_{\mathrm{L} t d}\right) \\
& \left.\mathcal{Y}\left[\frac{p}{r}, \frac{s}{t}\right]\right] \epsilon^{b c d}\left(d_{\mathrm{L} p a} C d_{\mathrm{L} r b}\right)\left(u_{\mathrm{L} s c} C u_{\mathrm{L} t d}\right)\left(\bar{d}_{\mathrm{L} u}^{a} e_{\mathrm{R} v}\right)
\end{aligned}
$$

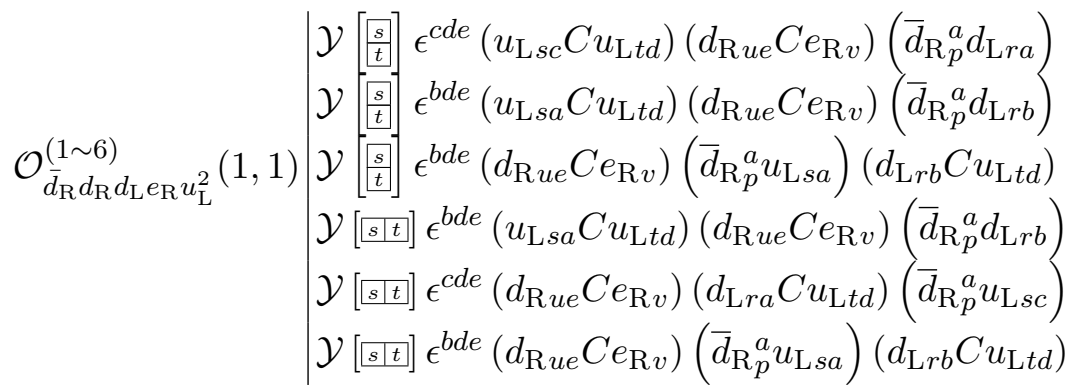

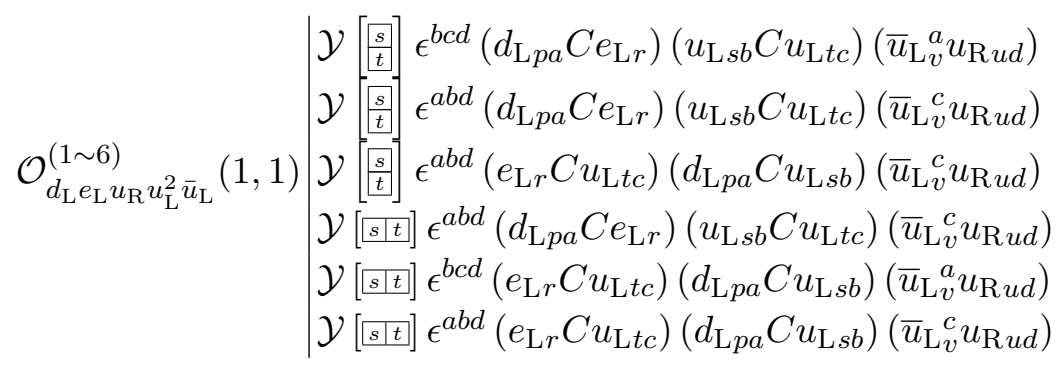




$$
\begin{aligned}
& \mathcal{Y}\left[\begin{array}{c}
s \\
\frac{s}{t}
\end{array}\right] \epsilon^{b c d}\left(d_{\mathrm{L} p a} C e_{\mathrm{L} r}\right)\left(u_{\mathrm{L} s b} C u_{\mathrm{L} t c}\right)\left(\bar{d}_{\mathrm{L} v}^{a} d_{\mathrm{R} u d}\right) \\
& \mathcal{Y}\left[\begin{array}{ll}
s \\
t
\end{array}\right] \epsilon^{a b d}\left(d_{\mathrm{L} p a} C e_{\mathrm{L} r}\right)\left(u_{\mathrm{L} s b} C u_{\mathrm{L} t c}\right)\left(\bar{d}_{\mathrm{L} v}^{c} d_{\mathrm{R} u d}\right) \\
& \mathcal{O}_{d_{\mathrm{R}} d_{\mathrm{L}} \bar{d}_{\mathrm{L}} e_{\mathrm{L}} u_{\mathrm{L}}^{2}}^{(\sim 6)}(1,1) \mathcal{Y}\left[\begin{array}{c}
s \\
t
\end{array}\right] \epsilon^{a b d}\left(e_{\mathrm{L} r} C u_{\mathrm{L} t c}\right)\left(d_{\mathrm{L} p a} C u_{\mathrm{L} s b}\right)\left(\bar{d}_{\mathrm{L} v}^{c} d_{\mathrm{R} u d}\right) \\
& \mathcal{Y}[s] t] \epsilon^{a b d}\left(d_{\mathrm{L} p a} C e_{\mathrm{L} r}\right)\left(u_{\mathrm{L} s b} C u_{\mathrm{L} t c}\right)\left(\bar{d}_{\mathrm{L} v}^{c} d_{\mathrm{R} u d}\right) \\
& \mathcal{Y}[s \mid t] \epsilon^{b c d}\left(e_{\mathrm{L} r} C u_{\mathrm{L} t c}\right)\left(d_{\mathrm{L} p a} C u_{\mathrm{L} s b}\right)\left(\bar{d}_{\mathrm{L} v}^{a} d_{\mathrm{R} u d}\right) \\
& \mathcal{Y}[s \mid t] \epsilon^{a b d}\left(e_{\mathrm{L} r} C u_{\mathrm{L} t c}\right)\left(d_{\mathrm{L} p a} C u_{\mathrm{L} s b}\right)\left(\bar{d}_{\mathrm{L} v}^{c} d_{\mathrm{R} u d}\right)
\end{aligned}
$$

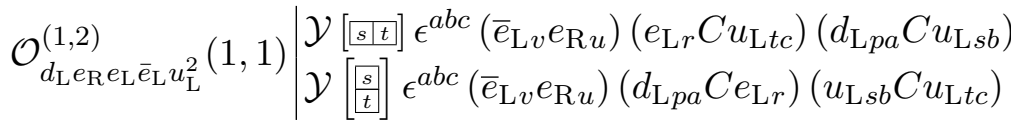

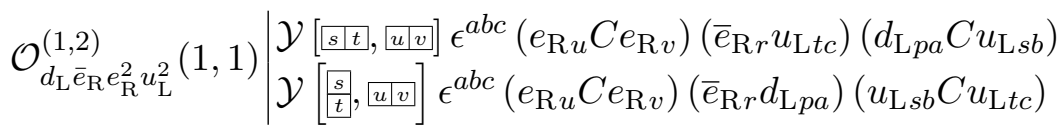

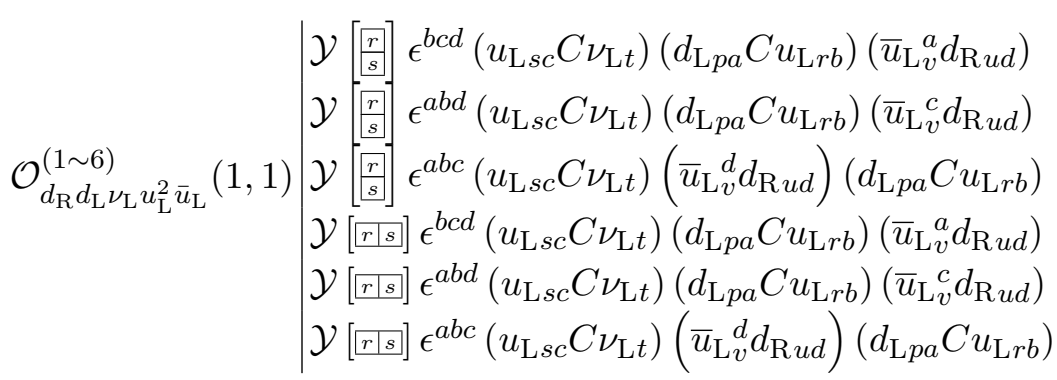

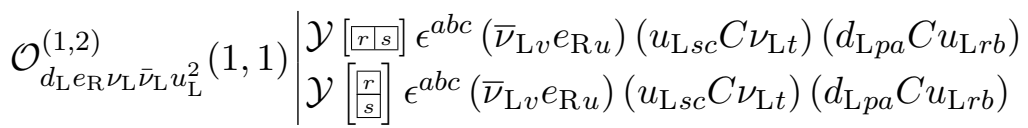

$$
\begin{aligned}
& \mathcal{Y}\left[\frac{s}{t},\left[\begin{array}{l}
u \\
v
\end{array}\right] \epsilon^{c d e}\left(u_{\mathrm{L} s a} C u_{\mathrm{L} t c}\right)\left(d_{\mathrm{R} u d} C d_{\mathrm{R} v e}\right)\left(\bar{d}_{\mathrm{R}}{ }_{p}^{a} e_{\mathrm{L} r}\right)\right. \\
& \mathcal{Y}\left[\begin{array}{l}
\frac{s}{t}, \\
\frac{u}{v}
\end{array}\right] \epsilon^{c d e}\left(e_{\mathrm{L} r} C u_{\mathrm{L} t c}\right)\left(d_{\mathrm{R} u d} C d_{\mathrm{R} v e}\right)\left(\bar{d}_{\mathrm{R} p}^{a} u_{\mathrm{L} s a}\right) \\
& \left.\mathcal{O}_{\bar{d}_{\mathrm{R}} d_{\mathrm{R}}^{2} e_{\mathrm{L}} u_{\mathrm{L}}^{2}}^{(1 \sim 6)}(1,1) \mathcal{Y}[s \mid t,, u v]\right] \epsilon^{b c d}\left(d_{\mathrm{R} u d} C d_{\mathrm{R} v a}\right)\left(e_{\mathrm{L} r} C u_{\mathrm{L} t c}\right)\left(\bar{d}_{\mathrm{R}}{ }_{p}^{a} u_{\mathrm{L} s b}\right) \\
& \mathcal{Y}\left[s \mid t, \frac{u}{v}\right] \epsilon^{c d e}\left(u_{\mathrm{L} s a} C u_{\mathrm{L} t c}\right)\left(d_{\mathrm{R} u d} C d_{\mathrm{R} v e}\right)\left(\bar{d}_{\mathrm{R} p}{ }^{a} e_{\mathrm{L} r}\right) \\
& \mathcal{Y}\left[s \mid t,\left[\begin{array}{l}
u \\
v
\end{array} \epsilon^{c d e}\left(e_{\mathrm{L} r} C u_{\mathrm{L} t c}\right)\left(d_{\mathrm{R} u d} C d_{\mathrm{R} v e}\right)\left(\bar{d}_{\mathrm{R} p}^{a} u_{\mathrm{L} s a}\right)\right.\right. \\
& \mathcal{Y}\left[\frac{s}{t}, u[v] \epsilon^{b c d}\left(d_{\mathrm{R} u d} C d_{\mathrm{R} v a}\right)\left(u_{\mathrm{L} s b} C u_{\mathrm{L} t c}\right)\left(\bar{d}_{\mathrm{R} p}^{a} e_{\mathrm{L} r}\right)\right.
\end{aligned}
$$

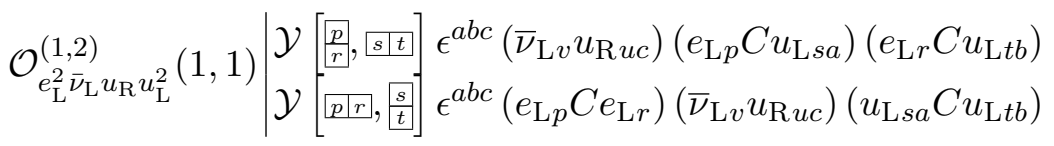

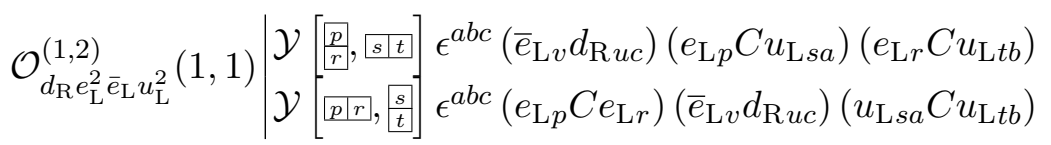

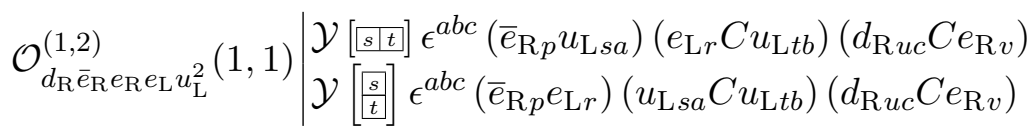

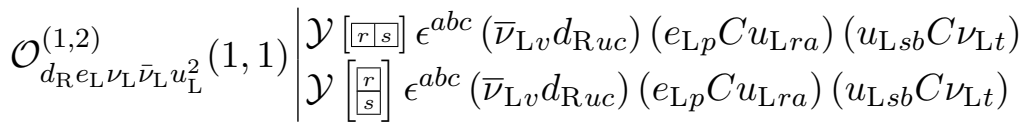




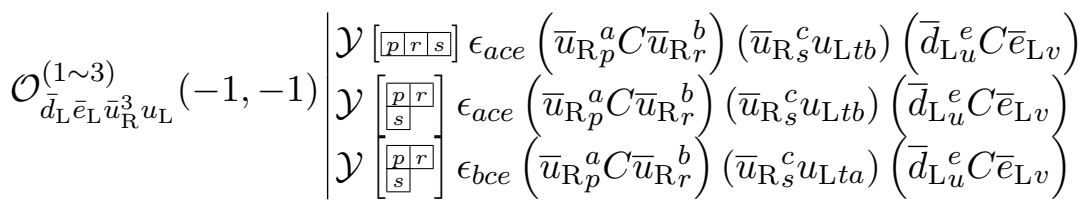

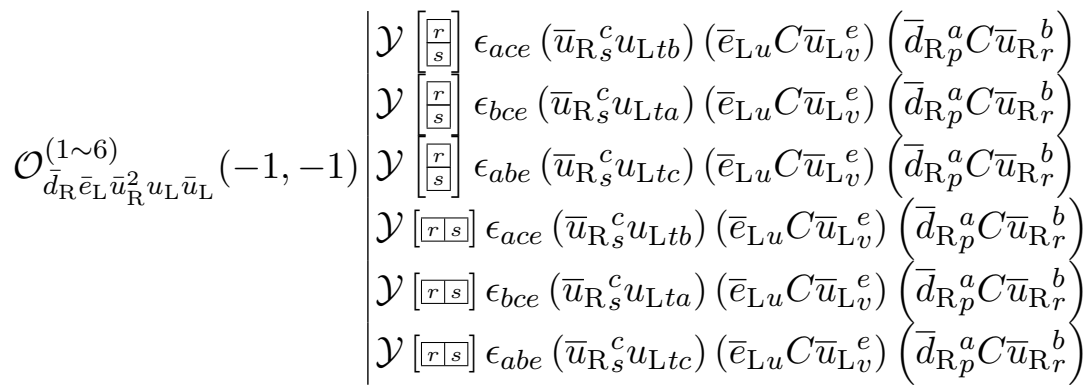

$$
\begin{aligned}
& \mathcal{Y}\left[\begin{array}{l}
{\left[\frac{r}{s}\right.} \\
\frac{1}{s}
\end{array}\right] \epsilon_{a c e}\left(\bar{u}_{\mathrm{R}}^{c}{ }_{s} u_{\mathrm{L} t b}\right)\left(\bar{d}_{\mathrm{L}}^{e} C \bar{\nu}_{\mathrm{L} v}\right)\left(\bar{d}_{\mathrm{R} p}^{a} C \bar{u}_{\mathrm{R}}^{b}\right)
\end{aligned}
$$

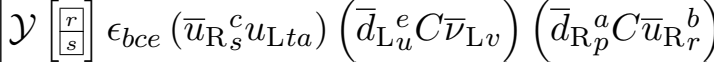

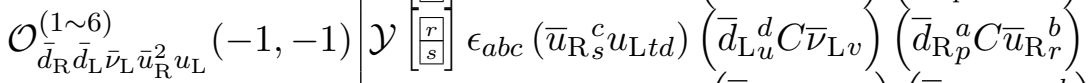

$$
\begin{aligned}
& \mathcal{Y}\left[[r \mid s] \epsilon_{a c e}\left(\bar{u}_{\mathrm{R}}^{c} u_{\mathrm{L} t b}\right)\left(\bar{d}_{\mathrm{L} u}^{e} C \bar{\nu}_{\mathrm{L} v}\right)\left(\bar{d}_{\mathrm{R}}^{a} C \bar{u}_{\mathrm{R}}^{b}\right)\right. \\
& \mathcal{Y}\left[[r s] \epsilon_{b c e}\left(\bar{u}_{\mathrm{R}}^{c}{ }_{s} u_{\mathrm{L} t a}\right)\left(\bar{d}_{\mathrm{L} u}^{e} C \bar{\nu}_{\mathrm{L} v}\right)\left(\bar{d}_{\mathrm{R}}^{a} C \bar{u}_{\mathrm{R}}^{b}\right)\right. \\
& \mathcal{Y}\left[[r s] \epsilon_{a b c}\left(\bar{u}_{\mathrm{R} s}^{c} u_{\mathrm{L} t d}\right)\left(\bar{d}_{\mathrm{L} u}^{d} C \bar{\nu}_{\mathrm{L} v}\right)\left(\bar{d}_{\mathrm{R} p}^{a} C \bar{u}_{\mathrm{R} r}^{b}\right)\right.
\end{aligned}
$$

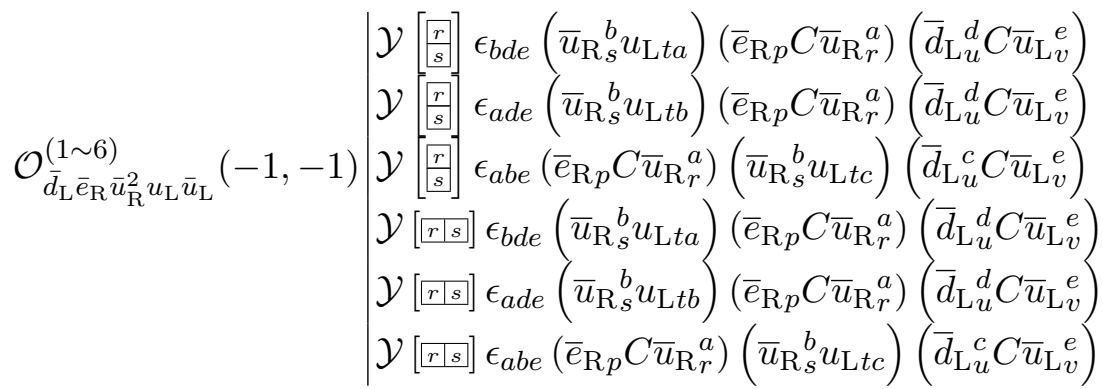

$$
\begin{aligned}
& \mathcal{Y}\left[\underline{u v v]} \epsilon^{a c e}\left(d_{\mathrm{L} p a} C e_{\mathrm{L} r}\right)\left(u_{\mathrm{R} u b} C u_{\mathrm{R} v e}\right)\left(\bar{u}_{\mathrm{R}}^{b} u_{\mathrm{L} t c}\right)\right. \\
& \mathcal{Y}[\underline{\underline{u} v]}] \epsilon^{a c e}\left(u_{\mathrm{R} u b} C u_{\mathrm{R} v e}\right)\left(e_{\mathrm{L} r} C u_{\mathrm{L} t c}\right)\left(\bar{u}_{\mathrm{R}_{s}^{b}}^{b} d_{\mathrm{L} p a}\right) \\
& \mathcal{O}_{d_{\mathrm{L}} e_{\mathrm{L}} \bar{u}_{\mathrm{R}} u_{\mathrm{R}}^{2} u_{\mathrm{L}}}^{\left(1 \sim{ }^{2}\right.}(1,1) \mathcal{Y}\left[\begin{array}{l}
u \\
v
\end{array}\right] \epsilon^{c d e}\left(d_{\mathrm{L} p a} C e_{\mathrm{L} r}\right)\left(u_{\mathrm{R} u d} C u_{\mathrm{R} v e}\right)\left(\bar{u}_{\mathrm{R} s}{ }^{a} u_{\mathrm{L} t c}\right) \\
& \mathcal{Y}\left[\begin{array}{c}
\frac{w}{v} \\
v
\end{array} \epsilon^{a c e}\left(d_{\mathrm{L} p a} C e_{\mathrm{L} r}\right)\left(u_{\mathrm{R} u b} C u_{\mathrm{R} v e}\right)\left(\bar{u}_{\mathrm{R}}{ }_{s}^{b} u_{\mathrm{L} t c}\right)\right. \\
& \mathcal{Y} \frac{\underline{v}}{v} \epsilon^{c d e}\left(e_{\mathrm{L} r} C u_{\mathrm{L} t c}\right)\left(u_{\mathrm{R} u d} C u_{\mathrm{R} v e}\right)\left(\bar{u}_{\mathrm{R} s}^{a} d_{\mathrm{L} p a}\right) \\
& \mathcal{Y}\left[\begin{array}{l}
\underline{u} \\
\underline{v}
\end{array}\right] \epsilon^{a c e}\left(u_{\mathrm{R} u b} C u_{\mathrm{R} v e}\right)\left(e_{\mathrm{L} r} C u_{\mathrm{L} t c}\right)\left(\bar{u}_{\mathrm{R} s}^{b} d_{\mathrm{L} p a}\right) \\
& \mathcal{O}_{d_{\mathrm{R}} d_{\mathrm{L}} \nu_{\mathrm{L}} \bar{u}_{\mathrm{R}} u_{\mathrm{R}} u_{\mathrm{L}}}^{(1 \sim 1,1)} \mid \begin{array}{l}
\epsilon^{c d e}\left(u_{\mathrm{L} s c} C \nu_{\mathrm{L} t}\right)\left(\bar{u}_{\mathrm{R} r}^{a} d_{\mathrm{L} p a}\right)\left(d_{\mathrm{R} u d} C u_{\mathrm{R} v e}\right) \\
\epsilon^{a c e}\left(u_{\mathrm{L} s c} C \nu_{\mathrm{L} t}\right)\left(d_{\mathrm{R} u b} C u_{\mathrm{R} v e}\right)\left(\bar{u}_{\mathrm{R} r}^{b} d_{\mathrm{L} p a}\right) \\
\epsilon^{c d e}\left(\bar{u}_{\mathrm{L} s b} C \nu_{\mathrm{L} t}\right)\left(d_{\mathrm{R} u d} C u_{\mathrm{R} v e}\right)\left(\bar{u}_{\mathrm{R}}{ }_{r}^{b} d_{\mathrm{L} p a}\right) \\
\left.\epsilon_{\mathrm{L} p a} C u_{\mathrm{L} s c}\right)\left(d_{\mathrm{R} u d} C u_{\mathrm{R} v e}\right) \\
\epsilon^{a d e}\left(\bar{u}_{\mathrm{R}}{ }_{r}^{b} \nu_{\mathrm{L} t}\right)\left(d_{\mathrm{L} p a} C u_{\mathrm{L} s c}\right)\left(d_{\mathrm{R} u b} C u_{\mathrm{R} v e}\right)
\end{array}
\end{aligned}
$$




$$
\begin{aligned}
& \mathcal{Y}\left[\frac{p}{r}\right] \epsilon_{\text {ace }}\left(\bar{d}_{\mathrm{R} p}^{a} C \bar{d}_{\mathrm{R}}^{b}\right)\left(\bar{u}_{\mathrm{R}}^{c}{ }_{s} u_{\mathrm{L} t b}\right)\left(\bar{u}_{\mathrm{L} u}^{e} C \bar{\nu}_{\mathrm{L} v}\right) \\
& \mathcal{Y} \frac{p}{r} \epsilon_{a b e}\left(\bar{u}_{\mathrm{R}}^{c} u_{\mathrm{L} t c}\right)\left(\bar{d}_{\mathrm{R} p}^{a} C \bar{d}_{\mathrm{R} r}^{b}\right)\left(\bar{u}_{\mathrm{L}}^{e} C \bar{\nu}_{\mathrm{L} v}\right)
\end{aligned}
$$

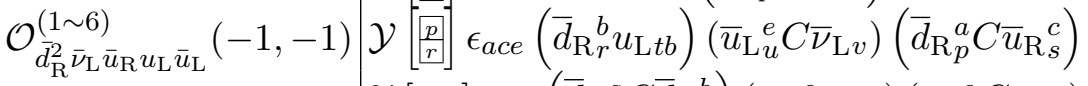

$$
\begin{aligned}
& \mathcal{Y}[\overline{p p r}] \epsilon_{\text {ace }}\left(\bar{d}_{\mathrm{R}_{p}^{a}}^{a} C \bar{d}_{\mathrm{R}}^{b}\right)\left(\bar{u}_{\mathrm{R}}^{c} u_{\mathrm{L} t b}\right)\left(\bar{u}_{\mathrm{L} u}^{e} C \bar{\nu}_{\mathrm{L} v}\right) \\
& \mathcal{Y}[\overline{p r r}] \epsilon_{\text {ace }}\left(\bar{d}_{\mathrm{R} r}^{b} u_{\mathrm{L} t b}\right)\left(\bar{u}_{\mathrm{L} u}^{e} C \bar{\nu}_{\mathrm{L} v}\right)\left(\bar{d}_{\mathrm{R} p}^{a} C \bar{u}_{\mathrm{R} s}^{c}\right) \\
& \mathcal{Y}\left[\underline{p p r]} \epsilon_{a b e}\left(\bar{d}_{\mathrm{R} r}^{b} u_{\mathrm{L} t c}\right)\left(\bar{u}_{\mathrm{L} u}^{e} C \bar{\nu}_{\mathrm{L} v}\right)\left(\bar{d}_{\mathrm{R} p}^{a} C \bar{u}_{\mathrm{R}_{s}^{c}}\right)\right. \\
& \mathcal{Y}\left[\underline{u v v]} \epsilon_{a b e}\left(\bar{d}_{\mathrm{R} p}^{a} C \bar{e}_{\mathrm{R} r}\right)\left(\bar{u}_{\mathrm{R} s}^{b} u_{\mathrm{L} t c}\right)\left(\bar{u}_{\mathrm{L} u}^{c} C \bar{u}_{\mathrm{L} v}^{e}\right)\right. \\
& \mathcal{Y}[\overline{u v v}] \epsilon_{a b e}\left(\bar{e}_{\mathrm{R} r} u_{\mathrm{L} t c}\right)\left(\bar{u}_{\mathrm{L} u}^{c} C \bar{u}_{\mathrm{L} v}^{e}\right)\left(\bar{d}_{\mathrm{R} p}^{a} C \bar{u}_{\mathrm{R}}^{b}\right)
\end{aligned}
$$

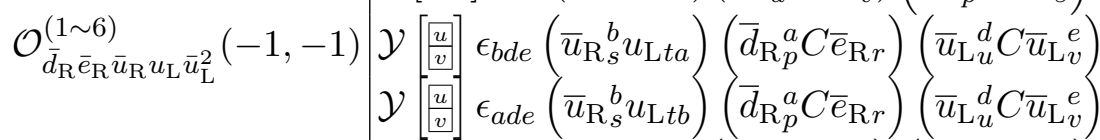

$$
\begin{aligned}
& \mathcal{Y}\left[\begin{array}{c}
u \\
v
\end{array}\right] \epsilon_{b d e}\left(\bar{e}_{\mathrm{R} r} u_{\mathrm{L} t a}\right)\left(\bar{u}_{\mathrm{L} u}^{d} C \bar{u}_{\mathrm{L} v}^{e}\right)\left(\bar{d}_{\mathrm{R} p}^{a} C \bar{u}_{\mathrm{R} s}^{b}\right)
\end{aligned}
$$

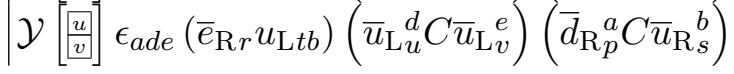

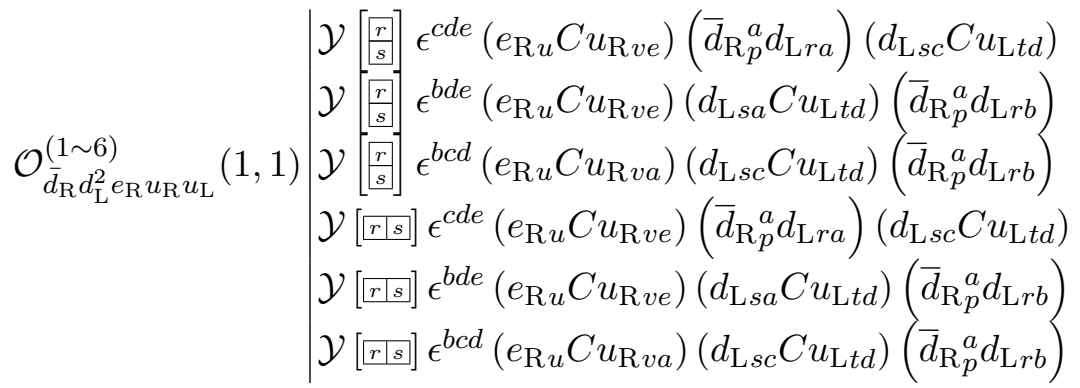

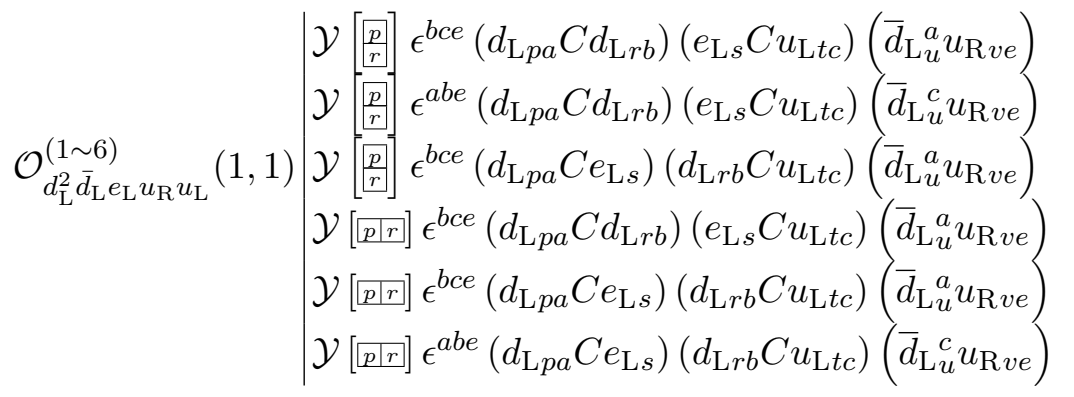

$$
\begin{aligned}
& \mathcal{Y}\left[\begin{array}{l}
\frac{p}{r} \\
{[}
\end{array} \epsilon^{b c d}\left(d_{\mathrm{L} p a} C d_{\mathrm{L} r b}\right)\left(u_{\mathrm{L} s c} C \nu_{\mathrm{L} t}\right)\left(\bar{u}_{\mathrm{L} v}^{a} u_{\mathrm{R} u d}\right)\right. \\
& \mathcal{Y}\left[\frac{p}{r}\right] \epsilon^{a b d}\left(d_{\mathrm{L} p a} C d_{\mathrm{L} r b}\right)\left(u_{\mathrm{L} s c} C \nu_{\mathrm{L} t}\right)\left(\bar{u}_{\mathrm{L}}^{c} u_{\mathrm{R} u d}\right)
\end{aligned}
$$

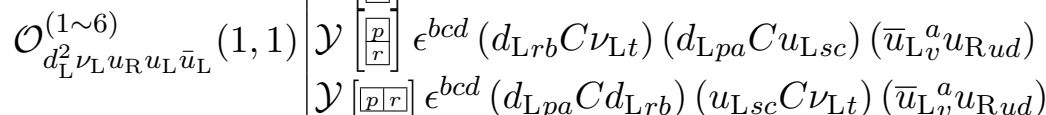

$$
\begin{aligned}
& \mathcal{Y}[\overline{p r r}] \epsilon^{b c d}\left(d_{\mathrm{L} r b} C \nu_{\mathrm{L} t}\right)\left(d_{\mathrm{L} p a} C u_{\mathrm{L} s c}\right)\left(\bar{u}_{\mathrm{L} v}^{a} u_{\mathrm{R} u d}\right) \\
& \mathcal{Y}[\overline{p r r}] \epsilon^{a b d}\left(d_{\mathrm{L} r b} C \nu_{\mathrm{L} t}\right)\left(d_{\mathrm{L} p a} C u_{\mathrm{L} s c}\right)\left(\bar{u}_{\mathrm{L} v}^{c} u_{\mathrm{R} u d}\right)
\end{aligned}
$$




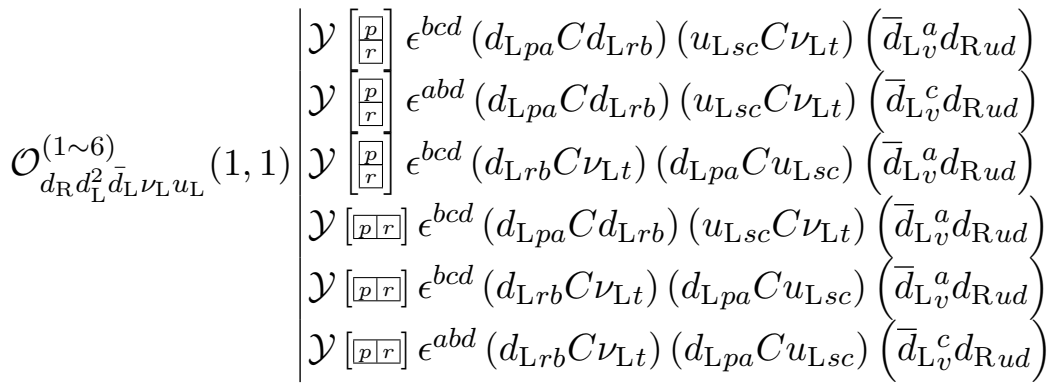

$$
\begin{aligned}
& \mathcal{O}_{d_{\mathrm{L}}^{2} e_{\mathrm{R}} \bar{e}_{\mathrm{L}} \nu_{\mathrm{L}} u_{\mathrm{L}}}^{(1,1)} \mid \begin{array}{l}
\mathcal{Y}\left[\begin{array}{l}
{[p r]} \\
\mathcal{Y}
\end{array} \epsilon^{a b c}\left(\bar{e}_{\mathrm{L} v} e_{\mathrm{R} u}\right)\left(d_{\mathrm{L} r b} C \nu_{\mathrm{L} t}\right)\left(d_{\mathrm{L} p a} C u_{\mathrm{L} s c}\right)\right. \\
\mathcal{Y}\left[\begin{array}{l}
p \\
r
\end{array}\right] \epsilon^{a b c}\left(\bar{e}_{\mathrm{L} v} e_{\mathrm{R} u}\right)\left(d_{\mathrm{L} p a} C d_{\mathrm{L} r b}\right)\left(u_{\mathrm{L} s c} C \nu_{\mathrm{L} t}\right)
\end{array} \\
& \mathcal{O}_{\bar{d}_{\mathrm{R}} d_{\mathrm{R}} d_{\mathrm{L}} e_{\mathrm{L}} u_{\mathrm{R}} u_{\mathrm{L}}}^{(1 \sim 1)}(1,1) \mid \begin{array}{l}
\epsilon^{c d e}\left(e_{\mathrm{L} s} C u_{\mathrm{L} t c}\right)\left(\bar{d}_{\mathrm{R} p}^{a} d_{\mathrm{L} r a}\right)\left(d_{\mathrm{R} u d} C u_{\mathrm{R} v e}\right) \\
\epsilon^{b d e}\left(e_{\mathrm{L} s} C u_{\mathrm{L} t a}\right)\left(d_{\mathrm{R} u d} C u_{\mathrm{R} v e}\right)\left(\bar{d}_{\mathrm{R} p}^{a} d_{\mathrm{L} r b}\right) \\
\epsilon^{b c d}\left(e_{\mathrm{L} s} C u_{\mathrm{L} t c}\right)\left(d_{\mathrm{R} u d} C u_{\mathrm{R} v a}\right)\left(\bar{d}_{\mathrm{R}}{ }_{p}^{a} d_{\mathrm{L} r b}\right) \\
\epsilon^{c d e}\left(\bar{d}_{\mathrm{R}}^{a} e_{\mathrm{L} s}\right)\left(d_{\mathrm{L} r a} C u_{\mathrm{L} t c}\right)\left(d_{\mathrm{R} u d} C u_{\mathrm{R} v e}\right) \\
\epsilon^{b d e}\left(\bar{d}_{\mathrm{R} p}^{a} e_{\mathrm{L} s}\right)\left(d_{\mathrm{L} r b} C u_{\mathrm{L} t a}\right)\left(d_{\mathrm{R} u d} C u_{\mathrm{R} v e}\right) \\
\epsilon^{b c d}\left(\bar{d}_{\mathrm{R} p}^{a} e_{\mathrm{L} s}\right)\left(d_{\mathrm{R} u d} C u_{\mathrm{R} v a}\right)\left(d_{\mathrm{L} r b} C u_{\mathrm{L} t c}\right)
\end{array}
\end{aligned}
$$

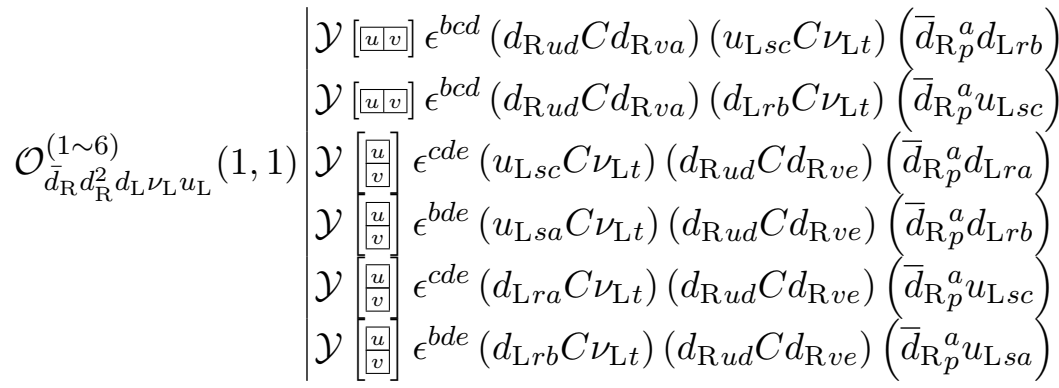

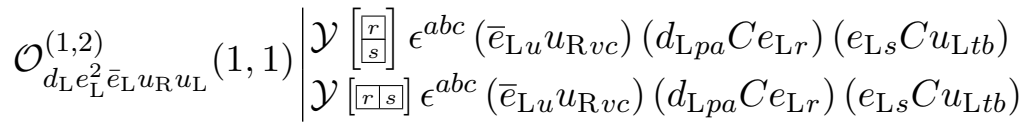

$$
\begin{aligned}
& \mathcal{O}_{d_{\mathrm{L}} \bar{e}_{\mathrm{R}} e_{\mathrm{R}} e_{\mathrm{L}} u_{\mathrm{R}} u_{\mathrm{L}}}^{(1,1)} \mid \begin{array}{ll}
\epsilon^{a b c}\left(\bar{e}_{\mathrm{R} r} d_{\mathrm{L} p a}\right)\left(e_{\mathrm{L} s} C u_{\mathrm{L} t b}\right) & \left(e_{\mathrm{R} u} C u_{\mathrm{R} v c}\right) \\
\epsilon^{a b c}\left(\bar{e}_{\mathrm{R} r} u_{\mathrm{L} t b}\right) & \left(d_{\mathrm{L} p a} C e_{\mathrm{L} s}\right)\left(e_{\mathrm{R} u} C u_{\mathrm{R} v c}\right)
\end{array} \\
& \mathcal{O}_{d_{\mathrm{L}} e_{\mathrm{L}} \nu_{\mathrm{L}} \bar{\nu}_{\mathrm{L}} u_{\mathrm{R}} u_{\mathrm{L}}}^{(1,2)}(1) \mid \begin{array}{l}
\epsilon^{a b c}\left(\bar{\nu}_{\mathrm{L} v} u_{\mathrm{R} u c}\right)\left(d_{\mathrm{L} p a} C e_{\mathrm{L} r}\right)\left(u_{\mathrm{L} s b} C \nu_{\mathrm{L} t}\right) \\
\epsilon^{a b c}\left(e_{\mathrm{L} r} C \nu_{\mathrm{L} t}\right)\left(\bar{\nu}_{\mathrm{L} v} u_{\mathrm{R} u c}\right)\left(d_{\mathrm{L} p a} C u_{\mathrm{L} s b}\right)
\end{array} \\
& \mathcal{O}_{d_{\mathrm{R}} d_{\mathrm{L}} e_{\mathrm{L}} \bar{e}_{\mathrm{L}} \nu_{\mathrm{L}} u_{\mathrm{L}}}^{(1,1)} \mid \begin{array}{l}
\epsilon^{a b c}\left(\bar{e}_{\mathrm{L} v} d_{\mathrm{R} u c}\right)\left(d_{\mathrm{L} p a} C e_{\mathrm{L} r}\right)\left(u_{\mathrm{L} s b} C \nu_{\mathrm{L} t}\right) \\
\epsilon^{a b c}\left(e_{\mathrm{L} r} C \nu_{\mathrm{L} t}\right)\left(\bar{e}_{\mathrm{L} v} d_{\mathrm{R} u c}\right)\left(d_{\mathrm{L} p a} C u_{\mathrm{L} s b}\right)
\end{array} \\
& \mathcal{O}_{d_{\mathrm{R}} d_{\mathrm{L}} \bar{e}_{\mathrm{R}} e_{\mathrm{R}} \nu_{\mathrm{L}} u_{\mathrm{L}}}^{(1,1)} \mid \begin{array}{ll}
\epsilon^{a b c}\left(\bar{e}_{\mathrm{R} r} d_{\mathrm{L} p a}\right)\left(u_{\mathrm{L} s b} C \nu_{\mathrm{L} t}\right)\left(d_{\mathrm{R} u c} C e_{\mathrm{R} v}\right) \\
\epsilon^{a b c}\left(\bar{e}_{\mathrm{R} r} \nu_{\mathrm{L} t}\right)\left(d_{\mathrm{R} u c} C e_{\mathrm{R} v}\right)\left(d_{\mathrm{L} p a} C u_{\mathrm{L} s b}\right)
\end{array}
\end{aligned}
$$

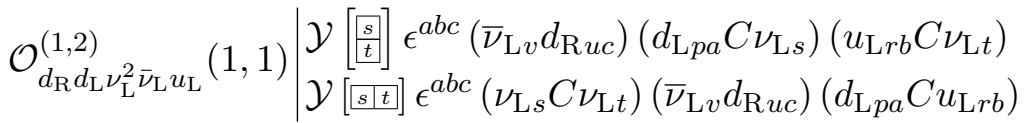

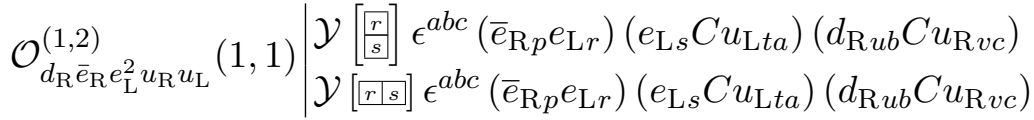




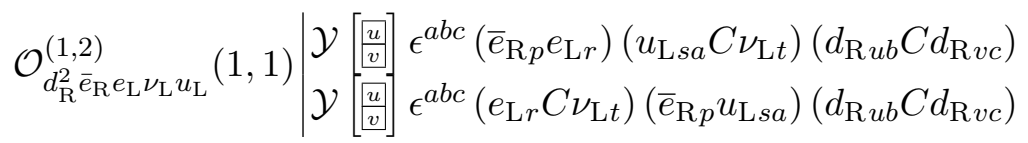

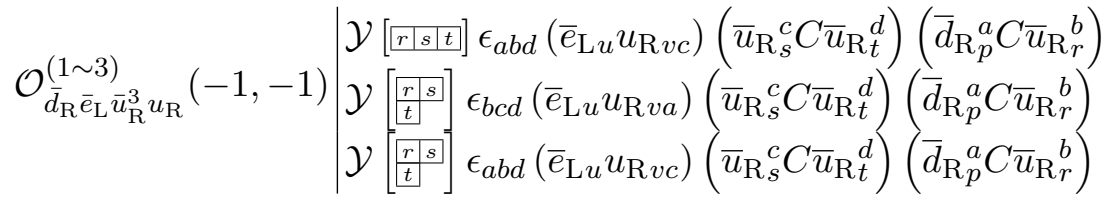

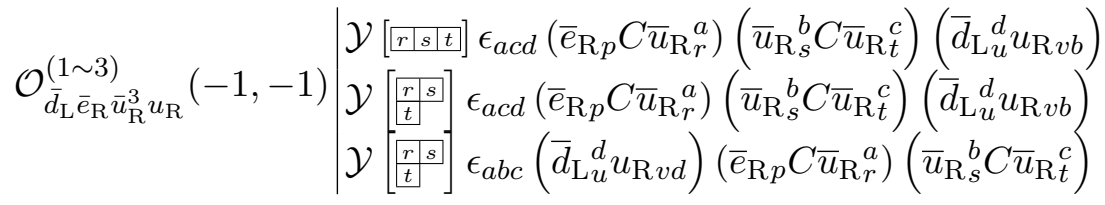

$$
\begin{aligned}
& \mathcal{O}_{\bar{e}_{\mathrm{L}}^{2} \nu_{\mathrm{L}} \bar{u}_{\mathrm{R}}^{3}}(-1,-1) \mid \mathcal{Y}\left[\frac{p r r}{s}, \underline{u v v}\right] \epsilon_{a b c}\left(\bar{e}_{\mathrm{L} u} C \bar{e}_{\mathrm{L} v}\right)\left(\bar{u}_{\mathrm{R}}{ }_{s}^{c} \nu_{\mathrm{L} t}\right)\left(\bar{u}_{\mathrm{R}}^{a} C \bar{u}_{\mathrm{R} r}^{b}\right) \\
& \mathcal{Y}\left[\begin{array}{l}
s \\
\underline{t}
\end{array}\right] \epsilon_{c d e}\left(\bar{d}_{\mathrm{R} p}^{a} d_{\mathrm{L} r a}\right)\left(\bar{u}_{\mathrm{R}}^{c} C \bar{u}_{\mathrm{R} t}^{d}\right)\left(\bar{d}_{\mathrm{L} u}^{e} C \bar{e}_{\mathrm{L} v}\right) \\
& \mathcal{Y}\left[\begin{array}{ll}
s \\
t]
\end{array}\right] \epsilon_{\text {ade }}\left(\bar{d}_{\mathrm{R} p}^{a} d_{\mathrm{L} r b}\right)\left(\bar{u}_{\mathrm{R}}{ }_{s}^{b} C \bar{u}_{\mathrm{R} t}^{d}\right)\left(\bar{d}_{\mathrm{L} u}^{e} C \bar{e}_{\mathrm{L} v}\right)
\end{aligned}
$$

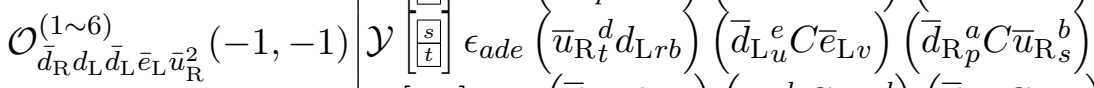

$$
\begin{aligned}
& \mathcal{Y}[s \mid t] \epsilon_{\text {ade }}\left(\bar{d}_{\mathrm{R} p}^{a} d_{\mathrm{L} r b}\right)\left(\bar{u}_{\mathrm{R}}^{b} C \bar{u}_{\mathrm{R} t}^{d}\right)\left(\bar{d}_{\mathrm{L} u}^{e} C \bar{e}_{\mathrm{L} v}\right) \\
& \mathcal{Y}[s \mid t] \epsilon_{c d e}\left(\bar{u}_{\mathrm{R} t}^{d} d_{\mathrm{L} r a}\right)\left(\bar{d}_{\mathrm{L} u}^{e} C \bar{e}_{\mathrm{L} v}\right)\left(\bar{d}_{\mathrm{R} p}^{a} C \bar{u}_{\mathrm{R}}^{c}\right) \\
& \mathcal{Y}[s \mid t] \epsilon_{\text {ade }}\left(\bar{u}_{\mathrm{R} t}^{d} d_{\mathrm{L} r b}\right)\left(\bar{d}_{\mathrm{L}}^{e} C \bar{e}_{\mathrm{L} v}\right)\left(\bar{d}_{\mathrm{R} p}^{a} C \bar{u}_{\mathrm{R}_{s}}^{b}\right) \\
& \left.\mathcal{Y}\left[\begin{array}{c}
s \\
\frac{s}{t},
\end{array}, \frac{u}{v}\right]\right] \epsilon_{c d e}\left(\bar{e}_{\mathrm{R} r} d_{\mathrm{L} p a}\right)\left(\bar{u}_{\mathrm{R} s}^{a} C \bar{u}_{\mathrm{R} t}^{c}\right)\left(\bar{d}_{\mathrm{L} u}^{d} C \bar{d}_{\mathrm{L} v}^{e}\right) \\
& \mathcal{Y}\left[\frac{s}{t}, \frac{u}{v}\right] \epsilon_{c d e}\left(\bar{u}_{\mathrm{R} s}^{a} d_{\mathrm{L} p a}\right)\left(\bar{e}_{\mathrm{R} r} C \bar{u}_{\mathrm{R} t}^{c}\right)\left(\bar{d}_{\mathrm{L} u}^{d} C \bar{d}_{\mathrm{L} v}^{e}\right) \\
& \mathcal{O}_{d_{\mathrm{L}} \bar{d}_{\mathrm{L}}^{2} \bar{e}_{\mathrm{R}} \bar{u}_{\mathrm{R}}^{2}}^{(1 \sim 6)}(-1,-1) \mathcal{Y}\left[s \mid t,[u v] \epsilon_{b c e}\left(\bar{u}_{\mathrm{R} s}^{b} d_{\mathrm{L} p a}\right)\left(\bar{d}_{\mathrm{L} u}^{a} C \bar{d}_{\mathrm{L} v}^{e}\right)\left(\bar{e}_{\mathrm{R} r} C \bar{u}_{\mathrm{R} t}^{c}\right)\right. \\
& \mathcal{Y}\left[s \mid t,, \frac{u}{v}\right] \epsilon_{c d e}\left(\bar{e}_{\mathrm{R} r} d_{\mathrm{L} p a}\right)\left(\bar{u}_{\mathrm{R} s}^{a} C \bar{u}_{\mathrm{R} t}^{c}\right)\left(\bar{d}_{\mathrm{L} u}^{d} C \bar{d}_{\mathrm{L} v}^{e}\right) \\
& \mathcal{Y}\left[s \mid t,,\left[\begin{array}{l}
u \\
v
\end{array} \epsilon_{c d e}\left(\bar{u}_{\mathrm{R}_{s}^{a}}^{a} d_{\mathrm{L} p a}\right)\left(\bar{e}_{\mathrm{R} r} C \bar{u}_{\mathrm{R} t}^{c}\right)\left(\bar{d}_{\mathrm{L} u}^{d} C \bar{d}_{\mathrm{L} v}^{e}\right)\right.\right. \\
& \mathcal{Y}[\underline{s}, \bar{t}, u \mid v] \epsilon_{b c e}\left(\bar{e}_{\mathrm{R} r} d_{\mathrm{L} p a}\right)\left(\bar{d}_{\mathrm{L} u}^{a} C \bar{d}_{\mathrm{L} v}^{e}\right)\left(\bar{u}_{\mathrm{R}}^{b} C \bar{u}_{\mathrm{R} t}^{c}\right)
\end{aligned}
$$

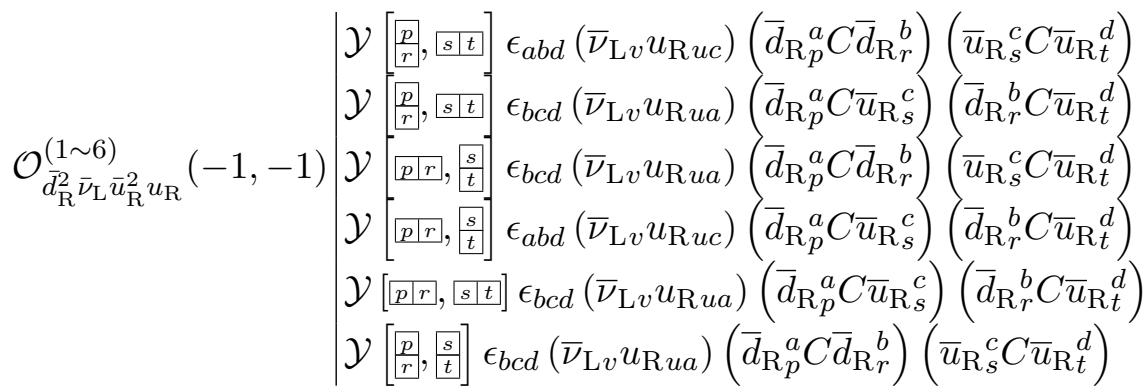

$$
\begin{aligned}
& \mathcal{Y}\left[\left[\frac{p}{r}, s \mid t\right] \epsilon_{a b d}\left(\bar{e}_{\mathrm{L} v} d_{\mathrm{R} u c}\right)\left(\bar{d}_{\mathrm{R}}^{a} C \bar{d}_{\mathrm{R}}^{b}\right)\left(\bar{u}_{\mathrm{R}}^{c} C \bar{u}_{\mathrm{R} t}^{d}\right)\right. \\
& \mathcal{Y}\left[\frac{p}{r}, s \mid t\right] \epsilon_{b c d}\left(\bar{e}_{\mathrm{L} v} d_{\mathrm{R} u a}\right)\left(\bar{d}_{\mathrm{R} p}^{a} C \bar{u}_{\mathrm{R}}^{c}\right)\left(\bar{d}_{\mathrm{R} r}^{b} C \bar{u}_{\mathrm{R} t}^{d}\right)
\end{aligned}
$$

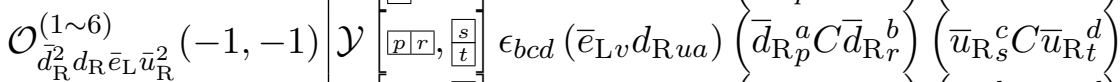

$$
\begin{aligned}
& \mathcal{Y}\left[\overline{p p r}, \frac{s}{t}\right] \epsilon_{a b d}\left(\bar{e}_{\mathrm{L} v} d_{\mathrm{R} u c}\right)\left(\bar{d}_{\mathrm{R} p}^{a} C \bar{u}_{\mathrm{R} s}^{c}\right)\left(\bar{d}_{\mathrm{R} r}^{b} C \bar{u}_{\mathrm{R} t}^{d}\right) \\
& \mathcal{Y}\left[\left[p \mid r,[s \mid t] \epsilon_{b c d}\left(\bar{e}_{\mathrm{L} v} d_{\mathrm{R} u a}\right)\left(\bar{d}_{\mathrm{R} p}^{a} C \bar{u}_{\mathrm{R} s}^{c}\right)\left(\bar{d}_{\mathrm{R} r}^{b} C \bar{u}_{\mathrm{R} t}^{d}\right)\right.\right. \\
& \mathcal{Y}\left[\begin{array}{c}
p \\
\frac{p}{r}, \frac{s}{t}
\end{array}\right] \epsilon_{b c d}\left(\bar{e}_{\mathrm{L} v} d_{\mathrm{R} u a}\right)\left(\bar{d}_{\mathrm{R}_{p}^{a}}^{a} C \bar{d}_{\mathrm{R}_{r}^{b}}^{b}\right)\left(\bar{u}_{\mathrm{R}_{s}^{c}}^{c} C \bar{u}_{\mathrm{R} t}^{d}\right)
\end{aligned}
$$




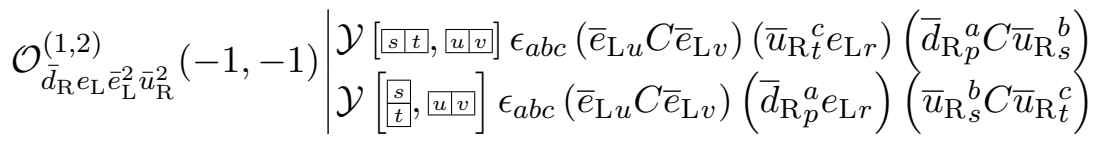

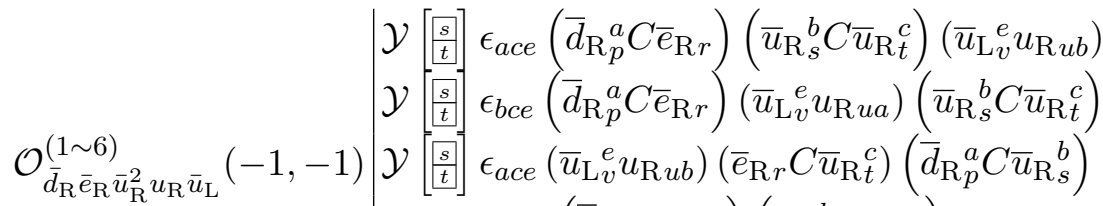

$$
\begin{aligned}
& \mathcal{Y}[s \mid t] \epsilon_{a c e}\left(\bar{d}_{\mathrm{R} p}^{a} C \bar{e}_{\mathrm{R} r}\right)\left(\bar{u}_{\mathrm{R} s}^{b} C \bar{u}_{\mathrm{R} t}^{c}\right)\left(\bar{u}_{\mathrm{L} v}{ }^{e} u_{\mathrm{R} u b}\right) \\
& \mathcal{Y}[s \mid t]] \epsilon_{\text {ace }}\left(\bar{u}_{\mathrm{L} v}^{e} u_{\mathrm{R} u b}\right)\left(\bar{e}_{\mathrm{R} r} C \bar{u}_{\mathrm{R} t}^{c}\right)\left(\bar{d}_{\mathrm{R} p}^{a} C \bar{u}_{\mathrm{R}_{s}^{b}}^{b}\right) \\
& \mathcal{Y}[s \mid t]] \epsilon_{b c e}\left(\bar{u}_{\mathrm{L} v}^{e} u_{\mathrm{R} u a}\right)\left(\bar{e}_{\mathrm{R} r} C \bar{u}_{\mathrm{R} t}^{c}\right)\left(\bar{d}_{\mathrm{R} p}^{a} C \bar{u}_{\mathrm{R} s}^{b}\right)
\end{aligned}
$$

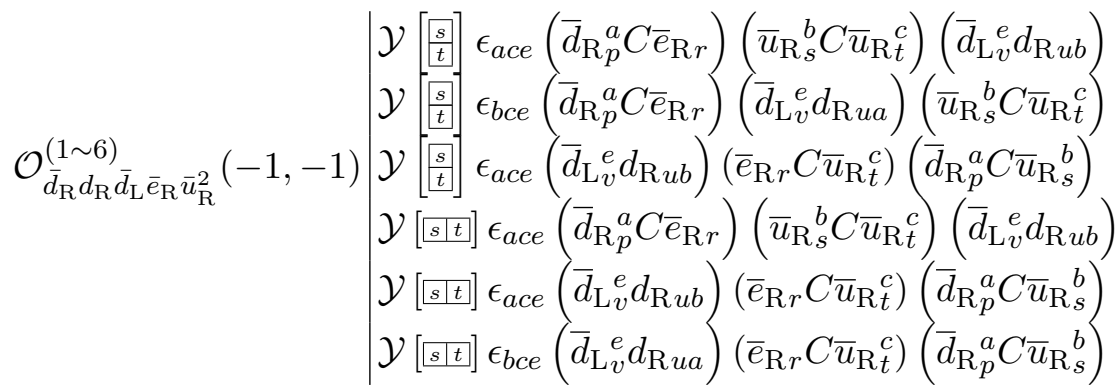

$$
\begin{aligned}
& \left.\mathcal{O}_{\bar{d}_{\mathrm{R}} \bar{e}_{\mathrm{R}} e_{\mathrm{R}} \bar{e}_{\mathrm{L}} \bar{u}_{\mathrm{R}}^{2}(-1,-1)} \mid \begin{array}{l}
\mathcal{Y}[s \mid t] \epsilon_{a b c}\left(\bar{e}_{\mathrm{L} v} e_{\mathrm{R} u}\right)\left(\bar{e}_{\mathrm{R} r} C \bar{u}_{\mathrm{R}}^{c}\right)\left(\bar{d}_{\mathrm{R}}^{a} C \bar{u}_{\mathrm{R}}^{b}\right) \\
\mathcal{Y}\left[\begin{array}{l}
s \\
\underline{t}
\end{array}\right] \epsilon_{a b c}\left(\bar{e}_{\mathrm{L} v} e_{\mathrm{R} u}\right)\left(\bar{d}_{\mathrm{R} p}^{a} C \bar{e}_{\mathrm{R} r}\right)\left(\bar{u}_{\mathrm{R} s}^{b} C \bar{u}_{\mathrm{R} t}^{c}\right.
\end{array}\right)
\end{aligned}
$$

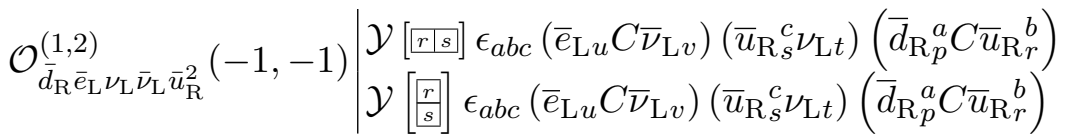

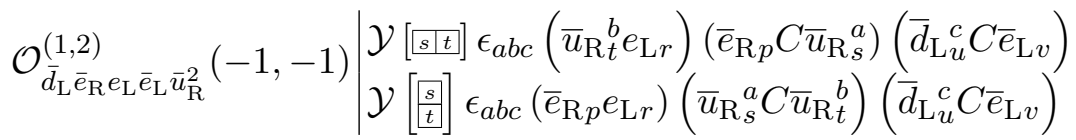

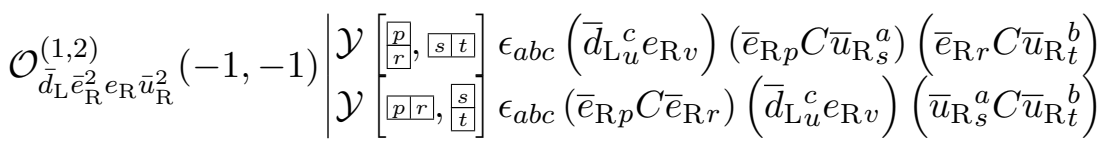

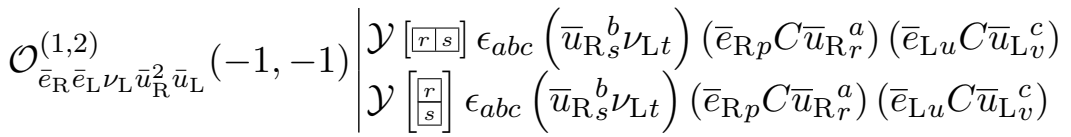

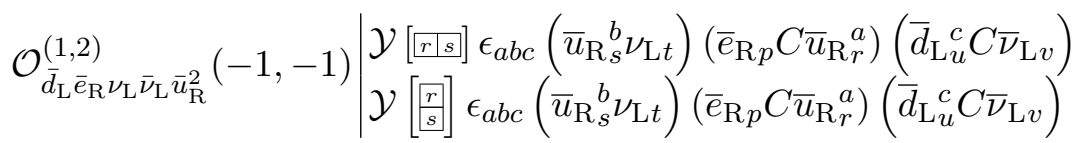

$$
\begin{aligned}
& \mathcal{Y}\left[\frac{p}{r}, \frac{u}{v}\right] \epsilon^{b d e}\left(d_{\mathrm{L} p a} C d_{\mathrm{L} r b}\right)\left(u_{\mathrm{R} u d} C u_{\mathrm{R} v e}\right)\left(\bar{u}_{\mathrm{R}}{ }_{s}^{a} \nu_{\mathrm{L} t}\right) \\
& \mathcal{Y}\left[\frac{p}{r}, \underline{u} v\right] \epsilon^{b d e}\left(d_{\mathrm{L} r b} C \nu_{\mathrm{L} t}\right)\left(u_{\mathrm{R} u d} C u_{\mathrm{R} v e}\right)\left(\bar{u}_{\mathrm{R} s}^{a} d_{\mathrm{L} p a}\right) \\
& \mathcal{O}_{d_{\mathrm{L}}^{2} \nu_{\mathrm{L}} \bar{u}_{\mathrm{R}} u_{\mathrm{R}}^{2}}^{(1 \sim 6)}(1,1) \mathcal{Y}[p \mid r, \overline{u v}] \epsilon^{a b e}\left(d_{\mathrm{L} r b} C \nu_{\mathrm{L} t}\right)\left(u_{\mathrm{R} u c} C u_{\mathrm{R} v e}\right)\left(\bar{u}_{\mathrm{R}}^{c}{ }_{s}^{c} d_{\mathrm{L} p a}\right) \\
& \mathcal{Y}\left[\overline{p \mid r}, \frac{u}{v}\right] \epsilon^{b d e}\left(d_{\mathrm{L} p a} C d_{\mathrm{L} r b}\right)\left(u_{\mathrm{R} u d} C u_{\mathrm{R} v e}\right)\left(\bar{u}_{\mathrm{R}}{ }_{s}^{a} \nu_{\mathrm{L} t}\right) \\
& \mathcal{Y}\left[p \mid r, \frac{u}{v}\right] \epsilon^{b d e}\left(d_{\mathrm{L} r b} C \nu_{\mathrm{L} t}\right)\left(u_{\mathrm{R} u d} C u_{\mathrm{R} v e}\right)\left(\bar{u}_{\mathrm{R} s}^{a} d_{\mathrm{L} p a}\right) \\
& \mathcal{Y}\left[\frac{p}{r}, u \mid v\right] \epsilon^{a b e}\left(d_{\mathrm{L} p a} C d_{\mathrm{L} r b}\right)\left(u_{\mathrm{R} u c} C u_{\mathrm{R} v e}\right)\left(\bar{u}_{\mathrm{R} s}^{c} \nu_{\mathrm{L} t}\right)
\end{aligned}
$$




$$
\begin{aligned}
& \mathcal{Y}\left[\frac{p}{r}\right] \epsilon_{b d e}\left(\bar{d}_{\mathrm{R} p}^{a} C \bar{d}_{\mathrm{R} r}^{b}\right)\left(\bar{u}_{\mathrm{R} t}^{d} d_{\mathrm{L} s a}\right)\left(\bar{e}_{\mathrm{L} u} C \bar{u}_{\mathrm{L} v}^{e}\right)
\end{aligned}
$$

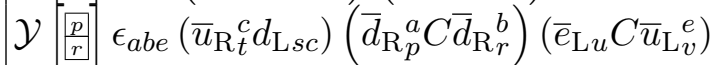

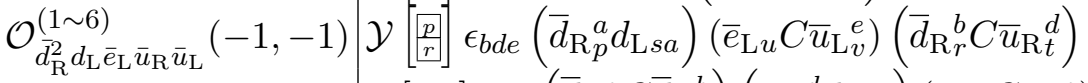

$$
\begin{aligned}
& \mathcal{Y}[\overline{p p r}] \epsilon_{b d e}\left(\bar{d}_{\mathrm{R}_{p}^{a} C} C \bar{d}_{\mathrm{R} r}^{b}\right)\left(\bar{u}_{\mathrm{R} t}^{d} d_{\mathrm{L} s a}\right)\left(\bar{e}_{\mathrm{L} u} C \bar{u}_{\mathrm{L} v}^{e}\right) \\
& \mathcal{Y}[\overline{p r r}] \epsilon_{b d e}\left(\bar{d}_{\mathrm{R} p}^{a} d_{\mathrm{L} s a}\right)\left(\bar{e}_{\mathrm{L} u} C \bar{u}_{\mathrm{L} v}^{e}\right)\left(\bar{d}_{\mathrm{R} r}^{b} C \bar{u}_{\mathrm{R} t}^{d}\right) \\
& \mathcal{Y}\left[\overline{p \mid r]} \epsilon_{a b e}\left(\bar{d}_{\mathrm{R} p}^{a} d_{\mathrm{L} s c}\right)\left(\bar{e}_{\mathrm{L} u} C \bar{u}_{\mathrm{L} v}^{e}\right)\left(\bar{d}_{\mathrm{R} r}^{b} C \bar{u}_{\mathrm{R} t}^{c}\right)\right.
\end{aligned}
$$

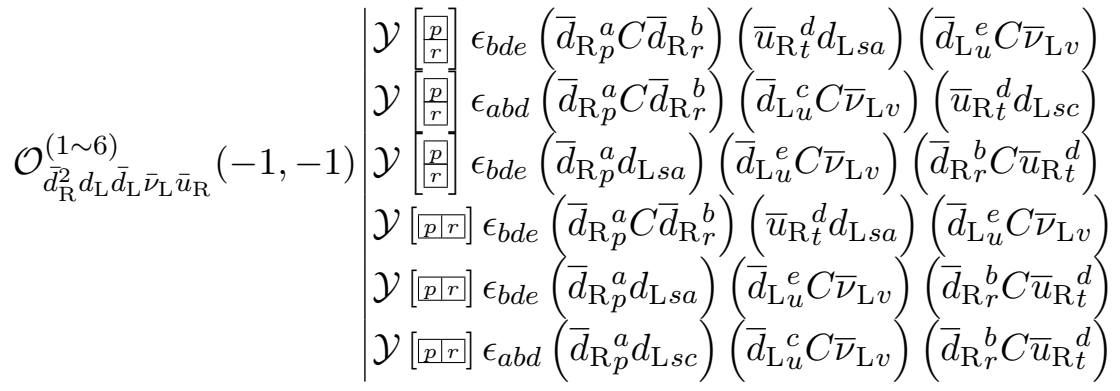

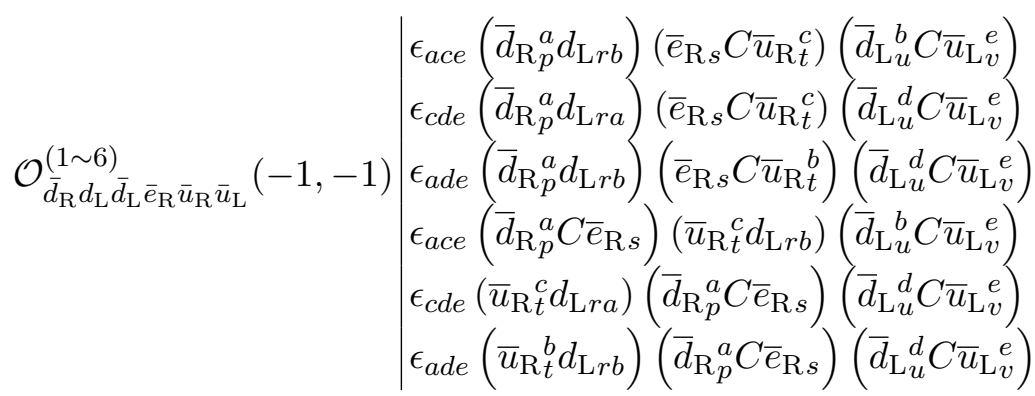

$$
\begin{aligned}
& \mathcal{Y}\left[[p|r| s] \epsilon_{b c d}\left(\bar{\nu}_{\mathrm{L} v} d_{\mathrm{R} u a}\right)\left(\bar{d}_{\mathrm{R}}^{a} C \bar{d}_{\mathrm{R}}^{b}\right)\left(\bar{d}_{\mathrm{R}}^{c} C \bar{u}_{\mathrm{R} t}^{d}\right)\right.
\end{aligned}
$$

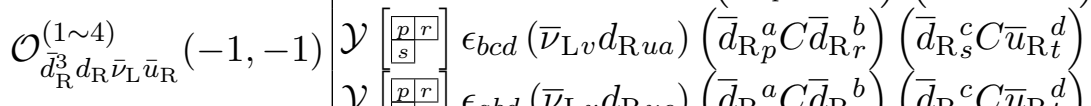

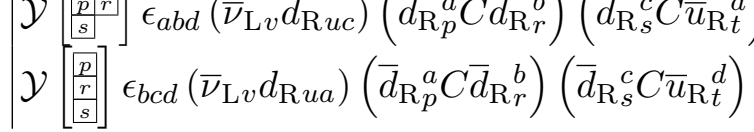

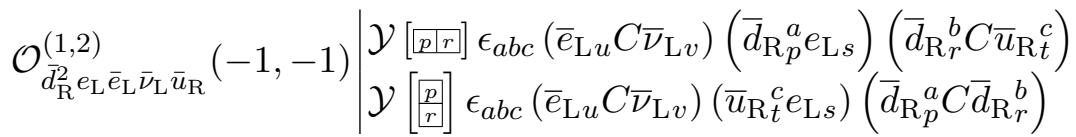

$$
\begin{aligned}
& \mathcal{Y}\left[\frac{p}{r}\right] \epsilon_{\text {ace }}\left(\bar{d}_{\mathrm{R}}^{a}{ }_{p}^{a} C \bar{d}_{\mathrm{R}}^{b}\right)\left(\bar{u}_{\mathrm{L}}^{e} d_{\mathrm{R} u b}\right)\left(\bar{e}_{\mathrm{R} s} C \bar{u}_{\mathrm{R}}^{c}\right) \\
& \mathcal{Y}\left[\frac{p}{r]}\right] \epsilon_{a b e}\left(\bar{d}_{\mathrm{R} p}^{a} C \bar{d}_{\mathrm{R}}{ }_{r}^{b}\right)\left(\bar{e}_{\mathrm{R} s} C \bar{u}_{\mathrm{R} t}^{c}\right)\left(\bar{u}_{\mathrm{L} v}{ }^{e} d_{\mathrm{R} u c}\right)
\end{aligned}
$$

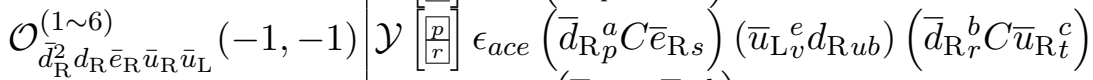

$$
\begin{aligned}
& \mathcal{Y}[\overline{p r r}] \epsilon_{\text {ace }}\left(\bar{d}_{\mathrm{R} p}^{a} C \bar{d}_{\mathrm{R}}^{b}\right)\left(\bar{u}_{\mathrm{L} v}^{e} d_{\mathrm{R} u b}\right)\left(\bar{e}_{\mathrm{R} s} C \bar{u}_{\mathrm{R}}^{c}\right) \\
& \mathcal{Y}\left[[p r r] \epsilon_{\text {ace }}\left(\bar{d}_{\mathrm{R} p}^{a} C \bar{e}_{\mathrm{R} s}\right)\left(\bar{u}_{\mathrm{L} v}^{e} d_{\mathrm{R} u b}\right)\left(\bar{d}_{\mathrm{R} r}^{b} C \bar{u}_{\mathrm{R} t}^{c}\right)\right. \\
& \mathcal{Y}\left[[\overline{p \mid r}] \epsilon_{a b e}\left(\bar{d}_{\mathrm{R} p}^{a} C \bar{e}_{\mathrm{R} s}\right)\left(\bar{u}_{\mathrm{L} v}^{e} d_{\mathrm{R} u c}\right)\left(\bar{d}_{\mathrm{R} r}^{b} C \bar{u}_{\mathrm{R} t}^{c}\right)\right. \\
& \mathcal{O}_{\bar{d}_{\mathrm{R}}^{2} \bar{e}_{\mathrm{R}} e_{\mathrm{R}} \bar{\nu}_{\mathrm{L}} \bar{u}_{\mathrm{R}}(-1,-1)} \mid \begin{array}{l}
\mathcal{Y}[\overline{p l r}] \epsilon_{a b c}\left(\bar{\nu}_{\mathrm{L} v} e_{\mathrm{R} u}\right)\left(\bar{d}_{\mathrm{R}}^{a} C \bar{e}_{\mathrm{R} s}\right)\left(\bar{d}_{\mathrm{R}}{ }_{r}^{b} C \bar{u}_{\mathrm{R} t}^{c}\right) \\
\mathcal{Y}\left[\begin{array}{l}
p \\
{[r]}
\end{array} \epsilon_{a b c}\left(\bar{\nu}_{\mathrm{L} v} e_{\mathrm{R} u}\right)\left(\bar{d}_{\mathrm{R}}^{a} C \bar{d}_{\mathrm{R}}^{b}\right)\left(\bar{e}_{\mathrm{R} s} C \bar{u}_{\mathrm{R}}{ }^{c}\right)\right.
\end{array}
\end{aligned}
$$




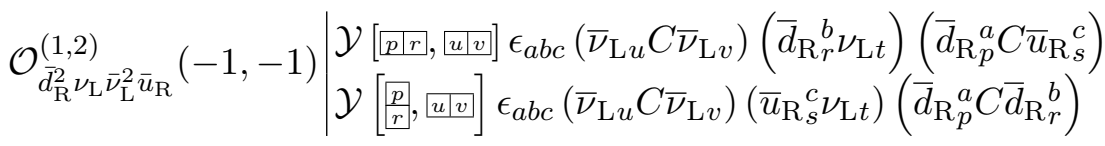

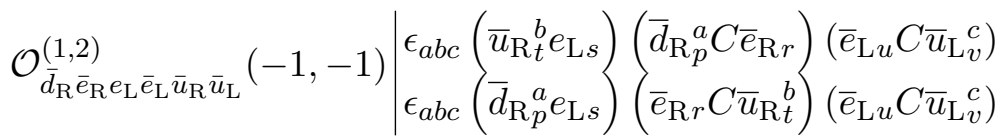

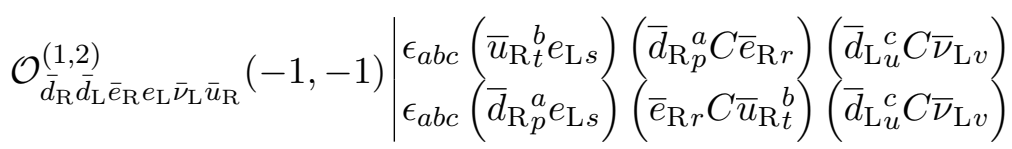

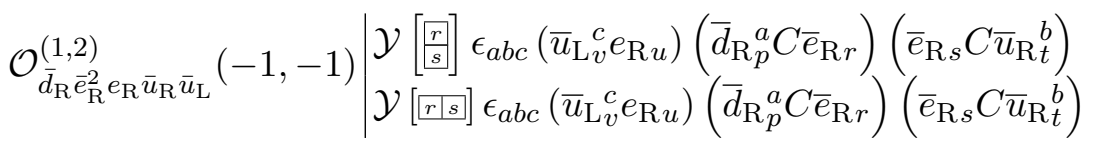

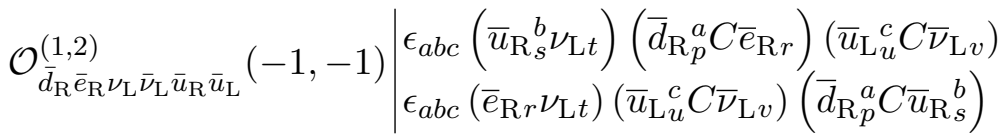

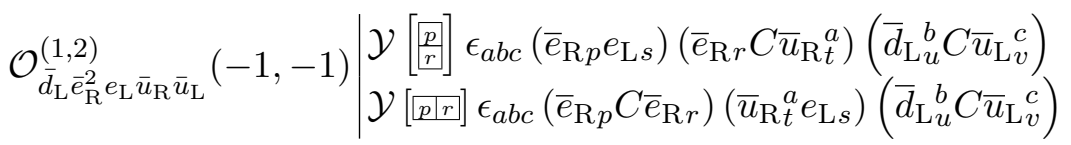

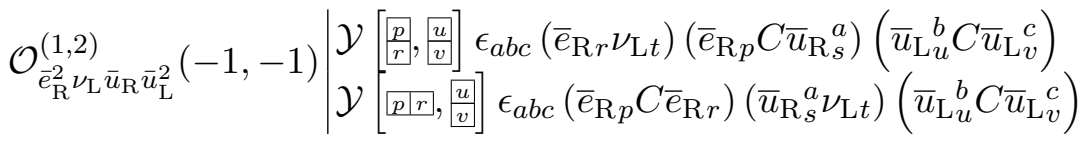

$$
\begin{aligned}
& \mathcal{Y}\left[\overline{p|r| s]} \epsilon^{b c e}\left(d_{\mathrm{L} p a} C d_{\mathrm{L} r b}\right)\left(d_{\mathrm{L} s c} C \nu_{\mathrm{L} t}\right)\left(\bar{d}_{\mathrm{L} u}^{a} u_{\mathrm{R} v e}\right)\right.
\end{aligned}
$$

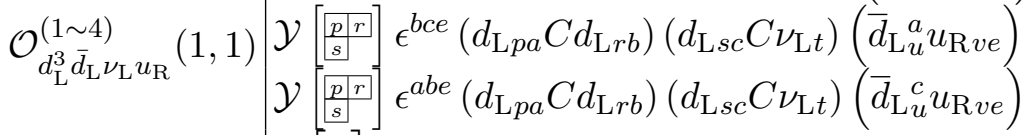

$$
\begin{aligned}
& \mathcal{Y}\left[\begin{array}{l}
\frac{p}{r} \\
s
\end{array}\right] \epsilon^{b c e}\left(d_{\mathrm{L} p a} C d_{\mathrm{L} r b}\right)\left(d_{\mathrm{L} s c} C \nu_{\mathrm{L} t}\right)\left(\bar{d}_{\mathrm{L} u}^{a} u_{\mathrm{R} v e}\right) \\
& \mathcal{Y}\left[\frac{r}{s}, \underline{u}, \frac{u}{v}\right] \epsilon^{c d e}\left(d_{\mathrm{L} s c} C e_{\mathrm{L} t}\right)\left(u_{\mathrm{R} u d} C u_{\mathrm{R} v e}\right)\left(\bar{d}_{\mathrm{R} p}^{a} d_{\mathrm{L} r a}\right)
\end{aligned}
$$

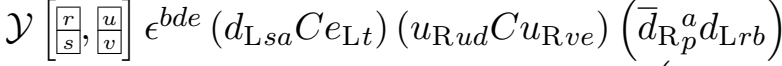

$$
\begin{aligned}
& \mathcal{O}_{\bar{d}_{\mathrm{R}} d_{\mathrm{L}}^{2} e_{\mathrm{L}} u_{\mathrm{R}}^{2}}^{(1,6)} \mathcal{Y}[\underline{r r s}, u v v] \epsilon^{b c d}\left(u_{\mathrm{R} u d} C u_{\mathrm{R} v a}\right)\left(d_{\mathrm{L} s c} C e_{\mathrm{L} t}\right)\left(\bar{d}_{\mathrm{R} p}^{a} d_{\mathrm{L} r b}\right) \\
& \mathcal{Y}\left[\overline{r r s}, \underline{u} \frac{u}{v}\right] \epsilon^{c d e}\left(d_{\mathrm{L} s c} C e_{\mathrm{L} t}\right)\left(u_{\mathrm{R} u d} C u_{\mathrm{R} v e}\right)\left(\bar{d}_{\mathrm{R} p}^{a} d_{\mathrm{L} r a}\right) \\
& \mathcal{Y}\left[\overline{r r s}, \frac{u}{v}\right] \epsilon^{b d e}\left(d_{\mathrm{L} s a} C e_{\mathrm{L} t}\right)\left(u_{\mathrm{R} u d} C u_{\mathrm{R} v e}\right)\left(\bar{d}_{\mathrm{R} p}^{a} d_{\mathrm{L} r b}\right) \\
& \mathcal{Y}\left[\frac{r}{s}, u v v\right] \epsilon^{b c d}\left(u_{\mathrm{R} u d} C u_{\mathrm{R} v a}\right)\left(d_{\mathrm{L} s c} C e_{\mathrm{L} t}\right)\left(\bar{d}_{\mathrm{R} p}^{a} d_{\mathrm{L} r b}\right)
\end{aligned}
$$

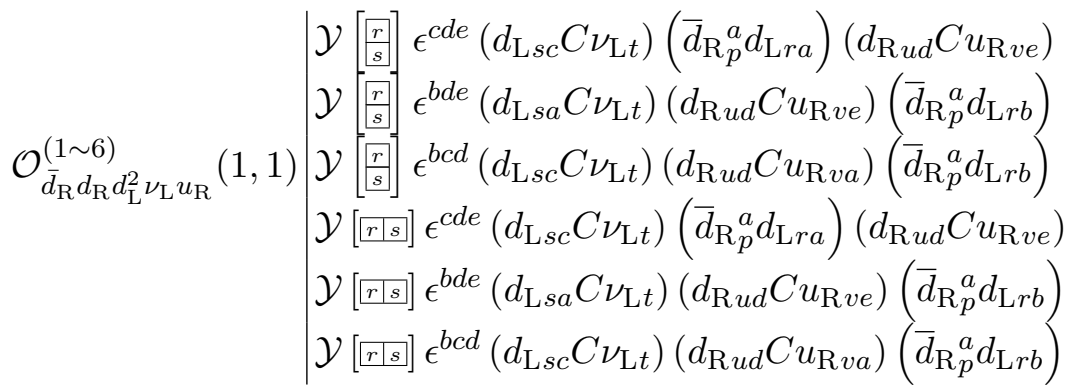

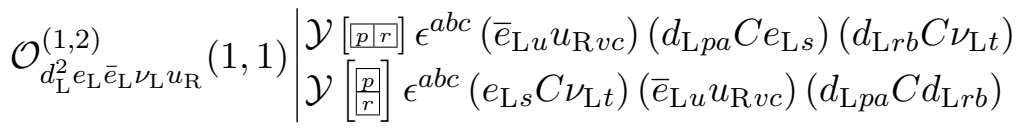




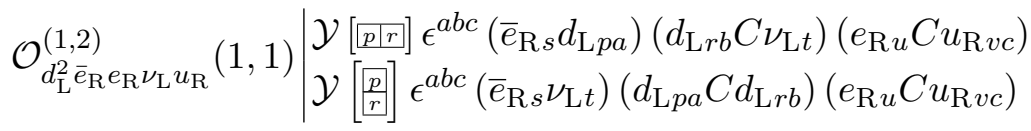

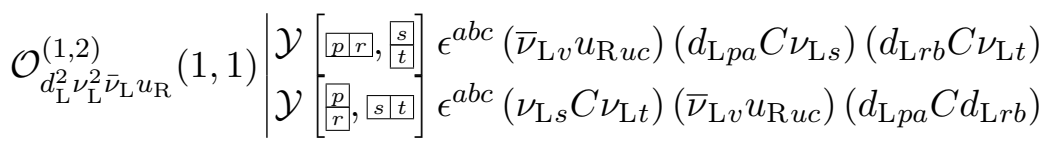

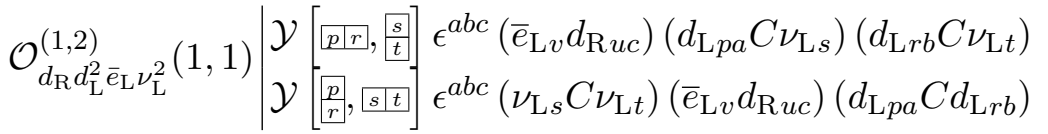

$$
\begin{aligned}
& \mathcal{Y}\left[[p|r| s] \epsilon_{a c e}\left(\bar{d}_{\mathrm{R} p}^{a} C \bar{d}_{\mathrm{R} r}^{b}\right)\left(\bar{d}_{\mathrm{R}}^{c} d_{\mathrm{L} t b}\right)\left(\bar{u}_{\mathrm{L} u}^{e} C \bar{\nu}_{\mathrm{L} v}\right)\right.
\end{aligned}
$$

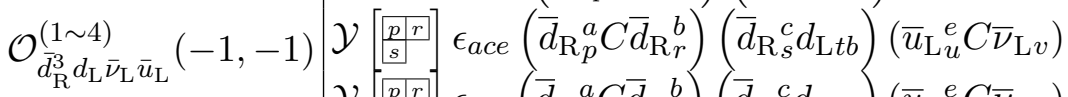

$$
\begin{aligned}
& \mathcal{Y}\left[\frac{p p r}{s s}\right] \epsilon_{b c e}\left(\bar{d}_{\mathrm{R} p}^{a} C \bar{d}_{\mathrm{R}}^{b}\right)\left(\bar{d}_{\mathrm{R}}^{c} d_{\mathrm{L} t a}\right)\left(\bar{u}_{\mathrm{L}}^{e} C \bar{\nu}_{\mathrm{L} v}\right) \\
& \mathcal{Y}\left[\begin{array}{c}
\frac{p}{r} \\
s
\end{array}\right] \epsilon_{a c e}\left(\bar{d}_{\mathrm{R} p}^{a} C \bar{d}_{\mathrm{R} r}^{b}\right)\left(\bar{d}_{\mathrm{R} s}^{c} d_{\mathrm{L} t b}\right)\left(\bar{u}_{\mathrm{L} u}^{e} C \bar{\nu}_{\mathrm{L} v}\right) \\
& \mathcal{Y}\left[\frac{p}{r}, \frac{u}{v}\right] \epsilon_{b d e}\left(\bar{e}_{\mathrm{R} t} d_{\mathrm{L} s a}\right)\left(\bar{d}_{\mathrm{R}}^{a} C \bar{d}_{\mathrm{R} r}^{b}\right)\left(\bar{u}_{\mathrm{L} u}^{d} C \bar{u}_{\mathrm{L} v}^{e}\right) \\
& \mathcal{Y}\left[\frac{p}{r}, \frac{u}{v}\right] \epsilon_{b d e}\left(\bar{d}_{\mathrm{R} p}^{a} d_{\mathrm{L} s a}\right)\left(\bar{d}_{\mathrm{R} r}^{b} C \bar{e}_{\mathrm{R} t}\right)\left(\bar{u}_{\mathrm{L} u}^{d} C \bar{u}_{\mathrm{L} v}^{e}\right) \\
& \mathcal{O}_{\bar{d}_{\mathrm{R}}^{2} d_{\mathrm{L}} \bar{e}_{\mathrm{R}} \bar{u}_{\mathrm{L}}^{2}(-1,-1) \mathcal{Y}[p p r, \mid u v]} \epsilon_{a b e}\left(\bar{d}_{\mathrm{R} p}^{a} d_{\mathrm{L} s c}\right)\left(\bar{d}_{\mathrm{R} r}^{b} C \bar{e}_{\mathrm{R} t}\right)\left(\bar{u}_{\mathrm{L} u}^{c} C \bar{u}_{\mathrm{L} v}^{e}\right) \\
& \mathcal{Y}\left[\overline{p p r}, \frac{w}{v}\right] \epsilon_{b d e}\left(\bar{e}_{\mathrm{R} t} d_{\mathrm{L} s a}\right)\left(\bar{d}_{\mathrm{R}}^{a} C \bar{d}_{\mathrm{R}}^{b}\right)\left(\bar{u}_{\mathrm{L} u}^{d} C \bar{u}_{\mathrm{L}}^{e}\right) \\
& \mathcal{Y}\left[p \mid r, \frac{u}{v}\right] \epsilon_{b d e}\left(\bar{d}_{\mathrm{R} p}^{a} d_{\mathrm{L} s a}\right)\left(\bar{d}_{\mathrm{R} r}^{b} C \bar{e}_{\mathrm{R} t}\right)\left(\bar{u}_{\mathrm{L} u}^{d} C \bar{u}_{\mathrm{L} v}^{e}\right) \\
& \mathcal{Y}\left[\frac{p}{r}, u \mid v\right] \epsilon_{a b e}\left(\bar{e}_{\mathrm{R} t} d_{\mathrm{L} s c}\right)\left(\bar{d}_{\mathrm{R} p}^{a} C \bar{d}_{\mathrm{R}}^{b}\right)\left(\bar{u}_{\mathrm{L} u}^{c} C \bar{u}_{\mathrm{L} v}^{e}\right)
\end{aligned}
$$

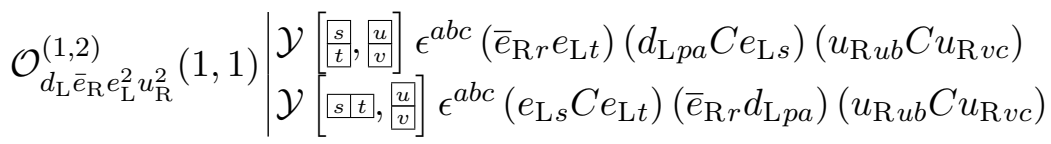

$$
\begin{aligned}
& \mathcal{O}_{d_{\mathrm{R}} d_{\mathrm{L}} \bar{e}_{\mathrm{R}} e_{\mathrm{L}} \nu_{\mathrm{L}} u_{\mathrm{R}}}^{(1,1)} \mid \begin{array}{l}
\epsilon^{a b c}\left(e_{\mathrm{L} s} C \nu_{\mathrm{L} t}\right)\left(\bar{e}_{\mathrm{R} r} d_{\mathrm{L} p a}\right)\left(d_{\mathrm{R} u b} C u_{\mathrm{R} v c}\right) \\
\epsilon^{a b c}\left(\bar{e}_{\mathrm{R} r} \nu_{\mathrm{L} t}\right)\left(d_{\mathrm{L} p a} C e_{\mathrm{L} s}\right)\left(d_{\mathrm{R} u b} C u_{\mathrm{R} v c}\right)
\end{array} \\
& \mathcal{O}_{d_{\mathrm{R}}^{2} d_{\mathrm{L}} \bar{e}_{\mathrm{R}} \nu_{\mathrm{L}}^{2}}^{(1,2)}(1,1) \mid \begin{array}{l}
\mathcal{Y} \\
\mathcal{Y}\left[\begin{array}{l}
{\left[\begin{array}{l}
s \\
-\frac{u}{t},
\end{array}\right] \epsilon^{a b c}\left(\bar{e}_{\mathrm{R} r} \nu_{\mathrm{L} t}\right)\left(d_{\mathrm{L} p a} C \nu_{\mathrm{L} s}\right)\left(d_{\mathrm{R} u b} C d_{\mathrm{R} v c}\right)} \\
s s t, \frac{u}{v}
\end{array}\right] \epsilon^{a b c}\left(\nu_{\mathrm{L} s} C \nu_{\mathrm{L} t}\right)\left(\bar{e}_{\mathrm{R} r} d_{\mathrm{L} p a}\right)\left(d_{\mathrm{R} u b} C d_{\mathrm{R} v c}\right)
\end{array}
\end{aligned}
$$

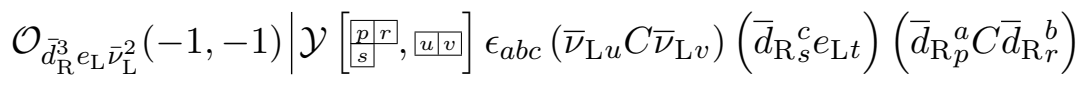

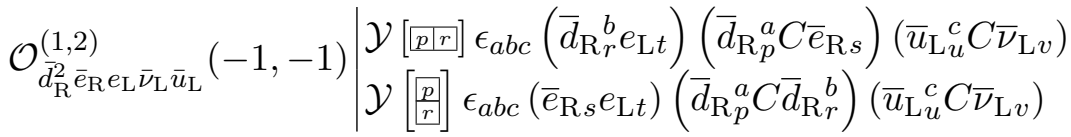

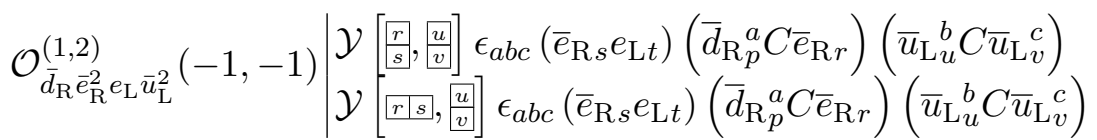


$(\Delta B, \Delta L)=( \pm 1, \mp 1)$

$$
\begin{aligned}
& \mathcal{Y}\left[\begin{array}{l}
s \\
t
\end{array}\right] \epsilon^{c d e}\left(\bar{\nu}_{\mathrm{L} v} d_{\mathrm{R} u e}\right)\left(u_{\mathrm{L} s c} C u_{\mathrm{L} t d}\right)\left(\bar{u}_{\mathrm{R} r}^{a} d_{\mathrm{L} p a}\right) \\
& \mathcal{Y}\left[\begin{array}{ccc}
s \\
\hline t
\end{array} \epsilon^{a c e}\left(\bar{\nu}_{\mathrm{L} v} d_{\mathrm{R} u e}\right)\left(u_{\mathrm{L} s c} C u_{\mathrm{L} t b}\right)\left(\bar{u}_{\mathrm{R}}{ }_{r}^{b} d_{\mathrm{L} p a}\right)\right.
\end{aligned}
$$

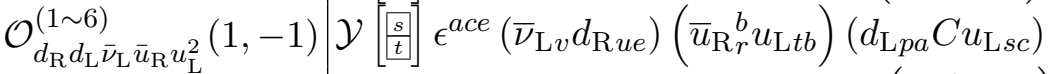

$$
\begin{aligned}
& \mathcal{Y}[\overline{s \mid t]}] \epsilon^{a c e}\left(\bar{\nu}_{\mathrm{L} v} d_{\mathrm{R} u e}\right)\left(u_{\mathrm{L} s c} C u_{\mathrm{L} t b}\right)\left(\bar{u}_{\mathrm{R}}^{b} d_{\mathrm{L} p a}\right) \\
& \mathcal{Y}\left[s_{s \mid t}\right] \epsilon^{c d e}\left(\bar{\nu}_{\mathrm{L} v} d_{\mathrm{R} u e}\right)\left(d_{\mathrm{L} p a} C u_{\mathrm{L} s c}\right)\left(\bar{u}_{\mathrm{R}}{ }_{r}^{a} u_{\mathrm{L} t d}\right) \\
& \mathcal{Y}[s \mid t]] \epsilon^{a c e}\left(\bar{\nu}_{\mathrm{L} v} d_{\mathrm{R} u e}\right)\left(\bar{u}_{\mathrm{R} r}^{b} u_{\mathrm{L} t b}\right)\left(d_{\mathrm{L} p a} C u_{\mathrm{L} s c}\right) \\
& \mathcal{Y}\left[\frac{p}{r}, s \mid t\right] \epsilon^{a b d}\left(d_{\mathrm{L} p a} C d_{\mathrm{L} r b}\right)\left(u_{\mathrm{L} s c} C u_{\mathrm{L} t d}\right)\left(\bar{u}_{\mathrm{L} u}^{c} C \bar{\nu}_{\mathrm{L} v}\right) \\
& \mathcal{Y}\left[\frac{p}{r}, s \mid t\right] \epsilon^{b c d}\left(d_{\mathrm{L} p a} C u_{\mathrm{L} s c}\right)\left(d_{\mathrm{L} r b} C u_{\mathrm{L} t d}\right)\left(\bar{u}_{\mathrm{L} u}^{a} C \bar{\nu}_{\mathrm{L} v}\right) \\
& \mathcal{O}_{d_{\mathrm{L}}^{2} \overline{\mathrm{L}}_{\mathrm{L}} u_{\mathrm{L}}^{2} \bar{u}_{\mathrm{L}}}^{(1 \sim 6)}(1,-1) \mathcal{Y}\left[p p r,, \frac{s}{t]} \epsilon^{b c d}\left(d_{\mathrm{L} p a} C d_{\mathrm{L} r b}\right)\left(u_{\mathrm{L} s c} C u_{\mathrm{L} t d}\right)\left(\bar{u}_{\mathrm{L} u}^{a} C \bar{\nu}_{\mathrm{L} v}\right)\right. \\
& \mathcal{Y}\left[p p r, \frac{s}{t}\right] \epsilon^{a b d}\left(d_{\mathrm{L} p a} C u_{\mathrm{L} s c}\right)\left(d_{\mathrm{L} r b} C u_{\mathrm{L} t d}\right)\left(\bar{u}_{\mathrm{L}}^{c} C \bar{\nu}_{\mathrm{L} v}\right) \\
& \mathcal{Y}[[p|r,, s| t]] \epsilon^{b c d}\left(d_{\mathrm{L} p a} C u_{\mathrm{L} s c}\right)\left(d_{\mathrm{L} r b} C u_{\mathrm{L} t d}\right)\left(\bar{u}_{\mathrm{L}}^{a} C \bar{\nu}_{\mathrm{L} v}\right) \\
& \left.\mathcal{Y}\left[\frac{p}{r}, \frac{s}{t}\right]\right] \epsilon^{b c d}\left(d_{\mathrm{L} p a} C d_{\mathrm{L} r b}\right)\left(u_{\mathrm{L} s c} C u_{\mathrm{L} t d}\right)\left(\bar{u}_{\mathrm{L} u}^{a} C \bar{\nu}_{\mathrm{L} v}\right)
\end{aligned}
$$

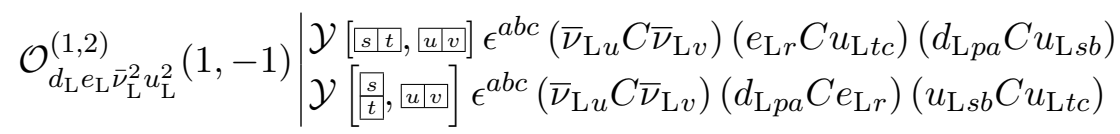

$$
\begin{aligned}
& \mathcal{Y}\left[\frac{p}{r}, \underline{u} v\right] \epsilon_{b d e}\left(u_{\mathrm{L} s a} C \nu_{\mathrm{L} t}\right)\left(\bar{u}_{\mathrm{R} p}^{a} C \bar{u}_{\mathrm{R}}^{b}\right)\left(\bar{d}_{\mathrm{L} u}^{d} C \bar{d}_{\mathrm{L}}^{e}\right) \\
& \mathcal{Y}\left[\frac{p}{r}, \frac{u}{v}\right] \epsilon_{b d e}\left(\bar{u}_{\mathrm{R} p}^{a} u_{\mathrm{L} s a}\right)\left(\bar{u}_{\mathrm{R} r}^{b} \nu_{\mathrm{L} t}\right)\left(\bar{d}_{\mathrm{L} u}^{d} C \bar{d}_{\mathrm{L} v}^{e}\right) \\
& \mathcal{O}_{\bar{d}_{\mathrm{L}}^{2} \nu_{\mathrm{L}} \bar{u}_{\mathrm{R}}^{2} u_{\mathrm{L}}}^{(1 \sim 6)}(-1,1) \mathcal{Y}[[p \mid r,, u v]] \epsilon_{a b d}\left(\bar{u}_{\mathrm{R}}{ }_{r}^{b} \nu_{\mathrm{L} t}\right)\left(\bar{u}_{\mathrm{R} p}^{a} u_{\mathrm{L} s c}\right)\left(\bar{d}_{\mathrm{L} u}^{d} C \bar{d}_{\mathrm{L} v}^{c}\right) \\
& \mathcal{Y}[\overline{p p r}, \underline{u} v] \epsilon_{b d e}\left(u_{\mathrm{L} s a} C \nu_{\mathrm{L} t}\right)\left(\bar{u}_{\mathrm{R} p}^{a} C \bar{u}_{\mathrm{R} r}^{b}\right)\left(\bar{d}_{\mathrm{L} u}^{d} C \bar{d}_{\mathrm{L} v}^{e}\right) \\
& \mathcal{Y}\left[\overline{p r r}, \frac{u}{v}\right] \epsilon_{b d e}\left(\bar{u}_{\mathrm{R}}{ }_{p}^{a} u_{\mathrm{L} s a}\right)\left(\bar{u}_{\mathrm{R}}{ }_{r}^{b} \nu_{\mathrm{L} t}\right)\left(\bar{d}_{\mathrm{L} u}^{d} C \bar{d}_{\mathrm{L} v}^{e}\right) \\
& \mathcal{Y}\left[\frac{p}{r}, u v v\right] \epsilon_{a b d}\left(u_{\mathrm{L} s c} C \nu_{\mathrm{L} t}\right)\left(\bar{u}_{\mathrm{R} p}^{a} C \bar{u}_{\mathrm{R} r}^{b}\right)\left(\bar{d}_{\mathrm{L} u}^{d} C \bar{d}_{\mathrm{L} v}^{c}\right) \\
& \mathcal{Y}\left[\begin{array}{l}
p \\
r
\end{array}\right] \epsilon^{b d e}\left(\bar{\nu}_{\mathrm{L} v} u_{\mathrm{R} u e}\right)\left(d_{\mathrm{L} p a} C d_{\mathrm{L} r b}\right)\left(\bar{u}_{\mathrm{R}}{ }^{a} u_{\mathrm{L} t d}\right) \\
& \mathcal{Y}\left[\frac{p}{r}\right] \epsilon^{a b e}\left(\bar{\nu}_{\mathrm{L} v} u_{\mathrm{R} u e}\right)\left(d_{\mathrm{L} p a} C d_{\mathrm{L} r b}\right)\left(\bar{u}_{\mathrm{R}}^{c} u_{\mathrm{L} t c}\right) \\
& \mathcal{O}_{d_{\mathrm{L}}^{2} \bar{\nu}_{\mathrm{L}} \bar{u}_{\mathrm{R}} u_{\mathrm{R}} u_{\mathrm{L}}}^{(1 \sim \bar{y}}(1,-1) \mathcal{Y}\left[\frac{p}{r}\right] \epsilon^{b d e}\left(\bar{\nu}_{\mathrm{L} v} u_{\mathrm{R} u e}\right)\left(\bar{u}_{\mathrm{R} s}^{a} d_{\mathrm{L} p a}\right)\left(d_{\mathrm{L} r b} C u_{\mathrm{L} t d}\right) \\
& \mathcal{Y}[\overline{p p r}] \epsilon^{b d e}\left(\bar{\nu}_{\mathrm{L} v} u_{\mathrm{R} u e}\right)\left(d_{\mathrm{L} p a} C d_{\mathrm{L} r b}\right)\left(\bar{u}_{\mathrm{R} s}{ }^{a} u_{\mathrm{L} t d}\right) \\
& \mathcal{Y}[\overline{p p r}] \epsilon^{b d e}\left(\bar{\nu}_{\mathrm{L} v} u_{\mathrm{R} u e}\right)\left(\bar{u}_{\mathrm{R}}^{a}{ }_{s} d_{\mathrm{L} p a}\right)\left(d_{\mathrm{L} r b} C u_{\mathrm{L} t d}\right) \\
& \mathcal{Y}[\overline{p r r}] \epsilon^{a b e}\left(\bar{\nu}_{\mathrm{L} v} u_{\mathrm{R} u e}\right)\left(d_{\mathrm{L} r b} C u_{\mathrm{L} t c}\right)\left(\bar{u}_{\mathrm{R}}^{c} d_{\mathrm{L} p a}\right)
\end{aligned}
$$

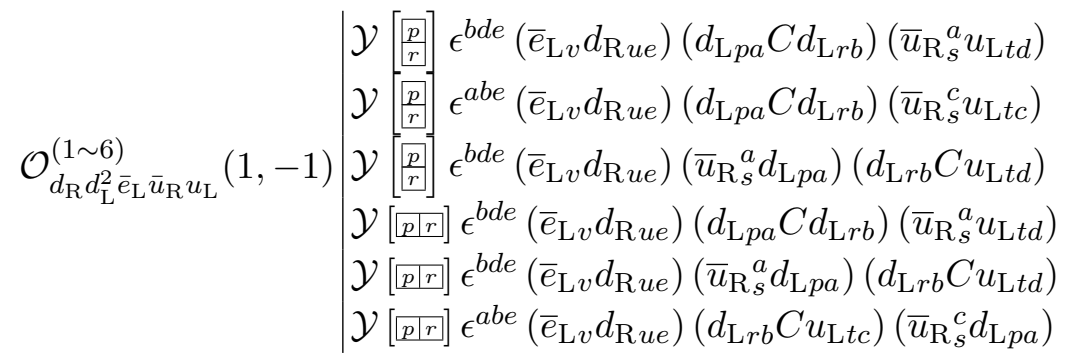




$$
\begin{aligned}
& \mathcal{Y}[\underline{u v v}] \epsilon^{a c e}\left(\bar{e}_{\mathrm{R} r} d_{\mathrm{L} p a}\right)\left(d_{\mathrm{R} u b} C d_{\mathrm{R} v e}\right)\left(\bar{u}_{\mathrm{R}}^{b} u_{\mathrm{L} t c}\right) \\
& \mathcal{Y}[\overline{u v v}] \epsilon^{a c e}\left(\bar{e}_{\mathrm{R} r} u_{\mathrm{L} t c}\right)\left(d_{\mathrm{R} u b} C d_{\mathrm{R} v e}\right)\left(\bar{u}_{\mathrm{R}}^{b} d_{\mathrm{L} p a}\right) \\
& \mathcal{O}_{d_{\mathrm{R}}^{2} d_{\mathrm{L}} \bar{e}_{\mathrm{R}} \bar{u}_{\mathrm{R}} u_{\mathrm{L}}}^{(1 \sim \overline{\mathrm{C}}}(1,-1) \mathcal{Y}\left[\begin{array}{l}
\underline{u} \\
\underline{v}
\end{array}\right] \epsilon^{c d e}\left(\bar{e}_{\mathrm{R} r} d_{\mathrm{L} p a}\right)\left(d_{\mathrm{R} u d} C d_{\mathrm{R} v e}\right)\left(\bar{u}_{\mathrm{R} s}{ }^{a} u_{\mathrm{L} t c}\right) \\
& \mathcal{Y}\left[\begin{array}{c}
a \\
\underline{v}
\end{array} \epsilon^{a c e}\left(\bar{e}_{\mathrm{R} r} d_{\mathrm{L} p a}\right)\left(d_{\mathrm{R} u b} C d_{\mathrm{R} v e}\right)\left(\bar{u}_{\mathrm{R}}^{b} u_{\mathrm{L} t c}\right)\right. \\
& \mathcal{Y} \underline{u}, \epsilon^{c d e}\left(\bar{e}_{\mathrm{R} r} u_{\mathrm{L} t c}\right)\left(d_{\mathrm{R} u d} C d_{\mathrm{R} v e}\right)\left(\bar{u}_{\mathrm{R} s}^{a} d_{\mathrm{L} p a}\right) \\
& \mathcal{Y}\left[\begin{array}{l}
u \\
v
\end{array}\right] \epsilon^{a c e}\left(\bar{e}_{\mathrm{R} r} u_{\mathrm{L} t c}\right)\left(d_{\mathrm{R} u b} C d_{\mathrm{R} v e}\right)\left(\bar{u}_{\mathrm{R} s}^{b} d_{\mathrm{L} p a}\right)
\end{aligned}
$$

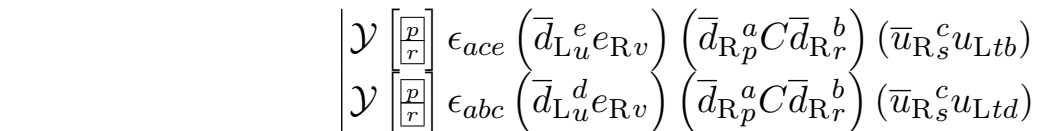

$$
\begin{aligned}
& \mathcal{O}_{\bar{d}_{\mathrm{R}}^{2} \bar{d}_{\mathrm{L}} e_{\mathrm{R}} \bar{u}_{\mathrm{R}} u_{\mathrm{L}}}^{(1 \sim 6)}(-1,1) \mathcal{Y}[\underline{\underline{p}}] \epsilon_{a c e}\left(\bar{d}_{\mathrm{R}}^{b} u_{\mathrm{L} t b}\right)\left(\bar{d}_{\mathrm{L} u}^{e} e_{\mathrm{R} v}\right)\left(\bar{d}_{\mathrm{R} p}^{a} C \bar{u}_{\mathrm{R}_{s}^{c}}^{c}\right) \\
& \mathcal{Y}[\overline{p r r}] \epsilon_{a c e}\left(\bar{d}_{\mathrm{L} u}^{e} e_{\mathrm{R} v}\right)\left(\bar{d}_{\mathrm{R} p}^{a} C \bar{d}_{\mathrm{R} r}^{b}\right)\left(\bar{u}_{\mathrm{R}}^{c} u_{\mathrm{L} t b}\right) \\
& \mathcal{Y}[\overline{p p r}] \epsilon_{\text {ace }}\left(\bar{d}_{\mathrm{R} r}^{b} u_{\mathrm{L} t b}\right)\left(\bar{d}_{\mathrm{L} u}^{e} e_{\mathrm{R} v}\right)\left(\bar{d}_{\mathrm{R} p}^{a} C \bar{u}_{\mathrm{R} s}^{c}\right) \\
& \mathcal{Y}[\overline{p p r}] \epsilon_{a b c}\left(\bar{d}_{\mathrm{L} u}^{d} e_{\mathrm{R} v}\right)\left(\bar{d}_{\mathrm{R} r}^{b} u_{\mathrm{L} t d}\right)\left(\bar{d}_{\mathrm{R} p}^{a} C \bar{u}_{\mathrm{R}}^{c}\right) \\
& \mathcal{Y}\left[\underline{u v v]} \epsilon_{a b d}\left(\bar{d}_{\mathrm{R}_{p}^{a}}^{a} e_{\mathrm{L} r}\right)\left(\bar{u}_{\mathrm{R} s}^{b} u_{\mathrm{L} t c}\right)\left(\bar{d}_{\mathrm{L} u}^{d} C \bar{d}_{\mathrm{L} v}^{c}\right)\right. \\
& \mathcal{Y}\left[\underline{u v v]} \epsilon_{a b d}\left(e_{\mathrm{L} r} C u_{\mathrm{L} t c}\right)\left(\bar{d}_{\mathrm{L} u}^{d} C \bar{d}_{\mathrm{L} v}^{c}\right)\left(\bar{d}_{\left.\mathrm{R}_{p}^{a} C \bar{u}_{\mathrm{R}}^{b}\right)}\right)\right. \\
& \left.\mathcal{O}_{\bar{d}_{\mathrm{R}} \bar{d}_{\mathrm{L}}^{2} e_{\mathrm{L}} \bar{u}_{\mathrm{R}} u_{\mathrm{L}}}^{(1 \sim 6)}(-1,1) \mathcal{Y}[\underline{u}] \underline{v}\right] \epsilon_{b d e}\left(\bar{d}_{\mathrm{R} p}^{a} e_{\mathrm{L} r}\right)\left(\bar{u}_{\mathrm{R} s}^{b} u_{\mathrm{L} t a}\right)\left(\bar{d}_{\mathrm{L} u}^{d} C \bar{d}_{\mathrm{L} v}^{e}\right) \\
& \mathcal{Y}\left[\begin{array}{ll}
u \\
v
\end{array} \epsilon_{a b d}\left(\bar{d}_{\mathrm{R} p}^{a} e_{\mathrm{L} r}\right)\left(\bar{u}_{\mathrm{R} s}^{b} u_{\mathrm{L} t c}\right)\left(\bar{d}_{\mathrm{L} u}^{d} C \bar{d}_{\mathrm{L} v}^{c}\right)\right. \\
& \mathcal{Y}\left[\begin{array}{ll}
\underline{v} \\
\underline{v}
\end{array} \epsilon_{b d e}\left(e_{\mathrm{L} r} C u_{\mathrm{L} t a}\right)\left(\bar{d}_{\mathrm{L} u}^{d} C \bar{d}_{\mathrm{L} v}^{e}\right)\left(\bar{d}_{\mathrm{R}_{p}^{a}} C \bar{u}_{\mathrm{R}}^{b}\right)\right.
\end{aligned}
$$

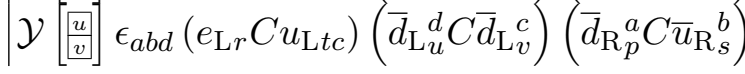

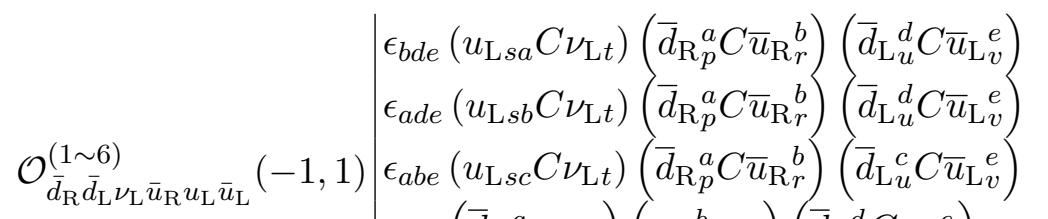

$$
\begin{aligned}
& \epsilon_{b d e}\left(\bar{d}_{\mathrm{R} p}^{a} u_{\mathrm{L} s a}\right)\left(\bar{u}_{\mathrm{R} r}^{b} \nu_{\mathrm{L} t}\right)\left(\bar{d}_{\mathrm{L} u}^{d} C \bar{u}_{\mathrm{L} v}^{e}\right) \\
& \epsilon_{\text {ade }}\left(\bar{u}_{\mathrm{R} r}^{b} \nu_{\mathrm{L} t}\right)\left(\bar{d}_{\mathrm{R} p}^{a} u_{\mathrm{L} s b}\right)\left(\bar{d}_{\mathrm{L} u}^{d} C \bar{u}_{\mathrm{L} v}^{e}\right) \\
& \epsilon_{a b e}\left(\bar{u}_{\mathrm{R}}^{b} \nu_{\mathrm{L} t}\right)\left(\bar{d}_{\mathrm{R} p}^{a} u_{\mathrm{L} s c}\right)\left(\bar{d}_{\mathrm{L} u}^{c} C \bar{u}_{\mathrm{L} v}^{e}\right)
\end{aligned}
$$

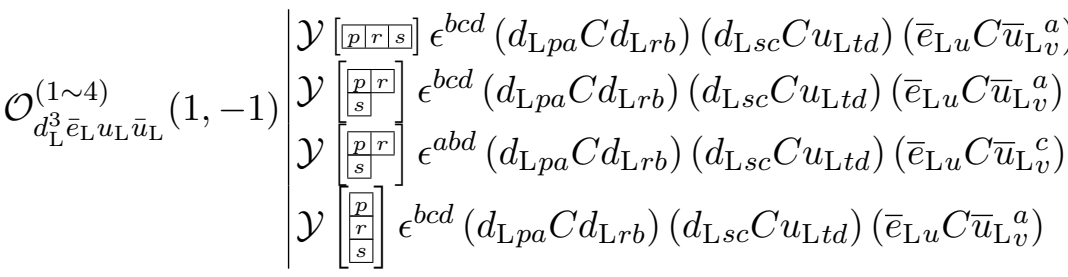

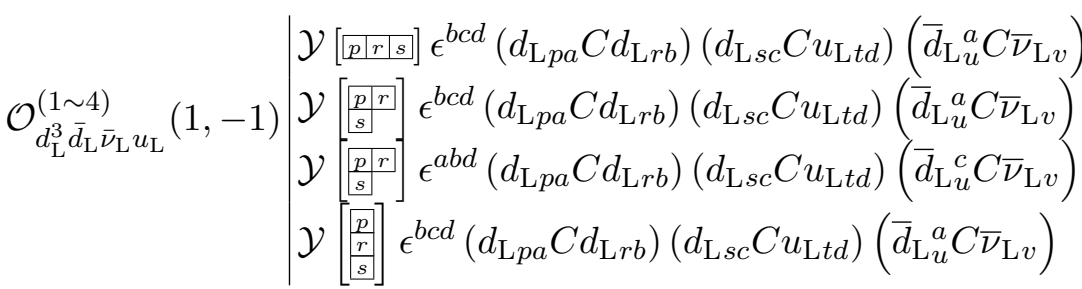




$$
\begin{aligned}
& \mathcal{Y}\left[\frac{r}{s}\right] \epsilon^{c d e}\left(\bar{\nu}_{\mathrm{L} v} d_{\mathrm{R} u e}\right)\left(\bar{d}_{\mathrm{R} p}^{a} d_{\mathrm{L} r a}\right)\left(d_{\mathrm{L} s c} C u_{\mathrm{L} t d}\right) \\
& \mathcal{Y}\left[\begin{array}{r}
r \\
s
\end{array} \epsilon^{b d e}\left(\bar{\nu}_{\mathrm{L} v} d_{\mathrm{R} u e}\right)\left(d_{\mathrm{L} s a} C u_{\mathrm{L} t d}\right)\left(\bar{d}_{\mathrm{R} p}^{a} d_{\mathrm{L} r b}\right)\right.
\end{aligned}
$$

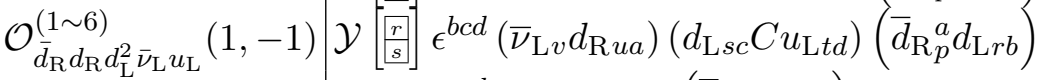

$$
\begin{aligned}
& \mathcal{Y}[[r] s] \epsilon^{c d e}\left(\bar{\nu}_{\mathrm{L} v} d_{\mathrm{R} u e}\right)\left(\bar{d}_{\mathrm{R} p}^{a} d_{\mathrm{L} r a}\right)\left(d_{\mathrm{L} s c} C u_{\mathrm{L} t d}\right) \\
& \mathcal{Y}[[r s]] \epsilon^{b d e}\left(\bar{\nu}_{\mathrm{L} v} d_{\mathrm{R} u e}\right)\left(d_{\mathrm{L} s a} C u_{\mathrm{L} t d}\right)\left(\bar{d}_{\mathrm{R}}^{a} d_{\mathrm{L} r b}\right) \\
& \mathcal{Y}[[r] s] \epsilon^{b c d}\left(\bar{\nu}_{\mathrm{L} v} d_{\mathrm{R} u a}\right)\left(d_{\mathrm{L} s c} C u_{\mathrm{L} t d}\right)\left(\bar{d}_{\mathrm{R} p}^{a} d_{\mathrm{L} r b}\right)
\end{aligned}
$$

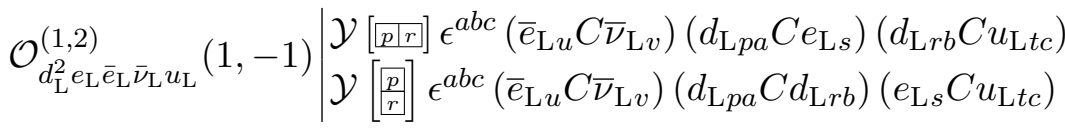

$$
\begin{aligned}
& \mathcal{Y}\left[\frac{p}{r}\right] \epsilon^{b c d}\left(\bar{e}_{\mathrm{R} s} u_{\mathrm{L} t c}\right)\left(d_{\mathrm{L} p a} C d_{\mathrm{L} r b}\right)\left(\bar{u}_{\mathrm{L} v}^{a} d_{\mathrm{R} u d}\right) \\
& \mathcal{Y} \frac{p}{r} \epsilon^{a b d}\left(\bar{e}_{\mathrm{R} s} u_{\mathrm{L} t c}\right)\left(d_{\mathrm{L} p a} C d_{\mathrm{L} r b}\right)\left(\bar{u}_{\mathrm{L} v}^{c} d_{\mathrm{R} u d}\right)
\end{aligned}
$$

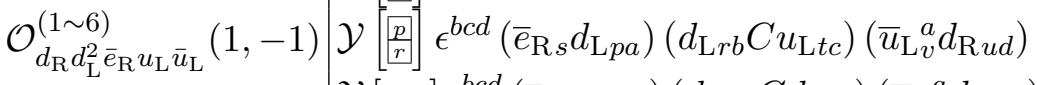

$$
\begin{aligned}
& \mathcal{Y}[\overline{p p r}] \epsilon^{b c d}\left(\bar{e}_{\mathrm{R} s} u_{\mathrm{L} t c}\right)\left(d_{\mathrm{L} p a} C d_{\mathrm{L} r b}\right)\left(\bar{u}_{\mathrm{L} v}^{a} d_{\mathrm{R} u d}\right) \\
& \mathcal{Y}[\overline{p p r}] \epsilon^{b c d}\left(\bar{e}_{\mathrm{R} s} d_{\mathrm{L} p a}\right)\left(d_{\mathrm{L} r b} C u_{\mathrm{L} t c}\right)\left(\bar{u}_{\mathrm{L} v}^{a} d_{\mathrm{R} u d}\right) \\
& \mathcal{Y}[\overline{p p r}] \epsilon^{a b d}\left(\bar{e}_{\mathrm{R} s} d_{\mathrm{L} p a}\right)\left(d_{\mathrm{L} r b} C u_{\mathrm{L} t c}\right)\left(\bar{u}_{\mathrm{L} v}^{c} d_{\mathrm{R} u d}\right) \\
& \mathcal{O}_{d_{\mathrm{L}}^{2} \bar{e}_{\mathrm{R}} e_{\mathrm{R}} \bar{\nu}_{\mathrm{L}} u_{\mathrm{L}}}^{(1,-1)} \mid \begin{array}{l}
\mathcal{Y}[[p \mid r]] \\
\mathcal{Y}\left[\frac{p}{\underline{r}}\right] \epsilon^{a b c}\left(\bar{\nu}_{\mathrm{L} v} e_{\mathrm{R} u}\right)\left(\bar{e}_{\mathrm{R} s} d_{\mathrm{L} p a}\right)\left(d_{\mathrm{L} r b} C u_{\mathrm{L} v c}\right) \\
\left.e_{\mathrm{R} u}\right)\left(\bar{e}_{\mathrm{R} s} u_{\mathrm{L} t c}\right)\left(d_{\mathrm{L} p a} C d_{\mathrm{L} r b}\right)
\end{array}
\end{aligned}
$$

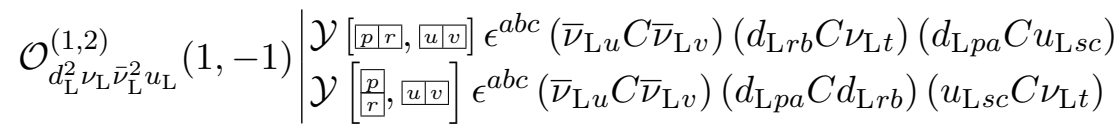

$$
\begin{aligned}
& \mathcal{O}_{d_{\mathrm{R}} d_{\mathrm{L}} \bar{e}_{\mathrm{R}} e_{\mathrm{L}} \bar{\nu}_{\mathrm{L}} u_{\mathrm{L}}}^{(1,-1)} \mid \begin{array}{l}
\epsilon^{a b c}\left(\bar{e}_{\mathrm{R} r} d_{\mathrm{L} p a}\right)\left(\bar{\nu}_{\mathrm{L} v} d_{\mathrm{R} u c}\right)\left(e_{\mathrm{L} s} C u_{\mathrm{L} t b}\right) \\
\epsilon^{a b c}\left(\bar{e}_{\mathrm{R} r} u_{\mathrm{L} t b}\right)\left(\bar{\nu}_{\mathrm{L} v} d_{\mathrm{R} u c}\right)\left(d_{\mathrm{L} p a} C e_{\mathrm{L} s}\right)
\end{array}
\end{aligned}
$$

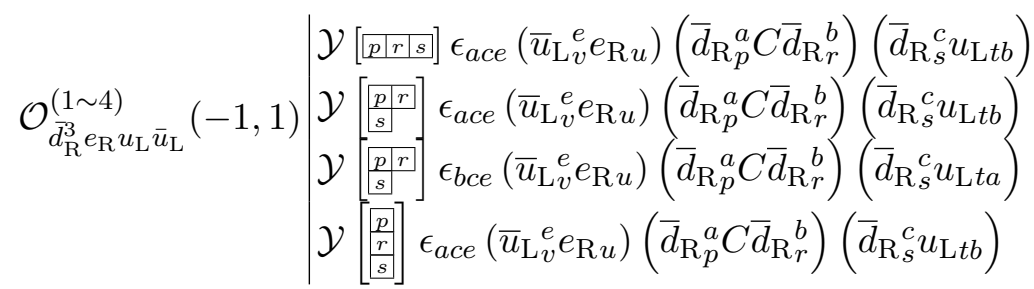

$$
\begin{aligned}
& \mathcal{Y}\left[\frac{p}{r}\right] \epsilon_{b d e}\left(e_{\mathrm{L} s} C u_{\mathrm{L} t a}\right)\left(\bar{d}_{\mathrm{R} p}^{a} C \bar{d}_{\mathrm{R} r}^{b}\right)\left(\bar{d}_{\mathrm{L} u}^{d} C \bar{u}_{\mathrm{L} v}^{e}\right) \\
& \mathcal{Y}\left[\begin{array}{ll}
p \\
r
\end{array} \epsilon_{a b e}\left(e_{\mathrm{L} s} C u_{\mathrm{L} t c}\right)\left(\bar{d}_{\mathrm{R} p}^{a} C \bar{d}_{\mathrm{R} r}^{b}\right)\left(\bar{d}_{\mathrm{L} u}^{c} C \bar{u}_{\mathrm{L} v}^{e}\right)\right.
\end{aligned}
$$

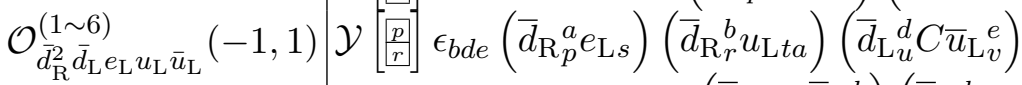

$$
\begin{aligned}
& \mathcal{Y}[\overline{p p r}] \epsilon_{b d e}\left(e_{\mathrm{L} s} C u_{\mathrm{L} t a}\right)\left(\bar{d}_{\mathrm{R} p}^{a} C \bar{d}_{\mathrm{R} r}^{b}\right)\left(\bar{d}_{\mathrm{L} u}^{d} C \bar{u}_{\mathrm{L} v}^{e}\right) \\
& \mathcal{Y}[\overline{p p r}] \epsilon_{b d e}\left(\bar{d}_{\mathrm{R} p}^{a} e_{\mathrm{L} s}\right)\left(\bar{d}_{\mathrm{R} r}^{b} u_{\mathrm{L} t a}\right)\left(\bar{d}_{\mathrm{L} u}^{d} C \bar{u}_{\mathrm{L} v}^{e}\right) \\
& \mathcal{Y}[\overline{p p r}] \epsilon_{a b e}\left(\bar{d}_{\mathrm{R} p}^{a} e_{\mathrm{L} s}\right)\left(\bar{d}_{\mathrm{R} r}^{b} u_{\mathrm{L} t c}\right)\left(\bar{d}_{\mathrm{L} u}^{c} C \bar{u}_{\mathrm{L} v}^{e}\right)
\end{aligned}
$$




$$
\begin{aligned}
& \mathcal{Y}\left[\frac{p}{r}, \frac{u}{v}\right] \epsilon_{b d e}\left(u_{\mathrm{L} s a} C \nu_{\mathrm{L} t}\right)\left(\bar{d}_{\mathrm{R} p}^{a} C \bar{d}_{\mathrm{R}_{r}^{b}}\right)\left(\bar{u}_{\mathrm{L}}^{d} C \bar{u}_{\mathrm{L} v}^{e}\right) \\
& \mathcal{Y}\left[\frac{p}{r}, \frac{u}{v}\right] \epsilon_{b d e}\left(\bar{d}_{\mathrm{R} p}^{a} u_{\mathrm{L} s a}\right)\left(\bar{d}_{\mathrm{R} r}^{b} \nu_{\mathrm{L} t}\right)\left(\bar{u}_{\mathrm{L} u}^{d} C \bar{u}_{\mathrm{L} v}^{e}\right) \\
& \mathcal{O}_{\bar{d}_{\mathrm{R}}^{2} \nu_{\mathrm{L}} u_{\mathrm{L}} \bar{u}_{\mathrm{L}}^{2}(-1,1) \mathcal{Y}[\overline{p p r}, \overline{u v}]}\left(\epsilon_{a b e}\left(\bar{d}_{\mathrm{R} r}^{b} \nu_{\mathrm{L} t}\right)\left(\bar{d}_{\mathrm{R}}^{a} u_{\mathrm{L} s c}\right)\left(\bar{u}_{\mathrm{L} u}^{c} C \bar{u}_{\mathrm{L} v}^{e}\right)\right. \\
& \mathcal{Y}[\overline{p p r}, \underline{u} v] \epsilon_{b d e}\left(u_{\mathrm{L} s a} C \nu_{\mathrm{L} t}\right)\left(\bar{d}_{\mathrm{R} p}^{a} C \bar{d}_{\mathrm{R}}^{b}\right)\left(\bar{u}_{\mathrm{L} u}^{d} C \bar{u}_{\mathrm{L} v}^{e}\right) \\
& \mathcal{Y}\left[\frac{p p r}{v}, \frac{u}{v}\right] \epsilon_{b d e}\left(\bar{d}_{\mathrm{R} p}^{a} u_{\mathrm{L} s a}\right)\left(\bar{d}_{\mathrm{R}}{ }_{r}^{b} \nu_{\mathrm{L} t}\right)\left(\bar{u}_{\mathrm{L} u}^{d} C \bar{u}_{\mathrm{L} v}^{e}\right) \\
& \mathcal{Y}\left[\frac{p}{r}, u u v\right] \epsilon_{a b e}\left(u_{\mathrm{L} s c} C \nu_{\mathrm{L} t}\right)\left(\bar{d}_{\mathrm{R} p}^{a} C \bar{d}_{\mathrm{R} r}^{b}\right)\left(\bar{u}_{\mathrm{L} u}^{c} C \bar{u}_{\mathrm{L} v}^{e}\right) \\
& \mathcal{Y}\left[\frac{r}{s}\right] \epsilon_{a c d}\left(\bar{u}_{\mathrm{R}}^{c} \nu_{\mathrm{L} t}\right)\left(\bar{d}_{\mathrm{L} u}^{d} u_{\mathrm{R} v b}\right)\left(\bar{d}_{\mathrm{R} p}^{a} C \bar{u}_{\mathrm{R} r}^{b}\right) \\
& \mathcal{Y}\left[\begin{array}{r}
r \\
s
\end{array} \epsilon_{a b c}\left(\bar{u}_{\mathrm{R}}^{c} \nu_{\mathrm{L} t}\right)\left(\bar{d}_{\mathrm{L} u}^{d} u_{\mathrm{R} v d}\right)\left(\bar{d}_{\mathrm{R} p}^{a} C \bar{u}_{\mathrm{R} r}^{b}\right)\right. \\
& \mathcal{O}_{\bar{d}_{\mathrm{R}} \bar{d}_{\mathrm{L}} \nu_{\mathrm{L}} \bar{u}_{\mathrm{R}}^{2} u_{\mathrm{R}}}^{(\sim 6)}(-1,1) \mathcal{Y}\left[\begin{array}{l}
\underline{r} \\
\underline{s}
\end{array}\right] \epsilon_{b c d}\left(\bar{u}_{\mathrm{R}}^{c} \nu_{\mathrm{L} t}\right)\left(\bar{d}_{\mathrm{L} u}^{d} u_{\mathrm{R} v a}\right)\left(\bar{d}_{\mathrm{R}}^{a} C \bar{u}_{\mathrm{R}}^{b}{ }_{r}^{b}\right) \\
& \mathcal{Y}\left[\overline{r r s]} \epsilon_{a c d}\left(\bar{u}_{\mathrm{R}}^{c} \nu_{\mathrm{L} t}\right)\left(\bar{d}_{\mathrm{L} u}^{d} u_{\mathrm{R} v b}\right)\left(\bar{d}_{\mathrm{R} p}^{a} C \bar{u}_{\mathrm{R} r}^{b}\right)\right. \\
& \mathcal{Y}\left[\underline{r r s]} \epsilon_{a b c}\left(\bar{u}_{\mathrm{R}}^{c}{ }_{s} \nu_{\mathrm{L} t}\right)\left(\bar{d}_{\mathrm{L} u}^{d} u_{\mathrm{R} v d}\right)\left(\bar{d}_{\mathrm{R} p}^{a} C \bar{u}_{\mathrm{R}}^{b}\right)\right. \\
& \mathcal{Y}\left[\overline{r r s]} \epsilon_{b c d}\left(\bar{u}_{\mathrm{R}}^{c} \nu_{\mathrm{L} t}\right)\left(\bar{d}_{\mathrm{L} u}^{d} u_{\mathrm{R} v a}\right)\left(\bar{d}_{\mathrm{R}_{p}^{a}}^{a} C \bar{u}_{\mathrm{R}}^{b}\right)\right.
\end{aligned}
$$

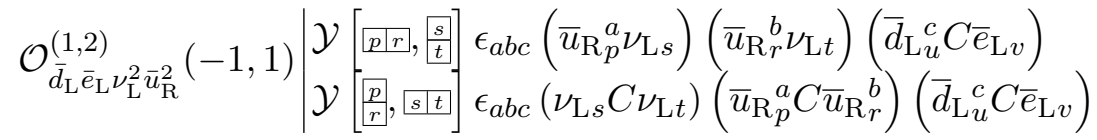

$$
\begin{aligned}
& \mathcal{Y}\left[\overline{p|r| s]} \epsilon^{b c e}\left(\bar{e}_{\mathrm{L} u} u_{\mathrm{R} v e}\right)\left(d_{\mathrm{L} p a} C d_{\mathrm{L} r b}\right)\left(\bar{u}_{\mathrm{R} t}^{a} d_{\mathrm{L} s c}\right)\right.
\end{aligned}
$$

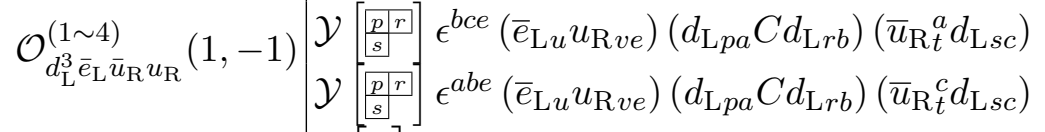

$$
\begin{aligned}
& \mathcal{Y}\left[\begin{array}{c}
\frac{p}{r} \\
s
\end{array}\right] \epsilon^{b c e}\left(\bar{e}_{\mathrm{L} u} u_{\mathrm{R} v e}\right)\left(d_{\mathrm{L} p a} C d_{\mathrm{L} r b}\right)\left(\bar{u}_{\mathrm{R} t}^{a} d_{\mathrm{L} s c}\right) \\
& \mathcal{Y}\left[\begin{array}{l}
\frac{p}{r} \\
r
\end{array}\right] \epsilon^{b d e}\left(d_{\mathrm{L} p a} C d_{\mathrm{L} r b}\right)\left(d_{\mathrm{R} u d} C u_{\mathrm{R} v e}\right)\left(\bar{e}_{\mathrm{R} s} C \bar{u}_{\mathrm{R}}{ }_{t}^{a}\right) \\
& \mathcal{Y} \mid \frac{p}{r} \epsilon^{a b e}\left(d_{\mathrm{L} p a} C d_{\mathrm{L} r b}\right)\left(d_{\mathrm{R} u c} C u_{\mathrm{R} v e}\right)\left(\bar{e}_{\mathrm{R} s} C \bar{u}_{\mathrm{R} t}^{c}\right) \\
& \mathcal{O}_{d_{\mathrm{R}} d_{\mathrm{L}}^{2} \bar{e}_{\mathrm{R}} \bar{u}_{\mathrm{R}} u_{\mathrm{R}}}^{(1 \sim 6)}\left(1, \mathcal{Y}\left[\begin{array}{l}
\underline{p} \\
\vec{r}
\end{array}\right] \epsilon^{b d e}\left(\bar{e}_{\mathrm{R} s} d_{\mathrm{L} p a}\right)\left(d_{\mathrm{R} u d} C u_{\mathrm{R} v e}\right)\left(\bar{u}_{\mathrm{R} t}^{a} d_{\mathrm{L} r b}\right)\right. \\
& \mathcal{Y}[\overline{p \mid r}] \epsilon^{b d e}\left(d_{\mathrm{L} p a} C d_{\mathrm{L} r b}\right)\left(d_{\mathrm{R} u d} C u_{\mathrm{R} v e}\right)\left(\bar{e}_{\mathrm{R} s} C \bar{u}_{\mathrm{R} t}{ }^{a}\right) \\
& \mathcal{Y}[\overline{p r}]] \epsilon^{b d e}\left(\bar{e}_{\mathrm{R} s} d_{\mathrm{L} p a}\right)\left(d_{\mathrm{R} u d} C u_{\mathrm{R} v e}\right)\left(\bar{u}_{\mathrm{R} t}^{a} d_{\mathrm{L} r b}\right) \\
& \mathcal{Y}[\overline{p r r}] \epsilon^{a b e}\left(\bar{e}_{\mathrm{R} s} d_{\mathrm{L} p a}\right)\left(d_{\mathrm{R} u c} C u_{\mathrm{R} v e}\right)\left(\bar{u}_{\mathrm{R} t}^{c} d_{\mathrm{L} r b}\right) \\
& \mathcal{Y}\left[\underline{u v v]} \epsilon_{a c e}\left(\bar{u}_{\mathrm{R} s}^{c} \nu_{\mathrm{L} t}\right)\left(\bar{d}_{\mathrm{R} p}^{a} d_{\mathrm{L} r b}\right)\left(\bar{d}_{\mathrm{L} u}^{b} C \bar{d}_{\mathrm{L} v}^{e}\right)\right. \\
& \mathcal{Y}\left[\underline{u v v]} \epsilon_{a c e}\left(d_{\mathrm{L} r b} C \nu_{\mathrm{L} t}\right)\left(\bar{d}_{\mathrm{L} u}^{b} C \bar{d}_{\mathrm{L} v}^{e}\right)\left(\bar{d}_{\mathrm{R} p}^{a} C \bar{u}_{\mathrm{R} s}^{c}\right)\right.
\end{aligned}
$$

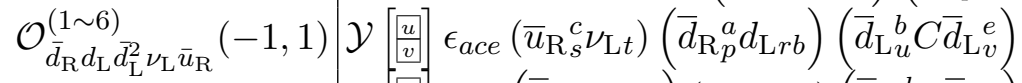

$$
\begin{aligned}
& \mathcal{Y}\left[\begin{array}{ll}
u \\
v
\end{array} \epsilon_{c d e}\left(\bar{d}_{\mathrm{R} p}^{a} d_{\mathrm{L} r a}\right)\left(\bar{u}_{\mathrm{R}}^{c} \nu_{\mathrm{L} t}\right)\left(\bar{d}_{\mathrm{L} u}^{d} C \bar{d}_{\mathrm{L} v}^{e}\right)\right. \\
& \mathcal{Y}\left[\begin{array}{ll}
u \\
\underline{v}
\end{array}\right] \epsilon_{a c e}\left(d_{\mathrm{L} r b} C \nu_{\mathrm{L} t}\right)\left(\bar{d}_{\mathrm{L} u}^{b} C \bar{d}_{\mathrm{L}}{ }^{e}\right)\left(\bar{d}_{\mathrm{R}_{p}^{a}} C \bar{u}_{\mathrm{R}}^{c}\right) \\
& \mathcal{Y}\left[\begin{array}{l}
\underline{u} \\
v
\end{array}\right] \epsilon_{c d e}\left(d_{\mathrm{L} r a} C \nu_{\mathrm{L} t}\right)\left(\bar{d}_{\mathrm{L} u}^{d} C \bar{d}_{\mathrm{L} v}^{e}\right)\left(\bar{d}_{\mathrm{R} p}^{a} C \bar{u}_{\mathrm{R} s}^{c}\right)
\end{aligned}
$$




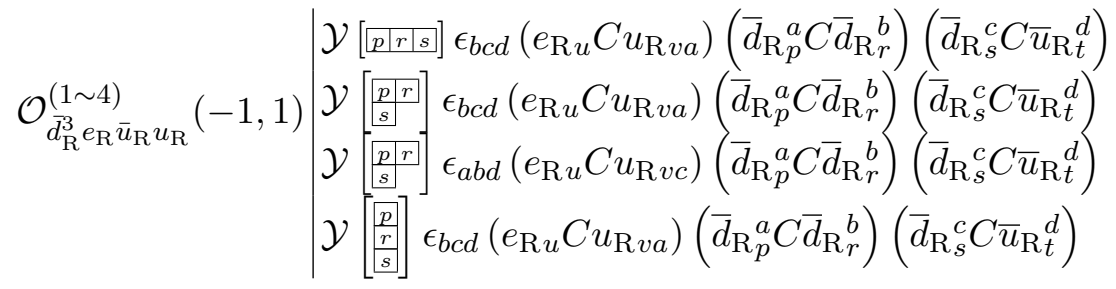

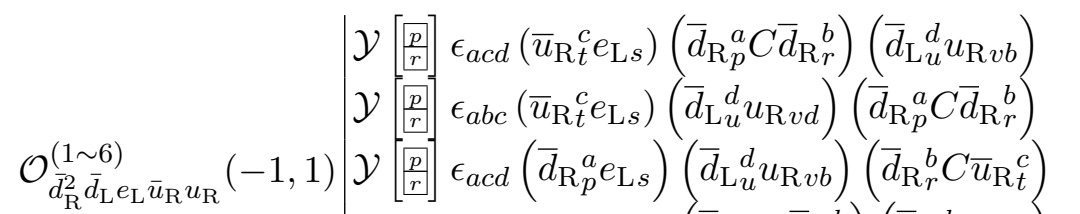

$$
\begin{aligned}
& \mathcal{Y}[\overline{p p r}] \epsilon_{a c d}\left(\bar{u}_{\mathrm{R} t}^{c} e_{\mathrm{L} s}\right)\left(\bar{d}_{\mathrm{R} p}^{a} C \bar{d}_{\mathrm{R} r}^{b}\right)\left(\bar{d}_{\mathrm{L} u}^{d} u_{\mathrm{R} v b}\right) \\
& \mathcal{Y}[\overline{p r r}] \epsilon_{a c d}\left(\bar{d}_{\mathrm{R}}^{a} e_{\mathrm{L} s}\right)\left(\bar{d}_{\mathrm{L} u}^{d} u_{\mathrm{R} v b}\right)\left(\bar{d}_{\mathrm{R}}^{b} C \bar{u}_{\mathrm{R} t}^{c}\right) \\
& \mathcal{Y}\left[\overline{p \mid r]} \epsilon_{a b c}\left(\bar{d}_{\mathrm{R} p}^{a} e_{\mathrm{L} s}\right)\left(\bar{d}_{\mathrm{L} u}^{d} u_{\mathrm{R} v d}\right)\left(\bar{d}_{\mathrm{R} r}^{b} C \bar{u}_{\mathrm{R} t}^{c}\right)\right. \\
& \mathcal{Y}\left[\begin{array}{l}
\frac{p}{r} \\
{[}
\end{array} \epsilon_{\text {ace }}\left(\bar{u}_{\mathrm{R}}^{c}{ }_{s} \nu_{\mathrm{L} t}\right)\left(\bar{d}_{\mathrm{R}_{p}^{a}}^{a} C \bar{d}_{\mathrm{R}}^{b}\right)\left(\bar{u}_{\mathrm{L}}^{e} u_{\mathrm{R} u b}\right)\right. \\
& \mathcal{Y}\left[\frac{p}{r}\right] \epsilon_{a b e}\left(\bar{u}_{\mathrm{R}}^{c} \nu_{\mathrm{L} t}\right)\left(\bar{d}_{\mathrm{R} p}^{a} C \bar{d}_{\mathrm{R}}^{b}\right)\left(\bar{u}_{\mathrm{L} v}^{e} u_{\mathrm{R} u c}\right)
\end{aligned}
$$

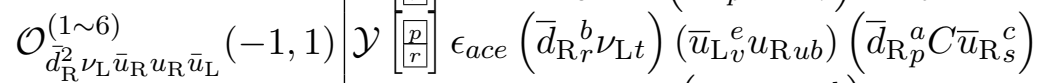

$$
\begin{aligned}
& \mathcal{Y}[\overline{p p r}] \epsilon_{\text {ace }}\left(\bar{u}_{\mathrm{R}}^{c} \nu_{\mathrm{L} t}\right)\left(\bar{d}_{\mathrm{R} p}^{a} C \bar{d}_{\mathrm{R}}^{b}\right)\left(\bar{u}_{\mathrm{L} v}^{e} u_{\mathrm{R} u b}\right) \\
& \mathcal{Y}\left[[\overline{p r r}] \epsilon_{a c e}\left(\bar{d}_{\mathrm{R} r}^{b} \nu_{\mathrm{L} t}\right)\left(\bar{u}_{\mathrm{L}}^{e} u_{\mathrm{R} u b}\right)\left(\bar{d}_{\mathrm{R}_{p}^{a} C \bar{u}_{\mathrm{R}}^{c}}^{c}\right)\right. \\
& \mathcal{Y}\left[\overline{p r r]} \epsilon_{a b e}\left(\bar{d}_{\mathrm{R} r}^{b} \nu_{\mathrm{L} t}\right)\left(\bar{u}_{\mathrm{L} v}^{e} u_{\mathrm{R} u c}\right)\left(\bar{d}_{\mathrm{R} p}^{a} C \bar{u}_{\mathrm{R} s}^{c}\right)\right. \\
& \mathcal{Y}\left[\begin{array}{l}
p \\
r
\end{array}\right] \epsilon_{\text {ace }}\left(\bar{u}_{\mathrm{R}}{ }_{s}^{c} \nu_{\mathrm{L} t}\right)\left(\bar{d}_{\mathrm{R} p}^{a} C \bar{d}_{\mathrm{R} r}^{b}\right)\left(\bar{d}_{\mathrm{L} v}^{e} d_{\mathrm{R} u b}\right) \\
& \mathcal{Y}\left[\begin{array}{ll}
r \\
r
\end{array} \epsilon_{a b c}\left(\bar{u}_{\mathrm{R}}{ }_{s}^{c} \nu_{\mathrm{L} t}\right)\left(\bar{d}_{\mathrm{L} v}^{d} d_{\mathrm{R} u d}\right)\left(\bar{d}_{\mathrm{R} p}^{a} C \bar{d}_{\mathrm{R}}^{b}\right)\right. \\
& \mathcal{O}_{\bar{d}_{\mathrm{R}}^{2} d_{\mathrm{R}} \bar{d}_{\mathrm{L}} \nu_{\mathrm{L}} \bar{u}_{\mathrm{R}}}^{(1 \sim 6)}(-1,1) \mathcal{Y}\left[\begin{array}{l}
\underline{p} \\
r
\end{array}\right] \epsilon_{a c e}\left(\bar{d}_{\mathrm{R}}^{b} \nu_{\mathrm{L} t}\right)\left(\bar{d}_{\mathrm{L} v}^{e} d_{\mathrm{R} u b}\right)\left(\bar{d}_{\mathrm{R}_{p}^{a}} C \bar{u}_{\mathrm{R}}^{c}\right) \\
& \mathcal{Y}\left[\overline{p \mid r]} \epsilon_{a c e}\left(\bar{u}_{\mathrm{R} s}^{c} \nu_{\mathrm{L} t}\right)\left(\bar{d}_{\mathrm{R} p}^{a} C \bar{d}_{\mathrm{R} r}^{b}\right)\left(\bar{d}_{\mathrm{L} v}^{e} d_{\mathrm{R} u b}\right)\right. \\
& \mathcal{Y}[\overline{p p r}] \epsilon_{\text {ace }}\left(\bar{d}_{\mathrm{R} r}^{b} \nu_{\mathrm{L} t}\right)\left(\bar{d}_{\mathrm{L} v}^{e} d_{\mathrm{R} u b}\right)\left(\bar{d}_{\mathrm{R} p}^{a} C \bar{u}_{\mathrm{R} s}^{c}\right) \\
& \mathcal{Y}[\overline{p r r}] \epsilon_{a b c}\left(\bar{d}_{\mathrm{R} r}^{b} \nu_{\mathrm{L} t}\right)\left(\bar{d}_{\mathrm{L} v}^{d} d_{\mathrm{R} u d}\right)\left(\bar{d}_{\mathrm{R} p}^{a} C \bar{u}_{\mathrm{R} s}^{c}\right) \\
& \mathcal{O}_{\bar{d}_{\mathrm{R}}^{2} e_{\mathrm{R}} \bar{e}_{\mathrm{L}} \nu_{\mathrm{L}} \bar{u}_{\mathrm{R}}}^{(-1,1)} \mid \begin{array}{l}
\mathcal{Y}\left[\frac{p p r}{[p]} \epsilon_{a b c}\left(\bar{e}_{\mathrm{L} v} e_{\mathrm{R} u}\right)\left(\bar{d}_{\mathrm{R}}^{b} \nu_{\mathrm{L} t}\right)\left(\bar{d}_{\mathrm{R}}^{a} C \bar{u}_{\mathrm{R}}^{c}{ }_{s}^{c}\right)\right. \\
\mathcal{Y}\left[\frac{p}{r}\right] \epsilon_{a b c}\left(\bar{e}_{\mathrm{L} v} e_{\mathrm{R} u}\right)\left(\bar{u}_{\mathrm{R}}^{c}{ }_{s}^{c} \nu_{\mathrm{L} t}\right)\left(\bar{d}_{\mathrm{R}_{p}^{a}}^{a} C \bar{d}_{\mathrm{R}}^{b}{ }_{r}^{b}\right.
\end{array}
\end{aligned}
$$

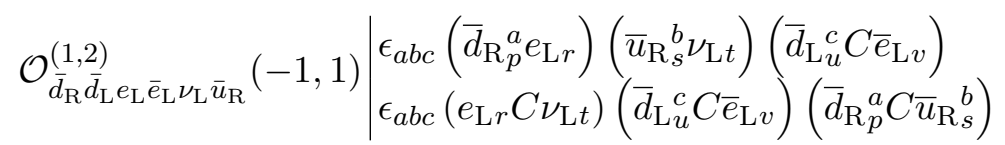

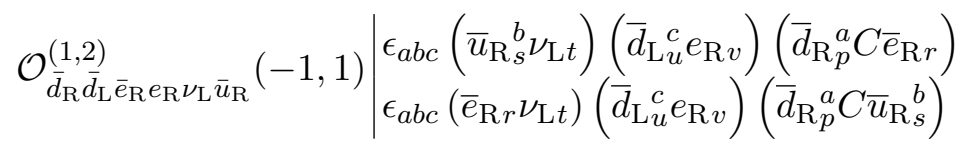

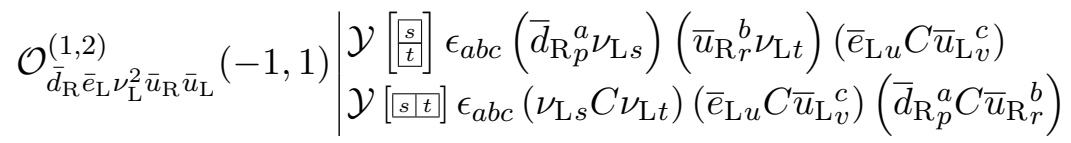

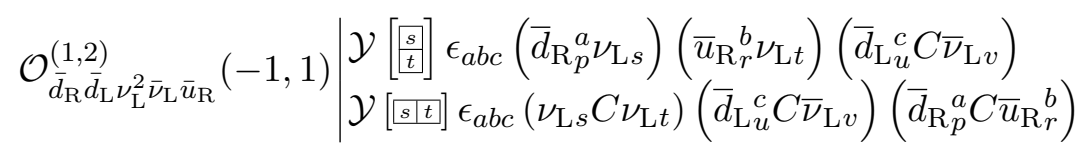




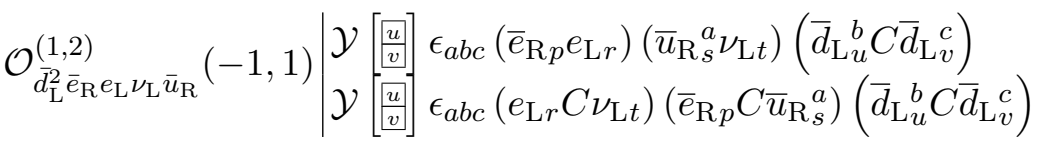

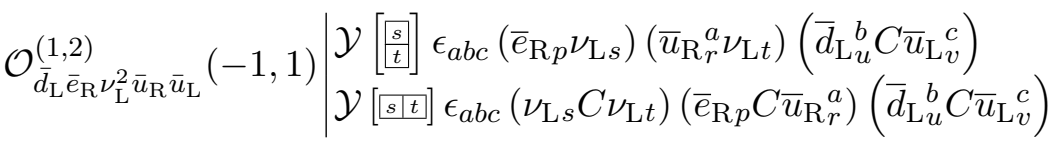

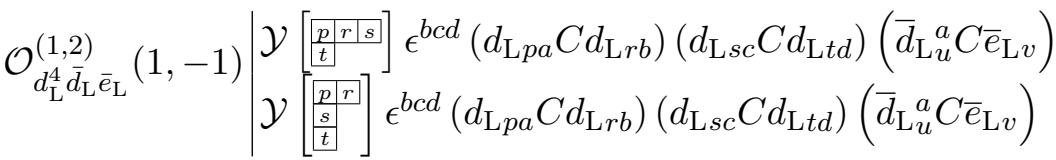

$$
\begin{aligned}
& \mathcal{Y}[[r|s| t]] \epsilon^{b d e}\left(\bar{\nu}_{\mathrm{L} v} u_{\mathrm{R} u e}\right)\left(d_{\mathrm{L} s a} C d_{\mathrm{L} t d}\right)\left(\bar{d}_{\mathrm{R} p}^{a} d_{\mathrm{L} r b}\right)
\end{aligned}
$$

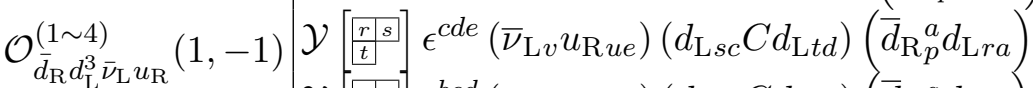

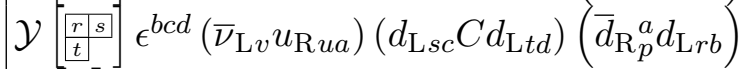

$$
\begin{aligned}
& \mathcal{Y}\left[\begin{array}{c}
\frac{r}{s} \\
t
\end{array}\right] \epsilon^{c d e}\left(\bar{\nu}_{\mathrm{L} v} u_{\mathrm{R} u e}\right)\left(d_{\mathrm{L} s c} C d_{\mathrm{L} t d}\right)\left(\bar{d}_{\mathrm{R}}^{a} d_{\mathrm{L} r a}\right)
\end{aligned}
$$

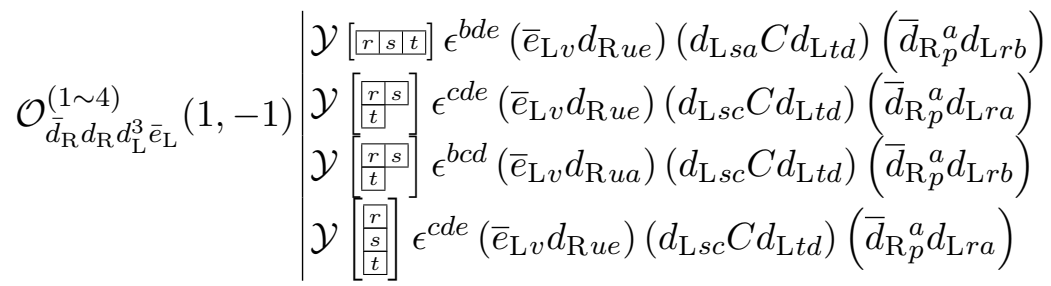

$$
\begin{aligned}
& \left.\mathcal{O}_{d_{\mathrm{L}}^{3} e_{\mathrm{L}} \bar{e}_{\mathrm{L}}^{2}}(1,-1) \mid \mathcal{Y}\left[\frac{p p r}{s s}, u v\right]\right] \epsilon^{a b c}\left(\bar{e}_{\mathrm{L} u} C \bar{e}_{\mathrm{L} v}\right)\left(d_{\mathrm{L} p a} C d_{\mathrm{L} r b}\right)\left(d_{\mathrm{L} s c} C e_{\mathrm{L} t}\right) \\
& \mathcal{Y}\left[\left[_{p|r| s}\right] \epsilon^{b c d}\left(\bar{e}_{\mathrm{R} t} d_{\mathrm{L} s c}\right)\left(d_{\mathrm{L} p a} C d_{\mathrm{L} r b}\right)\left(\bar{u}_{\mathrm{L} v}^{a} u_{\mathrm{R} u d}\right)\right. \\
& \mathcal{O}_{d_{\mathrm{L}}^{3} \bar{e}_{\mathrm{R}} u_{\mathrm{R}} \bar{u}_{\mathrm{L}}}^{(1,-1)}\left[\begin{array}{l}
\mathcal{Y}\left[\begin{array}{l}
{\left[\begin{array}{l}
p r \\
\frac{s}{s} \\
\mathcal{Y}
\end{array}\right] \epsilon^{b c d}\left(\bar{e}_{\mathrm{R} t} d_{\mathrm{L} s c}\right)\left(d_{\mathrm{L} p a} C d_{\mathrm{L} r b}\right)\left(\bar{u}_{\mathrm{L} v}^{a} u_{\mathrm{R} u d}\right)} \\
\left.\frac{p r}{s}\right]
\end{array} \epsilon^{a b d}\left(\bar{e}_{\mathrm{R} t} d_{\mathrm{L} s c}\right)\left(d_{\mathrm{L} p a} C d_{\mathrm{L} r b}\right)\left(\bar{u}_{\mathrm{L} v}{ }^{c} u_{\mathrm{R} u d}\right)\right.
\end{array}\right. \\
& \mathcal{Y}\left[\begin{array}{c}
\frac{p}{r} \\
s
\end{array}\right] \epsilon^{b c d}\left(\bar{e}_{\mathrm{R} t} d_{\mathrm{L} s c}\right)\left(d_{\mathrm{L} p a} C d_{\mathrm{L} r b}\right)\left(\bar{u}_{\mathrm{L} v}^{a} u_{\mathrm{R} u d}\right)
\end{aligned}
$$

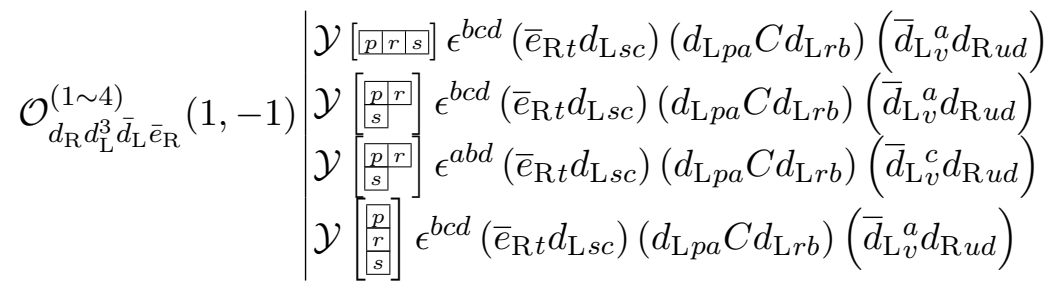

$$
\begin{aligned}
& \mathcal{O}_{d_{\mathrm{L}}^{3} \bar{e}_{\mathrm{R}} e_{\mathrm{R}} \bar{e}_{\mathrm{L}}}(1,-1) \mid \mathcal{Y}\left[\frac{p r r}{s}\right] \epsilon^{a b c}\left(\bar{e}_{\mathrm{L} v} e_{\mathrm{R} u}\right)\left(\bar{e}_{\mathrm{R} t} d_{\mathrm{L} s c}\right)\left(d_{\mathrm{L} p a} C d_{\mathrm{L} r b}\right) \\
& \mathcal{O}_{d_{\mathrm{L}}^{3} \bar{e}_{\mathrm{L}} \nu_{\mathrm{L}} \bar{\nu}_{\mathrm{L}}}(1,-1) \mid \mathcal{Y}\left[\frac{p r r}{s}\right] \epsilon^{a b c}\left(\bar{e}_{\mathrm{L} u} C \bar{\nu}_{\mathrm{L} v}\right)\left(d_{\mathrm{L} p a} C d_{\mathrm{L} r b}\right)\left(d_{\mathrm{L} s c} C \nu_{\mathrm{L} t}\right)
\end{aligned}
$$




$$
\begin{aligned}
& \mathcal{Y}\left[\frac{r}{s}, \frac{u}{v}\right] \epsilon^{c d e}\left(\bar{e}_{\mathrm{R} t} d_{\mathrm{L} s c}\right)\left(d_{\mathrm{R} u d} C d_{\mathrm{R} v e}\right)\left(\bar{d}_{\mathrm{R} p}^{a} d_{\mathrm{L} r a}\right) \\
& \mathcal{Y}\left[\frac{r}{s}, \frac{u}{v}\right] \epsilon^{b d e}\left(\bar{e}_{\mathrm{R} t} d_{\mathrm{L} s a}\right)\left(d_{\mathrm{R} u d} C d_{\mathrm{R} v e}\right)\left(\bar{d}_{\mathrm{R} p}^{a} d_{\mathrm{L} r b}\right) \\
& \mathcal{O}_{\bar{d}_{\mathrm{R}} d_{\mathrm{R}}^{2} d_{\mathrm{L}}^{2} \bar{e}_{\mathrm{R}}}^{(1 \sim 6)}(1,-1) \mathcal{Y}[[r \mid s, u v]] \epsilon^{b c d}\left(\bar{e}_{\mathrm{R} t} d_{\mathrm{L} s c}\right)\left(d_{\mathrm{R} u d} C d_{\mathrm{R} v a}\right)\left(\bar{d}_{\mathrm{R} p}^{a} d_{\mathrm{L} r b}\right) \\
& \mathcal{Y}\left[r r s, \frac{u}{v}\right] \epsilon^{c d e}\left(\bar{e}_{\mathrm{R} t} d_{\mathrm{L} s c}\right)\left(d_{\mathrm{R} u d} C d_{\mathrm{R} v e}\right)\left(\bar{d}_{\mathrm{R} p}^{a} d_{\mathrm{L} r a}\right) \\
& \mathcal{Y}\left[\overline{r r s}, \frac{u}{v}\right] \epsilon^{b d e}\left(\bar{e}_{\mathrm{R} t} d_{\mathrm{L} s a}\right)\left(d_{\mathrm{R} u d} C d_{\mathrm{R} v e}\right)\left(\bar{d}_{\mathrm{R} p}^{a} d_{\mathrm{L} r b}\right) \\
& \mathcal{Y}\left[\frac{r}{s}, u v v\right] \epsilon^{b c d}\left(\bar{e}_{\mathrm{R} t} d_{\mathrm{L} s c}\right)\left(d_{\mathrm{R} u d} C d_{\mathrm{R} v a}\right)\left(\bar{d}_{\mathrm{R} p}^{a} d_{\mathrm{L} r b}\right) \\
& \mathcal{O}_{d_{\mathrm{L}}^{2} \bar{e}_{\mathrm{R}} e_{\mathrm{L}} \bar{\nu}_{\mathrm{L}} u_{\mathrm{R}}}^{(1,-1)} \mid \begin{array}{l}
\mathcal{Y}\left[\frac{p p r]}{[p} \epsilon^{a b c}\left(\bar{e}_{\mathrm{R} s} d_{\mathrm{L} p a}\right)\left(\bar{\nu}_{\mathrm{L} v} u_{\mathrm{R} u c}\right)\left(d_{\mathrm{L} r b} C e_{\mathrm{L} t}\right)\right. \\
\mathcal{Y}\left[\frac{p}{[r]}\right] \epsilon^{a b c}\left(\bar{e}_{\mathrm{R} s} e_{\mathrm{L} t}\right)\left(\bar{\nu}_{\mathrm{L} v} u_{\mathrm{R} u c}\right)\left(d_{\mathrm{L} p a} C d_{\mathrm{L} r b}\right)
\end{array}
\end{aligned}
$$

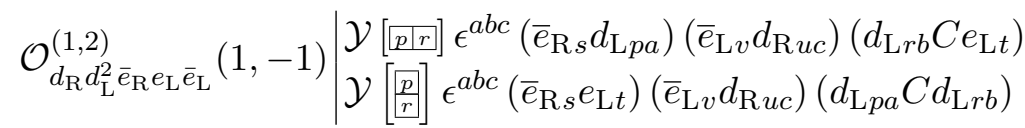

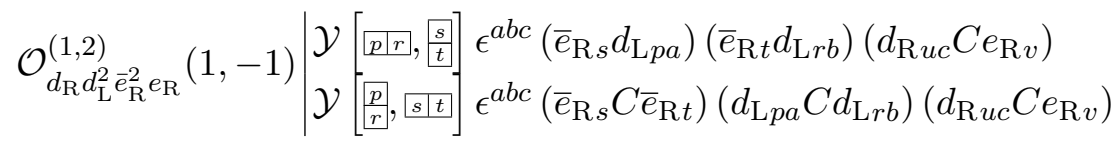

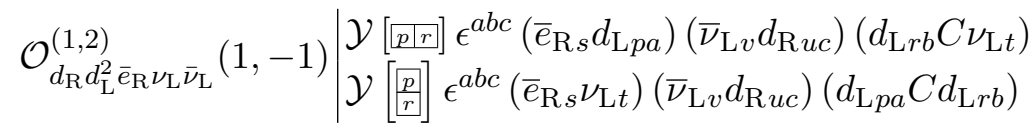

$$
\begin{aligned}
& \mid \mathcal{Y}\left[\overline { p p r | s ] } \epsilon _ { a c e } ( \overline { d } _ { \mathrm { L } u } ^ { e } e _ { \mathrm { R } v } ) ( \overline { d } _ { \mathrm { R } _ { p } ^ { a } } C \overline { d } _ { \mathrm { R } _ { r } ^ { b } } ^ { b } ) \left(\bar{d}_{\left.\mathrm{R}_{s}^{c} d_{\mathrm{L} t b}\right)}\right.\right.
\end{aligned}
$$

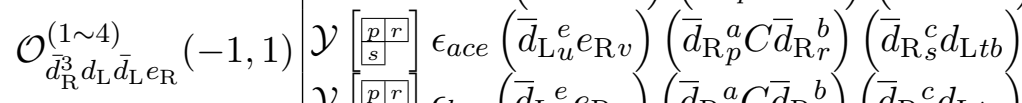

$$
\begin{aligned}
& \mathcal{Y}\left[\frac{p r r}{s-}\right] \epsilon_{b c e}\left(\bar{d}_{\mathrm{L} u}^{e} e_{\mathrm{R} v}\right)\left(\bar{d}_{\mathrm{R} p}^{a} C \bar{d}_{\mathrm{R}}^{b}\right)\left(\bar{d}_{\mathrm{R}_{s}^{c}} d_{\mathrm{L} t a}\right) \\
& \mathcal{Y}\left[\begin{array}{l}
\frac{p}{r} \\
\frac{r}{s}
\end{array}\right] \epsilon_{\text {ace }}\left(\bar{d}_{\mathrm{L} u}^{e} e_{\mathrm{R} v}\right)\left(\bar{d}_{\mathrm{R}_{p}^{a}} C \bar{d}_{\mathrm{R}_{r}^{b}}\right)\left(\bar{d}_{\mathrm{R}_{s}^{c}} d_{\mathrm{L} t b}\right) \\
& \mathcal{Y}\left[\frac{p}{r}, \frac{u}{v}\right] \epsilon_{b d e}\left(d_{\mathrm{L} s a} C e_{\mathrm{L} t}\right)\left(\bar{d}_{\mathrm{R}_{p}^{a}} C \bar{d}_{\mathrm{R} r}^{b}\right)\left(\bar{d}_{\mathrm{L} u}^{d} C \bar{d}_{\mathrm{L}}{ }_{v}^{e}\right)
\end{aligned}
$$

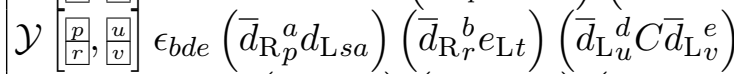

$$
\begin{aligned}
& \mathcal{O}_{\bar{d}_{\mathrm{R}}^{2} d_{\mathrm{L}} \bar{d}_{\mathrm{L}}^{2} e_{\mathrm{L}}}^{(1 \sim)^{2}}(-1,1) \mathcal{Y}[\overline{p r r}, u \mid v] \epsilon_{a b e}\left(\bar{d}_{\mathrm{R}}^{b} e_{\mathrm{L} t}\right)\left(\bar{d}_{\mathrm{R} p}^{a} d_{\mathrm{L} s c}\right)\left(\bar{d}_{\mathrm{L} u}^{c} C \bar{d}_{\mathrm{L} v}^{e}\right) \\
& \mathcal{Y}[p \mid r,, \underline{u} v] \epsilon_{b d e}\left(d_{\mathrm{L} s a} C e_{\mathrm{L} t}\right)\left(\bar{d}_{\mathrm{R} p}^{a} C \bar{d}_{\mathrm{R} r}^{b}\right)\left(\bar{d}_{\mathrm{L} u}^{d} C \bar{d}_{\mathrm{L} v}^{e}\right) \\
& \mathcal{Y}\left[\frac{p \mid r}{[-1}, \underline{v}\right] \epsilon_{b d e}\left(\bar{d}_{\mathrm{R} p}^{a} d_{\mathrm{L} s a}\right)\left(\bar{d}_{\mathrm{R} r}^{b} e_{\mathrm{L} t}\right)\left(\bar{d}_{\mathrm{L} u}^{d} C \bar{d}_{\mathrm{L} v}^{e}\right) \\
& \mathcal{Y}\left[\frac{p}{r}, u v v\right] \epsilon_{a b e}\left(d_{\mathrm{L} s c} C e_{\mathrm{L} t}\right)\left(\bar{d}_{\mathrm{R} p}^{a} C \bar{d}_{\mathrm{R}}{ }_{r}^{b}\right)\left(\bar{d}_{\mathrm{L}_{u}^{c}} C \bar{d}_{\mathrm{L} v}^{e}\right) \\
& \mathcal{Y}\left[\begin{array}{l}
\frac{p}{r} \\
r
\end{array} \epsilon_{b d e}\left(d_{\mathrm{L} s a} C \nu_{\mathrm{L} t}\right)\left(\bar{d}_{\mathrm{R}_{p}^{a}} C \bar{d}_{\mathrm{R} r}^{b}\right)\left(\bar{d}_{\mathrm{L} u}^{d} C \bar{u}_{\mathrm{L} v}^{e}\right)\right. \\
& \mathcal{Y}\left[\frac{p}{r}\right] \epsilon_{a b e}\left(d_{\mathrm{L} s c} C \nu_{\mathrm{L} t}\right)\left(\bar{d}_{\mathrm{R} p}^{a} C \bar{d}_{\mathrm{R}}^{b}\right)\left(\bar{d}_{\mathrm{L} u}^{c} C \bar{u}_{\mathrm{L} v}^{e}\right)
\end{aligned}
$$

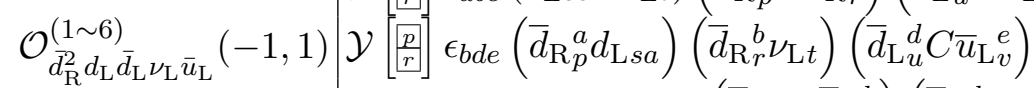

$$
\begin{aligned}
& \mathcal{Y}[\underline{p r r}] \epsilon_{b d e}\left(d_{\mathrm{L} s a} C \nu_{\mathrm{L} t}\right)\left(\bar{d}_{\mathrm{R}_{p}^{a}} C \bar{d}_{\mathrm{R}_{r}^{b}}^{b}\right)\left(\bar{d}_{\mathrm{L} u}^{d} C \bar{u}_{\mathrm{L} v}^{e}\right) \\
& \mathcal{Y}[\overline{p p r}] \epsilon_{b d e}\left(\bar{d}_{\mathrm{R} p}^{a} d_{\mathrm{L} s a}\right)\left(\bar{d}_{\mathrm{R} r}^{b} \nu_{\mathrm{L} t}\right)\left(\bar{d}_{\mathrm{L} u}^{d} C \bar{u}_{\mathrm{L} v}^{e}\right) \\
& \mathcal{Y}[\overline{p p r}] \epsilon_{a b e}\left(\bar{d}_{\mathrm{R} r}^{b} \nu_{\mathrm{L} t}\right)\left(\bar{d}_{\mathrm{R} p}^{a} d_{\mathrm{L} s c}\right)\left(\bar{d}_{\mathrm{L} u}^{c} C \bar{u}_{\mathrm{L} v}^{e}\right)
\end{aligned}
$$

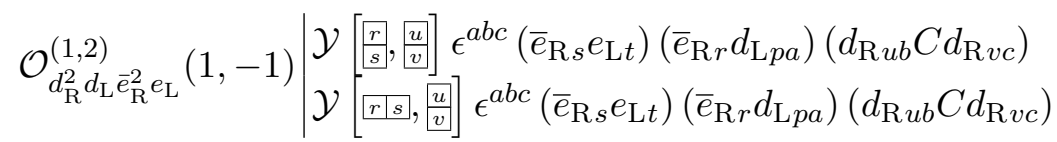




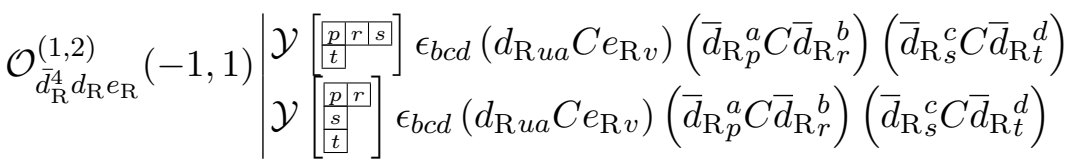

$$
\begin{aligned}
& \mid \mathcal{Y}\left[{ }_{[p|r| s]} \epsilon_{a c e}\left(\bar{d}_{\mathrm{R} s}^{c} e_{\mathrm{L} t}\right)\left(\bar{d}_{\mathrm{R} p}^{a} C \bar{d}_{\mathrm{R}}^{b}\right)\left(\bar{u}_{\mathrm{L} v}^{e} u_{\mathrm{R} u b}\right)\right.
\end{aligned}
$$

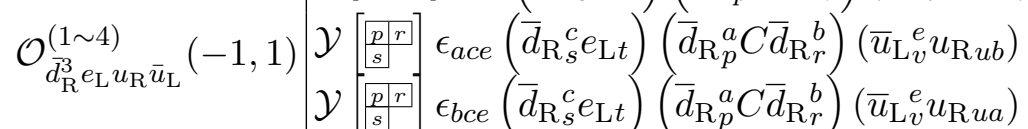

$$
\begin{aligned}
& \mathcal{Y}\left[\begin{array}{c}
p \\
\frac{p}{s} \\
s
\end{array}\right] \epsilon_{a c e}\left(\bar{d}_{\mathrm{R} s}^{c} e_{\mathrm{L} t}\right)\left(\bar{d}_{\mathrm{R} p}^{a} C \bar{d}_{\mathrm{R}}^{b}\right)\left(\bar{u}_{\mathrm{L} v}^{e} u_{\mathrm{R} u b}\right) \\
& \mathcal{Y}\left[[p|r| s] \epsilon_{a c e}\left(\bar{d}_{\mathrm{R}}^{c} e_{\mathrm{L} t}\right)\left(\bar{d}_{\mathrm{R} p}^{a} C \bar{d}_{\mathrm{R} r}^{b}\right)\left(\bar{d}_{\mathrm{L} v}^{e} d_{\mathrm{R} u b}\right)\right.
\end{aligned}
$$

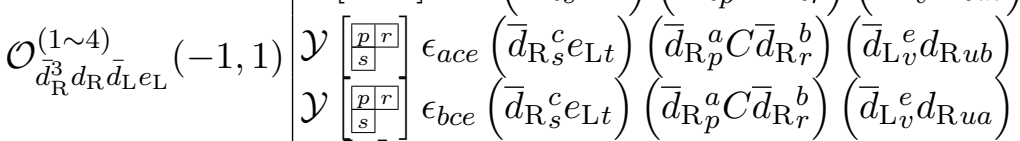

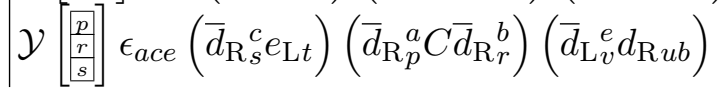

$$
\begin{aligned}
& \mathcal{O}_{\bar{d}_{\mathrm{R}}^{3} e_{\mathrm{R}} e_{\mathrm{L}} \bar{e}_{\mathrm{L}}}(-1,1) \mid \mathcal{Y}\left[\frac{p r}{s}\right] \epsilon_{a b c}\left(\bar{e}_{\mathrm{L} v} e_{\mathrm{R} u}\right)\left(\bar{d}_{\mathrm{R}}^{c}{ }_{s}^{c} e_{\mathrm{L} t}\right)\left(\bar{d}_{\mathrm{R} p}^{a} C \bar{d}_{\mathrm{R}}^{b}\right) \\
& \mathcal{O}_{\bar{d}_{\mathrm{R}}^{3} \bar{e}_{\mathrm{R}} e_{\mathrm{R}}^{2}}(-1,1) \mid \mathcal{Y}\left[\frac{p p r}{s}, u \mid v\right] \epsilon_{a b c}\left(e_{\mathrm{R} u} C e_{\mathrm{R} v}\right)\left(\bar{d}_{\mathrm{R}}^{a} C \bar{d}_{\mathrm{R} r}^{b}\right)\left(\bar{d}_{\mathrm{R}}^{c} C \bar{e}_{\mathrm{R} t}\right) \\
& \mathcal{Y}\left[{ }_{p|r| s}\right] \epsilon_{a c e}\left(\bar{d}_{\mathrm{R}}^{c} \nu_{\mathrm{L} t}\right)\left(\bar{d}_{\mathrm{R}}^{a}{ }_{p}^{a} \bar{d}_{\mathrm{R} r}^{b}\right)\left(\bar{u}_{\mathrm{L} v}^{e} d_{\mathrm{R} u b}\right)
\end{aligned}
$$

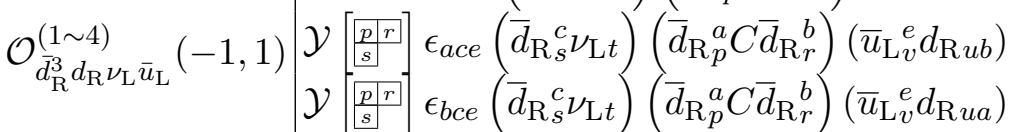

$$
\begin{aligned}
& \mathcal{Y}\left[\begin{array}{c}
\frac{p}{r} \\
\frac{r}{s}
\end{array}\right] \epsilon_{\text {ace }}\left(\bar{d}_{\mathrm{R}}^{c} \nu_{\mathrm{L} t}\right)\left(\bar{d}_{\mathrm{R} p}^{a} C \bar{d}_{\mathrm{R}}^{b}\right)\left(\bar{u}_{\mathrm{L} v}^{e} d_{\mathrm{R} u b}\right) \\
& \mathcal{O}_{\bar{d}_{\mathrm{R}}^{3} e_{\mathrm{R}} \nu_{\mathrm{L}} \bar{\nu}_{\mathrm{L}}}(-1,1) \mid \mathcal{Y}\left[\frac{p r r}{s}\right] \epsilon_{a b c}\left(\bar{\nu}_{\mathrm{L} v} e_{\mathrm{R} u}\right)\left(\bar{d}_{\mathrm{R}}^{c} \nu_{\mathrm{L} t}\right)\left(\bar{d}_{\mathrm{R}_{p}^{a} C \bar{d}_{\mathrm{R}}^{b}}^{b}\right)
\end{aligned}
$$

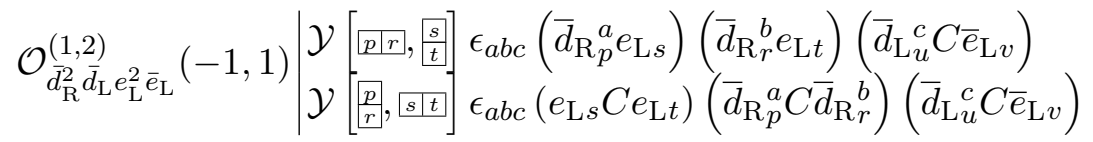

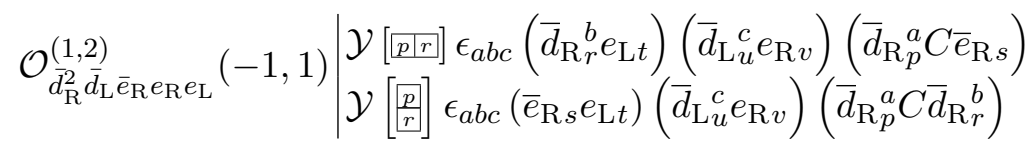

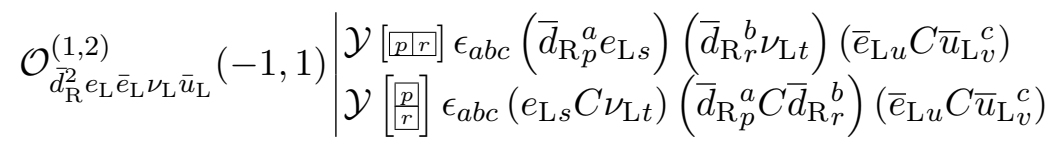

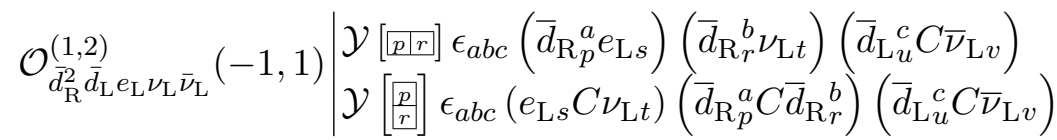

$$
\begin{aligned}
& \mathcal{O}_{\bar{d}_{\mathrm{R}}^{2} \bar{e}_{\mathrm{R}} e_{\mathrm{R}} \nu_{\mathrm{L}} \bar{u}_{\mathrm{L}}}^{(-1,1)} \mid \begin{array}{l}
\mathcal{Y}\left[\frac{p r r}{p}\right] \epsilon_{a b c}\left(\bar{d}_{\mathrm{R}}^{b} \nu_{\mathrm{L} t}\right)\left(\bar{u}_{\mathrm{L}}^{c} e_{v} e_{\mathrm{R} u}\right)\left(\bar{d}_{\mathrm{R} p}^{a} C \bar{e}_{\mathrm{R} s}\right) \\
\mathcal{Y}\left[\frac{p}{r}\right] \epsilon_{a b c}\left(\bar{e}_{\mathrm{R} s} \nu_{\mathrm{L} t}\right)\left(\bar{u}_{\mathrm{L} v}^{c} e_{\mathrm{R} u}\right)\left(\bar{d}_{\mathrm{R} p}^{a} C \bar{d}_{\mathrm{R} r}^{b}\right)
\end{array}
\end{aligned}
$$

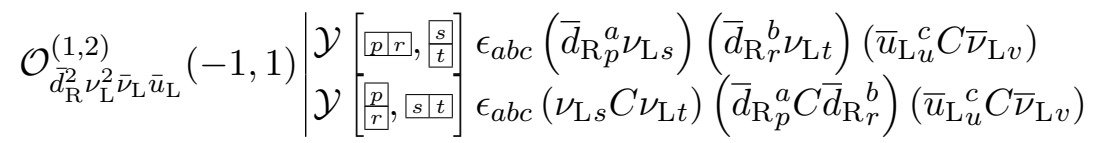




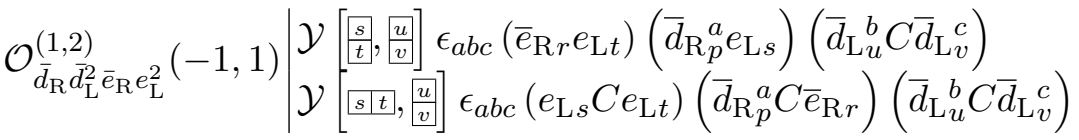

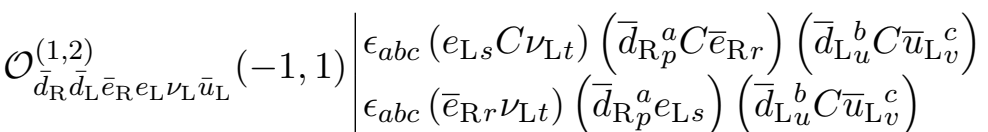

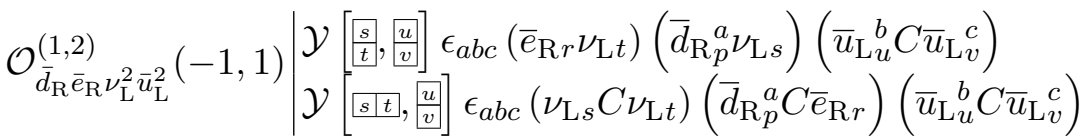

$(\Delta B, \Delta L)=( \pm 1, \pm 3)$

$$
\begin{aligned}
& \mathcal{O}_{e_{\mathrm{R}}^{2} \nu_{\mathrm{L}} u_{\mathrm{L}}^{3}}(1,3) \mid \mathcal{Y}\left[\frac{p r r}{s}, u \mid v\right] \epsilon^{a b c}\left(e_{\mathrm{R} u} C e_{\mathrm{R} v}\right)\left(u_{\mathrm{L} p a} C u_{\mathrm{L} r b}\right)\left(u_{\mathrm{L} s c} C \nu_{\mathrm{L} t}\right)
\end{aligned}
$$

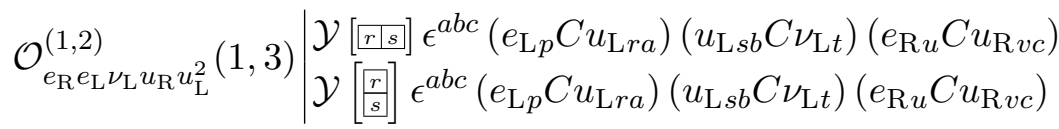

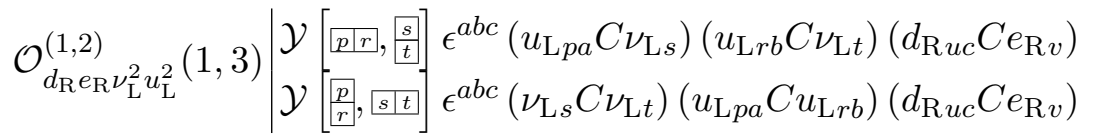

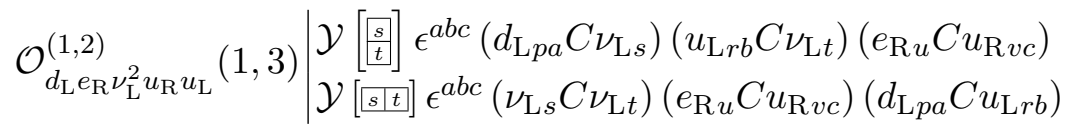

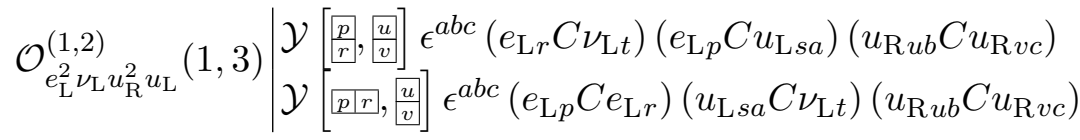

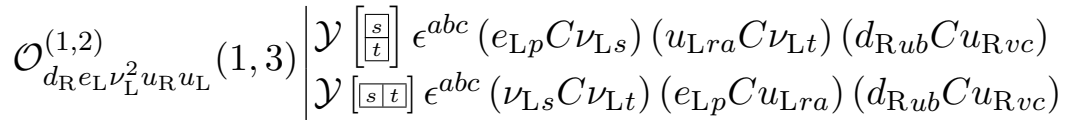

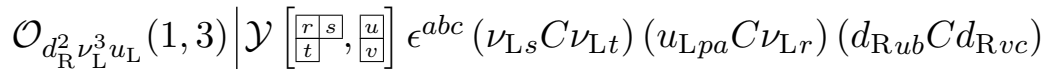

$$
\begin{aligned}
& \mathcal{O}_{\bar{e}_{\mathrm{R}} \bar{e}_{\mathrm{L}} \bar{\nu}_{\mathrm{L}} \bar{u}_{\mathrm{R}}^{3}}(-1,-3) \mid \mathcal{Y}\left[\frac{r s]}{t}\right] \epsilon_{a b c}\left(\bar{e}_{\mathrm{L} u} C \bar{\nu}_{\mathrm{L} v}\right)\left(\bar{e}_{\mathrm{R} p} C \bar{u}_{\mathrm{R}}^{a}\right)\left(\bar{u}_{\mathrm{R}}^{b} C \bar{u}_{\mathrm{R} t}^{c}\right)
\end{aligned}
$$

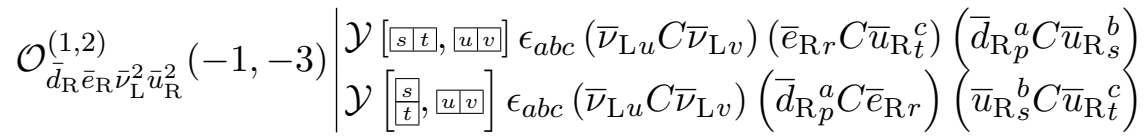

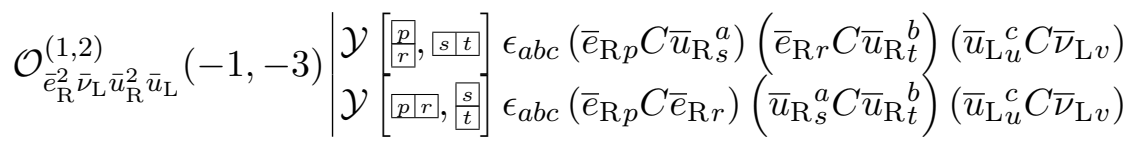

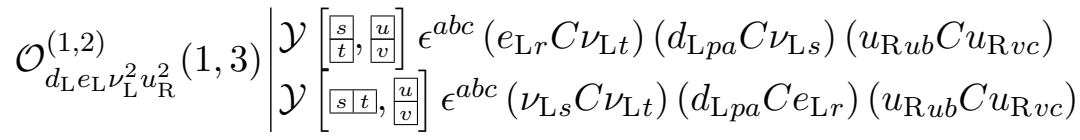

$$
\begin{aligned}
& \mathcal{O}_{d_{\mathrm{R}} d_{\mathrm{L}} \nu_{\mathrm{L}}^{3} u_{\mathrm{R}}}(1,3) \mid \mathcal{Y}\left[\frac{[r s}{t}\right] \epsilon^{a b c}\left(\nu_{\mathrm{L} s} C \nu_{\mathrm{L} t}\right)\left(d_{\mathrm{L} p a} C \nu_{\mathrm{L} r}\right)\left(d_{\mathrm{R} u b} C u_{\mathrm{R} v c}\right)
\end{aligned}
$$

$(\Delta B, \Delta L)=( \pm 1, \mp 3)$

$$
\begin{aligned}
& \mathcal{O}_{\bar{d}_{\mathrm{L}}^{2} \nu_{\mathrm{L}}^{3} \bar{u}_{\mathrm{R}}}(-1,3) \mid \mathcal{Y}\left[\begin{array}{c}
r s s \\
t \frac{u}{v}
\end{array}\right] \epsilon_{a b c}\left(\nu_{\mathrm{L} s} C \nu_{\mathrm{L} t}\right)\left(\bar{u}_{\mathrm{R} p}^{a} \nu_{\mathrm{L} r}\right)\left(\bar{d}_{\mathrm{L} u}^{b} C \bar{d}_{\mathrm{L} v}^{c}\right) \\
& \mathcal{O}_{d_{\mathrm{L}}^{3} \bar{e}_{\mathrm{R}} \bar{\nu}_{\mathrm{L}}^{2}}(1,-3) \mid \mathcal{Y}\left[\frac{p p r}{\underline{s}, u v]}\right] \epsilon^{a b c}\left(\bar{\nu}_{\mathrm{L} u} C \bar{\nu}_{\mathrm{L} v}\right)\left(\bar{e}_{\mathrm{R} t} d_{\mathrm{L} s c}\right)\left(d_{\mathrm{L} p a} C d_{\mathrm{L} r b}\right)
\end{aligned}
$$




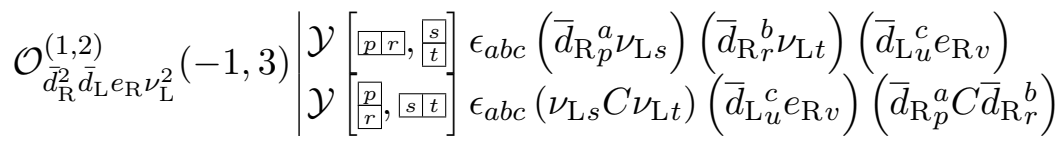

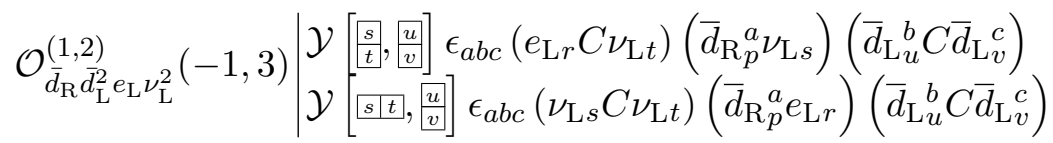

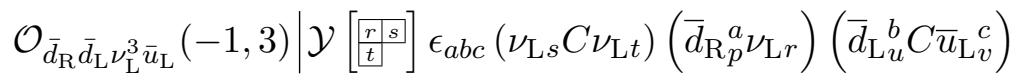

$(\Delta B, \Delta L)=( \pm 2,0)$

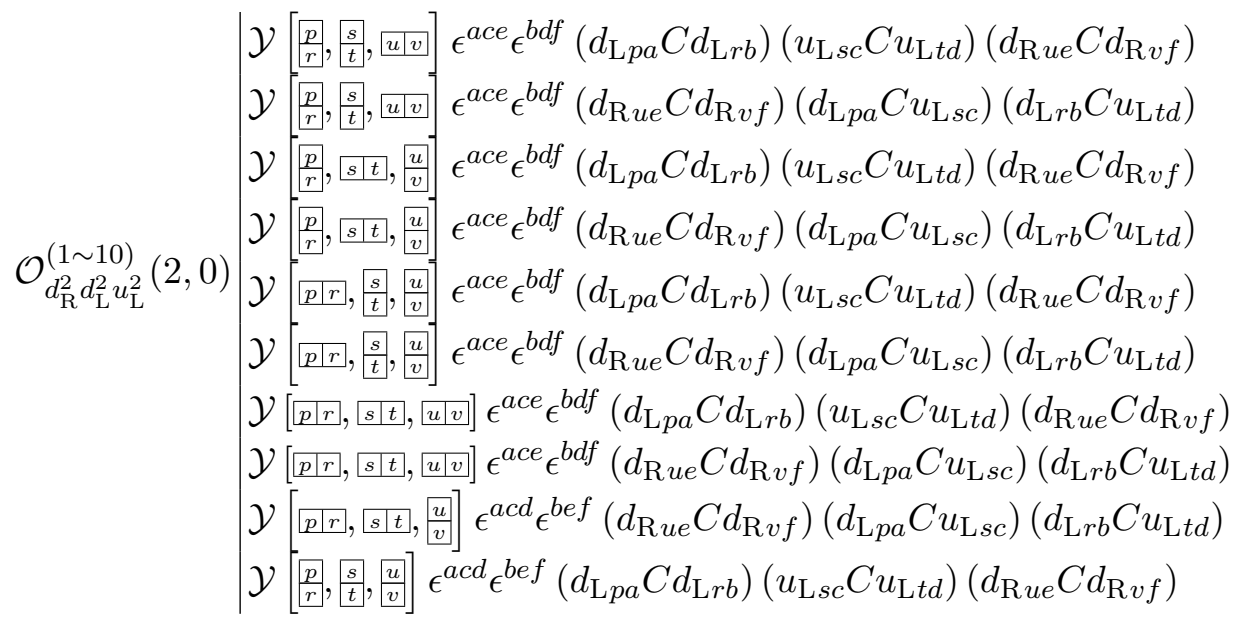

$$
\begin{aligned}
& \mathcal{Y}\left[[p|r| s] \epsilon^{a c e} \epsilon^{b d f}\left(d_{\mathrm{L} p a} C d_{\mathrm{L} r b}\right)\left(d_{\mathrm{L} s c} C u_{\mathrm{L} t d}\right)\left(d_{\mathrm{R} u e} C u_{\mathrm{R} v f}\right)\right. \\
& \mathcal{Y}\left[[p|r| s] \epsilon^{a c d} \epsilon^{b e f}\left(d_{\mathrm{L} p a} C d_{\mathrm{L} r b}\right)\left(d_{\mathrm{L} s c} C u_{\mathrm{L} t d}\right)\left(d_{\mathrm{R} u e} C u_{\mathrm{R} v f}\right)\right. \\
& \mathcal{O}_{d_{\mathrm{R}} d_{\mathrm{L}}^{3} u_{\mathrm{R}} u_{\mathrm{L}}}^{(1 \sim 7)^{\prime}}(2,0) \\
& \mathcal{Y}\left[\frac{p r}{s}\right] \epsilon^{a c e} \epsilon^{b d f}\left(d_{\mathrm{L} p a} C d_{\mathrm{L} r b}\right)\left(d_{\mathrm{L} s c} C u_{\mathrm{L} t d}\right)\left(d_{\mathrm{R} u e} C u_{\mathrm{R} v f}\right) \\
& \mathcal{Y} \frac{p r r}{s} \epsilon^{a c d} \epsilon^{b e f}\left(d_{\mathrm{L} p a} C d_{\mathrm{L} r b}\right)\left(d_{\mathrm{L} s c} C u_{\mathrm{L} t d}\right)\left(d_{\mathrm{R} u e} C u_{\mathrm{R} v f}\right) \\
& \mathcal{Y}\left[\frac{p r r}{s}\right] \epsilon^{a b c} \epsilon^{d e f}\left(d_{\mathrm{L} p a} C d_{\mathrm{L} r b}\right)\left(d_{\mathrm{L} s c} C u_{\mathrm{L} t d}\right)\left(d_{\mathrm{R} u e} C u_{\mathrm{R} v f}\right) \\
& \mathcal{Y} \frac{p}{\frac{p}{s}}\left[\epsilon^{a c e} \epsilon^{b d f}\left(d_{\mathrm{L} p a} C d_{\mathrm{L} r b}\right)\left(d_{\mathrm{L} s c} C u_{\mathrm{L} t d}\right)\left(d_{\mathrm{R} u e} C u_{\mathrm{R} v f}\right)\right. \\
& \mathcal{Y}\left[\begin{array}{c}
\frac{p}{r} \\
\frac{r}{s}
\end{array}\right] \epsilon^{a c d} \epsilon^{b e f}\left(d_{\mathrm{L} p a} C d_{\mathrm{L} r b}\right)\left(d_{\mathrm{L} s c} C u_{\mathrm{L} t d}\right)\left(d_{\mathrm{R} u e} C u_{\mathrm{R} v f}\right) \\
& \left.\mathcal{Y}\left[\frac{p}{r}, \frac{s}{t},, u v\right]\right] \epsilon_{a c e} \epsilon_{b d f}\left(\bar{d}_{\mathrm{R}}{ }_{p}^{a} C \bar{d}_{\mathrm{R}}{ }_{r}^{b}\right)\left(\bar{u}_{\mathrm{R}}{ }_{s}^{c} C \bar{u}_{\mathrm{R} t}^{d}\right)\left(\bar{d}_{\mathrm{L} u}^{e} C \bar{d}_{\mathrm{L}}^{f}\right) \\
& \mathcal{Y}\left[\frac{p}{r}, \frac{s}{t}, u[v], \epsilon_{a c e} \epsilon_{b d f}\left(\bar{d}_{\mathrm{L} u}^{e} C \bar{d}_{\mathrm{L} v}^{f}\right)\left(\bar{d}_{\mathrm{R} p}^{a} C \bar{u}_{\mathrm{R} s}^{c}\right)\left(\bar{d}_{\mathrm{R} r}^{b} C \bar{u}_{\mathrm{R} t}^{d}\right)\right. \\
& \mathcal{Y}\left[\frac{p}{r}, s \mid t,, \frac{u}{v}\right] \epsilon_{a c e} \epsilon_{b d f}\left(\bar{d}_{\mathrm{R}}{ }_{p}^{a} C \bar{d}_{\mathrm{R}}^{b}\right)\left(\bar{u}_{\mathrm{R}}{ }^{c} C \bar{u}_{\mathrm{R} t}^{d}\right)\left(\bar{d}_{\mathrm{L} u}^{e} C \bar{d}_{\mathrm{L}}^{f}\right)
\end{aligned}
$$

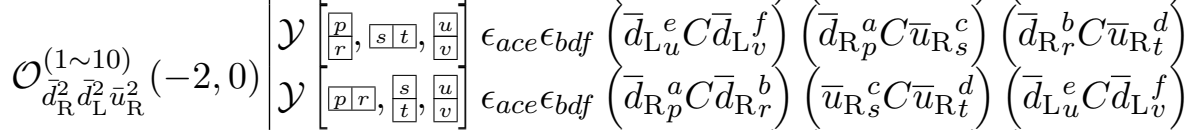

$$
\begin{aligned}
& \mathcal{Y}\left[\overline{p p r}, \frac{s}{t}, \frac{u}{v}\right] \epsilon_{a c e} \epsilon_{b d f}\left(\bar{d}_{\mathrm{L} u}^{e} C \bar{d}_{\mathrm{L} v}^{f}\right)\left(\bar{d}_{\mathrm{R} p}^{a} C \bar{u}_{\mathrm{R} s}^{c}\right)\left(\bar{d}_{\mathrm{R} r}^{b} C \bar{u}_{\mathrm{R} t}^{d}\right) \\
& \mathcal{Y}\left[\underline{p \mid r}, \underline{s \mid t}, \underline{u \mid v]} \epsilon_{a c e} \epsilon_{b d f}\left(\bar{d}_{\mathrm{R} p}^{a} C \bar{d}_{\mathrm{R} r}^{b}\right)\left(\bar{u}_{\mathrm{R}}^{c} C \bar{u}_{\mathrm{R} t}^{d}\right)\left(\bar{d}_{\mathrm{L} u}^{e} C \bar{d}_{\mathrm{L} v}^{f}\right)\right.
\end{aligned}
$$

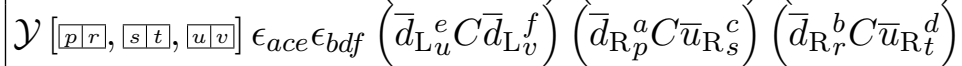

$$
\begin{aligned}
& \mathcal{Y}\left[\overline{p \mid r}, s \mid t,, \frac{u}{v}\right] \epsilon_{a b c} \epsilon_{d e f}\left(\bar{d}_{\mathrm{L} u}^{e} C \bar{d}_{\mathrm{L}}{ }^{f}\right)\left(\bar{d}_{\mathrm{R} p}^{a} C \bar{u}_{\mathrm{R} s}^{c}\right)\left(\bar{d}_{\mathrm{R}}{ }_{r}^{b} C \bar{u}_{\mathrm{R} t}{ }^{d}\right) \\
& \mathcal{Y}\left[\frac{p}{r}, \frac{s}{t},, \frac{u}{v}\right] \epsilon_{a b c} \epsilon_{d e f}\left(\bar{d}_{\mathrm{R} p}^{a} C \bar{d}_{\mathrm{R}}^{b}\right)\left(\bar{u}_{\mathrm{R}}^{c} C \bar{u}_{\mathrm{R}}^{d}\right)\left(\bar{d}_{\mathrm{L} u}^{e} C \bar{d}_{\mathrm{L} v}^{f}\right)
\end{aligned}
$$




$$
\begin{aligned}
& \mid \begin{array}{l}
\mathcal{Y}\left[\overline{p|r| s]} \epsilon_{a e f} \epsilon_{b c d}\left(\bar{d}_{\mathrm{R}}^{a} C \bar{d}_{\mathrm{R}_{r}^{b}}^{b}\right)\left(\bar{d}_{\mathrm{R}}^{c} C \bar{u}_{\mathrm{R} t}^{d}\right)\left(\bar{d}_{\mathrm{L}_{u}^{e}} C \bar{u}_{\mathrm{L}}^{f}\right)\right. \\
\mathcal{Y}[p|r| s] \epsilon_{a c f} \epsilon_{b d e}
\end{array}\left(\bar{d}_{\mathrm{R}_{p}^{a}} C \bar{d}_{\mathrm{R}_{r}^{b}}^{b}\right)\left(\bar{d}_{\mathrm{R}_{s}^{c} C \bar{u}_{\mathrm{R} t}^{d}}\right)\left(\begin{array}{l}
\bar{d}_{\mathrm{L} u}^{e} C \bar{u}_{\mathrm{L} v}^{f}
\end{array}\right)
\end{aligned}
$$

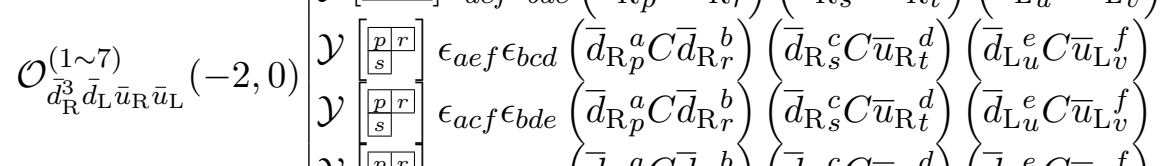

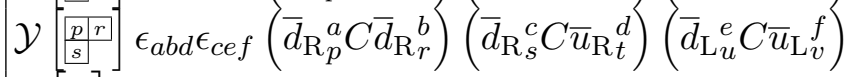

$$
\begin{aligned}
& \mathcal{Y}\left[\begin{array}{l}
\frac{p}{r} \\
\frac{r}{s}
\end{array}\right] \epsilon_{a e f} \epsilon_{b c d}\left(\bar{d}_{\mathrm{R}_{p}}^{a} C \bar{d}_{\mathrm{R}}{ }_{r}^{b}\right)\left(\bar{d}_{\mathrm{R}_{s}^{c}} C \bar{u}_{\mathrm{R}}{ }_{t}^{d}\right)\left(\bar{d}_{\mathrm{L}}{ }_{u}^{e} C \bar{u}_{\mathrm{L}}^{f}\right) \\
& \mathcal{Y}\left[\begin{array}{c}
\frac{p}{r} \\
s
\end{array}\right] \epsilon_{a c f} \epsilon_{b d e}\left(\bar{d}_{\mathrm{R} p}^{a} C \bar{d}_{\mathrm{R}}^{b}\right)\left(\bar{d}_{\mathrm{R}}{ }_{s}^{c} C \bar{u}_{\mathrm{R}}{ }_{t}^{d}\right)\left(\bar{d}_{\mathrm{L}}^{e}{ }_{u}^{e} C \bar{u}_{\mathrm{L}}^{f}\right)
\end{aligned}
$$

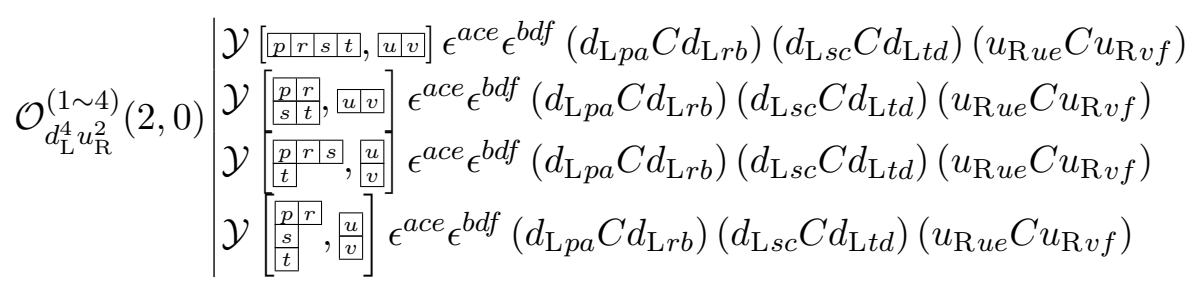

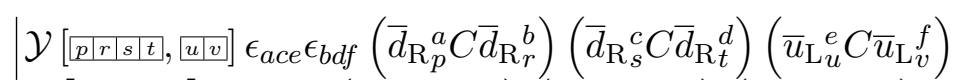

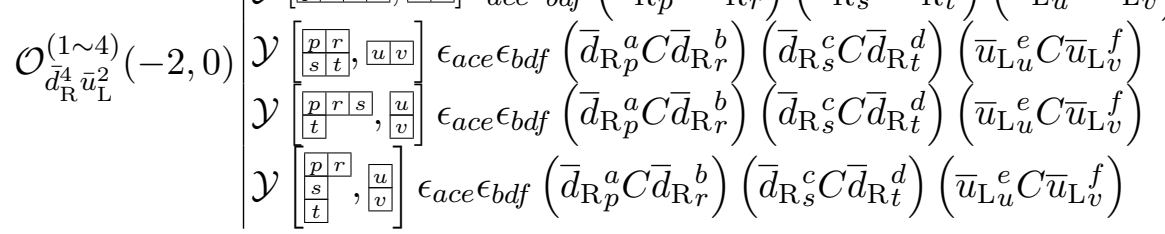

\section{Conclusion}

In this paper, we proved the validity of the on-shell method of operator enumeration $[1,2]$ for effective field theories including massive fermions. While the intuition is that fermion masses do not alter the possible form of operators, we also give a rigorous proof from the amplitude perspective. Hence we could apply the method to obtain the full operator basis at dimension 5 through 9 for LEFT, the effective field theory below the weak scale in the Standard Model where all fermions are massive. The completeness and independence of the basis are guaranteed by the method without subjective judgements.

At mass dimension 5, 6, 7, 8, 9, we conclude that there are 10, 118, 150, 756, 3686 independent operators respectively for one generation of fermions, and 76, 5875, 5118, 35058, 704584 flavor specified independent operators for three generation of fermions. The results of dimension 5 to 7 are consistent with previous existing results, with flavor symmetry specified. The total number of operators, as well as the number of operators with various lepton number violation $|\Delta L|$, are summarized for each dimension in table 2 and table 3 . In tables 4, 5, 6, 7, 8, we list the number of types (operators) for each baryon-number and lepton-number violation pattern with different kinds of flavors involved at different scales, ranging from the $\mathrm{MeV}$ scale to the bottom quark mass scale.

The low energy effective field theory has profound guiding significance, and a complete operator basis of it at high dimensions provides various phenomenological applications, especially for the exotic processes with baryon or lepton number violations. With the complete set of operator basis, it would be very interesting to see how these higher dimen- 


\begin{tabular}{|c|c|c|c|c|c|}
\hline \multicolumn{6}{|c|}{ Dim-5 operators } \\
\hline$(\Delta B, \Delta L)$ & $\left(n_{u}, n_{d}, n_{e}, n_{\nu}\right)$ & $(2,3,3,3)$ & $(2,2,3,3)$ & $(1,2,2,3)$ & $(1,1,1,3)$ \\
\hline$(0,0)$ & $10(70)$ & $10(50)$ & $10(28)$ & $10(10)$ \\
\hline$(0, \pm 2)$ & $2(6)$ & $2(6)$ & $2(6)$ & $2(6)$ \\
\hline
\end{tabular}

Table 4. The number of types (operators) of each baryon-number and lepton-number violation pattern with various flavors involved at dimension 5. $n_{u}, n_{d}, n_{e}$ and $n_{\nu}$ represent active number of up-type quarks, down-type quarks, charged leptons and neutrinos respectively. For $n_{u}=2$ and $n_{u}=1$, the corresponding active up-type quarks are $(c, u)$ and $(u)$ respectively, For $n_{d}=3,2,1$, active down-type quarks are $(b, s, d),(s, d)$ and $(d)$ respectively, $n_{e}=3,2,1$ corresponds active charged letpons $(\tau, \mu, e),(\mu, e)$ and $(e)$. The number of active neutrino are always set to 3 . The only type of $(0, \pm 2)$ at dimension 5 is $F_{L} \nu_{L}^{2}$, so the numbers of operators with different flavor numbers stay the same.

\begin{tabular}{|c|c|c|c|c|}
\hline \multicolumn{5}{|c|}{ Dim-6 operators } \\
\hline$(\Delta B, \Delta L)$ & $(2,3,3,3)$ & $(2,2,3,3)$ & $(1,2,2,3)$ & $(1,1,1,3)$ \\
\hline$(0,0)$ & $56(3631)$ & $56(2171)$ & $56(754)$ & $56(183)$ \\
\hline$(0, \pm 2)$ & $20(1200)$ & $20(870)$ & $20(390)$ & $20(120)$ \\
\hline$(0, \pm 4)$ & $2(12)$ & $2(12)$ & $2(12)$ & $2(12)$ \\
\hline$( \pm 1, \pm 1)$ & $18(576)$ & $18(324)$ & $14(86)$ & $12(20)$ \\
\hline$( \pm 1, \mp 1)$ & $14(456)$ & $14(156)$ & $14(86)$ & $4(12)$ \\
\hline
\end{tabular}

Table 5. The number of types (operators) of each baryon-number and lepton-number violation pattern with various flavor numbers at dimension 6. $n_{u}, n_{d}, n_{e}$ and $n_{\nu}$ represent active number of up-type quarks, down-type quarks, charged leptons and neutrinos respectively. For $n_{u}=2$ and $n_{u}=1$, the corresponding active up-type quarks are $(c, u)$ and $(u)$ respectively, For $n_{d}=3,2,1$, active down-type quarks are $(b, s, d),(s, d)$ and $(d)$ respectively, $n_{e}=3,2,1$ corresponds active charged letpons $(\tau, \mu, e),(\mu, e)$ and $(e)$.

sional operators are applied to various exotic processes and how the Wilson coefficients are constrained from current experimental data.

\section{Acknowledgments}

We thank Christopher Murphy point out an arithmetic error in counting numbers of the $F_{L}^{2} F_{R}{ }^{2}$ operators, although we list all the explicit operators (3.176)-(3.180) correctly which has been written in a systematical way. H.L.L. Z.R. and J.H.Y. were supported by the National Science Foundation of China (NSFC) under Grants No. 11875003 and No. 12022514. 


\begin{tabular}{|c|c|c|c|c|}
\hline \multicolumn{7}{|c|}{ Dim-7 operators } \\
\hline$(\Delta B, \Delta L)$ & $(2,3,3,3)$ & $(2,2,3,3)$ & $(1,2,2,3)$ & $(1,1,1,3)$ \\
\hline$(0,0)$ & $82(3168)$ & $82(1894)$ & $82(698)$ & $78(166)$ \\
\hline$(0, \pm 2)$ & $30(750)$ & $30(546)$ & $30(258)$ & $30(114)$ \\
\hline$( \pm 1, \pm 1)$ & $18(612)$ & $18(360)$ & $18(108)$ & $18(30)$ \\
\hline$( \pm 1, \mp 1)$ & $14(588)$ & $14(240)$ & $14(140)$ & $14(26)$ \\
\hline
\end{tabular}

Table 6. The number of types (operators) of each baryon-number and lepton-number violation pattern with various flavor numbers at dimension 7. $n_{u}, n_{d}, n_{e}$ and $n_{\nu}$ represent active number of up-type quarks, down-type quarks, charged leptons and neutrinos respectively. For $n_{u}=2$ and $n_{u}=1$, the corresponding active up-type quarks are $(c, u)$ and $(u)$ respectively, For $n_{d}=3,2,1$, active down-type quarks are $(b, s, d),(s, d)$ and $(d)$ respectively, $n_{e}=3,2,1$ corresponds active charged letpons $(\tau, \mu, e),(\mu, e)$ and $(e)$.

\begin{tabular}{|c|c|c|c|c|}
\hline \multicolumn{5}{|c|}{ Dim-8 operators } \\
\hline$(\Delta B, \Delta L) \quad\left(n_{u}, n_{d}, n_{e}, n_{\nu}\right)$ & $(2,3,3,3)$ & $(2,2,3,3)$ & $(1,2,2,3)$ & $(1,1,1,3)$ \\
\hline$(0,0)$ & $281(21144)$ & $281(11872)$ & $277(4140)$ & $267(954)$ \\
\hline$(0, \pm 2)$ & $78(5442)$ & $78(3810)$ & 78 (1668) & $78(516)$ \\
\hline$(0, \pm 4)$ & $4(48)$ & $4(48)$ & $4(48)$ & $4(48)$ \\
\hline$( \pm 1, \pm 1)$ & $78(4536)$ & $78(2592)$ & $74(720)$ & $72(180)$ \\
\hline$( \pm 1, \mp 1)$ & $58(3888)$ & $58(1440)$ & $58(816)$ & $48(132)$ \\
\hline
\end{tabular}

Table 7. The number of types (operators) of each baryon-number and lepton-number violation pattern with various flavor numbers at dimension 8. $n_{u}, n_{d}, n_{e}$ and $n_{\nu}$ represent active number of up-type quarks, down-type quarks, charged leptons and neutrinos respectively. For $n_{u}=2$ and $n_{u}=1$, the corresponding active up-type quarks are $(c, u)$ and $(u)$ respectively, For $n_{d}=3,2,1$, active down-type quarks are $(b, s, d),(s, d)$ and $(d)$ respectively, $n_{e}=3,2,1$ corresponds active charged letpons $(\tau, \mu, e),(\mu, e)$ and $(e)$.

M.L.X. is supported by the National Natural Science Foundation of China (NSFC) under grant No.2019M650856 and the 2019 International Postdoctoral Exchange Fellowship Program. JHY was also supported by the National Science Foundation of China (NSFC) under Grants No. 11947302. 


\begin{tabular}{|c|c|c|c|c|}
\hline \multicolumn{5}{|c|}{ Dim-9 operators } \\
\hline$(\Delta B, \Delta L) \quad\left(n_{u}, n_{d}, n_{e}, n_{\nu}\right)$ & $(2,3,3,3)$ & $(2,2,3,3)$ & $(1,2,2,3)$ & $(1,1,1,3)$ \\
\hline$(0,0)$ & $640(318062)$ & $640(144230)$ & $640(33046)$ & $634(4546)$ \\
\hline$(0, \pm 2)$ & $334(143808)$ & $334(75996)$ & $334(19964)$ & $334(3718)$ \\
\hline$(0, \pm 4)$ & $20(2136)$ & $20(1494)$ & $20(598)$ & $20(172)$ \\
\hline$(0, \pm 6)$ & $2(2)$ & $2(2)$ & $2(2)$ & $2(2)$ \\
\hline$( \pm 1, \pm 1)$ & $290(121590)$ & $290(52344)$ & $280(9646)$ & $266(1294)$ \\
\hline$( \pm 1, \mp 1)$ & $230(106494)$ & $230(33816)$ & $228(10330)$ & $194(1010)$ \\
\hline$( \pm 1, \pm 3)$ & $30(4746)$ & $30(3360)$ & $22(498)$ & $16(118)$ \\
\hline$( \pm 1, \mp 3)$ & $14(2730)$ & $14(840)$ & $14(510)$ & $8(46)$ \\
\hline$( \pm 2,0)$ & $16(5016)$ & $16(1064)$ & $16(288)$ & $16(28)$ \\
\hline
\end{tabular}

Table 8. The number of types (operators) of each baryon-number and lepton-number violation pattern with various flavor numbers at dimension 9. $n_{u}, n_{d}, n_{e}$ and $n_{\nu}$ represent active number of up-type quarks, down-type quarks, charged leptons and neutrinos respectively. For $n_{u}=2$ and $n_{u}=1$, the corresponding active up-type quarks are $(c, u)$ and $(u)$ respectively, For $n_{d}=3,2,1$, active down-type quarks are $(b, s, d),(s, d)$ and $(d)$ respectively, $n_{e}=3,2,1$ corresponds active charged letpons $(\tau, \mu, e),(\mu, e)$ and $(e)$.

Open Access. This article is distributed under the terms of the Creative Commons Attribution License (CC-BY 4.0), which permits any use, distribution and reproduction in any medium, provided the original author(s) and source are credited.

\section{References}

[1] H.-L. Li, Z. Ren, J. Shu, M.-L. Xiao, J.-H. Yu and Y.-H. Zheng, Complete Set of Dimension-8 Operators in the Standard Model Effective Field Theory, arXiv:2005.00008 [INSPIRE].

[2] H.-L. Li, Z. Ren, M.-L. Xiao, J.-H. Yu and Y.-H. Zheng, Complete Set of Dimension-9 Operators in the Standard Model Effective Field Theory, arXiv:2007.07899 [INSPIRE].

[3] W. Buchmüller and D. Wyler, Effective Lagrangian Analysis of New Interactions and Flavor Conservation, Nucl. Phys. B 268 (1986) 621 [INSPIRE].

[4] B. Grzadkowski, M. Iskrzynski, M. Misiak and J. Rosiek, Dimension-Six Terms in the Standard Model Lagrangian, JHEP 10 (2010) 085 [arXiv: 1008.4884] [INSPIRE].

[5] L. Lehman, Extending the Standard Model Effective Field Theory with the Complete Set of Dimension-7 Operators, Phys. Rev. D 90 (2014) 125023 [arXiv:1410.4193] [InSPIRE].

[6] C.W. Murphy, Dimension-8 operators in the Standard Model Eective Field Theory, JHEP 10 (2020) 174 [arXiv : 2005.00059] [inSPIRE]. 
[7] Y. Liao and X.-D. Ma, An explicit construction of the dimension-9 operator basis in the standard model effective field theory, JHEP 11 (2020) 152 [arXiv:2007.08125] [INSPIRE].

[8] J.F. Donoghue, E. Golowich and B.R. Holstein, Dynamics of the standard model, vol. 2, Cambridge University Press (2014) [DOI] [INSPIRE].

[9] S. Ando et al., Neutron beta decay in effective field theory, Phys. Lett. B 595 (2004) 250 [nucl-th/0402100] [INSPIRE].

[10] A. Falkowski, M. González-Alonso and O. Naviliat-Cuncic, Comprehensive analysis of beta decays within and beyond the Standard Model, JHEP 04 (2021) 126 [arXiv:2010.13797] [INSPIRE].

[11] A. Crivellin, S. Davidson, G.M. Pruna and A. Signer, Renormalisation-group improved analysis of $\mu \rightarrow e$ processes in a systematic effective-field-theory approach, JHEP 05 (2017) 117 [arXiv: 1702.03020] [INSPIRE].

[12] J. Aebischer, M. Fael, C. Greub and J. Virto, B physics Beyond the Standard Model at One Loop: Complete Renormalization Group Evolution below the Electroweak Scale, JHEP 09 (2017) 158 [arXiv: 1704.06639] [inSPIRE].

[13] M. González-Alonso and J. Martin Camalich, Global Effective-Field-Theory analysis of New-Physics effects in (semi)leptonic kaon decays, JHEP 12 (2016) 052 [arXiv:1605.07114] [INSPIRE].

[14] G. Prezeau, M. Ramsey-Musolf and P. Vogel, Neutrinoless double beta decay and effective field theory, Phys. Rev. D 68 (2003) 034016 [hep-ph/0303205] [INSPIRE].

[15] V. Cirigliano, W. Dekens, J. de Vries, M.L. Graesser and E. Mereghetti, A neutrinoless double beta decay master formula from effective field theory, JHEP 12 (2018) 097 [arXiv: 1806.02780] [INSPIRE].

[16] R.N. Mohapatra and R.E. Marshak, Local B-L Symmetry of Electroweak Interactions, Majorana Neutrinos and Neutron Oscillations, Phys. Rev. Lett. 44 (1980) 1316 [Erratum ibid. 44 (1980) 1643] [INSPIRE].

[17] S. Rao and R. Shrock, $n \leftrightarrow \bar{n}$ Transition Operators and Their Matrix Elements in the MIT Bag Model, Phys. Lett. B 116 (1982) 238 [inSPIRE].

[18] R.N. Mohapatra, Neutron-Anti-Neutron Oscillation: Theory and Phenomenology, J. Phys. G 36 (2009) 104006 [arXiv:0902.0834] [InSPIRE].

[19] D.G. Phillips II et al., Neutron-Antineutron Oscillations: Theoretical Status and Experimental Prospects, Phys. Rept. 612 (2016) 1 [arXiv:1410.1100] [INSPIRE].

[20] F. Wilczek and A. Zee, Operator Analysis of Nucleon Decay, Phys. Rev. Lett. 43 (1979) 1571 [INSPIRE].

[21] L.F. Abbott and M.B. Wise, The Effective Hamiltonian for Nucleon Decay, Phys. Rev. D 22 (1980) 2208 [INSPIRE].

[22] S. Girmohanta and R. Shrock, Improved Upper Limits on Baryon-Number Violating Dinucleon Decays to Dileptons, Phys. Lett. B 803 (2020) 135296 [arXiv:1910.08356] [INSPIRE].

[23] J. Engel, M.J. Ramsey-Musolf and U. van Kolck, Electric Dipole Moments of Nucleons, Nuclei, and Atoms: The Standard Model and Beyond, Prog. Part. Nucl. Phys. 71 (2013) 21 [arXiv:1303.2371] [INSPIRE]. 
[24] D. Chang, T.W. Kephart, W.-Y. Keung and T.C. Yuan, An Effective field theory for the neutron electric dipole moment, Nucl. Phys. B 384 (1992) 147 [INSPIRE].

[25] J. Heeck and V. Takhistov, Inclusive Nucleon Decay Searches as a Frontier of Baryon Number Violation, Phys. Rev. D 101 (2020) 015005 [arXiv:1910.07647] [InSPIRE].

[26] E.E. Jenkins, A.V. Manohar and P. Stoffer, Low-Energy Effective Field Theory below the Electroweak Scale: Operators and Matching, JHEP 03 (2018) 016 [arXiv:1709.04486] [INSPIRE].

[27] E.E. Jenkins, A.V. Manohar and P. Stoffer, Low-Energy Effective Field Theory below the Electroweak Scale: Anomalous Dimensions, JHEP 01 (2018) 084 [arXiv:1711.05270] [INSPIRE].

[28] Y. Liao, X.-D. Ma and Q.-Y. Wang, Extending low energy effective field theory with a complete set of dimension-7 operators, JHEP 08 (2020) 162 [arXiv:2005.08013] [INSPIRE].

[29] T. Ma, J. Shu and M.-L. Xiao, Standard Model Effective Field Theory from On-shell Amplitudes, arXiv:1902.06752 [INSPIRE].

[30] E. Conde and A. Marzolla, Lorentz Constraints on Massive Three-Point Amplitudes, JHEP 09 (2016) 041 [arXiv: 1601.08113] [INSPIRE].

[31] N. Arkani-Hamed, T.-C. Huang and Y.-t. Huang, Scattering Amplitudes For All Masses and Spins, arXiv:1709.04891 [INSPIRE].

[32] B. Henning and T. Melia, Constructing effective field theories via their harmonics, Phys. Rev. D 100 (2019) 016015 [arXiv: 1902.06754] [INSPIRE].

[33] G. Durieux, T. Kitahara, C.S. Machado, Y. Shadmi and Y. Weiss, Constructing massive on-shell contact terms, JHEP 12 (2020) 175 [arXiv: 2008.09652] [INSPIRE]. 